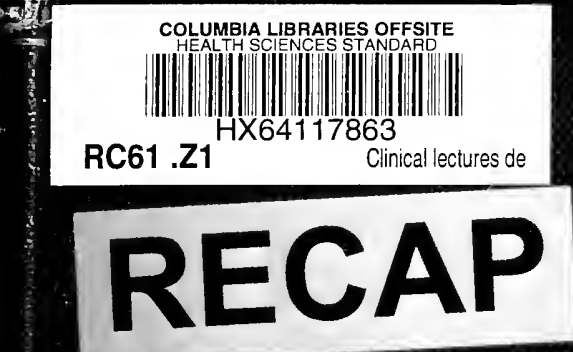




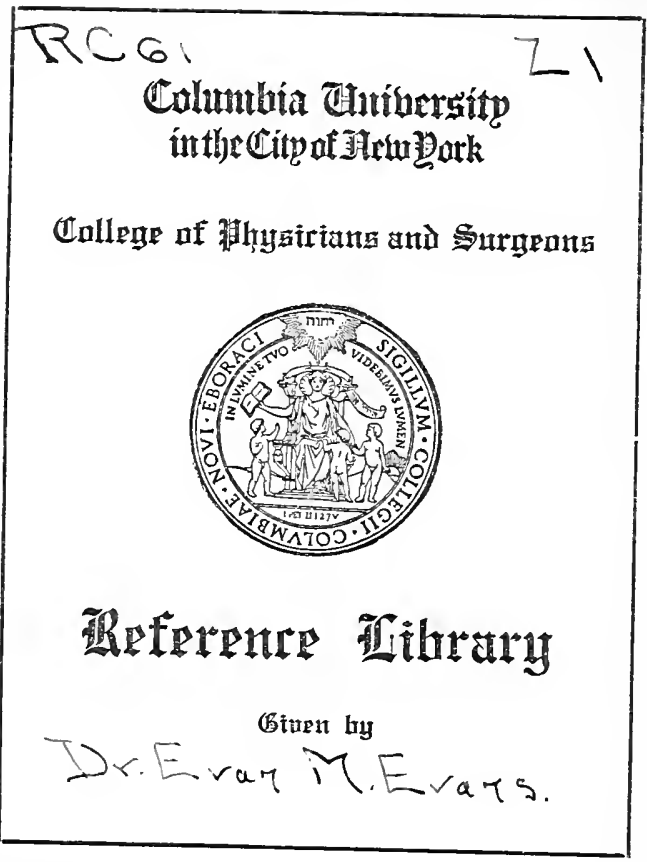




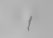

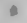






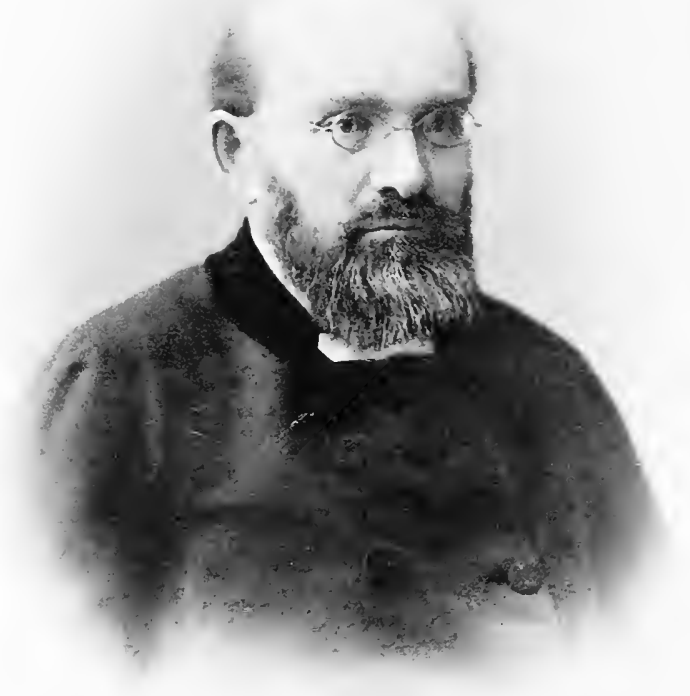

G. A. ZACHARIN. 


\title{
CLINICAL LECTURES
}

DELIVERED BEFORE THE STUDENTS

OF THE

\section{IMPERIAL MOSCOW UNIVERSITY}

\author{
BY \\ G. A. ZACHARIN, M.D.
}

Late Professor (Emeritus) of Clinical Medicine and Director of the Therapeutical

FaCulty Clinic of the IMPERIal MOSCOW UNIVERSity, HoNORARY MEMBER OF the Imperial ACAdemy of SCIENCES at St. Petersburg

TRANSLATED FROM THE FIFTH RUSSIAN EDITION

$\mathrm{BY}$

ALEXANDER ROVINSKY, M.D.

MeMber of the Massachtsetts Medical Society

BOSTON

DAMREL \& UPHAM

1899 
Digitized by the Internet Archive in 2010 with funding from Open Knowledge Commons 


\section{PREFACE.}

IT is the purpose of these lectures to serve, as far as it is possible to do so in print, the same aims as are pursued by clinical instruction, namely: by proceeding from the picture of the morbid condition as it presents itself in the patient and as obtained by interrogation, physical examination and from the collected data concerning his past, to diagnosticate this morbid state. in whatever peculiar and complex form it may appear, to prescribe a plan of treatment for the same, and to execute this plan before and with the participation of the students. In relation to these last and to the practising physician, the clinical lecture is also intended to serve as a specimen of methodical practical activity, "teaching the processes of interrogation. diagnosis, treatment and observation of the given disease $\left({ }^{\prime}\right.$ of the given class of diseases." Aiming first of all to make of his students practical phrsiciaus, the clinical instructor should offer to the future practitioners only what is essential. preëminently practical and absolutely requisite: methods of doubtful utility, whether of diagnosis or treatment, unnecessary superfluity of details and supposititious theorizing, have no place in the teaching of the true, conscientious clinician. The printed clinical lecture is distinguished by the same characteristics.

The following reasons induced the author to devote to the first case a much greater number of pages (35-120) than to any other: being the first case presented, it required ipso facto a detailed circumstantial analysis - as regards both diagnosis and treatment-and observation to the very end; it was. moreover, a complex case. presenting a combination of various morbid conditions; further, the space was largely devoted to the mineral waters, a subject of great importance in thera- 
peutics, which is unfortunately very little appreciated or even entirely neglected in the United States. The reader will find these reasons more fully explained by the author in his "Introduction to Clinical Exercises."

I have followed the author's original intention (as explained by him in the preface to the Russian edition) of omitting to designate at the head of each lecture the particular diagnosis of the disease under discussion. This is but in conformity with the general character of the work, which aims to be as like a lecture, or rather a demonstration, in the clinic, as possible. Says the author in his preface: "The clinical instructor, on presenting a patient before his class, does not announce the diagnosis of the morbid affection at once, but gradually unfolds it, as it were, in an evolutionary manner. If he were to announce the diagnosis at the beginning, and then proceed to examine the patient, he would only be proving a known proposition, instead of finding the unknown; whereas his students, as future physicians, will always have to find the unknown, which will demand solution. . . Moreover, to announce the diagnosis beforehand, thus giving the solution of the problem, would deprive the student of that mental stimulus, which spurs him on to seek for the solution." For the convenience of the reader I have marked the diagnosis of the cases in the list of contents, and have compiled an index of the same.

As regards the method of examination adopted by the author, the reader will find a detailed description of it in the author's Introduction. It is only necessary for me to state briefly the following: with the great progress of recent years in all the domains of the healing art, the various and rapidly increasing methods of what is known as physical examination have drivel farther and farther into the background the individuality of the patient himself; we are threatened with the approach of a period in medicine. when diseases will be treated per se. as such. without any regard to the characteristic peculiarities of the patient afflicted by them. It is a well-known fact. that one and the same disease will take on different forms in different individuals. all departing more 
or less from the type, though similar to it in the essentials. It is, therefore, necessary to become acquainted with the personality of the patient as thoroughly as possible, or, in other words, we must individualize each and every case. A systematic examination by inquiry, i. e. interrogation conducted according to a certain method, is the only means that will enable us to obtain a true and reliable insight into the characteristic peculiarities of the patient, which cannot be obtained by any means offered to us by the so-called physical methods of examination. This will frequently enable us to distinctly fix the ætiology of the disease (by interrogating the patient on his condition and mode of living), and greatly assist in arriving at a general as well as a minutely particular diagnosis; it will often also supply us with valuable material for treatment. This method of interrogation, which. as fully worked out by the author in his Introduction, was highly praised (and also adopted by many prominent clinical teachers) by the wellknown French clinician Henri Huchard, * is by no means a mechanical putting together of various facts, out of the summary of which a sort of mathematical deduction is made forming the diagnosis; it is, on the contrary, "an active. searching condition of mind": for while the facts are being collected, they are at the same time classified in the interrogator's mind, and the diagnosis is being evolved. so that by the time the data are fully collected, the diagnosis either formulates itself in the mind, or is easily formulated. It is true that the process of interrogating the patient will be in the beginning somewhat slow and laborious; however, once acquired, the practitioner will need but little time for interrogating his patient in as thorough a manner as the case may require. He will also soon be able to distinguish from among the data communicated to him by the patient, the necessary from the superfluous ones. There are, moreover, cases, as those of neurasthenia, hysteria. and the like, in which interrogation is the only means by the aid of which we may hope

* He calls the chief characteristic of Zacharin's school " l'interrogatoire, qui estélexé ì l'hauteur d'un art" (Exposé de l'Enseignement clinique, par Zakharine, Introduction par Henri Huchard. p. 3. Paris, 1891). 
to reach any diagnostic conclusions, for in these affections physical methods of examination play but a subordinate rôle. It is at the same time self-understood that we must avoid extremes in either relying altogether on interrogation to the neglect of physical methods of examination, or in allowing these last to entirely overshadow or exclude the interrogatory examination; a judicious combination of the two is a sine qua non of a successful diagnosis and treatment.

The author of these lectures, Gregory Antonovitch Zacharin, was connected, in the capacity of instructor and professor, with the Medical Department of the Imperial Moscow University for a period extending over thirty-five years (1861-1896).* A man of indomitable energy and very pronounced individuality, he is considered as the founder of what is known in the Russian medical world as the Zacharin school of practical medicine (we touched above on some of the prominent characteristics of this school), in whose tenets were brought up several generations of physicians scattered in every nook and corner of the Great Empire. He came of a poor, but very old family of nobility, and was compelled from his early studentyears to struggle with want and privation; but thanks to the possession of an iron will and unswerving determination, he rose to a position equal to that oceupied by Botkin and Pirogoff, the greatest medical minds of Russia. It may be truly said that Pirogoff, Botkin and Zacharin form the great triad of Russian clinical medicine of the nineteenth century.

Zacharin's most pronounced characteristic trait was probably his independence. "The physician should be independent, even more so than the poet or the artist, for to him we entrust our most valuable possession, namely, our health, our very life" ; and for the practical realization of this ideal he struggled unremittingly his whole life. Being a leader of men, his stand has greatly contributed to the elevation of the name and the position of the medical profession in Russia. To form an ap-

* His death, which occurred January 5, 1898, just when this translation was being completed, delayed its appearance until now. 
proximate idea of those obstacles which Zacharin had to overcome in establishing for himself and for his calling an independent, respected social position, it will suffice to introduce here the following few sentences from the address delivered by Prof. Sneguireff at the Zacharin Memorial Meeting of the Moscow Physio-Medical Society :

"We all remember the time when the physician was not allowed to sit down in the presence of his social superiors, how some of us were only allowed to treat serfs or servants, how others served on the gentlemen and the ladies, appearing every morning to inquire after their 'graces' ' health, for which they were paid by occasional gifts of flour, grain, fowls, etc.; a physician was considered as an accessory to the rich household. never as a necessity, and his labor, advice and time as trifling and superfluous."

Zacharin may justly be called a public hygienist, for he first of all insisted, and very rigidly at that, on the necessary changes in the patient's mode of life and surroundings, if any cure was to be obtained at all. In this regard his minute and precisely given instructions were not to be trifled with, and "Zacharin said so!" was a dictum not to be lightly disobeyed by his patients, whom he always took the trouble to personally instruct minutely in individual hygiene. As his personal practice was very extensive and his followers numerous, we can easily appreciate the influence of such instructions on the health of society at large.

He was a true therapist and a staunch believer in remedial agencies and their efficiency. It is said, that at his cleath-bed, when the question of consultation was being discussed (he died of acute bulbar paralysis caused by hæmorrhage into the medulla oblongata, which was early diagnosed by himself), he observed quietly, but firmly: "Why consultations? Have you not done all in your power? You have cleared the bowels, applied leeches and Spanish flies, and administered bromides; therapy has done everything ; the rest is beyond our power."

Although laconic, concise and precise in examining a patient, he would sometimes spend two or even three hours of closest scrutiny and inquiry, thus forming in his mind a per- 
fectly clear and distinct picture of the patient's condition and hiis surroundings, and thanks to his phenomenal memory this picture was stored up in his mental reservoir, to be sometimes called up in its entirety before the astonished patient after five or six years, thus inducing the latter to repose implicit confidence in his knowledge and skill. Besides being a great teacher (he was uninterruptedly professor at the University until 1896, when he was compelled to resign for reasons into which we cannot enter here), he was also instrumental in assisting the establishment of various special clinics, and especially the one in gynæcology.

He was the attendant physician to the late Emperor Alexander III in his fatal illness - acute interstitial nephritis.

Some time before his death Zacharin donated one quarter million dollars (one half million roubles) for the parochial schools in Russia.

I take here the opportunity to express my gratitude to Mme. E. P. Zacharin and to Prof. N. Th. Golouboff, of the Imperial Moscow Lniversity, whose valuable information and advice have greatly aided me in my work.

These lectures, which in the original passed through five editions in the period of a few years, were partly translated into both German (Klinische Abhandlungen: über den klinischen Unterricht, die Calomel-Therapie, etc. Von Prof. Dr. G. Sacharjin. Berlin, 1890, August Hirschwald; and Klinische Vorträge, 1892, same publisher) and French (L'Exposé de l'Enseignement clinique. par Zakharine, avec une Introduction par Henri Huchard. Paris, 1891, O. Doin, éditeur; and Leçons cliniques sur les Maladies abdominales, etc., traduit de la deuxième édition russe par le Dr. Oelsnitz (de Nice), 1893. Same publisher, also preface by $\mathrm{H}$. Huchard).

Alexander Rovinsky, M. D.

Boston, MAss., U.S.A., January, 1899. 


\section{CONTENTS.}

Preface, iii.

\section{INTRODUCTION TO CLINICAL EXERCISES.}

(THREE LECTURES.)

The importance of the clinic in medical education, 1 ; clinical exercises in their relation to medical education, 2 ; the necessary requisites of clinical instruction: a regular method and a system of individualization, 3 ; rarieties of clinics: conforming to the grades of medical studies, 3,4 ; by their contents; the general clinic, 4 ; the character of its content and its principal aims, 5, 6; the scope of clinical exercises. 6 : examination of the patient, 7 ; examination embraces status præsens and anamnesis, 8 ; status præsens consists of interrogation and objective examination, 8,9 ; interrogation about the patient's mode of living, 9,10 ; interrogation about patient's condition conducted according to an adopted plan, embracing: appetite and thirst, the tongue, teeth. etc., 10 ; circumstantial inquiry necessary regarding the condition of the evacuations, 11; the anus; the urinary blarlder, male genital organs; history of syphilis, and so on, 12-16; Anamnesis, also parents' health, 17; Objective examination, 17; avoid unnecessary minuteness and superfluity of details, 17,18 ; tact a requisite, 18 ; Diagnosis: how arrived at, 18; diagnosis of the disease (diagnosis morbi), of the patient (diagnosis ægri), and differential diagnosis (diagnosis per exclusionem), 19; Prognosis: its importance in the hospital as compared with that in private practice, 19; as regards the patient, 20, 21; as regards the patient's relatives, 21; conciseness in wording a prognosis: not serious. serious, dangerous and hopeless, 22 ; Treatment: method and a system of individualization necessary, 22; therapeutic nihilism and undue enthusiasm for certain drugs; neglect of hygienic treatment, 23; simultaneous employment of many drugs to be avoided, 24 ; characteristics of hospital therapy, 25; observations of the course of the malady, 26; the making up of deficiencies in the students' knowledge, 26: importance of autopsies, 27 ; the importance of the clinic in scientific medicine, 27,25 ; the duties of the clinical instructor: diseases taken up in order for clinical instruction, 23, 29: participation of students in the clinical exercises. 26, 30; duties of clinical assistants, 30 ; business of students: to conduct the history of the disease, and the description of the same; service in the clinic, 30, 31. 


\section{CLINICAL LECTURES.}

\section{ABDOMINAL DISEASES AND FUNCTIONAL DISTURBANCES OF THE NERVOUS SYSTEM. - THE INTERNAL USE OF MINERAL TATERS.}

Frrst CAse. - Chronic gastro-intestinal catarrh, biliary ealculi, renal colic, oxaluria, probably movable kidney, secondary nervous disturbances, etc., 35-121.

Anamnesis, 40, 41; Course of disease, 37-40, 45, 55, 66, 81, 97, 117, 120, 121; Diagnosis, 55-53, 60, 67-69, 70, 72; Diet, 37, 74, 75, 78, 81, 98; Hygiene, 43, 73, 74; Mole of living, 35, 36; Objective examination, 4549: Prognosis, 73; Treatment, 37, 45, 50-54, 67, 78-80, 82, 98, 117-120.

The internal use of mineral waters: sodium biearbonate and chloride in various eatarihal conditions, 86 ; the unreplaceability of the natural mineral waters by artificial ones, 87; the use of mineral waters at every season of the year, 83 ; the action of the curative agencies of the mineral waters: water, earbonic aeid, biearbonate and chloride of sodium, laxative salts, 90-92.

Table of mineral waters, 93; Ems, 94; Franzensbad, Kissingen, Karlsback, Viehy, 95; Yessentucki, Obersalzbrunnen, 96, 97; Marienbad, 95, 9. 101 ; compared to Ems, 101 ; compared to Viehy, 102 ; to Kissingen, 103; to Karlsbad, 102-104; Franzensbad Salzquelle, 104; indieations for Vichy, 104, 105; for Yessentucki, 105; Contrexéville, 97, 106.

The reliability of the combinations of remedial agencies, as contained in the mineral waters, 107; the routine method of drinking the waters at the resorts, 109,110 ; the quantity of tlie daily dose depending on the effect desired - Ems, Obersalzbrunnen, Vichy, 111, 112; Yessentucki and Karlsbad, 112, 113; Marienbar, Kissingen, 113; the length of the course of treatment, 113, 114; the distribution of the quantity of the water over the course of the day, 114; as regards meals, 114, 115; indications for cold and warm waters, 115 ; the diet while drinking the waters, 115,116 ; contraindications to the use of waters, $116,117$.

SEcoxu CAse. - Chponic gastric catarrh, gastric ulcer, nervous dyspepsia, dilatation of the stomach, 122-138.

Anamnesis, 12:, 124; Course of the disease, 125, 126, 138; Diagnosis, 127. 129; differential, 129; Diet, 124, 125, 138; Mode of living, 122, 12:3 ; Objective examination, 126, 127; Prognosis, 130 ; 'Treatmeut, 124. 130-132.

Gastric lavage: effects of, 132, 133; indications for, 133; not an indifferent proeedure, 133; mistaken employment of lavage, 134 ; ty pical cases in which it fails, 134, 135 ; wrongly applied, 136, 137.

Thirn Caś. - Nervous dyspepsia, 139-155.

Anamnesis, 139, 140 ; Course of disease, 140-142, 144, 154, 155; Diagnosis, 143; Diet, 142; Hygiene, 145, 146 : Mode of living, 139; Objective examination, 141; Prognosis, 144, 145; Treatment, 140, 142, $143,145-149,154,155$. 
Varieties of gastro-intestinal disorders in neurasthenia, 149; diet in nervous dyspepsia, 149, 150; treatment of constipation, 152, 154; douches, massage, etc., in nervous dyspepsia, 151; drugs, 151, 152 ; the various laxatives, 152, 153; clysters, 15:, 154 .

Fourth CAse. - Atrophic gastric catarrh, cancer of the duodenum, 156161.

Anamnesis and mode of living, 156, 157; Autopsy, 160; Course of disease, 157. 158; Diagnosis, 158; differential, 159, 160; Objective examination, 157; Treatment, 158, 160.

Fifth CAse. - Cancer of the pancreas, 162-165.

Anamnesis and mode of living, 162; Autopsy, 165 ; Course of disease. 163, 165 ; Diagnosis, differential, 163, 164; Prognosis, 164 ; Treatment, $163,164$.

Srxtr CAse.-Biliary calculi and cholangitis, 166-181.

Anamnesis and mode of living, 166, 167; Course of disease, 168, 175, 177, 178; Diagnosis, 169, 169, 171, 174; Objective examination, 168 ; Prognosis, 176; Treatment, 167, 176-181.

Biliary calculi : cause of formation, $169,170,176$; relation to neuralgia of the hepatic plexus, 171, 173, 174; differential diagnosis, 172, 17:3 ; symptoms aggravated at the beginning of treatment with Karlsbad, 175 ; duration of course of treatment, 179,180 .

Seventh Case. - Hypertrophic venous cirrlosis and cholangitis, 182-192.

Anamnesis and mode of living, 182, 183; Course of disease, 183, 184, 192 ; Diagnosis, 185. 186 ; differential, 187, 188, 190 : Objective examination, 18t-186; Prognosis, 191, 192 ; Treatment, 183, 184, 191, 192.

Hepatic diseases: differential diagnostication necessary, 187 ; nature of the various cirrhoses of the liver, 188,189 ; clinical data concerning same, 189, 190.

Eıgh⿻ Case. - Biliary caleuli, eholangitis and slight hypertrophic biliary cirrhosis, 193-196.

Anamnesis and mode of living, 193 ; Course of disease, 193, 194, 195; Diagnosis, 194; Objective examination, 194; Prognosis, 194; Treatment, 195. 196.

Ninth Case. - Echinococeus cyst of the liver, slight peritonitis, 197-201.

Anamnesis and mode of living, 197, 198; Course of disease, 198, 200 ; Diagnosis, 198, 199; Objective examination, 198; Prognosis, 199 : Treatment, 199, 200.

Tenth Case. - Syphilis of the liver, 202-205.

Anamnesis and mode of living, 202; Course of disease, 202, 203. 206, 207, 203; Diagnosis, 203, 204, 205; Objective examination, 203 : Prognosis, 205; 'Treatment, 205, 206, 208. 


\section{RHEUMATISM AND GOU'T.}

Éleventh CAse. - Acute articular rheumatism, 209-214.

Anammesis and mode of living, 207 ; Comrse of disease, 209, 210 , 211, 214; Diagnosis, 210; Prognosis, 210 ; Treatment, 209. 210, 211, 212.

Acute articular rheumatism an infectious disease, 212 ; its relation to gout, 213,214 .

Twelftr Case. - Polyarthritis chronica (chronic articular rhemmatism) and predisposition to gout, 215-224.

Anamnesis and mole of living, 215 ; Course of disease, 216, 221, 223 ; Diagnosis, 216, 217; Prognosis, 222 ; 'Treatment, 222, 223.

The inapplicability of the term chronic articular rheumatism, necessity for precise nomenclature, 217-219; nature of affections embraced under the eurrent name of chronic rheumatism, 219, 220; change of term recommended, 221 .

'Throtentir CASE. - Gont (arthritis urica), 224-230.

Anamnesis and mode of living, 224, 225; Course of disease, 225, $226,229,230$; Diagnosis 226; differential, 227: Prognosis, 227; Treatment, $225,220,227,228,229,230$.

Fourtentu Case. - Gout, nephrolithiasis, biliary caleuli, fatty liver, etc., $231-236$.

Anamnesis aud mode of living, 231, 232; Course of disease, 231, 232, 233, 234, 235, 236: Diagnosis, 233, 234, 235; Prognosis, 235; Treatment, 235,236 .

\section{DISEASES OF THE KIDNEYS.}

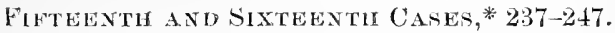

Fifteenth C'ase. - Chronie interstitial nephritis.

Anamnesis and mode of living, 236; Course of disease, 238, 247 : Diagnosis, 2tt, 2t5; Prognosis, 245; Treatment, 246.

sixteenth Case. - Parenehymatous nephritis.

Anamnesis and mode of living, 238, 239; Course of disease, 239, $240,2+1,24 \%$. 247 ; Diagnosis, 245; Prognosis, 245 ; Treatment, 239, 240,246 .

Nature of chronic nephritis, 242; symptoms of the interstitial as compared with the parenchymatous variety, 242,243 ; prognosis of either variety, 243,244 ; comse of, $243,244$.

\section{ANAMIA.}

SEVENTEEYTII CASE. - Chlorusis, 248-257.

Anamnesis and mole of living, 243; Course of disease, 249, 249: Diagnosis, 24?; differential, 249, 250; Prognosis, 253, 254: Treatment, $254-257$.

Firratum on p. 237 : Should be fifteenth and sixteenth cases, instead of sixteenth and serenteenth. 
Chlorosis: unsatisfactory classification of, in the text-books, 250 , 251; the nervous system in relation to chloros is, 251,252 ; sexual maturity in relation to chlorosis, 252: Brown-Sequard's theory, 252; hæmoglobin in chlorosis, 253; hysteria and chlorosis, 253; iron and arsenic in ehlorosis, 255,256 ; treatment of coustipation in chlorosis, 256 ; of the nervons system, 257.

Eighteenth Case. - Chloranæmia and ineipient pulmonary tuberculosis, 258-262.

Anamnesis aud mode of living. 258, 259; Course of disease, 259. 262; Diagnosis, 260, 261; Objective examination, 259: Proguosis, 261: Treatment, 261, 262.

\section{THORACIC AND INFECTIOUS DISEASES.}

Ninetenth Case. - Tuberculous pleurisy, 263-273.

Anamnesis and mode of living, 263, 264; Course of disease. 264, 270, 271, 272; Diagnosis, 265, 256: Objective examination, 264; Prognosis, 266; Treatment, 264, 270. 271, 272, 273.

Pleurisy: Eichhorst's treatment, 267; author's treatment, 267, 268 ; treatment of the iever with antipyretics and with calomel, 268 . 269 ; to further absurption of exudations, $269,270$.

Twexteth axd Twextr-First Cases. 27t-283.

Tuentieth Case. - Chronic pulnonary tuberculusis.

Anannesis and mode of living, 274, 275; Course of disease, 275, 276, 278, 281, 282; Diagnosis, 277, 278, 230, 231; Objective examination, 276; Prognosis, 276; Treatment, 275.

Twenty-first Case. - Chronic tuberular broncho-pneumonia and chronic bronchial catarrh.

Anamnesis and mode of living, 278, 279: Course of disease, 279, 230, 232, 283; Diagnosis, 282; Objective examination, 290; Prognosis. 289: Treatment, 279, 282. 28:3.

How the invasion of the lungs by tuberculosis takes place, 277 .

Genelat Reuares Concerning the Difryosis of Chronic TuberCUlosis of THE Lungs, 283-290. - Varieties of chronic tuberculosis and their complications, 284: pulmonary phthisis as compared with incipient tuberculosis, 285 ; the nature and cause of the hectic ferer, 255,286 ; pneumoniæ complicating chronic pulmonary tuberculosis: croupous, 286; catarrhal and caseous, 287; galloping phthisis. 287. 288: acute miliary tuberculosis, 288: peculiarities in the course of tuberculosis depending on the nature of the organs affected, 258. $2 \prec 9$; prognosis of tuberculosis pulmonum, 239,290 .

Treatuent of Chronic Puluonar Tuberculosis, 290-298.

Definition of a specific remedy, 291; symptomatic treatment: hæmoptysis, 291, 292; fever. 2.22 ; employment of koumyss, 292, 293; koumyss sanatoria, 294; cod-liver oil, keffir, ete., 294; arsenic, 295: creosote, guaiacol and guaiacol carbonate, 295, 296, 297; the last stage, 297; modern bacteriology in its relation to the treatment of tuberculosis. $297,298$. 
The Climatic Treathent of Curonic Pulgonar Tuberculosis. $298-316$.

Neglect of the subject, 293 ; climate in relation to climatic therapy , 298,299 ; the complexity of climatic influences, 299 ; Webber's classification of clinates. 299.

The Elements of Climate, 300-303. - The temperature, 300; sunlight, air, etc., 300-303.

Former climatic treatment of tuberculosis, 303; by residence in the mountains, 303, 304; the supposed curative agencies of high altitudes, 304; Brehmer and his sanatorium at Görbersdorf, 304, 305; his theories and their refutation; his success, 305, 303; Davos as compared with Görbersdorf and other sanatoria, 303, 307; change in the climatic treatment of tuberculosis, 307; Russia as compared with Western Europe as a resort for tubereulous patients, 309, 309; climatic treatment at home: cases illustratiug same, 309-315; cases for which trips are indicated, 316.

Twentr-second Case. - Croupous pneumonia, 317-324.

Anamnesis and mode of living, 317; Course of disease, 318, 323, 324; Diagnosis, 318; differential, 319; Objective examination, 318; Prognosis, 319: 'Treatment, 320, 323, 32t.

Treatment of high temperature in pneumonia, 320 , :321; of dyspnœa, pains and congh, $321,322,323$.

A Few Notes on ObJective Examixation, 325-331. - The importance of morbid symptoms in semiotics, as compared to those necessary for diagnosis, 325; their necessary differentiation, 326; the nature of the vesicular respiratory murmur, 327 ; of the bronchial respiratory murmur, 327 ; the indefinite respiratory murmur, rough breathing, 327 , $32 \mathrm{~S}$; varieties of râles, 828 , $: 20$; the percussion sound, 329 ; determination of dimensions of heart, liver and spleen, 329,330 ; the pulse, 330,331 .

\section{ELEMEN'TS OF BALNEOTHERAPY.}

Balneotinerapt for the. Practical Physician, 332-355.

Necessity for elinjeal individualization of bahneotherapy, 332, 333: the differences in the external and internal employment of mineral waters, 333 ; the effect of the temperature of the water, $335-338$; effects of the component parts of the waters: sodium chloride and carbonate, carbonic acid, sulphur, organic acids, etc.. 338, 339; the effect of mechanical influenees during the external employment of mineral waters, 339 ; the various classes of waters: 1 . the chemically indifferent, their effects and use (Schlangenbad, Ragatz. Gastein, ete.), 310; individualization necessary in their employment, 340 ; mineral moor, 341, 342; the influence of climate at the watering-places, 342 ; conditions of life there, $343 ; 2$. salt waters, their use and effects. (Kreutznach, Wiesbaclen, Soden, Ischl, Gmunden, Reichenhall, ete.), 343, 344; 3. carbonated waters, indications for employment, 345; 4. salt waters which are at the same time carbonated, 345; 5 . mineral muds employed in the form of mud-baths, indication for use in diseases of women, 345, 346: mineral murls employed directly as such. 345; sea- 
bathing, its effective agencies, 346,347 ; hot-air baths, fir-tree baths, 347 ; hydrotherapy, modes and effects of application, 347, 348; halfbaths, rubbings, etc., 349; possibility of balneotherapentic treatment at home, 349; the Russian bath, 349, 350: seasons of the year for balneotherapeutic treatment, duration of course of same, 350; outline of the diseases in which balneotherapy is employed, 350-355.

\section{CHOLERA, PARTICULARLY ITS TREATAENT. \\ Pages 356-384.}

Introductory notes, 356,357 ; the nature of cholera, 358 ; the former classification of infectious diseases and its unreliability at the present time, 358, 359; the three important factors in the development of a cholera epidemic, 359; Pettenkofer's theories concerning the influence of the soil-water, 359; Hueppe's investigations as to the spread of the epidemic in Hamburg, 360; Koch's failure to recognize the importance of the surrounding conditions of life, 361; Hueppe's theories concerning the influence of the intestinal tract on the growth of the bacteria, 361, 362; the unreliability of experimentation, 362 ; occurrence of cholera among washerwomen. 362; the basis for considering cholera as a contagious disease, 362,363 ; varieties and course of the disease: choleraic diarrhøa, cholerine, 363, 364; genuine cholera, symptomatology, 364, 365; causation of the algid stage, 365, 366, 367: leaction period and its attendant disturbances, 367, 368; preventive measures: quarantine in Europe and Asia, 368, 369; over the Suez Canal, 369 ; the individual quarantining of patients, 369,370 ; disinfective measures, 370: supervision over arrivals, 370,371 ; the results of these measures in various parts of Russia during the recent cholera epidemic, 371; the individual measures of precaution, 371,372 ; the treatment: the unreliability of statistics as regards the value of the various methods of treatment, 372, 373: absence of any specific, 373, 374 ; the necessity for distinguishing the symptomatic from the routine treatment, 374 ; treatment of diarrhna: its varieties, 375 ; laxatives, 376; calomel not an "anti-choleraic" remedy, 376, 377; opium in diarrhce and the reasons for its employment, 377, 378: bismuth, clysters, etc. 379, 380; Cantani's treatment of choleraic diarrhcea, 380 ; effect of enteroclysmata, 380, 381: warm baths, etc, 381; treatment of cholerine, 381,382 ; of the algid stage of cholera proper, 382,383 ; of the reaction period, 383 , 384; nature of cholera nostras, 384 .

\section{SUPPLEMENTS.}

1. Ox Bloodlettixg. 387-406.

The recent history of bloodletting and its present condition, 387 , 388 ; neglect of the subject, 388, 389; former failures in its employment and reasons for same, 389, 390; opposition to it, 300; depletive and derivative effects, 391 ; depletive bloodletting: venesection in disturbances of the cerebral circulation, 391, 392; in threatening apoplexy, 392; in diseases of the heart, 392; in croupous pneumonia, 393 ; local bloodletting, 393; method of application, 394; wet cups, 394; their effect, 394, 395; where applied, 395 ; care in applying necessary, 
395 ; local depletive blood-extraction, 396; derivative bloodletting: nature of hæmorthoids, 396; an angioneurosis, 397; case proving this supposition, 397; etiology of hæmorrhoids, 398; how derivative bloodletting from the anus originated, 398; effects of this bloodletting, 399; case where it was employed, 399, 400; care in using tamponade of nasal cavity for nosebleed, 400 ; indications for derivative bloodletting: 1. in the region of the head, 401,$402 ; 2$. in the region of the spinal column, 402,403 ; 3 . in the region of the chest, in various hæmoptyses, 403, 404; in the abdominal region, in inflammation of large liæmorrhoidal nodes, 404 ; the method of employing derivative bloodletting, 404, 405; the time of the day, position of the patient, site of operation, number of leeches, etc., 405, 406 ; repetition of derivative bloodletting, 406.

2. CALOnEL. - In hypertrophic cirrhosis of the liver and in general therapy, 407-435.

History of calomel therapy in England, Germany and Russia, 407, 408; anthor's experience with calomel, 408; physiological action of the drug according to Binz, Shiff, Weiss and Sokoloff, 408, 409; the English practice as regards calomel, 409, 410 ; calomel in severe, especially febrile cases of colic from biliary calculi: cases, 410, 411-415 ; Methorl of employing the drug, 415, 416, 417; calomel compared with castor oil, 417; case of hypertrophic cirrhosis of the liver, 417-427; calomel in facial erysipelas, 427 ; in typhoid fever, 428 ; in croupons pneumonia, 428; in acute nephritis, 429 ; in diseases of the biliary passages, 430, 431; riaguosis of biliary calculi, 431, 432; calomel in catarrhal jaundice, 432-435.

3. STPHilis of THE LUNGs, $436-445$.

Pulmonary tubereulosis in syphilitie patients, 437 ; cases of syphilitic pneumonia, 437; diagnosis of, 441; syphilitic pneumonia complicated by pulmonary tuberculosis, 441-444; treatment of, 445 ; case exemplifying this treatment, 445 .

4. Syphilis of the Heart, 446-459.

Lack of knowlerlge on the clinical aspect of cardiac syphilis, 447 ; cases of syphilis of the heart (syphilitic myocarditis, syphilitic affection of the cardiac muscle), 447,448; character of the majority of cases of cardiac syphilis, 449; case presenting a type of syphilitic cardiac affection (contemporaneous syphilitic affection of the cardiac muscle and nerves), 449-451; its treatment, 451; failure of digitalis in same, 452 ; condition of the nelvous system of the patient, 452 ; data for diagnosis of cardiac syphilis, 454; its treatment, 454, 455; the milk treatment of disease of the heart, 455 ; Spanish flies in angina pectoris, 456 ; case of aneurism of the aorta due to syphilitic aortitis, $457-459$.

i. Treatuext of Fever, 460-46:3.

Certain symptoms, among them fever, as protecting agencies to the organism, 460: treatment of fever in acute infectious diseases, 460 , 461 ; use of cool baths, 462 ; cold sheet, 462 ; nse of antipyrine, antifebrine and phenacetine, 462: use of quinine, 463 ; fever in pulmonary tuberculosis, 463; in septicxemia and pyrmia, 463 . 


\section{N TRODUCTION}

\section{TO \\ CLINICAL EXERCISES.}

THREE LECTURES.

\section{Gentlumen:- -}

BEFORE we begin our clinical exercises, it is necessary that we understand the nature of the clinic and its importance in the matter of medical education and in scientific medicine. The duty of explaining this importance devolves principally on me, as the teacher at the Faculty Therapeutical Clinic, this last being, as we shall see later, the first general, as contrasted with the special, clinic, which you come to attend.

The Importance of the Clinic in Medical Education. You desire to become physicians, that is, to learn the art of treating and preventing diseases. - It is impossible to learn this without the knowledge of disease and of therapeutic means. You cannot interpret disease, unless you know what constitutes the normal condition. The course of medical studies is therefore such as to teach you first what constitutes the normal condition (Anatomy and Physiology), and later the nature of diseases, their treatment and the nature of the remedies (general and special Pathology, and Therapeutics with Pharmacology). Until the present you have occupied yourselves with the above-mentioned sciences; now, in due order, follows your acquaintance with diseases and their treatment in practice: this necessity of medical education is fulfilled by the clinic, i. e. by clinical instruction and clinical exercises.* What is the in-

* "Clinic" is derived from the Greek $\kappa \lambda i v \eta$, a bed, a couch, on which the patient reposes; as used in these lectures it refers to a hospital connected with the medical school for purposes of practical teaching. 
fluence of clinical exercises or generally of observation and treatment of the sick, even when outside of the clinic, on the course of medical education?

The student, on his coming to the clinic, is well informed, but in a purely abstract manner, of all the possibilities of a pathological process, and the potentialities of therapeutic action. All this information is, as it were, evenly distributed in bis mind: there is no occasion for one certain part of it to become more prominent than another.

In the clinic. as generally in practice, the phenomena of the pathological processes, and the reasons for therapeutic action, as well as the action itself, present themselves not in an abstract form, but under all the conditions of a given case; they may appear to one physician in a greater number than they do to another, but they never will appear to one in such numbers and varieties as to exhaust all the possibilities indicated by pathology and therapeutics. Under such an influence of the clinic or of practical activity in general, certain medical information will take precedence over others in the mind, and will thus come forward, at the same time grouping itself otherwise than in the systematic study of pathology and therapeutics: for, whereas in this last the etiological and anatomo-pathological points of view are chiefly predominant, in clinical and in practical work in general the diagnostic and therapeutic considerations are chiefly taken into account. Systematic exposition starts with the definition of the disease in the direction of its picture; whereas in the clinic we begin with the picture of the disease and deduct its definition therefrom. This difference creates the practical physician, who. knowing the real nature of the disease and the influence of medical means thereon, appreciates correctly the extent of the pathological process and the degree of effectiveness of the remedial agencies; he is thus enabled to quickly concentrate his mind on the two most important aims of practical medicine, namely, the recognition of the disease and its treatment. On the other hand, this same difference may naturally become the foundation of a future routine tendency in medical practice.-C (omprehending then the influence of clinical and of practical activity in general, and 
the possibility of the two above-named results therefrom - a desirable and an undesirable one - what must be the ains of clinical teaching? To educate the students into practical and as perfect physicians as possible, the teacher must see to it that they acquire a regular method, following a laid-out plan, according to which the clinical exercises, that is, practical medical activity, are to be conducted, thus avoiding irregular clinical teaching. To prevent the development of a routine habit, he must individualize his cases, that is, point out their special characteristics. Of course, the mastering by the future physician of a method and the acquisition of the skill in individualizing is a rather slow process at the beginning; but once acquired, their possessor finds them at his command sooner and to greater advantage, than the man who is not habituated to them. The acquisition by the students of a method and of skill in individualizing must be the chief aim of the teacher, as contrasted with the tendency to convert the clinic into a kaleidoscope, and to show in a shortspace of time - always limited - all the diseases of the special branch of pathology in all their varieties, combinations, and under all conditions. It is impossible to show all in one year, - not even in ten; and to insist upon an impossibility is unreasonable. - Whoever has aequired a method and the habit of individualizing, will quickly familiarize himself with any new case which may present peculiarities unknown to him before; and such new cases are met with quite frequently even by the very experienced physician, the more so by the beginner: for such is the peculiarity of the medical, as of every other practice, that is, of an activity amidst conditions of real life. The teacher should, surely enough, introduce to his students the principal morbid varieties and typical cases of that particular branch of pathology, to which his clinic is especially devoted.

In accordance with their aims the clinics are divided into different varieties:-

\section{A. Those conforming to the grades of medical studies. -}

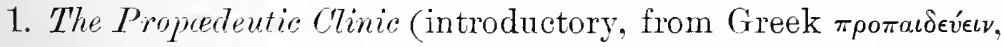
teach beforehand), which is, properly speaking, practical semiotics, i. e. it familiarizes the students with the signs of diseases and with the groups of signs in real life. In such a clinic the 
teacher cannot, while devoting his time to this subject, conduct any thorough clinical investigation concerning the diagnosis, prognosis, and treatment of a case; besides, the students are not sufficiently prepared to profit by it.

2. The Faculty Clinic is the first complete clinic, in which the teacher conducts before his hearers the above-mentioned medical acts, methodically and individualizing each case, and presents, therefore, a smaller number of patients.

3. The Hospital Clinic. - In this the teacher can do his work more rapidly, and goes over a greater number of cases, thus increasing the experience of the students, who had been prepared for this by the preceding clinic.

4. Clinic for the out-patients (ambulatory.) - While the more serious affections are usually to be seen in the hospitals, in the out-patient department we see a great many others; namely, the less serious ones, that do not go to the hospital, and the serious forms at their beginning. The out-patient department affords also opportunities for observing the course and treatment of diseases not under hospital conditions, but under the variable surroundings of every-day life; as their attendance requires a certain degree of practical maturity, they should be in charge of those students who have gone through the stationary clinics (the preceding ones); but this is not always adhered to, nor is it always possible to do so.

B. As to their contents, clinies are divided into general and special. The reason for the existence of the special clinic lies in the great general law of progress : the subdivision of labor. As a result of the clinician's ability to confine his work to one special branch of pathology, he perfects himself in it, becomes better able to teach it, and assists in advancing it more rapidly as a science. The number of special clinics is therefore continually on the increase : thus in the last few decades the former special clinics in surgery, obstetrics and ophthalmology, were increased by the addition of those in gynæcology, nervous diseases, insanity, skin diseases, venereal and diseases of the genito-urinary tract.

It is rather more difficult to define the character of the general clinic. What is it, and what are its aims? The name 
general clinic can only be applied to the clinic of internal diseases. The ailments usually treated in such a clinic refer to organs whose functional activity forms the basis of animal metabolism, namely, the organs of nutrition, respiration, circulation and of the secretions, - then disturbances of the processes of the animal metabolism itself, acute and chronic contagious diseases, functional disturbances of the nervous system which are inevitably met with in all these disorders (we can leave aside the organic diseases of the nervous system that belong properly to the clinic of nervous diseases), and at times diseases belonging to the special clinics, particularly affections of the female genital organs.

What conditions have secured for the clinic of internal diseases its significance and its contents? The question naturally arises, whether it were not possible and more advantageous to distribute all that the clinic of internal medicine claims, over special clinics for diseases of the chest, abdomen, etc.? There is not the slightest doubt but that clinics of such a nature would be just as useful as any other special clinics; but they could not possibly replace the clinic of internal diseases in its present form. The fact is, that the special clinic suffers from one radical defect: the difficulty that the specialist clinician experiences even in satisfactorily determining, in the given case, the general condition, the condition of other parts of the organism, after he had recognized in the most thorough manner the defect of the organ of his specialty; and the more perfect the specialist, the greater this difficulty, as devotion to his speciaity must needs have alienated him from the others. The specialists are well aware of this defect, point at and struggle against it, but, as every-day experience proves, cannot remove it, because it is organically connected with the very nature of specialization.

What would become of medical teaching and of medical science, if, possessing only special clinics, we were deprived of one, that would have as its principal aim the teaching of the importance of connecting all the phenomena of a given case, so that a general conclusion about it may be arrived at, and thus lead us to the physician's chief duty - application of remedial agents? 
Writhout such a clinic the education of a physician is not to be thought of. And such a clinic is the one of internal diseases with the above-mentioned contents; occupying itself with the simultaneous observation of disorders of those portions of the organism that are chiefly concerned in connecting all its parts in common; that is, the simultaneous observation of disturbances of functions that form the basis of animal metabolism, of the metabolism itself and of the functions of the nervous system. This it is that characterizes the peculiarity of the clinic of internal diseases and entitles it to the name of a general clinic. Notwithstanding the importance of the surgical clinic. it does not have the character of a general one, as the subjects of its treatment do not possess the same importance in the comprehension of the common connection of pathological phenomena, as do the subjects treated of in a clinic of internal diseases. You will see from the above, that the pediatric clinic has the character of a general one. Our university, as also the majority of universities, has two clinics of internal diseases - the faculty clinic and the hospital clinic. As you attend the faculty clinic first, it - the faculty clinic of internal diseases - consequently is the first complete and at the same time general clinic which the student sees: it is the true clinical school of the medical department. From this fact it derives its importance and in it is laid the basis of your future medical activity.

While discussing the importance of the clinic and of clinical exercises in the course of medical education, we must make some necessary remarks of a general nature on the method of conducting these exercises.

What is principally embraced by clinical exercises? IVe usually have to define the nature of the disease (examination and diagnosis), its future course and its termination (prognosis), and to order a plan of treatment and execute it, being guided by the course of the disease (observation). The clinical exercises, as we explained above, must be conducted according to a method and to a process of individualization. As regards a method, I have, after several attempts in the begimning of my clinical and practical activity, adopted the following one as the best : -- 
I. Examination. - I begin my inquiry with: What ails the patient, and for how long a time? The patient, in the majority of cases, does not distinguish in his history the present from the past, confounds the symptoms referable to diseases of one portion of the body with those of another, the course of the disease with the results of the applied treatment, supplies you with unnecessary information, and is generally incoherent.

In the beginning of my practice I used to listen to these tales, and have concluded that they are of no value and only tend to fatigue both the physician and the patient. On those rare occasions when your patient is either an intelligent layman or a physician, stating his case in a connected, sensible manner, there is the danger that the examiner may accept, alongside with the history of the case, also a conclusion about the disease and its treatment, - a conclusion which had been formulated either by the patient himself or by his physicians, as even the manner of relating the history of the case reflects this preconceived opinion. Such a preconceived conclusion will interfere with your further investigation and may prevent you from arriving at a correct and proper idea about the disease and its treatment.

With this in view, I usually interrupt the patient's narrative, explaining to him that I desire to be informed in a concise manner of the most prominent symptoms he suffers from (as dyspnoea, pains, weakness, etc.), and of their duration (in weeks, months, etc.), that I will later conduct the inquiry in due order, after which I shall be ready to listen to his story. With this I beg the patient to answer as explicitly as possible, thus: first, to affirm or deny only what he is certain of, what he remembers well, otherwise to say: "I do not know, I do not remember;" and second, to answer the question, and that only, without mixing in foreign matter not related to it, and not to be carried away by his own tale, thus distracting the physician's attention from the principal subject. From among the innumerable multitude of inaccurate answers let me instance for clearness' sake such a one: "Do you drink any tea, and if yes. how much?" Answer: "I am not a particular lover of tea"; - and only after two, three or more questions the physician finds that, although not a "lover of tea," the patient imbibes so many 
glasses or cups per day. More time still is wasted uselessly in listening to answers which become tales of subjects unconnected with the case. I advise the beginner not to permit any such distractions. As a circumstantial examination requires time and energy, the above-named deviations will bring it about that at the end, when the important point will have been reached as to what treatment is to be applied by the physician, the latter will have become mentally fatigued just at the moment when his whole energy, judiciousness and mental clearness are to be put to test. However, in the majority of cases it is only necessary at the beginning to correct the patient's answers, to state explicitly what is wanted of him, namely, as concise and direct answers as possible, and the further interrogation will proceed smoothly. It is self-evident, that to receive such answers the physician in his turn must put direct, concise and simple questions.

The examination of the patient consists of inquiries as to his present condition (status prcesens) and inquiries relating to his past (anamnesis). When examining a chronic case, I usually begin with the status prcesens, as the information obtained facilitates the inquiries about the past, and makes the data concerning anamnesis itself more intelligible. In recent cases, where, for instance, the patient has been ill only for a few days, and where the anamnesis is on this account short, it is much more convenient to begin with the latter. It would, however, be mere pedantry to strictly cireumscribe each method of examination: particularly so, when in examining the present state we are sometimes compelled to make special inquiries concerning the past without going into a full examination of the latter.

The examination of the present condition (status prosens) consists of interrogation and the so-called objective examination, or examination through the sense-organs. At the beginning both interrogation and to some extent objective exanination are resorted to simultaneously, the latter by easily accessible means, as, for instance, in inspecting the tongue, feeling of the pulse, or while talking to the patient, the physician cannot help noticing the latter's condition, the state of nutrition (whether fat or thin), the appearance of the superficial tissue 
(whether plethorio or anæmic, cyanotic, icteric, cachectic, etc.), his physical and mental condition (movements of the body, facial expression, mode of speaking, etc.). But a complete objective examination, when it becomes necessary to undress the patient or to resort to such means as the examination of the urine, sputum, etc., is much more convenient to make after you will have finished your inquiries about his present and past condition.

In interrogating about the status presens I first of all seek for information about the most important conditions surrounding the patient's life, and about his mode of living.

1. The locality. - Whether damp, malarial, or dry, dusty: whether open to winds or not, and so on.

2. The dwelling-place. - Dimensions, flooring, position of bedroom, temperature and ventilation, condition of water-closet. and so on. Where is the work of the day performed?

3. How does the patient wash himself? Outdoor bathing, public hot baths, simple house-bathing, sponging?

4. Clothing in general and particularly the protection of the abdomen (sashes and corsets) and feet (covering - warm or cold, roomy or tight).

5. What nervines is the patient addicted to: tobacco, tea. coffee, wine, whiskey, beer?

6. What drinks: ordinary water, or alkaline (sodia, seltzer), kvass, ${ }^{*}$ milk?

7. Food - meat or otherwise; light or heavy, frequency of meals?

8. Family life, single, retired.

9. Children, abortions.

10. Does the patient get sufficient sleep or not? Duration of the sleeping hours. How often is he sleepy?

11. Mental and physical activity? Rest?

12. How much of the day is spent out of doors and hou much in confined rooms?

* A fermented drink in general use in Russia, taking the place of beer of other countries. Common kvass is made from an infusion of raised rye-flour or dough, or of other flour or baked bread, with malt. Fines kinds are made from apples, rasplerries. or other fruit. without malt. Century Dic. 
We next inform ourselves of the patient's condition by carrying our inquiries along an adopted plan, which is, of course, the essence of systematic examination. The beginner, without having acquired this method, and not appreciating the necessity of it, conducts his inquiries without order, and is therefore liable to be influenced in arriving at the diagnosis by the first impression produced on him by the patient's complaints (as, for instance, difficulty in breathing, pain in the side, cough), on the strength of which he is led to think of a certain disease; and without going into details about the condition of the whole organism, he hopes, to quickly diagnosticate the case with the aid of a few questions relating to his assumed ailment. But bitter experience will teach him - - if he only be capable of selfimprovement_- that such reasoning is deceptive and is justly condemned by experience, and that the only reliable--although somewhat slower and more laborious - method of examination, is the complete one, conducted in accord with a certain, once established order. In the basis of $\mathrm{my}$ system of examination, which experience has taught ne to consider the best, I put the union of two principles: the physiological one (systems and organs), and the topographical one (neighboring parts). There may be others, but the first condition of every method requires that the questions include all that is most important.

1. Appetite and thirst.

2. The tongue. teeth, mouth and throat.

3. The stomach. Is the introduction of food followed by belching, burning. heaviness. pain. nausea, vomiting; any pains when the stomach is empty?

4. Intestines. Character of stool, regular (i. e. daily), of sufficient quantity, evacuation of normal consistency and form, - or irregular? If the latter, do not content yourself with the general statement of an existing constipation or diarrhœa, but inquire fully as to just what irregularity there is. With this in view, take a certain period of time previous to the examination, say a week or more, if the patient can call it to his memory, and inquire about the conclition of the stool on each day of this period: whether a eonstant diarrhoe, or a constant constipation, that yielded only to injections and laxatives, or. 
what is most frequent, both constipation and diarrhoea alternating with each other. If the movements of the bowels were of late exceptionally regular, or constipated, or loose, inform yourself of their usual condition. If the patient resorts to laxatives, find what they are and in what doses. If he uses enemata,of what and at what time: at bed-time, when the system is fatigued, and the nerro-muscular apparatus in general, as well as that of the intestinal tract, reacts less readily:- or in the morning, after a night's rest and the morning tea or coffee. when the patient feels an inclination to stool not of itself sufficient to clear the bowels, but strong enough to do so effectually with the aid of an injection. Does not the patient, as a general thing, resist the first inclination to stool, thus preventing the emptying of the bowels (the most important and frequent cause of constipation)? Does he empty at stated times the bowels, when constipated. so as to aroid overloading of the same and the possibility of a consequent diarthuea? What kind of food or drink, or what conditions in general produce constipation or diarrhcea? Such a circumstantial inquiry will at once lead to a full recognition of the irregularities in the case, - irregularities. that are so important by their frequency and influence on the rest of the organism: you will seldom indeed find a patient, whatever the disease he may be suffering from, who has perfectly regular stools. The same inquiry will also furnish you with very valuable information regarding the therapy and, even more important, in respect to the hygiene of the given part of the organism. How many times, for instance, have I succeeded in effecting a cure of a rebellious constipation. by simply enjoining upon the patient not to resist the first inclination to stool; or in persons habituated to no other drinks but warm tea, by adrising to diminish the quantity of the latter and to take half a glassful of water of ordinary temperature. from one to three times a day, each time one and one-half or two hours before a meal. The reason for my dilating on the subject of irregular rectual evacuations lies in the fact that we are often liable to pass over this subject and its treatment in a rather hasty and superficial manner.

5. The anus. Any lumps? Do any hannorrhages take place 
from the anus, and by what phenomena in the rest of the organism are they accompanied?

6. How does the urinary bladder empty itself? Is the urine ever red and turbid? It is here that I inquire about swelling of the lower extremities (the parts of the body in which the phenomena of dropsy make their first appearance).

7. The male genital organs: the condition of potentia viritis. How frequent are the connections? Does the patient feel weak after them (this is very important in neurasthenia)? Masturbation, past or present; pollutions. Also inquire about diseases (past or present) that originate most frequently through sexual contact, as gonorrhea, chancre, syphilis. As regards this last, I consider it of paramount importance to be convinced as to whether the patient is syphilitic or not; and it is quite superfluous, I think, to dispute the necessity for this. If conrenient. I inquire of the patient whether or not he had syphilis, but do not rely entirely upon either the positive or the negative reply, but verify it by inquiring as to whether the patient (if a woman) has had any children, or abortions and miscarriages; whether he had any falling of the hair (particularly from the eye-brows and beard), not ascribable to any cause; any eruptions, and of what nature; are there any scars left? Were there before or are there now any nocturnal pains in the bones and articulations; any chronic catarrh, etc.? If, on the other hand, it is either inconvenient or useless to put the question directly, as, for instance, to a woman, in whom the infection might have passed unobserved, then I at once resort to the above verifying questioning. If such inquiry furnishes me with accurate information. I continue it to the end. But if it leads me back into the anamnesis, - if the history of the apparent or doubtful syphilis has been intimately connected for a long time with the history of other morbid processes in the given case, I then postpone the inquiry until I have collected complete information covering the past, until the investigation of the anamnesis. This observation, as to how far into the anamnesis we may go while investigating the present condition, not only refers to the inquiry about syphilis, but to that about any other disease of the organism. 
8. Female genital organs. Former deliveries or abortions. Condition of menstruation before and now. Leucorrhoea.

9. The abdomen in general. IVell-marked changes in the size of the abdomen, observed even when the patient is dressed, impress themselves on one's memory. But the most important inquiry, regarding the abdomen in general, consists in informing oneself as to the presence or absence of pains in that region; if present, whether constant or paroxysmal, and chiefly, where localized; this last gives us important information as to the source of the pains. In case the patient finds it difficult to define the seat of the pain, we assist him in finding an answer by asking him whether the pains are felt all over the abdomen or more to the middle part of it; whether they are accompanied by rumbling, and whether they are relieved by the escape of gases per os or per anum, by vomiting or by a loose stool (gastric and intestinal pains). Is there more pain in the upper as compared with the lower part of the abdomen, -- more on its right than on its left side? With what functional activities do they coincide (from this we infer as to their connection with the liver, kidneys, ovaries, uterus and urinary bladder)? Are there any pains in the posterior wall of the abdomen, in the loins, and with what do they coincide: with constipation, with pains along the tract of the ureters or with movements of the back, as in lumbago in general (i. e. in various myopathies, neuropathies and affections of the vertebræ and their articulations in the lumbar region)?

10. The chest in general, and also the organs of respiration and circulation. If there are pains in the chest, find their localization and with what they coincide: as, for instance, pain in the region of the heart, of paroxysmal nature, transmitted to the left arm or to both arms, accompanied by a sensation of fright and anguish (as in angina pectoris); pains in the sides, aggravated by coughing, as in pleuritis of tuberculous and other nature; pains along the tract of the intercostal nerves (as in affections of the same, and in liver and kidney colics): pains in one half of the chest and simultaneously in the corresponding arm, with this also tenderness on pressure in the supraclavicular cavity of the same side (neuritis of the bra- 
chial plexus); pains all over the chest, aggravated by pressure on the sternum and ribs (as, for instance, when lying on the side), coincidently occurring with rheumatic pains in the extremities and aggravated at the same time as these last on catching cold (usually gout or syphilis, which are easily differentiated: or gout and syphilis of the ribs and sternum, at times with a neuritis of a similar origin) and so on. - Then inquire about difficulty in breathing (dyspnoua), paroxysms of choking (asthma). palpitation of the heart, conghing, expectoration, spitting of blood. the condition of the nose and larynx (alterations in the voice). Examine the pulse and inquire about the condition of the circulation: is the patient subject to chilliness in general or in particular parts of the body (the extremities, feet. and particularly the soles of the feet)? Is he subject to flashes of heat in general or in some certain part (particularly the head)?

11. Is there any febrite corrlition (chill, heat. or only weakness. loss of appetite. and thirst)? If not. inquire if patient is subject to any habitual feverish condition, and what does it accompany: for instance. catarthal conditions. most frequently of the respiratory tract. less so of the intestines and urindry tract (febris cartarrhalis); or it may accompany rheumatic pains in the extremities, in the back, chest and head (febris rheumatica); i. e. most frequently it is a febrile condition accompanying an exacerbation of gouty or syphilitic pains. or both of these together. on account of a cold or for some other reason. Is he not subject to febrile states without catarh and "rheumatics," of a more or less clearly defined intermittent nature. coincident with a residence in a malarial region. especially during autumn and spring? Is there a fever of an exhaustive character. accompanying serious diseases of important organs, most frequently tuberculosis, cancer or syphilis (particularly frequent with syphilitic liver). etc.?

12. Condition of nutrition and of the hematopoietic system. Does the patient tend to grow full and fat. or thin and pale? Does he look cachectic. etc.?

13. Steer. whether quiet or not: if disturbed, why so: is there cerebral hyperemia. nenrasthenia. pains. cough. dyspncea. constipation and an overloated stomach and intestines. febrile condition, etc. ! 
14. Mental condition: memory and the faculty of reflection: state of mind, - hopeful, pleasant or oppressed, sad, ete.

15. Headache. Important questions to ask: does your head ache or not? If it does ache, then, the whole or only part of it, whether the temples. the back, the front, the top; and what is the character of the pain - is it constant or paroxysmal, and what is the nature of the paroxysms? Before proceeding further I must make here the following observation: while the examination, as stated above, is being conducted, information must not only be collected, but also made as clear to the mind as possible; it is not sufficient to learn the existence of certain morbid conditions; we must at the same time endeavor to find their cause, at least the proximate one. The nearer we come to an explanation of the latter, the clearer to one's mind become the data obtained by examination, the easier and more fruitful is this examination. It is self-understood, that such a procedure is more possible for an experienced physician than for a beginner: but the latter will soon gain the experience, if he but acquire the habit, instead of simply collecting facts mechanically, to classify the gathered information, to look for the causes and thus prepare valuable material for the erection of an organic whole - the diagnosis of the whole morbid condition. It is of course also necessary at the same time to acquire a certain tact in such attempts at immediate elucidation of the matter, so that the once established order of examination is not interfered with: if a few questions suffice to make clear the nature of the morbid phenomenon, then an immediate explanation of it is not only desirable, but even obligatory ; but if it become necessary to multiply the number of questions, and enter further and further in to the inquiry about the condition of organs not yet examined, and even into the history of the past, - then we must postpone the explanation of the phenomenon until we shall have collected all the information, i. e. until after our full examination. Attention and exercise on the part of both the student and the instructor will result in the speedy acquisition of the above-named tact.

Let us now resume the question of headache. There is hardly a morbid phenomenon which is so frequent and brought 
about by so many and so various causes as the unpleasant sensations in the head: pain and heaviness. Morbid conditions of the soft parts of the head and of the bones of the skull and face, of the numberless nerves of the head, of the organs of the higher senses, and, the most important of all, of the cerebrum itself, which, outside of disturbances of its own substance, reflects in itself in various ways the influence of disturbances of other parts of the organism, - these are all causes of frequent headaches and heaviness. If the picture of the morbid phenomenon, as seen from the above necessary questions, points directly to some certain disease (as, for instance, migraine, masked malaria, syphilis, etc.), and if the completed part of our examination - and this latter will have been finished, if conducted in a certain order, by the time we reach the inquiries about the headache - contains data that may be considered as the causative agents of the pain in the head, then we certainly should make inquiries verifying our assumption. Otherwise we must leave the explanation to the end of our investigation, although it may happen, especially with an inexperienced practitioner, that he will traverse almost the whole region of pathology, before he will have found the causation of the pain in the head.

16. Vertigo. If present, find whether accompanied by a flushed or pale face, by an overloaded stomach or other dyspeptic phenomena, by constipation, before hæmorrhoidal bleeding, before menstruation or after it, by uræmic manifestations, etc.

17. Pains in the neck, spine. and extremities. If present, localize them (in the articulations, along the bones, nerves and muscles), and determine their character: constant, aggravated by pressure (in the arthrites, periostites and perichondhites, neurites and myosites), or of an opposite nature (as, for instance, lancinating pains in tabes); aggravated or not at night, etc.

18. Parcesthesice and ancesthesioe.

19. The nervo-muscular apparatus: condition of muscular strength, disturbances of locomotion, condition of reflexes.

20. Sight and heroring.

21. General integument: abnormal dryness of the skin, or sweating, itching, eruptions. 


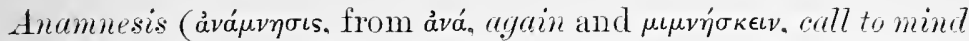
- a recalling to mind). The information obtained from the patient concerning his present condition will serve as a guide in conducting the inquiry concerning his past: we must inform ourselves of the history, of the origin. course and treatment (if there was any) of those departures from the normal health, the presence of which was elicited while inquiring about his present condition. WVe must naturally also inquire abont ailments which, as the patient thinks (and often wrongly so), have no connection with his present disease. We also inform ourselves at this stage of the health of the parents and relatives in general.

Haring learned the patient's past and present condition. we invite him to add anything he may desire to the physicians inquiries. It is rarely that a patient will find it necessary to add anything after a physician s thorough questioning.

Objective investigation. Palpate the head (if necessary): inspect, palpate, percuss and auscultate the chest; inspect. percuss, and, what is most important, paIpate the abdomen: inspect and palpate the neck, back and extremities; examine the excreta, chiefly the urine (quantity, specific gravity. color, sediments, reaction, presence or absence of almormal constituents) and the sputum, and at times the intestinal excreta and the gastric contents: examine. if necessary. the blood; determine the temperature of the body; institute a special examination, if necessary, of the organs of sight and hearing: of the larynx, of the urinary bladeler. ete.

Examination of the patient serves as the basis for the diagnosis, prognosis and treatment. Following the method as above outlined, the phrsician need not fear that he may fail to observe or may omit anything of importance for these nedical aims. But I must also warn you against another possible mistake - that of hunting for superfluous details, and unnecessary minuteness in investigating both the present and the past conditions of the patient. Not everything by far, obtainable by investigation, is necessary or important for our medical aims: we must especially distinguish the diagnostic from the semiotic aims: not everything interesting to the semiotician is necessary 
to the diagnostician. It is important that the beginner acquire the requisite tact in his examinations, so as to avoid fatiguing himself and his patient; and to preserve the energy which is so necessary in arriving at serious, sober and therefore correct conclusions, he must leave out umnecessary and minute details, as well as everything that is confusing and superficial. The full possession of such a tact is. of course, only a question of time and experience; but at the same time a regular clinical teaching, free from confusion, superficiality and excessive mimuteness of detail in investigation, without deviating from its direct aim, will undoubtedly assist, by its example, in acquiring this tactful habit. - If the patient's condition be such that an examination of it will be difficult or even painful to him,-- as in cases of great weakness. hæmoptysis, dyspnoea. congh, pains. etc., then we must ask the most necessary questions, such as he himself will be able to answer, and so formulate them that a word or even a sign, positive or negative, will sufficiently answer them. For other information we look to the people surnounding the patient. They are naturally the ones to turn to in cases of loss of consciousness. It is self-understood, that the objective examination in such cases must be conducted with the utmost care and must include only what is very essential. The continuous improvement in the condition of the patient offers us the opportunity to fill in the gaps in the investigation.

II. Diagnosis. - It is a mistaken idea, that a diagnosis is arrived at only after the investigation has been completed, as if this latter were but a mechanical process of collecting information in a certain order. On the contrary, as we have endeavored to prove above, the process of investigation is a rery active, searching conditon of mind : the data obtained by inquiries and objective examination are bound to create certain suppositions in the mind of the physician; these he endeavors to prove by verifying questions and oljective investigations, olserving at the same time due moderation, so as not to allow himself to be carried away beyond what he has already obtained nor change the order which he decided to follow. Thus the diagnosis is being continuously evolved while the investigation is going on. so that in the majority of cases, after the latter will have 
been concluded, we only have to sum up the total and we get a complete diagmosis, i. e. that of the principal disease (dingmosis morl $i$ ), as well as that of the secondary disorders and of all the peculiarities of the patient (diagnosis agri). Less frequently. in cases which do not seem to be clear, or in complex cases. we must resort, after the investigation is gone through with and its sum total is macle up, to another special process - to the socalled differential diagnosis, or diagnosis by exchusion(diagnosis differentialis sen dirgnosis per exchsionem); in this we andyze in turn all the suppositions that have arisen concerning the nature of the giren morbid phenomena, and throwing aside the less probable ones. we retain what is most probable.

III. Prognosis. Diagnosis is a conclusion based on the present; prognosis is a presupposition of the future based on the diagnosis. referring to the course of the disease. to the changes to be expected at first and later on; to the termination of the disease: whether restoration to health. complete or partial. or death: to the nature of the effects of the prescribed treatment. While following the course of the disease, we verify the suppositions, we verify the promosis. It is thus evident how important the prognosis is for the physician; the more correct the prognosis, the stronger it is verified by further observations, the more self-confident becomes the physician, the less wavering he is, and the more positive he acts; i. e. the nearer he comes to being a truly practical worker, a practical physician.

None the less important is the prognosis as regards the putient and his immediate people and relatives. The importance of a correct prognosis in the hospital is as nothing compared to that in private practice: the reputation of and the confidence in the physician depends on a prognosis which is correct, faithful and in accordance with the course of the disease. It is hardly necessary to enlarge on the painful position of the practitioner in whom the patient reposes no confidence: and worse even is the condition of the patient, if in a small community, in the absence of any other physician, he is compelled to take treatment at the hands of such a physician. I therefore consider it my duty, and the duty of every clinieal teacher. to explain in each given case the prognosis not only as regards 
the patient, but also his relatives, although in the clinic, or in the hospital in general, these last are absent. I will here also make a few general remarks concerning the value of the prognosis for the patient and for his friends.

As regards the patient. You must always remember that, with very few exceptions, those affected with a serious ailment are, by the fact of the presence of the morbid condition itself, in an oppresserl mental state and look rather gloomily and with but little hope into the future. For the very sake of succeeding with his treatment the physician must infuse hope into the patient, promise a complete return to, or at least, in accord with the case, an improvement of his health, calling his attention to those favorable signs in the patient's condition which the latter in his despair fails to see or appreciate. Quite frequently such an intentional assurance will induce the much needed sleep which was absent; and it certainly needs no explanation to comprehend the importance of a quiet sleep for the functional activity of the nervous system and through it of the whole organism. Nor is this the only result of the change from an oppressed mental condition into a cheerful one; if we but keep in mind the facts relating to the phenomena of what is known as suggestion, we will easily perceive that here the prognosis coincides with the treatment. But to apprise the patient of all the apprehensions arising in the physician's mind is always a blunder on the part of the latter, and sometimes even a crime : in dangerous cases, where the least significant influence may decide one way or another (at times either death or a complete return to health, as, for instance, in the stage of collapse in acute pneumonia), to strengthen or to weaken the patient's courage-may bring fatal consequences. I may add that, following, as I do, the above outlined plan as a rule, I find that it will assist the physician in requiring of the patient a strict adherence to the prescribed measures, disobedience being threatened with a change for the worse.

But it may be asked, what will be our conduct in those cases where the patient directly puts to us the questions about the prognosis of the malady, desiring an answer because of the necessity of arranging lis worldly affairs and of fulfilling his 
religious duties? Let us take an extreme case: there is no hope for prolonging life, the end is approaching. In such a case, our reply must be to the effect that there need be no connection in the patient's mind between the approaching dissolution and the necessity for arranging the affairs and attending to the religious rites : the performance of either does not necessarily imply a nearing end; that the fulfillment of either will conduce to the patient's quietude, and will therefore also strengthen him, and the physician of course sees no hindrance to their being performed. As to what regards the direct answer to the question about death and its imminence, one may admit that the condition of the patient is very serious (not hopeless), and at the same time point out the possibility on the part of the physician to err in regard to his suppositions in general and to that of the exact time of death in particular. If those around the patient desire him to attend to the requirements of religion and his private affairs, then the physician must never put any obstacles in their way, choosing an opportume moment in the condition of the patient; but the physician is not the one to remind the patient of it: the latter will justly consider it as his sentence of death. It is the business of those around the patient to remind him of it, they again pleading the necessity for his doing so, not because of the approaching end, but because the fulfilling of the necessary duty will exert a quieting and strengthening effect on the patient.

If the condition of the patient is dangerous and even hopeless, and neither he nor his friends approach the physician with regard to the questions as above, then it becomes the duty of the physician to opportunely remind them to settle his affairs and attend to the religious rites, and through them the patient is made aware of the necessity of the case.

Prognosis as regards the patient's relatives. - The responsibility that rests on the physician, and the necessity for the patient's friends to be informed of the true condition of the patient, so that measures in accord with it may be taken, make it incumbent upon the physician to apprise the patient's nearest relatives of his condition without hiding anything. Of the persons named, only those are to be informed whose health will not 
materially suffer from the commmication of the expected fatality.

In a general way it is necessary to be concise and precise in wording a prognosis. I am in the habit of employing the following formula, which may he applicable to the immense majority of cases: 1. Case (or condition) of no serious import, where danger is neither present, nor to be apprehended. 2. Serious rases, in which the danger, althongh absent at present, is liable to appear. 3. Dougerous arses, but not hopeless ones, where danger is present, but where there is a possibility of a favorable termination. so that hopes for recovery may be entertained. $t$. Hopeless cases. — Thus when using the expressions: "a light (not serious) case," "serious," "dangerous," and "hopeless" one. I distinctly state what these expressions stand for.

IV. Treatment. - The principal rule to follow in ordering treatment is the same as in other clinical exercises, namely, the observance of a method and of a system of individnalization. The begimer, in whom clinical exercises failed to inculcate the habit of strictly following this rule. usually proceeds thus: he diagnosticates the most striking morbid phenomenon in the given case, and then, either from memory or ly the aid of his text-book, he selects one ont of several recommended remerlies. the success of the choice depending entirely on chance. Whereas, by observing the rule above allnded to. we select a path which is much more deroid of chance, and is more certain ; it is both methodical. not allowing us to omit anything, and individnalizing, making prominent all the preculiarities of the case and consequently pointing to the indications and contraindications to treatment indicated by them. The rule is as follows: keeping in mind the identical order of procedure which was observed in diagnosis and prognosis, we must discuss consecutively the hygienic measmes, the remedial means indicated by the diagnosticated disturbances of the digestive and other abdominal organs; then the indications for the present disturbances of the organs of respiration, circulation, etc.. taking in disorders found in all parts of the organism; - then we nut see whether the measures and remedies indicated ly disorders in certain organs are not contraindicated by disturbances of 
other organs, and then after considering all the indications and the contraindications, we give the preference to the most important ones, and out of several means recommended for the chief morbid condition we select the one that is the most iudicated and the least contraindicated by the general condition of the olganism.

To this principal rule to be observed in ordering treatment, I must add a few very necessary remarks concerning the properties which, in my opinion, therapy must possess.

1. The history of medicine betrs witness to the fact that periods of enthusiasm for certain remedies are succeerled by periods of therapeutic nihilism. Thirty years ago I had the opportunity of witnessing the decarlence of such a period of nihilism in Germany, - a period, the beginning of which may be traced back to the time and partly to the influence of the founder of the so-called homeopathy - Hanneman; the termination of this period was due to the efforts of the best representatives of German medicine - as, for instance, of Virchow - who have arisen in protest against therapentic nihilism. At the present time, thanks to the discovery of a great many new and partly very useful remedies, we olserve a certain enthusiasm for drugs, that is kept up by a still greater, everywhere olserved failing of medical practice, - mamely, the neglect of hygienie treatment. Not that the importance of hygiene is not fully appreciated; it is, on the contrary, appreciated better than at any previous period, but the temptation to consider our medical duty fulfilled after we give a prescription that costs so little labor, and thus avoid a thorough hygienic investigation of the case and the medical advice based thereon, this temptation is frequently the cause of the above-named neglect of hygiene. Steering between therapeutic nihilism and an undue enthusiasm for drugs, we must conscientiously and clearly keep in mind that a true, real, and not only seeming medical advice is one which is based on a full recognition of the mode of living, as well as on the history of the past and present condition of the patient; such an arlvice embraces not only the plan of treatment, but it aquaints the patient with the causes, perpetuating the morbid condition and found in the mode of his living; it makes it clear 
to the patient, that treatment will only assist him in returning to health; but to make this return secure the above-named causes must be remored - in slort, the patient must be taught personal hygiene. I may as well add here, that as the majority of patients are of weak will-power, it is the duty of the physician to assist them by his strength of character; and in prescribing for treatment and mode of living only the necessary measures, he must insist upon these latter being strictly carried out.

2. The simultaneous employment of many remedies is to be avoicled as much as possible, especiaily by the beginning practitioner. When one remedy is being employed, its effect is more prominent, and the beginner is sooner able to distinguish it from the changes in the condition of the patient not dependent on the employment of the drug. If it becomes necessary to employ simultaneously two or three remedies, then they must not be administered in one mixture, but each must be given separately, distinctly designating the time when they are to be taken : at bedtime (for instance, to quiet a congh that prevents sleep, or simply as a sommifacient), or in the morning, or with a meal, at certain attacks, etc. We thus meet the therapeutic indication more exactly, the effect of the drugs becomes more apparent and consequently the practitioner becomes more experienced in the application of the same. The prescriptions we frequently see containing four, five and more drugs are positively incorrect: whoever knows that we must carefully weigh all the changes in the patient's condition to be able to determine exactly the effect of even one drug, and that it becomes more difficult to determine the effect of two and especially of three simultaneously employed clrugs, will understand that it is impossible to determine with any degree of exactness the effect of a mixture of a great number of drugs, and that there is no reasonable excuse for employing such mixtures, unless it be in exceptional cases and even then for a limited period of time. As a matter of fact, the number of old mixtures that are preserved for employment at the present time is insignificant as compared with what it formerly was. The creation anew of such combinations - as for instance in the form of pills containing seven ingredients, - with our modern legitimate and 
necessary tendency for accuracy in medicinal effects, is a sad anachronism indeed.

3. Hospital therapy is generally of a one-sicled character, depending upon preference in the emplorment of the apothecary's means, "drngs." Such a one-sidedness, when in the clinic, will be transmitted to the students. if the cinician be not careful enough to avoid such a defect. It is therefore the duty of the instructor, - having first of all satisfied all the requirements of the patient's personal hygiene, as much as the hospital conditions permit, - to apply along with the apothecary's means also all others, and to acquaint the students with the so-called special therapies: balneotherapy (the mineral waters for drinking and bathing purposes. hydrotherapy, etc.). climatic therapy, the emplorment of compressed air, and the inhalation therapy in general, electricity, kinesotherapy (massage and gymnastic exercises), treatment by a special diet, etc.: either by directly applying the named methods of treatment, or: in case it be impossible to do so (as, for instance, in climatic therapy), by directing attention to the indications for them as found in the given diseased condition. If it is impossible to require of every physician, that he be as skilled in the various special ther"apies as every specialist therapentist is in lis respective specialty, we ean and must at least insist, that every pliysician should know where and when to apply this or that special therapy, just as he knows where and when to employ this or that drug. Besides, the most important methods and applications of special therapies are usually acquired easily by the ordinary practitioner, as soon as they have been worked out by the specialists.

V. Observation of the course of the malady. - It is selfunderstood, that the duty of the physician is not at an end after the diagnosis and the prognosis are made and a plan of treatment is laid out: he must put this last into execution. The duties of the clinical instructor run along similar lines: he must not limit himself to diagnosis, prognosis and plan of treatment. but must execute the latter before his students, familiarizing them with all the difficulties and peculiarities of medical practice which become fully apparent only while treatment is being carried 
out. With this in view the elinical instructor must impart to the students the art of olserving the patient, the knowledge of comprehencling the changes taking place in the condition of the latter, teach them to distinguish those of the changes which clepend on the course of the disease itself from those due to the treatment, discuss the results of this last, and in accord with them to either keep on with the once ontlined plan of treatment. or to change the latter in accord with the observation, reminding his students that the physician is but the minister nature. I can not help remarking here, that the clinicians do not often give due consideration to the subject of observation, although, withont this last, clinical instruction commenced and never brought to an end always fails of its aim. without bringing the benefit that it could and should bring. At the same time it must be added that a certain amount of experience is required of the instructor as well as due care in exploiting the time designated for clinical instruction. which is always too limited, to enable the clinician to fulfill all his duties. especially in such a rast clinic as that for intermal diseases; namely, to acquaint the students with the investigation, diagnosis, prognosis and plan of treatment in typical cases in all the branches of internal medicine as equably and sufficiently as possible, and with this to find time for familiarizing them with the execution of the treatment, and with the observation of the patient. and also to attract the students to participation in all the above-named exercises and quite frequently to fill up the deficiencies in their knowledge: of this we will speak presently.

\section{The making up of the deficiencies in the information} of the students is often very necessary, because these latter, on account of the impossibility of observing a thorough consecutive ness of instruction, come to the clinic, especially at the begimning of the year, without a sufficient knowledge of certain branches of pathology, and more frequently of therapeutics, particularly in the domain of the special therapies. Again, it is often necessary to inform the student of some particularly important changes or acquisitions in the teaching of that disease, or of that department of diseases, which is moler observation, - changes and acquisitions which have taken place only very recently, and which the student is naturally unable to follow himself. 
VII. Autopsies have an extraordinary importance for the clinic, as an instrument of progress in scientific medicine. They give additional data to the ante-mortem olservations. that shed light on the matter, thus furthering diagnosis and general pathology, and consequently also therapeutics. The history of medicine bears eridence to the fact, that it was rnly the success of pathological anatomy that made possible diagnostic progress. and assists in the development of special pathology. and through it of special therapeutics. From the standpoint of clinical instruction, the antopsy is important as a rerification of antemortem conclusions, as a means of conrincing the students the future physicians - of the possibility of a correct diagnosis. and consequently of a correct theraps. Still we must not forget that autopsy is by far not the only means for verifying medical conclusions; that a constant verification is to be had while observing the course and termination of the disease. It would therefore be incorrect and a slirking of his duty - that of teaching diagnosis and treatment of diseases - if the clinical instructor were to select by preference such cases for the students as would promise an autopsy, i. e. in which treatment would be powerless.

The importance of the clinic in scientific medicine. We have seen from the foregoing. that in the matter of medical education the clinic occupies a very important place. No less important and significant is its rôle in medicine, as a science. As at one time the necessity for assisting the sick created medical practice and later medical science, so at present the clinical, that is the medico-practical activity, continues to supply and stimulate medical arlvance, perfecting semiotics. diagnosis, general pathology and therapentics, inducing experimental investigations aiming to determine the very nature of diseases and their treatment. As regards the relation of the scientific clinical work to the subject of clinical instruction, the instructor, without deviating from his principal aim, and without loss of time, can always embrace the opportunity of pointing out to the students the importance and the value of clinical observation for the progress of medicine, and thus acquaint them also with this rôle of the clinic. He must, however, al- 
ways bear in mind, that his first dnty consists in clinieal instruction, in educating scientific, practical physicians, and that a considerable expenditure of time on anything outside of clinical work will lead to a prematme and therefore useless diversion of the attention of the imprepared students from their most necessary exercises, withont satisfying their most essential. requisites.

In conchuding the introduction to clinical exercise, I must say a few words regarding the interpelation between the work of the clinical professor, that of the clinical assistants, and of the sturtents.

The professor must, first, himself follow a method and system of individualization in his clinical work, and thus inculcate in the students habits for the same.

Secondly, he must carefully apportion the time allotted 'for' clinical instruction, so that it may be possible to fulfill all the duties of the clinician in an equable manner. These dnties have been defined above, while discussing the observation of the patient. As regards, in particular, familiarizing the students in the course of the academic year with the typical cases in all the departments of internal medicine as fully and as equably as possible, I must here mark ont for yon the order which my experience, extending over many years, has taught me to consider as the best. I begin the exercises in the clinic for internal diseases the first half of the academic year (serenth semester) with diseases of the digestive organs (stomach, intestines, liver. etc.), as they are the most important ones, becanse of their frequency and their comparatively greater eurability. As among the very first cases presented to the students we are also sure to run across some functional disturbances of the nervous system, these are likewise discussed during the same half-year. We at the same time take up the diseases of the peritoneum, the less important renal diseases (renal gravel and catarh of the renal pelvis) and gont, which are so frequently met with in diseases of the digestive organs and with which they are so closely connected genetically. The second half of the academic rear (eighth semester) is taken up with diseases of the organs of respiration and circulation (their cansation and partly their diagnosis is some- 
what familiar to the students from the propædeutical clinic), and also with the more serious renal diseases (as inflammations. degenerations, etc.). Syphilis of the internal organs is preferably taken up the first half-year, because of the frequency of syphilis of the liver. The acute infectious diseases, - as typhoid, typhus. relapsing fever, malaria, pneumonia, acute articular rheumatism. facial erysipelas, etc., the disturbances of nutrition (diabetes, anremix, obesity, etc.), and the disturbances of the organs of locomotion, that we are liable to meet with in the clinic for internal diseases (as diseases of the muscles and peripheral nerves, of the articulations and bones) - all these are taken up in both halfyears. It is impossible to strictly subdivide the contents of the clinic for internal medicine between the two half-years, principally because we usually meet complex cases, in which, besides the principal disease under consideration, belonging to that part of pathology with which we are occupied in the given half-year, there are otler morbid conditions, which, although belonging to the other half-year, must be discussed as fully as the principal disease, for the sake of the correct investigation and individualization of the given case. As regurds the special therapies, we discuss the internal use of mineral waters in connection with abdominal diseases, the inhalation and climatic therapy in connection with diseases of the organs of respiration. the dietetic therapy in abdominal diseases in connection with obesity and failure in mutrition, kinesotherapy and treatment by electricity in comnection with various cases; balneothexapy at the end of the academic year, in the course of which the students have had the opportunity of olserving the various applications of hydrotherapy.

Thirdly, it is the duty of clinical instructors to constantly attract the student to participation in the clinical exereises. As far as objective exumination is concerned, all that can be seen and partly (as percussion) heard, can usually be demonstrated before the whole class. But whatever needs demonstration by palpation and anscultation would require so much time to be demonstrated before the whole class, that it is impossible to do it in the clinic of intemal diseases, and as a subject for instruction, it belongs to the propedeutic clinic ; but I nevertheless con- 
sider it necessary to always, in every case, afford an opportunity to as many students as practicable, of personally convincing themselves through palpation and auscultation of the most important data - those on the presence of which the diagnosis is based. Thus in the course of the year many acquire experience in objective examination under the supervision of the instructor (others under the supervision of the assistant, of which later): and what is most important, the whole class gains the conviction, that the diagnosis is based on data obtainable not only through investigation by a skilled physician, but through that by a beginning practitioner. In conducting the investiyation, diaynosis, proynosis and plan of treatment, as well as in the preliminary anilysis of the given case and in further investigating it, before I pronounce my conclusion, I usually look for the opinions of some of the students, and in the cases of greater difficulty I turn to the whole class, requesting any one to answer the question asked. Such a procedure not only attracts the students to participation in elinical exercises, but soon excites in them an interest toward these last.

The clinical assistants, being independent and responsible physicians, must fulfill their medical tasks, namely, to conduct the investigation, diagnosis, prognosis, treatment and observation in the presence and with the participation of the students in the clinic, strictly following the precepts of the school - that is, methodically and individualizing - thus continuing, as it were, the work of the teacher, assisting the students by exercises in fortifying themselves in the acquisition of a method and a habit of individualization, and consequently assisting the clinical institute in perfecting its aims. The personal self-advancement of the assistants - at times the future clinical instructors - as well as the treatment of the patients in clinic, gains not a little by this.

It is the lnsiness of the stulents, besides participating in the clinical exercises of the professor and his assistants, to conduct and describe the histories of diseases and to be on duty in the clinic.

How to conduet the history of a disease. - The history begins with the description of what was found on the patient's admission to the clinic: the principal complaints, the conditions and 
mode of his living, the result of the investigation into the present and past condition of the patient. Then follows the diary; namely, the daily note of the treatment and of the alterations in the patient's condition.

The description of the history of the disease, which is presented at the end of the half-year and which, with the conduct of the history of the disease, serves as a certificate of the student's diligence in erediting him with a semester, embraces everythiug found at the preliminary investigation, and then based on this the diagnosis, prognosis and plan of treatment, and finally the diary in a connected, historical statement. In cases of autopsies their results are noted down. Epicritical notices are desirable, but not requisite, as not every history of a disease will present a reason for them.

Service in the clinic enables the student to freely observe pictures of diseases and become acquainted with the application of various methods of investigation, as well as of treatment (as, for instance, hydrotherapy, massage, electrotherapy, etc.). 

CLINICAL LECTURES. 



\section{CLINICAL LECTURES.}

FIRST CASE.

\section{LECTURE DELIVERED SEPTEMBER 19, 1889.}

Gentlemen : -

We concluded last time the introduction to clinical exercises, in which we explained in what order the various departments of internal diseases would be taken up by us. At the beginning of our clinical work I present to you the first patient. He has been in the clinic since last week (from Sept. 13th), and is suffering from several diseases. It is a complex case, selected by me by reason of its being more suitable for demonstration than the others we have in the clinic at present.

The patient, a peasant, 18 years of age, when asked as to what ails him, and how long he has been sick, complains, as you see, of pains in the abdomen and loins, of diarrhoea and vomiting, dating his illness from Sept. 8th, inst.

Patient is so emaciated, pale and backward in stature and general growth (he hardly looks to be 15 years old), that we can scarcely believe that he was completely well up to Sept. 8th. And indeed, when asked abont it, he replies that he has been ailing for a long time, but began to feel very bad since Sept. 8th.

In view of this, we must begin, as we explained in our introduction, the investigation of the patient from his present condition; after this the interrogation about his past becomes easier, and the anamnestic data more intelligible.

Here is the information about the conditions and mode of living of our patient before his admission to the clinic: he lives in a village of the Tversk Government, in a healthy local- 
ity, free from malaria; his dwelling is an ordinary peasant's hut; the privy is in the barn for the cattle, - not a very cold place; he takes a hot, steaming bath once a week; does not bathe in the sea during summer; does not smoke, nor drink whiskey; drinks some four cups of hot, unsweetened tea daily; has three meals a didy (early breakfast, midday dinner, and supper), keeps all the fast-days. His food consists of rye bread. cabbage soup, potatoes, occasionally buckwheat gruel, and, except fast-days, meat three times a week. The patient is single, has lived the past year with his parents, and does not work on account of illness and weakness; in fact, goes out-of-doors but little, exercises little, and keeps his bed most of the time. Some three years before, he had been employed as an apprentice to a village tailor, living under the same conditions as when at home; but with the exception of seven hours for sleep and the time taken up by the three meals, he would work the whole day in the close and vitiated atmosphere of the peasant's hut; leisure for a daily rest or a daily walk was not allowed: this could be had only on Sundays and important holidays; although he had at his disposal seven hours for sleep while working, and as much time as he wanted when living with his family. the patient had suffered for the last few years so constantly from abdominal pains that he can sleep but three hours out of the twentyfour; besides, he sleeps on almost naked boards (on a bench).

Even from this collected information a great deal will become clear.

It is only by the assistance of considerable muscular activity out of doors that such heavy food as our patient used can be readily digested: whereas, his only bodily exercise consisted in this: that he would sit a whole day in a close room, in one position - that of a tailor at work, - with bent body and crossed legs, his left hand holding the garment. while the right one is engaged in monotonously plying the needle. Not to mention other possible causes, such conditions alone are sufficient to give rise to a disordered digestion. The disturbed sleep extending over several years has naturally tended to contimuously undermine his health, etc. 
Now as to the conditions surrounding the patient in our clinic. This latter, as you are aware, is situated in a healthy location, on a hill, and surrounded by an extensive yard and garden. The ward where our patient is placed is satisfactory. The water-closet is warm. For the six days that he has been in the clinic, he got daily two soft boiled egg's, two glasses of milk, two glasses of soup with chicken-meat well minced, and one-fourth of a pound of lread without the crust. This quantity of food was given in six portions, in the following order: 1. At about 8 A.x., two soft-boiled eggs with some bread; 2. Two-thirds of a glass of soup and some bread; 3. A glass of milk and some bread; t. Two-thinds of a glass of soup with some bread; 5. A glass of milk with bread; and 6 , at about 8 P.x., two-thirds of a glass of soup with a little bread. For a drink, only tea which is neither strong nor hot, three to four glasses (not full ones) a day. unsweetened, * for which two small lumps of sugar were given during the whole day.

For treatment the patient diank daily two half-glassfuls (a glass contains about 8 ounces. or about 16 tablespoonfuls) of natural Ems water (Kessellorunnen), warmed to $30^{\circ} R \cdot\left(99.5^{\circ}\right.$ $\boldsymbol{F}$.), the first in the morning, one hour before the first meal, and the second, one hour before the fourth meal (the second soup); some five minutes after the first and the fourth meals, he took a tablespoonful of decoction of condurango ( 3 ij to $₹$ iij), with five drops of the tincture of nux romica; after each soup, that is three times a day, the patient was given a tablespoonful of strong white Crimea wine; he was given in the course of the six days, for severe abdominal pains, codeine. gr. $\frac{1}{4}+$ gr. v sacchari albi per dose. His abdomen was all this time wrapped in a double layer of flannel. The patient himself preferred lying, and he was also advised to avoid in any way becoming fatigued, - to repose, and to walk and sit but little. Being literate, the patient was allowed to read a little.

Status to-day, September 19, 1889.-- Appetite, very poor on patient's admission into the clinic, is somewhat better now; his former thirst, although not great, has disappeared. Tongue, somewhat dry on admission, is now normal. Teeth healthy:

* The sugar is bitten off with each sip of tea. 
swallows freely. Before armission, he always suffered from a constant feeling of heaviness and pain at the pit of the stomach, which would become aggravated considerably after eating, when there would appear belching of gases and of sour liquid, with a feeling of burning, as well as nausea, and lately almost constant vomiting. The ejecta usually contained a moderate quantity of the gastric contents; at one time some bloor (of this latter in the anammesis). The pains and heariness, as well as the belching and heartburn, have now considerably diminished; nausea is rare, and there has been no vomiting since his sojourn in the clinic. On his admission the patient had loose bowels, some four to five morements a day, little at a time (about a glassful for 24 hours), watery, mixed with mucus, but of normal color and without blood. For the last two days the patient had two movements a day (the whole quantity for one day also about a. glassful), less watery and without mucus. He had, on admission, frequent paroxysms of severe pain in the abdomen every day, also at night, accompanied often by rumbling, and relieved by a movement of the bowels or by emission of gas per anum. These pains are at present of rarer occurence and much less severe. We observe no icteric phenomena, nor does the patient acknowledge any in the past. Emptying of the bladder takes place regularly; quantity of urine $1400 \mathrm{ce}$. in 24 hours; spec. gr. 1.01t, of a strong acid reaction and pale color; when fresh it is limpid, but soon becomes turbid; microscopic examination has shown this turbidity to consist exclusively of crystals of oxalate of lime, with some very few epithelia of the urinary bladder and a residue of sodium urate; albumen and sugar absent. Condition of genitatia normal; no history of either coitus or masturbation.

Asked as to whether he suffers from any other pains in the abdomen besides the constant pains we know of at the pit of the stomach, aggravated by eating, and the severe paroxysms of pains all over the abdomen (evidently of intestinal origin), the patient informs us that there is a constant severe pain to the left of the umbilicus, that is intensified by riding in a jolting wagon (an ordinary peasant's vehicle), and is radiating into the left groin and into the loins; also some pain in the chest (patient points 
with his hand to the lower half of the sternum and the adjacent portions of the anterior surface of the chest). This pain, as it appears from investigation, coincides in time exactly with the pain at the pit of the stomach; it too is aggravated by eating. and it has also diminished since the patient's sojourn in the clinic; it depends most likely on the same cause: disease of the stomach. The pain in the left half of the abdomen is evidently referable to the left kidney, but may certainly depend also on disease of the descending portion and sigmoid flexure of the colon.

When resting, the patient's respiration is 21 to 24 per minute; pulse normal (was weak on admission, stronger now), rate 65 to 70 ; movements, and especially rising, induce dyspncea and palpitation. Patient has never expectorated any blood, does not cough now, and cannot recollect that he has ever suffered from cough, any throat trouble, or a prolonged coryza.

As mentioned above, the patient is very pale and thin (his weight on admission amounted to $91 \mathrm{lbs}$ ). He is also very sensitive to chills. Febrile phenomena are absent: temperature lowered -36.4 to $36.6^{\circ} \mathrm{C} .\left(97.5\right.$ to $97.9^{\circ} \mathrm{F}$.) ; the tendency to sweating, from which he suffered while at home, has disappeared; the urine is pale, pulse not frequent, appetite is improving.

He slept before admission not more than three hours a day; now he enjoys six or more hours of quiet sleep, thanks to the diminution of the pains, partly to the wine and the more comfortable bed.

Condition of mind, much oppressed on his admission, is now more spirited; there are positively no signs of a morbidly exaggerated mental impressiveness ("nervousness"); on the contrary, the patient, as you see, is very quiet, reserved, and, we must add, to judge from his answers, very sensible.

He suffered before admission from headaches and vertigo; these have considerably diminished and are relieved now, as could be expected, by better sleep and improved circulation (pulse became stronger).

Asked about the pains in the back, the patient replies that the whole back aches, but particularly the loins (he puts his 
hand on the lumbar regions of the spine and the adjoining lateral parts). As the pains are increased not only by riding in a jolting carriage, but also by any movement of the trunk, they point not only to the kidneys, but also to the motor agencies of the spine (bones, muscles and ligaments). The objective examination gives us a clearer idea of the localization of the pain. There is no pain in either arms or legs, -- their movements are free; but the patient is very weak and easily fatigued. Sight and hearing are normal.

Anammesis. - It would be very desirable to be informed of the condition of health enjoyed by our patient's parents. The patient, I must say again, is so pale and emaciated, is so puny and backward in his physical development, that one finds it difficult. without further verification, to ascribe these peculiarities solely to the recent infirmities, to which the so numerous and so prominent abdominal symptoms point. One can not help thinking that his generally weak condition and the local disorders may be but an expression of a general cause (such a cause is most frequently syphilis or tuberculosis); is the patient a victim of hereditary syphilis or does he come from a tuberculous family? On inquiry. as you hear, we find that his parents are alive and enjoying good health; i. e. they do not ail generally and particularly from any chest troubles (they suffer neither from cough nor from any difficulty in breathing). Out of a family of seven children, five died in childhood; those remaining include himself and his younger brother, who is not ailing. We can, however, arrive at $n 0$ positive conclusions from this information; the fact that out of seven children five died may lead us to suspect syphilis, if it were not for the considerable mortality of children. which is unfortunately of ordinary occurrence amidst the deplorable circumstances surrounding the life of the peasant.

The patient himself has no recollection of having been sick until his fourteenth year, but remembers that he was neither thin nor weak. When fomrteen, he was apprenticed to a village tailor. Of the severe life with the latter we have already spoken. After two years of such a life his health began to fail: there appeared belching, heartburn, nausea, heaviness at the pit of the stomach, and, later, pain that kept continually in- 
creasing; also vomiting, at first rare, then more frequent; difficult stool; patient grew emaciated, became weak and sleepless. After another year's work in such a condition, patient became very thin and very weak, had to give up work altogether. and over a year ago (in Nay, 1888) returned home, where, although idle, he still lived under much the same circumstances as before, without any treatment, so that his health was not at all improved for this last year. Some six weeks ago his former constipation gave place to diamhoea, — slight at the begimning. Eleven days ago, September 8th, the patient having eaten some mushrooms, cabbage and cucumbers, he soon experienced severe pains in the region of the stomach, then there set in profuse vomiting of a dark-colored mass (the first and only time), that resembled snuff dissolved in water, and somewhat later, accompanied by acute pains in the abdomen, he had some six loose stools of the color of tar (indicates the possibility of the presence of blood in the evacuations per os and per amum). Since then, and up to the time he entered the clinic, September 13th, he suffered continuously from daily vomiting, diarrhcea and aggravating pains at the pit of the stomach and in the abdomen generally. He does not recollect the exact time when he first felt pains generally in the back and particularly in the loins, as well as in the abdomen to the left of the umbilicus, but thinks it must be long ago.

It were now time to enter upon the final objective examination of the patient; but the lecture is at end, and without finishing this examination and establishing a final diagnosis, I must take up the treatment of the patient; I must also explain the necessity for such a procedure in certain cases in clinical teaching: The case before you is the first that we examine jointly, and besides, as far as can be seen already, it is a complex one. To judge by the information obtainerl we must expect disturbances of various parts and functions of the organism : of the stomach, intestines, kidneys, of nutrition. Either of these two circumstances (the more so when both together) necessitates a lengthy preliminary analysis that would require many lectures; and as we have but three lectures a week, it would take us too long to complete our preliminary analysis. The patient, meanwhile, 
cannot be left without treatment: this last begins from the time he enters the hospital, so that considerable changes may have taken place in the condition of our patient by the time we finish our extensive examination. Let us assume, that he will improve, will regain his health, will be cured; what profit can you derive from the fact that $I$ was able to cure the patient, if you yourselves will not acquire the skill to do it, if you will not be constantly informed as to how such a desirable consummation was attained, and if you will not follow with me the treatment and learn why we use certain remedies, and why we replace them with or add others to them, as the changes in the condition of the patient may demand? I therefore, in such cases, conduct simultaneously the analysis of the patient and the course of treatment, and devote one part of each lecture to a continuation or conclusion of the primary analysis, and the other to observation, that is, to the discussion of the changes that take place and to the direction of a further course of treatment.

It is true that without arriving at a positive diagnosis it is impossible to fully explain just why a certain course of treatment is to be adopted. But, as you know from my introduction to clinical work, and as you see it verified in this case, a diagnosis is arrived at, not immediately at the end of the investigation, but gradually while this is being conducted; if we have not as yet established a positive diagnosis, we nevertheless can not say that nothing in the condition of the patient is clear to us. This enables me, with the aid of the information obtained by you from the systematic study of internal diseases, to gradually acquaint you, although at first not fully so, with the treatment adopted even before the conclusion of the primary analysis, and thus to attract your participation from the very beginning in such an important matter for you as conducting treatment and investigation. The farther we advance in our primary analysis and generally in our familiarity with the case, the easier will such a method of teaching become; some such method, however, is inevitable. From my long experience, I will add here, that such a method is not only free from inconvenience, but is indeed useful; the teacher must at times repeat himself — repeat the same subject twice; but first, such a repetition is usually a 
more lucid explanation of the subject, and forms an addition to one's knowledge; and secondly, a repetition of those impressions and information most important for the practical physician that should always be fresh in his mind - will result in this, that all the impressions and information will not be superficial and ephemeral, but will implant themselves lastingly in the memory of the students. It is well understood that, when in analyzing the patient's condition the plan of treatment will have been discussed, all the gaps will be filled, and whatever could not be clearly understood in the begiming will be explained then.

When beginning the discussion of the treatment, as well as the hygiene of the patient, we must first explain to you what has already been done for him since his admission into the elinic, and what has already produced, as you have heard, a perceptible improvement in the patient's condition. It is hardly necessary to explain the importance of a satisfactory habitation, a warm water-closet, and of flannel around the abdomen of a patient subject to acute abdominal pains and diarrhea ; also that he was given food often and a little at a time, - almost exclusively of a semi-liquid nature, and, I may add, lukewarm (tea, milk, soups. everything at the temperature of freshly drawn milk). I shall speak however, more fully later on of such an important subject in any disease, - and especially for our patient, — as diet. You understand the indication for codeine for pain. The chief indication for the wine was the feebleness of the heart's action as evidenced by the weak pulse and by dizziness; besides, the wine he got was strong and not sour, such being useful in diarrhora. I will also remind you that the rapid combustion of the alcohol of the wine in the blood protects from combustion the tissues of the body, thus checking the tendency to exhaustion. To be sure, such effect is inconsiderable, in view of the small quantity of wine the patient gets; but taking into consideration his extreme emaciation and the impossibility, on account of disease of the alimentary organs, of rapid improvement in nutrition. even such a trifling advantage is not to be neglected.

I will not stop at this moment to explain the indication for the Ems-Kesselbrumnen mineral water. I know, from years 
of experience, that the students attending the clinic are informed theoretically, by their text-books, that such and such waters are used in such and such diseases; while the systematic study of internal pathology teaches them that for such and such diseases such and such waters are ordered; but I also know that they are completely at sea as regards the clinical, that is, the practical, part of balneotherapy - lacking as they do the ability to select, for a given case, from several, often many, waters recommended for a certain diserse, the one most suitable for the case, as well as the technical skill in the use of such mineral waters (how much and at what times to be taken, etc.). But then I consider it the duty of the clinician to familiarize the students with this subject; and therefore, from almost the very beginning of my clinical practice, appreciating highly the great importance of mineral waters as an agent of gastric therapy, and not of that alone either - I have considered it my duty to see to it that the students acquire skill in selecting mineral waters, and that they become accustomed to their practical use. With this in view I utilize the first case, which presents an indication for the use of mineral waters; but such a case must be clear in all its peculiarities, and must be fully diagnosticated. Thus only after having established a positive diagnosis and plan of treatment will I be able to show you the indication, in our present case, for mineral waters, - for Ems-Kesselbrumen in particular, - its quantity and the time of its administration (one hour before meals, etc.). Once this elucidation is made and somewhat extended by suitable examples, no difficulty will present itself in explaining the indication for mineral waters in other cases that we may come across.

The decoction of condurango and the tincture of nux vomica are not to be compared with the mineral waters in their importance; but their use will be fully explained in the complete course of treatment later on.

It remains for us to designate the treatment for to-day. The undoubted and equable improvement of all the symptoms in the condition of the patient points to a correct course of treatment, and it will, therefore, be adhered to without alteration; but in order the sooner to put an end to the abdominal pains and the 
diarrhœa, which produce a depressing and weakening effect on the patient, I shall replace the codeine with a stronger preparation, - namely, the tincture of opium (tinc. opii simplex, $\tilde{5}$ drops in case of more or less severe pains) and a warm bath; not quite a warm one, a lukewarm bath will rather tend to aggravate the didrrhoea, consequently also the pains; a hot bath may weaken the patient, whose heart is already weak. As we aim to relieve the pains and the diarrhora without weakening the patient, he will be given a bath that will produce in him a feeling of agreeable warmth. In the determination of the temperature of such a bath we are guided by the following: the more exhausted and the more sensitive to chills the patient is, the higher must be the temperature of the bath to produce the sensation of agreeable warmth; if this sensation is induced in a healthy man by a bath of the temperature of 27 to $28^{\circ} R$. (92.7 to $95^{\circ} \mathrm{F}$.), then a sensitive patient will require one of 29 to $30^{\circ} R$. $\left(97.2\right.$ to $99.5^{\circ} \mathrm{F}$ ) and higher. We will order for our patient a bath of $29^{\circ} R$. $\left(97.2^{\circ} \mathrm{F}\right.$.) so that, in case he feels chilly, we may be able to at once raise it to a higher temperature by adding some hot water.

\section{LECTURE OF SEP'TEMBER 20, 1889.}

The patient's condition. The gastric and intestinal pains were relieved (opium was needed only once), but he had one loose stool; there is also less pain in the back. The bath at $29^{\circ} R$. $\left(97.2^{\circ} F\right.$.) did not seem comfortably warm to the patient, but when its temperature was raised to $30^{\circ} R .\left(99.5^{\circ} \mathrm{F}\right.$.) he felt well and remained in it for ten minutes. Was somewhat weak after the bath, but slept well-longer (seven hours) and sounder than before. Feels himself generally better to-day.

Let us now turn to the unfinished part of our joint investigation of the patient, to the objective examination. The results of this are as follows:

Inspection of the whole body. - On the back we find the characteristic scaly eruption of pityriasis versicolor, the microscope 
shows the presence of the parasite in the scales causing the eruption (microsporon furfur), so generally met with in emaciated persons, and particularly in those suffering from or predisposed to tuberculosis. Extreme emaciation; skin thin, with almost no subcutaneous fat. Cervical, axillary and inguinal glands normal.

Chest - flat and generally poorly developed; scapula stand out. Percussion and auscultation elicit nothing abnormal in the respiratory organs, the heart and the great thoracic arteries.

Abdomen, on inspection, seems to be sunken, and equally so on all sicles, also in the region of the stomach (at the pit of the stomach and in the left hypochondrium).

Palpation of the abdomen. - I must first make a few remarks concerning the method of palpating the abdomen, as I practised it before you, and as I usually do it, without going into a particular discussion of this method of investigation, which is the business of the propedeutic clinic, $i$. e. of practical semiotics. Palpation of the aldomen must be conducted with the utmost care, so as not to cause harm to the patient nor obtain misleading data, and not to induce, by rough manipulation, pain in localities where there is none. I will also add that abdominal palpation should be practised as infrequently as possible, as even the most careful examination may react unfavorably on the patient; and moreover, the less frequent the palpation, the more appreciable become any changes in the data obtained by this method of investigation. I first determine the presence or absence of cutaneous hyperasthesia. I then proceed to palpate the patient as he is usually seen, in the lying position, on the back, at first on the median part of the abdomen, from the ensiform cartilage down to the os pubis, then laterally from the hypochondrium to the inguinal region. I always make a double palpation - first superficially, and afterwarks a deep one down to the posterior wall of the abdomen if possible. From the data obtained by such a method of examination (always obligatory, if the inquiry as to the condition of the abdomen demands it) it may be learned whether it is necessary to resort to other means, such as palpation of the abdomen with the patient in the lateral or in any other position, or to some special method 
of palpation. I would also remark that in the case of a doubtful, not clearly defined tenderness to pressure in some part of the abdomen, I palpate, for comparison's sake, the corresponcling locality on the opposite side, so as to aroid a possible error that may be caused by an unequal pressure. I palpate the diseased side somewhat more lightly and the healthy side somewhat stronger. If this should indicate a greater tenderness on the former, there is, of course, no doubt of its presence there.

I will now recapitulate the data obtained by palpation: cutaneous hyperesthesia absent; quite strong compression of the folds of the skin in rarious parts of the abdomen do not cause any pain; patient says he feels the forcible squeezing. but experiences no pain. From the upper edge of the liver and spleen, located while percussing the chest and by the palpation of both hypochondria, it becomes apparent that the dimensions of these organs are normal. The kidneys are inaccessible to palpation, either with the patient in the lying or in any other position. The region of the spleen is not sensitive to pressure (pressure under the left margin of the ribs causes no pain). There is considerable tenderness in the region of the stomach (it was stronger on his admission) and some tenderness on the side of the liver, sharply defined by the region of the gallbladder; the remaining portions of the right hypochondrium are not tender. Taking into account all these data, together with the dyspeptic symptoms learned by interrogating the patient, and the simultaneous relief of these symptoms and of the pain in the region of the stomach, we must accept the stomach as the seat of this pain, and not the left lobe of the liver. As you will recollect, we found on inquiry, that the patient had severe intestinal pains, which have now diminished. Palpation has shown the region of the descending colon and of the sigmoid flexure to be sensitive, while that of the creum and of the ascending colon were not sensitive - a good sign, indicating, in the presence of diarrhrea, of a frequent desire to defecate, and of mucus in the evacuations, a simple catarrh of the large intestine. Tuberculous ulcerations of the intestines, which one might suspect in view of the extreme emaciation of the patient, 
his weak constitution and the eruption already mentioned, are usually located at the termination of the small and the beginning of the large intestines, thus causing tenderness in the right, and not, as in our patient, in the left, iliac region. In the other portions of the abdomen, that correspond to the position of the intestines, there is no sensitiveness on palpation. There is, to be sure, some very slight tenderness along the median line of the abdomen, but it must evidently be referred to the abdominal aorta, which is surrounded by a network of nerves, as the pain is felt by the patient only along the course of that artery, and only when its pulsation is perceptible through palpation. This sensitiveness to palpation in the vicinity of the abdominal aorta is of common occurrence in exhausted and irritable subjects, as our patient had become from long-continned insomnia. Thus also the considerable painfulness on the left side of the abdomen, which is sharply defined by the locality of the left kidney and its ureter, is evidently referable to these two organs. and not to the intestines, the more so because. while palpating the left kidney from the front the patient complains of pain simultaneously in the left loin. Sensitiveness on palpation, although in a lesser degree, is also felt in the region of the right kidney and ureter. We must exclude the participation of the peritoneum, that is. the presence of peritonitis, in the production of the abdominal pains : the character of the pains (which are neither as severe nor as sharp as in peritonitis), the freedom of motion, and the absence of febrile conditions, render it improbable.

You may see, therefore, that a cireumstantial inquiry and an exact objective investigation make it possible, notwithstanding the existence of many causes for pains and the great variety of them dependent thereon, not to be confused by this variety, but to exactly determine the sources of the pains, the affected organs. As such we find, in this ease, the stomach, part of the intestinal canal, both kidneys - particularly the left one - and the gallbladder.

Back. - On his admission the patient complained of pains all over the back, particularly in the loins, aggravated by movements of the spine. Objective investigation has shown then 
the absence of hyperesthesia cutis, but on pressure there was tenderness in the prominent bony portions (the spinous processes of the vertebre and scapulæ), and some in the muscles of the spine and a few intercostal nerves; directly and acutely painful was the region of the left kidney, that of the right one less so. At present the patient does not complain of pain all over the back, but only in the region of the loins, especially on the left side. By objective examination we find absence of cutaneous hyperæsthesia, the tenderness in the bones. muscles and nerves of the back disappeared, with the exception of some in the lumbar region of the spine, the motions of the spine became freer, but the sensitiveness in the kidneys, especially the left one, has diminished but little. Considering the fact that while at home the patient slept on almost naked boards, so that his back was subjected to almost continuous traumatism, and that with the removal of these latter (the patient reposing on a soft bed while in the clinic) and with the improvement in his nervous condition, thanks to a more quiet sleep, relief of the abdominal pains, warm bath and the like, the pains over the whole back have rapidly diminished and almost disappeared, - we must refer these pains to the abovenamed external parts of the back which suffered from traumatism. We must, of course, exclude any affections of the spinal cord or it membranes owing to the absence of any symptoms of such affections. The pains in the back in the region of the kidneys are unquestionably to be seferred to the kidneys, as is evident from the data obtained through inquiry and objective examination of the abdomen.

The tendon, knee and other reflexes are normal.

I have already had occasion to olserve that the weak constitution and extreme emaciation of the patient, in conjunction with the pityriasis versicolor, excite a suspicion of the possibility of his suffering from tuberculosis. Of the organs affected in our patient, we would in all probability expect the intestines and the kidneys to be the seats of tuberculosis; we therefore had the freces and urine examined for tuberculous bacilli; but in neither of them was their presence demonstrated.

Having finished our investigation, we should now pass over 
to the positive diagnostication of the case; but in accordance with the method of teaching adopted by me for cases requiring a lengthy discussion, as I explained last tine, I must devote the rest of the lecture to the treatment required by the condition of the patient to-day.

Wre will certainly not change the hygienic conditions. Patient's appetite is improving; he would fain eat more than he is allowed; but for the sake of caution we will wait another day or two before increasing his quantity of food. The Ems water, the bitters and the wine we will continue in view of their favorable action.

In the treatment of the diarrhora one addition is to be made: notwithstanding the improvement of other symptoms, the evacuations continue fluid; this is naturally detrimental to the condition of our emaciated patient and hinders his improvement.

What are we to add to our treatment in order to overcome this difficulty? If the evacuations up to now had been not only fluid, but also frequent and contained mucus, - which would point to a continuing catarthal condition of the large intestine, then it were better to introduce some therapentic means per rectum - as tannin suppositories. But the frequent tenesmus has ceased, and there is no mucus in the freces; so that the continnous fluid evacuations point rather to a poor absorptive power of the small intestine, depending, perhaps, on a catarrhal condition of the same.

The introdnction of remedies per anum in diseases of the small intestine is recommended as yet only for special conditions (as, to destroy microles, to render ptomaines harmless, and to remove accumulated contents), in cholera, typhoid fever and other diseases ; it remains yet for practice to prove the usefulness of such a procedure; but in such cases, as in that of our patient, of an exidently simple catarrh, practice speaks for the introduction of remedies per os.

What then shall we prescribe for our patient per os? Shall we increase the dosage of opium? No. Opium is indicated (always after a preliminary emptying of the bowels) in more acute cases of catarrh; the chief indication for its use is intestinal 
pain. In chronic cases, where the intestinal pains have already disappeared, opium seldom cures the diarrhoea, and moreover, it is harmful, masmuch as it tends to derange the appetite and weaken the musculo-nervous apparatus of the intestines. The intestinal pains in our patient are very slight, and the appetite is just beginning to improve, while the nervo-muscular apparatus of the intestines is very weak, as is evident from the poorly developed muscular system generally and the constant constipation during the whole course of the disease, until the present diarrhoa. We will, therefore, give him the tincture of opium as heretofore only in the case of more or less acute intestinal pains which disturb the patient: five drops per dose. while for the diarrhua proper we shall prescribe tincture of coto. at first in ten-drop doses in a spoonful of warm water. three times a day, in the intervals between meals, and increase the dose if necessary. Why do we give just this, and no other remedy? First, in cases like the present one, tinct. coto has proved to be useful, and I can testify to this from my own experience; and secondly, because in this case tinct. coto seems to be more suitable than the other remedies recommended for diarthoa, as, for instance, to mention the principal ones. tamnin, lead acetate. silver nitrate, and bismuth. Tannin and lead acetate derange the appetite. while silver nitrate and bismuth subnitrate are less reliable, in my experience, than is tinct. coto. Besides, the simultaneous use of mineral waters, which is necessary for our patient, as you see by the success attending the treatment, and as you will find later from the detailed explanation of our therapy, makes it easier to bear tinct. coto than tamin and the above enumerated metallic salts.

Shall we continue the warm baths? Patient slept particularly well last night after a warm bath - a quiet, sound sleep. As a patient's sleep is better the less the pain he suffers from. and vice versa. we may assume that the warm bath has tended to relieve the pains and at the same time to diminish the pathological processes that cause these pains. But experience teaches us that a warm bath may also loring about better sleep in another way, - - by changing the distribution of blood in the periphery of the body and in the internal organs, - in this case in the 
cerebrum: our patient is always chilly, and particularly so on sleepless nights; but last night he was not chilly. The favorable action of the warm bath on the pains and on the sleep is of the greatest importance to our patient, who has so long suffered with insomnia. Withont improving the sleep it is impossible to bring the nervous system, and consequently the whole organism, to a normal condition. The good effects of a warm bath are the more to be appreciated, as it does away with the necessity for the intemal administration of hypnoties, which, besides inducing sleep, exert other and even harmful influences either on the alimentary or other organs or on the nerrous system itself.

But the warm bath has also its drawbacks. The patient felt weak some time before and after leaving the bath; it was at the same time evident, from the feebleness of the pulse, that the general debility was a result of weakened heart-action, which was manifested quite clearly in the cerebral cirenlation. To resort to frequent warm baths. subjecting the patient repeatedly to such weakening of the heart's action, temporary and evanescent though it be, is certainly hardly desirable. Besides, the frequent use of the warm bath may establish a predisposition to colks, which must especially be taken into consideration in view of the approaching winter and the severe conditions of life surrounding our patient. We will therefore order for him baths not daily, but, as circumstances may require, one every second or thind day; we shall continue using them as long as we obtain favorable results. We ma expect that, with his improvement, the patient will be able to bear baths of a lower temperature, $29^{\circ} \mathrm{F} .\left(97.2{ }^{\circ} \mathrm{F}\right.$ ) or even $28^{\circ} \mathrm{R} .\left(95^{\circ} \mathrm{F}\right.$ ); in case of his complete recovery, he will have lukewarm baths, and later cool rubbings to diminish the susceptibility to cold.

Finally, I consider it necessary and, in the present condition of the patient, possible, to give him a general massage; I say possible in his present condition, becanse on lis admission, a week ago, the numerous pains were so severe, so aggravated by active and passive morements (as, for instance, eren when turned over very carefully). his sensitiveness on account of the insommia was so great, that he could hardly have borne the slightest massage. Now, as yon know, all the pains have dimin- 
ished, the motions are freer, and there is a feeling of comfort, thauks to the quiet sleep for the past week; the application of general massage is therefore timely. But why and for what purpose?

General massage for cases not exactly like ours, but similar, was recommended by the American physician S. I'eir Mitchell, and gave excellent results. His observations were soon verified and his method of treatment was accepted everywhere. I personally can attest to its undoubted usefulness. and in certain cases, I think, it cannot be replaced by any other method. The idea occurred to Dr. Nitchell in connection with a case like the following: a woman, the mother of a numerous family, exhausted by pregnancies, births, and sometimes by lactation, begins very decidedly to lose her health; the appetite and nutrition are impaired. she loses sleep, grows rery thin and weak; with these there are usually associated disturbances of the sexual and nervous functions: too frequent and weakening losses of blood during menstruation, excruciating pains in the head and back, palpitation. despnote etc. The usual remedies such as arsenic, iron, the bromides, forced feeding and others - have no effect, principally because the houtsehold cares and the children require intense physical and mental activity and do not leave sufficient time for sleep, poor though it be, but tend to keep up the disturbance of the health. It is in such cases, that Dr. Weir Mitchell, leaving medicines aside (later, when there is improvement in the patient's condition he administers iron, and in very large doses at that), attacks directly the cause of the disturbed health, the ummatural physical and mental exertion and the fatigue resulting therefrom; he prescribes for the patient absolute rest-physical (remaining in bed six weeks at first withont getting up at all) and mental (complete abstinence from household duties for the same length of time, and even, as far as possible, to be kept free from all disturbing impressions; when not being given massage, rubbing and frequent nourishment. the patient is allowed to listen to some light reading, but is not permitted to read herself). In the absence of physical activity, the only means of inducing a constantly good appetite and sleep - the first requisites in the 
improvement of mutrition and of the nervous system - she is ordered general massage.

The general idea of Mitchell's method of treatment being clear, without going into particulars, for which this is not the time, let us tum to the use of massage in the case of our patient. We were to prescribe for him bodily rest — to rest in a lying position, not only because any movement would produce pain, and does so to some extent even now, but also on account of his extreme weakness and fatigue. I may as well add here, that mental rest is also secured for the patient: his youthfulness, particularly in connection with his sojoun in the clinic, preclude any care; his only anxiety - about his health - we succeeded in allaying by promising a complete restoration of health. In the absence of physical exercise we will give him general massage over the whole body except the head, the condition of which presents no indication for massage, and the abdomen, in which there are so many tender and painful organs, that even very careful massage mat cause harm. Besicles securing a good appetite and sleep, massage will be useful, firstly, in diminishing the chilliness of the patient's body, by inducing an equable distribution of the peripheral circulation. and through this of the circulation of the rest of the body ; secondly, by removing the remaining pains in the muscles and other parts of the back, it will chiefly tend to better nutrition and to develop the muscles and the other organs of locomotion. which circumstances, in comnection with the general improvement in his health, can not but exert a favorable influence on the future of the whole organism : thirdly, by becoming the means of removing the important distubance of nutrition, as evidenced by the oxaluria (the constantly abundant urinary sediment consists almost entirely of oxalate of lime). The exact nature of oxaluria is not known, but its close relation to diabetes mellitus is probable; the therapy of both has very much in common, and we are therefore led to think that general massage, so useful in diabetes, will also be so in oxaluria.

The application of massage in our patient we shall alternate with the use of baths: two or three days massage and then a bath. 
We treat the pityriasis versicolor by rubbing the affected parts with cotton-wool saturated in a solution of corrosive sublimate (1 to 1000$)$.

\section{LECTURE OF SEPTEMBER 22, 1889.}

Condition of the patient. - Appetite better, patient begs insistently for more food; the dyspeptic gastric phenomena, with the former quantity of food, consist only of a slight eructation devoid of pain; stool once a day and of thicker consistency; intestinal pains very slight; the pains in the left kidney are diminishing, but not to an equal extent. Respiration less frequent ( 20 against 23 to 25 as previously), eridently on account of the diminished abdominal pain, and partly also because of a sounder sleep. Pulse, 60, stronger than formerly; patient's weight $92 \mathrm{lbs}$. (one pound more than before) : sleep better always seven hours and quietly; pains in the back diminished. He bears massage very well; feels better than usual at the time of, and a few hours after the massage, - at times he sleeps for about one-half hour after it. There seems to be no call for a change in the treatment, while the quantity of food must be increased.

Diagnosis. - Let us now turn to positive diagnostic conclusions, while we follow the same method as we have already explained in our introduction to the clinical work - as we did in our investigation.

I have already had occasion to observe that the patient's weak constitution and extreme emaciation, as well as the presence of pityriasis versicolor, excite a suspicion of tuberculosis or syphilis. From the data obtained in regard to his parents, we could not, as you will recollect, decide as to his coming from a tuberculous family, nor was there foundation enough for suspecting hereditary syphilis. As far as the patient himself is concerned, everything contradicts his suffering from either syphilis or tuberculosis. As regards the intestines and the kiclneys, those organs in which we would suspect the existence of tubercles the soonest, the careful examination 
of the freces and urine for the nine days of his sojourn in the clinic failed to elicit anything to justify the suspicion, while the continuous and comparatively rapid general improvement, as well as the improvement of these organs. speaks decidedly against their being invaded by tuberculosis. Nothing indicates the existence of syphilis: the hair on the head, quite thick, although falling out a little in the course of the ailment, does so on account of the patient's lack of nutrition, but there never was a decided falling of the hair ; teeth healthy; outside of the pityriasis versicolor there is no other eruption, nor is there any scar from any previous ulcerations; there are no evident traces of any former affection of the mucous membranes; lymphatic glands are normal, no history of any obstinate chronic catarh in the past, nor is there one at present: no pronounced nocturnal pains in the tract of the tibice or other bones, no weakening of the memory.

IVe must therefore conclude, that the patient has neither syphilis nor tubereulosis, but that, in his present condition, he is predisposed to this latter, or in fact to any other severe disease, as for instance. chronic nephritis (in view of the decided oxaluria and the evident renal colic) and others.

The Stomach. - This organ, which seems from the anamnesis to have been affected the first of all, presents a complete picture of a chronic catarh: there developed consecutively anorexia, heaviness in the stomach, gaseous ernctation, sour eructation with heartbun, pain in the stomach, nausea and vomiting, constipation; all these symptoms were aggravated by eating. But will the coineidence of the gastric symptoms in our patient with the type of a chronic gastric catarrh be sufficient proof for us to establish a final diagnosis of the latter without further discussion? Certainly not: first, because the disease is of such duration and has so weakened our patient. that we are led to think of something nore serious than a chronic gastric catarih, especially as there was some bloody voniting, which does not occur in the latter; and secondly, because, this being your first and withal complex case, it must be gone into as particularly as possible, so that there may be a complete explanation of all points, not hindered by any doubts (the more 
so, as all the gastric diseases present many similar symptoms). With this object in view we will call in the aid of differential diagnosis.

Does not the patient suffer from nervous gastric dyspepsiu? This may call into existence the same symptons as a gastric catarrh: heaviness and pains in the region of the stomach, eructation and heartburn, nausea and vomiting. It chiefly differs from catarrh in that in the latter all the above enumerated symptoms are constant; whereas in nervous dyspepsia, in uncomplicated cases, they are liable to sharp changes in accord with the changes in the nervous condition generally (uncomplicated nervous dyspepsia is rarely seen without a general disturbance of the nervous system); if the nervous condition is not normal. then even small quantities of very light food will tend to produce the dyspeptic phenomena; but so long as the condition of the nervous system is good, then even abumdant quantities of heavier food will be digested without any inconvenience; besides. these changes may occur rapidly, daily. 'The gastric contents in cases of catarrh contain mueus and, according to the investigations of our clinical assistant, Dr. Popoff, conducted in the laloratory of Prof. Babuchin, also lencocytes. whereas neither is present in nervous dyspepsia. But all this refers to prere nervous dyspepsia, which is not seen quite often; if continued for a long period. it will become associated with a catarrhal condition, which is partly caused by it. Pure gastric catarrh without simultaneous symptoms of nervous dyspepsia is observed more frequently; but the most frequent cases are probably the complicated ones; viz., catarrh, and at the same time, to a greater or lesser extent, nerrous dyspepsia. The presence therefore of mucus and lencocytes in the gastric contents indicates only a catarrh, but at the same time does not exchude the possibility of a simultaneous nervous dyspepsia. Turning now to our patient. the total absence of the above-mentioned sudden changeability in the gastric symptoms. previously (as I learned in my inquiry) and before us in the clinic, refutes the idea of his suffering from nervous dyspepsia. Later, when we come to examine the general condition of his nervous system, we will find proofs to justify such a conclusion. 
Does the patient have an atrophic gastric catarh? Did not the long continued gastric catarh induce an atrophy of the gastric glands, which would result in a diminution, or even in a suspension, of the secretion of hydrochloric acid and pepsin, i. e. in the impossibility to digest albuminous bodies? The rapid diminution of the gastric symptoms in our patient since his admission to the clinic and their total absence at present while digesting a goodly quantity of largely nitrogenous food (as eggs, soup with broken-up meat, milk), speak decidedly against it.

Does our patient suffer from gastric cuncer? The patient's age and the long continuance of his trouble certainly contradict this supposition, but still not unconditionally. Cancer is possible even at his age; and as far as the long duration of the disease is concerned, there might at first be a chronic eatarrh, while the cancer could appear later. But against cancer speak positively the same data as against atrophy of the gastric glands: rapid improvement of the gastric symptoms and the unimpeded digestion of a tolerable quantity of food.

We can not be so positive with regard to ulcer of the stomach. The apparently bloody vomiting and the frequently occurring (especially before admission to the clinic) attacks of severe pains in the abdomen, accompanied by vomiting, speak for it. The fact that this bloody vomit happened only once in the long run of the disease, and even then only after he had eaten a considerable quantity of mushrooms, speaks against ulcer. There are known cases where the use of mushrooms, as a food, was followed by a bloody vomit. The attacks of acute pain in the abdomen accompanied by vomiting might be ascribed to an exacerbation of the gastric eaturh, and also to symptoms of liver and kidney colic (of which I will speak later). Although the rapid improvement in the gastric symptoms and their absence during the digestion of a goodly quantity of food do not speak as decidedly against ulcer as against cancer and atrophy of the glands of the stomach, still they make the existence of an nlcer in our present case hardly probable, because even if improvement and return to health is possible in gastric ulcer, it could not be so decided in such a short time.

It is beyond doubt, then, that our patient suffers from a chonic gastric catarh. 
In a chronic gastric catarh the mucous membrane of the stomach is found to be hyperæmic, thickened and covered with mucus. As regards the microscopic appearances, Dr. Popoff"s investigations * give the following results: there is observed everywhere a hyperplasia of the tissues of the mucous membrane, particularly of the connective tissue, a mass of leucocytes and mast-cells; the epithelium is not only not detached. but is in a condition of active karyokinesis, while its cells are enlarged and full of mucus: the number of parietal cells is increased and the process of karyolinesis is olserved in some: then there are a great many chief cells and also a number of cells which it is difficult to distinguish as belonging to either one or the other (Nussbaum's cells).

As regards the hydrochloric acid and pepsin, the investigations of Dr. Popoff, both from experiments on animals and from observations on patients, show that in the condition of chronic gastric catarrh these substances are secreted in quantities sufficient for digestion, so that the peptic (digestive) power of the stomach remains unimpaired, except at the period when fatty degeneration and atrophy of the gastric glands have aheady begun. Where. then, one may ask, do the dyspeptic symptoms in catarrh come from? The causes of these are: 1. weakened gastric morements, (as evidenced by the insufficient emptying of the stomach, that usually occurs in gastric (atarrh) which prevent the ford from being intimately mixed with the gastric juice (this may also be caused by the mucus). 2. As Dr. Popoff assumes the numerons microbes of the stomach, which remain in it. when the latter is in a catarnal condition, for a longer time on account of being surrounded by the mucus corering the wall of this organ, and also because of the shnggishmess of the gastric movements. The classification and study of these microbes in the future will surely shed some light on their possible rôle in causing the dyspeptic phenomena in gastric catarrh, as well as their part in the origin of the catarrh itself. 3. Finally, the addition to the catarh of a nervous dyspepsia, - of prssible changes of nervous origin. in the secretion of the gastric juice. However that may be, the contents of the stomach in a" catar* In his doctoral thesis, "Catarrh of the Stomach." 
rhal condition contain an abnormal quantity of gases and acids; then there is besides, as already observed, an insufficient emptying of, and at times even a stretching of the stomach by the accumulated contents, and as a result of the retarded passage of the gastric contents into the intestines, there is constipation. This stretching of the stomach, quite often met with, but usually yielding readily to correct treatment, must not be confounded, as is often done, with another, a much less frequent but more obstinate and difficultly removable condition of the stomach, viz., dilatation of the stomach, as it is observed, for instance, in diseases of the gastric pylorus, that prevent the passage of the gastric contents into the intestines.

Does our patient suffer, besides the gastric catarth, also from stretching of the stomach? The data obtained so far fail to verify this, at least for the later period of the disease : neither on his admission nor at present can we notice the least distention in the left hypochondrium; the attacks of vomiting, before his admission, with the catarh in a condition of exacerbation, were of daily occurrence and the vomiting non-abundant; whereas in the condition of gastric distention, vomiting is abundant in quantity, but of rarer occurrence. But it is principally the rapid improvement in the appetite that speaks against gastric distention, because in the latter condition, as long as this distention is not removed, as long as the stomach is not emptied (for this. lavage is best), there is anorexia. You will observe, that in discussing the question as to whether our patient suffers from a distended stomach or not, I do not mention the so-called gastric splash, as I do not consider this a reliable symptom of distention of the stomach; it is true that it usually accompanies distention, but may be found to take place also in the absence of this condition, even in perfectly healthy people; it is even probable that it may occur also in the intestines, at least in the large one. It were unreasonable, in view of the unreliability of such a symptom, to attempt to cause it in our patient, whose abdomen, including the stomach itself and the neighboring very tender left kidney, is so highly sensitive. In the discussion of cases which will present a therapentic indication for lavage, and which will later probably be brought before you, I shall touch also on the gastric splash. 
Particular attention has been drawn lately to the stomach and its diseases, to the investigation of the walls of the stomach, to the condition of its glands at the time of digestion. to the observations of the first stages of gastric catarnh, to the investigation of alterations in the secretions of the gastric juice in diseases of the stomach and in other ailments. As might be expected. the results obtained are taken advantage of in perfecting diagnosis: not only is the matter romited or that obtained by gastric lavage (where this last is used therapeutically) examined, - all this was certainly also done before; but the gastric contents are directly obtained with a riew of examining them and the introduction of sample food is resorted to ("trial breakfast," "trial dinner"). This consists in introducing into the previously emptied and washed-out stomach food of a certain quantity and quality, and later. at certain intervals. the gastric contents are withdrawn and examined: by the results of this investigation we judge of the peptic power of the stomach, of the quality of the gastric juice (whether or not it contains a sufficiency of hydrochloric acid and pepsin) and of the strength of the gastric movements (whether or not the stomach is emptying its contents regularly). Some insistently recommend such procedures in practice, and require their application almost in every case of gastric disease, claiming that diagnosis, without these, is either impossible, or at least insufficient.

Without drawing at present any general conclusions as to the importance of the above-mentioned methods of investigation, and the judiciousness of their being insisted upon in practice, let us see what data we might obtain, were we to apply these methods to our present case. whose seriousness warrants the use of any method that would give any special results. If our patient vomited or if there were any necessity for washing out his stomach, then it would certainly be singular not to examine the romiter matter or that obtained by lavage. But while in the clinic our patient did not romit, and lavage was not indicated (why it was not, is partly apparent to you from what I said of the complete absence in the patient of any signs of a distended stomach, and will later, in discussing the treatment. be made clear). IVe consequently would have to resort to obtaining the contents 
of the stomach and to the experimental introduction of food, that is, to the frequent introduction of the gastric sound. And what would we learn by this? Ire would learn, whether the food is well digested or not, whether or not the hydrochloric acid and pepsin are secreted in sufficient quantity. But we have learned this without the introduction of a somnd, and moreover with greater certainty and without any ineonvenience or harm to the patient. By introducing easily digestible food in small quantities. we observed that the dyspeptic phenomena began to diminish rapidlyand in a few days disappeared altogether: at the present time our patient, having six meals a day, not only does not observe any dyspeptic symptoms after each meal, but on the contrary soon has a desire to eat again - also a sure indication of a previously completed digestive act. Consequently. we also introduced "trial " meals, and several times a day at that and we not only learned that the secreted gastric juice is of normal quility. and that normal digestion can take place. but we also constantly keep under observation the condition of the digestion and its improvement, not by resorting to the continual introduction of the sound and to the investigation of the gastric contents, - and how inconrenient this would be for both the phrsician and patient? — but by being guided by the most reliahle criterion, natmely, the absence of drspeptic phenomena and the improvement in the appetite. with which the data obtamed by the gastric sound will stand comparison, these being trequently misleading and.questionable. Besides, these last data can only point to the condition of the gastric digestion. and it would therefore be one-sider and erroneous to judge by them - as one is often tempted to do - of the condition of digestion in general. because digestion takes place not in the stomach alone. but to an equal. if not to a greater. extent also in the small intestine.

But this is only one side of the question; let us look at the other. Does not the introduction of the stomach-tube entail hardships on the patient, - may it not even harm him? It is only after persistent persuasion on the part of the physician. that patients overcome the feeling of extreme disgust towards this procedure. As far as harm is concerner. not to speak of the unfortunate accidents in the past that were due partly to 
the imperfectness of the method and partly on account of the physcian's negligence, even now. with the most perfect method and extreme care, the introduction of the sound is often not devoid of harm to the patient. Thus, in our patient, in the presence of the exacerbation of the gastric catarrh, with which he was admitted to the clinic (daily vomiting with acute pain), the introduction of the sound might have caused a fresh attack of nausea and pain. that is, it might have aggravated the condition of the stomach, that was just beginning to improre when the patient in general and his stomach in particular were surounded as far as possible by farouble conditions. This would take place in a catarrhal condition of the stomach; the risk of injuring the patient would be greater still in gastric ulcer or cancer. The introduction of the sound, in cases of nerrous drspepsia, may also considerably aggravate the condition of the patient. More than once, at medical consultations. hare I seen serere cases of nerrous dyspepsia and general nerrous disturbance. in which the proposition to introduce the somd ras under discussion. - a proposition that in the majority of cases had to be set aside. and the patient improsed despite or rather on account of its non-introduction. It can not be denied. that the thoughtless introduction of the sound, without sufficient reasons. unfortunately quite frequent with practitioners who are carried away by novelty and fashion. deserves strong condemnation.

From the foregoing it becomes clear which method would be the more reliable in determining the condition of our patient's stomach and its peptic power, - the one that we have chosen. creating for the patient and his stomach possible farorable conditions, and remoring whatever aggravated the condition of the stomach and made digestion difficult, or the other. in which we would add to the abore the introduction of a gastric sound, that might exert a contrary influence.

Let us now consider the importance of the gastric sound in the differential diagnosis of our case. In regard to the atrophic condition of the gastric glands. the rapid decrease of the dyspeptic phenomena and the improrement in the appetite speak so strongly against it. as to obriate the necessity for using the sound. While its use with a diagnostic purpose in the case of 
cancer would not only fail to give as good results as were obtained by observation. but would even lead to vacillations in the diagnosis. In fact. the rapid decrease of the dyspeptic phenomena and the improvement in the appetite. which are not to be thought of in a case of a fully developed cancer (the patient has been ailing for a long time). enabled us to positively exclude cancer: whereas the examination of the gastric contents obtained by the aid of the sound. by showing the presence of hydrochloric acid, would lead us to doubt as to whether the case was that of a simple chronic catarn or whether cancer might not also be present; for. although in the majority of cases of cancer the contents of the stomach contain no free hydrochloric acid. appreciable under certain color reactions. still there are frequent exceptional cases. in which the free acid is found. In ulcer of the stomach the introduction of the sound, not to speak of special risk of injuring the patient. would give nothing of positive value in the way of a differential diagnosis between this condition and chronic catarh. As regards nervous drspepsia, we have said before. that the complete absence of sudden changes in the local (dyspeptic) phenomena, and as we shall see later, the general nervous condition of the patient, permitted us to positively exchule it without necessitating a supplementary investigation.

But some will say. this is all clear to me now ; but how was it at the begimning? In the begimning it was only probuble, that the patient had a chronic gastric catarth. and no other disease of the stomach: but the probability became stronger every day, so that in a few days the probability became a certainty. To be sure we had to wait a few days; but washing of the stomach. the introduction of a " trial " meal and, subsequent to this, the frequent introduction of the sound, as well as the repetition of this procedure (which would be necessary in such a serious, complicated case of long (uration), would also require rather more than less time, because. as was explained above. observation of the patient when surrounded by tavorable conditions only would clear up the matter sooner than if he were surroumded by both favorable and unfarorable (the introduction of the sound) conditions. The possibility of keeping the patient under 
observation after he had been placed among favorable circumstances, the causes keeping up the diseased condition having been removed,while strong medical agents are not resorted to before the matter is clear, - this possibility is of great value in arriving at a correct diagnosis, and consequently, also, correct therapeutics, especially in chronic cases.

Now that we have considered the value that the gastric sound and the introduction of a "trial" meal would have in our case, I can express my opinion concerning the importance of this method of investigation in its present state for the diagnosis of gastric diseases in general: only rare cases present a judicious reason for the application of this method; it may be otherwise when in the course of time the reliability of the data obtained by this method will be increased.

To avoid misunderstandings, I will add that we must not confound two different matters: it is one thing to work out a new method, which is always a matter worthy of respect. but is only a question of the personal investigations of the clinician, and not a subject of instruction for his students ; to these he must communicate only tried methods, that deserve to be applied in practice on account of their valuable results; nor must they be burdensome to either patient or physician. It is another thing to present to the students methods that are incomplete and may burden them with procedures that offer nothing new or valuable. at the same time inconveniencing the patient and leading to waste of time and energy on the part of the physician, besides distracting. his attention from facts that are important and yet easily olstainable. Many a time have I seem the fruitlessness of the labors of physicians brought up under such influences : such a physician would collect a mass of minute and worthless data (not to speak of the present time, let me recall the "titration" epidemic of sad memory, the exhaustive examination of the urine, useless for practical purposes, etc.), without knowing what to do with them: he would spend time and energy in collecting these and, not having passed through a regular clinical course, he fails to ofserve the simple, patent and nevertheless very important facts: and even if he does observe, he does not take advantage of them. and thus remains, until tanght by bitter experience, if not for- 
- ever, a finical semiotician, a poor diagnostician, and consequently an inefficient therapeutist. Such a physician prides himself on his "scientific" methorl of practice in that he applies the minutest and latest results of investigations, not comprehending that science - the highest common-sense - can not be contradictory to the ordinary reasoning, according to which we must select from a great mass of clata only what is of utility, and must resort to methods of investigation that are really necessary.

I must remind you that whatever has been said of the gastric sound refers to its use for diagnostic purposes; its application in therapeutics, where it constitutes a useful, unreplaceable method of treatment, is a totally different matter, of which we shall speak later.

\section{LECTURES OF SEPTEMBER 27 AND 29 AND OF OCTOBER 4, 1889.}

Patient's condition September 2i. - Former treatment continued; the quantity of food increased: up to the 22d the patient got two glasses of milk, two glasses of soup with finely broken up meat, two eggs, and about one-quarter pound of white bread; but now he gets besides these two glasses of milksoup with mama, one-fourth of a chicken, and another onequarter pound of bread. This quantity of food the patient takes in seven portions. The appetite continues improving and the patient craves for more food. The only dyspeptic symptom is slight retching. The pains in the stomach and on the side of the gall-bladder have disappeared entirely: pressure in these regions elicits very little tenderness. Stool - once a day, painless (since the 2.2d he has been given the opium drops only twice); evacuations thicker. not liquid, but soft and somewhat formed, without mucus. The urine is less pale. with a smaller sediment of oxalate of calcimm. The pains in the kidneys, and especially in the left one, persist as yet and become aggravated on moving and walking, if the abdomen be not suffiently supported by the flannel bandage; the left kidney is still very sensitive to pressure. The pains in the back are limited to the region of the kidneys, the others have disappeared after mas- 
sage. The organs of the chest are normal, as before. For five days the patient gained another pound in weight (his weight is now 93 pounds). Sleeps quietly, some seven and one-half hours at night and one-half hour in the day-time; mental condition better, physical weakness less. The former headaches and dizziness absent.

There is evidently no need of changing the treatment; we must only increase the dose of the tincture of coto, as the evacuations are as yet changeable, at times formed, at others not: besides, the patient might become habituated to the previons dose: we will give him now fifteen drops three times a day (instead of the former ten). We will also add some cognac to the milk - a teaspoonful to a glass. The quantity of food will be carefully increased.

We will turn now to the diagnosis:

The diagnosis of the condition of the stomach was finished last time. We will only add to-day, that the increase of the appetite and the continually improving digestion tend more and more to verify our diagnostic conchsions concerning the condition of this organ.

The intestinal tract. - Until last August the patient suffered with a constant constipation. The causes of this might be: first, the retarded passage of the gastric contents into the intestines because of the weakened movements which usudly. accompany gastric catarrh (the patient on admission had no signs of dilatation of the stomach, probably on account of the constant vomiting before admission. which emptied this ongan); and second, a weakening of the nervo-muscular activity of the stomach and intestines, due to the reakness of the nervomuscular apparatus in general and to his sedentary life. Since August the constipation has been replaced by a diarthoa - a sign of a superadded intestinal catarrh. Both the large intestine (frequent painful straining, mucus in the evacuations. pains in the region of the sigmoid flexure) and probably the small one (as was explained when ordering tincture of coto) are effected by the catarrh. The patient's reak constitution and poor mutrition, as well as his pityriasis versicolor, excited a : uspicion of tuberculosis in general, and of that of the intestines 
in particular, although the absence of fever and pains in the ileo-coecal region spoke against it. Repeated examination of the freces failed to prove the presence of intestinal tuberculosis, while the rapid improvement in the general condition as well as in the condition of the intestines also positively exclude it.

The liver and the biliary passages. - The liver presents nothing abnormal: the pain elicited by pressure at the pit of the stomach was referable, as explained during the objective examination, not to the left lobe of the liver, but to the stomach, There is slight but constant tenderness on pressure in the region of the gall-bladder, pointing to some diseased condition of the bladder. Which is it? From the data at hand it points only to biliary calculi in the bladder; the slight intensity of the pains speaks against pericystitis, while the entire absence of swelling excludes any new formation. Catarth of the gall-bladder, as part of the general catarn of the biliary passages, must also be eliminated, on account of the absence in the past and at the present time of any symptom of this condition, namely jaundice, as a result of the obstruction of the biliary passages, due to their catarhal condition. There remain then biliary calculi. Whether or not our patient had attacks of biliary colie brought on, under accidental circumstances (as by traumatism, by riding in a jolting carriage, by faulty diet, mental excitement, cold), by the presence of calculi in the bladder, we can neither deny nor affirm. We have not observed them in the clinic; but then every exacerbation and every attack of pain ceased in the clinic. Concerning the past history of the patient it is impossible to decide as to what extent the attacks of pain eventuating in vomiting depended on the exacerbation of the gastric catarrh and to what extent they were due to liver and kidney colies. As regards the last two diseases, we may infer, judging by the present condition of the patient, that the principal colic was of renal origin.

Kinlneys. - The left kirlney is very tender on pressure; the right one much less so. Besides, at home, after a jolting ride. and. to a very slight extent. in the clinic after walking, there appeared typical attacks of renal colic, viz., pains in the re- 
gion of the left kidney, radiating upward and into the left loin, as well as downward into the left groin and left testicle. The cause of this is evidently ascribable to concretions of oxalate of lime in the pelves of the kidneys (nephrolithiasis), especially in the left one. We say of oxalate of lime, because our patient has a typical oxaluria: urine pale, but strongly acirl on his admission it gave an abundant sediment of crystals of oxalate of lime, with scarcely any uric-acid deposit. Further than this the kidneys are not affected: there is neither hematuria nor any catarsh of the renal pelves, (the urine contains no mucus, no lencocytes, no epithelium from the renal pelvis, nothing but a small quantity of bladder epithelium), nor nephritis (aibumen and cylinders are also absent in the urine). The patient himself presents a typical oxaluric aspect, as clescribed by the well-known neapolitan clinician, Cantani: mental and nervous oppression (oppressed mental conclition and bodily weakness on his arlmission), emaciation in general and of that the muscular system in particular, and severe pains in the loins. I will add that, with the improvement in general and with that of nutrition in particular (as seen by the gain in weight), there was also observed a turn for the better in the course of the oxaluria : the urine is now less pale, and gives a less abumdant sediment of oxalate of lime crystals, but, instead, more of the uric-acid deposits (amorphous sodimm-urate and even crystals of uric acid). Physicians resident at the mineral springs, such as Contrexéville, where so many sufferers with oxalic acid nephrolithiasis congregate, have observed that, with the improvement in health in these patients, a diminution in the oxalic acid sediments in the urine takes place, while the uric-acid sediments increase proportionately.* However, the improvement in our patient is only noticed in the oxaluria as yet; the kidney pains decrease rery slowly. Experience has shown that the concretions of oxalate of lime, formed in the pelvis of the kichey, are the most difficult of all to treat, and require a long time before they disappear.

Does not our patient suffer from morable kichneys, especially the left one, which is so sensitive on motion (although this symp-

* Debout d'Estrées: Des indications des eaux de Contrexéville, Paris, 1889, p. 8 . 
tom is also found with renal calculi)? Notwithstanding the patient's extreme emaciation and his sunken abdomen, we were unable, while examining him, to even once feel the left (or the right) kidney; however, this fact would speak against a material displacement, but would not exclucle limited mobility of the kidney, for the existence of even this latter condition is sufficient to account for severe renal pains while in motion or while being jolted. Considering the extreme failure of nutrition, the weakening of all the tissues of the body and the number of traumata, to which our patient was subjected (riding in a wagon), mobility, especially of the left, painful kidney, is very probable.

Patient's chest, as we said before, is poorly developed. The lungs and the respiratory tract present nothing abnormal; the heart is normal except for the weakness of its contractions. The weakness of the pulse, which is now decreasing, depends partly on the anæmia, partly on the weakened heart-action, that accompanies failure of nutrition in general and of the muscular system in particular.

Nutrition and Hamatopoiesis. We have pointed more than once to the extreme failure of nutrition and to the poor derelopment of the patient, as well as to the causes of these conditions; we have also called your attention to another important symptom of a greatly disturbed nutrition, namely, oxaluria. As regards hrematopoiesis, the patient certainly presents anremic symptoms - a weak pulse, paleness, dizziness with a pale face, chilliness, etc. ; but his anemia is only a part of the general failure of nutrition, and not an independent affection, as chlorosis, pernicious anremia, lenkremia. We draw this conchusion, first, from the complete absence of any symptoms of the above-named diseases. and secondly, from the fact that neither the spleen nor the lymphatic glands present anything abnormal.

His nerous system, when he was admitted to the clinic, presented, as yon will recollect, the following abnormal phenomena: poor sleep, dizziness, pains in the head, back and abdomen, oppressed mental condition and bodily weakness - an aggregation of symptoms which is usually observed in persons suffering from nervous debility, - neurasthenia. Must we conclude from 
this that our patient has neurasthenia? We constantly hear in our practice nowadays of cases diagnosed as "neurasthenia," and we find that under this name entirely different cases are included, which are similar only in their nervous manifestations, and not eren in all of these. Such a custom will eventuate in the term neurasthenia losing its distinctive significance and becoming a name only for a group of symptoms, and this not always the same; whereas in reality it defines a certain disease.

Here are the characteristic features presented by persons suffering from a typical, pure, uncomplicated neurasthenia : ofttimes they are men sturdily built, long-lived, with perfectly healthy pectoral and abdominal organs of a normal (up to a certain period) functional activity, and an excellent nutrition; and still they are complaining of nervous disturbances, that at times they sleep well, at other times poorly, without being able to assign any reason for it; of a rapid changeability of the mental condition - now oppressed, now excited; both their mental energy and physical strength quickly give way to weakness. To all this there is superadded pain in different parts of the body, for which there would, at the painful spots, seem to be no apparent local cause; also hyperesthesia cutis and an increase in the tendon reflexes; later on disturbances appear in the functions of different organs, usmally first of all constipation, later (occasionally a long time after) nervous dyspepsia, palpitation of the heart, and other symptoms. Investigation of the causes of such a diseased condition usually brings to light hereditary predisposition, - weakness and susceptibility of the nervous system from their very birth, and also affections of the latter acquired through the agency of noxious influences.

Is it thus with our patient? The poor sleep in his case was not the result of unknown causes, but was due to the pains, upon the relief of which it soon became normal; with better sleep and with the improvement of the pulse (of the heart-action) the headaches and dizziness also soon disappeared; the other pains were very clearly traceable to various diseased conditions in parts where they were felt; our patient has neither skinhyperæsthesia and increased tendon reflexes, nor any apparent signs of nervous dyspepsia, nor palpitation of the heart; nor is 
his mental condition changeable; on the contrary, he is very reserved; the oppressed mental state in which he was when admitted soon passed away with the relief of the pains, improvement in the sleep and with the revival of hope in our patient; the considerable and slowly disappearing physical weakness is easily explainable by the extreme emaciation of the muscles and the unsatisfactory development of the skeleton. Considering the general failure of nutrition in our patient, which would naturally reflect itself in the nutrition of his nervous system, then the poor sleep for a prolonged period of time, and the acute pains in the many affected organs for a similar period, which in its turn would affect the nervous system injuriously, and observing at the same time, how rapidly the latter improves simultaneuosly with the general improvement in the patient's health, with the relief of local pains and better sleep, we must conclude, first, that our patient's nervous system presents not only a particularly weakened condition, but that, on the contrary, it appears to be the strongest part of his whole organism; and secondly, that our patient has no real, primary neumsthenia; that his nervous symptoms were developed in consequence of and owing to diseases of other organs (witness the anammesis), and they pass away so quickly with the removal of the canses, that they evidently disprove the existence of obstinate neurasthenia, even of secondary origin.

As is evident from the preceding, the summary of our diagnostic conclusions is as follows: our patient has a chronic gastrointestinal catarih, that assumed an acute form before he entered our chinic; also biliary calculi. renal colic, very probably a movable left kidney, serious failure in nutrition, with oxaluria. pityriasis versicolor, a series of secondary nervous disturbances of a passing nature, extreme emaciation of the muscles, and a poorly developed skeleton. We can not state the degree of depenlence of the last two conditions on heredity, as we know but very little of the constitution and health of the patient's parents; but it is beyond doubt that at first the continuous sitting in the position of a tailor at work, and later on the minterrupted lying posture. on account of the pains and weakness. which means a continuous lack of regular physical exercise and fresh air in the 
course of the last four years, in the period of adolescent growth of the organism, could not but play an important part in the development of the diseased conditions above mentioned.

Prognosis. - The patient's condition is serious, that is, such (vide Introduction to Clinical Exercises) as not to present any immediate danger to life, but such a danger may arise either because the various affections of our patient, becoming aggravated on the retum to his former surroundings of life, may bring him into a condition beyond remedial action, or, rather, because of the possible development in the exhausted organism of tuberculosis, chronic nephritis, etc.; or finally, some accidental cause, such as a severe cold, may bring about a rapidly developing affection that may prove fatal to the weakened organism. The diseased conditions of the patient are not of a fatal character per se, and are curable. Whether or not they are easily curable is another question: it must be apparent even to you, beginners though you be, that they are difficultly curable conditions, - particularly so, if we are to apply treatment as thoroughly as our science demands it; i. e. not only to effect the removal of local affections, but also to improve the patient's nutrition and constitution, without which treatment will be far from thorough; nor would the improvement in health be lasting. But the chief difficulty lies in this, that if we even succeed in removing the local disturbances while he is in the clinic, in improving his nutrition and in directing into a healthy path the future well-being of the patient, we must teach him to live, is as far as possible, under such conditions of life, that he may, after leaving the clinic and returning to the former unfavorable surroundings of life, remain in the possession of what he gained and thus strengthen his health the more.

Such is, in the given case, the physician's view of the future. Of this the patient must be informed only so far as will enable him to appreciate his own condition and his individual hygiene. in order to combat noxious influences, and by avoiding them to preserve his health; but not so much of it as would discourage him, depriving him of the energy so essential in the struggle.

Treatment. - To meet all the requirements of hygiene is the essential condition of every successful treatment. You know 
by this time the nature of and the reasons for the hygienic conditions under which we placed our patient; you also know that, partly with a hygienic purpose in view (to make up for the lack of exercise) and partly on accomnt of certain therapeutic objects, as already explained, massage was ordered for our patient. It remains now, while we occupy ourselves with the hygiene, to speak of the diet.

As a drink, the patient was given only tea, lnkewarm, neither hot nor strong. Water, especially if not warmed, would only tend to prolong the diarrhoa. Lukewarm tea, as a slightly astringent drink, is more suitable for diarrhœa. But tea is not only slightly astringent, it is chiefly a stimulant and the patient is habituated to its use; this is to be taken into account. I will not enter now into a detailed discussion of the important question, as to the limits to be permitted by the physician in the use of stimulants, as our case does not present a sufficient reason for such a discussion: our patient not only does not abuse the most important of these, - wine, brandy and tobaceo, - but does not use them at all. I will only observe that, as the normal course of the nervous and consequently of the other functions of the body does not, strictly speaking, require the aid of coffee or tea, and as a resort to such beverages will therefore only throw out of balance the regular operation of these functions, the physician can certainly not advise those not accustomed to the use of such stimulants to become habituated to them, but, on the contrary, he must dissnade them from acquiring such a habit. It is a different matter when one is already haljituated to the use of tea or coffee. If these have been used for a long time in moderate. quantities without exerting any particularly noticeable unfavorable influence, then there is no reason for insisting on abstinence from them, because their discontinnance (not to mention the fact that in the majority of cases the physician's prohibition will be like unto the voice Glamoring in the wilderness) is often accompanied by important and, in the case of an active life of the habitué, very distressing disturbances to his health. To be sure, cases of abuse of these beverages with evident bad consequences therefrom, require either linitation in their use or even total abstinenee; the latter, as is evident from what was just said 
concerning their moderate use, is to be accomplished very carefully. The use of wine, brandy, and to a certain extent of tobacco must certainly be regarded much more seriously, and their limited use or their discontinuance must be urged more insistently ; but I must say again, their total discontinuance is in certain cases to be undertaken with great caution, after weighing all the circumstances pro and contra.

To cause our patient to discontinue the use of tea and to compel him, in his present condition, to give up an accustomed and a most permissible stimulating beverage, would be not only inexpedient, but positively erroneous. Our patient needs the tea not only as a drink suitable for diarrhoea, but also as a gentle stimulant. The indications for stimulants are so strong in this case that we have even prescribed wine for him, the indications for which have been pointed out above.

We will now take up the question of fool. The patient was admitted with a chronic gastric catarrh, and in a state of exacerbation at that. We first of all took care to see to it, that the foód did not irritate the stomach, and with this in view we ordered it preferably in a liquid form (particularly on account of the possible, although hardly probable presence of ulcer of the stomach), viz., such as would not irritate mechanically; and warm, so as not to produce any thermic irritation — neither hot nor cold (especially in view of the diarrhoea): and finally such as not to irritate the stomach chemically (the patient, of course. gets no vinegar, mustard. or pepper, etc.).

Now come the most important questions concerning the quantity and quality of food and the time for taking it. You know that we instructed our patient to eat little at a time and often; you also know the nature of the food. The general success of the treatment stands witness to the fact that it also has been administered correctly. You are, doubtless, desirous of knowing why such an apportionment of food was successful and what guided us in prescribing it. We will take it up in order.

The quantity and the apportionment of the food. - If a patient with a catarrhal condition of his stomach is given, at his usual meal-times, for breakfast or dinner, one-half or even one-thircl of his ordinary quantity of food, he will, on taking it, fail to ol- 
serve any dyspeptic symptoms at all or he will have them in a much lesser degree; but he would usually complain of either being hungry or at least of not experencing a feeling of satiety, that is, a sufficient filling of the stomach. This fact is the basis of the commonly accepted and very useful method of administering food to such sick - little at a time and at frequent intervals. How is this to be ordered?

A sensible, careful patient, with a strong will-power, is advised thus: Eat little at a time, - enough to still the hunger, but never gorge yourself, not even to complete satiety; but as soon as you feel not merely hungry, but a lively appetite, eat again the same quantity, that is, without complete satiation. It is usually necessary to add: eat as slowly as possible; do not hurry (a besetting sin); you will thus be able to better chew the food and will observe that your hunger is the sooner satisfied: you finish eating before being over-sated. To be sure, even with such patients and with such instructions we do not always at once get good results; but we at once establish the proper quantity of food to be taken at one time. Patients are liable to either overeat, or to eat too little at a time, if frightened by too energetic instructions from the physician, particularly if they be over-scrupulous: they will not repeat the meal often enough, and by going hungry interfere with the success of the treatment. But if the physician follows attentively the course of treatment and repeatedly offers additional explanatious, then the patient will acquire the habit of taking his meals regularly, thus guaranteeing a healthy condition of the stomach also for the future.

On the other hand, in some cases — as, for instance, in our patient - such instructions are not sufficient. He was habituated to poorly nutritious, bulky and difficultly digestible food; here he gets food that is nourishing, of small bulk, easily digested, and besides more palatable and more appetizing. It is, therefore, rather difficult for him to ohserve the principal requirement in eating, namely to eat till hunger is satisfied, and not to satiety. If the quantity of food be not clearly limited, he will always overeat. In such cases the physician must do as we have done: having explained to the patient the above-mentioned 
requirement, define exactly the quantity of food and the frequency of the meals, and then verify their correctness by observation, increasing or diminishing the quantity of and the intervals between the meals in accordance with the degree of the dyspeptic phenomena and the condition of the appetite. The gradual increase in the general quantity of food runs parallel to the improvement in the condition of the stomach, as you see clearly from the present case.

It is even more difficult to properly prescribe the quality of the food. To be guided only by general hygienic and physiological data will not bring the desired results, as even little experience will prove: isolated cases are too often met with presenting too great idiosyncrasies. In consideration of this it only remains to thoronghly study the given case. Our patient had a gastric catarrh while ingesting poorly nourishing food, that was bulky and not frequently taken; we proved successful with him while giving him food of an exactly opposite nature. In other cases, as for instance, in those of pure nervous dyspepsia, uncomplicated by catarth, nourishing but too delicate food is often successfully replaced by a coarser food. To be sure, we may run across some rery difficult cases; but a careful investigation of every given case, of the habits and peculiarities of the patient as regards his food (as to which kind of food agrees with him and which not) will always enable us to prescribe, in accord with the hygienie and physiological data, the first, so to speak, trial food. and later, grided by the results obtained. to gradually formulate a correct selection, thus at the same time teaching the patient how to preserve the stomach in a healthy condition. I must here enter my protest against the use of the stomach sound for verifying the correctness of the prescribed food still more forcibly than I did while we discussed its use for diagnostic purposes in gastric diseases: first, because the results of verification obtained by the sound, with the methods at present in use, are less reliable than those obtained by ordinary observation; and then, the introduction of the sound, especially if frequent, may undoubtedly hinder digestion and become prejudicial to the favorable course of the disease.

The food must be varied within limits admissible for the pa- 
tient, but it must be prepared plainly, without suspicious sauces and dressings, but palatable withal.

In prescribing a diet for our patient, it was not only the condition of the stomach that was taken into consideration. The intestinal catarrh, as apparent from the diarrhcea, required certain peculiarities in the prescription, as has been noted above; the prescription would be different in the case of constipation, which is seen oftener than diarrhoea in gastric catarrh. The disturbances in the patient's nutrition also require a different diet. We have been able to a certain extent to conform our treatment to these, especially as regards the oxaluria, having prescribed for him preferably a nitrogenized food with a prohibition of sweets (excepting a very trifling quantity of sugar with his tea); this, as has been proved, was not only permissible, but even requisite in the condition of his stomach and intestines. The present condition of his digestive organs certainly does not permit the use of a fattening diet, which is indicated by his extreme leanness, as for instance, cod-liver oil or large quantities of milk or its preparations. Time will show how far such a diet may be necessary and when its use is possible. It will also be our task to gradually change his present food, in accordance with the rapidity of his convalescence, to his habitual coarser diet, which he will return to after leaving our clinic.

Taking up the treatment proper, we will follow the same order as laid down in our Introduction to Clinical Work, and which has been followed in our investigation and diagnosis.

The stomach. - The indication for Ems water will be spoken of separately, for reasons we have already explained. Why did we prescribe for onr patient decoction of condurango and tincture of nux-vomica? The so-called bitters are useful in chronic catarn of the stomach. Without discussing the pharmacological reason for their action, I will say that clinically their use is of undoubted utility: when taken at meal-times they alleviate the dyspeptic symptoms. Conclurango, notwithstanding its slightly bitter taste, acts like the bitters (by diminishing the dyspeptic phenomena) even better than those formerly used. Besides. condurango is very useful in diarrhea, which will sometimes 
pass off by the use of this drug alone. The nux-vomica drops are added, - first, because they are strongly bitter (the slightly bitter, insipid taste of condurango is even somewhat unpleasant to patients, who usually prefer the addition of nuxvomica), and secondly, this drug, because of the strychmine, will act as a tonic to the weakened nervo-muscular apparatus of the intestines, and our patient, as you will recollect, suffered from almost constant constipation until last August. It is also convenient to administer condurango and nux-vomica in the following formula : $\mathrm{R}$ - Extracti fluidi condurango $\bar{j} \mathrm{j}$, tincturæ nucis-vomicæ 3 ij. M. D. S. $20-25$ drops in one-half wineglass of warm water twice a day after meals.

Intestines. - The measures to combat the diarrhoa, the intestinal catarrh, are as follows: rest in recumbent position, warmth (warm food, a warm abdominal supporter, warm baths), improvment in the condition of the stomach, of the gastric digestion, and, as mentioned above, condurango. We have already explained why we prescribed codeine and the tincture of opium, (for pain), why we give coto drops and not tannin or other astringents, - and why we did not resort to tamnin or any other clysters.

The gall-bladder. - Ems water was prescribed against biliary calculi, because clinical and partly pharmacological data lead to the conclusion that the alkaline mineral waters, to which class, as you know, the Ems rrater belongs, have the tendency to reduce the size of and break up the calculi, thus facilitating their expulsion. This will also be expedited in our patient by the improvement in the gastro-intestinal digestion and in the nutrition. The correction of faults in diet, rest, the avoidance of traumatic influences, warmth (warm abdominal bandages and warm baths), and the strengthening of the nervous system brought about by the relief of the various pains and the improvement in sleep -.. all these will tend to diminish the constant tenderness of the gall-bladder and to avert attacks of biliary colic.

The Kidneys. - For the oxalic acid gravel we prescribed the Ems water, although alkaline mineral waters are properly more suitable for renal gravel of another nature - namely, the uric 
acid gravel, which is usually found not with pale urine, as that of our patient and of oxaluria in general, but in urine that is more strongly colored than normal, namely, orange-yellow or even orange-red. Against oxalic-acid gravel are particularly indicated the earthy (containing lime) mineral waters, as, for instance, Contrexéville; but we could not prescribe these waters, as they would act, especially in our patient, as laxatives, thus prolonging the exhausting diarrhcea. It is the meat diet, the improvement in nutrition and in digestion, general massage, and the improvement in the condition of the nervous system, that will diminish the production of the oxalic-acid gravel and stop the oxaluria. The diminution in the tenderness of the kidneys, and the cessation of attacks of renal colic, will be effected by the same measures, as were recommended for the gall-bladder symptoms, namely, rest, warmth and a strengthened nervous system. As it is quite probable that the patient's left kidney is somewhat movable, it will become necessary for him, later on, when he moves about more freely, to wear a special kidney bandage, in place of the insufficient simple abdominal band.

The heart. - The weak cardiac activity required stimulation; we have spoken above of the indication for wine in connection with this.

Nutrition. - We just now mentioned the measures for combating the oxaluria. While discussing the diet we spoke of what could be done for the patient in his present condition for the extreme emaciation.

The nervous system in general, the musculo-nervous apparatus. the constitution of the patient. - We first of all attempted to improve the patient's sleep, for as long as there is sleeplessness we cannot expect any improvement either in the nervous system or in the condition of the whole organism. IVe succeeded in gaining a considerable improvement in this direction by relieving the various pains and by the use of warm baths and of general massage; all this has already been spoken of. Besides, the special object in the use of massige lies in the improvement of nutrition and in the development of the nervo-nuscular apparatus, in the increase of muscular strength, which, after the local ailments shall have been removed and improvement in the 
general nutrition having been attained, will become the startingpoint for the possible improvement in the constitution of the patient.

It now remains for us to explain why we prescribed Ems water for our patient, and coincidently we shall speak of mineral waters in general.

\section{LECTURE OF OCTOBER 6, 1889.}

The patient's condition is improving constantly; the prescribed treatment is justified by the obtained results and requires almost no alterations; there is therefore no necessity for bringing the patient before you as frequently as before. 'The patient was given, as you remember, at the beginning, daily, two glasses of milk, two glasses of soup with broken-up meat, two eggs, less than one-fourth pound of white bread and three spoonfuls of wine. He still gets two glasses of milk with cognac, two glasses of soup with meat, two eggs, more than one-half pound of white bread, two glasses of milk soup with manna, one-fourth of a chicken, two meat cutlets, and the wine as before; but the appetite is constantly increasing and the patient begs for more food. This quantity of food is well digested, withont any dyspeptic phenomena, unless it be some belching at times, which is easy, painless, odorless and non-acid. Tery rarely, when the patient feels strong hunger. there appears a slight pain in the region of the stomach, but this ceases at once on taking some food. This pain is but an indication of a greater necessity for nourishment. a necessity that appeared simultaneously with the improvement in the condition of the stomach. Considering the patient's extreme emaciation and the fact that he is as yet growing (he is but eighteen years old), this necessity is certainly not to be wondered at. The evaeuations, once a day, are normal. more frequently of a solid consistence (we have given no opium since September 27th). The abdomen is not distended. notwithstanding the abundant quantity of food and the not rer'y copious excretions, which fact points to a normal intestinal ahsorption; this is also apparent from the cessation of the formerly frequent rumbling. The spontaneous abdominal pains are 
insignificant, appearing only on walking-_in the region of the left kidney. Examination of the abdomen elicits a marked tenderness in the same region, and a trifling sensitiveness in the region of the sigmoid flexure and in that of the gall-bladder. The urine is not pale, but of normal color, does not become turbid so readily as it did formerly: the oxalate of lime sediment is continually decreasing, while that of sodium urate has increased a little, but ceased to increase further. The pulse is continuously improving. Patient's appearance is better than before; he has gained three pounds since admission (he weighs ninety-four pounds). The pityriasis versicolor is passing away - is less scaly and paler. The sleep is better; the mind is clear, the strength increases little by little: the patient is able to stand up longer (this used to produce dizziness) and to walk more.

How shall we continue the treatment? We will add another half glass of Ems water (so that the patient will be taking half a glass three times a day, each time about one hour before a meal) with a view to more effectively diminish the kidney pains, which may be due to the oxalic-acid gravel. To use the Contrexéville water for this would be rather risky, because, as we explained before, it might cause a return of the diarrhøa. Even a somewhat larger dose of Ems water, though it be lukewarm, would tend to prolong an existing diarrhœea. Wre have therefore limited the dose until now to two half-glassfuls of this water; but now, with the improved condition of the intestines, we can give some more of the Ems for the above-mentioned purpose. The condurango and the nux vomica will be continued for a long time; experience has proven the utility of such a procedure in cases similar to ours, in a gastric eatarrh of such duration and with a simultaneous intestinal catarrh. Guided by experience, we will continue the coto drops some time even after the cessation of the diarrhoea, and then leave them off gradually. As is apparent from what was said, we must continue the wine, massage, and at times the baths.

May we recommend for our patient walking ont-of-doors? He has grown somewhat stronger, the weather is pleasant, and the fresh air would certainly be very beneficial for him. But it is better to abstain, as yet, from recommending such walks. Although 
surely better than before, the patient's strength is still not very great; besides, he still takes at times the warm baths so necessary for him. There is therefore a risk of jeopardizing the patient's condition through fatigue from walking and a possible cold; this might bring on a return of the diarrhcea, or it might increase the renal pains. Besides, the walks are not necessary for him as yet: the patient's appetite is excellent and his sleep satisfactory.

Before passing on to the mineral waters, I consider it necessary to say a few words about those methods of treating gastric disease to which I have not resorted in the present case. A review, if not of all, then at least of the most generally used methods of treatment of gastric diseases in connection with our first case, will be advantageous in explaining the relative usefulness of the various methods of this therapy, and will make still clearer the reason why we have treated our patient as we did and not otherwise.

Hydrochloric acid was proposed for facilitating gastric digestion some thirty year's ago; and since it has been shown experimentally that the secretion of this acid by the stomach diminishes at times, or is even entirely suspended, its therapeutic application seemed to become even more judicious. During the first year of my clinical practice (also some thirty years ago) I made use of this acid; but the results obtained compelled me to dismiss it, so that since then I do not resort to it (I shall mention later the only exception where I use it still). At consultations, however. I have had, and have now, opportunities to see it employed in diseases of the stomach. As before, so at the present time, with the condition of the stomach so carefully investigated by the modem methods, the results of its use are identical. First, they are very inconstant, the acid benefiting the patient at times, but oftener not, on the contrary impairing the appetite, causing pains in the region of the stomach, - in short, tending to himder digestion; second, even when it does benefit the patient, the relief is only temporary, i. e., it assists in promoting the digestion of the meal with which it was taken, but does not cure the disease that causes the dyspepsia. If this latter diseave 
be a catarrh of the stomach, then it will be necessary to cure it with alkalines, bitters, lavage; if the dyspepsia be of nervous origin, then we must resort to a radical treatment of the general nervous disturbance. At the same time, hydrochloric acid, being but a palliative remedy, while helping (which it rarely does), will disguise the results of the radical treatment, and will prevent the formation of a correct idea as to the success of the latter; while in case it does not relieve, it may become harmful, causing, as we said above, a disturbance of digestion and even of untrition, if used for a long time.

We alluded above to the employment of hydrochloric acid in diseases, in which the secretion of this fluid by the stomach, or rather, in which the action of the acid secreted by the stomach on food, is made difficult, in catarrhal conditions by the presence of mucus, in gastric dilatation by the accumulated contents. (Dr. Popoff, whose work I have already mentioned, found the secretion proper of hydrochloric acid by the stomach in these diseases to be normal in a very great number of both experimental and clinical cases investigated by him.) While in cases where the secretion of this acid is entirely absent and cannot be made to reappear, that is, in atrophy of the stomach, when its glands are destroyed, the palliative results obtained through the employment of the acid are trifling and not to be relied on as tending to prolong to any extent the life of the patient. At all events, as you may know, hydrochloric acid is neither employed nor valued in diseases of the stomach as much at present as it was formerly.

Of the employment of pepsin and trypsin, in the form of an extract of the pancreas, I must say the same that I said of the employment of hydrochloric acid, and again with the same exception.

Such a conclusion concerning these three remedial agents refers to the condition of things at present only, without, of course, making any predictions in regard to their future application.

The exception, which was spoken of before, refers to cases of gastric dyspepsia in chlorosis and other anemic conditions, in which the secretion of the gastric juice is very much decreased, and where hydrochloric acid administered after meals sometimes gives good results. The complete cessation of dyspeptic phe- 
nomena even in these cases can only be obtained however by a radical cure of the above-named diseases.

Here I must also mention the various food-compounds, prepared so as to make them very suitable for digestion, as meatjuice, (beef-juice), meat powder, peptonized albuminates and the like. I personally do not employ them, but as a consultant have seen them used in quite a number of cases. I could never persuade myself as to the necessity for their employment. In the majority of cases their use is not followed by any particular results; while in those rare cases where the patients bore them well — when unable to bear ordinary food - and even improved on their use, investigation has shown that either the "ordinary" food was unsuitable, or that the treatment followed at the time of its administration was wrong, and the food could not therefore be borne. I will also add that, through the careless preparation and preservation of these so-called " especially digestible " foods, which are unfortunately much more frequent than their conscientious manufacture, they, being generally unpalatable, become repugnant to, and may even harm, the patient, probably on account of the ptomaines developed in them. The relatively high price of these food preparations must also be taken into account.

We employed no lavage of the stomach in the present case, as there was no indication for it, viz., distention of the stomach by the accumnlater contents (as we pointed ont in our diagnosis), - not to speak of the exacerbation of the catarrhal condition, just before he was admitted (pains in the stomach and daily romiting), which was a strong coutra-indication for gastric lavage. The success of our treatment is a sufficient proof that there was in reality no necessity for the employment of this method of treatment. I hope to present to you at my next lecture a case with indications for gastric lavage, and I will then discuss thoroughly the necessity for its employment in its correct application, in which it is a most useful method of treatment. 


\section{LECTURES OF OCTOBER 10 AND 11, 1889.}

It remains to be explained why we prescribed for our patient a mineral water; why particularly the Ems; why in the quantity designated; and why at certain times (one hour before the meal), etc.

Of all known remedies for catarrh of the stomach, and also of the upper portion of the intestines, of the biliary, urinary and respiratory tracts, for biliary calculi, renal gravel and gout, sodi$\mathrm{um}$ in the form of the bicarbonate and the chloride is considered the best. What is the most convenient form for administering these combinations of sodium in gastric catarrh? At the beginning of my medical practice I oceasionally saw sodium bicarbonate administered even in pills, - the least suitable form: for along with the medicament itself, the bicarbonate, there was introduced into the stomach in large quantities a pillular mass (extr. taraxaci, etc.) that uselessly burdened the organ and only served as an obstacle to the efficiency of the sodium. The best form is without doubt a solution, in which the above named compounds of sodium come in contact with the mucous membrane of the stomach in the easiest and most equable manner.

Sodium bicarbonate and sodium chloride are often given, with the addition of laxative salts, if necessary, in powders, the patient being directed to dissolve them in a certain quantity of water. The chief recommendation for such a for'm is its cheapness; but it has, infortunately. numerous drawbacks : the patient may not always take of the supply prepared by the druggist a uniform quantity, nor does he always dissolve it in a like quantity of water (the degree of concentration of the solution is quite important); and. what is most important, he dissolves the powders in water of an uncertain composition. 'This water may contain substances either directly harmful to the stomach (usually an abundance of lime compounds), or such as will change the composition and through this the effectiveness of the powder dissolved in it. To have powders prepared in a drugstore, weighed ont for each dose (often several times daily), would destroy the principal advantage of this method - its 
cheapness - considering the value of the apothecary's labor; while to have an exactly prepared solution made with carefully selected soft water (containing but few hard particles; not to speak of distilled water) for daily use, would make such treatment very expensive. It thus remains to prepare exact solutions in great quantities in factories, which is certainly much cheaper, and which we see in establishments for artificial mineral waters.

Can artificial mineral waters replace the natural ones? My long experience teaches me to prefer the natural ones, used either at the places they are found, or in the patient's house when imported. We certainly can not deny the efficiency of the artificial waters, which may, in the alsence of the natural ones, suffice, if they are carefully prepared. Unfortunately this latter circumstance is rather a rarity. The difference in the prices of the natural and artificial waters is very small, owing to their mutual competition. Besides, the mineral waters are being employed now in much smaller doses than they were formerly, (of this later), and treatment by them can by no manner of means be designated as more expensive and less accessible than treatment by other drugs, - rather the contrary. As regards the efficiency of the natural mineral waters as compared with the above deseribed powders, for the composition of which they usually serve as specimens, the superiority of the former is so immense as not to admit of any comparison. Many a time have I seen a complete cure by the regular employment of mineral waters in cases of gastric catarh, constipation, renal and biliary colic, in which the long continued use of the powders was productive only of failure.

At the beginning of my medical practice the customary course was as follows: during the summer, patients were either sent directly to the mineral springs, or, if not sent there, they were ordered to take artificial waters and at times natural imported ones. At the other seasons of the year the above-named powders and pills were prescribed. The difference in the results of the treatment, made more prominent by the dissimilar conditions of life during the warm and cold seasons of the year, at the place of the patient's regular residence, or, in case of his having gone to the waters, anid completely changed surroundings, was very 
striking indeed. I therefore, from the very beginning of my clinical and independent medical practice began to use the mineral waters, mostly natural ones, all the year round, exercising due caution, of course; - the routine habit was so strong, and the possible dangers from the use of mineral waters, especially warm ones, in the cold season, were so great. Physicians who quietly looked on while their patients imbibed, both in winter and summer, some five to ten eups and more of hot tea daily, felt anxious lest these patients should eatch cold by drinking three times a day half a glass of Ems or Karlsbad water, which, although somewhat warmed, was still of a lower temperature than the customary beverages. But the force of circumstances, aided by my practice and that of my assistants and former students at the clinic, has triumphed at last, so that now in Moscow, as well as in Russia generally, the use of mineral waters at every season of the year has replaced the pills and to some extent the powders; although even at the present time in some university cities with clinics, and partly within the circles of their medical influence, the improved practice is either altogether absent or is only slowly being introduced.

Appreciating highly, for reasons just explained, the importance of mineral waters, I lecture on them every year to my students.

I consider it the business of the clinic to impart to the future physicians the skill in selecting and the technique of using the mineral waters; and the personal experience of my former students, testifying to the benefit derived from these lectures, impels me to continue in the same line.

It becomes apparent from the above why we prescribed for our patient the best remedies against gastric catarrh - bicarbonate of soda and sodium chloride - in the form of a mineral water. The question arises now, why have we selected the Ems water and no other? To answer this and at the same time to show by what we are guided in selecting a mineral water, we will compare the Ems water with others containing the above-named combinations of sodium. But I must beforehand say a few words in explanation of the difficulties encountered by the young physician in selecting a mineral water 
in any given case, and thus explain to you the duty of the instructor in impressing upon you the acquisition of the ability to make this selection. Our physician opens his text-book of pathology and therapeutics and looks for the indications for the employment of the mineral waters in the given case-say catarrh of the stomach: the text-book recommends Ems, Vichy, Yessentucki, Karlsbad, etc. ; for biliary calculi, Karlsbad, Vichy, Yessentucki, etc; for renal gravel, Vichy, Yessentucki, Karls. bad, etc; but which water is he to order? No answer. He then looks in his text-book of balneotherapy for advice, and finds that Ems is recommended for gastric catarrh, biliary calculi and renal gravel; Vichy and Yessentucki for gastric catarrh. biliary calculi and renal gravel ; and Karlsbad for gastric catarrh. biliary calculi and renal gravel. The text-books evidently do not help one. It is only in the clinic, that is, in presence of the given cases, that we can show in a correct way, in the very best. manner, how the choice of a mineral water is to be made. The clinic certainly does not exclude the text-books on balneotherapy. nor those on special pathology and therapeutics, - they are surely necessary; but the clinic supplies the key to them, and teaches how to make use of the material therein contained.

Let us take for comparison the mineral waters of Ems, Obersalzbrunnen, Vichy, Yessentucki, Karlsbad, Franzensbad, Marienbad and Kissingen. We will also have to discuss the so-called bitter waters, which contain principally laxative salts (such as Fiedrichshall, Püllna, and our Caucasian spring “ Maria Theresia") as well as the earthy waters (as Contrexéville), since the first named, although of course employed separately. are sometimes used in conjunction with the latter ones; while the indications for the others are at times not clearly distinguishable from those we have mentioned. We will thus speak of the most important mineral waters employed for internal use, except those containing iron and arsenic, which we will have occasion to discuss in the treatment of chlorosis and anæmia. I have selected the above enumerated waters because they are the best known and the most studied from a chemical, clinical and to some extent physiological standpoint. and because, if correctly employed, they are undoubtedly efficacions. 
Experience of many years - for some of them, of centuries has proved that they contain a happy combination of curative agencies, which are very beneficial in many frequently occurring and important diseases; besides, they present types for comparison with many other mineral waters, so that in discussing these we discuss not the individual waters, but whole classes of them. I have just said that the mineral waters selected by me for comparison contain a happy combination of curative agencies. What are these agencies? Water, carbonic acid, bicarbonate of soda, sodium chloride. and laxative salts (sulphate of soda and sulphate of magnesia). 'The constituents of the mineral waters enumerated are found in a comparatively large quantity ; they contain besides some other solid components in a small. eren infinitesimal quantity, as compared with those mentioned. The importance of the latter is not absolutely known. In selecting a mineral water we generally take into account the constituents found in a comparatively large quantity; although we should not, nor is there any reason to, deny the importance of the components found merely in minimal proportion. It is quite likely that some differences in the action of the various waters depend on these latter, though there may be similar composition as to the quantity and quality of their chief ingredients.

You have been taught by pharmacology the pharmaco-dynamics of these chief ingredients. I will only remind you of what is most important for an understanding of the therapeutic action of mineral water.

Water increases all the secretions; if warm. it particularly increases perspiration; if of lower temperature. then the urine, bile. etc: by increasing secretion it influences the animal metabolism. Cold water causes increased peristalsis in the gastro-intestinal canal. removes constipation. but may tend to cause diarrhora and pains. intestinal and gastric. whereas warm water. on the contrary. soothes the pains and diminishes the diarrhera.

The action of carbonic acid. whether free or liberated from the carbonates. in the stomach. is similar to that of cold water in the gastro-intestinal canal: it increases peristalsis. removes constipation. but may also canse diarrhrea, pains and. at times. vomiting.

It is the biculomate of sold and the solium chlorite which are 
the principal curative ingredients of the alkaline mineral waters, as proven by the experience of centuries. The mechanism of their action is not wholly known. We know this much: first. they assist in dissolving and removing the mucus in catarrhal conditions, an effect which is of very great importance. In gastric catarrh the mucus prevents the access of food to the mucous membrane, so that this is stimulated to a lesser degree by the food, and consequently secretes the gastric juice less actively, which in its turn reaches the food less readily on account of the mucus; on removing this, the normal condition is reëstablished. In catarrhal conditions of the respiratory tract the accumulated mucus causes difficulty in breathing, conghing, etc. The excellent action of the combinations of sodium mentioned is not limited to the removal of mucus only, but we know nothing more of an exact nature. Secondly, they increase the secretion of the gastric and other alimentary juices, of the urine, etc. They alter secretions; for instance, they convert a strongly acid urine, with uric acid serliments, into a slightly acid, neutral or even alkaline one, and thus they prevent the formation of fresh gravel and assist in dissolving and remoring that already formed. They very likely act in a similar manner on the bile. Thirdly, in small quantities they act favorably on nutrition (contrariwise in large quantities, - of this later), diminishing the quantity of the excreted nitrogen. To both bicarbonate of soda and sodium chloride a similar action is ascribed, especially as the bicarbonate, on reaching the stomach, must be converted into sodium chloride in the presence of the free hydrochloric acid of the gastric juice. But licarbonate of soda is particularly valuable in practice, as principally those waters are used internally - in catarrhal conditions, biliary calculi. renal gravel, gout, diabetes mellitus, etc., - which contain the bicarbonate, usually also the sodium chloride; and not the ones containing any sodium chloride without the bicarlomate of sodit.

The laxutive salts increase peristalsis and may cause a transudation from the mucous memlinane of the intestines. By emptying the intestines, in cases of constipation, they cause a diminution in the size of the alsdomen, facilitate breatling and circulation, which is very beneficial in difficult circulation of 
the head and chest. Decreasing, by the aid of laxation, the absorption by the intestines, and inviting a transudation from their mucous membrane, they diminish the fullness of the portal venous system (which action may be very serviceable in certain liver troubles), and of the venous system in general; they also influence nutrition and reduce obesity.

The following table, compiled by me from the most reliable analyses obtainable, so kindly placed at my disposal by Dr. A. I. Tsherbakoff, shows the total amount of the solid components, and, separately, the amount of those that are found in large (in heavy type) and medium (in ordinary type) proportions (the very small components are omitted so as not to impair the general aspect of the table) in the mineral waters which we have selected for comparison, as well as in the bitter ones. The table also shows the quantity of carbonic acid contained in the various mineral waters, and their temperature in degrees of both Réaumur and Fahrenheit. The carbonic acid, whether free or combined, is shown in its total amount, because when in the stomach, if combined, it will be liberated under the influence of the hydrochloric acid of the gastric juice.

As regards the temperature of the water, I will say this: some of you will probably practice at the places where mineral springs are located, but the majority will doubtless employ imported waters (patients are not very frequently sent to the watering places). The imported waters have not, of course, their natural temperature, but that of the surroundings in which the vessel containing them is placed. Nevertheless, the table shows the natural temperature of the waters, that long years of observation have shown to be so very important a part of their efficacy. What shall be the exact temperature of the water we are employing in the given case will be explained later on. I will only state now, that even while employing the waters at their source the extremes of their natural temperatures are to be avoided ; as, for instance, in case of a too low temperature, like that of the Marienbad Kreuzbrunnen $\left(53.1^{\circ} \mathrm{F}\right.$.), the water requires to be warmed; in case of too high a temperature, as that of the hot Karlsbad spring, the water is allowed to cool. But at the same time, the Marienbad water is used and prescribed 


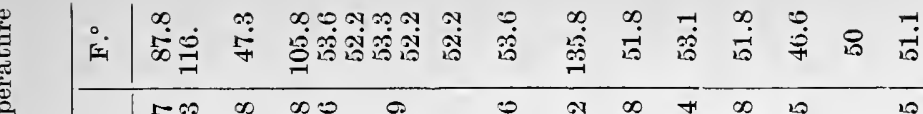

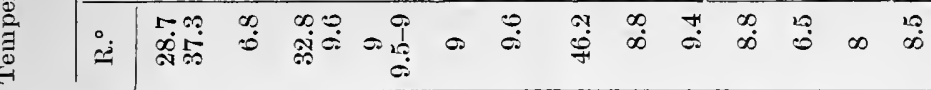

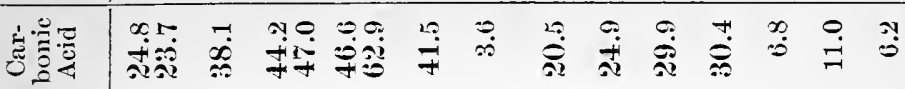

$\Xi \nsubseteq$

蛋

密空

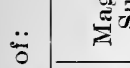

吾紊

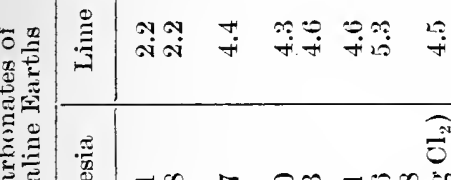

ה每

舟登 焉

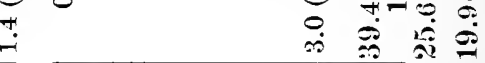

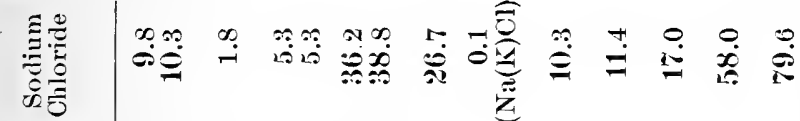

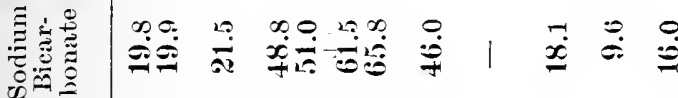

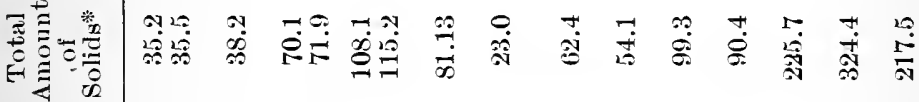

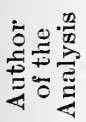

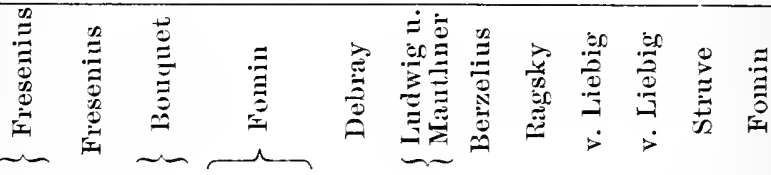

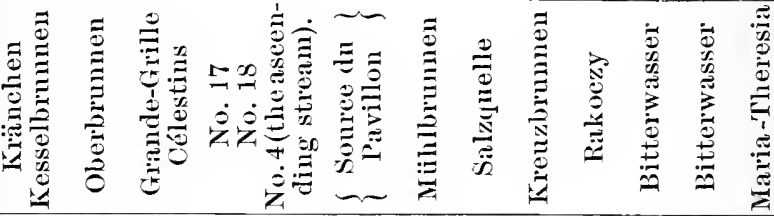



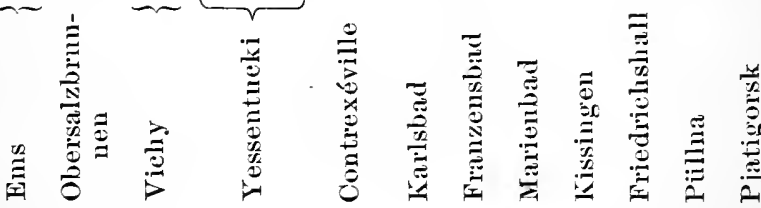


because of its being comparatively cold, and the Karlsbad for its being a comparatively warm water, and the benefit obtained by the use of these waters, as shown by experience, depends on their being used at the above temperatures. In prescribing the temperature of the imported waters we must also be guided by these experiences.

Having become familiar with the composition of the mineral waters selected by us for comparison, let us see why we ordered for our patient the Ems water and why we consider it more suitable for our case than any other water.

The diagnosis has shown our patient to be suffering from a chronic gastro-intestinal catarrh, in a state of exacerbation just before he was admitted to our clinic, from biliary calculi, renal colic, oxaluria, extreme failure of nutrition and of physical development, and from consequent nervous disturbances of a temporary nature. He chiefly complained, on admission, of general weakness, of pains in the abdomen and of a diarrhoea.

As can be judged by the table, the advantages of Ems water for such a patient are at once apparent: the warm temperature of the water is very beneficial in pains and in diarrhoea; the moderate quantity of bicarbonate of soda and of sodium chloride is much more suitable for pains, diarthota and failure in nutrition, than a large amount, as. for instance, that amount of the bicarbonate in Vichy and Yessentucki, and of the chloride in Kissingen; then the trifling amount of other component parts besides the principal ones (the Kesselbrumnen spring contains, out of the total amount of 35.5 of solid parts per $10,000,30.2$ parts of bicarlonate of soda and sodium chloride, so that on the rest fall but 5.3 parts) is always of great importance, and especially so in a case like ours. where the stomach and the intestines are so severely affected, because such " pure " mineral waters are much easier borne than waters of an opposite composition.

The course of the illness has proved, as you know, that we were not in error in prescribing Ems for our patient. Let us consider now what would have been the action of other mineral waters in the present case.

Marienbad is evidently contra-indicated, — first, because, being a cold water $\left(53.1^{\circ} \mathrm{F}\right.$.), it would aggravate the diarrhea and 
all the pains; and secondly, containing, as it does, great quantities of sodium sulphate ( 49.5 per 10,009$)$, it would act as a strong laxative.

The same must be said of Franzensbad, which is also a cold $\left(51.8^{\circ} \mathrm{F}\right.$.) water, and which also contains, although in a smaller amount, sodium sulphate (28.0 per 10,000$)$.

Kissingen is contra-indicated in our patient for many reasons : it is also a cold water $\left(51.8^{\circ} \mathrm{F}\right.$.), but, although the sodium sulphate is absent, it contains such a quantity of the sodium chloride (58.8 per 10,000) that it acts as a laxative, the more so as it also contains, small though the quantity be, some magnesium sulphate, a strongly laxative salt; finally, it contains no bicarbonate of soda, but on the contrary a great deal of lime salts (15.3 per 10,000).

Karlsbad, with one important exception, is very similar to Ems : it, too, is a warm $\left(135.8^{\circ} \mathrm{F}\right.$.) water, containing the same amount of sodium bicarbonate (18.1 per 10,000) and sodium chloride (10.3 per 10,000) as Ems. It is also a very pure water ; but among the chief component parts it contains a considerable quantity of sodium sulphate (23.9 per 10,000) - a laxative salt. Some physicians employ Karlsbad, as a warm, or hot, water, and in diarrhcea.

In cases where the constipation is alternated often by diarrhoea, but where the first usually predominates, I have had opportunity to see some successful results with the Karlsbad; but for cases similar to that of our patient I positively prefer the Ems.

Vichy. - The Célestins spring is cold $\left(53.6^{\circ} \mathrm{F}\right.$ ) and consequently unsuitable for our patient. The Grande Grille spring is very similar to Ems : the water is also warm $\left(105.8^{\circ} \mathrm{F}\right.$.), does not contain any laxative salts (except a trifling quantity of sodium sulphate), is also very pure, but has a greater quantity of sodium carbonate ( 48.8 per 10.000$)$, and is therefore contraindicated in our patient, or is at least less suitable for him than Ems, partly because of his diarrhora, but principally on account of the extreme failure in his nutrition. The long-continued use of great quantities of bicarbonate of soda has been shown by clinical investigations and experimental data to injuriously 
affect the nutrition, especially in patients as emaciated as ours is.

The Yessentucki springs are cold, contain even more bicarbonate of soda than the Vichy springs, and also a great deal of sodium chloride, and are therefore even more contra-indicated in our patient than those last, on account of the diarrhoea and of the failure in nutrition.

Obersalzbrunnen ("Silesia water") approaches Ems in the quantity of the bicarbonate of soda ( 21.5 per 10,000$)$, but is unsuitable for our patient because it is a cold water $\left(47.3^{\circ} \mathrm{F}\right.$.), containing a great deal of carbonic acid (38.1 per 10,000), and, although in a small quantity, also sodium sulphate (4.6 per 10,000 ), and is consequently liable to aggravate the diarrhoea and the pains. I will take occasion to say a few words concerning the employment of Ems and Obersalzbrunnen in diseases of the respiratory tract, in which these waters are used more frequently than any others; I will do so now, so that I may not have to touch upon this subject later on when we come across patients suffering from these diseases. The Ems waters, from the Kränchen and Kesselbrunnen springs, are employed at their location in the following mamer: Kränchen for diseases of the organs situated above the diaphragm, and Kesselbrunnen in diseases of parts below the diaphragm. The reason for this is evident: in the diseases of organs below the diaphragm, as we see it so well exemplified in our patient, with his (on admission) torturing pains and diarrhœa, the warmer Kesselbrunnen (37.3 $R ., 116^{\circ} \mathrm{F}$.) is more suitable than the cooler Kränchen $\left(28.7^{\circ} \mathrm{R}\right.$, $87.8^{\circ} \mathrm{F}$.) ; on the contrary, in diseases of "parts above the diaphragm," namely, of the respiratory organs, Kränchen is more suitable, because the much warmer water of the Kesselbrumnen might rather tend to call out hæmoptysis. In the imported Ems water there is certainly no such difference in the temperature of Kränchen and Kesselbrunnen, and they can therefore both be used indifferently, in view of the similarity of their composition, heating them up to the temperature required by the presenting case.

Ems and Obersalzbrunnen. - Ems is usually prescribed in catarrhal conditions of the respiratory tract, Obersalzbiunnen in 
tuberculous affections of the same: thus in the ordinary most frequent form of tuberculosis of the lungs, that is, in chronic tuberculous broncho-pneumonia. Ems is not prescribed for tuberculosis pulmonum for fear of causing hrmoptysis. The cause of hrmoptysis when brought on by the employment of Ems in patients suffering with pulmonary tuberculosis, is to some extent found in the very locality of Ems. which is very warm during summer ; while the locality where Obersalzbrumen is situated is much cooler. Another cause lies in the fact, that Ems. being warm and containing less of carbonic acid, rather tends to constipate, and constipation creates a predisposition to hæmoptysis ; whereas Obersalzbrumnen, being cold and containing a great deal of carbonic acid and also little of a laxative salt (sodium sulphate), is rather of a laxative nature. When using the imported Ems and Obersalzbrumen waters. the differences of locality and temperature are of course not taken into account. and both waters may be employed indifferently for catarh and tuberculosis of the respiratory organs, Obersalzbrumnen being prescribed for cases inclined to constipation, and Ems for those with a tendency to diarrhoea.

As has been explained above, the Contrexéville water, containing lime, would be the most suitable for the oxaluria and renal colic in our patient. but as it would tend to prolong the diarrhoea we were compelled to abstain from employing it and had to resort, in combating the above-iramed diseased conditions. to the use of the alkaline Ems waters.

It becomes apparent from the above. why we preferred above others the Ems water for our patient; but to make still clearer the matter of selecting mineral waters and at the same time to characterize, even to a slight extent, the most importint of those selected by us for comparison, I shall again return to this subject in the next lecture.

\section{LECTURE OF OCTOBER $13,1889$.}

Condition of the patient. - A week has passed since we saw the patient last time. I) ring this week he continued taking the warm Ens rater (one and one-half glasses per day). the 
decoction of condurango with drops of nux-vomica, drops of coto, and wine; continued taking baths at times and had massage constantly. The patient's condition is steadily improving. The quantity of food is slightly increased by the addition of a glass of milk and of about one-fourth of a pound of white bread, and the food itself is somewhat changed; on account of the difficulty in obtaining fresh eggs, they were discontinued and replaced by an additional quantity of chicken meat. The patient gets now per diem the following: about one pound of white bread (the whole bread with the crust is eaten), one-half chicken, two meat cutlets, two glasses of soup with broken up meat, two glasses of milk soup with manna, and three glasses of milk with cognac. This quantity of food is digested by the stomach withont any dyspeptic phenomena. The intestinal evacuations once a day, the first days after the third half-glass of Ems was added, were formless and even somewhat liquid, however without pains on defecation, but these last few days they again became quite solid, althongh the patient continues, as I said, drinking three half-glasses of the water. The abdomen is not distended. The spontaneous pains appear only at the side of the left kidney, more frequently on motion, and are weaker than formerly. Examination of the abdomen also elicits tenderness only on the side of the left kidney; at the other places, among them in the region of the gall-bladder and sigmoid flexure, where a week ago pressure elicited tenderness, there is at present neither sensitiveness nor tenderness to be noted. The urine became normal, — it is neither pale nor turbid, outside of the usual and normal slight cloudiness due to mucus, and gives absolutely no sediment of calcium oxalate crystals. The pulse continues strong. Patient keeps gaining in weight. the latter being now ninety-eight pounds (seven pounds more than he weighed on admission). His sleep is satisfactory, his strength grows steadily.

After what was said of the plan of treatment and in view of the just enumerated results of this last. it is evident that nothing is to be changed as yet in the treatment. The drops of coto must be continued till the intestinal eracuations become normal - steadily, without oscillations; the dose of these drops 
must be slightly increased, as the patient could become habituated to them; instead of fifteen drops as heretofore he will be given twenty drops three times a day.

\section{I return to the mineral waters.}

In what cases is Marienbad to be prescribed? This water in the quantity of bicarbonate of soda (16 per 10,000) and of sodium chloride ( 17 per 10,000) approaches Ems water, but contains a great quantity of a laxative salt, sodium sulphate $(49.5$ per 10,000$)$ and besides it is a cold water $\left(53.1^{\circ} F\right.$.). I will attempt to give you a description of typical patients who require treatment by Marienbad. They are usually people of good constitution, over forty years of age, well-to-do, leading generally an indoor life, brain-workers; their amusements (reading, cards, theatre, society) also of a mental character, with little out-door exercise, - mostly city inhabitants. The following collection of diseased phenomena is usually to be found with them: 1st, considerable dyspeptic phenomena principally constant or frequently occurring symptoms of an over-loaded stomach (due usually to a temporary overdistention of the organ, and not to an established dilatation of the same): a feeling of fulness and lieaviness in the epigastrium, belching, often painful, with a sensation of burning, of gases, liquids, or of mostly undigested food; but constant and severe pains, as well as vomiting, are usually absent. Investigation ordinarily finds the causes of this condition to be in the majority of cases as follows: the patient over-eats constantly; being well-to-do. he usually has very palatable food, often also wine, and he therefore eats much more than is plainly necessary for satisfying hunger; added to this is a nervous dyspepsia, as the patient is either a neurasthenic, or, as we shall see later, becomes such: oftentimes there is also associated with this a gastric catarrh. but such is usually trifling, without pain or vomiting. Then such patients usually suffer from a constant constipation (or at least from an insufficient emptying of their bowels), partly on account of their neurasthenia, partly becinse of the clistencled stomach, that is on account of the retarded passage of the 
gastric contents into the intestines. The abdomen is distended, the diaphragm is pushed upward. The urine is more colored than normally, and gives frequently some uric acid sediments. There appear at times slight symptoms of renal colic, and some gouty pains in the small articulations. The liver is usully enlarged and sensitive on account of hyperæmia. This latter is due either to the fact that the patient is addicted at the same time to alcoholic drinks, or because the passage of the food-stuffs from the intestinal canal into the vence porte is more abundant than normally, or. as some suppose, also because these stuffs contain a great number of ptomaines (which are also supposed to cause a hyperæmia of the liver), which in such a condition of digestion are produced in abundance by the nsual microbes of the stomach and of the intestines. There are at times also produced slight symptoms of liver colic. The organs of respiration are either normal or there is a slight catarthal condition of the upper respiratory organs; the organs of circulation are either healthy or present some slight signs of a chronic arteritis. The patients, howerer, usually complain of drspnora and palpitation, which may in part be due to purely mechanical causes. as the high position of the diaphragm, or partly to reflex action. through the nervous system. by the abdomimal disturbances, and perhaps on account of the above mentioned ptomaines. The patients are usually not lean, but stout. and sometimes even obese; they usually complain of "iufluxes of blood to the head." or of the following morbid s.mptoms (partly simply of a vasomotor origin. partly probably of ptomaine origin): a feeling of leat in the head, flushed face. a general heaviness in the head, at times paim at the nape of the neck, dizziness. troublesome sleep, fatigue while employed mentally, and an irritable and somewhat gloomy mental state. Such patients. if they were not neurasthenic before. hecome such now to a greater or lesser extent. on account of the abore-mentioned abdominal and mental disturbances, especially under the influence of the above-described mode of living. If this morbid condition is not clearly defined as yet. heing only in the beginning of its derelopment, then it is, of comre. possible to bring the patient to his normal health by the 
aid only of a strictly regulated diet. But if this condition be completely, even if not extremely dereloped, then it would be erroneous to attempt to improve the patient's condition by hygiene only, without a resort to treatment: it is doubtful whether such an improvement could ever be reached; at any rate only after a considerable length of time, in the course of which the patient would remain needlessly in a very unsatisfactory or even painful condition. It is here that we must prescribe Marienbad in commection, of conrse, with hygiene.

Before we speak of the action of Narienbad, let us consider what would be the action of Ems in such a case. Being a warm water and not containing any laxative salts, Ems would tend to costiveness, thus aggravating the constipation, and, on being absorbed, would increase the overloading of the rena portie and the hyperemia of the liver; as a consequence both the chest and chiefly the head symptoms would become aggravated. Whereas Marienbad. being a cold water and containing a laxative salt, if used correctly in quantities sufficient to cause abundant, gruel-like evacuations (but not watery, of which later), would sufficiently empty the gastro-intestinal canal and would thus remove the above-named consequential disturbances. If the Marienbad water be arministered correctly, that is, so as to produce abundant, gruel-like evacuations, but not always watery, then it is likely that its Jicarbonate of soda and its sodium chloride are absorbed either completely or partially, because with the general improvement of the patient the former slight symptoms of liver and kidney colic, as well as the gouty symptoms, are seen to disappar. Patients, especially if obese, grow thin by the use of Marienbad.

If $i$ addition to the use of Marienbad, there is also a radical change instituted, even for a short time, in the mode of living and in the surrounding circumstances; as, for instance, a trip to Marienbad itself, and later, to combat the neurasthenia, a trip to the sea-lyatling places (for those who are younger, bo the more northern ones, as Lamanche or the Baltic Sea; for the older ones - to Biarritz or the Black Sea), or, without leaving the immediate locality, a remoral from the city to the country (in connection with this, Marienbad is taken in May, while the 
younger patients bathe in the river during June and July, and the older ones take salt-baths at a temperature of $27^{\circ} \mathrm{R}$. and lower), then the result of snch a treatment may be a complete return to health; - the patients feel rejuvenated. If, besides, the physician be careful and strict in his requirements and make it clear that the obtained result can be lasting only in case the hygienic rules are strictly adhered to, and the former careless mode of life is avoided. then the issue will be perfectly satisfactory, especially if the patient possess a strong will and be careful of his health. In the contrary, unfortunately more frequent, cases, when the patient falls back to his irregular hygienic habits, a repetition of the Marienbad or of some other similar water becomes necessary. These repetitions do not, of course, give the same satisfactory results, as when the water was used the first time, and besides a repeated and, particularly frequent, employment of mineral water is not without its undoubted harm, for it deranges digestion and nutrition.

What would be the action of the Vichy springs on a case, as described above, so suitable for Marienbad? Grande Grille, being a warm water and containing almost no laxative salts, would aggravate the constipation and with this also the condition of the patient. The cold Célestins would be less constipating; but not containing any laxative salts, it would not act upon the bowels sufficiently and would consequently be inferior to Marienbad. In such cases it is the degree of costiveness that decides the choice; if the tendency to constipation is not great. then even the cold Célestins will act as a laxative, if assisted by a temporary or a frequent use of a small quantity of some bitter, so that together they will exert an effect approximating the action of Marienbad: (the other diseased conditions, outside of constipation and its consequences, found in a patient described above. as the dyspeptic, liver and kidney symptoms, will be certainly favorably acted upon by the Célestins, so rich in licarbonate of soda). This latter is then evidently better indicated for such patients than Vichy.

What would be the action of the Karlstad springs on such a patient? They contain sodium sulphate, but in half the quantity, contained by Marienbad. and besides they are partly 
warm and partly hot: the warmth of the water may, especially in conditions of constipation, inhibit the laxative action of the moderate quantity of the sodium sulphate and consequently, by aggravating the costiveness or its effects (particularly the head symptoms), will aggravate the patient's condition or at least by not inducing a sufficient evacuation of the bowels, it will not produce the necessary curative effect.

A few words regarding the indication for Kissingen. It has much in common with that for Marienbad, because, although Kissingen does not contain any sodium sulphate and but a little ( 5.9 of the 10,000 parts) of magnesium sulphate, it still is a laxative, thanks to its low temperature $\left(51.8^{\circ} \mathrm{F}\right.$.), the great amount of carbonic acid ( 30.4 per 10,000) and the enormous quantity of sodium chloride (58.8 per 10,000). But the laxative action of Kissingen is not as strong as that of Marienbad, and it is therefore suitable for such patients as were described in discussing Marienbad, but somewhat older and not in the best of nutrition; besides, also for such patients as do not present any clearly expressed indications for the use of bicarbonate if soda (as uric acid gravel, etc.), as this last is absent in the Kissingen water.

For what cases is Karlsbad prescribed? Principally for severe gastric catarrh, accompanied by pains and vomiting, and for severe liver colic, depending on biliary calculi; in both such cases there is, to a greater or lesser extent, constipation, or constipation intermittent with diarrhoea of short duration: but this latter is neither constant nor considerable (for such a case, as was explained above, we would employ Ems). The warm or even the hot water of the Karlsbad springs, containing a moderate amount of sodium bicarbonate, of sodium chloride and of sodium sulphate, and as little of the remaining ingredients as the water of the Ems springs, and being just as " pure," acts splendidly in the above mentioned cases. Marienbad would evidently be unsuitable for such cases, because, being a cold water, it would tend to aggravate the gastric pains, the romiting and even the attacks of the liver colic, or because it would purge the patient strongly; whereas in these cases severe purging is positively undesirable, as it hinders the treatment, while our aim is to empty the bowels regularly ; this would not prevent absorption 
and consequently the further action of the mineral alkaline water on bile, biliary calculi and also on the urine and urinary gravel, if such is to be found at that time. On the contrary, Vichy, namely, the warm Grande Grille, which would be suitable in such cases because of its warmth and of the abundance of its sodium bicarbonate, is contra-indicated by the constipation, that particularly aggravates the head symptoms, because, not containing any laxative salts, the water would aggravate the constipation and its consequences. 'The same must be said of Ems. In the above described cases where Karlsbad is inclicated, if the constipation be severe and the head symptoms considerable, then a laxative salt (usually the "Karlsbad" salt itself, obtained from Karlsbad water and containing sodium sulphate) is added to the water; while in old persons, in whom there may be any apprehension of apoplexy, derivative bloodletting is to be resorted to, leeches applied to the region of the coccyx, before Karlsbach is employed.

A few words concerning the indications for the Franzensbad Salaquelle (lit. salt-spring). Fuanzensbad is famous for its mineral-mud baths (Moorbäder). Thither flock in great numbers women suffering from diseases of the genital organs and from failure in nutrition and strength, and also exhausted and neuasthenic men. Such persons often, almost always, suffer with dyspeptic symptoms, gastric and intestinal, due to a slight catarh or to nerrous dyspepsia, - usually with constipation,-who require the use of slightly alkaline and laxative waters. Salzquelle, as you may see by the table, is just such a water, and is in addition very pure, containing, outside of the principal ingredients, very little of other component parts. Marienbad would be too strong for such patients. Ems is also unsuitable. as it would not remove the constipation. Finally the cold Salzquelle is preferred to the hot Karlsbad for women with diseases of the generative organs, because of the liability of the latter to either cause or to aggravate intemal hemorrhage.

The chief indication for the richy springs is gout (arthritis urica) and renal gravel (in the form of urates) with or without dyspeptic symptoms, but without obstinate constipation, and besides in patients with a florid, or at least a good uutrition. 
Marienbad would be unsuitable for such patients, for acting as a laxative, it would hinder absorption and consequently also any further action of the alkalies; while Karlsbad, becanse of the warmer temperature of its water, would have a weaker diuretic effect, and besides would act as a laxative, which again would comnteract its diuretic effect. Finally, Vichy contains bicarbonate of soda - the principal active agent for the abovenamed cases - in a much larger amount than either Karlsbad or Marienbad. Howerer, Karlsbad is prescribed for renal gravel, as well as Vichy is for biliary calculi. we being guided. besides other considerations, usually by the fact that if, in the presence of both the liver and the renal colics, the first predominates, then Karlsbad is given, if the latter - then Vichy.

Yessentucki. - There is a generally prevalent opinion to the effect that the Yessentucki springs are equivalent to those of Vichy. This opinion is wrong; the number of indications embraced by the Yessentucki springs is much greater and much more varied than the number of indications for Vichy springs. Théy are alike in the quantity of the predominant bicarbonate of soda, although the Yessentucki springs Nos. 17 and 18 are richer in it than the Vichy springs. But the chief difference between the springs of both groups consists in the quantity of sodium chloride, of which there is little in Vichy and much in the Yessentucki. The considerable quantity of the sodium chloride imparts to the Yessentucki springs a laxative action, which is lacking in the Vichy springs; although this laxative action is not as strong as that of the waters containing sodium sulphate or magnesium sulphate. We shall see later that in this quality of the Yessentucki springs lies their chief preference. Finally the three Yessentucki springs. Nos. 17,18 and $t$ (the ascending stream), so very similar in their composition qualitatively. present a considerable quantitative difference, which again forms a great advantage. enaluling us to order this or that spring, according as to whether the case requires a stronger or weaker action. taking into account the degree of development of the diseased conditions, the constitution and nutrition of the patient.

In all the cases for which the Vichy springs are prescribed (vide above), we also prescribe the Yessentucki with eren greater 
success, if there be, as there is liable to be in the majority of cases, a tendency to constipation; if there be an inclination toward diarrhoa, which is rather of rarer occurrence, then the Vichy, as is apparent from what was stated above, would be more preferable. In view of their low temperature and the considerable quantity of the contained sodium chloride and carbonic acid, i. e. because of their laxative effect, the Yessentucki springs are successfully prescribed for the same cases, in which Kissingen-Rakoczy is indicated (vide above), if there is simultaneously an indication for the introduction into the organism of bicarbonate of soda, which is lacking in the Kissingen spring. With the aid of the simultaneous use of a small quantity of some bitter water, as, for instance, of the Maria Theresia spring (near Pyatigosk), the water of the Yessentucki springs Nos. 17 and 18 can in many cases fully replace Marienbad, and, when warmed sufficiently, also Karlsbad.

For urinary gravel, composed not of urates, but of phosphates and oxalates, as also for gouty patients with failure in nutrition, in the so-called atypical gout, the earthy mineral waters are indicated; the best of these, as far as is known now, is Contrexéville, which I have already mentioned above.

\section{LECTURE OF OCTOBER $18,1889$.}

While comparing the mineral waters of which I spoke above, I presented typical examples where they are to be employed, but I certainly did not intend to exhaust the whole variety of cases which may present some preëminent indication for the use of this or that water. This comparison of the most important mineral waters has made clear to you their chief individual characteristics, the knowledge of which will enable you to correctly prescribe these waters; I repeat, the most important waters, because they have been the most investigated, the most useful, and because in the great majority of the occurring cases we see them meet all the indications; not to mention the fact, that they, as I said once already, present types of whole classes of mineral waters. This comparison has also made it 
clear to you as to how the selection of a mineral water for a given case is made, which knowledge will aid you in prescribing also other mineral waters not discussed by us.

When speaking of treatment (in the Introduction to Clinical Exercises) I pointed out the mistake of employing complicated medication. You might ask whether it would be correct to use such combinations of drugs as presented by a mineral water. You might also ask why I selected for comparison only the foreign waters, except the Yessentucki.

The knowledge of the effectiveness of the mineral waters has been obtained in a purely empirical way, at a remote period, when medicine and balneology were far from their present perfected condition. To reach this perfection there were required centuries of labor, gigantic progress in diagnostication, general pathology and therapeutics, as well as in the chemical and clinical (and partly physiological) knowledge of the mineral waters. These tests of centuries the waters stood nobly: the more they were studied, the greater and not lesser, became their importance, until it is now enormous as compared with what it was before. They stand above comparison with the various complex decoctions, powders, pills. drops, etc., -- fruits of experience. but oftener of the fancy of the physician, - fruits of but ephemeral existence, as time shows, burying them into oblivion.

For comparison we took only foreign waters, except Yessentucki. We must first of all remember that these foreign waters are the very useful ones, and that a familiarity with these and the knowledge of their employment is just as necessary as the knowledge of the employment of opium, quinine and others, and these also are foreign drugs. We have selected these waters because they present the best examples known to us of a happy natural combination of curative agencies, and because the indications for their use are best known to us (it is for the same reason that we chose Yessentucki from among our own waters). However, I do not in the least doubt, but that the considerable employment with us of the foreign mineral waters is but a matter of temporary duration. I am perfectly certain, that our great land is very rich in various mineral waters possessing just as happy, and even much happier, combinations of curative 
forces. Time will bring it about, that we will become acquainted with these waters and that we will investigate their composition. Knowing this last and the various combinations which centuries of experience in foreign waters, and some experience in our own, has shown to be effective, we shall soon learn for what cases these our waters will be beneficial, and we will employ them on the spot as well as export them, instead of the foreign ones, as we do now.

I pass now to the very important subject of the mode of employing the mineral waters: what shall be the daily quantity, at what time of the day administered, and for how long a period.

At the begimning of my medical practice the mineral waters were employed only during summer and the patient usually departed, with the purpose of drinking them, for the places where the springs were located. Imported mineral waters were prescribed but seldom. Artificial mineral waters were used also during summer in those few places, so rare then, where such waters were prepared. But for the principal object, I will repeat, of drinking the water, summer excursions were undertaken to the mineral water resorts.

The general character of practice at that time was such that waters were used then in much larger quantities than they are used now. Dr. Debout d'Estrées (" Les indications anx eaux de Contrexéville," Paris, 1889 ) mentions the fact that the famous writer Madame Sérigné (he refers, as you see, to a long goneby past) was ordered daily at Tichy 12 glasses of the water of the Grande Grille spring, she being previously bled and given a laxative. We now generally give less. Dr. Debout d'Estrées is rather inclined to ascribe this to the fact that, as he expresses it, the struggle for existence has made our generation anæmic and nervous.**

Without denying, but on the contrary considering it quite probable, that the percentage of men of strong constitution and health in certain social strata is now smaller than it was formerly, I still think that there was another reason for the former

* Tos cerveaux, surmenés par le strugrele for lifé nous ont domné l'anémie et les uévooses, que ne eonnaissaient pas nos pères (page 11). 
use of water in greater quantities, namely, this : that the arrivals at the springs, never having the necessary time at their disposal, the physician attempted to obtain the desired effects sooner, and therefore administered the water in great quantities. But at present, when the waters are much more used, while remaining at one's own constant residence, this lack of time, and with it the necessity for haste, are absent. It is well understood that the progress in diagnosis, general pathology and therapeutics could not but have a certain influence on the diminution of the prescribed quantity of the water for drinking purposes.

However, even now the routine method of drinking the water at the resorts in summer differs greatly from the manner in which they are used at the other seasons of the year, and even now preserves a great many of the former procedures. The water is usually drunk in the early morning, when it is not yet hot, so that the prescribed walk to be taken after the water is made less burdensome; the water is taken on an empty stomach and in lesser quantity than formerly, but always in a greater quantity than when the water is prescribed not in summer, but at other seasons of the year and at the place of the patient's residence. Such a routine treatment often gives excellent results. Picture to yourselves a city inhabitant of strong constitution, who is habituated to late rising and of course to retiring late to bed, who commits errors in his diet, lives a mental life, has almost no exercise, and but seldom goes out into fresh air,-imagine such a person leading a totally different life at the watering place: he rises early and therefore goes to bed early, observes a correct diet, spends considerable time out-doors, does a great deal of moving around, rests from his mental labor, and frequently, in addition to all this, removed from the place of his permanent residence and free from the many constant and unpleasant impressions, he drinks the correctly preseribed water and usually takes his baths regularly. We certainly get in such cases strikingly good results. But unfortunately such is not always the case. Routine, that has always something of good with it, - namely, that which created it, remains but routine. that is, it is at times beneficial, at other times harmful. Not to speak of the cases, when the physician s advice concerning a trip to the watering place is incorrect, when without individualizing 
the given case, without considering all its peculiarities, he orders a trip not for the one for whom it may be convenient and beneficial, but for a person who can only with difficulty tear himself from his near relatives and wonted surroundings of life, and for whom, besides, such a trip is beyond his means. Not to speak of such a wrong and useless advice to undertake a trip, I will only point to the impracticability of the above alluded to routine even for many such cases, where the trip is both indicated and possible. Here is one of the cases that occur quite frequently. A woman, somewhat exhausted and anæmic, subject to chills, nervous, and a poor sleeper, comes to the watering place in accord with the advice of the physician, begins the above described routine method of living and of drinking the waters. She cannot fall asleep early in the evening but sleeps quite soundly in the morning hours. She is waked up to go to the spring, to take a walk, and to listen to the inevitable music. Without having a good night's sleep she starts out, soon becomes chilled, owing to the coolness of the morning air, experiences a sharp sensation of hunger and is soon fatigued; and in such a condition _- hungry, chilled through and tired out, she must drink a cold water and walk for a long time. Her condition has, of course, become even worse than it was before, and the water was of no benefit. The patient then consulted one of my former students, who lived at the watering-place and who was acquainted, through my clinic, with the use of mineral waters. He first of all advised her to sleep in the morning as long as she felt sleepy, not to interrupt the sleep, not to drink the water on an empty stomach, but after the morning coffee, before breakfast and before dinner, and in a lesser quantity; to be out in the fresh, open air, for a longer time, but at the same time to walk only as long as her strength permits it, and by no manner of means until she becomes fatigued. After this the patient began to improve.

I alluded to the trips to the watering places with the purpose of acquainting you, at least to some extent, with this medicinal agency, and to present examples in which this agency is suitable and where not, and finally to point out the necessity, while employing this remedy, for individualizing your cases, so that you may not fall into routine liabits and that you may 
change the routine order of living and of drinking the waters in accord with the peculiarities of the given case. I cannot discuss the subject much longer, the more so, as you will all undoubtedly have to prescribe the mineral water much oftener for the patients in their places of residence, without sending them to the watering places. We will now endeavor to answer the questions as to the quantity of the water to be used per day, for how long a time, and at what periods of the day. I have already said that at the present day mineral waters are employed in lesser quantities than formerly, but the manner of employing them at the different watering places, or even by several physicians at one and the same watering place, is very varied; I will therefore, in view of the absence of any standard or commonly accepted rule, speak only of that method of employing the mineral waters to which I adhere on the ground of my own experience extending over many years.

The quantity of the daily dose of the waters depends, of course, on what you have in view when prescribing the water, and consequently on the character of the water chosen for the purpose. Within the limits of the effects of the waters selected by us for comparison, we may note the following most important objects, that we most frequently strive for:

1. We strive to secure a direct effect on the mucous membrane of the stomach and the upper portion of the intestines, or, besides this, by way of absorption of the mineral water by the intestine and by its entrance into the blood, on the mucous membrane of the biliary, urinary and respiratory tracts, on biliary calculi and urimary gravel; provided the intestinal evacuations of the patient take place regularly. In such cases we usually prescribe Ems, Obersalzbrunnen, Vichy and Yessentucki, in doses, for adults, from two to six times a day (the usual dose is one-half glass, i. e. four ounces, or three-fourths of a glass, i. e. six ounces, but then only four glasses a day): for weak and exhausted persons two or three half-glassfuls of Ems or Obersalzbrunnen; for strong persons with a good nutrition three to six half-glassfuls of Vichy or Yessentucki. In case there is a tendency to diarrhwa I prescribe a smaller number of doses and the warm spring, or water warmed up to the required 
temperature. If I find an already existing diarrhoea, then I order only Ems, in smaller doses, and at times, at the beginning, only half-doses, one-fourth of a glass, or two ounces of the water, still more warmed. In cases with a tendency to constipation I use Yessentucki springs and in a greater number of doses. If in addition to an inclination to costiveness the patient be exhausted, weak and suffering with pains, that serve as an indication for warm water (gastric pains and liver colic), then I prescribe Ems, warmed, in less frequent doses (for instance three or four half-glassfuls) and at the same time evacuating clysters. I will add here, that both for cases to which I allude now and for those of which I shall speak later, we can prescribe in summer generally more water than at the cold season of the year; because movement in the free air assists the organism in better sustaining the mineral waters; and light summer dress makes out-door movement certainly easier, than the heavy winter overcoat. Besides, the use especially of warm water is rather risky in winter: at the hours of drinking one must not go ont of doors, but must walk around the room. In ordering movement, whether out-of-doors or within the room, while water is being taking. you will clearly see from what I said just now and from the examples alluded to above. that every given case must be individualized, i. e. we uust take into considerstion the season of rear, the weather, the quantity of the ordered water, the strength of the patient and indeed the whole course of treatment.

2. We have in view the same aims as in the first cases, but we deal with patients who either suffer constantly from constipation, or from a constipation that is at times intermittent with a diarhura, and in whom we must therefore take pains to secure a regular evacuation of the bowels, but at the same time to prevent severe purging, as that would destroy our chief object, namely. the absorption of the mineral water and its passage into the circulation for further effect. In such cases I either order Tessentucki (as I do for cases with a tendency to costiveness) and. moreover, in larger doses (up to six half-glassfuls), at times with the addition of a bitter water. or, if in case of severe pains (of gastric origin and liver colic) there is also required a warm 
or even a hot water, then Karlsbad - in winter three to four half-glassfuls (if necessary I add Karlsbad salt), in summer from four to six doses of three-quarters of a glass each, also with the addition of the salt, if necessary. As referred to above (vide Yessentucki), Karlsbad water may in such cases be often replaced by the warmed Yessentucki, with the addition of some bitter water.

3. Finally we aim not only at evacuating the bowels, but at inducing a more or less considerable looseness of the same for a certain period of time, as, for instance, for several weeks. For such cases I order Marienbad or Kissingen or Yessentucki (with the addition of a bitter water), three-quarters of a glass per dose. from three to six doses a day, in accord with the desired and obtained results, and usually only during the summer. The use of such a quantity of water in winter or generally at a cold season of the year is inconvenient and risky, as it absolutely requires considerable movement or at least a prolonged stay outof-doors. In the winter and at the cold season generally we can reach the desired effect for such cases, even if somewhat slower. by ordering simultaneously with Yessentucki some bitter water. - of both together some four half-glassfuls per day — artministering more of the one or of the other, in accord with the desired and the obtained effect.

For how long a period is the prescribed water to be taken? (The length of the course of treatment.) Certainly, up to the time the desired object is effected; but I usually do not continue treatment with mineral waters for longer than six weeks at a time. If they are prescribed for a recent catarrh of the stomach and intestines, or of the biliary, urinary and respiratory tracts, then the above length of time, or even a shorter period of five, four or three weeks, is quite sufficient; just as in the cases in which the waters are ordered as constant laxatives. But for cases of chronic catarnh, biliary calculi, renal gravel and gout a six weeks' course proves frequently insufficient; but this notwithstanding I often interrupt for a time the use of the prescribed water after six weeks or before - for two reasons: 1 . A longer, uninterrupted administration of mineral waters tends to disturb digestion and nutrition, as there appear dyspeptic 
phenomena, and the patients grow thin and pale. 2. As the effects of the water last for some time after its employment has been discontinued, we give an opportunity for the results of the treatment to show themselves, after we have ceased employing the water after a period of six weeks; then, guided by these results and by the condition of the patient's digestion and nutrition, we are enabled sooner or later to return to the treatment by waters.

The distribution of the prescribed quantity of the water over the course of the day must be made in accord with the aim we have in view. If the water be employed for its action on the mucous membrane of the stomach and of the intestines and also for its absorption and passage into the circulation and for further action, then we must assign the ordered quantity over the whole day for instance, before morning coffee or tea, midday lunch and dinner (at about 6 P. M.; it is better to avoid the use of the waters late at night, as that may disturb the sleep). But if the water be taken for its laxative effect, then all the prescribed doses must be taken, in intervals between fifteen and twenty minutes, in the morning on an empty stomach; this is quite convenient as it requires at the same time movement during the summer and in vigorous persons of strong constitution and fair strength; for contrary cases we must order one-half of the doses before the morning tea and coffee, and the other half before the midday meal.

A mineral water is always to be taken on an empty stomach (the last dose at least one hour prior to a meal), because its action on the mucous membrane of the stomach and intestines, as well as its absorption and its laxative effect, have then a much more favorable influence, not being hindered by the contents of the stomach and intestines. A mineral,water taken soon after a meal will have, if an alkaline, only a palliative effect on the dyspeptic symptoms. It will diminish pyrosis, alleviate belching and the feeling of heaviness in the stomach, but, as I said once already, will with difficulty exert its principal, most important action, in fact may completely lose it. The practice of some of administering the mineral waters with the meals, — usually Vichy or some other alkaline water for renal 
gravel and gout - deserves positive condemnation. Notwithstanding its constant administration, the gout and the attacks of renal colic are not influenced at all, while the patient's nutrition and digestion suffer from the constant introduction of a mineral water; besides, the use of the mineral waters in the ordinary regular manner, as during a course of treatment, does not bear as good results in these patients, as it does in patients not habituated to the constant use of waters. The employment of alkaline mineral waters in cases of gout and renal gravel for a continuous period of time, but with interruptions, in a small daily quantity and in the usual manner, i. e. on an empty stomach. deserves undoubted preference over the above-mentioned practice of constant drinking of waters during meals. The quite extensive employment at the present time of the alkaline mineral waters, natural and artificial, as a constant beverage, is prejudicial on the same ground.

From a great deal of what I said concerning the internal use of mineral waters, it is easy for you to comprehend, where the cold water is indicated and where the warm one. We must now give more precisely the temperature of the prescriber waters. Cnder a cold water we mean water of such a temperature as can be borne comfortably by the stomach, usually not below the temperature of an ordinary apartment in winter, i. e., about $14^{\circ}$ $R$. $\left(64^{\circ} \mathrm{F}\right.$.). Of the waters we discussed, Marienbad, Franzensbad, Kissingen and Yessentucki are given at such a temperature (sometimes a little higher) for their laxative effect. Alkaline waters, indicated for their action on the mucous membrane of the stomach and intestines and for absorption, are administered to persons inclined to constipation, at a temperature of about $18^{\circ}$ to $20^{\circ} \mathrm{R}$. $\left(74^{\circ}\right.$ to $78^{\circ} \mathrm{F}$.), and to those with a tendency to diarrhora at the temperature of freshly drawn milk, i. e. about $25^{\circ}$ to $27^{\circ} R$. (88. $2^{\circ}$ to $92.7^{\circ} \mathrm{F}$ ), while for diarrhou with severe gastric and intestinal pains as well as for kidney gravel, they are given warmer still.

While drinking the mineral waters such a riet is ordered as is, firstly, required by the condition of the patient; and secondly, all the very sour articles of food are to be excluded. as the use of vinegar with meals, lemon, also cucumbers. mush- 
rooms, berries and fruits, so as to avoid a possible diarrhœa, frequently of a severe nature, accompanied by acute intestinal pains, and so obstinate, as to require not only an interruption in the treatment, but a prolonged cessation of the same, because a return to the use of the mineral water brings on a recurrence of the diarrhœa. Ripe, sweet berries are sometimes borne well while the mineral waters are employed, but this is always risky, as together with the sweet ones there may be some that are unripe and sour.

The mineral waters, used as they are at the present time in smaller and moderate, as compared with the past, quantities, are contraindicated only in considerable disturbances of the circulation, hoematopoiesis and nutrition, in conditions of cachexia and marasmus. - We have often seen anemic and exhausted persons, as, for instance, our patient, or, still more frequently, women exhausted by pregnancies and diseases of the sexual apparatus, but with an undoubted gastric catarrh, for whom, in view of their anæmia and exhaustion, one rather hesitates to order a mineral water, hoping to cure them by directly ordering forced alimentation, iron and arsenic, but without first improving the condition of the stomach. Such attempts - I repeat again, in the presence of an undoubted gastric catarrh - always fail: the "nutritious" food does not nourish the patient, as it is not digested; on the contrary, introduced in large quantities (at the advice of the physician - the so-called forced alimentation) and in conjunction with iron and arsenic, it tends to derange digestion still more and through that the general condition. On the contrary, - by regulating strictly the hygiene, and, particularly, the diet and physical exercise (i. e., prohibiting any fatigue), and by ordering general massage, it becomes possible, even in such cases, by the moderate use of the light mineral waters and bitter drugs, to so improve the condition of the patient's stomach, as to make it possible to further improve the general condition and, consequently, that of the stomach itself. Our patient may serve as a good example of the results of such a mode of treatment.

Nervous dyspepsia certainly does not present any contraindication for the use of mineral waters, if it be accompanied by 
certain morbid conditions presenting an indication for their use, as, for instance, gastric catarrh, renal gravel, gout, etc.; in the absence of such indications it would be erroneous to rely on the mineral water alone as a cure for nervous dyspepsia. Of the treatment of this so frequent and so important morbid condition we shall speak when discussing cases of patients subject to it.

Condition of patient October 18th, 1889. Patient is given the same food. His condition is as good as it was five days ago, when we saw him last. During these five days he gained one pound in weight, - he weighs now ninety-nine pounds, eight pounds more than he weighed on admission. Of the morbid symptoms there remain, although constantly diminishing, the former pains in the left kidney on motion, leanness and physical weakness.

What is to be done further? Haring finished the analysis of the present case, and the plan of treating it, and having brought the patient to a very satisfactory condition, we must proceed to the analysis of other patients; it will thus be impossible for us to return to him as frequently as heretofore, and it is therefore necessary to outline the plan of future treatment for a longer time, which is at present, after our treatment has been justified by the obtained results, certainly more possible than it was at the beginning.

The patient has boen taking the Ems-Kesselbrunnen for the last five weebs; notwithstanding the complete absence of dyspeptic phenomena, still caution requires, that we do not discontinue at once the use of this water, but continue it for another week. First, because the patient has had the catarrh for such a long time; secondly, the dyspeptic phenomena are absent when very light food is taken with due care (little at a time and frequently), whereas the patient has to return to his habitual rougher food; and, thirdly, to meet as much as possible the renal indications (oxalic acid gravel).

We will also continue for another week the drops of coto, diminishing the dose continually for the same reasons. - so as to secure the obtained result, the cessation of the diarrhoea.

The bitters (condurango and tinctura nucis vomicæ) we will continue for a long period, very likely up to the time he leaves 
our clinic, as experience has shown the utility of their prolonged administration after an obstinate catarrh of the stomach and intestines of such duration, especially in emaciated patients, and also in view of the approaching change in his food.

The baths, which continue to influence the patient favorably, will be ordered for him only at rare intervals, in view of the fact, that he will soon begin taking out-door walks in the free air.

We will continue massage up to the time when the patient will become strong enough to be able to walk and to generally move around in a measure satisfactory to his health. The pains in the region of the left kidney depend, as was explained above, partly on the oxalic acid gravel and partly on the displacement of this organ. Instead of the former abdominal bandage the patient put on yesterday a kidney truss, and to judge by the considerable relief thereafter of the above-named pains on motion, we may well hope to remove them entirely.

What shall we do to further improve the patient's nutrition and his physical vigor?

We cannot think of resorting to any fattening means; the use of koumyss at this season of the year and under hospital conditions is out of the question. It were besides risky to order koumyss, kephir or cod liver oil on account of the only recently passed dyspeptic phenomena and the diarrhora that ceased not long ago. We must then confine ourselves to the correct regulation of the food.

For the same reasons it would be risky to give arsenic and, although not to such an extent, also iron. But then, our patient's appetite is so good, and his weight increases so considerably, that there appears to be no necessity for resorting to these drugs.

After he will have discontinued the use of the Ems water, we will think of prescribing for him silver nitrate, in view of the considerable weakness of the patient's nervo-muscular apparatus. and also in view of the recently ceased intestinal catarrh, so that we may the better secure the now present good condition of the intestinal tract. Until now we have not yet ordered any outdoor walks for our patient, although, considering his pres ent 
condition, he could take these to some extent, and they would certainly benefit him; but we did not allow them on account of the extremely unfavorable weather - it being damp, windy and cold. It would be beneficial for the patient to spend the winter in the South, in a warm climate, where he would be able to stay out-of-doors for a long time and move around clad in a light garment, convenient for a weak patient: this would tend to rapidly improve his nutrition and strength, and through these also his constitution (growth). It certainly would be cruel irony, in view of the patient's poor or even wretched circumstances, to recommend him a trip to the South; but my duty, as that of a clinical instructor, requires that I point out to you the indications for climatic therapy, that you may take advantage of them in treating other patients.

\section{FROM THE LECTURE OF NOVEMBER $28,1889$.}

Almost six weeks have passed since we saw the patient last. He continued taking after that, for a whole week, the Ems water and the coto drops, and then left them off. Five weeks have passed since, and his digestion remains good, notwithstanding the fact that his food is coarser now. He continues the use of the bitters. In accord with our propositions he was given three weeks ago pills of silver nitrate, of one-twentieth grain per dose (with extr. trifolii), at first three times a day, the last ten days four times a day, every time immediately before a meal. We discontinued the baths recently, and instead of these he is sponged twice a week, in the morning, with a mixture of brandy and water, half and half, at first of the temperature of fresh milk and later somewhat cooler, followed by rubbing with flannel; this he bears well, does not feel chilly, but feels hale and hearty. Massage is continued less frequently than before, as the patient walks around a great deal. Three days ago (November 25th), in a light frost and during pleasant, dry and quiet weather, the patient began his fresh-air walks, at first twice a day, half an hour each time - and this seemed to do him good: he is not chilly, does not become fatigued and sleeps 
better after it. Some three weeks previous to these walks, which could not be taken on account of the uninterruptedly bad weather, the patient, being confined in the room and deprived of fresh air, began to sleep poorly, but he was improved by franklinization (static electricity), which was resorted to rather than the use of hypnotics internally.

Patient's condition at present. He gets rougher food: more than a pound of bread - half of white bread, the other half pound of rye-bread; instead of chicken and beef cutlets - a pound of beef; instead of chicken soup - lately, cabbage-soup, besides - six eggs, three glasses of milk with cognac, two spoonfuls of wine. Digestion entirely normal. With the kidney truss on, pains in the left kidney are experienced only when making very abrupt movements, and even then they are slight, while in ordinary walking, and especially when at rest, none at all are experienced. Examination of the abdomen elicits but slight sensitiveness in the region of the left kidney. Pulse of normal strength. The patient has grown considerably in body (his weight now is 111 pounds, or 20 pounds more than what he weighed on admission) and looks hale; there appeared even a slight flush on his cheeks. His sleep is good, mental condition splendid and physical strength very much improved.

The patient, or rather the convalescent, desires to leave our clinic in about two weeks, before Christmas, so as to visit his parents. Up to that time he will continue the bitters, the silver nitrate pills and the sponging, and also, at intervals, franklinization and massage, till the gradually increasing walks will permit us to dispense with them. We will also continue to gradually introduce our patient to food that approaches his habitual one.

Patient left the clinic December 15th, strong and having become stout (his weight 115 pounds - 24 pounds more than he weighed on admission), and digesting freely, for the last week, cabbage-soup, buckwheat-gruel and rye-bread. On his departure he was advised not to eat much at a time, to avoid fatigue, and not to go to any bathing establishment in bad 
weather, but to sponge himself instead with a mixture of water and brandy.

Our clinical assistant, Dr. Nikulin, received two letters from the former patient, one in the middle of February, and the other in the middle of April, 1890. He writes in the first. that he is well and bears the wintry weather well (does not catch cold), and also the ordinary peasant's food, and in the second, that at the time of the great fast days (before Easter) he ate well and has borne well the fast meal, has not experienced with this any abdominal pains, has neither grown thin nor weak. 


\section{SECOND CASE}

\section{LECTURES OF OCTOBER 20 AND 24, 1889.}

The patient before you has been in the clinic for three weeks already. The detailed analysis of the first patient I presented to you terminated only with the last lecture, preventing me until now from presenting before you this one. Meanwhile the condition of this patient has undergone a considerable change for the better; but the case is so instructive that I consider it necessary to acquaint you with it.

The patient is forty years of age, was admitted to the clinic September 29th, complaining of constant heaviness and pain in the stomach, aggravated by meals, of belching of gas of the odor of rotten eggs, of pyrosis, nausea and romiting of large masses.

Conditions and mode of living. Patient spends about three summer months in the country, the rest of the year he lives in the eity; in either of the places in a healthy locality and a goor house. Is not habituated to open air bathing (sea or river), but takes his bath in the public bath-house once a month. Smokes a great deal (about forty cigarettes per day). does not drink any brandy, and but little and seldom wine and beer. Used to drink formerly a little very sweet tea, but discontinued it lately on account of the pyrosis, and drinks only water, four glasses a day. Has three meals daily: is fond of fat. sweet and cold articles of food (as ice-cream). dislikes meat. nor can he, as he says. bear it. Patient is married, denies erer having been affected with syphilis, and presents, neither from the investigation of his present condition nor from his past history, no indication of the latter. Patient is occupied during the summer, in the capacity of a manager of a country place. with rural economy; has mich to do, and is subjected to 
a great deal of worry; never sleeps enough, is constantly fatiguing himself, eats irregularly and always in a hurry; in winter, the other extremes: has no responsible occupation, leads a secluded life, plays cards, reads, moves very little in the open air and has little exercise.

Anamnesis until his admission to the clinic and for the time he has been there. - Patient comes from a healthy family. Does not remember having had any sickness until his fifteenth year; in his sixteenth year he had some febrile affection; in his twenty-first had passed through an attack of typhoid fever. Since his twenty-fifth year he began his activity as a country manager, leading the above-described mode of life. It is to this period. that he refers the beginning of lis present illness, the appearance of heaviness (but no pains) in the stomach, of belching, heartburn, nausea and, very seldom, of vomiting. Some three years later these phenomena became aggravated and to them was added constipation. Two years later, that is, about ten years ago, along with the aggravation of the above-named symptoms, there appeared severe pains in the stomach. and vomiting of dark, ground-coffee like masses. Patient began to take Yessentucki water and improved considerably; but the morbid symptoms again became worse in the course of a year. Patient drank Karlsbad water, was treated with silver nitrate, and another period of improvement, lasting one and a half years, followed; but returning to his former mode of living, the morbid condition became again aggravated, and since then until the present time there took place no considerable improvement of even a temporary nature, but, on the contrary. the condition grew steadily worse. Patient began to grow thin and weak, slept badly, became low-spirited and irritable. He always felt better during summer than in winter, thanks to the constant outdoor occupation and notwithstanding the irregularity of his life. Since his treatment with Karlsbad water, or since more than seven years ago, the patient has not been treated any more with mineral waters, but has been taking various pharmaceutical preparations, withont deriving any substantial benefit therefrom. Nor was he treated by gastric lavage. For the last six weeks the morbid symptoms 
have become especially aggravated: the pains in the stomach became excruciating; to obtain a relief from them, the patient at times caused romiting intentionally; besides, spontaneous vomiting of large, coffee-like masses took place once in ten days; the vomited matter usually contained particles of food, more frequently of meat, ingested several days previously.

Directly on the second day of his admission the patient's stomach was washerl out with Ems water; this brought away an enomons quantity of mucus - through the sound and along its sides. The washing was repeated on the next day, but in view of the great quantity of mucus the Ems water was replaced by a stronger (two per cent) solution of sodium bicarbonate, so as to more certainly clear the mucous membrane of the stomach of the accumulated mucus. This second washing also brought away a great quantity of mucus. After the washings the pains and heaviness were considerably relieved. He was given internally Ems-Kesselbrunnen at the temperature of freslily drawn milk, a half glassful three times a day, each time one hour before the meal; five drops of tinct. of nux rom. twice a day, after each meal (the decoction of condurango, prescribed at the beginning, was set aside, as it tended to increase the pyrosis); for severe pyrosis magnesia usta, about one-fourth of a teaspoonful for a dose; for the relief of the severe abdominal pains we tried first bismuth subnitrate (in $5 \mathrm{gr}$. doses) and tinctura cannabis indica (in doses of 10 drops), but they were of little avail, and we replaced them successfully with one-fourth gr. doses of codeine ( $+\tilde{j} \mathrm{gr}$. of (arbonate of sodium): For constipation a clyster of two to three glasses of water at the temperature of warm milk, every other day. In view of his weakness and the exacerbation of the pains on motion, the patient was advised to repose (which he preferred himself) and to avoid sudden changes of position. The abdomen was protected by a flamnel bandage. A preferably liquirl food was ordered - neither cold. nor hot, at the temperature of wam milk, of a bland character and in small quantities at a time. It first the patient was given, for a whole dity, only two ghasses of chicken houllon, one glass of milk, about one-hali a glass of milk-manna gruel. 
two eggs and about one-fourth pound of white lread, without the crust. The quantity of food was increased proportionately with the improvement in the patient's condition.

Strong Crimea wine. which the patient drank rather reluctantly, but which had to be given on account of some weakness of the pulse, was administered in tablespoon doses once or twice a day: to the milk was arded some cognac. a teaspornful to a glass of milk. All these inchuded his food and drink the first time. He was advised to smoke as little as possible.

During the early time of his residence in the clinic. the patient had tro attacks of romiting. but of less abundant contents than formerly. Examination proved the romited matter to contain lencocytes, sarcina rentriculi. hamatin and free $\mathrm{HC}$. the latter in a quantity eren greater than nomal (three and onehalf in one thousand). while a specimen portion of fibrin was completely and rapidly digested. Another washing of the stomach after the second romiting. with a two per cent solution of bicarbonate of soda. brought away much less mucns than the first two washings. Notwithstanding the fact. that he felt rerr much relieved after each washing. which apparently exerted it favorable influence on the comse of the disease, the patient subjected himself to them rather reluctantly: but after the third washing the disease toris such a farorable comse. that there appeared no necessitr to insist on further mashings and we are satisfied with the above ontlined treatment.

The present condition of the patient is. as I sair before. much hetter than it was on his admission. The appetite is keener. The first days the patient ras given for a whole day two glasses of chicken bouillom. a glass of milk with cognac. about one-half glass of milk soup with mannd. two eggs. and about one-fourth pound of white brearl: he gets now one-half pound of white bread. four eggs. two glasses of milk soup with nama. two glasses of bouillon with manna semolino and fom glasses of milk with cognac (outwide of this no bererage but one and onehalf glasses of Ems water) and. as you will hear presently. he is able to digest all this food much easier than before. He gets his wine, as heretofore, one or two spoonfuls per dar. As he takes meat rather reluctantly and observes after its ingestion ais 
aggravation of the gastric symptoms, we do not insist upon his eating it. 'The gastric symptoms may at the present time be smmmed up as follows: at about five P. M. the patient experiences pains at the pit of the stomach, (he takes his dinner at one P. x. and drinks a glass of milk at about three P. x.), which are quite bearable while he is resting, but increase on his moving about, and which are relieved by an easy belching of odorless gases: they disappear at seven P. м.., when food is taken again. During the rest of the day the patient is almost free from any gastric symptoms; whereas on his admission he almost continuously suffered from painful belching of fetid gas and sour liquid, conjoined with a feeling of very severe hearthmrn, nansea, and acute, at times even excruciating, pains in the upper part of the abdomen simultaneously with pain in the opposite part of the lack. The improvement of the patient is also evident from the fact, that for the last ten days there appeared no necessity for resorting to the above-mentioned palliative means against pyrosis and the pains. There was no vomiting for about two weeks. The obstipation also begins to disappear: there were normal morements for the last three days without the aid of clysters. The urine is somewhat pale, but, outside of this, normal. The organs of both respiration and circulation present nothing abnormal: pulse 60 to 70 , was somewhat weak on admission, but is of almost normal strength now. The patient, who never was fat, naturally became very thin while ailing. The temperature is below normal, about $36^{\circ}$ (about $98^{\circ} \mathrm{F}$.) seldom a little higher. The sleep is short, about six to seven hours, but much more sound, than it was on his admission, when it was disturbed by abdominal pains. Mental condition now better. Dizziness and the pains at the nape of his neck, to which he was subject while suffering with obstinate constipation, do not trouble him any more. The patient is stronger than he was on admission.

The objective examination of the abdomen gives, as you see, the following results. Abdomen not distended, whereas on his admission there was considerable bulging of the upper part of the abdomen, and especially so in its median portion and left half. There is no cutaneous hypercesthesia. Tenderness on pressure 
along the median line, from the xyphoid cartilage to the umbilicus, is more noticeable in the direction upwards; below the umbilicus along the same line there is no tenderness. Both hypochondrial regions, especially the left one, are tender on pressure: the right at a point that corresponds more to the position of the pylorus than to the gall-bladder. There is sensitiveness on pressure in the region of the cremm and the ascending portion of the colon. No other abdominal morbid condition is elicited by the objective examination.

Diugnosis.* It is evidently the stomach that is chiefly affected in our patient, and, besides, not by one, but by several morbid conditions. The great accumulations of mucus and the presence of leucocytes in the gastric contents point to an unquestionably severe catarrh of the stomach. Vomiting of dark liquid, that accompanies the attack of acute pains over the whole epigastric region, and especially at the pit of the stomach, and the presence of hrematin in the above-named liquid, speak in the present case for gastric ulcer; we shall see later, why cancer is excluded. We also olserve symptoms of nervous dyspepsia; that is, a greater or weaker development of the dyspeptic phenomena under the influence of a respectively better or worse mental condition, though this latter, as well as the general nervous symptoms (poor sleep, general weakness) depend evidently in their turn on the affection of the stomach and the pains and the insomnia resulting therefrom : from the anamnesis and from observation of the patient we learn, that a gloomy and irritable mental condition, insomnia and general weakness run parallel to the condition of the gastric symptoms, and that they increase or diminish in proportion as these are better or worse. Finally, the patient has a considerable dilatation of the stomach. This is shown by the following: the very marked inflation of the whole epigastrium, especially at the pit of the stomach and in the left hypochondrium, which was so noticeable before the washings, but has disappeared since; the vomiting of great masses, that has troubled the patient for such a long time, the great quantities of

*After the investigation is finished, further explanations, regarding diagnosis, prognosis, etc., are always, in every case, conducted in the patient's absence. 
the gastric contents. that were bronght up by the first washing made on an empty stomach; the presence in these masses, as observed by the patient more than once. of food ingested sereral days ago: the painful belching of great quantities of fetid gases and som liquids. which pointed to stagnation and dyspeptic decomposition of the gastric contents. On patient's moving about, the abdominal splashing was also clearly heard. I mention this srmptom simply because it was present; but I rould certainly not attempt to cause its appearance intentionally: first. because it would be inexcusable, in riew of the former attacks of acute pains and the great sensitiveness in the region of the stomach, to call out this phenomenon, as there would be a risk of simultaneously causing the appearance of such an attack: and secondly, because, as has been explained before, I do not consider this phenomenon as a reliable symptom of dilatation of the stomach. I will add here. that I know of cases, where the splash was caused intentionally and where the sound, introduced immediately after that, showed the stomach to be empty, while after the sound was taken out, the splashing could again be produced. In view of the above undoubted symptoms of considerable gastric dilatation it would be a profitless task to resort, for diagnostic purposes. to such doubtful methods of investigation, as the introduction into the stomach of gas-producing mixtures and the like: not to speak of inconveniencing the patient or even of subjecting him to dangers, that may arise from such attempts. As regards the introduction into the stomach of salol and the consequent determination of the length of time, when salyciluric acid could be detected in the wine, - the application of this method of inrestigation in our case, in the presence of the determined diagnosis of gastric dilatation, would be but a ". scientific ". onnament to an already erected structure; not to mention the fact. that time must ret prove the degree of reliability and practicability of such a diagnostic method. I must also add. that not only does our patient present undoubted srmptoms of considerable gastric dilatation, but that the absence of such a morbid condition would be unintelligible in his case. Dilatation of the stomach may appear as a result either of gastric catarh or of nerrous drspepsia. Our patient has a severe 
gastric catarrh of long duration, and also nervous dyspepsia. The symptoms of gastric ulcer in the given case have also been observed for a long time, some ten years, but they would at times disappear for a year or a year and a half, which fact would lead us to think of former cicatrization of the ulcers and of present scars. However, these latter seem to be so located, that they do not, at least not to a great extent, embarrass the egress of food: we are led to think thus by the rapid relief from the constipation, which indicates a sufficiently free passage of the gastric contents into the intestines.

Does not the patient also have a cancer of the stomach? There is certainly much that speaks against it: the sum total of the gastric symptoms is sufficiently explained by the undoubtedly existing affections, namely: by the catarrh, the ulcer and the gastric dilatation, and also by the nervous dyspepsia; the duration of the illness, the absence of a swelling in the region of the stomach, and the abundance of free $\mathrm{HCl}$ in the gastric contents all speak against cancer. But all the enumerated data make the absence of a gastric cancer in this case only very probable, but not fully certain: there is also in gastric cancer observed sometimes an abundance of free hydrochloric acid in the contents of the stomach; nor is it rarely, but in fact frequently, that we are unable in cases of cancer to detect a swelling in the region of the stomach; it is not at all impossible, that to the long continued gastric suffering of a non-cancerous character in our patient, there was also superadded of late a cancer of the stomach. But then, such apprehensions could only be entertained on the pacient's admission to the clinic; at present, after a considexable improvement has taken place, an improvement that is generally absent in cases of gastric cancer, and would be so particularly in one as broken down as our patient is, - at present such apprehensions are untenable.

As regards the other organs in our patient, we will only note the tenderness on pressure in the region of the crecum and the ascending portion of the colon, surely depending on some slight typhlitis and colitis, as results of the prolonged obstinate constipation. The liver, spleen, kidneys and the urinary bladder, as well as the organs of respiration and circulation. 
present nothing abnormal. We have already alluded to the failure of nutrition and the disturbances of the nervous system.

The prognosis is certainly more favorable now, than it was on the patient's admission to the clinic, when we apprehended the possibility of a gastric cancer. But even now his condition must be considered as very serious, that is, as one that presents no immediate danger, but a great possibility of the same, thus: the obstinate gastric ailment, that has repeatedly reappeared after improvement has taken place, - to be sure, on account of the patient's negligence, - may in the end completely destroy his now already failing health; some accidental cause may bring about a rupture of the thimned-out gastric walls at the sites of the ulcers and of the cicatrices. We have, therefore, while giving him hopes of a return to health, enjoined upon our patient the necessity of a strictly regular mode of living and circumspection in movements and exertions, so as to avoid dangerous consequences.

Treatment. - The gastric therapy of the present case differs from that of the preceding one, chiefly in that in this case we ordered lavage of the stomach, that has given such good results. I shall discuss this important method of treatment, after I will have explained the other therapeutic means; these latter will take but little of our time now, after our discussion of the first case. It is certainly not necessary for me to explain to you why the patient was ordered the above-described diet, the warm abdominal bandage and rest, and why he was given a mineral water (I shall speak later of my reasons for prescribing the Ems-Kesselbrunnen), and the tincture of nux vomica. We have explained above why we gave codeine and magnesia usta. We did not order any warm baths for the relief of the abdominal pains, first, because the codeine relieved the pains sufficiently, and secondly, that he might avoid movements incident to undressing, immersing in the bath and dressing again; these, as any other movements, would tend to aggravate the pains.

Why have we selected of all the mineral waters the EmsKesselbrunnen? Within the limits of the waters chosen by us for comparison, we apparently had to avoid the cold springs and 
those rich in earbonic acid, as such would tend to aggravate the gastric pains and the vomiting in our patient; and we also had to avoid springs rich in alkaline and laxative salts, because of the patient's exhausted condition. For these reasons we had to exclude, first of all, Marienbad, Kissingen and Franzensbad, they being cold springs, and to select from among Ems, Karlsbad, Vichy (the warm spring of Grande Grille) and the warmed Yessentucki springs. In view of the patient's debilitated condition we gave the preference to Ems, as it does not contain any laxative salts (like Karlsbad) and is less rich in alkaline salts, than Vichy and Yessentucki; though if the condition of the nutrition were better, the other three waters could be given. And as a matter of fact, it appears from the anamnesis, that the patient, when in a better condition of nutrition and strength, was treated with success by the Yessentucki and the Karlsbad waters.

All this refers to treatment of the gastric catarrh. How is ulcer of the stomach to be treated? We know of no directly curative remedy, but experience teaches, that an ulcer heals under the same treatment and the same (or even stricter yet) diet, that is so successful in gastric catarrh.*

How shall we treat dilatation of the stomach? After having removed the accumulated gastric contents and having cured the catarrh and the ulcer, it would be necessary to attempt to strengthen the nervo-muscular apparatus of the stomach by the aid of massage, electricity and hydrotherapy (douches in the region of the stomach). The ulcers and cicatrices of long standing in the present case would make it very risky to resort to such measures, as their employment would bring about strong contractions of the stomach, and consequently a liability, not to

* A later supplemental note: For the last few years I add to this treatment the use of bismuth subnitrate with undoubtedly successful results. I give at first twenty-grain doses, increasing the dose, later on, to $3 \mathrm{j} s \mathrm{~s}$ and more, once or twice a day, - always on an empty stomach (in the morning before breakfast. and one hour before dinner). The powder of bismuth is directly put on the tongue and is washed down with the warm alkaline water, which is indicated for the given case. The patient lies on his back, then turns on the belly, and from side to side, so as to enable the remedy to more certainly come in direct contact with the ulcer. 
speak of the pains, of rupturing the walls in the thimned-out portion of this organ. However, to juclge by the favorable course of the disease, we may hope that, on removing the accumulated gastric contents and on curing the catarrh, the ulcer and the nervous dyspepsia, the strength of the nervomuscular apparatus of the stomach will reëstablish itself to a sufficient extent.

For the treatment of the slight typhlitis and colitis, it will in all probability be sufficient to establish a regular emptying of the bowels, to see that the abdomen is kept at rest and warm, in connection, of course, with the contemporaneous improvement in the gastric ailment.

The improvement in the nervous disturbances of the patient is attained as yet through the improvement of the gastric trouble, through the alleviation of the pains consequent upon this last and of the insomnia. Later on, after we will have finished the treatment with the mineral waters, we will think of combating the nervous disturbances and, consequently, the nervous dyspepsia, with silver nitrate, which the patient had used before with great success. Arsenic and iron are contraindicated by the condition of the stomach. We will besides order for our patient general massage (except massage of the abdomen), in accord with the indications, which we followed in our first analyzed case. We hesitate as yet to order massage under the apprehension, that the movements of massage might aggravate the abdominal pains. The treatment by cold water, with a view of improving the patient's nervous state, is contra-indicated by his exhausted condition.

I will now take up the subject of gastric lavage. The present case may be called a typical example of the class of cases, in which lavage of the stomach is an unconditional indication of such importance. that without its previous employment no other kind of treatment can be instituted. In such cases even one or two washings are sufficient to rapidly bring about important results: by carrying away a mass of dyspeptically decomposed gastric contents, absorption of the products of such a decomposition - certainly very injurious to the patient - is put a stop to, as well as the irritating effect produced by them on the walls 
of the stomach, causing vomiting and pains; consequently, as a lesult, a rapid alleviation of the symptoms, is effected; further, the mucous membrane of the stomach is cleared of the mucus, and is made accessible to the radical treatment of the catarrh by mineral waters and by bitters ; finally, the nervo-muscular apparatus of the dilated or distended stomach is able, on the latter being emptied, to act better, namely to expedite the gastric contents into the intestines and thus prevent a fresh accumulation. Therefore, not only in cases of such a prolonged and obstinate morbid condition, gastric dilatation, as seen in our patient, but even in more lecent cases of overloading of the stomach, in which the indications for emptying this last are as positive as they are in our present case, I begin the treatment with gastric lavage, repeating it several times till the patient obtains undoubted relief; and at the same time I begin the radical treatment, if possible, of the gastric disease, most frequently of the catarrh or of the nervous drspepsia, that has caused the dilatation or distention of the stomach; and as soon as the acute symptoms, the attacks of pain, and romiting, and pain on pressure in the region of the stomach, have ceased. I add to the foregoing such remedial means, as would strengthen the weakened nervo-muscular apparatus of the stomach, namely, electricity, massage, or douches, according to the idiosyncrasy of the case. As soon as the sound improvement in the patient's condition can last without lavage, I at once discontinue it; because experience teaches that a prolonged employment of lavage may generate a habit for it. of which it may become difficult to get rid; an example of this will be presented later. But if the indications for lavage are neither so positive, nor so necessary, particularly not in cases of old dilatation, but in those of recent distention, then, without resorting to washings, I order radical treatment in conjunction, of course, with regulation of the hygiene in general and of the diet in particular.

If the success is prompt and lasting. then I surely content myself with the adopted treatment; otherwise I add to it lavage. Wherever possible I avoid lavage, as it is never, especially at the beginning, an indifferent process and far from being harmless for the patient. not to speak of the feeling of disgust entertained towards it by him. 
We often observe in practice, how lavage - which was formerly and is to an extent even now a fashionable procedure - is employed quite frequently without success. Let us consider the causes, as I have observed them, of such failures.

The most frequent cases are as follows: the patient suffers from a gastric catarrh in a condition which does not present any indication for lavage. At first the physician does not resort to lavage, and orders the radical treatment, but he prescribes it either incorrectly or carelessly, without regulating the hygiene and particularly the diet. Failure follows. The physician now resorts to lavage, without having correctly instituted his former treatment and regulated the hygiene, and not having rectified his former errors and omissions, failure is sure to come again. - Or take such a case. The patient has a gastric catarrh in a condition presenting positive indications for lavage : this is ordered by the physician, but he neglects the radical treatment of the catarrh, forgetting that lavage, though an excellent, is at the same time but a symptomatic, palliative measure. The patient is not only not cured, but acquires the habit of washing his stomach, "cannot get along without it," just as one suffering with constipation and habituated to clysters is unable to get along without these. I will tell you in this connection of one very instructive case from my practice. The patient, thirty-five years old, of good constitution, called on me in February, 1888, complaining of pain and heaviness in the region of the stomach, nausea, poor sleep and oppressed mental condition.

Anamnesis. - Up to his twenty-fifth year the patient enjoyed good health; but has since then abused alcoholic drinks (whiskey and beer), took only one meal a day, but an abundant one, and was at times intensely preoccupied with his affairs (he is a tradesman). He soon grew restless, of a sad mood, and costive. Some five years ago there appeared dyspeptic gastric symptoms and pains at the pit of the stomach. The patient continued drinking and leading his former mode of life. Two years ago the gastric pains became aggravated, and there appeared once or twice a week abundant vomiting. containing considerable quantities of mucus. He left off drinking and called on his physicians : these ordered daily gastric lavage with plain water, but no other 
treatment, cautioning him only to avoid heavy food. The first two months the patient experienced considerable relief, but later the improvement not only became stationary, but gave way to a more aggravated condition.

Present state. - Patient smokes much and drinks a great deal: nine to ten glasses of tea daily with cream, and four to five glasses of soda or artificial seltzer water; drinks neither beer, wine, nor brandy. Washes his stomach daily for the last year with plain water on an empty stomach; this brings up mucus, at times in considerable quantity. The lavage procures a relief from the heaviness and pain in the stomach, from the nausea and oppressed mental condition for one or one and one-half hours (in the beginning the relief lasted one-half day or even longer), but later he returns to his usual condition. He is constipated: movements of bowels every other day - evacuations small and hard. Once or twice a month patient takes castoroil or some bitter water. Abdomen distended. The liver, spleen, kidneys and the organs of circulation and respiration present nothing abnormal. Nutrition poor. Sleep unsatisfactory, mental condition oppressed. Treatment: Ems water, one-half glass three times a day, one hour before meals, at the temperature of freshly drawn milk; decoction of condurango and the tincture of nux vomica; watery clysters; once during the day fifteen grains of potassium bromide in the last half-glass of the Ems water, and in the morning sponging of the whole body with water at the temperature of $22^{\circ}$ to $18^{\circ} \mathrm{R}$. (about $82^{\circ}$ to $67^{\circ} \mathrm{F}$.). $\mathrm{Hy}$ giene in general and the diet in particular strictly regulated: quantity and quality of food and drink is limited and precisely designated - to be taken frequently, but little at a time. He was advised to gradually rid himself of the habit of washing his stomach : to do gastric lavage at first twice a week, then once, and later to discontimue it altogether. In about eight weeks of such treatment (the potassium bromide was discontinued before that time), the patient could get along without lavage, and improved considerably: there remained only slight retching and some inclination toward constipation; the pain and heaviness in the gastric region, as well as the nausea, disappeared; both sleep and mental condition improved. A prolonged sail 
on a Volga steamer brought about a complete return to health.

Finally, there are cases, where the employment of gastric lavage, in nervous dyspepsia, is wrongly applied.

Take such a case. Some ten years ago there was admitted to our clinic a medical student of the senior class, with a strongly developed neurasthenia and nervous dyspepsia. The physician whom he consulted before his admission to the clinic, found a "splash" in his abdomen, and concluded that the patient suffered with dilatation of the stomach, requiring gastric lavage. Examination of the patient failed to elicit any symptoms of dilatation of the stomach: the abdomen proved to be equably and pronouncedly sunken everywhere; but to quiet the frightened patient a sound was introdnced: the stomach proved to be empty, which fact raised the patient's spirits considerably; while the subsequent treatment, with cold water douches and silver nitrate, soon removed the phenomena of neurasthenia and of nervous dyspepsia. Or here is another case (a later supplement). Patient, thirty-six years old, of good constitution, was admitted to our clinic April ith, 1890. complaining of heaviness in the region of the stomach and belching, of constipation and pains in the abdomen, which proved on inquiry to be of intestinal origin, of poor sleep and oppressed mental state. Anamnesis: Until his twenty-ninth year patient enjoyed good health; at this age he passed through an attack of typhoid, which left him in a weaker condition than he was before. Later there were superadded some other influences unfavorable to health: the patient, who formerly used to bathe with apparent benefit to his health, had to give up bathing on account of change of residence and absence of a suitable bathing place; he began to be intensely preoccupied in the capacity of a manager of an estate, and to all this were finally adder certain family troubles. Under such conditions there appeared, two years ago. belching, heavimess in the stomach. constipation and. later, intestinal pains. Patient began to be treated, took at various times hydrochloric acir, pepsin, bismuth, carbolic acid, resorcin, socka powders, for a short time silver nitrate, and. for a shorter period still (eight days), arsenic - but all these failed to bring any relief. During 
December, 1889, and in January and February, 1890, he had been given about sixty washings of the stomach, which failed to alleviate his condition in the very least. During February and March the patient drank Vichy, cold and after meals, also without success.

Mode of living. - Patient lives in a healthy locality and resides in a good house. Does not bathe in the sea, takes baths at home but rarely. Smokes little; does not drink any beer. wine or brandy; drinks some five to six glasses of weak, warm and unsweetened tea per day; drinks neither water nor kvass; eats two light meals a day; keeps no fast days. He is single. This last year, on account of indisposition or, rather, considering himself sick, he gave up his occupation, which fact tends to more aggravate his oppressed mental state.

Status presens. - Appetite somewhat worse. There is a constant heaviness at the pit of the stomach and light belching of odorless gases, both after meals and on an empty stomach; there were neither in the past, nor are there at present, any pyrosis. nausea, vomiting, and pain at the pit of the stomach, neither spontaneous nor on pressure. Being costive, patient resorts often to laxatives, as pulvis liquiritiæ compos. Intestinal pains. Abdomen somewhat distended. The liver, spleen, the kidneys, the organs of respiration and of circulation present nothing abnormal. Patient grew thin. The sleep is poor, the mental condition oppressed.

Treatment. - The symptoms of neurasthenia and of nervous: dyspepsia, so marked even on his admission, became so prominent during the first days of the patient's stay in the clinic, that we ordered hydrotherapy (douches, from 22 to $18^{\circ} \mathrm{R}$. (81.5 to $72.5^{\circ} \mathrm{F}$.), to the exclusion of any other treatment. There followed a rapid simultaneous improvement in all the symptoms: as heaviness at the pit of the stomach, belching, constipation. intestinal pains, insomnia and oppressed mental condition. Patient left the clinic April 30th, almost completely improved.

He was advised to make this improvement durable by summer bathing and by a correct mode of living. and among other things. also by a return to his occupation, but without unduly exerting himself 


\section{FROM THE LECTURE OF NOVEMBER 28, 1889.}

Five weeks passed since we saw the patient last. Up to November 17th, he drank the Ems water and took drops of the tincture of nux vomica; had at times clysters, and but very seldom resorted to codeine. Since the $17 \mathrm{th}$, he is given pills of silver nitrate, at first one-twentieth-grain twice a day at mealtime, and since November 220d, three times a day. For the last several days he has had general massage. Patient desires to leave the clinic to-morrow. His condition has improved considerably. The appetite is good; patient gets during the day a pound of bread, six eggs, two glasses of bonillon. three to four glasses of milk-soup with manna (patient refuses meat), and at times begs for more food. Of the gastric symptoms there remained some heaviness at the pit of the stomach, at times easybelching, and, very seldom, slight, rapidly passing pains, that do not require any codeine. He is somewhat costive, and must at times resort to clysters. The tenderness on pressure in the region of the cœeum and ascending portion of the colon disappeared. Pulse is good. His nutrition has improved markedly. Has eight hours of quiet sleep. Mental condition better; he feels stronger. Patient was advised, besides a strict perseverance in a correct mode of living and circumspection in movements and exertion, to continue the treatment by silver nitrate and the general massage. 


\section{THIRD CASE.}

\section{LECTURE OF NOVEMBER 3, 1889.}

The patient, a student, twenty-two years old, complains of abdominal pains and constipation, which is at times replaced by a diarrhœa; of palpitation and of unpleasant sensations in the region of the heart, of pains in the head and spine, and of poor sleep; he is quickly fatigued by physical and mental exertion and is in a despondent mood: he is always disturbed by various anxieties, but chiefly by the apprehension, that he suffers from an "organic heart trouble."

Circumstances and mode of living. - For the last four years patient lived continuously in Moscow, summer and winter. Lodgings fair, water-closet cold; he formerly used to bathe himself, but has not done so lately, as bathing would chill him and cause attacks of cardiac palpitation. He does not go to public baths, but washes himself at home. Drinks but little tea, and neither coffee nor wine. Smoked before, but has discontinued it now, as it brought on palpitation of the heart. Order of meals : breakfast, tea with bread in the morning, dinner and supper; his board was always good, with the exception of a period of two weeks, of which more later. The patient is, as I said, a student, and also a private tutor. He goes out of doors for two hours daily. Has enough time for sleep, from 11 P. M. to 8 A. M.

Anamnesis. - His parents, according to his statement, are healthy. While yet in the gymnasium (high school), some ten years ago, the patient suffered from constipation, otherwise enjoyed fair health up to the time he took up his residence in Moscow, when the constipation increased and the patient began to grow somewhat thin and weak. He passed through an attack of acute articular rheumatism some three years ago, but it was of 
a light character (he not being confined to bed during the attack) and it passed away in a few days with the aid of sodium salycilate; since then he suffers with palpitation of the heart. About one year ago his sleep became poor, mental condition gloomy, the weakness more pronounced, the attacks of palpitation were accompanied by umpleasant sensations, and at times even by pains in the region of the heart. All these phenomena became aggravated because of an affliction, which the patient had suffered some three months ago. A month ago the patient was compelled to have bad meals, which caused a diarrhoea ; this was stopped at first by tra. opii, but it reappeared again; since then the patient would either be constipated for three or four days, or would have three or four stools a day, with pain in the abdomen, of liquid consisteney and with an admixture of mucus. He had a diarthea when he was admitted to our clinic, October, 29th. He was given five drops of the tincture of opium; the abdomen was wrapped in flannel, and one-half glass of hot Ems-Kesselbrunnen water was prescribed for him to be taken in the morning on an empty stomach, one hour before his tea, and a tablespoonful of the decoction of condurango (3ij to 5 iij) with five drops of the tincture of nux vomica after dinner and supper; a clyster in case of constipation and, after the diarrhea stopped, one day static electricity ("the bath" - i. e. sitting on the insulated chair at the time of the séance), the next day general massage, excepting the abdomen; later on, cold water treatment. He was enjoined to avoid physical and mental fatigue - to read but little; while the diarrhøe lasted he had tea for a drink; of the food we will speak later.

Status. - Patient is of average physical stature and, contrary to his assertions that he grows thin, his nutrition is quite satisfactory: the subcutaneous adipose tissue is quite considerable, and the muscles are well developed. The appetite and the gastric digestion, as the patient claims, are very changeable: on the days when he has a good rest, and when his mental condition is good, he eats well without experiencing any umpleasant aftereffects; but after a poor sleep, and when feeling poorly, he eats little and still experiences a painful sensation at the pit of his stomach, that passes away only after painful and prolonged belch- 
ing. Until the appearance of the diarrhcea he experienced no other unpleasant sensations in the abdomen; but with the diarrhœa there appeared also intestinal pains. After his admission to the clinic the diarrhoea ceased and the intestinal pains have almost disappeared. He had yesterday a water clyster, that induced a satisfactory evacuation after a constipation of two days' duration; the abdomen, however, is somewhat distended and slightly sensitive to examination, which latter causes some gurgling. We are unable to detect any particular tenderness or generally anything abnormal in the regions of the stomach, liver, spleen and kidneys. The urine is normal. The patient was for a long time very intemperate in his sexual relations; the erections are now weaker, he himself feeling weaker after the acts of copulation, although he performs them less frequently than formerly. He had two attacks of gonorrhœa, which passed without leaving a trace, but had aggravated his disturbed mental state. $\mathrm{He}$ is free from syphilis. - Some dyspnœa on walking. Pulse normal, except during the above-mentioned attacks of palpitation with pain in the region of the heart, when it is considerably quickened. Objective examination elicits nothing abnormal in the respiratory organs, heart and blood-vessels, as well as in the chest, with the exception of a small area of cutaneous hyperæsthesia around the left nipple, an area smaller than that of the region of the heart, where the patient experiences pain during the severe attacks of palpitation. Fever absent. The nervous phenomena are exactly pictured in the complaints of the patient and in the anamnesis. Headaches, when the patient is constipated, are experienced in the occipital region, while after mental exertion, in the frontal part; at times slight migraine. The pains along the spine seem to be due to cutaneous hyperæsthesia in the region of several vertebræ. All the movements of the spine are perfectly free and painless. Reflexes considerably increased.

As the lecture draws to a close, we will take up the diagnosis later; and now a few words about the treatment. After having ingested poor food, the patient suffered from an attack of diarrhoea, evidently due to an intestinal catarrh, for the painful evacuations were mixed with mucus: it is likely, that the gastric catarrh also began then, and that the dyspeptic gastric 
phenomena depend, to some extent at least, on the catarih; we therefore prescribed Ems, but in view of the diarrhæa, only onehalf glass in the morning, and very warm. The indication for condurango and the tincture of nux vomica, which proved of such advantage in both constipation and diarhoea, as you know from the analysis of the first case, requires no explanation, when employed for a patient, who suffers alternately from diarrhora and constipation. The indications for the use of electricity, massage and hydrotherapy will be spoken of later, after the final diagnosis has been arrived at.

\section{LECTURE OF NOVEMBER 7,1889.}

Status. - Patient's condition improved all around. Appetite and digestion are such that for the last three days he is given daily two plates of chicken soup, three-fourths of a chicken, over onehalf pound of roast meat, six eggs and about one pound of white bread; he takes all this at four meals, and, outside of slight heaviness and belching, and even this rarely, not after each meal, he suffers from no dyspeptic phenomena. Such a condition after only a five days' use of Ems, and of but one-half glass of it per day, speaks against catarrh of the stomach, the more so against ulcer or cancer, especially if you recollect the slow improvement in the appetite and in the nutrition with the more energetic and continuous treatment and very strict diet in our previously discussed case of a real catarrh of the stomach; it also excludes dilatation of the stomach, as the evacuations. though with the aid of clysters, are sufficient. The abdomen is less distended and not tender. He had almost no cardiac attacks during these days. He sleeps better, although not every night. His mental state is calm, especially after I examined and positively assured him that he had no organic heart lesion, and that his malady was perfectly curable. There is also less weakness.

Before taking up the final diagnosis, we will say a few words about the treatment, as indicated by the present condition of the patient. As there is no gastric catarrh, we will discontinue the Ems water, but will continue the condurango and the nux vo- 
mica, for there is still at times a feeling of heaviness in the stomach and some belching; besides, the diarrhoea has only ceased recently and the constipation continues as yet. Franklinization and massage acted well on the patient; but the cessation of the diarrhœea requires hydrotherapy instead. We will also prescribe for the patient silver nitrate (one grain in twenty pills with extract trifolia), at the beginning twice a day, immediately before each meal. As the sleep is not as yet always sound, and the disappearance of the diarrhøa permits of the use of bromides, we will resort to these if necessary. The reason for the employment of silver nitrate and of the bromides will be made clearer after the final diagnosis, when we will take up the whole plan of treatment.

Diagnosis. It is evident, from what was pointed out above, that catarrh, dilatation, ulcer and cancer of the stomach may positively be excluded in this case; while the change in the appetite and in the dyspeptic phenomena, and their close connection with the general nervous condition go to show, that the patient suffers from nervous dyspepsia. Besides the gastric there are also present morbid intestinal, cardiac and general nervous symptoms. We will begin with the general nervous symptoms, as even you, though beginners, will easily perceive from the data collected by observation and objective investigation, that they are the most important in this case.

To better elucidate the importance of the morbid nervous phenomena in this patient, I must.refer you to what I said regarding the condition of the nervous system in our first case. (Tide pp. 70-72.) From a careful comparison of the two, you will perceive that our present case is just the opposite of that one: in the presence of a good constitution and nutrition, of a normal condition of all the organs, except the nervous system (the trifling diarrhoea appeared only recently, while the constipation and the cardiac phenomena are, as we shall see later, of a neurasthenic origin). The patient leading a life amidst incomparably better surroundings, there appeared a primary, obstinate, progressive neurasthenia, its sum total of symptoms having been made clear by inquiry and by objective examination. It is a case of genuine, typical, although not extremely developed, neurasthenia. 
As far as its origin is concerned, we know nothing definite of the influence of heredity, while the factors that caused its appearance are patent; thus: a three years' residence, during both winter and summer, in a great city, conjoined with considerable uninterrupted mental labor (private tutoring even in summer), lack of bathing all this time, worries, but certainly most important of all, extreme intemperance in sexual relations and also the attacks of urethritis. In extreme cases of genuine neurasthenia, it is necessary to call to aid the differential diagnosis from hysteria and even from multiple sclerosis. But in this moderately developed ease, where the distinction from these diseases is so very evident, such procedure, a differential diagnosis, would be but a waste of time. Althongh our patient had an attack of acute articular rheumatism - a very slight attack and of short duration - but in the presence of the perfectly normal condition of the organs of circulation, the morbid phenomena met with in this case in the sphere of the plexus cardiaci, as palpitation and pain in the region of the heart, must be referred to the neurasthenia, of which they constitute a very frequent symptom.

The constipation, that is, the weakening of the nervo-muscular apparatus of the intestines, was at the beginning due to his neglect to attend to the calls of nature while yet in high school. With the development of general neurasthenia this condition of the intestines naturally became more aggravated. The intestinal catarrh, which appeared about a month ago, was the cause of the origin of the diarrhœa, and since that time the intestines presented a picture of two contemporaneously existing morbid conditions - of the weakness of their nervo-muscular apparatus and of a catarrh of their mucous membrane.

Prognosis. - Whether or not our patient has an innate predisposition to neurasthenia cannot be asserted with certainty; but even if it be present, it is hardly of any considerable extent: the data, obtained by the investigation, show, that up to a certain period, almost up to the time he entered the university, the patient had no symptoms of weakened nervous activity; these latter began to develop only after the following evident causes appeared, tending to neurasthenia, namely: constant residence 
and constant occupation (also during summer) in a great city, lack of bathing, to which he was habituated before, worries of various nature, and chiefly - sexual intemperance. In such cases, that is, where there is no imnate and, besides, obstinate, difficultly removable weakness of the nervous system, but where the neurasthenia is aequired and is, as in our patient, not extremely developed, a perfect cure is possible; but the stability of the cure, and the absence of recurrent attacks will certainly depend on the will-power and the ability on the part of the patient to avoid the influences, that give rise to neurasthenia.

Treatment. - IVe will begin with the principal ailment in the present case, the disease of the nervous system, neurasthenia. As what concerns his hygiene, we will chiefly advise the patient, first of all, to shorten at once his occupations and return to his native place, there to stay until the beginning of the future semester, in the middle of January; then to entirely discontinue his work for at least one month every summer, while residing as long as possible in a pleasant suburban locality, to bathe in the sea or river, to take due care of his sleep, not to interfere with it (as for instance, by an unnecessary meal or fatiguing mental work immediately before going to bed), nor to shorten it, - to sleep just as long as necessary ; to work only after a sufficient rest, and never to fatigue himself either physically, or mentally. We will advise complete abstinence from sexual intercouse the most important cause of the morbicl condition in the present case. Unfortunately the advice of the physician concerning this matter is not always followed; but it were erroneous to think. that it is never followed, and that it would therefore hardly be worth while giving it. On the contrary, a sensible, decisive and friendly advice will not be followed rather in the minority of cases, but if followed, it will be of inestimable value to the patient, which is the most important aim of the physician's practice in such cases. Nor is the fulfilling of this advice as difficult or impossible, as the patients, of whom we speak, usually think, if the physician but teach them to avoid sexual excitement through physical influences (as constipation, soft bedding, tight clothing, alcoholic drinks, etc.), as well as through influences acting in a psychical manner (lascivious thoughts, cer- 
tain books, certain male and female society), and if he explain to the pationts the harm in such cases of insufficient physical activity, of mental idleness, of lack of æsthetic impressions and, first of all, impress upon them their moral and religious duties. Besides, our patient is twenty-two years old and in a year and a half he will graduate from the University; consequently in the near future we may expect that he will marry.

The best of all the non-apothecary means for the treatment of neurasthenia is hydrotherapy, and in proportion as the patient is young and the condition of the internal organs and that of nutrition is good, and the more the nervous disturbances have the character of nervous depression, the more applicable is cool or cold water; on the contrary, if the patient is older, and the apprehensions excited by the condition of this or that internal organ are great, while the condition of nutrition is bad and the more the nervous disturbances have the character of excitement, - mental (total insomnia, anxious mental state) and physical (hyperæsthesia, pains and spasmodic attacks of nausea and palpitation, hampered and quickened respiration), - the more applicable is warm water. I usually devote two lectures, at the end of the year, to clinical balneotherapy — to balneotherapy for the practical physician, and I will discuss then the subject in a more detailed manner; but what I have already said is sufficient to show, that for our patient cool (and later cold) water is more suitable than warm water. One of the best, if not the best, way of applying cold water to patients of quite strong constitution, as in the case of our patient, is in the form of fan-like douches under high pressure, applied over the whole surface of the body, except the head, on which a cold compress is placed. The diarrhoea did not permit until now of the employment of these douches, and we therefore, out of the non-apothecary means, used only gen eral massage and electricity.

General massage is by far not as reliable in the treatment of neurasthenia, as hydrotherapy is: for cases of neurasthenia of the nature of excitement, with hysteroid symptoms, massage is as unsuitable as it is in pure hysteria, and sometimes simply impossible of application, for instance, in cases with considerable hyperresthesia. But in our patient, in view of the fact, that the symp- 
toms bearing the character of depression prevail, that the hyperæsthesia is insignificant, either by its intensity, or by the extent of the affected area, in view of the chillines of the patient and the trifling amount of physical exercise prescribed for him, and consequently partly to replace this last, we resorted to general massage, and, as we said before, the results have justified our expectations.

Electricity, particularly static and galvanic, is shown from experience to be beneficial in neurasthenia, but not always so. We cannot give any precise indications for its employment: experience decides as to whether it is beneficial or not, and as to which form is to be employed - static or galvanic (either one or the other); we have tried the static. and as it proved to exert a good effect on the nervous disturbances from which our patient suffers, we did not change it.

At present, when the diarrhora has ceased, the intestinal pains have finally disappeared and the abdomen became free from pain. we will leave off massage and electricity and will use the douches, in the beginning at $28^{\circ} \mathrm{R}$. (about $97^{\circ} \mathrm{F}$.) and later at a lower temperature.

The chief apothecary remedies used in neurasthenia are silver nitrate, arsenic and iron. The last is suitable in cases of neurasthenia with a strongly pronounced anxemia, which is more frequently seen in women than in men. For our patient iron is not only not indicated, as he has no symptoms of anæmia. but is rather contraindicated, as it may easily impair the appetite and cause constipation, from which the patient is already suffering. Arsenic is suitable for cases of neurasthenia in which the patient's appetite is poor and his nutrition has failed greatly; our patient's appetite, although changeable, is nevertheless most of the time good, his nutrition suffered but little. while the recently ceased diarrhoea constitutes a contraindication to the employment of arsenic, as this last causes a diarrhcea so easily. Silver nitrate is particularly beneficial in those cases of neurasthenia in which the morbid phenomena of the nature of depression prevail, as in our patient; it too tends somewhat to costiveness, but not at all to the extent and frequency, as is the case with iron, nor does it impair the appetite in the least. It 
therefore becomes apparent to you why we employed silver nitrate.

Is it always necessary to employ two or more remedial agents in neurasthenia, as we do in the present case - silver nitrate and hydrotherapy? Certainly not always : for cases of moderately dereloped and recent neurasthenia in robust young men it is sufficent - especially in summer, when a prolonged stay out-of-doors is possible - to observe the hygiene and hydrotherapy, for example, in the form of river, or still better, sea-bathing. The neurasthenia in our patient is quite severe and of long standing, the season of the year now is the beginning of winter; besides, the patient contemplates returning soon to his own home, where he will not be able to be benefited by the use of such strong fan-like douches and under high pressure, as he has in our clinic, but will replace them by a hand-rlouche from a pump, acting certainly much weaker; this was the reason for our prescribing for the patient hydrotherapy and silver nitrate at one and the same time.

If the above-named renedial agencies, very good though they be for neurasthenia, will not act with sufficient rapidity - which is very possible in the given case of such obstinate neurasthenia, - and will not with sufficient rapidity remove certain particnlarly burdensome morbid phenomena, dependent on neurasthenia and in their tum prolonging it, principally the poor sleep, then we will order symptomatically bromides ( 15 grains of sodium bromide in case of insomnia), until the principal treatment, hydrotherapy and silver nitrate, which we may call radical, will securely remove also the insomnia together with the other synptoms of neurasthenia. The bromide preparations, some of the best. if not the best, somnifacient agencies, are particularly indicated in the given case also by the gloomy and worrying mental state as well as by the morbidly excited sexual sphere (lasciviousness and a tendency to pollutions). I must also observe, that the bromides, as any other symptomatic and palliative means, must be ordered with extreme caution, just in quantities required by the necessity of the case. The abuse of symptomatic and palliative remedies is the true sign of a poor physician. who is either unable to comprehend the case and to order the 
radical treatment, or, what is more frequent, who is rather annoyed at the necessity of fulfilling this, his duty.

The cardiac phenomena, since the patient's apprehensions about his heart had been allayed and he generally feels better, have become so quiet, that they require no particular treatment.

The diarrhoa has disappeared, and there remain only some trifling dyspeptic phenomena, at times slight heaviness and belching, and some constipation. Of the treatment of the constipation next time.

\section{LECTURE OF NOVEMBER 28, 1889.}

Before discussing the treatment of the gastro-intestinal disturbances in neurasthenies, let us see whether there is only one variety of gastric nervous. dyspepsia, and whether this variety is always to be met in the form we see it in our patient. The theoretical attempts to create various, very numerous forms of nervous dyspepsia, basing their classification upon the assumed heightened or lowered activity of the gastric nerves sensory, motor, and secretory, - have all failed; but practice has proved the existence of at least two varieties of nervous dyspepsia : one, the most frequent, such as seen in the present case (its principal symptoms - a feeling of heaviness after a meal and belching), observed in neurasthenia with the character of depression; the other, less frequent, of which the chief symptoms consist of severe pains in the region of the stomach (cardialgia and gastralgia proper), followed by tormenting, very sour belching and nausea, accompanied frequently by contemporaneous attacks of severe migraine. This variety is usually seen in neurasthenia with the character of excitement. The treatment and partly the diet differ in either of the varieties of nervous dyspepsia.

When ordering a diet for nervous dyspepsia, we must be guided, as has already been explained in discussing our first case, besides the general physiological and dietetic considerations. also by the individuality of the given case. Generally speaking, for cases of pure nervous dyspepsia, uncomplicated by any 
other disease of the stomach, the "delicate" food given for gastric catarrh is unsuitable; rather, on the contrary, we must order food which is more prone to excite a sufficient excretion of the gastric juice; however, in cases of the above-named second variety of nervous dyspepsia, careful observation in the administration of such food becomes necessary. In this last. variety there is quite probably an increased secretion of the gastric juice or, at least, of the hydrochloric acid contained in it; and a still greater excitation of this secretion will bring about an aggravation of the heartburn and sour belching, as well as of the pains in the stomach and of the sour vomiting. Moreover, while directing the neurasthenic patients to eat, though not as frequently nor as little at a time, as we advise patients with gastric catarh, but still not very much at a time, so as not to overeat, we must bear in mind that the neurasthenic patients are just the ones who are liable to fall into the other extreme of not eating sufficiently, of starving, and thus prolonging, through an insufficiency in the nutrition of the nervous system, their neurasthenia. In prescribing the diet we must also consider the constipation, from which the neurasthenic usually suffers, especially in the beginning; later on there is superadded a catarrh of the large intestine and there appear intermittently both diarrhoea and constipation; and to comteract the costiveness we must vary the food and prescribe, as far as possible, such food to which the patient is not habituated: for instance, in patients who habitually drink tea and no water, costiveness is frequently removed by drinking two to three half glassfuls of water, one half glass in the morning on an empty stomach and in the course of the day, one or two hours before a meal; in others the same effect is produced by replacing the morning tea with weak coffee with milk and with chicory; in others again by the use of raw milk or of curdled milk, but without taking at the same time of any other food and particularly of bread; by the employment of vegetables, (as, for instance, a half or a full glass of carrot juice in the morning on an enpty stomach), of berries and fruits, eaten not together with the other food, but taken on the previously more or less emptied stomach.

The treatment of gastric nervous dyspepsia and constipation 
begins naturally with the treatment of the general neurasthenic condition. Then the same non-apothecary agencies are applied also to the digestive organs. For the variety of dyspepsia seen in our patient, the above-described douches are suitable (in these the douche on the abdomen is allowed to play for a somewhat longer time than on the other portions of the body), also abdominal massage, as well as the induced and interrupted galvanic current applied directly to the abdomen. In the other variety of nerrous dyspepsia, accompanied by gastric pains and acid vomiting, these means may tend to aggravate the morbid condition, and we therefore employ warm baths, static electricity (" the bath " and the "fan " to the region of the stomach) and the constant galvanic current (one electrode over the stomach, the other on one or the other side of the neck, at the region of the vagus and the great sympathetic nerves).

If with the nervous dyspepsia there is also abdominal suffering, causing attacks of pain and sensitiveness of the affected organs (for instance, liver and kidney colic, acute or subacute catarrh of the biliary and urinary tracts, as well as of the stomach and intestines), then only warm baths are suitable and probably also franklinization; douches, massage, the induced and the interrupted galvanie current will only tend to aggravate the condition of such patients (and at times even to a dangerous extent, as douches and massage by causing hæmaturia and acute exacerbation of pyelitis).

Silver nitrate is apparently suitable in an equal degree to either variety of nervous dyspepsia. Bitters, and particularly nux vomica, are beneficial in the variety of neurasthenia seen in our patient, but are unsuitable where there is pyrosis and severe belching, gastric pains and acid romiting, as they aggravate these symptoms, evidently by creating a still greater excitation of the secretion of the gastric juice or at least of its hydrochloric acid. It was thus in the second case discussed by us (vide p. 122). In such patients, if there be at the same time a gastric catarrh, requiring the use of an alkaline mineral water, for instance of Ems, this last must be given after a meal, say in about an hour; it is then borne well (probably because the secretions of the gastric juice caused by it is used up for digestion), whereas, if 
given one hour before a meal it tends to call out pyrosis, acid belching and even pain in the stomach.

At times the constipation is rapidly put a stop to by the radical, above-outlined method of treating general and gastro-intestinal neurasthenia; but at other times it is slow in disappearing and we then must resort to temporary means, to clysters, and. much less frequently, to laxatives; but we first of all insist upon, that the patient respond promptly to the inclination to stool. I have spoken elsewhere of the principal indications for the regular employment of clysters. I will add here, that it is usually sufficient to make a clyster of two to three glasses of water of the temperature of $20^{\circ} \mathrm{R}$. (about $79^{\circ} \mathrm{F}$.). The best time to employ a clyster is in the morning, after the first meal (especially after the coffee) and before beginning one's usual work. which may weaken the already lessened inclination to stool. Having put in the clyster, some would do better to at once respond to the consequent inclination to stool, while for others it is better to resist for a few minutes : experience will teach in which case one or the other advice is more beneficial. The apprehension to become habituated to clysters is without any ground, if we employ at the same time all the therapeutic and hygienic measures for curing the general and the gastro-intestinal neurasthenia.

In case the clysters do not sufficiently empty the intestines, then, until the radical treatment of the constipation has not done its work completely, resort must be had to the employment of laxatives. In view of the great number of laxatives recommended, I consider it necessary to enumerate those that have proved themselves preferable over others, in my experience. 1. Magnesia usta-burnt magnesia - a light laxative. 2. Potassium tartrate - much stronger. 3. Soctium sulphate (Glauber's salt), still stronger (it is best to administer this with the addition of some sodium bicarbonate. or in some alkaline mineral water, otherwise it may cause intestinal pains and unnecessary looseness of the bowels. t. Poudered rhuburb-either by itself or, in case of pyrosis, in conjunction with half its quantity of burnt magnesia; it acts moderately. but effectually. 5. Alor is probably the best laxative because of the reliability of its action. 
which, besides, approaches normal laxation ; nor does one become habituated to its use for a long time; (the best formula: $\mathrm{R}$. Extr. Rhei comp., extr. aloes āâ. 3ss. M. f. 1. a. pilulæ 20 v. 30. Consp. lycopodio). 6. Folia semae - acts well and not strongly in a cold infusion (when hot will rather tend to cause pain), which is readily prepared at home. 7. Infusum sennae salinum (i. e., with the addition of Glauber's salt) is a strongly acting laxative, especially suitable when a sure and rapid effect is desired, as, for instance, in cases of dangerous cerebral attacks (congestion of the brain threatening apoplexy): it is then given every hour in wineglassful doses (containing two tablespoonfuls), till the desired effect is obtained. 8. Podophyllin, 9. Cascara sagrada, and 10. Cortex rhamni fiaugulce are all very useful, if it become necessary to temporarily replace the rhubarb, aloe and the senna leaves, to which the patient became habituated, and which have therefore ceased to be effective. 11. Calomel; this is probably borne by the gastro-intestinal organs better than all the other laxatives, and is particularly suitable in diseases of the biliary passages and in acute infectious diseases of the intestinal canal, but it has also its contra-indications. (This will be spoken of in another lecture.) 12. Castor-oil acts effectively, but is unsuitable for frequent use. The doses of the laxative remedies are approximately given by pharmacology: each given case must be individualized, beginning, in case there are no urgently necessary indications, with a small dose.

To judge by the course of his health, it will not be necessary to employ the clysters in our patient for a long time, nor will there hardly be any urgency for the administration of laxatives; but there occur cases, usually in elderly persons whose muscular apparatus is not only in a condition of asthenia, but already in that of atrophy, where there are found at one and the same time morbid conditions of the abdominal and thoracic organs absolutely contra-indicating the employment of hydrotherapy, massage, and of the induced and interrupted galvanic currents, and where the exhaustion of the patient constitutes an obstacle in the way of applying correct hygiene, as, for instance, sufficient exercise; in such cases the business of the physician consists wholly in the skilful contemporaneous employment of clysters 
and of laxatives, in the dexterous selection and careful interchanging of these last.

In our first analyzed case we had to treat diarrhoea, in the present case. constipation; either of the patients have, comparatively speaking, simple cases of diarrhœea and constipation. Quite different is, of course, the treatment of constipation, that intermits with a diarrhoa, in cases of intestinal nemrasthenia with, at the same time, a chronic, obstinate catarrh of the large intestine; this presents a wide field for the systematic employment of clysters, simply evacuating and medicated ones - with tannin, bismuth, boric acid, etc.; different also is the treatment of diarrhoea in acute and chronic infectious diseases of the intestinal canal, in stagnation of blood in the portal vein because of hepatic diseases, in the varions neuroses, as in Basedow's disease ; different is the treatment of constipation, if along with it there are found other morbid conditions, thoracic, abdominal and cerebral.

The general question: "how to treat constipation," or " how to treat diarrhcea "is just as unreasonable as the question "how to treat a cough": in each given case the ordering of treatment must be preceded by a precise diagnosis.

We will now turn to our patient, who is leaving the clinic today, intending to go to his home.

For the eleven days which passed since we saw the patient the last time, he took bitters and silver nitrate in pills (onetwentieth grain twice a day), when necessary he had watery clysters and the daily employment of the fan-like douche under high pressure, in the beginning at $20^{\circ} R$., the last days at $18^{\circ} R$.; on the abdomen the douche played somewhat longer than on the other parts of the body. The patient bore the douches very well: he rapidly warmed himself and felt heartier after the douche. For this period we had to resort to the bromides four times (fifteen grains at bedtime): he slept sounder after this.

Status. - The patient, as you hear, declares himself much better than what he was formerly ; the appetite is good, the dyspeptic gastric phenomena disappeared, the stool is mostly regular, the clyster is used only at times, the former painful sensations in the abdomen passed away; the former cardiac attacks hardly 
at all trouble him now; the physical strength is good (patient began taking out-door walks), but the sleep is not always sound, while mental occupation still causes headache, although not a severe one, and a feeling of fatigue.

On the moming of the day before yesterday, the patient, while rising awkwardly from his bed, felt a sudden pain in the right inguinal region. The cause of the pain was shown by investigation to be due to a slightly displaced kidney; after this was pushed back into its place, the pain disappeared. There is no doubt but that the kidney was movable before (before the appearance of pain the patient did nothing that could displace and make movable a kidney that was until now normally implanted in its place), but it was supported in its place by the intestines, which, on account of the constant constipation. were considerably distended with gases. At present, when with the regular evacuation of the bowels, the meteorism passed away (the abdomen is not distended), a trifling cause, such as an awkward movement, was sufficient to displace the now movable kidney. However, the displacement is not great and it is quite likely that he will be able to get along without a kidney truss, and a belt worn either separately or attached to the under-dress may suffice. While moring about, the patient will have to tighten the belt, and when at rest to loosen it, otherwise the constant tightening of the part of the abdomen under the belt will aggravate the constipation.

Our advice to the patient: after leaving the clinic to take silver nitrate (it apparently does not increase the constipation in our patient) for another month, gradually increasing the dose (the first two weeks three pills daily, the next two, four a day, while the last clays to reduce by one pill daily) and then continue for another six weeks the fan-like douche from a hand-pump, gradually lowering the temperature to $16^{\circ} R$.; to resort to potassium bromide as rarely as possible, and when necessary to use a clyster.

We have spoken already of the hygienic instructions. 


\section{FOURTH CASE.}

\section{FROM THE LECTURE OF NOVEMBER 24, 1889.}

The patient, of whom we will speak presently, since his admission to our clinic was so exhausted and suffered from such torturing pains, that we could hardly think of transferring him from his ward to the class-room for demonstration. However, not only were all the hospital attendants well informed abont his condition and our views concerning it, but almost all of you, while visiting the ward, had the opportunity to see everything that could possibly be demonstrated before a large audience: chiefly the emaciated and cachectic appearance of the patient and the attacks of tormenting vomiting, from which he suffered; therefore in view of the interest in the case I will take it up now.

The patient, a retired military man, fifty-nine years old, was admitted to our clinic complaining of oppressive pain in the stomach, pyrosis, belching of the odor of rotten eggs, of vomiting and constant, tormenting nausea (which compelled him at times to induce vomiting by irritating the pharynx by tickling), and also of emaciation and debility.

Anamnesis and mode of living. - Since his youth patient has lived almost his whole life in the Caucasus, where he frequently suffered from malaria, for which he was treated with quinine. For the last ten years patient was always subject to costiveness, and he frequently experienced after a meal slight heaviness in the stomach and belching, that annoyed him but little. For the rest, until last summer (1889), our patient, being of strong constitution and leading a quiet life under favorable conditions, could hardly complain of anything. The chief irregularity in his mode of living consisted in a lack of exercise, constant sitting, while reading, writing or at times playing cards, in a 
very plentiful table and considerable use of alcohol - two to three wine-glasses of whiskey daily, about two glasses of local Caucasian wine and tea with cognac. During June the patient passed through the customary attack of malaria, which quickly yielded to quinine, but after this the usual gastric symptoms, heaviness and belching after meals, became aggravated and were accompanied by others - pyrosis and nausea; the constipation became more obstinate. Until the middle of August the patient's condition was bearable enongh and he did not resort to treatment. But since then the condition became rapidly aggravated : the appetite disappeared, the sensation of heaviness in the stomach gave way to oppressive pain, belching (now malodorous, of rotten eggs) and pyrosis increased; patient began to vomit, the nausea became a torture, and the patient was compelled to intentionally provoke romiting; he grew thin and weak. He then called in his physician. The treatment (at first $\mathrm{HCl}$, later the bitters, condurango and nux vomica, and Vichy water in one-half glassful doses three times a day, then gastric lavage) brought but little relief and for a short time: the gastric symptoms kept on growing worse, while his nutrition and strength failed with ever increasing rapidity, till in this condition he was admitted into our clinic.

The clinical investigation elicited the following. The nervous system in a normal condition (inquiry has failed to establish the existence at any time even of neurasthenia); and so are the organs of respiration. There are signs of arterio-sclerosis; the arteries are somewhat hard; there is a slight systolic mumur over the sternum and a moderate enlargement of the left ventricle: the heartbeat is felt the clearest in the mammillary line; the pulse is frequent and somewhat weak; the temperature of the body is subnormal, and there is extreme emaciation. The urine contains some sugar (one per cent) and some albumen. The inquiry failed to elicit any clearly pronounced symptoms of diabetes mellitus, as immoderate thirst and appetite, in the past. By objective examination nothing abnormal is to be noted in the liver, spleen and kidneys. Patient had no syphilis.

The digestive organs. - All the above enumerated symptoms, that progressively increased before the patient's admission to the 
clinic, continued to augment rapidly, accompanied by a corresponding failing of the strength; almost from the very first day the patient failed to retain the administered trifling quantity of food and of medicine, the latter consisting of some Ems water and condurango, tra. valer. æther., and morphine. The food was ejected completely undigested. The ejecta, as proved by repeated examination, contained, besides the undigested food, very little mucus, very little hydrochloric acid (0.33 per 1,000) and usually some bile; there was never any blood. The subgastric region was slightly distended and somewhat sensitive; no swelling in the abdomen could anywhere be palpated. Gastric lavage done the very first day with a solution of soda, brought but a trifling relief from the gastric symptoms, while lavage on the next day failed even in this. The clyster brought away a small quantity of dry exereta.

In speaking of the diagnosis, I will take up only the organs of digestion; on the others I will touch but lightly. The importance of abnormal phenomena in the organs of circulation was already alluded to. The constant presence of albumen in the urine (although cylinders are absent) depends, most likely, on the presence of a slight, so-called senile, interstitial nephritis, that usually accompanies arterio-sclerosis in aged people. The failure in nutrition and strength must certainly be considered as a result of the disease of the digestive organs, and not of diabetes, which never presented any pronounced symptoms, while the percentage of sugar was trifling. We will have time to discuss in detail the importance of the symptoms and diagnosis of arterio-sclerosis, as well as of the nephrites and of diabetes mellitus later on in suitable cases, in which these diseases play the chief rôle.

What disease of the digestive organs is our patient suffering from? It is evident from the first that it is a disease of the stomach. Which is it? Nervous dyspepsia can surely be excluded; not to speak of the fact, that nervous dyspepsia can haritly ever bring one to the condition in which we find our patient, there is nothing that would speak for it: neither did the patient ever suffer from general neurasthenia, nor do the gastric symptoms present the important feature of nervous dyspepsia, 
namely, changeability; on the contrary, they are very constant.

Does our patient have an ordinary gastric catarh? To cause the gastric symptoms to the degree seen in our patient, the catarrhal condition should have been extremely developed, consequently there would have been a great accumulation of mucus, whereas, on the contrary, there is very little of it.

Gastric ulcer may evidently also be excluded, as there are no attacks of acute pain so peculiar to this condition (cardialgia), nor was there any blood in the vomited matter.

Our patient has, first of all, atrophic gastric catarh an atrophy of the gastric glands; this is apparent from the fact, that the vomited food is undigested and the quantity of hydrochloric acid is very much diminished (0.33 per 1,000 instead of the normal 2.00 per 1,000). But atrophic catarrh of the stomach is observed in various diseases, which lead to exhaustion, to marasmus of the organism. Which of these diseases is the most probable in this case? It is cancer, because the patient, who only four or five months ago enjoyed his ordinary, for the last ten years, good health and even a florid nutrition, was reduced in such a short time to extreme marasmus; of chronic diseases, leading to marasmus, it is the malignant growths which have a particular tendency to such a rapid course. The absence of signs of other chronic diseases, that may bring about a condition of marasmus (as, for instance, inveterate syphilis or tuberculosis, etc.), as well as the patient's age, also speak for cancer. The localization of this, in view of the obstinate vomiting, is most likely to be in the pylorus of the stomach (no swelling is anywhere to be palpated), although the almost constant presence of bile in the vomited matter speaks somewhat against a difficult passage in this place. Cancer pancreatis is to be excluded to some extent by the absence of permanent jaundice, which could be expected in a cancer of this organ, of sufficient size to compress the duodenum, on account of the pressure exerted by the cancerous growth on the ductus choledochus (not to mention the possible ascites from pressure of the same swelling on the vena portx). We are generally unable to obtain any definite symptoms of a pancre- 
atic cancer, that would result from the influences created by a cessation of the functional activity of this organ, nor can we certainly do so in the present case, as everything introduced into the stomach is vomited up.

The treatment, in view of the impossibility of introducing anything into the stomach and of the rapidly approaching dissolution, could only consist of nutritive enemata (two to three raw eggs with the addition of some water and table salt, the whole contained in a glass and warmed to the temperature of $30^{\circ} R$.), to which were added some eight drops of laudanum, so that the clyster could be better retained, and a dessertspoonful of cognac as a stimulant; for the excruciatingly painful romiting re solietimes injected morphine hypodermically (one-eighth grain at a time).

Patient died on the thirteenth day after his admission to the clinic. Autopsy showed: sclerosis of the arteries, among others also of the aorta; left ventricle dilated, its walls pale and flabby, but presenting at some places traces of hypertrophy; a moderate nephritis, the liver, spleen and pancreas present no changes; catarrh and atrophy of almost the whole mucous membrane of the stomach (it is very much thinned out and is perfectly smooth, its surface not presenting that normal mammillary appearance, surface mamelonnée), the muscular layer of the stomach is also atrophied; the duodenum is so constricted in its lower third, that it hardly admits an ordinary pencil: on the immer surface of the posterior wall of the intestine in this locality there is a cancerous ulcer the size of a ten cent piece - situated in the cancerous growth of the intestinal wall, this last - the tumor - being the size of a walnut: the neighboring mesenteric glands present a series of cancerous metastases.

Thus in the four patients demonstrated by me, you had the opportunity to observe, besides a great many other ailments, the principal varieties of diseases of the stomach, namely: pure nervous dyspepsia (in the third case), - a pure chronic gastric 
catarrh (in the first), - simultaneously (in the second), chronic catarrh, ulcer and gastric dilatation, with additional phenomena of nervous dyspepsia, where, however, notwithstanding the co-existence of several diseases of the stomach, it was possible to diagnose each of them, - and, finally, an atrophic catarrh of the stomach, and cancer, if not of the pylorus, then of the duodenum, which is so similar to it in its symptoms. 


\section{FIFTH CASE.}

\section{FROM THE LECTURE OF DECEMBER 7, 1890.}

The patient, a peasant, fifty years old, was admitted to the climic November 24th, complaining of pain in the epigastric region and in the right hypochondrium, of jaundice, emaciation and general weakness.

Anamnesis and mode of living. - Until his twentieth year patient lived in a village, since then in Moscow, in the capacity of a janitor. His lodgings are fair. He bathes himself during summer in the sea, at other seasons of the year takes his bath a sweat bath - every week. Drinks a great deal of hot tea, a moderate quantity of whiskey during the last year, but used to drink to excess formerly. His food is the ordinary food of a workingman. Until the appearance of the disease he did not find his occupation fatiguing. Is married and has many children.

When nine years old, patient passed through an attack of variola; when twenty-one years he had some febrile disease from which he completely recovered, enjoying good health since and until last August. Patient had no syphilis. Last May patient had a severe affliction. Last August there appeared some pains in the epigastric region and in the right hypochondrium, the appetite became impaired, and he grew costive. Three weeks ago - in the beginning of November - there appeared jaundice and the patient grew markedly emaciated and weak. While in the clinic he continued costive (every one or two days he had some dry excreta, not in the least colored by bile), and the abdomen was somewhat distended. Patient was given, November 30th, seven powders of calomel, each containing one grain (the first five every hour, the last two every two hours): the stools were a little loose, and only slightly colored by bile, but they 
were not the characteristic calomel stools; patient was not weakened after this, but neither did he experience any relief (the pains remained as heretofore). Besides calomel the patient took daily (except November 30th) three half-glassfuls of warm Ems water, once or twice (during the exacerbation of the pains) one-quarter grain of codeine, and during the day some four tablespoonfuls of strong wine (the pulse was all the time somewhat weak). As the evening temperature rose to $38.5^{\circ}\left(101.3^{\circ}\right.$ $F$.), he was sometimes given quinine (five grains per dose once a day); this apparently diminished the febrile condition. Besides, he had some warm baths, that tended to relieve somewhat the pains and the itching of the skin.

Status. - The patient is of good constitution, but very emaciated; the skin and the sclerotics are severely jaundiced. The appetite is very poor. There are almost no dyspeptic phenomena, except some slight heariness after a meal: he never had, nor has he now any constant and painful belching, pyrosis, nausea or vomiting. The pains in the epigastric region and in the right hypochondrium do not become aggravated after a meal, but do so on pressure on the painful parts. The abdomen is distended. The liver is enlarged and sensitive to pressure, but does not present any palpable unevenness or any protuberance; the gall-bladder is distended to the size of a considerable swelling, but is almost insensitive to pressure. The spleen is somewhat enlarged. Patient is constipated: after the calomel he had every other day watery clysters, which brought away a small quantity of uncolored by bile evacuations. The urine is very much jaundiced, contains neither sugar nor albumen. The arteries are slightly stiff, the pulse is frequent and somewhat weak. The organs of respiration present nothing abnormal. Patient, as I said once, is feverish; his evening temperature ranges between $37.5^{\circ} R .\left(99.5^{\circ} \mathrm{F}\right.$ ) and $38.5^{\circ} \mathrm{R} .\left(101.3^{\circ} \mathrm{F}\right.$.). His sleep is poor, he is at times dizzy; his skin itches.

Diagnosis. - A morbid process, which develops in a man no longer young, approaching old age, but of strong constitution and good health heretofore, - especially if developed after a severe affliction, _- and leading the patient to a rapid failure of nutrition and of strength, is usually a cancer (generally a malig- 
nant new formation), less frequently diabetes mellitus. Our patient has neither diabetes nor any other pathological process (except a cancer) which could explain his dangerous condition. Syphilitic gumma, compressing the ductus choledochus, is most certainly out of the question : not only does the patient deny infection, but he presents absolutely no signs of syphilis (besides, his wife had eleven children and not a single abortion). $\mathrm{Hy}$ pertrophic biliary cirrosis and multilocular echinococcus of the liver, which one may be led to suspect in view of the enlargement of the liver and because of the jaundice, are to be excluded, besides other considerations, by their much slower courses; so that only cancer remains. Which organ is affected by it? It is evidently not the stomach, because there are neither severe, nor in fact any gastric symptoms, and besides it is apparent, that the ductus choledochus is compressed (the evacuations are not in the least colored by bile, there is jaundice, the gall-bladder is very much distended), and consequently the tumor must be located about it. It is evident that the tumor is most likely to be assumed as lying on the lower surface of the liver (in the porta hepatis), or that it is a cancer of the pancreas. We have already stated in connection with the previous case that we have no reliable symptoms, outside of a localized swelling, that would indicate a cancer of the pancreas, nor can we detect any such swelling in our patient. Cancer of the liver is to be excluded - though not absolutely so - by the fact that in the part of the liver accessible to palpation there is observed neither any unevenness, nor any protuberance, and besides, its seusitiveness is not great and is everywhere equable, and not severer in some places than in others. This sensitiveness and at the same time the enlargement of the liver is fully explained by the stagnation of the bile in this organ and the consequent distention of its capsule.

Prognosis - is but too evident.

Treatment. - Notwitlistanding the improbability of the presence of such a process in the biliary tract in our patient, on which treatment could produce an effect, we nevertheless attempted in the beginning to treat the case by calomel and Ems, but failed to obtain any results. It only remains now to sus- 
tain the patient's strength and relieve the particularly painful symptoms; the means for this were spoken of before.

\section{FROM THE LECTURE OF JANUARY 18, 1891.}

The condition of the patient grew continually worse and January 16th he died; the last two days of his life were marked by a surprising lowering of the pulse and strength, and by extreme paleness.

The autopsy made to-day showed the following: cancer of the pancreas, that extended by ulceration into the duodenum and caused a severe hæmorrhage; the intestines (except the sigmoid flexure and the rectum) are filled with clots of blood; the ductus choledochus is very much distended above the seat of pressure, and so are all the other biliary ducts, as well as the gall-bladder (this last to the size of an ostrich egg). 


\section{SIXTH CASE.}

\section{LECTURE OF OCTOBER 25, 1889.}

The patient, a peasant, twenty-nine years old, was admitted to the clinic October 20th, complaining of jaundice, weakness and pains all over the abdomen, but particularly in the right hypochondrium; he claims, that he took sick one week before his admission here.

Anamesis and mode of living. - Patient comes from a healthy family; until his twentieth year he lived in a village, then served in the militia, and for the last two years, as a member of the reserve army, he made Moscow his place of residence. His lodgings are fair; the water-closet cold. While in Moseow, he does not bathe in the river (bathed formerly and felt very good); goes frequently to the public bath-house; drinks a great deal of hot tea; is subject to sweating. Twice a week drinks some three wineglassfuls of whiskey. Order of his meals : in the morning tea with bread, later dimner, and supper. His board is good (fish on fast days), but patient is very much occupied-he supervises the forwarding of goods and accompanies the wagons packed with them - so that he frequently takes his dimner not at home, nor in time, eating whatever he may get hold of (as sausages, salted fish, etc.) ; nor does he sleep enough on account of his occupations. For the last two years patient had a great deal of worry partly on account of the very responsible nature of his occupation, partly on account of some family troubles. Patient is single, had no venereal aftections.

With such data on hand, we certainly could not rely on the patient's statement, in reply to the first question, that until the present illness he had never been sick. As a matter of fact. the inquiry about the patient's condition, about the functional activities of his organism immediately before the present illness. 
has elicited the fact, that although his appetite, digestion, urination, respiration and circulation were perfectly normal, still the patient while residing in Moscow, in better rooms and eating better food than formerly, lost flesh and his hale and hearty mental state, became impressionable and irritable ("began to take things too seriously to heart"); besides, since October 1st. or some two weeks previous to the present illness, he began to experience, most frequently while riding on the street pavement (as he often rode in a wagon while forwarding the goods), some pain in the right hypochondrium (patient pointed directly to the region of the gall-bladder).

October the 13th patient had eaten some fat soup of the flesh of white sturgeon, and the same day, having remained for a long time out of doors, he became very much chilled; in the evening he felt pain at the pit of the stomach and weakness. The next day the pain grew worse, and the appetite became impaired; on the third day the white of the eyes became yellow. October 16th patient took some very salt and fat cabbage soup made of kidney, and after this drank some cold beer. There appeared on the same day severe pains around the umbilicus and an attack of diarrhoea. which continued into the next day. October 18th the pains around the umbilicus diminished and the diarrhora ceased, but the pains in the right hypochondrium grew worse and the urine became dark. October 20th the patient entered our clinic. For the last few days the patient excreted daily small quantities of dry fetid evacuations entirely uncolored by bile.

October 21st the patient was given seven grains of calomel (in powder, one grain every hour): patient had good loose stools the 21st and the 22d, copious and characteristic calomel dark-green evacuations, after which the pains around umbilicus completely disappeared, and those in the right hypochondrium were relieved, the urine became clearer, the heaviness in the head was relieved, and the appetite returned. The $23 \mathrm{~d}$ the patient was given one-half glass of warm natural Karlsbad water (Mühlbrunnen spring), one hour before dinner, and the $22 \mathrm{~d}$ two half-glassfuls, one an hour before dinner, the other an hour before supper. 
Yesterday, the 24th, the patient had a severe attack of pain, lasting from five to seven P. M., in the right hypochondrium and under the right scapula, and at the same time the temperature, which was normal until then, rose to $38.5^{\circ} R$. $\left(101.3^{\circ} \mathrm{F}\right.$.).

Status. - The temperature is as yet $37.6^{\circ} R$. (99.7 $\mathrm{F}$.), but the patient, as you may hear, claims to feel much better now than he did before admission. The appetite is very good, but he is given as yet but little to eat. Dyspeptic phenomena are absent; a clyster, administered yesterday, brought away a sufficient quantity of evacuations markedly colored by bile. The urine is much more limpid, than it was before, but examination shows, that it contains still some biliary pigment and the biliary acids, but neither albumen nor sugar. The abdomen (protected since his admission by flannel) is sensitive only in the region of the gall-bladder, and is not distended. The liver is somewhat enlarged, the gall-bladder does not permit of palpation. Nothing abnormal found in the region of the spleen and of the kidneys. The organs of respiration and of circulation are in a normal condition. Patient's sleep is generally better, than it was before admission, but still it is not perfectly normal, partly on account of the abdominal pains, and partly - in the absence of these last - simply because of the insomnia caused by worrying about his illness. IVe alluded in the anamnesis to the somewhat gloomy and irritable mental state of the patient.

Diagnosis. - Outside the light nervous symptoms, - of the significance of which we have spoken already in connection with the discussion of neurasthenia in the first and third cases, - we observe in the patient at present only symptoms of an affection of the biliary tract, namely: jaundice, sensitiveness on pressure and pains in the region of the gall-bladder, usually not severe, but at times very severe and acute, in the form of clearly definable attacks. For a week before the patient's admission to the clinic he suffered from gastro-intestinal disturbances (loss of appetite, intestinal pains and diarrhea), which passed away rapidly in the course of two days. Up to that time the appetite and digestion were perfectly normal, and after that the 
appetite returned quickly, the dyspeptic phenomena disappeared, the intestinal pains passed away and there are, properly speaking, no intestinal symptoms : if the patient is costive and if his evacuations are not bile-colored, it is to be ascribed to the small quantity of food and the damming back of the bile; that is, to the affection of the biliary tracts, which proves itself to be a more obstinate morbid condition, than the rapidly disappearing gastro-intestinal disturbances. It were therefore erroneous to consider the present case as one of a gastrointestinal catarrh, that spread into the biliary tracts: the above-mentioned quickly passing away gastro-intestinal disturbances could hardly originate from any more or less firmly established gastro-intestinal catarrh; while the affection of the biliary tracts, I must say again, proves itself to be very firmly established. Besides, its symptoms, as pains in the region of the gall-bladder, appeared even two weeks previously to the before mentioned gastro-intestinal disturbances, at a time when both the appetite and the digestion were as yet perfectly niormal. It is evident that the disease of the biliary tract existed before the appearance of the enumerated gastro-intestinal disturbances and only became aggravated after them.

What disease then of the biliary passages does our patient suffer from? Guided by your knowledge of the special pathology of the internal organs and by what I said of biliary calculi in connection with the first case discussed by us, you have probably concluded, by this time, that our patient is suffering with calculi in the gall-bladder, and you are not in error: our patient is really suffering from this disease, which, although not completely developed, presents, nevertheless, all its characteristic symptoms; - a disease of great importance, because of its frequent occurrence and because it is so easily cured by a correct treatment and regimen (hygiene), while otherwise it may ruin one's health and become dangerous. I consider it, therefore, necessary to stop here to discuss the importance of its symptoms, its diagnosis and therapy.

What is the formation of gall-stones due to? All are agreed on one point, namely, that it is promoted by the sluggish passage of the bile along the biliary tracts. This fact explains the 
frequency of biliary calculi in women (the wearing of a narrow corset and pregnancies limit the movements of the diaphragm, which assist in propelling the bile along its passages and its entrance into the intestines) and in men of sedentary habits of life (in these also the movements of the diaphragm are hindered, and are neither as free, nor as extensive as in men leading a lively, physically active mode of life). It was further assumed that the cause of the formation of biliary calculi is to be found in the secretion of a bile of an altered, as compared with the normal, character; but exact investigations failed to substantiate this assumption. It is more probable, as supposed at the present time, that biliary calculi are formed within the normally secreted bile, through the influence of causes which emanate from the intestinal canal and which call forth a precipitation of the cholesterin, of the biliary pigments and of the lime salts - the chief component bodies of biliary calculi. According to some, these causes (it is most likely assumed, that it is the microbes, so abundant and various in the intestinal canal) call forth a catarrhal condition of the gallducts and of the gall-bladder. which impedes the passage of the bile and which, through its products, occasions the alterations in this fluid, and the formation within it of the above-named precipitates; while others assert that these causes are directly responsible for the changes produced in the bile and its precipitates. All these assumptions require further investigation and verification.

The clinical facts coincide in so far with these suppositions, that they substantiate the frequency of the co-existence at one and the same time of gall-stones and of a catarrhal condition of the biliary passages (it must in this comnection be kept in mind, that the irritation of these last by the calculi may also lead to a consequent catarrh of the same): constant tenderness, and at times direct sensitiveness on pressure in the region of the gall-bladder, and frequent jamdice are the characteristic symptoms of biliary calculi. But as the peculiarly characteristic symptoms of this disease are to be considered attacks of biliury colic accompanied by sharp, at times excruciating pain, which develops rapidly, and which disappears 
after a certain period of time just as rapidly. I will not stop here to describe an attack of colic: that you know well from your study of special pathology. The attacks of biliary colic are considered as due to neuralgia of the hepatic plexus, probably in conjunction with a contemporaneous tonic spasm of the gall-bladder. It is assumed that these attacks are caused by the passage of the calculi through the narrow portions of the biliary tracts, which occasions, as a consequence. an irritation of these last; but as we shall see later, this is hardly always the case. I said just now, that as the most characteristic symptoms of biliary calculi are considered the attacks of biliary colic; but it would be very erroneous to think that their absence would speak against the presence of biliary calculi. On the contrary, we very frequently come across cases of this disease which do not present attacks of sharp pain, i. e. of biliary colic, but in which a constantly present pain of only moderate severity in the region of the gall-bladder is observed, of which the patient complains himself, or which may be elicited only on investigation by pressure. Sometimes, in these cases, in their further course, we observe also the appearance of attacks of biliary colic, at other times we do not. These cases yield to the usual treatment of biliary calculi, as do cases characterized by attacks of liver-colic. It may be stated, that if we consider the attacks of biliary colic as the most characteristic symptom of biliary calculi, then we may say that the most frequent, and the most constant symptom of this disease, is a constantly present pain in the region of the gall-bladder, aggravated by pressure.

It appears, then, that our patient has unmistakable symptoms of biliary calculi and of a catarrh of the biliary ducts. Even now there is some pain in the region of the gall-bladder, aggravated on pressure, while on his admission it was much more severe. He had yesterday quite an acute attack of biliary colic, and slight attacks, as seen from the anamnesis, made their appearance since the beginning of October. While the jaundice, which developed in connection with the trifling and rapidly disappearing gastro-intestinal disturbance (it presented on the patient's admission a complete failure of the 
bile to enter the intestine), proves the fact, that, up to the time this disturbance made its appearance, there was a catarrhal condition of the biliary ducts, severe enough to become exacerbated from a trifling cause.

Some consider it necessary for the diagnosis of gall-stones to search for passed calculi in the evacuations. I consider such a search an idle task for practical purposes. In the great majority of cases, to which may be referred the present one, the diagnosis is easy, and does not present any difficulties. In the rare cases of a difficult diagnosis, the detection of calculi in the excreta is, firstly, of such casual occurrence, and fails so frequintiy, that it can not be relied upon, and, secondly, even when successful, it is of but small assistance in the matter. Let us touch upon these, generally quite interesting, cases of difficult diagnostication.

It is at times necessary to decide as to what the patient suffers from - biliary calculi or cancer of the liver. We usually have to do in such cases with very emaciated patients, who present a slight jaundice and who have severe pains in the region of the liver (which is also sensitive on pressure, especially at the seat of the gall-bladder), but without any pronounced objective symptoms of cancer of that organ. A search is made for the stones in the evacuations, and, let us assume, it is successful - the stones are found; but will this fact exclude a contemporaneous affection by a cancer (patients who suffer for a prolonged period with gall-stones become quite frequently victims of cancer of that organ)? And to settle the fact of the presence or absence of this last is usually the most important diagnostic task in such cases. It is not the detection of calculi in the excreta, but the careful objective examination and attentive analysis of the patient's past and present conditions, of the course of the disease, - at times it is the success or failure of the treatment, - that clear up the diagnosis in these cases.

We further meet patients with symptoms which excite a suspicion of both gall-stones and of disease of the stomach, usually of an ulcer (less frequently of cancer) about the pylorus: such patients have attacks of severe pains, with vomit- 
ing (but without the characteristic icteric phenomena and without blood in the vomited matter) and tenderness on pressure to the right of the middle abdominal line, between the umbilicus and the costal edge, at a seat corresponding at times to the gall-bladder and at other times to the gastric pylorus. Here also, even if the search for gall-stones be successful, the question, as to whether there is not also with the biliary calculi a gastric ulcer, remains unanswered; here also the diagnosis is cleared up by the aid of the same procedure as in the foregoing case (least of all, however, by the results of the treatment, that may be similar in both diseases). As regards the objective examination in such cases, I will say, that if the pain is felt distinctly by pressure on the mentioned region in the direction upward, toward the right hypochondrium, it points to sensitiveness of the gall-bladder, but if the pain is felt in the direction downward and inward (to the umbilicus), then it is sensitiveness of the gastric pylorus.

Finally, while speaking of cases in which the diagnosis of biliary calculi is made with difficulty, I must mention the difference between them and the attacks of neuralgia of the hepatic plexus caused by them (i. e. of attacks of biliary colic) and attacks of pure neuralgia of the plexus. We first of all ask whether there is such a thing as a pure neuralgia of this last? Some assume that there is: thus the well-known clinician, and the author of a capital work on diseases of the liver, Frerichs, described a case of biliary colic, for which repeated treatment at Karlsbad failed, while a strengthening and anti-nervine treatment was successful, and he considers this case as one of pure neuralgia of the hepatic plexus (hepatalgia). But Frerichs' case is, in my opinion, not convincing, chiefly because the anti-nervine treatment in the case was preceded by the repeated treatment at Karlsbad: it is probable that this last removed the biliary calculi, while the attacks remained, having become an independent hepatalgia, which in its turn yielded later on to the anti-nervine treatment. An example of this we see in the neuralgia of the facial nerve, caused by aching teeth, which persists (though not always. but at times for a long period) after these are cured or ex- 
tracted. I have not met with cases which could be considered as presenting a genuine and withal primary, idiopathic hepatalgia, but I have frequently seen such in which the period of undoubted symptoms of gall-stones, during which the usual treatment was available (calomel and mineral waters), was followed by one in which the attacks of biliary colic continued, although somewhat weaker, and only yielded to treatment directed towards strengthening the condition of the nervous system (and local treatment to the region of the gall-bladder with static and galvanic electricity). It is likely that the calculi in these cases have already disappeared and there remained only an independently established biliary colic in the form, consequently, of a hepatalgia not of a primary, but of a secondary origin, consequent upon the formerly present calculi. The diagnostic difficulties for me consisted not in my inability to make up a decision, as to whether the biliary colic in the given case presented symptoms of gall-stones, or whether it was a primary genuine hepatalgia, but in deciding as to the period of the disease, whether there are as yet present biliary calculi to be treated accordingly, or whether the attacks of biliary colic presented an established neuralgia, which was to be treated as such. It is not always easy to decide on this point, and a conclusion can be reached through a careful analysis of the condition of the patient, the course of the disease and the results of the preceding treatment; but at times only fresh therapeutic attempts decide the matter.

The present case, as I said already, does not belong to those in which a diagnosis is difficult. In view of the so distinctly prominent data in the sphere of biliary tracts and the slight nervous symptoms in our patient, pure hepatalgia is positively to be excluded, as well as ulcer of the stomach, in view of the condition of the digestion, and finally also, cancer of any organ. in view of the patient's youth and physical strength as well as on account of the rapid improvement in his condition.

Again, there are cases in which the diagnosis, properly speaking, is easy, but the physician - certainly if negligent - may commit an error. The fact is, that patients suffering from biliary calculi, who do not have any attacks of biliary colic, but 
only a constant pain in the region of the gall-bladder, complain frequently of pain in "the pit of the stomach." without distinctly designating the seat of pain, which is so easily done in view of the vicinity of the gall-bladder to the "pit of the stomach."

The physician, yielding to the first impression, investigates, makes pressure only in the region of the stomach, asks the patient about it, receives in reply that the pain is aggravated, and concludes that the patient has a gastric catarrh; whereas a careful investigation, i. e. such as would also include the neighboring organs, would show in such cases the seat of the maximum sensitiveness in the region of the gall-bladder. There may certainly be present at one and the same time both biliary calculi and a gastric catarrh, but in such a case we must find a complete summary of the symptoms of both diseases.

In speaking of the diagnosis, we must say a few words concerning yesterday's attack of biliary colic. The patient felt véry much relieved by the action of calomel: the sensitiveness on pressure in the region of the gall-bladder has considerably diminished since then and remains so at the present time. But he had another attack of colic yesterday. What could have caused this? The usual causes, which call out such attacks, - as traumata (as we saw in our patient when it resulted from riding in a jolting wagon over the pavement), mental disturbances, dietetic errors, and colds - were all absent in the clinic. We can only say this much, that such attacks are frequently observed at the beginning of the treatment of biliary calculi with Karlsbad (or generally with any alkaline) water, which fact, however, prevents in no way its further curative action.* The above-mentioned attack was accompanied by a slight febrile condition - a not infrequent phenomenon: in my article on calomel I have described a case of

* A similar phenomenon is observed in the treatment of renal gravel by mineral waters, namely, the attacks of renal colic appear or become aggravated at the beginning of the treatment; so that in either case, that is, in the treatment of either biliary calculi or of renal gravel by mineral waters, it is advantageous to warn the patient of the possibility of such an initial aggravation of the attacks. 
gall-stones, in which the attacks of biliary colic were almost always accompanied by a febrile condition.

Prognosis is favorable: the disease is curable; but the stability of recovery and the absence of recurring attacks depend on the patient's will and ability to fulfil all the requirements of hygiene and therapy.

Treatment. - The patient was at first given calomel, which produced such a considerable and usual improvement in these cases.* We then prescribed the warm alkaline and slightly laxative mineral water (Karlsbad Miihlbrunnen). Why did we prescribe it? Surely not because we were guided by theoretical considerations, which change in accord with the changes in the views concerming the formation of gall-stones: thus, when the cause of these last was supposed to be found in the alterations of the secreted bile, then the effect of alkaline mineral waters was attributed to the influence on the secretion of bile, to the fact, that by the aid of the alkaline, the secretion of the bile becomes more abundant and more fluid (this was evidently also substantiated by experimental investigation), and it was consequently thought that it would thus tend to dissolve and remove the stones; at the present time, when the chief cause of the formation of biliary calculi is considered to be due to alterations in the already secreted but sluggishly (stagnatory) passing bile on account of the catarrhal condition of the biliary tracts, the beneficent influence of the mineral alkaline waters on the gall-stones is chiefly ascribed to their action on this catarrhal condition. There is perhaps to an extent some truth in either of the views, but the chief guide for the employment of mineral alkaline waters in gall-stones are not all these assumptions, but the facts that practice has proved the utility of the employment of these waters for this disease and has taught the method of employing them.

Why did we prescribe Karlsbad water, and no other alkaline water, as Ems, Marienbad, Vichy and Yessentucki? I had occasion to go into particular explanations regarding the selec-

* The indications for calomel for biliary stones have been fully discussed by me in my article on calomel, and to avoid repetitions, I must refer the reader to it. 
tion of mineral waters; I would therefore, for the sake of a short explanation of the present case, say but a few words. If the patient were subject to diarrhœa, we would give him Ems; but as he is slightly costive, he was given Karlsbad. As a strongly laxative water Marienbad is not indicated in this case, and being a cold water and rich in carbonic acid, it would tend to aggravate the pains, and is consequently contra-indicated. If the hepatic colic and the gall-stones would play but a subordinate part in our case, and the principal trouble would consist in renal colic and renal uric-acid-gravel, while the patient himself would present unmistakable symptoms of gout (arthritis urica), then we would prescribe Vichy, Yessentucki (warmed), etc.

The patient declares, that as he apprehends the loss of his occupation, he would like to leave the clinic as soon as possible; we will therefore prescribe for him a somewhat larger close of Karlsbad than what is usually administered during the cold season of the year with constant confinement within the house: thus, the day before yesterday our patient drank one half-glassful of the above-named water, yesterday he had two half-glassfuls; we will prescribe to-day three, and from to-morrow he will take four half-glassfuls, two doses one hour before the early (mid-day) dinner, in the interval of one-quarter of an hour one from the other, and the other two one hour before supper ( 7 P.M.) in the same order. The gradual increase in the quantity of the mineral water, beginning with one half-glassful per day, is indicated in the present case by the fact, that first the patient had recently a diarrhea, and secondly, he had only lately loose stool from calomel: four half-glassfuls of Mühlbrumnen from the very first day would have caused a diarthoa, which is far from being desirable.

Besides the Mühlbrunnen we shall order for the our patient a warm bath, which we are likely to repeat, as a very beneficial agency in pains caused by biliary calculi and by catarrhal jaundice. In case the patient has another attack of biliary colic, he will get one or two doses of one-fourth of a grain of codeine, which, to judge by yesterday's attack, is sufficient to subclue the pain (in very acute attacks a resort to morphine becomes necessary). 'The patient, as I said before, is somewhat 
nervous and subject to sleeplessness : he will be given at times a few drops of the æther-valeriana preparation (the more so, as the pulse becomes weak at times), and at bedtime, to overcome the sleeplessness, daily doses of fifteen grains of potassium bromide.

\section{FROM THE LECTURE OF OCTOBER 27, 1889.}

Status. - There were no attacks of biliary colic, the temperature is normal, there is improvement all around. The former treatment will be continued.

\section{FROM THE LECTURE OF NOVEMBER 15.}

Status. - The patient, as you hear, declares that he is completely cured. As a matter of fact, his appetite and digestion are normal (stool for the last few days without a clyster), the jaundice disappeared, attacks of biliary colic are absent, there is neither pain, nor tenderuess on pressure in the region of the gall-bladder, the sleep is sound (he was given the bromide only during one week), and the mental condition is hale (we found it necessary to give him only two warm baths, while for the few last days he was given cool spongings $20^{\circ} \mathrm{R}$. (about $77^{\circ} \mathrm{F}$.), with a mixture of water and brandy, which exerted a beneficial influence on his nervous system). Until to-day, patient drank of Mïhlbrunnen four half-glasses daily; to-day we will give him only two half-glassfuls, because his affairs require that he leave the clinic to-morrow.

The patient was made to understand that only the visible symptoms of his disease had disappeared, that time would show how lasting his recovery would be, and that to avoid the return of the morbid attacks, he must beware of irregularities in his mode of living, which we discovered by our investigation (his sojourn in our clinic has served as a good hygienic schooling for our patient). We told him that it may very likely become necessary to resort to repeated treatment by Miihlbrunnen, and that this treatment must be postponed till the warm season of the year, (i. e. so as to avoid at least until then a return of the morbid 
attacks), when he would be able to undergo treatment by mineral waters, without being compelled, at least altogether, to give up his occupation.

I do not advise our patient to drink any Mühlbrunnen at home, first, because at the present season of the year, with his occupation out-of-doors, it would subject him to a risk; and secondly, because the drinking for twenty-four days of the Mühlbrunnen, which he finishes to-day, may perhaps suffice; already for the last ten days there is no jaundice, nor any pain in the region of the gall-bladder, while no attack of biliary colic took place since the one of which we spoke October 25th. In other cases it is frequently necessary to prolong the course of treatment with mineral waters for six weeks. Further than that I usually do not continue the treatment with mineral waters, but make an interruption, and if necessary, repeat it; I do this because, first, a more prolonged, uninterrupted employment of mineral waters may exert a deleterious influence on nutrition and digestion, and secondly, to enable me to clearly define the results obtained by the treatment. If, during this necessary interruption the patient be suffering with pains in the region of the gall-bladder, I then prescribe ether, - - ten, fifteen or twenty drops three times a day (exercise special care, so as to administer the smallest dose to men already advanced in age, who may have a hypertrophy of the heart, or in whom it is generally easily excitable), or, for men with good digestion, ether ( $3 \mathrm{j})$ and oleum terebenthinæ rectificatum (3j) in the above-named dose. These remedies (the remedy of Durande consists, properly speaking, of one part of oleum tereb. and four parts of ether) are sometimes undoubtedly effective.

But ordinarily I continue the treatment of gall-stones until all their symptoms have disappeared (and even somewhat beyond that time); chiefly not the attacks of biliary colic (which appear so vaguely, that they can hardly serve as a guide), but the constant pains and sensitiveness on pressure at the seat of the gall-bladder. It is always a most reliable way to be guided by these last symptoms; the attacks of biliary colic, however. cease usually before the disappearance of the above-named constant pains and of the sensitiveness on pressure. 
While discontinuing the treatment, as per above-named indications, I am far from assuming that the gall-stones have been "cured," removed, or are absent: we frequently observe cases, where the above enumerated symptoms have disappeared, and the patient feels absolutely no pain in the region of the gallbladder, no attacks of biliary colic have taken place for a very long time, and the patient leads a faultless hygienic life, when all at once, through an accidental cause (most frequently following strong mental excitement) an acute attack of biliary colic breaks out unexpectedly, showing the presence as yet of one or many stones in the bladder. It is true such cases are very rare; ordinarily the return of the attacks of biliary colic is preceded by a return of a constant, although slight, pain and sensitiveness on pressure in the region of the gall-bladder, that reminds us of the necessity for a repetition of the treatment and usually of the necessity for a stricter observance of hygienic rules. But, I repeat again, the treatment must be conducted only till the disappearance (and somewhat longer) of the above-named symptoms. To continue treatment until " the stones have completely disappeared," is unreasonable (not to speak of the possible danger from a too prolonged treatment), because, what can guide us in prolonging the treatment, and for how long a period can we do it? Then there is no necessity for it: experience shows, that with the observance of hygiene, with regularity in all the functional activities of the organism, calculi may remain in the bladder without giving rise to any morbid phenomena; but if the hygienic rules be neglected and there be superadded gastro-intestinal, nervous and other disturbances, then even the complete removal of the stones (if such be successfully accomplished) will be of no avail, as others will be formed again. I alluded once already to the fact, that on the return of the symptoms at the seat of the gall-bladder it is necessary, in order to anticipate an attack of biliary colic, to repeat the treatment with mineral water.

Sodium salycilate and olive oil, recommended recently for the treatment of biliary calculi, do not, in my opinion, deserve any consideration.

The present case does not present sufficient cause for the 
discussion of the operative treatment of biliary calculi, which is at times (to be sure, in conjunction with correct therapy and regimen, in very rare cases) the sole means for the relief of the unbearable sufferings or even the means of saving the patient's life. 


\section{SEVENTH CASE.}

\section{LECTURE OF MARCH 24, 1892.}

The patient, a private of the reserve army, complains of jaundice, enlargement of the abdomen, cedema of the legs, a febrile condition and general weakness.

Mode of living and anamnesis. - Patient lives in a healthy, nonmalarial locality (a village) of the Vladimir Government. The residence is an ordinary peasant's house, the stable serves as a water-closet. Used to bathe during summer until the appearance of his sickness. Takes a hot bath every week. Smokes some and is a moderate tea-drinker, but drinks daily a goodly quantity of whiskey and at times considerable of it. His board is good. He is single. Denies syphilitic infection, and investigation substantiates this denial. In view of his sickness he has had no occupation lately, but served in the army formerly.

Patient comes from a healthy family. He began drinking whiskey when eighteen years old and suffered since then with burning and belching after meals. Entered the military service in his twenty-first year (served as an artillery man in IVarsaw), when he was compelled to abstain from drinking, and noticed that both the burning and the belching disappeared; but in June 1891, he seems to have passed through an attack of typhus (during six weeks he had suffered from high fever, debility and unconsciousness), after which, at the end of July, he was given a leave of absence "to recover his health." He began drinking while at home, when there soon reappeared belching, burning and pains in the stomach, especially after a cold drink and rough food. By the end of September there appeared the jaundice, and at the same time the gastric symptoms became aggravated, especially the pains, which were this time accompanied by vomiting. The patient left off drinking, became more care- 
ful with his food, and took some Karlsbad salts and some powders (apparently containing rhubarb) ; by the middle of November the pains and dyspeptic phenomena were relieved and the jaundice, as he claims, disappeared. Patient again took to drinking, and again there appeared jaundice, pains and vomiting (now of daily occurrence), and also a slight febrile condition, enlargement of the abdomen, cdema of the legs and general weakness. Patient entered our clinic the first time December 6th, with the following most important data (besides those above enumerated): the liver is very much enlarged, firm, smooth (edge sharp), not tender to pressure, nor is the region of the gall-bladder sensitive; the fæces are but slightly colored by bile, the urine is very much jaundiced, contains neither albumen nor sugar; the spleen is slightly enlarged, there is moderate ascites; the heart is somewhat raised by the high position of the diaphragm, its tones are clear, the pulse is weak; the organs of respiration and the nervous system are in a normal condition. The patient was prescribed a diet, Ems, the bitters (extr. condurango fl. and tra. nux vom.) and stimulating drops (tra. valer. æther. and liqu. anod. Hoff. $\bar{a} \bar{a})$; the vomiting ceased, and the dyspeptic phenomena were relieved.

December 11th he was given ten powders of calomel āa gr. j, after which he had abundant loose characteristic calomel stools, followed by almost normally colored evacuations, while the urine became more limpid and the febrile condition passed away. After the calomel we continued the former treatment (Ems, bitters and stimulants), but December 20th, the patient, before the termination of the laid-out plan of treatment, left our clinic, considerably improved however: the jaundice and the dyspeptic phenomena have almost completely passed away, as well as the odema of the legs, the ascites diminished, and his strength began to return. After leaving the clinic the patient again took to drinking and began eating indiscriminately, but this notwithstanding he felt comfortably well until the middle of February. Since then his condition grew rapidly worse : there appeared considerable jaundice, almost daily vomiting and diarrhœa (three to four very fluid, painless stools per day), the abdomen enlarged rapidly, the legs became oedematous, 
there appeared a febrile condition with daily chills, weakness and later on hæmorrhage from the nose and gums, and hemeralopia. He was again admitted to our clinic March 15th.

Since admission patient has been taking all the time remedies to strengthen the heart's action (wine, tra. valer. æther. + liqu. Anodyn. Hoffm, twenty-five drops four times a day, and frequently caffeinum natro-salycil., five grains three times a day), and December 17th he was given five one-grain powders of calomel with good effect. The febrile condition, which reached in the evenings $40.2^{\circ}(104.5 \mathrm{~F}$.), diminished after the administration of the calomel and later disappeared altogether, so that it was entirely absent these last three days; but to-day he had again a slight chill. The urine became more limpid after the calomel, while the evacuations are tolerably colored and became less frequent and more solid (twice a day). The dyspeptic phenomena passed away. The pulse and general condition of the patient became better, but the ascites is on the increase.

Status. - A slight appetite appeared. With the disappearance of the fever the thirst diminished and the dryness of the tongue passed away. The gums are spongy and bleed. The pain in the stomach, the vomiting and the other dyspeptic phenomena passed away. The stools are as above. The urine is less jaundiced, than it was on the patient's admission, contains neither albumen nor sugar, its quantity amounts to $1,500 \mathrm{c.}$ c. There are no pains in the abdomen, the ascites is very considerable, the superficial abdominal veins appear very much distended. The ascites does not permit of palpating the liver and the spleen at present, but on his admission investigation showed, that the liver was very much enlarged (its free edge is some four fingers' width below the normal), but otherwise in the same condition as it was during the patient's first sojourn in the hospital; it is also perfectly insensitive to pressure (and so is the region of the gall-bladder), although the spleen emerges one and one-half to two fingers' width from under the edges of the ribs (patient never had malaria), it is firm and also insensitive. The heart is raised by the high position of the clidphragm, but it is not enlarged; at both the apex and the base there is a perceptible systolic murmur of equal intensity. 
Pulse 112, regular, stronger than it was on his admission, but still somewhat weak. There is no pain in the chest: dyspnura on moving about (when at rest, respiration 30 per minute). a slight cough with a trifling amount of mucous expectoration; auscultation elicits some dry râles here and there; the voice is very hoarse (laryngoscopic examination shows a catarrhal condition of the vocal cords). Patient is very thin and weak. We alluded above to the febrile condition; with its disappearance the sleep became better, while the formerly occuring (in the evening during the increase of the fever) headaches have almost completely disappeared. Movement gives rise to dizziness. The consciousness is clear, but the memory has weakened somewhat during the last half-year. The mental condition is oppressed. There is hemeralopia. Hearing is normal. No pains in the back. arms and legs. The legs up to the knees are considerably redematous. Severe jaundice: the sclerotics and the visible mucous membranes are colored yellow, the skin brown-yellow.

Diagnosis. - The dyspeptic phenomena as well as the pains in the stomach, which appeared with such regularity while the patient was drinking and committing dietetic errors, and which passed away as soon as the patient gave up drinking and exercised due care in his food and which so rapidly yielded to the treatment by Ems water and bitters during the first sojourn of the patient in the clinic, point undoubtedly to a catarrh of the stomach. Caused at the beginning by excesses in drinking whiskey and by dietetic errors, this sickness is now perpetuated by the so markedly apparent impediment in the circulation of the vena portæ due to the disease of the liver. The diarrhcea, too, is chiefly to be ascribed to this impediment in the circulation: the trifling admixture of mucus in the evacuation points only to an insignificant intestinal catarrh, while the evacuations, although infrequent, are abundant, watery and entirely unaccompanied by pains (in a catarrh. on the contrary, they would rather be frequent, not so abundant, rather more of a mucous than of a liquid nature, and to a greater or lesser extent accompanied by pains). The urinary organs. outside of the presence of biliary elements in the urine are healthy. Except for the pains in the stomach, which were ac- 
companied by vomiting and which disappeared now, the abdomen is entirely painless and has always been so; in view of this we must consider the great ascites in our patient as a result of the impeded circulation of the vena portæ, which fact is also substantiated by the considerable distention of the subcutaneous abdominal veins, by the above-named diarrhoea and by the enlargement and thickening of the spleen.

This last condition cannot be referred to anything else: the patient never suffered with malaria, while there is no sufficient. reason to ascribe this enlargement to the results of an (assumed) former typhus; such an enlargement and thickening of the spleen is rather unusual after typhus. The sharply pronounced symptoms speak clearly for an affection of the liver and of the biliary passages, but this we shall discuss at the end, after we will have analyzed the conclition of the other parts of the organism. The catarrhal condition of the pharynx, larynx and the slight bronchial catarrh, so usually seen in drinkers, requires no special explanations. The cause of the displacement of the heart upward is clear. The weakness of the pulse and the systolic murmur may depend partly on the displacement of the heart, partly on trophic changes in the cardiac muscle (which it would be so natural to find in a patient, who passed but recently through a severe febrile affection, and who, this is most important, has so ruined his health in general, and particularly his nutrition, by drinking) and partly on account of some disturbance of the innervation of the heart caused by cholæmia, the presence in the blood of biliary elements, because the patient presents already unmistakable signs of the presence of cholæmic phenomena - bleeding of the gums and hemeralopia.

There is not the slightest foundation for considering the systolic murmur as a symptom of affection of a cardiac orifice or of a corresponding valve, first because the most important proof of the existence of such an affection - a resulting enlargement of the heart - is absent, and secondly because in December, during the patient's first sojourn in the clinic, the tones of the heart were clear, there were no murmurs, although the principal morbid phenomena in our patient (the abdominal) were already at that time in evidence, though to a slighter degree. The 
frequency of the pulse is due to the same causes, and also undoubtedly to the febrile condition. The oudema of the legs is kept up by the weakness of the pulse, but it certainly depends principally on the pressure of the fluid (which accumulated in the peritoneal cavity and distended the abdomen), exerted on the inferior vena cava and the great veins of the abdominal cavity terminating in it. We can find no other causes for the febrile condition outside of disease of the liver and of the biliary passages. After all that was said, we hardly need to stop here for the explanation of the nervous symptoms.

What disease of the liver does our patient have? I must first of all observe, that cases of hepatic diseases, in which the diagnosis is directly apparent by the collection of the symptoms, are less frequently seen, than such which for their diagnostication require a resort to differential diagnosis; as an example of the first class, we may instance a case in which a large, painless liver with undoubted fluctuation at some point on its surface in conjunction with a complete absence of any other morbid data 'either in the liver itself. or in the rest of the organism, points at once to an echinococcus cyst of this organ, or when an enlarged, tuberous and extremely sensitive liver in a cachectic patient, who is apparently free from syphilis, speaks for cancer or generally for a malignant new formation in this organ. Our present case belongs to the second class of cases, and we will therefore apply to it the above-named method of differential diagnosis. It is evident that the sum total of the symptoms in our patient can not be ascribed to either hyperæmia of the liver, or to fatty degeneration of this organ. Amyloid degeneration of the liver is to be excluded loy the considerable ascites, by the phenomena of icterus and the absence of albuminuria, which could be expected as a symptom of a contemporaneous amyloid affection of the kidneys, if we admit such an advanced amyloid degeneration in our patient as would be necessary to assume in order to explain the great enlargement of the liver as well as of the spleen. Syphilis of the liver could give rise to the symptoms observed in our patient (with the addition, however, of pains in the hepatic region, which usually accompany syphilis of this organ, but which were and are now absent in the present 
case); but this is not only to be excluded by the fact that the patient, not by any means a man of deceitful nature, denies syphilitic infection, but chiefly by the fact, that neither the anamnesis nor the present history give the least clue as to its presence. Against cancer of the liver speak the patient's youth, the absence of roughness, and complete insensitiveness of this organ, as well as the repeated improvement (and even disappearance) of the phenomena of icterus and the parallel cessation of the febrile condition after the employment of calomel, which could not be thought of, were they conditioned by cancer of the liver. Echinococcus eyst could not give rise to the symptoms observed in our patient, not to mention the fact of the absence of any symptoms of this affection (fluctuation). Multilocular echinococens, which emigrated into the biliary passages and caused the chronic jaundice, differs from the present case in this, that it occasions a very pronounced alteration in the shape of the liver (the right lobe is particularly enlarged), that it takes a more chronic course, is usually unaccompanied by ascites, and especially of such magnitude, but principally by the fact, that a diminution, the more so the disappearance, of the icterous symptoms caused by it, is never effected by the employment of calomel. There remains, therefore, that disease of the liver, which, in view of the etiology of the given case - abuse of alcohol in conjunction with an assumed attack of typhuswould first of all suggest itself, namely cirrhosis of the liver, as a result of an interstitial inflammation of the organ (hepatitis interstitialis).

Our knowledge of the cirrhoses of the liver is far from being perfect. The pathologo-histological data concerning these affections are interpreted in varions ways. As based on these, some assume the following cirrhoses: venous, in which the interstitial inflammation begins from the branches of the vena portæ; biliary, in which it progresses from the small biliary passages, and the mired, when the disease advances contemporaneously from both the venous and the biliary branches; others again assume that there is only one kind of cirrhosis, that the interstitial inflammation of the liver involves at the same time both the branches of the vena portie and the biliary ducts and that it 
only predominates to a greater extent in one case around the first, in the other case around the second, but that eventually it spreads everywhere. The clinic proves the following: there are, first, cases of cirrhosis, which present only results of impeded venous circulation in the liver (as ascites, enlarged spleen, diarrhœa, intestinal as well as gastric hæmorrhages) without an arrest in the flow of bile; second - cases, in which, on the contrary, the last condition is observed, without any disturbance of the circulation of the portal vein; and third, - such, where both classes of symptoms are to be seen. It appears then, that the clinical data are in greater accord with the first of the above-named views, which was first promulgated by the French investigators Charcot, Hanot and others; the second one is defended by the Germans.

It is also from an etiological point of view more probable, that in some cases the exciting agencies of the inflammation reach the liver through the blood vessels, in others through the biliary ducts (from the intestinal canal) and in others again contemporaneously through both tracts. The fact, that the interstitial inflammation, originating at one point, for instance at the biliary ducts, and accompanied at the beginning only by signs of an arrested flow of bile, may later on spread also over the circumference of the system of the vena portæ and give rise to phenomena of disturbed circulation in this last, can surely not serve as a proof that the process commenced everywhere at one and the same time. I will also say that in cases of cirrhosis accompanied by disturbances of the portal venous circulation, the liver is sometimes very much diminished in size (the cirrhosis first described by Lænnec, called by his name), at other times it is greatly enlarged. Some think that such an enlargement takes place only at the beginning, and that later on it is followed by a diminution in size, and that if this decrease does not take place, it is to be ascribed to the fact that the exhausted patient does not last long enough for this process to develop.

As a matter of fact, it has been proved by various investigations that the liver, enlarged at the beginning, undergoes a process of shrinking later on. But we much more frequently 
meet cases, in which the liver remains enlarged up to the patient's death, although the patient may live long enough, even longer, than those with a small liver, and we cannot therefore assert, that such an enlarged liver always undergoes diminution later on. The pathologo-histological investigations fail as yet to clearly elucidate this point.

Thus the chinic presents the following forms of cirrhosis: 1. Venous, or cirrhosis accompanied by the phenomena of disturbed venous portal circulation, in which the liver is either extremely diminished in size (Lennec or atroplic cirrhosis) or very much enlarged (hypertrophic venous cirrhosis, by which we mean a hypertrophy of the connective tissue of the liver, while its glandular element, the hepatic cells, are always affected in cirrhosis). 2. Hypertrophic biliary cirrhosis, accompanied by phenomena indicating the retention of the bile, and 3. Mixed cirrhosis, with symptoms of both. What form of cirrhosis does our patient suffer from? The first impression of the case, the jaundice and the ascites would suggest a mixed cirrhosis; but a further investigation will lead us to another conclusion. As evident from the anamnesis, there appeared repeatedly in our patient after a drinking debanch and a faulty diet, an attack of jaundice contemporaneously with pronounced signs of a severe gastric catarrh (dyspepsia, pains, voniting), while a corrected diet and treatment caused their disappearance at one and the same time, so that it (the jaundice) undoubtedly depends on a catarrhal condition of the great biliary passages. It is true, that this last, being present for a long time or recurring frequently, may also spread over to the small biliary ducts and thus give rise to inflammation of the connective tissue around them, i. e. it may bring about a condition of biliary cirrhosis. but the investigation of the given case speaks against the presence of this last, for the jaundice has so rapidly yielded to the regimen and treatment, and particularly to the calomel, which it usually fails to do in biliary cirrhosis (the phenomena of impeded flow of bile in this yield to successful treatment much slower). Consequently our patient has venous cirrhosis and of the hypertrophic form at that (the liver is much enlarged) and at the same time also catarrh of the biliary passages, cho- 
langitis catarrhalis. There are no symptoms of biliary calculi. The febrile condition of the patient, which accompanied the jaundice and disappeared with it at the previous, as well as at the present, sojourn of the patient in the clinic, seems evidently to be due to the just mentioned cholangitis.

Treatment. - In view of the discussions conducted in the previous lectures, it is, of course, superfluous for me here to enter into particular explanations regarding the use of calomel, of Ems, the bitters and the stimulating drops during the patient's first sojourn in the clinic, as well as of calomel and the stimulants (together with the diuretics, as wine, the above-named drops and the caffeine) employed at the present time. After the calomel the high temperature fell rapidly, and for the last few days the patient had no fever at all; at the same time there took place a pronounced and considerable diminution of the icterous phenomena and the dyspepsia disappeared. Thanks to the effect of the stimulants the pulse became stronger, but the quantity of urine was not perceptibly increased, while the most threatening symptom-the ascites. so distressing to the patient, causing such a disturbance of the breathing and of circulation - became more aggravated, so that in the near future we shall have to resort to operative evacuation of the peritoneal cavity. We will again administer to-day to our patient some calomel, as he again had a slight chill and the jaundice has not passed away; we may also expect a ditiretic effect from the calomel. We will, of course, continue the wine, the above-named drops and the caffeine in increased doses. The other diuretics, as potassium acetate and the strong ones (as adonis vernalis, squilla, ete.) are unsuitable for the present case on account of the diarrhoa (after the calomel the patient's bowels move better, not worse). However, the irresistibly advancing ascites can hardly encourage us to hope for a considerable effect of the diuretics, and the performance of paracentesis abdominis is inevitable.

Prognosis. - A fully developed venous cirrhosis of the liver, hypertrophic and atrophic, is incurable in the present condition of therapy; not one of the tried remedies, among them also the iodine preparations, realized our expectations: the rarely ob- 
served cases of effective action of these last remedies are very - likely due to the fact, that these cases present a mixed cirrhosis, from alcohol and syphilis. Given a relatively favorable condition of the other organs and good surromnding circumstances, we may succeed in prolonging the patient's life by keeping in good order the digestion, circulation and the secretion of urine and by operative evacuation, at times, of the peritoneal cavity. The greatly disturbed condition of our patient's health gives us but small encouragement for consiclerably prolonging his life.

\section{FROM THE LECT'URE OF MARCH 27, 1892.}

After the second administration of calomel there was no fever, the secretions are sufficiently colored, the biliary pigment disappeared from the urine and the sclerotic became clear, but the ascites progresses further and it becomes necessary to tap the abdomen to-day.

The further history of the disease. — March 27th we obtained by tapping 2500 c.c. of limpid, lemon-colored fluid of a purely transudative character; this afforded the patient considerable relief. Since April 1st patient became delirious (the delirium simulates delirium potatorum). At the entreaties of his parents patient left the clinic, April th, for his home. At the end of the month he again returned to Moscow and was examined by the clinical assistunt under whose care he was formerly: the delirium disappeared, there is not a trace of jaundice (the hemeralopia and the systolic murmur disappeared); there is enormous ascites and cedema of the legs; by tapping 3000 c.c. were drawn; investigation showed that the liver is just as enlarged and solid as it was during the patient's sojourn in the clinic. Patient went home, returned to Moscow at the beginning of June again with an enormous ascites and cedema of the legs. Again 2500 c.c. were drawn, he was given sodium iodide (20-50 gr.) and six glasses of milk per day: the result of this treatment in two weeks amounted to absolutely nothing, and the accumulated ascites again necessitated tapping, by which 3000 c.c. were drawn. Patient has gone home and we have lost track of him since. 


\section{EIGHTH CASE.}

\section{FROM THE LECTURE OF DECEMBER I, 1889.}

The patient, a peasant eighteen years old. complains of pain in the right hypochondrium. of jaundice of one and one-half years' duration and of general weakness.

Hode of living and andmnesis. - For the last ten years pattient has lived constantly without intermission in Moscow (formerly lived in a village), all the time in damp and cold rooms (usually in a basement); does not bathe in the river, takes a weekly steam-bath. His board is tolerably good. Has been addicted to drinking whiskey for the last two years. His occupation is that of a shoemaker, he works from morning till evening, and. except holidays, is seldom out-of-rloors.

Patient enjoyed good health until two years ago. Since then, as I said before, he began to drink whiskey: six montlı after this he began to feel pain in the right side and soon after there appeared jaundice, which is sererely aggravated at the present time. The pain in the right side is of a double nature: it is either constant and bearable. or at times very acute, lasting three to four hours. The attacks of this last pain became more frequent and more acute in the comse of time. and have been accompanied of late by vomiting (evidently attacks of biliary colic). Patient grew thin and weak and was admitted to our clinic November 2 Tth ; he was never treated before. He denies syphilitic infection and presents no signs of it.

Status. - Patient is of fair constitution : there is very considerable emaciation and jaundice of the skin and of the scleræ. The appetite is good: patient digests daily about three-fourths of a pound of meat, one-half pound of hread, two egg's and two plates of soup, without the least rypeptic signs. and even begs for more food. He is somen hat constipated: the 
evacuations are dry, fetid and apparently not at all colored by bile. The urine contains a moderate quantity of biliary pigment. but neither sugar nor albumen; to judge by the absence of color in the secretions, pointing to a failure of the bile to pass into the intestines, we should expect a darker discoloration of the urine (an indication that the glandular elements of the liver, the hepatic cells, are already affected and hindered in their activity). The spleen is normal. The liver is enlarged, more solid than normally, but of regular outlines and smooth, and very sensitive to pressure. The organs of respiration and circulation, as well as the nervous system, present nothing abnormal: the sleep is good. Fever absent.

Iniagnosis. - It is evident, that our patient has biliary calculi and a severe catarrh of the biliary passages, - cholangitis catarrhalis, - - to which must be ascribed the pains, the failure of the bile to pass into the intestines, its retention in the liver (this again caused the enlargement of the organ and probably an impediment also of its bile-formative function and through this of its other activities by compressing the hepatic cells) and general jaundice. But is this all? Is there no further affection of the liver, and if so, what is its nature? The etiology, the course of the disease and the condition at present show, that such a further affection could be an interstitial inflammation around the minute biliary ducts of the liver, that is, a development of a hypertrophic biliary cirrhosis (I shall not review the differential diagnosis applied in the former case). We can neither deny nor affirm the presence of such a cirrhosis in this case; on one hand the disease has lasted for a time long enough for a cirrhosis to develop; but on the other hand it may safely be assumed that the observed symptoms were caused by cholangitis and biliary calculi. We shall wait for further observations.

We must also wait with our prognosis, the more so as it always depends not only on the diagnosis (even if clearly defined), but also on the degree of success of the treatment. We cannot, however, deny the fact, that in view of the patient's youth, his good constitution and the healthy condition of his other organs (outside of the liver), as well as because the 
patient dirl not subject himself to any treatment, his future prospects are favorable.

Treatment. - IVe will first prescribe calomel in a slightly laxative dose, without inducing a diarrhcea; later on we may employ a mineral water, namely Karlsbad, as the patient is inclined to be costive; in the former case, with a tendency to diarncea and weakness on the part of the patient. Ems was prescribed. We will also order for the patient one or probably more warm baths.

\section{FROM THE LECTURE OF DECEMBER 12, 1889.}

Status. - December 3d, th and 5th patient took four onegrain powders of calomel daily with the best of results: the bth. 7th and 8th, he harl, once a day, sufficient. soft. normally colorert stools. The the wain observed that the evacuations were poorly colored, and he was therefore given. the day before yesterday and yesterday. form powders of calomel. The effect was again good, but there appearerl slight salivation, which we combat with the usual means of rinsing his mouth with potassium chlorate and painting the gums with tra. gallarum; outside of this, the patient's condition is entirely satisfactory: the appetite is normal, the dyspepsia disappeared, the circulation. respiration and the nervous system are in good order, he feels much stronger; the urine contains almost no biliary pigment. the acute pains in the region of the liver have almost passer away, the liver itself is almost insensitive. but it has diminisherl. though markedly, not much so, which circumstance speaks for the presence of a cirrhosis, though of small extent.

We will certainly give no calomel to-day, but from to-morrow on he shall take Karlsbad; later on, in case of necessity. we will again resort to calomel.

The further history of the disease. - Patient drank four halfglassfuls of Karlsbad water daily until January 2d, when he left the clinic; we did not administer calomel any more. By the time he left not only were the evacuations all the time entirely colored and the mine always limpid. containing no biliary pigment, but the general jaundice harl almost disap- 
peared. the patient gained eleven pounds in weight (he weighed 121 pounds on admission, his weight when he left was 132 pounds), and gathered strength: the liver is entirely insensitive to pressure, but it remained of a rather more enlarged size than it would be normally.

At our insistent advice patient gave up drinking, and about six months later he appeared before our clinical assistant in the same satisfactory condition, as he enjoyed when leaving our clinic.

Thus the sixth case presents biliary calculi and cholangitis. the seventh-hypertrophic venous cirrhosis and cholangitis, the eighth-biliary calculi, cholangitis and a slight hypertrophic biliary cirrhosis. In my article on calomel the reader will find not only a fully characteristic, but also a very severe case of hypertrophic biliary eirrhosis, which however, terminated favorably. 


\section{NINTH CASE.}

FROM THE LECTURE OF NOVEMBER 16, 1890.

The patient, a peasant twenty-nine years old, was admitted to the clinic a week ago. complaining that the abdomen "swells up." and that he is weak; he added, that he was once before taken care of in the hospital, where he was told, that his disease is called echinococcus of the liver.

Mode of living and anamesis. - During the last year, on account of his illness, patient has been living in a village of the Ryazan Government, in a healthy locality; formerly he lived altemately in both Moscow and in the village, under the ordinary conditions of life of a peasant and of a workingman. While in the country his occupations are those of a peasant; in the city he is a joiner. He drank but little whiskey. He is married and has several children; his wife never aborted.

Patient comes from a healthy family and until the last four years enjoyed good health. Four years ago he began to notice a swelling in the right hypochondrium, which was painless, but kept constantly growing, until he was finally so much hindered in his occupation and movements, that three years ago he entered the hospital, where they performed an operation, drew the fluid and told him (he has a distinct recollection of the name) that his disease was called echinococcus of the liver. He remained in the hospital for seven months and left without a trace of the former swelling. The next year and a half he enjoyed good health; but about a year ago he again observed a swelling in the right hypochondrium, somewhat painful at that time, which also began to grow in size and interfered with his work; at the same time patient began to grow thin and weak. Two weeks ago the swelling suddenly diminished, and almost disappeared, but at the 
same time the abdomen began to swell rapidly, it hecame painful and there appeared chills, fever and cliarhura: he had some ten liquid stools daily; in the course of two days the fever and the diarrhoea passed, and soon the abdominal pains quieted down, but the abdomen began to increase in size; the patient then came to Moscow and entered our clinic.

Status at the day of admission. - The appetite is good, but the patient eats little at a time; otherwise he feels uncomfortable, as respiration grows difficult. Outside of this there are no dyspeptic phenomena; stools regular. The quantity of urine is 1100 c. c., containing neither albumen nor sugar. There is a pronounced ascites of considerable size (distinct fluctuation): the circumference of the abdomen at the level of the umbilicus is $98 \mathrm{c}$. m., the distance between the anterior superior spine of the ileum and the umbilicus is $28 \mathrm{~cm}$. The tension of the stretched abdominal walls does not permit of palpating the liver. There are neither pains nor sensitiveness on pressure in the abdomen. The diaphragm is very much raised. There is dyspnoea on moving around, breathing while in a state of rest is 26 ; pulse 76 , regular, but weak ; outside of this there is nothing abnormal in the organs of respiration and circulation, as well as in the nervous system, except that the sleep is not sound on account of the large size of the abdomen, which hinders motion and freedom of abdominal breathing. Ferer absent. The patient, as I said before, has grown weak and thin. He denies syphilitic infection, nor are there any indications of it.

The patient was placed amidst normal conditions of life, the abdomen was protected by flannel, and he was given internally potassium acetate as a diuretic, and six to eight tablespoonfuls of port wine per day.

Status to-day. - Patient feels somewhat hetter: his breathing became freer, his strength increases, and the sleep is also better; the dimensions of the abdomen do not present any marked decrease; the quantity of urine has almost doubled ( 2000 c. c.).

Diagnosis. - It is evident, that within the last year there again formed in our patient an echinococcus bladder, which burst into the peritoneal cavity, and probably, at the same time, also 
into the intestines. The absence of severe pains and of fever for the time - one year - the disease lasted speaks against its being an abscess of the liver: the pains were very trifling, while there was no fever at all; the appetite remained always good, but the patient could not eat as much as he desired, in view of the pressure exerted by the enlarged liver on the stomach, and he therefore grew thin and weak. The emptying of the contents of the echimococcus bladder into the peritoneal cavity caused a slight peritonitis, which soon passed, but it produced ascites.

Treatment. - Accumulations in the peritoneal cavity do not in the majority of cases yield to internal treatment, but require operative interference - either radical, or, in case of impossibility, palliative, namely, the drawing off of the fluid. Fortunately in the present - very rare - case, the condition is more favorable. The phenomena of peritonitis were so trifling (the pains were not of an acute character, there was no vomiting, the fever was slight), and passed away so rapidly, that we hope for a favorable. hardly at all changed condition of the peritoneum, and consequently may expect a possible absorption of the accumulated fluid. That part of the liver, which escaped the invasion of the echinococens, performs its functions normally, as evidenced by the regular digestion and the fact, that the intestinal evacuations are well colored by the bile; consequently we may hope for a sufficiently free circulation in the liver, and therefore also in the portal system. The appetite is good, the stomach, intestines, kidneys and the other organs are healthy.

All these circumstances encourage us to hope for the possible absorption of the fluid accumulated in the peritoneal cavity; it would therefore be erroneous to resort at once to the operation, before first attempting internal remedies.

Having surrounded our patient by correct hygienic conditions, we prescribed a diuretic to encourage the absorption of the accumulated fluid. Sudorific treatment, as, for example, warm baths, pilocarpine, would tend to weaken the heart's action and would thus, instead of encouraging absorption, rather add to the accumulation; not to speak of the fact, that in the patient's condition, with his difficultly performed respiration and circulation, such treatment would become dangerous; while treatment by 
the aid of laxatives would tend to disturb digestion and nutrition, thus aggravating, instead of improving, the patient's condition. In prescribing the diuretic treatment, we aimed at the two principal agencies of urinary secretion - the heart and the kidneys: for the first we gave the wine, as the patient's pulse was weak, for the second potassium acetate (strong diuretics, as adonis vernalis, squilla, ete., would disturb digestion, and weaken the patient), half a drachm per day, thus: R. _ Potas. acetatis $3 j$, aquæe petroselini ${ }^{2} \mathrm{vj}$, for two days, in tablespoonful doses; this was given during four days, after which it was stopped for two days.

The condition of the patient is apparently improving, and the plan of treatment will therefore remain as heretofore; but as the dose of the potassium acetate - 3ss. for a whole day- is rather small, and the patient bears it well, we will increase the dose to one drachm per day: R. - Pot. acetatis $3 \mathrm{ij}$, aqua petroselini $z_{i j}$, for two days, in tablespoonful doses. As before, we will interrupt the administration of the drug after four days for two days: thus the power of digestion will more certainly be preserved in a good condition, nor will there be formed a habit for the drug, which would bring on a weakened effect of this last on the patient.

\section{FROM THE LECTURE OF DECEMBER 12, 1890.}

Strutus. - Patient declares that he feels entirely well. As a matter of fact, his appetite is good, digestion regular, the quantity of urine 2000 (it even reached 3000 ), the spleen is normal, the abdomen is of normal dimensions, its cirenmference is $72.5 \mathrm{~cm}$, the distance from the superior spine of the ilem to the umbilicus is $15 \mathrm{~cm}$., the diaphragm is at its normal position, there are no traces of any former aecumulation, the respiration, eirculation and the nervous system are in a normal condition, the patient became stout and quite strong. The liver is entirely insensitive and is markedly enlarged in its left lohe; nothing abnormal to be noted in the right one. Eehinococeus hladders are most frequently fomm 
in the right lobe of the liver: limited functional activity of this portion of the organ may bring on an increase in the functional activity and hypertrophy of the left lobe.

The patient was told of the possible failure of a fresh formation of the swelling, but in case of its reappearance he is to apply at once for medical aid. 


\section{TENTH CASE.}

\section{FEBRUARY, 1890.}

The patient is thirty-three years old and was admitted to our clinic February 3, 1890, complaining of pains in the right side and of difficulty in breathing.

Mode of living and anumesis. - Patient is a resident of Moseow, inhabits fair lodgings, the water-eloset is cold. Frequents public baths; is subject to sweating. His ocenpation is that of a forenan at a raihoad station: he is a bookkeeper and superintends the reception and delivery of goods; this occupation is not a fatiguing one. He is married, but his wife had no ehildren. Has strongly abused alcohol since his twentythird year, but since his illness, from July of last year, he gave up drinking altogether.

In December 187\%, patient became infected with syphilis, and was treated for two months with potassium iodide, and one year later he entered the hospital in St. Petersburg, where he was treated with mercurial innnction, and also with potassium iodide. Since then, in the course of the next ten years, up to the summer of 1889, he observed nothing to require a resort to treatment. During the summer of 1889 he began to experience heaviness in the abdomen and dyspnoa, and soon after that pains in the right hypochondrium aggravated at nighttime and on moving around. The patient left off drinking abruptly; after this the appetite grew worse, and he began to grow thin and weak, the dyspnoea increased, and he became costive. At the advice of his physician, who ahready at that time detected a considerable enlargement of the liver, he drank, during one month, Yessentucki waters No. 17 and took laxatives, but withont any avail. Since September 1889, patient was treated during three weeks in the hospital with meremrial 
inunctions - altogether about ten times - and with potassium iodide internally, and later on took the same, without increasing the dose, at home for another three weeks; the pain improved for some time. From that time on until his admission to the clinic the patient was not treated, and his condition continued growing worse.

Status on February 3d.- Patient is of a strong constitution. Appetite fair, but patient eats little, as the feeling of heaviness in the abdomen becomes aggravated after a meal. There is no dyspepsia. He is very costive. Urine shows ab sence of albumen and sugar. The abdomen is enlarged. there is slight ascites. The spleen is somewhat enlarged; the liver very much so: on percussion the superior border is higher than normally, while the free border is palpated at a distance greater than the width of the palm below the costal edge ; it is moreover not sharp, but dull to the touch : there are no other alterations in the shape of the liver; it is more solid than normally, and besides, it is sensitive to pressure all over; the mobility of the liver in respiratory movements is of slight extent. There was no jaundice before, nor. is there any at present. The heart is displaced upward, its tones are clear, the pulse - 106 - is weak, there is some dyspncea. The organs of respiration present nothing abnormal; there is no fever. The patient is very weak and thin. The imperlerl breathing and the nightly exacerbation of pains on the side of the liver prevent sound sleep. The head, and especially the occiput, aches while patient is constipated, but the pain is relieved by a good evacuation of the bowels.

Diaynosis. - If certain persons, who are habituated to drinking strong alcoholic drinks for a long time, leave off the habit abruptly, then as a consequence there appears a whole series of disturbances: the appetite is imprired. the patient has either constipation or diarrhea instead of the former regular evacuation of the bowels, the pulse grows weak, there appears dyspnøa, the patient grows thin. sleeps badly and weakens physically and mentally. As seen from the anamnesis, some of the disturbances observed in our patient are undoubtedly of such an origin, while the others depend on an evident disease 
of the liver: this last is very much enlarged and sensitive, and the ascites and the enlarged spleen speak of impeded circulation in the portal system.

What hepatic disease is to be found in this case? The anamnesis, and partly the symptoms, as the regular aggravation of the hepatic pains at night. point to syphilis as the immediate cause of the affection of the liver; we cannot at the same time deny the influence of a prolonged abuse of alcohol. Irith such data on hand and in view of all that we said, while discussing the foregoing cases, regarding the diagnosis of diseases of the liver in general and their differential diagnosis in particular, there seems to be no reason to further discuss the exclusion of other affections of the liver in this case. Of the two affections, the presence of which is possibly indicated by the anamnesis, which is more probable, the syphilitic or the alcoholic: The data which speak for syphilis of the liver are incontestable: the liver is painful, which is only to a slight degree the case in alcoholic affection of the organ; the pains are aggravated at night-time, speeific treatment relieves the pains, and the liver is very much enlarged (this again is but to a slight extent the case in the alcoholic affection of the liver); there are no direct indications of the existence also of an alcoholie affection of the liver (as impeded portal venous circulation is also peculiar to syphilis of the liver). but we simply can not deny the possibility of its existence in the given case.

What is the nature of the syphilitic affection in the given case? There is tirst of all an indication of a syphilitic perihepatitis, namely: tenderness of the liver, the aggravation of pains at night-time, and the restriction of the respinatory movements of this organ; however. the value of this last symptom is lessened by the fact. that in the presence of an enlarged lirer and of tension of the abdominal walls, the respiratory movements of this organ are liable to be less free than under normal conditions, even if we exclude the possible adhesions due to the perihepatitis. There are no indications of any considerable gummatons swelling, as the surface of the liver is smooth. There remain, therefore. out of the number of rarious syphilitic affections of the liver, ninute disseminated gummatous new 
formations, disseminated interstitial hepatitis and its result in the form of cirrhosis of the liver, to which in this case we must ascribe the enlargement of this organ. Which of these just mentioned affections prevails is rather difficult to say: the symptoms of impeded portal venous circulation point to an already existing circhosis.

Prognosis. - The pains in the liver, caused by the perihepatitis, yield readily to specific treatment, especially to the iodide preparations. But the prognosis is most favorable, when there are gummatous swellings, even if considerable, but without affections of other parts of the organ; in such a case complete cure is possible. Much worse is the prognosis in diffuse interstitial hepatitis and in the cirrhosis of the liver resulting therefrom: here the success of the treatment is liable to be rather insignificant and of short duration; if aggravated, after a temporary improvement, the disease yields with greater difficulty to treatment and usually terminates in death. The same process evidently takes place in the liver, as in the nervous system (and probably also in the other organs), namely, an outspoken syphilitic affection (gummatous? - as in the nervous system, in case of lues of the brain and spinal cord), which yields completely to specific treatment, and also such, in which the connection with syphilis is apparent (as shown from the statistics of the affections of the nervous system, progressive paralysis and tabes dorsalis), but in which the specific treatment falls short of having this efffect. The prospects, as regards the termimation of the disease in our patient, who presents such evident symptoms of affection of the whole liver and together with this also symptoms of an already progressive impediment in the portal venous circulation, are certainly not favorable; but as the patient was never suljected to a continuous, sufficiently persistent and intensive treatment, the last word in the prognosis will depend on the treatment.

Treatment. - Having surrounded the patient by regular hygienic conditions, we will prescribe for him some strong wine. as the pulse is weak and the patient at the present more than at any other time is in need of his habitual stimulus: for the constipation, - watery clysters, and for the principal affection 
specific treatment, at the beginning iodine in the form of sodium iorlide. We will give him the iodide, and not mercury, first, because in the last stage of syphilis, which, as seen from the anamnesis and from the very nature of the syphilitic affection, our patient is passing through, iodide is more applicable; and secondly, because the patient's health has become greatly impaired, and the mercurial treatment by itself is more liable to weaken, than the treatment with iodides; and thirdly, because the syphilitic pains particularly yield readily and rapidly to the iodine treatment. We will administer the sodium iodide in an alkaline mineral water, as in Vichy, one and one-half glasses per day because the iodides are better borne by the organism when dissolved in it, and also because our patient's urine is red, very acid and saturated with uric acid salts. In such cases as the present one, the iodine preparations are to be administered, as experience teaches, in constantly increasing and generally large quantities. We will be guided in our further treatment by the results of the iodine treatment.

Status on February 28th. - The patient took sodium iodide as follows:

February 4th, $20 \mathrm{gr}$; February 5th and 6 th, 30 gr. each day: February 7 th to 10 th, 40 gr. each day; February 11 th to 15 th. $50 \mathrm{gr}$. each day; February 16 th to 20 th, $60 \mathrm{gr}$. each day ; February 21 st to $28 \mathrm{th}, 70 \mathrm{gr}$. each day.

The iodide was administered in solution (3ij in इrj aque dest.) in tablespoonful (loses, with the addition in the beginning: of a small quantity of Vichy-Célestins, and later - in view of a beginning looseness of the bowels - of Ems-Kesselbrunnen. about one and one-half glass per day.

To-day the patient announces that he feels so well, that, apprehending the possible loss of his situation, he intends to leave the clinic. His condition is as follows: the appetite is very good. bowels move regularly (the olysters were necessary only during the first days of the patient's sojourn at the elinic), the quantity of urine is greater, it is limpid and not red. all the pains disappeared, the pulse is better, the sleep in good. patient gathered considerable strength, the liver is entirely painless. but it has diminished in size but very little. and so did the ascites. 
The patient was informed of the seriousness of his condition; he was told, that, although the pains have disappeared, the appetite and sleep improved and he became stronger physically, still the most important and the most dangerous symptoms namely, the enlargement of the liver and the ascites. have not yielded markedly to the iodide treatment; that the treatment must be continued persistently. and that at present he must begin the mercurial treatment: he was made to understand, that a correct employment of such a powerful drug as mercury, to avoid any risk of harm. is only possible under immediate medical supervision and under suitable circumstances, namely, in the Hospital, and that any postponement of this treatment will exert a dangerous tendency as regards a favorable termination of the disease. The patient insisted that he must return to his occupation and that he mould try to be treated at home; he left the clinic the next day.

The further history of the disease. - Patient continued taking sodium iodide during March in small quantities and irregularly, but up to June he felt almost as well as he did on leaving the clinic. Since then there reappeared the serere pains in the right side, and the patient began to take calomel, four powders a day (the dose is unknown; he had slight looseness of the bowels) in the following manner: one week calomel, the next week none; during this time rinsed his mouth with pot. chlor. and his gums were not affected. This treatment was continued through June, July and August. The patient improred somewhat and his tolerable condition continued up to February 1891. when the right side became again painful, the abdomen began to enlarge rapidly, and later on the legs began to swell. February 18th patient again entered our clinic.

Status on February 18th. - Appetite very poor; there is properly speaking no dyspepsia, but the nausea and romiting are rather of uremic nature, as they take place not after a meal, but in connection with heaviness in the head and with pain. There is at times constipation, at other times diarrhoea. The urine contains a considerable quantity of albumen. and also some cylinders - hyaline and granular; the quantity of urine in twenty-four hours is 500 c. c. The ascites is great. while the 
wedema of the legs extends up to their middle; the spleen does not admit of palpation. The liver is in the same condition in which it was a year ago, the tenderness on pressure is not considerable. The heart is displaced upward, the pulse is 96 and weak. The respiratory organs are normal. Fever is absent. The sleep is very poor. The patient became very much emaciated and weak.

Treatment. - Correct hygienic conditions, wine, and an injection when necessary. As the patient has been treated for a long time with mercury, and has not taken any iodide for almost a year, we prescribed for him sodium iodide, at the beginning in a small quantity of Ems water, and later on in boiling milk. As the tendency to diarrhœa increased while the iodide was being administered, we also gave at the same time ten to twenty drops of tra. coto four times a day, which enabled us to continue the administration of the iodide for six weeks, and to increase the dosage to ninety gr. per day; but there was no improvement: February 27 th we had to draw 3000 c. c. of fluid of a purely transudative character from the peritoneal cavity. Since March 19 th we added to the sodium iodide also mercurial inunction - a half-drachm of mercurial ointment per day. Narch 25th, however, we were again compelled to draw 3,000 c. c. By the end of March the specific treatment with the iodide, as well as with the mercury, was discontinued (we made altogether about ten inunctions) and we continued to strengthen him as much as possible. April 12th patient left the clinic in the same condition in which he was admitted; the accumulation in abrlominal cavity, after the last tapping, did not as yet reach the former dimensions, but it continued on the increase. 


\section{RHEUMATISM AND GOUT.}

\section{ELEVENTH CASE.}

\section{LECTURE OF NOVEMBER $29,1889$.}

The patient, a peasant water-carrier, twenty-five years old, was admitted to the clinic, complaining of severe pains in the articulations, loss of appetite and weakness.

Mode of living and anamesis.-- Patient lives in Moscow. His lodgings are very warm. the water-closet cold. Goes often to public bathing house and drinks a great deal of hot tea; is very much subject to sweating. Is a moderate whiskey drinker. His food is that of an ordinary workingman. He works hard and is very much fatigued at the close of the day. He is single.

Until the present sickness the patient always enjoyed good health, except that he suffered with headaches. which appeared at the hot season of the year during field labor, and which passed away accompanied by nasal hæmorrhages. A week ago, November 21st, the patient became strongly chilled, and in the evening he experienced chills. fever and pain in the articulations; he took no treatment, and until his admission to the elinic, November 27th, he grew worse and worse. November 27th and 28th the patient was given seven ten-grain powders of sodium salycilate in Ems-Kesselbrunnen water, after which he had considerable timnitus aurium, but the pains were very much relieved and he was not given any more of the salycilate. The painful articulations were rubbed with warm olive oil and wrapped in cotton.

Status. - The appetite has reappeared, there is no dyspepsia. stool regular. The urine, formerly red and quite saturated. became limpid; it contains neither sugar nor albumen. Thie 
liver and spleen are in a normal condition, as well as the organs of respiration, circulation and the nervous system. His temperature on admission was $38.2^{\circ} R$. (100.7 $F$. $)$; at present it is $36.8^{\circ} R .\left(98.2^{\circ} \mathrm{F}\right.$.). When admitted patient perspired freely, now he perspires but little. On admission, all the joints (except that of the hip) of the lower extremities were affected, also both the elbow joints: the pains were acute and the patient avoided any movement; a slight swelling only was noticed at the articulations of the knees. At the present time all the pains are considerably relieved, but they are felt as yet to some extent whereever they were before; the swelling of the knee-joints disappeared. The noise in the ears continues as yet, but to a very much lesser degree.

Diagnosis. - It is evident that our patient suffers from acute articular rhemmatism. This is shown, outside of the characteristic picture of the clisease, by the acute beginning of this last in an organism previously healthy, in the presence of the equally peculiar in this disease predisposing conditions, as a warm lorging, frequent hot baths, hot tea, proclivity to perspiration, constant fatigne from physical labor, and finally a cold, and also by the decided effect of the salycilate of sodium, which serves in acute articular rhemmatism as hardly less of a specific remedy, than quinine does in malaria. We know of no other disease, that would call out so suddenly such an agglomerate of symptoms and which would so rapidly yield to the salycilate of sodium.

Prognosis. - The disease is slight and is already passing away, but it is very liable to return, especially under the circumstances surrounding our patient and his mode of living.

Treatment. - The patient was given the most reliable remedy against acute articular rheumatism, namely, sodium salycilate: you have seen its effects. I usually administer sodium salycilate in a small quantity (in one or two glasses per day) of the alkaline water Ems-Kesselhrunnen, or, to persons of a strong constitution and with good nutrition and who are. moreover, inclined to gout, in some Vichy-Celestins, because this remedy is borne, if thus administered, in the best manner. The patients, who dislike the unpleasant taste of sodium salycilate, 
may take this last in cachets and wash them down with an alkaline water. For acute articular rheumatism I never fail to give sodium salycilate until tinnitus aurium is produced (just as in the case of quinine for malaria), at times quite strong, at other times, in very severe cases, till very pronounced, watching of course carefully the action of the heart, in which large doses of salycilate of sodium, especially if given for a long time without an intermission, may induce a condition of collapse. Experience taught me to regard such a mode of employing sodium salycilate in acute articular rhemmatism (just as quinine is used for malaria) as giving the best results: the disease is interrupted rapidly and the interruption is lasting, the disease is not protracted, and does not return, as in cases where the remedy is given timidly and in small doses, without exerting any positive effect; this is very important, because the earlier and the more completely the disease is interrupted, the more certain may the patient be of avoiding the development of its dangerous complications, cardiae and otherwise. As soon as the tinnitus appear's to be sufficiently pronomnced, as judged by the severity of the disease, I at once discontinue the use of the salycilate. It happens frequently that with the appearance of a sufficiently strong tinnitus aurium the articular pains and the fever disappear not to return, so that the remedy is not given any more. But if the tinnitus has markedly diminished, but the pains and the fever continue, although to a lesser extent. then immediately prescribe again sodium salycilate. I begin in adults with ten-grain doses every two hours; but if the case is very severe and this dose be well borne, I rapidly increase it. give fifteen and later twenty grains every two hours, carefully watching, I repeat again, the condition of the pulse, so that in case of necessity to administer stimulants, as tinctura valer. æether. and liqu. anodyn. Hoffm. āà.

As I said before, the fever and the swelling of the knee-joints left our patient, and the pains have very much diminished in intensity, but they are still felt to a slight degree wherever theywere formerly, while the tinnitus aurium, on the cessation of the administration of the salycilate, has continually diminished and is now very slight. In accord, therefore, with the above-men- 
tioned rule, we will again return to this remedy, but without increasing its dose, because its effect is satisfactory as it is, and the case is a light one.

Antipyrin is also valuable in the treatment of acute articular rheumatism. There have even been described several cases of the disease in which the salycilate failed, while antipyrin was successful. I have not met with such cases, and guided by the number of my own observations and those of others, I still consider the salycilate of soda as the most reliable drug in the treatment of acute articular rheumatism ; besides, antipyrin in large doses is even more dangerous for the heart than is salycilate of soda.

The severity of some cases of acute articular rheumatism with cardiac and other complications may, of course, be determined by the particularly severe effect of the disease-causing agent, - very likely of a parasitic origin, because acute articular rheumatism is characterized by all the features of an infectious and withal acute affection; but, as I said before, such cases are chiefly to be observed, when the treatment of the clisease has been long neglected, the disease not being interrupted at the begimning, and the patient continues to subject himself to colds (as, for instance, by living in cold rooms, or having a cold water-closet), and to traumatic influences (can not obtain sufficient rest, gets up, or generally moves around). In such cases the sudden breaking up of the disease does not take place and there is no immediate return to health, so that a chronic affection of one or, more frequently, of several articulations is established, which goes under the current name of "chronic rheumatism," - a name which is not correct, is vague and prevents the formation of a positive diagnosis, and consequently also treatment, of the various affections, which are all without any differentiation embraced under this term (I shall have yet occasion to return to this subject). The affection, usually of one joint, which was left over, passes often into a condition of severe inflammation, which eventuates into pus-formation; this last circumstance points to a superadded secondary infection by pyogenic microorganisms.

Chronic affections of the joints, remaining after acute articu- 
lar rheumatism, are often found also in cases in which it attacked not a healthy, but a previously diseased organism, as for instance a tuberculous subject (thus in cases of acute articular rheumatism in youthful "scrofulous" persons), or more frequently one suffering with syphilis or gout, or even one with only a predisposition to gout. In view of the coming lectures on "chronic rheumatism" and gout, I wish to stop here to discuss the relation of acute articular rheumatism to the latter disease.

As proved by facts, gout is the result of an accumulation of uric acid salts in the blood and their deposition - principally that of the acid sodium urate - in various, or perhaps in all the tissues and organs, most frequently in the articulations, in their cartilages and other tissues; this deposition is the cause of various affections, according to the variety of the affected organs. Whence these uric acid salts accumulate is a debatable question; but this fact is certain, that such an accumulation is promoted by excessive eating and by the use of wine in the absence of active physical exercise, which means by conditions which weaken in the organism the processes of oxidation. The well-known clinician Ebstein assumes, not without ground, that the deposition of uric acid salts in the joints is chiefly favored by an abundant formation of uric acid in the organs adjacent to the joints, in the bone-marrow and the muscles. Acute articular rheumatism is accompanied by a febrile condition (which causes an increased formation of uric acid) and by abundant sweating, so that the urine is greatly diminished in quantity, becomes very red and saturated, and gives on standing an abundant sediment of sodium urate. It is well understood, that if such a morbid process, which brings about an increase in the formation and a decrease in the excretion of the uric acidthrough a decrease in the quantity of the urine - will affect not only a gouty person, but even one with a predisposition to gout, then this last is bound to make its appearance at once. And as a matter of fact, we often observe the following:

A man, say, forty years old, of good constitution, with superabundant nutrition and good digestion, a good eater, who imbibes freely and is physically inactive, and who has for a long time past observed that his urine was red, in short a candidate for 
gout, without, however, presenting a single local symptom of the same, is suddenly affected by acute articular rheumatism, grows feverish, sweats, and passes some red urine with an abundant brick-dnst sediment. Salycilate of sodium is administered correctly and the disease is rapidly interrupted; the fever and the. pains clisappear. The patient feels well, but in about two to three days he returns complaining that the "rheumatism" returned in the great toe of the right foot and has caused him excruciating pain during the whole night. The painful spot turns out to be swollen and red, but all the other joints of the body are entirely free from pain and there is no fever; in short the patient has a typical attack of acute gout and not the least return of acnte rheumatism. In other cases of this last in persons suffering with gout or predisposed to it, the fever and the greater number of articular affections disappear on correct treatment, but in one or more of the affected articulations there will remain a chronic disease by this time of a gonty nature.

The further course of the disease. - The patient took six tengrain powders more of the sodium salycilate, after which the tinnitus increased, but the pains disappeared and there remained only the general weakness. With the aid of a good appetite the patient improved rapidly, and December 11th left our clinic. During the last days, before dismissal, his whole body was sponged several times with water and brandy, and this strengthened him. On leaving, he was advised not to take any hot baths during cold or damp weather, but instead to sponge himself as above, and also to drink less of hot tea. 


\section{TWELFTH CASE.}

\section{LECTURE OF FEBRUARY 19, 1891.}

The patient, a peasant woman, 58 years old, entered our clinic Jannary 29th, complaining of pains and swelling in the lower and upper extremities, chiefly in the articulations of the wrist and shoulder, the knee and the ankle, and also in the small articulations of the hand and foot on either side. She has had the pains there for the last year and a lialf; they are aggravated by movements and make these last extremely difficult.

Mode of living and anamnesis. - She has been living for the last eighteen months in Moscow; lived formerly in a village. The lodgings are tolerable, the water-closet cold. Takes a hot báth once a month. Drinks neither tea nor brandy now, nor did she drink any before. The food is that of an ordinary working woman. Was married and had several cdildren; no abortions; has been a widow for a long time. For the last year and a half has been unable to work on account of sickness; formerly she did a great deal of very hard labor in orchards. Has always enjoyed good health up to the appearance of the present illness. In the autumn of 1889 , while working during cold weather in the orchard, she felt pains in the finger-joints of both hands; she continued at her work, but the pains increased considerably and to them was superadded swelling of the painful spots, so that it became impossible for her to work. She consulted a physician, who gave her some ointment, and for internal use some sweetish powders, evidently of sodium salycilate. She felt relieved, but the disease did not disappear entirely, and prevented her from working; in such condition it was protracted until the beginning of the last winter (1891), when, with the cold weather, the former pains became worse, while fresh ones appeared, accompanied by swelling in the remainder of the aboveenumerated affected joints. 
During the three weeks' sojourn of the patient in the clinic she drank daily of Yessentucki water No. 17, - at first one and a half, and later one glass per day ; she took several times salycilate of soda, at first every other day and later every third day, in quantities of twenty and later of forty grains daily, which caused some tinnitus aurium; and since February 6th, as soon as the affected joints, thanks to rest, warmth and the above-mentioned treatment, became much less painful, they were subjected to massage, and later we applied the electric static current: the patient felt much better.

Status to-day, February 19th. - The usual appetite is moderate. The stomach, intestines, liver and spleen are in a normal condition. The urine is reddish, without albumen and sugar. Menopause established fifteen years ago. The organs of respiration are normal (the slight bronchial catarrh, with which she suffered on entering and which caused some cough, passed away), as well as the organs of circulation, except that the arteries feel somewhat stiff. The patient, somewhat spare for a long time past, has not grown much thinner during the sickness. The temperature on admission was slightly raised, $37.6^{\circ}\left(99.7^{\circ} \mathrm{F}\right.$.), but it soon became normal. The sleep is good. There are no pains in the head, nor anywhere else outside of the abovenamed articulations. In these last the pains have considerably diminished and the movements become much more free. The swelling, which was particularly consicterable (there is also noticeable some fluctuation) in the wrist and shoulder-joint, as well as in the knee-joints of both sides, has diminished, while the slight redness observed on her admission, and the higher temperature in the last-named articulations, have disappeared.

Diagnosis. - The patient presents a type of what is usually denoted in the text-books of special pathology, in literature in general, as well as in practice, as chronic articular rheumatism; or, if besides the articulations there are also affected other organs of locomotion, as the muscles, nerves and the bones (i. e.. all the portions of the bones, and not their articular surfaces only), then it is simply designated as chronic rheumatism. I had already occasion to observe that I consider this designation incorrect, vague, and one that prevents the for- 
mation of a positive diagnosis (and consequently treatment) of the various affections, which are all, without any differentiation, embraced under this name. I will take advantage of the opportunity presented by this case, the only one of its kind on hand at the present time (although there occur others, of a more complicated nature and therefore more suitable for my purpose) to elucidate the basis of my opinion.

The term rheumatism, having long lost its ancient definition. has even at the present time no concise meaning: by it is meant on the one hand an acute articular rheumatism, - which. is a sharply differentiated disease, no less definite than are measles, variola, typhoid, typhus, etc., a disease which must not be confounded with any other, - and on the other hand. cases of the nature of the present one (of the importance of which I shall speak later), as well as more complicated ones that are diversified by the affected organs as well as, principally, by their etiology, and which are, besides. approaching by their many interstitial (in the character of their symptoms) manifestations the affection known as arthritis nodosa sire deformans. Nomenclature must of course avoid unnecessary destruction, it must not intrude on solidly based and universally intelligible definitions, nor introduce unnecessarily, before explaining fully the nature of the subject, any new terms; it should, however, strive for precision. If we must preserve the name rheumatism. then it can only be attained on the condition that we give it a definite designation: and this requisite is fully effected if we preserve this name solely for acute articular rheumatism, a disease, as I said before, of a definitely distinguishable character. The term rheumatism. as employed formerly in its old definition, will certainly not correspond with exactness either to what we know already, or to what we will probably learn in the future concerning the nature of acute articular rheumatism; we find the same facts as regards the terms measles, small-pox, scarlatina, plague, etc.. and in their correspondence to the relative diseases. Precise definitions will be possible only when the nature of the enumerated diseases will be known with precision, but until that time the implanted appellations have more rights to existence and they 
must therefore remain : the name rheumatism for the above alluded to discussed (in the elerenth case) acute affection of many articulations is no less firmly implanted than are the names measles. small-pox, etc.. for the diseases designated by them, and, I must say again, it refers to a sharply differentiated. definite disease, just as do these terms.

But if we were to preserve the name rheumatism for acute - articular rheumatism. may we then apply it, without depriving it of a defunite signiticance, to cases like the present one and to others still more complicated, which I mentioned and of which I shall yet have occasion to speak? Evidently not - first, because these cases differ one from another, presenting, as we shall see later, different affections originating from various causes, and secondly. because they are not in the least identical in their nature with acute articular rhemmatism. Thus the present case: at the beginning the joints of the fingers were affected and became swollen, and they have remained so until now: one year later the other joints became affected, and they remain thus now. Where is here the picture of acute articular rheumatism?

Do we find here a contemporaneous affection of many articulatious of a changeable, jumping character, accompanied by a febrile condition, by various complications, cardiac and otherwise, which it would be so natural to expect. if the disease were in reality what passes under the name of acute articular rheumatism, and one withal of such long duration! Finally, the salycilate of soda, which acts in such a specific manner, breaking up so decidedly an attack of acute articular rheumatism. acted in this case - to judge by the history of the disease and by our observations in the clinic - solely as a symptomatic remedy, as a sliglit pain-stilling agent, neither interupting nor curing the disease.

There occur, it is true. cases where an evident, typical, acute articnlar rheunatism does not terminate in a rapid breaking up and cure of the disease (usually on account of the fact, that the patient is surounded by unfavorable circumstances, or is not treated. or is treated wrongly and not sufficiently energetically), but leaves a chronic disease of the affected joints. which 
is dragged along through months and years, and is presented in exactly the same form as the present case, namely, without any characteristic peculiarities of acute articular rheumatism, unyielding to treatment by the salycilate. which fails to cure it. To denominate such cases by the term of chronic articular rheumatism, i. e., to consider them as eases of acute articular rhemmatism - a disease having all the peculiarities of an acut" infectious process - which passed into a chronir condition, would be the same, as if we were to consider chronic bronchial catarrh, which is sometimes left after measles, as chonic measles, or the diarhere left after an attack of typhoid fever. as chromir typhoid. It is apparent, that in all these cases it was not the acute infectious disease which passed into a chronic condition, but there were left loeal affections. which are caused by and. under favorable conditions, disapjent with the rlisease, and under. unfavorable circumstances become chronic.

In the chronic affection of the joints. which remains after an attack of acute articular rhemmatism, there take place at times apparent recurrences of this last: thus there appears a febrile condition, the affection of the joints becomes aggravated and takes on a migrating character, and the salycilate of soda exerts anew its beneficial effect. But such recurrences of acute artic ular rheumatism, which are generally so very peculiar to this disease and which occur frequently also in cases where its attacks leave no trace of any chronic affection of the joints, cannot go to prove the fact, that the chronic articular affection, which in the intervals between the attacks extended over months and years, is identical with the acute infections rlisease, which passes under the name of acute articular rhemmatism.

What then do these cases of chronic joint affection, of polyarthritis chronica, as our present one and others mentioned by me, present? Here it becomes particularly necessary to employ careful clinical analysis: I usually make in snch cases a double investigation and a double diagnosis - the anutomical, defining the affected organs and tissues, and the etiologfical, referring to the causation; I resort to this principally becanse the therapy presents differences not only in accord with the causes, but also with the affected organs, as joints, bones, muscles and nerves. 
The anatomical investigation gives the following: the most frequently and preëminently affected parts are the joints; in these we must determine the condition of the articulating ends of the bones and of their cartilages. the condition of the articulating capsule and of the soft parts outside of it. Less frequently are observed affections - nsually with pain and swelling of other parts, besides the joints; it then becomes necessary to determine just what part is affected: the skim, as evidenced by a hyperaesthetic condition, by dermatitis, etc., or the bones, most frequently in the form of a periostitis, accompanied by pain. which becomes aggravated on pressure, - and sometimes by swelling; or the cartilages, as seen, for instance, in a perichondritis of the costal, laryngeal and aural cartilages, - most frequently in gout; this condition also is accompanied by pain, which becomes worse on pressure; or it may be the muscles, as proved by the existence of a myositis: a high temperature, tension and sensitiveness on pressure and motion, and, later on, emaciation of the affected muscles, in which cirrhotic indurations may form: or the neress. in the form of a neuritis: pains, tendemess on pressure and various functional disturbances.

The investigation of the causes brings to surface most frequently the following: acute articular rheumatism, and also, although much less frequently, other acute infectious diseases, gonorrhoea (I cannot help observing. that the name polyarthritis gonorrhoica is much more preferable to the meaningless, vague term "gonorrhøeal rheumatism"). syphilis, tuberculosis, gout, a cold. traumatic influences, (of these last not so much those of an acute nature, - contusions, wounds, etc., - as the less severe, but constantly acting ones, as intense, fatiguing physical activity) and abuse of alcoholic drinks; for although alcohol does not cause directly any joint affections, it undoubtedly creates a predisposition to them.

Chronic affections of the joints, as I said once, are usually not of a simple. but of a complicated origin, depending on the effect not of one, but of sereral, eren of many causative agencies, as, for instance, of the conjoined action of a former acute articular rheumutism and at the same time of gout, syphilis, of a cold and of traumatic influences. 
What general name then is more suitable for those chronic affections of which we are speaking at present? If we desire to preserve the briefness so essential in a term, then it is best to call them polyarthritis chronica, because the affections of the joints are usually predominant, and such a name neither determines beforehand the concise diagnosis - and therefore the treatment - of the given case, nor does it render it obscure, as is the case with the term chronic "rheumatism."

We will tum now to the analysis of the present case. Persons of our patient's age, — fifty-eight years, _ living in the midst of miserable conditions, full of privations, suffer often from a particular form of gout, the so-called atonic gout, as distinguished from the other, the ordinary form, to which we alluded in our previous case. In our patient there are no characteristic manifestations of gout, as. for instance, affections of the joints of the great toes, neither the generally preëminent affection of the small joints (both the small and the larger ones are affected), nor any apparent gouty deposits around the articular ends of the bones; but she presents indications of a predisposition to gout - the constantly red urine and, as observation in the clinic made it evident, the favorable action on the patient of an alkaline (Yessentucki) mineral water. This predisposition to gont explains the fact. why the certain influences, traumatism and cold, to which the patient subjected herself with impunity during the whole of her laborious life. have at last, in her old age, caused the appearance of an articular disease. As regards the so much apparent influence of cold (she was taken sick twice during the beginning of cold weather), it is worth while noting, that precisely those joints became affected, which were either uncovered-as those of the fingers, and the wrist, or but insufficiently protected - as those of the knee and of the ankle (as the patient wears neither drawers nor a petticoat, while the soles were protected). As mentioned above, in the diseased joints the affection is found in the soft parts, located outside of the articular capsule, as evidenced by the swelling and by the higher temperature and slight redness on admission, and in the capsule itself (fluctuation detected); there are no signs of any affection of the bones. 
Prognosis. - Judging by the favorable course of the treatinent, we may hope for a satisfactory issue of the disease; we may expect a return to a considerable extent of freedom of motion, in case, certainly, the patient succeeds in aroiding in the future any deleterious influences. which are liable to cause a recurrence of the disease.

Treatment. - Why we prescribed for our patient, with her predisposition to gout, an alkaline water, and. in view of her age and poor nutrition. a small quantity of the same, you certainly understand well. I will only add that when atonic gout be found in patients, who have been ailing for a long time before with the usual form of gout, who drank a great deal of mineral alkaline waters, and whose nutrition suffered considerably, then we must administer to them either the lightest alkaline waters (Ems), or the earthy waters (C'ontrexéville). To be sure, our patient's nutrition is not of the best, but still we cannot call it very poor, and as she never drank any mineral waters. we therefore prescribed for her a very active alkaline water (Yessentucki, No. 17). but, as I said before, in a small quantity, and the course of the treatment justifies completely this conduct of ours. The salycilate of soda, as a pain-stilling agent. has relieved the patient sufficiently. Massage and the constant eleetric current seem to exert a favorable influence by diminishing the articular swelling and by making motion easier. Wre will therefore continue the Yessentucki water. a half-glass twice a day. the application of massage and of electricity; but there is no more need for the salycilate. nor for any other pain-stilling clrugs in general.

$\mathrm{IV}^{\mathrm{e}}$ e limit ourselves to the above-named means. because they seem to be suffieient. An excellent remedial agent for chronic joint affections we have in the salt baths. warm or even. in the absence of contra-indications, hot: we do not resort to them in the present case, because we can seemingly get along: without them. but principally because they are contra-indicated by the season, it heing winter time: the patient. after leaving our clinic. would subject herself to a greater risk in atching cold. A good remedy for chronic articular affections is car- 
bolic acid (in the form of fomentations of a two to four per cent solution and hypodermic injections); there is no need for it in the present case, and, besides, injections of carbolic acid cause severe pains. The iodine, as well as the mercurial preparations, are undoubtedly useful in articular affections when complicated by syphilis.

In prescribing local tratment attention must be paid as to what organs or tissues are affected. For muscles the best thing is massage. For the articulations - Spanish fly, massage, electricity and carbolic acid. For affections of the periosteum only general treatment, usually anti-syphilitic or anti-gonty, or both together, as, for instance, iodine preparations in alkaline mineral waters; massage and electricity increase the pains and aggravate the disease. For the nemrites - rest, Spanish fly. irritating and pain-stilling salves, and, at the end of the acute stage, a careful massage, not of the nerve itself. but with a derivative aim in view, of the neighboring parts. chiefly of the muscular masses; the action of electricity is not certain. Finally, I repeat again, salt baths, warm or hot, are useful in all the affections.

\section{FROM THE LECTURE OF MARCH $\mathfrak{l}, 1891$.}

The patient leaves the clinic to-day. All the functions are normal, the nutrition as well as the strength has improved. The swelling remains, although much less than it was before, only in the wrist-joints, while from the others it has almost passed away. There is an entire absence of any pains on pressure, or on passive and short active movements. A prolonged walk gives rise to a feeling of fatigue, and later also of pain in the knees, which, however, soon pass away after rest.

Patient was advised to avoirl certain harmful influences and to continue massage. 


\section{THIRTEENTH CASE.}

\section{LECTURE OF MARCH 3, 1892.}

The patient, a widow of an official, is forty-seven years old, and entered our clinic February 27th complaining of severe pains in the loins and in the thighs down to the knees; the pains are very much aggravated on motion, so that this last is almost impossible; she also complains of pains in the chest and in the left half of the abdomen.

Mode of living and anamnesis. - The patient is an inhabitant of one of the governments around Moscow, lives in a healthy locality and occupies a good house, but the water-closet is cold. Has not bathed in the river for the last five years, takes a hot bath twice a month, perspires very freely. Drinks no coffee, wine or whiskey, only some four glasses of hot tea per day; generally drinks but little: besides the tea only water, not more than one glass during the whole day. She breakfasts and dines at a good table, eats many sweets. Has been a widow for the last five years; was never pregnant. Goes out but little into fresh air, has but little exercise. Her occupations are those of a small housekeeper.

Patient comes from a healthy family and is herself of a strong constitution; in her seventeenth year she passed through an attack of typhoid, in her twenty-seventh had malaria. Has enjoyed good health since, up to the climacteric period, which began five years ago: the first three years the menstruation was irregular, but two years ago it ceased entirely. With the advance of the climacteric period there began to appear attacks of heat in the head and pronounced perspiration all over the body; they were of frequent occurrence during the first year, several times daily; since that time the quantity of the urine diminished, the mine became red and left frequently a reddish 
precipitate. Four years ago she had for the first time a very severe attack of renal colic on the left side, as evidenced by pains in the left loin transmitted to the left groin, which lasted, with intervals, for four days. A year later she had another attack of lesser severity, lasting two days. After that the attacks of renal colic took place frequently, always on the left side, but they were not of severe intensity. Since October 1891 there appeared a constant severe pain in the left loin and on the corresponding side of the abdomen, and since then began all those pains with which the patient entered the clinic, and which kept continually on the increase. The treatment was as follows: at the beginning sodium salycilate until the production of timnitus aurium during one month, but without any success; then, for one month, potassium iodicle (dose unknown) in solution, three spoonfuls per day; no results whatever. Later, cool spongings with a wet towel for one month, also without any success; finally, for two weeks before she entered the clinic, the patient drank Vichy-Célestins (she never used any mineral water before), about one glass and a half per day, and felt slightly relieved. While in the clinic the patient continues drinking the same water warmed to the temperature of freshly drawn milk, three half-glasses during the day, each time one hour before a meal.

Status. - The appetite is impaired. While observing a strict diet and using Vichy in the clinic, she has no dyspeptic symptoms, but formerly she felt heaviness in the stomach and pyrosis almost constantly. The patient is usually constipated and takes castor oil twice a month; in the clinic the rectum is sufficiently emptied with the aid of watery clysters. The liver and the spleen are in normal condition. The pains on the left side of the abdomen are due to the considerable tenderness of the kidney and the ureter; on the right side the kidney and the ureter are also tender on pressure, but much less so. For a few days before her admission to the clinic, the patient discontinued drinking Vichy and the quantity of urine on her admission was small, -500 to 700 c.c. in twenty-four hours, the urine very red, of a strongly acid reaction, with a considerable deposit, principally of sodium urate; at present, when the patient resumed drinking 
Vichy, and, as we advised, not very hot tea (she thus perspires less) and a greater quantity of ordinary water, the amount of urine increased from 1000 to 1200 c.c. in the twenty-four hours, the urine is not so red, and the sediment is much smaller. Urine contains neither albumen, nor sugar. The organs of respiration are healthy. The dimensions of the heart are normal, its tones clear, but the pulse is somewhat weak, which points to an insufficient activity, and probably to the already affected nutrition of the cardiac muscle, a condition so very natural in a sick woman who takes so little exercise. 'The patient was formerly stout, but grew thin during the disease, and yet she cannot be said to be emaciated. There is no fever. The pains prevent sleep to some extent, but yet it is quite sound and sufficiently prolonged. The pains in the chest of which the patient complains, are proved hy investigation to depend on the sensitiveness of the sternum, ribs and scapulx; but most sensitive are the pelvic bones (especially the sacrum) and both femurs; the movements of the acetabular articulations are extremely painful, which condition causes great difficnlty in turning over in bed, and makes getting up and walking about almost impossible : the patient, as you saw, was carried into the auditorium on a chair.

Diagnosis. - It is evident, that we have here to deal with a typical case of gout, arthritis urica. The patient, of strong constitution, with a hitherto good digestion, lived always in affluence, ate more than was necessary, had but little exercise, no children, grew stout, and with the approach of the climacteric period with its peculiar vasomotor disturbances (frequent "bathing" of the whole body in sweat, which increased the patient's usnal tendency to perspiration), she began to excrete a diminished quantity of red urine with an abundant uric-acid deposit and began to suffer from renal colic, a disease to which gouty persons are so liable to be subject, as it is connected with the very nature of gont: then there appeared the abovementioned pains in the bones, gonty periostites.

Is not there also syphilis in the case, as the pains prevent quiet sleeping at times? It is true that the pains interfere with the sleep, but only when the patient while asleep makes 
a movement causing pain; this last causes the patient to start up, but after a rest the pain passes away and the patient again goes fast asleep. But the nocturnal exacerbations of syphilitic pains are of a different nature, they last longer, for many an hour, and are not relieved by quiet posture; on the contrary, such patients at times get up and prefer to walk about during the nocturnal attacks of these pains. I will not stop here to discuss the point, that the bones which are most frequently affected in syphilis, as for instance, the tibix, the bones of the forearm, of the skull, are not affected here; nor are there any, specially characteristic of gout, affections of the small joints, and particularly of the great toes. But whereas the anamnesis and the present condition point with such positiveness to gout, they fail to give any indications of syphilis. Finally, one month's treatment with potassium iodide brought absolutely no improvement, while a two weeks' use of Vichy water has produced an inconsiderable but nevertheless undoubted relief. As to what concerns the fact, that in our patient it is the pelvic bones and the femur which are preëminently affected by the gout, it is quiet possible, that this was aided by a cold while sitting down in the water-closet in the beginning of the cold weather, last October, when the buttocks and the thighs were exposed to chilling blasts.

Prognosis. - Experience proves that patients like the one before us become usually free from the severe symptoms of gout and its recurrences, after an ordinarily repeated use of mineral waters (at the beginning alkaline, later alkaline and earthy) and a strict observance of hygienic regulations.

Treatment. - As a cause of failure of the former treatment we must apparently consider the vagueness in the diagnosis. The physician saw what he considered as "chronic rheumatism," and during a whole month he was administering sodium salycilate till the production of timnitus aurium, but without any success; salycilate of soda is perfectly inert in cases of gouty affections proper, and especially in gouty periostites ; it acts solely as a pain-stilling agent, and then in connection with alkaline mineral waters, only in gouty articular affections complicated by acute or subacute articular rheumatism or by 
the affection of the joints consequeut upon this, as well as by a catarrhal condition of these last. The failure of the treatment and a suspicion of syphilis led to the employment of potassium iodide, althongh it would have been more judicious to administer at first an alkaline mineral water, because, whereas the presence of syphilis was suspected, the presence of gout was beyoud doubt. An indication for the employment of cool sponging could be found either in the nervousness of the patient, who was harassed by pains, or again, in the vague idea about "chronic rheumatism;" but the nervousness could certainly not be removed without setting aside its causes, namely, the continuous pains; as for pains in general, and particularly for those of renal origin, warm baths are more suitable than the cool spongings (whether or not warm baths are suitable in the present case, we shall see later on).

The patient was given Vichy, as experience proves that the strong alkaline waters, as Vichy and Yessentucki, are the best remedial agents in such a typical gont. as found in our patient (later on, in atonic gont, Ems or an earthy water like (contrexéville is more suitable). We administer Vichy in a moderate quantity, although it would be desinable to give it in a larger, because the patient is not only mable, in view of the winter, to go out of doors, but she can not as yet even walk about the room: such being the case, a considerable quantity of an easily absorbable water, which has no tendency to loosen the bowels, would increase the raso-motor disturbances in our patient, the influxes of blood to the head, and the perspiration, which last would diminish the secretion of the urine. As we cammot administer any considerable quantity of the mineral water, we shall be compelled to give it for a longer period of time.

What else shall we prescribe for our patient to hasten her relief and improvement? As was said once, and as proved by experience, we have nothing to expect from the salycilate of soda, of which the patient took so much. The same may be said of antipyrin and other similar medical agencies, the action of which is so similar to that of the salycilate. Besides, these remedies are not only useless in the given case, but even harm- 
ful, as they increase the tendency to sweating, which prevents the excretion of a sufticient quantity of urine, and weakens still more the cardiac activity, weak as it is at present.

To give for the present case the preparations of opium for the relief of the pains would be very erroneous, as they would aggravate the above-mentioned vasomotor disturbances, which so distress the patient (as the influxes of blood to the head). and prevent sufficient urinary secretion (as the perspiration). It were most desirable to diminish the pains in a radical manner by removing their causes. which consist, namely, in those peculiar gouty deposits of sodium urate in the painful parts.

Lithium, used for this purpose, is undoubtedly effective. but not to a particular extent. We will prescribe for our patient piperazine recommended recently, which seems to promote solution and removal of uric acid deposits; there exist investigations which speak much in favor of piperazine. We will prescribe it in solution, (R. piperazini $3 \mathbf{j}$. aque destil. $\tilde{\xi} r j$ ) in tablespoonful doses three times a day. consequently fifteen grains a day. immediately before taking her Vichy water.

Of external means, in the present case with gouty periostites, electricity is useless, while massage is even harmful. as it may aggravate the pains. Wrarm baths are contraindicated by the extreme sensitiveness on moving about: the acts of undressing. getting into the bath. emerging therefrom and dressing anew. would aggravate the pains to a greater extent. than they would be relieved by a warm bath. Moreover, for this case we should have to employ, in view of the great tenderness of the kidney and of the ureter in the left side. very warm baths, which again would increase the tendency to perspiration so harmful for our patient.

\section{FROM THE LECTURE OF MARCH 27, 1892.}

The patient continues to drink Vichy, and from March th to 25th she had been taking piperazine. Her condition has improved considerably, all the pains in the bones as well as in the kidneys have very much diminished: the patient walkerl with 
ease into the auditorium. All her functions are regular, the urine is not red. gives no sediments and is of a weak acid reaction, the sleep is sound, there are no nocturnal pains, as the motions are painless.

As to what concerns the action of piperazine, it would seem, that after it was administered the relief of the pains appeared sooner, in fact much sooner; however, we caunot be sure about this, as the patient drank at the same time Tichy water, the use of which we could not, to make the experiment clear, set aside, on account of the patient's aggravated condition. The stomach bore the piperazine well in the above-naned dose, and the drug has in general failed to cause any umpleasant signs.*

As we were unable, as said before, to give the mineral water in large quantities, we must continue its use for as long a time as possible.

The further history of the disease. - April ith patient left the clinic almost without suffering any pains. Until the time that she left the clinic she drank Vichy in the former quantity; the urine became pale and had at times a neutral reaction. Patient was advised to discontinue drinking the mineral water, but during the summer, in case of a recurrence of the pains, even if they be not severe, to renew driuking Vichy, in the same quantity, but for a shorter period, say three or four weeks, and also, if possible. to take lukewarm baths of $27^{\circ} R$. (92. $7^{\circ} F$.) with salt and soda, of the first some six, of the last some three pounds per bath; experience has shown the utility of such baths for gouty persons; she must certainly observe most carefully her individual hygiene, as explained to her. She was advised to take more exercise, but with due rest, never fatiguing herself, and especially aroid fatiguing the formerly diseased parts.

* Further observations proved that piperazine cannot be relied upon: that its action, if it has such, is insignificant and uncertain, and that it belongs to the class of drugs, which have recently been pushed to the front by speculation and advertised by pseudo-scientific methods. 


\section{FOURTEENTH CASE.}

\section{LECTURE OF OCTOBER 11, 1891.}

The patient, the wife of a tradesman, is thirty-two years old; she entered our clinic October 2d, complaining of pains all over the body, and particularly in the abdomen, in the right side, in the chest, arms and legs ; of dyspncea, palpitation, œedema of the legs, poor sleep and general weakness.

Mode of living and anamesis. - Patient lives in one of the governments around Noscow, in a good locality and in a good house. Never bathed in the river much, and for the last five years has not bathed at all, as it caused attacks of severe dyspncea. Visits once a week the public bath-house; is not subject to sweating. She drinks two to three cups of tea and a glass of coffee; has never used any alcoholic drinks. Her order of meals - breakfast, dinner and supper; patient was always very intemperate in her eating, and is very fond of fatty, starchy and particularly of sweet articles of food. She was pregnant twice, last confinement nine years ago. She has some trifling household occupation; during the cold season of the year patient almost never goes out-of-doors; in summer she is out-of-doors some, but on account of ill-health hardly ever walks at all; which, however, she did not do even while well, because she never liked walking.

Patient comes from a healthy family and is herself of very robust constitution. Has always lived in the above-described manner, always ate much and exercised little; with the cessation of pregnancies she became very stout, and five years ago began to suffer with biliary colic, in the form of characteristic attacks of severe pains in the right side accompanied by vomiting and jaundice. October 1886 she entered our clinic the first time, and we found that besides general obesity and 
biliary calculi there was also a clearly pronounced gout, expressed in renal colic, the urine giving an abundant sediment of sodium urate, and in gouty periostites of the various bones and especially of the ribs (there was neither then, nor is there now any sign of syphilis). The patient observed the diet, drank Vichy, took several warm baths, and in the course of a month left the clinic greatly improved; but notwithstanding the fact, that we pointed out to her clearly what her mode of living would bring her to, she did not in the least alter it, and in January 1887 was again compelled to reënter our clinic, in which she remained for a month, was treated in a similar manner and left the clinic again improved. But being of a weak will-power, she returned to her former mode of living again, and in October 1887 she applied to the clinic for the third time, with all her former troubles, which became worse this time, and were especially accompanied by a severe renal colic. The treatment consisted in giving her calomel several times, warm baths, and later on Karlsbad and Vichy. Patient again improved considerably, and notwithstanding her former irregular mode of living, but little changed for the better, she enjoyed comparatively good health during almost a whole year, and only in October 1887 she was again compelled for the fourth time to apply for treatment; she was given calomel once, and later drank, during a period of six weeks, Karlsbad-Mühlbrumnen, two doses of one-half glass each in the morning, and Vichy-Célestins, two doses of one-half glass each the latter part of the day. She improved much, led a somewhat more regular life and felt comfortable for about a year and a half; then with her former disorderly habits of life there reappeared the former affections, both kinds of colics and particularly the gouty affections of the bones and articulations became much aggravated, and nine days ago patient entered our clinic, three years after her fourth sojourn in it.

Status. - Patient is very stout; the color of the skin, formerly normal, is at present dark, olive-like. The appetite is poor, there is no thirst; patient generally drinks sparingly. Slight dyspeptic phenomena, as pyrosis, belching, nausea, appear only when the diet is neglected, otherwise they are absent. 
She is costive; has daily stools, but insufficient ones; the excretions are normally colored by bile. The abdomen is largefrom an evident deposition of fat as well as from intestinal meteorism. Menstruation appears in time, but is small in quantity, lasting only one day. The urine, although small in quantity 900 c. c. - is pale and turbid; the sediment contains a great many crystals of oxalate of lime, epithelial cells from the renal pelvis, and leucocytes; albumen and sugar absent. The kidneys and the ureters are very sensitive. The liver is enlarged, but of normal consistency and form, and is not tender; the region of the gall-bladder is tender. There is considerable tenderness in the sternum, ribs, scapulæ and vertebræ; dyspnœea. There occur attacks of palpitation with slight signs of angina pectoris, as a sensation of oppression at the side of the heart and simultaneously in the left scapula and left arm. The objective examination of the respiratory tracts elicits nothing abnormal. There is no cough. The dimensions of the heart cannot be defined, on account of the great thickness of the subcutaneous fat; its tones are clear, but very weak; the pulse is regular, but very weak. At present, as at other times, there is no fever; but during a cold all the pains become aggravated, and at such times the patient has some fever, but it is slight and of short duration. There is no headache. Dizziness is of frequent occurrence, especially when she gets up suddenly or stands for some little time, then the face becomes pale.

The sleep is always very poor on account of the general pains. For the last year slight hysterical attacks began to appear: a sensation of oppression in the chest and in the throat, and weeping; there are no convulsions or loss of consciousness. All the bones and the joints of the extremities are more or less sensitive; in the lower ones it is especially the joints of the great toes, in the upper ones it is the shoulder-joints. There is a pronounced hyperæsthesia cutis. Hearing and sight are normal. Extreme muscular weakness. The legs are odematous up to the middle.

Diagnosis - The greater part of the affections which are observed in our patient were already discussed by us in connection with the foregoing cases. The stomach is as yet in 
good condition. The costiveness and the intestinal meteorism depend on the weakened condition of the muscular apparatus of the intestines, so natural with the inactive mode of life and the general muscular weakness of the patient. The urinary tract presents signs of nephrolithiasis and of catarthal pyelitis. There are biliary calculi. The enlargement of the liver, in the presence of insensitiveness and normal consistency, and also in view of the general obesity, must be referred to a fatty condition of this organ; this is also evidenced by the olive color of the skin, which points to some difficulty in the secretion of bile, caused by a narrowing of the biliary passages through fatty deposits. The weak cardiac activity, as seen by the weak pulse, dizziness in connection with a pale face and wedema of the legs, in the presence of a pulse of normal frequency and of normal rhythm, must be ascribed to the weakened activity of the cardiac muscle, very likely due to a fatty condition of this last; this condition, in connection with the developed hysterical phenomena, may also be the cause of the slight attacks of angina pectoris and of palpitation. The dyspncea, in the presence of a normal condition of the respiratory tracts, depends evidently also on cardiac weakness, and is partly due to the high position of the diaphragm on account of the enlarged abdomen. The slight hysteria developed in our patient under the influence of an inactive life in confinement, continuous insomnia and prolonged severe pains. The cutaneous hyperæsthesia may partly be due to the hysteria; however, it is of usual occurrence in severely painful affections of subcutaneons parts ; the hysteria in our patient is slight, whereas the cutaneous hyperesthesia is extremely pronounced and is observed not over one-half of the body, as is frequently the case in hysteria, but all over, and particularly over the deep painful parts, as the gall-bladder, urinary passages and over almost all the bones and cartilages. The disturbances of nutrition are very typical in this case: under the influence of the above depicted mode of life and with the cessation of pregnancies, our patient developed general obesity, later typical gout, arthritis urica, with the uric-acid nephrolithiasis, and later still, under the influence of muscular inactivity and the superadded functional nervous disturbances, thanks to a great 
quantity of sweet food, there appeared oxaluria. As is well known, such a combination of disturbances of nutrition, or with the addition yet of diabetes mellitus, is to be frequently met with.

Prognosis. - The condition of the patient is evidently very serious: in the presence of so many and such great disturbances of the organism, any accidental illness - of no consequence in a healthy person - may take on a fatal significance. As to what concerns the cure, or at least a considerable improvement in health, even this, as we shall see later, is beset with great difficulties, not to mention the patient's weak will, which paralyzes all our efforts in the direction of restoring her health on a durable basis.

Treatment and course of disease. - Diet, watery clysters, in accord with the necessity of the case, and stimulants (a mixture of the rether-valerianate drops with the Hoffman's drops with coffee). At the beginning, in view of the great sensitiveness of the gall-bladder, we gave calomel in eight one-grain powders, one every hour; this brought about considerable improvement, but álso a considerable, although only temporary, weakness, so that we had to give up the further use of calomel. For the same reason, after the first warm bath. which relieved the abdominal pains - those of the kidneys and of the gall-bladder — but which has very much weakened the cardiac activity, we were also compelled to give up warm baths. Massage of the affected joints and general massage, as indicated by the oxaluria, even if very slight, proved to be impossible, on account of the cutaneous hyperæsthesia. We had to limit ourselves to prescribing the Contrexéville water - for the oxaluria — in half-glassful doses three times a day, and to small doses (ten grains twice or three times per day, but not every day) of sodium salycilate as a painstilling agent, and fifteen grains of sodium bromide at bed-time (but not every night) as a somnifacient; both drugs were arlministered in the above-mentioned doses of Contrexéville water: but this relieved the patient but little; while larger doses of the salycilate were contra-indicated by the weak heart. Continuing the use of the mineral water we now took up antipyrine, in five-grain doses twice a day; this acted well and relieved the pains to such an extent, that sleep became possible, without weakening the cardiac activity. 
The patient left the clinic October 27 th, markedly relieved in all her sufferings. For further treatment she was given the following advice: while resorting as far as necessary to watery clysters, to stimulant and pain-stilling drugs (as antipyrine and, later, if she become habituated to it, phenacetine in the same dosage), she must drink alternately Vichy-Célestins, for the uric-acid deposits, and Contrexéville for oxaluria, - in small quantity (one half-glassful twice a day) and intermittently, and also to drink milk; when possible to resort to massage, as soon as the pains and the hyperæsthesia diminish, and to warm baths, when the weakness is diminished and during the warm season of the year.

The treatment by milk was advised as follows: while continuing the drinking of the mentioned mineral waters, and while resorting as far as necessary to the use of clysters, of stimulants and anodynes, during the days of the milk treatment to use, besides milk, only coffee with some sugar and a little tea without sugar, and absolutely nothing more, no other food or drink; to drink milk which is neither fat nor skimmed, one half-glassful at a time, during the whole day, in such a quantity as not to feel hungry, but not more than this; it is ordinarily sufficient to drink five to seven glasses per day; to drink for several days at a stretch, till there appears a feeling of weakness; then to leave off the milk, and to take up for a few days continuously another food, in accord generally with the prescribed diet; (various foods are recommended - meat, fish, eggs, and also starchy articles; excluded - sweet and very fatty food, as well as various desserts, sauces, gravies, etc.) ; as soon as the sensation of weakness will pass away after the use of such food, then return at once to the sole use of milk for a few days, until a satisfactory result is obtained. For indications for this treatment by milk in such cases, I refer to my article on syphilis of the heart. I will add, that in cases of atonic gout similar to our present one, when even the mineral waters prove ineffective, or effective but to a slight extent, good results are frequently obtained by a continuous use of a tea made of herbs and root of fragaria vesca (strawberry tea); for a pinch of this tea use a glass of boiling water, let it steep well and drink this quantity once or twice a day. 


\section{DISEASES OF THE KIDNEYS.}

\section{SIXTEENTH AND SEVENTEENTH CASES.}

\section{LECTURE OF FEBRUARY 19, 1893.}

The patient, a peasant, age thirty-nine, entered the clinic one week ago, complaining of swelling of the legs and face, difficulty in breathing, headache and general weakness.

Mode of living and anamesis. - Patient grew up in a village of the Moscow Gorernment. but has been living in Moscow for a long time. His lodgings are rather cold, the water-closet quite so. Takes a hot bath once a week. Is a hard smoker. hás been an intemperate alcoholist for a long time and is addicted to drinking hot tea. His table is that of a workingman. He is married, has children; no history of syphilis. His occupation is that of a machinist in a printing establishment, his work-day from 7 A. x. to 8 P. x. (with an interval of one hour for dinner). has enough time to sleep, but not much of an out-door life.

Until ten years ago patient had nothing to complain of, but later began to observe, after drinking heavily, swelling of the legs and face, which would pass away within a few days without treatment. Some six years ago, also after a debauch, he spat considerable blood, but this passed without leaving a trace and never reappeared. In the begimning of this winter there appeared the usual cedema of the face and of the legs, but it did not pass away; and recently it increased and to it was superadded a slight enlargement of the abdomen. diarrhoea, dyspncea, headache and general weakness. all of which compelled him to seek medical aid in the clinic.

During a week's sojourn in the clinic, patient began to improve markedly, thanks to the warm room and to the warm 
water-closet, to rest in a comfortable bed and to the use of strong wine (six tablespoonfuls per day), and of the stimulant drops (tra. valer. æeth. and liqu. anod. Hoffm. āā gttas. xxv, four times a day), his food consisting of milk (boiled, in view of a tendency to diarrhoea), milk soup of grits (semolino) and chicken soup.

Status. - The appetite is better than it was on admission, the former diarrhoa ceased. The liver and spleen present nothing abnormal. The region of the kidneys is somewhat tender on pressure. On admission to the clinic his urine amounted to 500 c.c. for the twenty-four hours, containing 0.3 per cent. of albumen by Essbach's albuminometer, and a sediment consisting of a few granular cylinders and red blood-corpuscles, and many lencocytes; the quantity of urine is now 2000 c.c. with 0.2 per cent. of albumen, a few leucocytes and with almost no cylinders and red blood-corpuscles; some accumulation of fluid can be observed in the abdominal cavity. The dyspnoea, although easier, is still considerable; there is a slight cough with some expectoration; objective examination of the respiratory tracts elicits nothing abnormal, outside of some dry râles here and there. The heart is enlarged: the apex beat is felt one finger's width to the left of the left mammillary line, and the sound on the inferior part of the sternum is markedly duller than on the superior; the tones are clear. The pulse is 88 , of regular rhythm, but somewhat weak; still it is already stronger than it was a week ago. The cedema of the legs and of the face diminished somewhat. There is no fever. Sleep is prevented by pains in the head which, althongh quite severe as yet, have abated some. The general weakness is less at present.

Before we turn to the diagnosis and treatment of this case, I will present to you another for a parallel investigation. This patient, a peasant woman, thirty-nine years old, entered our chinic October 31st, 1892, complaining of considerable swelling in the face, legs and abdomen, of extreme dyspnoea, of cough, constant headache, and extreme weakness.

Mocle of living and anamnesis. - The patient is a constant resident of a village in the government of Moscow, situated in a very damp locality. She lodges in an ordinary peasant's hut, with ' 
a water-closet in the barn. Patient takes a weekly steam-bath orer an oven, after which she dashes water over herself standing at the front of the house, even in winter.* Never drank any whiskey or wine - drinks a great deal of tea; food, ordinary peasant's. Patient is a widow, had neither children nor any miscarriages. Judging from her anamnesis as well as her present condition, syphilis may be excluded. She does house-work and field-labor.

Until the last five years our patient always enjoyed good health. Five years ago there appeared for the first time swelling of the legs and face, which passed away in the course of two weeks without any treatment. Since then the eyelids would swell occasionally. Last August the legs and face became swollen; during antumn and winter the swelling continued increasing and the abdomen hegan to enlarge rery markedly; to this were soon added headaches. general weakness, dyspncea and cough, and at times a diarrhea of abundant fluid evacuations, accompanied now and then by slight pain in the abdomen.

Patient has been in the clinic for the last three and a half months. Her surroundings and diet were the same as in the foregoing case, but on account of the diarrhora, milk was given in small quantities - two and a half glasses during the twentyfour hours. The treatment was as follows: the looseness of the bowels was kept under control, but not stopped suddenly, first, because it could assist in decreasing the cedema and especially the abdominal dropsy, which was very considerable, as we shall see later, and secondly, because the dyspnoea, caused by the cedema of the lung's and by uræmia, of which we will speak later, would become intolerable if the patient were constipated. To modify the diarrhcea, which took place occasionally, and not to check it suddenly, fifteen drops of tra. coto, three times a day, proved to be sufficient; opium, which is generally not indicated in cases of chronic, slightly painful diarchoea, is evidently contra-indicated in the present case by the general weakness as well as by that of the heart, but especially by odema of the lungs. To strengthen the cardiac activity we gave the same drops as in the foregoing case, twenty-five drops three

* A custom prevalent among a great part of the Russian peasantry. Note of the translator. 
times per day; we were compelled to discontinue the use of wine, as it tended to aggravate greatly the pains in the head. When the latter became very severe we resorted at times with success to fire-grain doses of phenacetine. As a diuretic we tried at first diuretin (theobrominum natrobenzoicum, $3 \mathrm{j}$ in $\bar{j} \mathrm{vj}$ of aqua destil., in tablespoonful doses), but had to give it up after the fifth dose, as it increased the headache and caused nausea and vomiting. For the same reason we left off caffeinum nitrobenzoicum, which we prescribed after the diuretin in fivegrain doses three times per day. We then gave her calomel, which was administered in one grain doses four times a day and was well borne, without increasing the diarrhae; but it did not in the least increase the quantity of the urine nor relieve the uræmic phenomena (the headaches), and it also was therefore given up. We have had thus to limit ourselves, for purposes of acting upon the kidneys, to the employment of baths since the end of November. The patient, who has acquired of late a susceptibility to cold. felt chilly in a bath at the temperature of $30^{\circ} \mathrm{R} .\left(99.5^{\circ} \mathrm{F}\right.$ ) and $31^{\circ} \mathrm{R} .\left(101.7^{\circ} \mathrm{F}.\right)$; but at $32^{\circ} \mathrm{R} .\left(104^{\circ} \mathrm{F}\right.$.) and later at $33^{\circ} R \cdot\left(106.2^{\circ} \mathrm{F}\right.$.) the bath induced an agreeably warm sensation. The patient had such baths, with slight interruptions, from the time she entered the clinic until December 10th; they brought about a slight improvement, the quantity of urine being increased temporarily and all the morbid phenomena having diminished; but this improvement was rather unstable and passed off rapidly. so that by December 10 th the average quantity of the urine was about the same as when she was admitted, about 1200 c.c. in twenty-four hours, and the condition of the patient improved but little. From that date on, we began the employment of dry hot-air baths, from $45^{\circ} R .\left(133.5^{\circ} \mathrm{F}\right.$.) to $60^{\circ} R$. $\left(167^{\circ} \mathrm{F}\right.$.), in an apparatus conveniently applied, as you see, to the bed; these baths called out profuse perspirations, and the condition of the patient began to improve steadily since; by January 10 th the amount of urine was increased to 2000 c.c. in twenty-four hours, by February 10 th to 3000 , which amount remains at present; the oedema and the ascites have also diminished greatly, and the patient has since enjoyed the good condition in which you find her now. 
Status. - The appetite, which was poor on the patient's admission to the clinic, is good now. The dyspeptic phenomena, as heaviness in the stomach and belching, also nausea and vomiting accompanied by severe headache evidently of uræmic origin, observed on her admission, have passed away; so has, since the middle of December, the diarrhoea. Ascites is not apparent at present: the circumference of the abdomen on a level with the umbilicus was $172 \mathrm{~cm}$. before, but at present is $89 \mathrm{~cm}$. The liver and the spleen, which did not admit of palpation on patient's admission, present on examination nothing abnormal now. No menstruation since August; it was formerly regular and painless; she never had any leucorrhea; the gynæcological examination shows that the climacteric period is approaching. The quantity of urine on admission was 1,000 c.c. in the twenty-four hours, red, turbid, with an abundant sediment, consisting of a great quantity of renal epithelium, granular hyaline cylinders. red blood-corpuscles and leucocytes; there was 0.6 per cent. of albumen; the amount of urine is at present 3,000 c.c., it is pale, slightly turbid, with a trifling sediment, in which the quantity of the above-named elements is much smaller, while the red blood-corpuseles, the renal epithelium and the epithelial cylinders are absent altogether; there is only 0.2 per cent. of albumen. When admitted, patient was suffering from a severe dyspnoea, which would not allow of sleeping in a recumbent posture, and a severe cough, that would raise with considerable difficulty a small quantity of expectorated matter, accompanied by mucous râles in the inferior portions of the lungs; at present the râles, as well as the cough and dyspncea, have almost disappeared, and the patient is able to sleep in a recumbent posture. The apex impulse, which could not be palpated on her admission, is clearly felt now along the left mammillary line; at the same time the pulse, which was formerly very weak, although not quite frequent (80) and rhythmical, became stronger and less frequent ( 72 to 76 ), the sound over the sternum is everywhere clear, the tones of the heart clear. The formerly enormous cedema has almost disappeared; there remains yet some on the legs. Fever neither before nor now. Patient, formerly very pale, is not so pale now. The sleep, 
which was formerly prevented by headaches and the patient's inability to sleep in a recumbent position, is now good; the headaches are less frequent and milder, the general weakness is much less.

These gathered data will certainly make it clear to you, that it is the kidneys which are principally affected in both cases, and that the morbid processes are of a chronic nature. Entering to-day on the discussion of diseases of the kidneys, namely, chronic ones (as there do not happen to be in the clinic just at present any pure cases of acnte nephritis), I selected from among the clinical patients the two whom I presented before you, because I considered their clinical analysis, conducted along parallel lines, as particularly useful in acquainting you with the chronic nephrites ; but before going any further, before considering the diagnosis and the treatment of both cases, I think it necessary to take a brief survey of the varieties of chronic nephritis recognized by modern pathology.

Pathological anatomy has proved that a chronic nephritis is always the same in the sense, that there always takes place a contemporaneous affection of the glandular element, - the urinary tubules, - and of the interstitial substance, but that in a great number of the cases one or the other of the affections is more predominating. The clinical data prove, that the variety of the disease and its course in cases in which the affection of the glandular tissue predominates, are clearly distinct from the variety and course of the disease with a predominance of the affection of the interstitial tissue, and the clinic therefore distinguishes two varieties of chronic nephritis: the parenchymatous and the interstitial. In the interstitial variety there is a great quantity of pale and limpid urine with a trifling sediment, consisting usually, although not constantly, of hyaline cylinders, the albuminuria is inconsiderable and inconstant; the various cedemata are either absent, or there may be some slight cedema of the legs, while the left ventricle of the heart is hypertrophied and usually dilated. In parenchymatous nephritis the urine is scanty, very turbid, and gives a considerable sediment, containing leucocytes, frequently also red blood-corpuscles, renal epithelium and epithelial, granular and hyaline cylinders; the albu- 
minuria is considerable; with this there occur severe anasarca and accumulations in the cavities, and a frequently recurring. obstinate diarrhøa, while the left ventricle of the heart, although enlarged (dilated), is not at all, or very little, hypertrophied, probably because the nutrition of such patients fails rapidly, whereas the nutrition of patients suffering with interstitial nephritis may remain for a long time in a good condition.

In interstitial nephritis death is brought about either by apoplexy in the brain, caused by hypertrophy of the dilated left ventricle in conjunction with a chronic arteritis, or even an atheromatous condition of the arteries ; or by uramia, or through disturbances of compensation (when the heart is further affected) or through inflammation of the mucous membranes, to which last these patients are so predisposed. Parenchymatous nephritis causes cleath through ascites and uræmia, as well as through the above-named inflammations, to which such patients are even more predisposed. Botl varieties of the fully developed nepliritis are incurable; but patients with interstitial nephritis, enjoying moderately good health, may live for a long time, much over ten years, while those with the parenchymatous variety will only live a few years and that in a condition of great debility and severe suffering. Prognosis is therefore worse for cases of parenchymatous, than for those of interstitial nephritis. In the course of both varieties of chronic nephritis there take place changes which point to their close relationship, to the fact, that they belong to one process.

In interstitial nephritis the urine will frequently diminish in quantity all of a sudden, become turbid, throwing down an abundant sediment, containing the same formative elements, as it does in parenchymatous nephritis, and show a great quantity of albumen, and at the same time there will appear considerable œdema; but all these phenomena will pass away rapidly in a few days under correct regimen and treatment (usually cardiac stimulants and warm baths), and the physician - certainly, the one who is very unexperienced or rather careless - who first saw the patient in the above-depicted condition of exacerbation, will be compelled later on to change his diagnosis (and consequenily also the prognosis), and instead of what seemed to 
him a parenchymatous nephritis, will recognize " an exacerbation" of the interstitial variety. It is true, that this "exacerbation" is in reality but a rapidly passing parenchymatous inflammation, complicating the original variety; but this original variety, with all the peculiarities in its course and termination, and consequently in the prognosis, is in such cases after all an interstitial nephritis. Such "exacerbations" of interstitial nephritis may pass away even if untreated, as is seen, for instance, from the history of our first patient.

Parenchymatous nephritis terminates in the majority of cases fatally; but at times, rather rarely however, a more favorable termination takes place, which is known as the "transition into interstitial nephritis": thus the amount of urine increases, its turbidity, the sediment of abundant formative elements and the considerable albuminuria, all these being the chief symptoms of a severe affection of the renal parenchyma, decrease and become insignificant. Alongside with these the various odemata and exudations into the cavities disappear, the diarrhœa ceases, while the nutrition improves, the patient gathers strength, and there is developed a hypertrophy of the left ventricle of the heart; thus the picture of a typical parenchymatous nephritis changes to that of a typical interstitial variety with its more benign course. It becomes evident, that this "transition of a parenchymatous nephritis into an interstitial" is but the cessation or extreme diminution of the more dangerous parenchymatous process, and a continuation, in the form of a further extension, of the interstitial variety, which always accompanies the parenchymatous form.

Diagnosis. - From the above it becomes apparent, that our first patient suffers from a chronic interstitial nephritis (as evidenced by the fact, that the disease had existed for ten years, that the cedemata are of a slight and rapidly passing character, and that the heart is enlarged) with an occasional "exacerbation," i. e. complicated by a rapidly passing inflammation of the renal parenchyma. That this last is of a rapidly passing nature is seen by the rapid improvement in the patient's condition: during the course of one week, with the aid of a correct regimen and the employment only of cardiac stimulants, 
even without warm baths, the quantity of urine was raised from 500 c.c. in the twenty-four hours to 2000 , and the formative elements in the urinary sediment began to disappear. For the sake of completness in the diagnosis we must say a few words on the condition of the heart; its dilatation is indicated by its enlargement: the dilatation of the left ventricle, as apparent from the long duration of the disease, is of a permanent nature, while the dilatation of the right one (as can be judged by the dull sound on the inferior portion of the sternum) is probably of a temporary character, dependent on the weakened activity of the heart, and is likely to diminish with the improvement of the latter. The rapid increase in the quantity of the urine points to a hypertrophy of the left ventricle. The dyspnœa, headache and insomia, which appeared together with the developed celema and the diminished quantity of the urine, and which decrease with the increase in the amount of this last, are evidently of an uræmic character.

Our second patient, from what we are led to judge by the abovementioned two varieties of chronic nephritis, presents not a rapidly passing inflammation of the renal parenchyma that occurs incidentally during the course of a chronic interstitial nephritis, but a furmly established parenchymatous nephritis; this is indicated not only by the characteristic picture of the disease, as by obstinate dropsical phenomena of anasarca, ascites and œdema pulmonum, by persistent diarrhœea, characteristic urine, and the condition of the heart, but also by the unyielding nature of the disease to treatment: warm, and even hot water-baths at the temperature of $32^{\circ}\left(104^{\circ} F\right.$.) to $33^{\circ} R$. $\left(106.2^{\circ} F\right.$.) employed during a whole month, failed to bring about the least improvement in the patient's condition, and only energetic application of air-baths at a high temperature induced a decided turn for the better.

Prognosis. - As seen from the colirse of the disease and its treatment, we may hope in the first case for a speedy disappearence of the "exacerbation" and for the return of the patient to his ordinary tolerable condition of health, while in the second case we may expect the "transition" of the disease into a tolerable condition of interstitial nephritis, i. e. the cessation of the more dangerous parenchymatous process. 
From the analysis of these two patients and from their further observation you will become acquainted with typical cases of both varieties of chronic nephritis and with the most important changes in their courses and also with the most efficient means for their treatment.

Treatment. - We gave as yet no hot baths to our first patient on account of his weakness, while internally he was given stimulant drops in view of the diarhoea. At present the patient is somewhat stronger and we will therefore order for him some baths and internally, outside the abore-mentioned stimulant drops (for the diarrhcea stopped), also some caffeine, as a diuretic which acts through the heart. by strengthening the latter's activity; as a diuretic agent on the kidneys, we have only milk: the "strong" diuretics are contraindicated by the condition of the kidneys, while the less irritating ones — as the alkaline mineral waters, - cannot be used on account of the only recently ceased diarrhua. The indication for prescribing caffeine - in the condition of cardiac activity as observed in the present case-for its diuretic effect through the heart, and no other " cardiac" drug, is certainly sufficiently clear to you now, but it will become clearer still, when, in analyzing clinically diseases of the heart. we will discuss the comparative effect of different cardiac drug's. In the second case, after what was shown by its observation, we must continue the air-baths at a high temperature and also the stimulating drops. For interual use, in view of the patient's pallor, there is an indication for iron; arsenic is contra-indicated, ontside of other considerations, by the former obstinate diarrhcea.

The male patient left the clinic March 11th. From February 19 th to March 6th he was given seven baths at the temperature of $31^{\circ} \mathrm{R}$. (about $101.7^{\circ} \mathrm{F}$.) : internally he was given from February 19 th to the 27 th caffeine natrobenzoate (in five-grain doses, at first twice a day, and later three, four and five times), and from February 2ith to Mareh bth he had theobromine natrobenzoate, in drachm quantities daily ( $j \mathrm{j}$ in $\bar{j} \mathrm{ij}$ of water, in tablespoonful doses). There was no appreciable difference in the action of either of the drugs, but, as is well known. and as you 
may also judge by the present case, we have to give greater quantities of theobromine than of caffeine; and the first costs more than the second. Already by March 6th the principal morbid phenomena, as the cedemata, the dyspnœea, headache, insomnia and weakness, disappeared, and the amount of urine increased; the patient left the clinic strengthened, suffering with a trifling albuminuria and an enlarged, but functionally efficient left ventricle of the heart; the right ventricle has evidently returned to its normal dimensions, as the somd on the inferior part of the sternum became clear. Our advice to the patient when leaving: not to abuse brandy and hot tea, not to go during the cold season of the year into a cold water-closet, nor to take a steam-bath in winter, and to wear woolen undergarments.

The female patient left the clinic April 17th. The very small quantity of iron (tinctura tonico-nervina Bestujeff, * five drops three times a day) which was prescribed for her February $23 d$ caused a diarrhnea on the very next day and it was therefore discontinued. Under the use of tra. coto the diarrhoa soon ceased. The treatment then consisted of the use of air-baths at a high temperature, together with the use of stimulating drops. By the beginning of April the baths became unnecessary: the anasarca, ascites and the cedema of the lungs passed away, there was no recurrence of the diarrhoa, the headache and insomnia disappeared, the patient left the clinic with an albuminuria ( 0.2 per cent), but very much strengthened and with a functionally active heart. Our advice on taking her leave: to take no steam-baths over the oven, nor dash any water over the body during a frost, and to wear woolen undergarments (with this last, being indigent, she was supplied from the clinic).

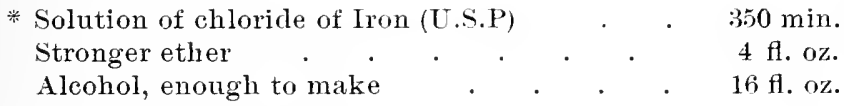

Each fluidrachm contains about $1 / 2 \mathrm{gr}$. of metallic Iron.

(U.S. D.) 


\section{ANAEMIAE.}

\section{SEVENTEENTH CASE.}

\section{LECTURE OF MARCH 16, 1893.}

The patient, a merchant's wife, age twenty, not desiring to enter the clinic, is being treated at home and comes here only for advice. She first applied here one week ago, complaining of difficulty in breathing, palpitation of the heart and dizziness on moving around, a constant noise in the head, general weakness and a slight cedema of the face and lower extremities.

Mode of living and anamnesis. - Patient moved two weeks ago to Moscow, into a good residence, and before this she lived in a healthy country locality, also in a good house. Is not used to sea-bathing, but takes a bath (in the public bath-house) weekly. Does not smoke, drinks neither wine nor coffee, and but a little tea. Has good meals (fish on fast-days). Her occupation is light, of a household nature, but fatiguing on account of her weakness, this last also keeping her most of the time in-doors. Was married two months ago.

The patient enjoyed good health until last summer, but on account of the cholera epidemic she spent that summer in a condition of great mental anxiety and suffered also a severe shock from the loss, by cholera, of persons dear to her. Since autumn the patient began to grow pale and weak; and during December the above-enumerated morbid phenomena made their appearance and since her marriage have become particularly aggravated.

Status. - The patient, as you see, is of average constitution and very pale, while a week ago the pallor was striking and had a greenish tint. The pallor, as well as the redema of the legs and face, have decreased considerably for the last week, because 
the patient began taking iron, which we prescribed for her in the clinic. Up to the time that she began taking iron her appetite was poor and she suffered from nausea after meals; the appetite is now better and the nausea disappeared. She is costive, but watery clysters empty the bowels sufficiently. The urine is pale, of a specific gravity of 1007 , but contains neither albumen nor sugar, nor peptones. The liver, the spleen and the abdomen in general present nothing abnormal. Menstruation appeared first when she was seventeen years old and had been quite regular formerly, but since summer it began to appear once in three months and very scantily, last time in December; it was always painless. Gynxeological examination shows the uterus rather under-developed, considering patient's age.

Both the dyspnoa and palpitation are now less than they were a week ago. The respiratory organs are in a normal condition. The dimensions of the heart are normal. All over the region of the heart there is heard a systolic murmur, while over the veins of the neck the murmur is constant. The number of the red blood-corpuscles is 50 per cent. of the normal, of the hæmoglobin but 12 per cent. of the normal quantity; there is neither poikilocytosis nor leucocytosis. The pulse is 80 , as yet weak, but already stronger than formerly. Patient is not feverish. The sleep is fair. She complains less of noise in the head, of dizziness and general debility.

Diagnosis. - We evidently have here before us a typical case of chlorosis in its extreme development. No part of the organism, outside of the blood and the sexual apparatus, presents any signs of a primary affection: the observed morbid phenomena, which in their entirety form such a complete picture of chlorosis, depend apparently on a clearly pronounced alteration in the blood; the systolic murmur in the region of the heart in the presence of normal dimensions of this last depends certainly on the same cause. Pernicious anæmia may be excluded by the absence of any signs of increased disintegration of the red blood-corpuscles, namely the absence of poikilocytosis, peptonuria and urine of a high specific gravity; against leukæmia speaks the absence of leucocytosis and of symptoms of any affection of the spleen and of the lymphatic glands; this last 
also excludes pseudo-leukæmia ; that the disease is not an anæmia resulting from failure in nutrition and hæmotopoiesis as due to unfavorable conditions of life, is evidenced by the fact, that the disease developed under the identical material conditions, under which her health was formerly satisfactory, while a depressed mental state - as in the present case - is shown by experience to favor the development of just the condition of chlorosis.

What is the nature of chlorosis? The investigation of this question lies outside of the domain of clinical exercises proper, as conducted for beginners, but I consider it important to take it up now, so that we may define the disease more fully and consequently be able to distinguish it with greater precision from other cases of anæmia that we may run across later on.

In the text-books of internal pathology chlorosis is described in the part treating of diseases of the blood; but this part forms one of the weakest features of every text-book. Let us take, for example, the well-known German text-book of Eichhorst, translated into the most important European languages: "Chapter TIII. Diseases of the blood and of the blood-forming organs. Part first. Diseases of the blood." And now, without any explanatory introduction, there follow one after the other descriptions of leukæmia, pseudo-leukæmia, pernicious anæmia, chlorosis, melaniemia, purpura simplex, purpura rheumatica, purpura hæmorrhagica, scorbutus, hæmoglobinuria and hæmophilia. What a variegated collection! How can we consider as belonging to one class such affections as purpura simplex - a but insignificant symptom to be met with in a great many different diseases, and Werlhoff's disease, which, when fully developed, presents all the symptoms of an independent affection, of a disease suigeneris, in all probability of an infectious nature. On the other hand, if we place among diseases of the blood purpura hæmorrhagica, in which the primary affection of the blood is perhaps probable, but has not been proved as yet, then why not describe in the same chapter also malaria, in which disease the affection of the blood is so apparent, and very probably of a primary origin? If the ohapter of the diseases of the blood 
treats of leukæmia and melanæmia, in which the alterations of the blood are but consequent on affections of the other parts of of the organism and on other diseases, then why not place there also uræmia, cholæmia, etc.? To treat thus of diseases of the blood tends to produce a vague idea about them in the beginner's mind and puts them within narrow limits. A short introduction should at least delineate fully the limits of this department of diseases, should distinguish the primary affections of the blood from the changes consequent thereon, and should keep it prominently before the beginner's mind, that it was only for the greater convenience of exposition, that either has been described in other parts of the text-book.

It is evident, that placing chlorosis in the chapter that treats of diseases of the blood, will assist but little in explaining the nature of this disease, especially if we consider the modern account of the same.

The changes in the blood in chlorosis and their consequences are evident, and to judge by the present case, may be very great: but what produces them? We do not observe in our patient any signs of an insufficient development of the general circulatory system, as the presence of a narrow calibre of the vessels, etc., to which Virchow called attention in cases of chlorosis. though they occur very rarely. The influence of unfarorable material conditions and of disturbances of digestion on the nutrition and hrematopoiesis have to be excluded by the very fact of their absence in the present case. One thing is certain the presence of a disease-causing influence on the nervous system, namely, the depressing mental condition. Influences of such a nature play an important part in the ætiology of chlorosis. Some physicians, as for instance, the late Prof. Botkin, in his clinical lectures on chlorosis, are even inclined to classify chlorosis with nervous affections. While I recognize fully, from my own experience, the truth of his observations as well as of those of others, - observations of which the present case niay serve as an example, - I still think that the morbid influence on the nervous system serves but as the exciting cause, which calls to the surface the hidden, but nevertheless already existing disease. 
I base my opinion, first, on the fact, that in many cases chlorosis is developed without any such influences on the nervous system; secondly, because such influences on the nervous system cause chlorosis only at the age when sexual maturity is in the process of being formed, while at any other age no such effect takes place; and thirdly, because even at this age they may call out the existence of the disease in some, and fail to do so in others. It is reasonable to assume that the immediate cause of the disease lies in the process of development of sexual maturity, and in the condition and functions of the sexual organs at this period of life. The signs of incomplete development of the sexual sphere and the anomalies of menstruation, usually observed in chlorosis, as well as the remarkable facts which have become known lately, all tend to strengthen this assumption still further.

The well-known physiologist Brown-Séquard promulgated the idea that the glandular organs of our body secrete not only externally, but also internally, thus exerting an influence on the life of the organism. As a particular confirmation of his idea, he pointed to the inter-relation between the activity of the male generative organs and the general condition of the organism, and on this fact he based the application, with a therapeutic aim, of his well-known injections. These injections were at first compromised by being thoughtlessly applied, and partly also through invidious exploitation, but at present they are considered as worthy of experimentation by serious investigators. Similar observations were at the same time made known, which tended to prove the hitherto unsuspected connection between the condition of certain parts of the body and that of the body as a whole: I refer to the investigations, which prove a relation of the thyroid gland to myxœedema, of the suprarenal bodies to Addison's disease, probably of the pituitary bodies to acromegaly, of the pancreas to diabetes. One cannot help thinking that there may be a similar relation between some special condition of the sexual apparatus during the period of the formation of sexual maturity and chlorosis; this condition being such, that only in its presence may influences on the nervous system cause the development of chlorosis, while the other circumstances, as 
failure in nutrition and blood-formation from various reasons, may favor this development.*

I must say here a few words on the relation of chlorosis to hysteria, in the rtiology of which morbid conditions of the genital sphere also play such an important part. The combination of chlorosis and hysteria is seen, according to my experience, not only infrequently, but rather rarely. The influence of marriage in chlorosis and hysteria is as follows: other condiditions being equal, for example, with a similar and withal good constitution, amidst similar and withal favorable conditions of life, hysteria, even if strongly developed, often passes away completely after marriage, or is at least considerably diminished; while chlorosis, if slight, becomits neither better nor worse after marriage and requires treatment; but if considerable, it always becomes worse, so that for chlorotic girls late marriage is more advisable, for the phenomena of chlorosis will then cease to recur and will finally disappear, and sexual maturity as well as that of the whole organism, will be fully established.

The prognosis in the present case is favorable, considering the patient's fair constitution, her good conditions of life, and also

* A later addition [from the article of the well-known (by his works in the domain of pathology of metabolism) Prof. Noorden : "Altes und Neues uber Pathologie und Therapie der Chlorose." - Berliner Flinische Wochenschrift, Nos. 9 and 10, 1895]. What causes the diminution of hæmoglobin in chlorosis, - the increased disintegration of the blood, or the weakened power of hæmatopoiesis? The bilirubin of the bile is formed from the hæmatin. From the bilirubin there is formed hydrobilirubin of the feces and urobilin, identical with hydrobilirubin; this urobilin is absorbed by the intestines and passes into the urine, of which it forms the principal coloring matter. Therefore, the general quantity of urobilin in the urine and of hydrobilirubin in the feces may serve as an index of the disintegration of the hæmoglobin. Not only the pale urine and the usually pale color of the feces in chlorosis, but also direct measurements of the quantity of urobilin in the urine and of hydrobilirubin in the feces, which measurements show their diminution in this disease, - go to prove that the disintegration of the hæmoglobin is not increased in chlorosis, but diminished, and that consequently the decrease of this body in the blood depends on the weakened power of hrmatopoiesis. On the contrary, in other anæmiæ, - as in the pernicious, leukæmic, febrile, malarial, - where the existence of an increased disintegration of the blood is too certain, there are constantly found greater quantities of urobilin in the urine and of hydrobilirubin in the feces. 
in view of the fact that she has hardly been subjected to any treatment. The prognosis would certainly be less favorable, if her constitution and mode of living were poor and if we had found in her such an extreme development of the disease after a considerable and frequent use of iron and arsenic; and such a thing does occur. Whereas now, especially in view of the fact that iron is well borne and is evidently effective, we may well hope to bring the patient soon out of her chlorotic condition. It is true, that this last is liable to recur and seldom yields to treatment instituted the first time; but with the aid of a good constitution and favorable conditions of life repeated treatment is usually successful in preventing a recurrence and in establishing the health on a firm basis. Otherwise the condition of health is liable to undergo different changes, while the organism may acquire a predisposition to various diseases, especially to pulmonary tuberculosis.

Treatment. - First of all, be sure to create as favorable hygienic conditions as possible; the principal therapeutic means are iron and arsenic.

Before prescribing these drugs you must carefully investigate the condition of the stomach: it is just as erroneous to consider the dyspeptic phenomena due to chlorosis as caused by a gastric catarrh and to hesitate in prescribing iron and arsenic, as it would be, on the contrary, to prescribe at once these remedies without previously removing such a catarrh, in case this last condition were present. The dyspeptic symptoms, as seen in our patient with extreme chlorosis, were trifling, (vomiting after meals) and appeared only during the course of the chlorosis; we therefore prescribed iron at once, and, to judge by the course of the disease, rightly so. Eight pills a day (the wellknown Blaud's pills: iron sulphate, potassium carbonate āā, tragac. q. s.), four after dinner and four after supper, each of them containing one and a half grain of iron sulphate, diminished the phenomena of chlorosis, improved the appetite, while the nausea after the meals disappeared. If, on the contrary, with a moderate chlorosis there were present considerabie dyspeptic symptoms which had existed prior to the appearance of the chlorosis, then it would have become necessary at first to 
remove them (most frequently by the use of alkaline mineral water and of bitter remedies).

When shall we administer iron and when arsenic in cases of chlorosis? In proportion as the phenomena of chlorosis are more prominent and the condition of nutrition favorable, the sooner must we give iron, and that in large, frequently even in very large doses, beginning with moderate ones and increasing them gradually; the best form is that of pil. Blaud. On the contrary, if the character of the chlorosis is less prominent, and if the nutrition has suffered greatly, and the more evidence there is of the case being one of simple anæmia, as a result of failure of general nutrition and that of hæmatopoiesis in particular, - then it is better to administer in the beginning arsenic, especially if the appetite be poor, thus: R. acidi arsenicosi gr. j, extr. trifolii q. s. ut f. l. a. pillulæ thirty or forty - one twice a day, after a meal; and later arsenic combined with a small quantity of iron, as: R. acidi arsenicosi gr. j, ferri hydrogenio reducti grana $x x x$ extr. trifolii q. s. ut f. l. a. pil. $x x x$, in the same dosage. Still, there occur also cases of pure chlorosis with fair nutrition, but of a very obstinate frequently recurring nature, when the patients become habituated to the use of iron, this latter ceasing to exert any effect (so that it is unable to prevent a recurrence of chlorosis); in such cases the administration of arsenic - as mentioned above — instead of the iron, usually prevents the recurrence of the disease, after which iron, in case of another recurrence, will again act favorably.

There is no need of interrupting the administration of arsenic itself, or in combination with some iron, during the menstrual periods; but we must certainly interrupt the administration of large quantities of iron during these periods. We frequently have to stop the iron even a few days before the appearance of menstruation, otherwise this latter will be very profuse, thus weakening the patient. The attempt to continue the administration of iron up to the very time of the appearance of the menstrual flow (so as to avoid the necessity for delaying the treatment), and then, by using egortine, tra. hydrastis and such like, to diminish this flow, usually miscarries; and it is better not to hurry on with the treatment and interrupt it a few days 
before the menstruation appears. The condition of the digestive apparatus may also require intermissions during the treatment by iron and arsenic. But in a general way the treatment is continued until a complete and certain removal of chlorosis is effected.

I consider it also necessary to remind you of the fact, which is unfortunately liable to be easily forgotten, that while taking arsenic every sour article of food is to be forbidden, as meals with vinegar, lemon, and all somr or soured fruits and berries, sour sances, as well as horse-radish and mustard, which are usually prepared with vinegar; otherwise a diarrhoa may start up, usually of a painful and lasting nature, delaying the success of the treatment.

The iron mineral waters play but a subordinate part in the treatment of chlorosis, principally becanse they do not stand transportation as well, for instance, as the alkaline waters, and can be successfully used only on the spot; but the treatment of chlorosis and of anæmiae in general by the aid of ferrous mineral waters is not more successful than the treatment by medicinal preparations of iron, but is in fact less so.

The treatment of chlorosis and of anæmiæe in general by import$e d$ arsenical and arseno-ferrous mineral waters, so widely advertised of late (Bourbonle, Levico, Roncegno) does not, from my observations, present any advantage over the treatment by the above-named correctly prepared medicinal preparations of arsenic or of arsenic with iron.

The constipation is easily overcome in our patient by watery clysters. These are efficient in the majority of cases; in exceptional cases we have to administer, and that rarely, light laxatives. There seems to be no reason for any other symptomatic treatment in the present case; in some cases we do resort to it; but the more the physician refrains from such treatment, and particularly from the use of the various nervines, as, for instance, the bromides, and the more he insists on the radical treatment, the more successful will he be in establishing a condition of sound health. I cannot help telling you that a predominating palliative therapy in practice is a sure sign of a poor physician. 
The condition of the nervous system in chlorosis requires certainly the greatest attention on the part of the physician, as is apparent from what we said concerning the rtiology of the disease: it is necessary to strengthen this system as much as possible; but to effect this purpose we must not direct our efforts against individual nervous symptoms, but we must act on the whole system. It is true that both iron, by improving the condition of the blood, and arsenic directly, improve the state of the nervous system as a whole; but the application of hydrotherapeutic and of balneotherapeutic means in general is also very beneficial in this connection. If the patients are not predisposed to chills, then bathing in the river, or, better still, in the sea, is the simplest means. Unfortunately, in cases of considerable chlorosis, patients are usually chilly and become weak after bathing; for such cases we must order baths at the temperature of $27^{\circ} R .\left(92.8^{\circ} \mathrm{F}\right.$.) and lower, so that the patients while in the bath, would feel refreshed, but not chilled, and after the bath would become quiclily warmed and feel vigorous. Such baths are best prepared with table-salt and soda. Warm baths $\left(29^{\circ} R\right.$. and over; $97.2^{\circ} F$.) tend to weaken such patients.

As our patient is not habituated to bathing in the river it will be more suitable to advise her to take the above-named baths for the coming summer season.

The patient was treated under the supervision of one of our clinical assistants: the result of the treatment was entirely successful. 


\section{EIGHTEENTH CASE.}

\section{LECTURE OF MARCH 5, 1893.}

The patient, a young girl nineteen years old, entered our clinic March 2d, complaining of dyspnœa, palpitation and dizziness on moving around, and of general weakness.

Mode of living and anamesis. - Up to within two years ago the patient lived in a small country town in a healthy locality and in farorable circumstances; but since then she has been living constantly, summer and winter, in Noscow. Her residence is fair. She bathes outside, but during the last year bathing tended to weaken her and cause dizziness. She neither smokes, nor drinks coffee or wine; drinks some four glasses of tea daily, sweetened and with lemon. Has three meals a day, but they frequently consist of lunches only, as bologna, cheese, etc. She is a student of midwifery and attends her lectures from nine o'clock in the morning to three o'clock in the afternoon; her evenings are spent in sewing and reading; she has sufficient time for sleep; goes out for about an hour and a half in the fresh air, but during her service as interne in the lying-in hospital (six days every month) she is entirely confined to the house. During the last year she is becoming very much fatigued from her studies.

In her childhood patient passed through attacks of measles, scarlatina and inflammation of the lymphatic glands on the right cheek, which suppurated and left a scar. Since then and up to the time she moved to Moscow, she enjoyed good health, with the exception of an attack of malaria, which was of short duration and yielded to quinine. During the first winter that the patient spent at Moscow, she began to feel weakness, dyspnoea and palpitation; the appetite became impaired, there appeared dyspepsia and an inclination to costiveness, 
and menstruation became very irreguiar ; later on, in May of last year, during laborious preparations for the examinations, there appeared considerable hremoptysis. During the summer the patient remained at Moscow, and the condition of weakness, the dyspncea, palpitation and dizziness kept increasing. In October patient, having canght cold, began to cough, and at this time she also had hæmoptysis for two days, felt some pain in the left side of the chest, and was feverish. In the course of a month all these phenomena disappeared but the weakness, dyspnœa, palpitation and dizziness became aggravated. In January patient was taking, during two weeks, tra. nervina Best. without any appreciable results.

Status. - Patient is of a weakly constitution, of tall stature, but with an insufficiently developed somewhat flat chest, and pale. The appetite is poor. Eating is accompanied by belching, pyrosis, nausea and by a sensation of heaviness in the stomach. She is usually costive, but there occur at times loose stools; thus, during the last twenty-four hours she had three loose stools, accompanied by pains in the abdomen. The urine contains neither albumen nor sugar, its specific gravity is 1021. The liver and spleen present nothing abnormal. The menstruation, which, previous to her coming to Moscow, was regular and painless, has become very irregular since, appears only once every two or three months, and is accompanied by abdominal pains; she menstruated last time six weeks ago. The heart is not enlarged, its tones are clear, there are no murmurs, pulse is very variable, between 60 and 84 , and weak. Movement causes palpitation and dyspnoea. 'The number' of the red-blood corpuscles is 50 per cent., that of the hremoglobin 35 per cent. of the normal. Lencocytosis and poikilocytosis absent. There is a slight enlargement of the thyroid gland. Patient does not cough, but has at times pains in the left side of the chest, especially after she has been sitting for a long time in a stooping position. Careful olojective investigation of the lungs elicits nothing abnormal. Patient grew thin; she is slightly feverish - 36.4. ${ }^{\circ} R$. $\left(97.5^{\circ} \mathrm{F}\right.$.) in the morning, $37.4 R$. $\left(99.5^{\circ} \mathrm{F}\right.$.) in the evening. Sleeps poorly; is dizzy; has at times attacks of migraine with vomiting. After sitting for a long 
time, experiences heaviness in the head and pain in the loins. There is considerable general debility.

Diagnosis. - The simultaneous appearance, about a year and a half ago, of general weakness, dyspnœa, palpitation, dizziness, of a sensation of noise in the head, and of the irregularity of menstruation, and the occurrence of changes in the blood, and the enlargement of the thyroid gland, all point to chlorosis; but we have here evidently something more to deal with than chlorosis only. The hæmoptysis during last May and especially the above-described disease of last autumn point to tuberculosis of the lungs. The absence of pronounced objective signs in the chest, as well as of cough and expectoration, do not certainly speak against it. Not to speak of the literature of the subject, even a limited personal experience is sufficient to prove the fact, that such rapidly passing, incidental illnesses, as that from which our patient suffered last autumn, are of frequent occurrence during the incipient stage of pulmonary tuberculosis; they pass away apparently, but usually precede the development of the real disease and serve therefore as a true indication of the presence of this last. It would be an unpardonable mistake to hesitate in such cases in taking the necessary measures against pulmonary tuberculosis and to wait for local symptoms - begimning with the cough and objective data to the finding of bacilli inclusive. For the rest, our patient presents also some local symptoms, as pains in the left half of the chest, and general indications, as slight fever, the most probable cause of which in the present case is tuberculosis of the lungs.

A question may arise as to whether the group of symptoms presented by our patient. so peculiar to chlorosis and appearing at the beginning, did not depend also on an incipient tuberculosis of the lungs, i. e., whether our patient had before and has now chlorosis, or only pulmonary tuberculosis. Against the last supposition speaks experience which proves that, although at the beginning of pulmonary tuberculosis emaciation, pallor and weakness are apt to occur, such a simultaneous collection of symptoms peculiar to chlorosis. as observed in our patient, does not take place; besides, we note considerable alterations in the blood 
and an enlargement of the thyroid gland. We must, however, take into account the fact, that the above-mentioned signs of anæmia are due not to chlorosis alone, but depend certainly on failure in nutrition and haematopoiesis amidst the unfavorable conditions under which our patient lived while in Moscow, and, later on, also because of the incipient tuberculosis pulmonum; so that we may call the disease chloranaemia, which, having attacked a weak constitution, created a predisposition to pulmonary tuberculosis.

Prognosis. - It will be more convenient for us to discuss the prognosis of chronic tuberculosis of the lungs after we will have analyzed a few cases of this disease; as far as the present case is concerned, I can only say that it is curable.

Treatment. - We will first of all advise our patient to give up her studies and to return to the former favorable conditions of life, until she is well again.

As regards treatment, we must certainiy first of all remove the dyspeptic symptoms. The patient is already taking Ems water - one-half glassful twice a day, warmed, one hour before a meal - and bitter drops (extr. fluidi condurango guttas xxx and trae.nucis vomicae guttas $v$, twice a day after a meal), and for overcoming constipation she resorts to the use of watery clysters. In view of the diarrhoea of the last few days we will prescribe ten grains of bismuth once or twice a day pro re nata

What shall we give her after the dyspeptic symptoms will have been removed? Shall we treat the chlorosis or the tuberculosis? We must certainly treat the tuberculosis, - first, because of the greater danger it presents, and secondly, because the principal remedy for chlorosis, namely iron, especially in large doses, is clearly contraindicated in the present case, as it may give rise to haemoptysis, to which the patient is so much predisposed, and which may favor the further development of the pulmonary trouble. Perhaps later on, after this last will have been completely and securely interrupted, we may be able, if necessary, to prescribe iron, certainly not in large doses, and with due care. Concerning arsenic, it is, firstly, contraindicated partly by the long-standing dyspepsia and the frequently occurring attacks of diarrhoea; and secondly, although it is used in the 
treatment of tuberculosis, it is much inferior in its effects in this disease to creosote and guaiacol. Of all the apothecary drugs at our disposal, these last two, in the present condition of our knowledge, are the most successful ones in the treatment of tuberculosis. I shall discuss these and the treatment of tuberculosis in general when we come to analyze some cases of this disease; but I will only state here, that not only in incipient tuberculosis, but also in eases presenting a failure in nutrition or anaemia, or slight chlorosis with only a predisposition to tuberculosis (on account of heredity, or of a poorly developed chest), I have frequently witnessed much more benefit from the use of these remedies, than from the employment of arsenic alone or in combination with iron.

In view of all this we will prescribe for our patient, after the dyspeptic symptoms will have been removed, guaiacol carbonate, it being borne by the stomach readily, in five-grain doses, after each meal, at other times in a half-glass of milk; we will constantly increase the number of the doses, and administer the drug for a long time, but with interruptions, till we will have secured a complete and lasting cessation of the pulmonary trouble.

\section{FROM $M_{33}$ THE LECTURE OF MARCH 16, 1893.}

Until March 12th we continued the above-outlined treatment of the digestive troubles; and we were enabled since then to leave off this treatment and to prescribe guaiacol carbonate, in five-grain doses, the first two days one dose a day. and later two doses daily. The condition of the patient at present is as follows: the appetite is better; there is no dyspepsia, stools regular; during the period of menstruation that occurred recently after a prolonged alosence of the same, there was an evening rise in the temperature (as is frequently to be seen in tuberculous women during their menstruation) up to $37.6^{\circ} R$. $\left(99.7^{\circ} \mathrm{F}\right.$.), and now it is $36.6^{\circ} \mathrm{R} .\left(97.5^{\circ}\right)$; the dizziness, heaviness in the head and the general weakness have diminished considerably. Patient leaves our clinic to-morrow and will continue the treatment with guaiacol in constantly increasing doses, guided by the results of the treatment and the condition of the stomach. 


\section{THORACIC AND INFECTIOUS DISEASES.}

\section{NINETEENTH CASE.}

\section{LECTURE OF JANUARY 29, 1893.}

The patient, a merchant's clerk, thirty-seven years of age, entered our clinic yesterday, complaining of severe pain in the left side, of dyspnœa, cough and general debility.

Mode of living and anamnesis. - Patient has lived for the last twenty-two years constantly in Moscow; formerly lived in the country. His lodging-rooms are good, with a warm watercloset; but he spends the day in a cold store. He takes a hot bath once a week; does not bathe during the summer; drinks as many as fifteen glasses of hot tea per day; is subject to sweating. Has been drinking whiskey, half a bottle daily, for a long time; somewhat less for the last two months. Does not smoke. Has a good board. Is married and has a few children. Never had syphilis.

Four years ago, after a blow over the nape of the neck, on which dropped a bale of goods, there appeared on the injured part a small swelling, which soon disappeared, accompanied by pain, which was also relieved in the course of time, but which, on his catching cold, reappears even now (periostitis of the occipital bone). Outside of this and of some slight colds, which, as he claims, were relieved by hot baths, patient has no recollection of any other diseases. The present trouble began six days ago: patien $\bar{t}$ was severely chilled by a draught and in the evening felt pain in the head and weakness, but this notwithstanding he went out to take a hot bath; on his return be began to shiver and felt a pain in the left side. On the next day he grew worse still, the pain was aggravated, there appeared a cough, patient was compelled to take to his bed, and 
remained in a condition which was growing worse all the time, till his admission to the clinic ; for the last five days he has been taking quinine powders, one in the morning and one in the evening. Yesterday he was given in the clinic a watery injection, the painful side was rubbed with butter and chloroform and wrapped in cotton and flannel, and internally we began to administer the mixture of Hoffman and valeriana drops, twenty-five at a dose, and also some wine; besides, he was given in the evening one-fourth grain of codeine.

Status. - Patient is spare and of weakly constitution; his chest is flat and narrow. Until the advent of the disease his appetite was good, he suffered from no dyspeptic symptoms, and had regular movements of the bowels. At present he has almost no appetite at all; a meal, even if light, causes difficulty in breathing; he had no movements of the bowels for four days previous to his admission to the clinic, but yesterday a clyster brought on a sufficient evacuation. The urine flows freely, is red, and contains neither albumen nor sugar. No abdominal pains. The regions of the liver, spleen and kidneys present nothing abnormal. There is dyspnca of the same character as yesterday, thirty-six respirations per minute, much more frequent on moving around; the pains in the side are somewhat quieter, and so is the cough; there is very little expectoration (twice during twenty-four hours). Objective investigation on the right side elicits nothing abnormal ; on the left side posteriorly, from the middle of the scapula downward, there is dullness, as well as over the lateral surface; the vocal fremitus is very much weakened; on auscultation, all over the left half of the chest, the respiratory murmur is weaker than on the right ride and non-vesicular, but of an indefinite character; the respiratory murmur is especially weak at the area of the dull sound; besides this, posteriorly, over the surface of the dull-sound area there are heard along a narrow zone some râles of an atelectatic character, while anteriorly, almost on the same level, there can be perceived a pleuritic rubbing sound. The intercostal spaces on the left side of the chest are sensitive to pressure. The heart is neither displaced nor enlarged, its tones are clear; the pulse is 110, regular, of fair volume (patient gets through the day 
four tablespoonfuls of wine and seventy-five drops); it was weak yesterday. The temperature yesterday morning, $38.1^{\circ} R$.

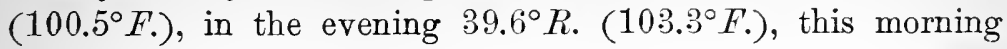
again $38.1^{\circ} R . \quad\left(100.5^{\circ} \mathrm{F}.\right)$. Patient slept somewhat better than he did while at home, but still poorly, and was slightly delirious. The whole head aches, but especially the injured spot. There is slight dizziness on getting up; the weakness as formerly.

Diagnosis. - We have apparently to do here with an acute thoracic disease, which causes the febrile condition and, through this, some disturbance in the organs of digestion and in the nervous system. The disease itself is evidently not located in the thoracic wall, which, outside of the sensitiveness of the intercostal spaces, presents a healthy condition, but within the chest, in the pleura or in the lung. What is it then - pleuritis or pneumonia? All the symptoms speak against pneumonia and for pleuritis : the severe pains, the weakened respiratory murmur in connection with a considerable area of a dull sound (if there were pneumonia in the case we would have, according to the period of the disease, either pronounced bronchial respiration, or, in the beginning, crepitation, and, at the end, subcrepitation), the pleuritic friction sound, weakened vocal fremitus and a dry cough; the expectoration is trifling in quantity and does not have the pneumonic character. The considerable difference between the morning and evening temperature - 38.1 $R$. (100.5 $\mathrm{F}$.) and $39.6^{\circ} R$. (103.2 $2^{\circ}$.) - is not usually peculiar to pneumonia. Thus the case is one of pleuritis, and to judge by the considerable area and intensity of the dull sound, by the weakened respiratory murmur and vocal fremitus, and partly also by the degree of the dyspncea, there must be a considerable effusion.

With this the diagnosis is, however, not at an end. Pleuritis may be of variable origin : it may be due only to a cold, and although this causative agency is disputed by many, my observations lead me to think that its existence can hardly be denied; it is frequently due to tuberculosis, to pyogenic microbes (the streptococci and the staphylococci), to acute articular rheumatism, to inflammatory conditions of the kidneys, sometimes to syphilis, actinomycosis and other diseases. What is the origin of the pleu- 
ritis in the present case? To attempt to prove that the disease in the present case is not due to acute articular rheumatism, because this last is absent, or to nephritis, on account of the absence of this disease, or to syphilis, because of its absence, etc., as would be attempted by a clinician who zealously believes in "the usefulness of exercises in differential diagnostication" (and there are such), would be but a profitless task, which, moreover, lowers the utility of a method really useful in certain cases and to a certain extent. From the collection of data referring to the case before us it becomes apparent, first, that cold has played undoubtedly an important part in the retiology of the pleuritis, and secondly, that notwithstanding the absence of indisputable symptoms of pulmonary tuberculosis, it would be rather rash on our part to conclude, in view of the patient's weak constitution and poor nutrition, as well as becanse of the long-continued abuse of whiskey so ruinous to health, and without further observation and examination of the sputum, that the pleuritis was entirely due to cold, and that the patient was free from tuberculosis.

What is the character of the effusion in our patient? In cases of pleuritis caused by cold only, and also in pleuritis of this nature in tuberculous persons, the effusion is usually, at least at the beginning, of a serous character. To decide the question of the nature of the pleuritic exudate we have to resort to a trial aspiration; but this procedure has to be adopted only when the disease yields to no other treatment, whereas our treatment is only just begun.

Prognosis. - Pleuritis caused by a cold, as well as pleuritis with pulmonary tuberculosis, may eventuate in a complete return to health; but the condition of our patient must nevertheless be recognized as serious, namely, as such, which, without presenting any immediate danger, may lead to it in the future, as we learn from the pathology of the disease.

Treatment. - Before discussing the treatment of our patient, I must make a few general remarks concerning my treatment of pleurisy, as it differs from the treatment recommended by the most widely read text-books on internal pathology and therapeutics. 
Thus we find in Eichhorst's Handbuch d. speciell. Pathol. $u$. Therapie ( fourth edition, vol. I, p. 590):

"In a recent and uncomplicated serous pleuritis we must, in the beginning, quietly wait" (Bei einer frischen, nicht complicirten serösen Pleuritis, warte man zunächst ruhig zu). Later on, if during the second and third week the disease presents no changes, we are to employ internal remedies to aid in the absorption of the exudate; but if by the fourth or fifth week we were unable to achieve any success, and the effusion is considerable or is even increasing in quantity, then we must resort to thoracentesis to effect its removal. Of internal drugs to aid absorption, Eichhorst recommends iodine (with due care also externally, over the painful side of the chest), diuretics, seldom laxatives, rather strong diaphoretics (subcutaneous injections of pilocarpine hydrochlorate and per os salycilic acid and sodium salycilate), while for debilitated and anaemic patients, nutritious food and strengthening measures.

But I, on the contrary, consider it erroneous to " wait quietly" until the second or third week, limiting one-self to hygiene and palliative measures. My observations teach me that the sooner we succeed in breaking up the acute stage of the pleurisy, the more favorable will be the further course of the disease, and the sooner will occur absorption of the effusion and the possibility of aiding it by therapeutic measures. Even in those cases, as for instance, in acute pleurisy in syphilitic or tuberculous patients, in which, later on, the cure of the pleurisy and the return to health in general will be effected by other treatment, having a particular, special relation to the principal disease, as iodide and mercury in syphilis, creosote and its preparations in tuberculosis, - even in such cases the acute stage of pleurisy, if pronounced clearly, requires a preliminary treatment of a particular character for itself.

The principal symptoms which characterize the acute stage of pleurisy, and which form an indication for such a treatment are fever and pain in the chest; with these there are, of course, dyspnoa and a dry cough, which are the more embarrassing the more prominent the first two symptoms: until these last are removed, or at least considerably relieved, we cannot hope for the absorption of the effusion to take place. 
For the treatment of the acute stage of pleuritis, for the removal of the above-named symptoms, I resort to the following: To relieve the pains I employ blood-letting; ${ }^{*}$ certainly in patients who are not anæmic, but in those with good nutrition and strength, and consequently, as we can see by the rtiology of the disease, in cases of rather unfrequent occurrence. Next to blood-letting, or, if this be contra-indicated, then at once, keep the painful side warm, as in the present case, or in case of necessity, give hypodermic injections of morphine, and internally codeine, Dover's powder, or again morphine to quiet the cough that tends to aggravate the pains in the chest.

The best remedy against the fever I consider to be sodium salycilate; at the same time I administer stimulants (to guard against collapse), which are, moreover, generally indicated in pleuritis. It is here, in the acute stage of pleuritis, that the great usefulness of this drug becomes apparent, as it causes a fall in the temperature and at the same time induces a pronounced relief of the pains, of the dyspnoea and cough. I administer the drug in ten-grain doses, and during twenty-four hours I begin with thirty or forty grains and increase the quantity as required; in moderately severe cases, as the present one, three or four days of such treatment are sufficient to bring about the desired result.

I will not stop to discuss here the various drugs, the effect of which is similar to that of the salyeilate of soda (as antipyrin, antifebrine, phenacetine), to which, in pleurisy, I prefer this last, but will say a few words on the use of calomel and quinine in this disease.

I administer calomel in pleurisy only in those very rare cases, in which this disease is complicated by certain morbid conditions in the abdomen, which form an indication for calomel (of this I spoke before); consequently calomel is given not against the pleuritis itself, but with a view of meeting such indications.

Quinine does not have even a shadow of the effect produced by the salycilate of soda in pleurisy. While speaking of the comparative effects of quinine and sodium salycilate in pleurisy,

*Vide article on "blood-letting." 
I will mark down the following difference between them, as observed in my experience: quinine acts well in febrile affection of the respiratory tract (of the nose, pharynx, larynx, trachea, bronchi) of a catarrhal nature caused by colds, better than the sodium salycilate; this last acts well in febrile diseases of the organs of locomotion due to colds, and especially of the muscles, aponeuroses and articulations (in the healthy organism, as well also as in the gouty, syphilitic, and in the one generally diseased), in which quinine exerts almost no effect whatever.

I will take up now the further treatment of pleuritis. Having removed the above-mentioned acute symptoms so as to further absorption of the effusion, I take particular care to establish a regular functional activity of all parts of the organism, a good nutrition and a farorable condition of the nervous system; as what concerns therapy, I apply flying blisters, so as not to keep up the irritation later on, over the painful half of the chest. With the exception of in syphilitics, I do not use iodine internally in pleuritis, as I did not obtain any benefit from it, but on the contrary harm, the general condition becoming worse; iodine externally fails to produce any marked effect, while it annoys the patient and hinders the application of the blisters. Except when necessary laxatives are certainly harmful, as they tend to weaken the patient. The diuretics can not be relied upon to a great extent. The strong diaphoretics are very risky, and may be used only for exceptional cases, and even then they can hardly be preferred to the operative method for removing the effusion.

As what concerns this last procedure I resort to it, first, when the exudation, if considerable, is not undergoing any absorption for a long time; but still I do not hasten, and do not limit myself to the four or five weeks' period recommended by the text-books; I had occasion to observe that a considerable effusion which had existed longer than this period, and which in the opinion of some physicians required operative abstraction, but which was not thus removed at the advice of other physicians, underwent absorption with the improvement in the general condition of the patient, thanks to better hygienic conditions and to a more careful surveillance over the regularities of 
functional activity and over the condition of nutrition and of the nervous system of the patient; secondly, in case of rapid increase in the effusion, and of the danger to life accompanying this increase because of the pressure on and displacement of the neighboring organs; thirdly, in cases of purulent and putrid effusion, which tends to keep up the exhausting fever.

I turn now to the treatment of our patient. Blood-letting is contra-indicated by his debilitated constitution and nutrition, and we therefore had to limit ourselves to keeping warm the painful side of the chest and to quieting the cough. As the temperature was high last night, $39.6^{\circ} \mathrm{R}$. (103.3 F.), we will prescribe to-day sodium salycilate, beginning with four tengrain doses per day, each dose in one-fourth glass of warm Ems water. The stimulants, which were indicated even yesterday by the weakness of his pulse, it is the more necessary to continue giving to-day, in view of the fact that we are administering sodium salycilate, as far as necessary and in a larger dose.

As what concerns hygienic conditions, the principal ones in pleuritis are rest and warmth. The "ambulance" treatment of pleuritis, even if this last be inconsiderable. will lead, particularly in the cold season of the year, to a more dangerous aggravation of the disease.

\section{FROM THE LECTURE OF FEBRUARY 3, 1893.}

The course of the disease. - January 29th we gave him forty grains of the salycilate, the evening temperature was $38.4^{\circ} R$. $\left(101.1^{\circ} \mathrm{F}\right.$ ) ; the night of January 30th was spent somewhat better. The 30th he was given fifty grains of the salt, the evening temperature was $37.5^{\circ} R$. $\left(99.5^{\circ} \mathrm{F}\right.$.) ; had a very good night the 31st; January 31st and February 1st he was given forty grains of the drug each day, and the temperature fell then for good, while the condition of the patient improved at once. Yesterday, February $2 d$, he was not given any of the salt at all.

Status. - He feels very well; has an appetite. Had regular stools for the last two days withont clysters. The urine increased in quantity, and is not red. The pain on the left side 
of the chest is much relieved and he can breathe more freely; the number of respirations is twenty-four per minute, instead of thirty-six as formerly. He coughs much less. The quantity of expectoration has not diminished, and it contains tuberculous bacilli. The pleuritic friction murmur disappeared. The area of dullness has diminished. In the area of dullness, as well as all over the left side of the chest in general, the respiratory murmur, which was formerly weak and of an indefinite quality, became much clearer, while in the region of the apex of the left lung, in front, under the clavicle, as well as behind, there appeared moist râles. The pulse is 80 and of fair volume. Last evening's temperature, $36.6^{\circ} R$. (97.9 $\mathrm{F}$.). This morning, $36.5^{\circ} R$. $\left(97.6^{\circ} F\right.$. . His sleep is good. There is neither headache nor dizziness on getting up. He is gathering more strength.

The presence of the tubercle bacilli in the sputum and of the râles in the superior apex of the left lung, which could not be heard when the patient was unable to fully expand the left lung, as he could not breathe sufficiently deep, and which became noticeable now when breathing became free, speak for tuberculosis of the lungs.

The beneficial effect of the sodium salycilate is but too evident in the present case. After the first few days of the patient's sojourn in the clinic under entirely normal circumstances, which were more favorable than during the first six days of the disease before his admission to the clinic, and with the aid of the necessary symptomatic treatment, the improvement in the patient's condition was but trifling; but from the very first day that we began the use of the salycilate of soda the temperature began to decline rapidly, and in four days a complete breaking up of the disease took place: the temperature fell finally to the normal and the local symptoms decreased very markedly.

At present, while keeping continually the left half of the chest warm, we will apply a blister, the size of the palm of the hand, under the left scapula, and for internal use we will give him creosote, beginning with five drops three times a day, and increasing the dose gradually. 


\section{FROM THE LECTURE OF FEBRUARY 17, 1893.}

The course of the disease. - Febuary $3 \mathrm{~d}$ we applied the first blister under the left scapula; the 9 th, the second one on the lateral surface of the left side of the chest. Since February $14 \mathrm{th}$, patient is treated by inhalations of compressed air. He takes creosote in eight-drop doses, three times a day.

Status. - The appetite is good, there is no dyspepsia, the stools are regular, the urine normal. At times patient experiences a trifling pain in the left side. The breathing is free. There is almost no cough, and there is but very little expectoration, which appears at times, and is absent at others. Objective examination shows that there is only under the scapulæa a slight difference in the percussion sound and that the respiratory murmur is but slightly weaker on the left side. In the region of the apex of the left lung there are sometimes heard a few râles, at other times they are absent. The temperature is normal, the sleep quiet; he gathers strength.

Treatment. - The blisters are not necessary any more. The creosote must certainly be continued. The benefit derived from inhalations of compressed air during the period of the absorption of the pleuritic exudate, is but too well known. The patient asserts, that after the inhalation of compressed air breathing became easier and the sensation felt by him heretofore, outside of the pain, of tightness in the left side, was relieved (as the patient expresses it, "the side was loosened"). Our patient, however, presents some contra-indication to this treatment, in view of the tuberculous nest in the left lung, and it was only because of the inconsiderable size of this nest that I ordered, three days ago, previous to the absorption of the exudate, the inhalation of compressed air. In the presence of more or less considerable tuberculous nests such inhalations are not only not beneficial, but, on the contrary, rather harmful, probably because they assist in aspiration of tuberculous expectoration by the healthy portions of the lungs. In our apparatus (Waldenburg's) for inhalation of compressed air, the air is conducted, as you see, from outside through a win- 
flow-frame and is warmed in a special apparatus. The air for our patient. conducted from the outside and warmed before being admitted to a Waldenburg apparatus. is passerl through a Wolff jar containing eucalyptus oil.

The patient, considerably strengthened. left the clinic February 22d. He was instructed as to the necessary regimen, and was advised to continue the creosote treatment. 


\section{TWENTIETH AND TWENTY-FIRST CASES.}

\section{LECTURES OF APRIL 9, 13 AND 14, 1893.}

Patient, a sergeant-major of the reserve army, age 37, entered our elinic. Warch 8, 1893, complaining of dyspnoea. cough with expectoration, pain in the left side and general weakness.

Mode of living and whamnesis. - Patient resides in a damp. marshy, woody locality. His rooms are very small, damp ami cold; the water-eloset is eold. For the last three years he has almost never taken a hot bath, although he washes himself at times at home; does not smoke or drink whiskey (he drank and swoked much formerly), drinks only a little warm tea.

His food is that of an ordinary workingman. He is single. never had syphilis. Our patient is a clerk in a country house. is much oceupied, does not sleep sufficiently, is often fatigued and subjected to colds, as he is compelled to talk much and londly out-of-door's in every season of the year.

Patient comes from a healthy family. Since lis nineteentls yeu, he was addicted to the abuse of whiskey. During his twentieth year he passed through an attack of malaria, which lasted nine months. On lis twenty-second year he entered the military service, in which he spent five years, during which time he did not drink any liquor, and enjoyed excellent health. Becoming a reserve, he began to drink again, and seven years ano. while employed in rafting, he began to congh, at first only slightly, but later on much more. Two and one-half years later, in the autumn of 1888 , he suffered for the first time from hamoptysis, which lasted for about one month (about one half-glassful of blood daily) and weakened the patient consiclerably: he grew thin, and began to eomplain of dyspmen. Dur- 
ing the autumn of 1889 there occurred another attack of hæmoptysis, which lasted a week; patient at the same time began to grow feverish and to feel a pain in the left side. Then, at the advice of his physician, he gave up whiskey drinking and smoking, and subjected himself to treatment. Of the drugs employed for treatment he remembers that creosote was of the greatest benefit of all, and he had been taking it during the winter of 1891-1892, up to forty drops a day, in milk; as a result of this treatment he gathered considerable strength, and became somewhat stout, while the fever, cough and the pain in the side diminished. Last January all the morbid symptoms reappeared again, as the result of a cold. Pa+ tient has been taking since cod-liver oil (three tablespoonfuls a day), at times powders of codeine, and applied blisters, but there was no improvement.

During the month that the patient resided in the elinic, the treatment was as follows: as he was costive, he was given. when necessary, watery clysters; during the morning eough, which was most embarrassing, as the expectoration was then brought up with great difficulty, patient sipped about one glass of a mixture of Obersalzbrumnen water and warm milk; this assisted materially in loosening the secretion; at bed-time, in case the cough prevented sleep, patient was given one-quarter grain of codeine; the left side of the chest was keptwarm; as the pulse was somewhat weak, he was given four tablespoonfuls of wine daily.

From the very first day of the patient's sojourn in the elinic, he has been taking creosote, commencing with five drops up to thirty drops a day. Besides this, patient took, March 29th and-30th, some ipecacuanha (infusi rad. ipecac. e gr. $\mathrm{rj} 5 \mathrm{vj}$. altogether twice this quantity) which was indicated by the difficult expectoration with the aggravated dyspnota and râles, and also with the rise in temperature, up to $38.8^{\circ} \mathrm{R}$. $\left(101.9^{\circ} \mathrm{F}\right.$.), while before this the temperature was below $38^{\circ} \mathrm{R} .\left(100.5^{\circ} \mathrm{F}\right.$.): after having taken the ipecac. patient expectorated very ireely, the dyspnoea was relieved, the râles diminished and the temperature reached its previous height.

Status. - Patient is of average constitution. The appetite, 
which was poor on admission, is good now. There was no dyspepsia before, nor is there any now. The stools are regular at present. The urine flows freely, contains neither sugar nor albumen, was reddish on admission. but is of a normal color now. The pains in the left side of the chest, not severe before, are trifling now. The dyspncea and cough are much better at present than they were when the patient was admitted to the clinic.

There are no pains in the abdomen, nor does objective examination discover anything abnormal in it. At the time of admission he had a muco-purulent expectoration, of about onehalf-glassful per day, mixed with blood; at present it is just half of what it was formerly. Repeated examinations of the expectoration always showed the presence of tubercle bacilli. On percussion the sound over the left side anteriorly from the clavicle to the fourth rib, as well as posteriorly from above down to the middle of the scapula, is duller than from the middle of the scapula downwards and on the right side, where the percussion sound is normal; on auscultating the left side, orer the area of dullness, we hear bronchial breathing and abundant moist râles; on the right side. under the clavicle, there are also at times heard a few râles; at the other parts of both the left and the right sides of the chest we hear the normal respiratory murmur, either vesicular or indefinite, in accordance with the depth of the respiratory movements. The heart is neither enlarged nor displaced, its tones are clear, the pulse was at the begimning 100 and somewhat weak, but is now 88 and of fair volume. When admitted, his evening temperature was about $38^{\circ} \mathrm{R}$. (100.5 $5^{\circ} \mathrm{F}$ ), and the molning below $37^{\circ} \mathrm{R}$. (98.6 $\mathrm{F}$.) ; now in the evening it is $37.6^{\circ} \mathrm{R} .\left(99.7^{\circ} \mathrm{F}\right.$ ) or $37.5^{\circ} \mathrm{R}$. $\left(99.5 .^{\circ} \mathrm{F}\right.$.) and in the morning below $37^{\circ} R$. $\left(98.6^{\circ} \mathrm{F}\right.$.). Patient is very thin: his weight when admitted was 147 pounds, it is now 149 . His sleep is good now; it was prevented formerly by cough and dyspncea. There is no headache. He has become markedly stronger than he was on admission.

Diagnosis. - It is evident, that we have here before us it pure case of chronic tuberculosis of the lungs, which causes all the morbid symptoms observed in our patient, the local as weil 
as the general ones, as fever, emaciation, and weakness; the other organs seem to be unaffected. The lungs may become affected by tuberculosis, first, from the neighboring organs; such cases are of comparatively unfrequent occurrence generally, and are particularly rarely seen in the clinic for internal diseases; they are more frequently met with, as tuberculosis of the vertebre. in surgicial and peediatric clinics; besides, they play but a secondary part in the prineipal affection; secondly, through the blood, when there is a general infection of this last: these are also, comparatively speaking. rare cases of miliary tuberculosis of the lungs; and. thirdly, most frequently. through the respir"dtory passages, when the apices of the lungs become first affected ${ }^{*}$ (either both at once or at the beginning one, and then the other), and later when the affection spreads by contiguity or through aspiration of the tuberculous expectoration by the hitherto healthy portions of the lungs ; these are the cases, which are known in pathological anatomy as broncho-pneumonia tubereulosia chronica, and which are called in common parlance consumption of the lungs, and in the clinics, hospitals and by physicians chronic tuberculosis of the lungs. The present case belongs eviclently to this last cliass : the tubercular affection of the lumgs. in view of the other unaffected organs, is present beyond doubt and to a considerable extent: both the superior lobe of the left lung and the apex of the right one are affected; the anamnesis gives sufficient proofs of its chronic course. while the absence

* This is, however, a disputed question. Thus Volland (Zeitschrift fïr clinische Medicin, 1893), denying that the presence of the tubercle bacilli in the air was ever proved, assumes, that infection by tuberculosis takes place in infancy, when the children are not on the arms of the mother or nurse, lyut unable as yet to walk, they crawl about, rub their hands over the floor, on which there may be some tuberculous expectoration, and then over their faces, and thus infect themselves through accidental lesions of continuity, so frequent around the mouth and nose. The infection by tuberculous and other microbes gives at first rise to eruptions ("scrofulous"), and later to an affection of the lymphatic. glands; then in the presence of a predisposition to tuberculosis, be it hereditary or acquired, the disease will sooner or later spread from the primary focus in a general way, but most frequently along the lymphatic vessels and the glands of the neck and pleura, the apices of the lungs, etc. Resting on these grounds and on some other data, which he eites. Volland insists upon prophylactic measures consistent with these last. 
of the exhaustive fever (namely, chills, constant and considerable rises and falls of the temperature as well as abundant sweating) shows, that the period of consumption. phthisis proper, has not as yet arrived.

The prognosis will be more conveniently discussed, when we speak of the prognosis in chronic pulmonary tuberculosis in general; while the treatment. in view of the success attained by it, will of course remain the same.

Patient left the clinic by the end of April, considerably strengthened and markedly grown, his weight being one hundred and fifty-six pounds, as against one hundred and fortyseven on admission; he had no fever. no pains in the side, almost no dyspnœa, and a slight cough with abont one-fourth of a glass of expectorated matter per day; he was adrised to continue the creosote in constantly increased doses.

This patient is a woman, forty-six years old, who entered the clinic April 2, 1893, complaining of dyspnœa, cough, and general weakness.

Mode of living and anamesis. - Patient has been living for a long time, during both winter and smmmer, in Moscow. Her residence was always a poor one, of late she lived in the basement of a stone building, which was cold and damp, the watercloset cold. Takes a hot bath every week. For the last halfyear patient gave up smoking and whiskey drinking, but used to smoke before, and drank a wineglassful of whiskey with both dinner and supper; drinks but little tea; her board is that of a working-woman. Her occupation - in the line of housekeeping - is burdensome, so that patient frequently does not sleep enough and is overworked. Goes but rarely out-of-doors. Has been maried for twenty-six years, has had eleven deliveries, all normal, last time eight years ago; has fire children living; presents no indications of syphilis.

Patient comes from a healthy family, and enjoyed during her childhood fair health. When fifteen years old she prssed through an attack of typhoid fever. Later on she caught fre- 
quent colds and coughed at times. Twelve years ago she had a slight attack of acute articular rheumatism. Eight years ago a pleurisy on the right side, after which the cough became constant, more severe in the winter than in the summer, accompanied at times by fever and pain in the right side. Last January (1893) the patient's condition became considerably aggravated, as a result of vexatious troubles and of a cold: the cough became very severe; there appeared considerable dyspnora and a feverish condition, the patient grew thin and lost strength, and her voice became very hoarse and weak. As far as treatment is concerned, patient resorted lately, as well as before, to the use of Dover's powders, and at rare times to ipecacuanha; she never took any creosote.

During her residence in the clinic patient was given, when necessary, a watery clyster, and every morning a glassful of Obersalzbrunnen water with milk, to be sipped during the cough; at bed-time a powder containing one-fourth grain of codeine, and during the day twenty drops three times a day of the mixture of liq. anod. Hofmanni and liq. ammonii anisati* $\bar{a} \bar{a}$. Besides this, patient took, on her admission, छrj of infusion of ipecac (e gr. vj), then terpine hydrate, five grains per dose, at the beginning twice. and later on three times per day, and for the laryngeal catarrh she inhaled atomized silesian water.

Status to-day, April 9th. - The appetite. which was poor on her admission, is better now. There is no dyspepsia. The former costiveness is somewhat relieved. The urine, which was reddish formerly, is almost of normal color, without albumen or sugar. The abdomen in general and the regions of the liver, spleen and kidneys present nothing abnormal. Menstruation is unfrequent - once in two or more montlis (last time one month ago) - quite abundant, but painless; no leucorrhcea.

When admitted, she had considerable dyspncea, pains all over the chest, and especially in the right side, severe cough, some expectoration, mostly of a muco-purulent character, partly foamy and tinged with blood, more than a glassful during

* Liq. ammon. anisatus is a mixture consisting of liq. ammon. 19.5 per cent, anise oil 2.4 per cent, and alcohol (90 per cent.), 78 per cent. 
twenty-four hours ; the expectoration shows, at times, the presence of the tubercle bacilli; percussion elicits nothing abnormal anywhere in the chest, on auscultation we hear everywhere a great number of râles, dry and moist, and over the apex of the right lung, in front and in the back, some slight bronchial breathing (the percussion sound is not altered in those places). At present the dyspnoea, cough and expectoration, as well as the râles. are much less prominent, the pain in the chest is greatly relieved. The voice, which was very hoarse and weak on her admission (laryngoscopic examination showed the presence of a laryngeal catarrh), is clearer and stronger now. 'The heart is neither displaced nor enlarged, its tones are clear, the pulse, 100 and weak on admission, is now 84 and stronger.

The highest temperature the patient reached during her stay in the clinic, took place the day before yesterday : namely, $37.8^{\circ} R$. $\left(100^{\circ} \mathrm{F}\right.$.) in the morning, $38.2^{\circ} R$. $\left(100.7^{\circ} \mathrm{F}\right.$.) in the evening; this moruing the temperature was $37.6^{\circ} R$. $\left(99.6^{\circ} \mathrm{F}\right.$.), last evening, $37.8^{\circ} R$. $\left(100^{\circ} \mathrm{F}\right.$.) Her sleep, which was formerly prevented by the cough, is at present much more quiet. At rare intervals patient suffers from migraine, and, when fatigued, from dizziness. She is much stronger than she was when admitted.

Diagnosis. - The presence of tubercle bacilli in the expectoration is an undisputable proof that the patient has pulmonary tuberculosis, while the other data, concerning both her present condition and her past history, as the bronchial breathing and the râles in the region of the apex of the right lung, the pains in the right side of the chest, and the chronic course of the disease, strengthen this supposition and point to that form of tuberculosis from which our previous patient also suffers, namely, chronic tuberculous broncho-pneumonia. But the two cases differ from each other by the great variance in the local, as well as in the general symptoms. and by the course of the disease.

Let us take the local data first. Our male patient presents pronounced symptoms of a complete affection of the whole upper half of the left lung, as evidenced by dullness in this region, by bronchial breathing and abundant râles, while the other parts of the chest, with the exception of the few râles heard at times on the right side, under the very clavicle, pre- 
sent nothing morbid, as evidenced by a normal percussion sound, and a normal respiratory murmur; in our female patient the percussion sound is everywhere normal and there are râles in abundance all over the chest. The male patient has evidently a complete tubercular affection of a consiclerable extent of the upper portion of the left lung, while the remaining parts of the lungs, outside of the right apex, are unaffected either by tuberculosis, or by a simple bronchial catarrh; in the female patient, if we consider the bronchial breathing and the râles in the region of the right apex as a sign indicating the presence of a tuberculous nest (which is shown by the presence of bacilli in the sputum) in this region, what then must be the condition of the remaining parts of the bronchi and of the lungs? We may suppose in this connection two morbid conditions: first, an ordinary bronchial catarrh, secondly, a chronic tubercular bronchopneumonia diffused all over the lungs, not, however, as a complete process, but in small islands surrounded by pulmonary tissue containing air, and which do not therefore cause any dullness nor bronchial breathing; such cases are usually observed during the further course of the disease (as their development requires some time), at its very termination, at the period of phthisis, when the exhaustive fever and the extreme emaciation of the organism foretell the approach of the fatal issue; but even at this time, when the aspect of the patient itself is a clear indication of the diagnosis and of the prognosis, even then the physician can only hear râles on auscultation, but can nowhere obtain a sure alteration in the precussion sound, nor generally any positively certain symptoms of a compact thickening of the pulmonary tissue.

Before we decide as to which of the two diseases our female patient suffers from, whether it is a chronic, at times exacerbated, bronchial catarrh, or a chronic tubercular broncho-pneumonia diffused all over the lungs in its last stage, let us compare the course of the disease and the general condition of both patients. The male patient is thirty-seven years old, he comes from a healthy family, lived under unfavorable conditions of life and abused alcoholic drinks, but for the last three years he has not been drinking or smoking, and has subjected himself to 
quite a radical treatment; but this notwithstanding, having been ill only seven years, he loses his health steadily.

The female patient is forty-six years old, she comes from a healthy family, has been surrounded by similar poor circumstances, has also been a drinker (although a rather moderate one, as she asserts) and a smoker, has had eleven children, has been ailing for eight years, had never subjected herself to radical treatment, and still her health is not failing as rapidly as it does in the male patient; on the contrary it is rather in a condition of a tolerable statu quo, worse in the winter as regards the cough and the dyspncea, and better during the summer, but it is not accompanied by such periods of fever and such a continuous emaciation, as we find in our male patient. Let me add, that while in the clinic, the woman improves more rapidly than the man (thus, the fever, generally trifling and without having the slightest symptom of an "exhaustive" nature, disappears itself without any resort to antifebrile remedies); still we did not as yet give her any creosote, while the man began to take it from the very day of his admission to the clinic.

We are justified then in concluding from the above statements, that our female patient has a moderately developed broncho-pneumonia tuberculosa chronica and a diffuse chronic bronchial catarrh, but not a broncho-pneumonia tuberculosa chronica disseminated over the whole lungs, in its last stage. This conclusion is strengthened also by the fact, that the muco-purulent expectoration, peculiar to bronchial catarrh, as observed in our patient, contained only at rare intervals the tubercle bacilli, whereas the sputum of phthisical patients contains them usually in abundance.

Prognosis. - The chronic bronchial catarrh, as well as the broncho-pneumonia tubereulosa chronica as developed in our patient, are both yielding to treatment, which is the more encouraging, as the patient up to this time was treated solely in a palliative way.

Treatment. - To explain why we gave the patient Obersalzbrunnen mixed with milk in the morning, codeine at bedtime, the mixture of liq. anod. Hofmanni and liq. ammonii anis., as a cardiac stimulant and as a means to promote expectoration, 
during the day, and why we gave on her admission ipecac; to explain all these after what was said in connection with the treatment of the foregoing case, appears to me superfluous; as regards terpine hydrate, I will say, that on a par with the alkaline mineral waters, it forms the best remedy, of those for internal use, for the treatment of chronic bronchial catarrh; as soon as the exacerbation of the disease, which compelled the patient to come to the clinic, has quieted down, we will begin the treatment by creosote.

(The female patient continued the above outlined treatment up to the time she left the clinic, April 23d; when she left her condition was as follows: appetite normal, stool regular, the dyspnœa, cough, expectoration and râles have diminished greatly, there are no pains in the right side, nor is there any fever; the sleep is sound, the patient gathered strength and became somewhat stout; she weighs 133 pounds as against 129 she weighed when admitted. She was prescribed creosote treatment.)

\section{GENERAL REMARKS CONCERNING THE DIAGNOSIS OF CHRONIC TUBERCULOSIS OF THE LUNGS.}

We have analyzed four cases of chronic pulmonary tuberculosis.

First, episodic, transient affections of the lungs by tuberculosis, which precede the development of the permanent disease in a person predisposed to tuberculosis because of a weakly constitution and the presence of chloranæmia.

Second, a small tuberculous focus in the apex of the left lung, complicated by pleurisy of the left side.

Third, a complete considerable affection of the left lung and an incipient affection of the right one, without any complications.

Fourth, a moderate affection of the lungs (apparently, to a greater extent, of the right one), complicated by a chronic bronchial catarrh, which at times becomes greatly exacerbated.

The cases of pulmonary tuberculosis, which are to be seen at this time in the clinic, present, outside of the two last 
discussed cases, the same condition as the third case analyzed by us, namely, chronic tuberculous broncho-pneumonia in various stages of development, but without any complications and in general without any pronounced peculiarities, and there appears therefore no reason for taking them up for discussion. There is one case of chronic pulmonary tuberculosis in its last stage, in that of phthisis declarata, but the patient is so exhaust$\mathrm{ed}$, and the final issue is so near at hand, that it is impossible to transfer him from his small ward into the auditorium for demonstration before the class.

However, the four cases discussed by us represent practically the most important clinical forms of chronic pulmonary tuberculosis - those which are particularly accessible to treatment, and in which treatment is of the greatest benefit. The complications which occurred with them-chloranæemia, pleurisy and bronchial catarrh - are also very important, as they are observed particularly often.

It is true, that the multiplicity of forms of chronic tuberculosis of the lungs, which is to be seen in medical practice and which depends on the peculiarities in the course and development of the tuberculosis itself, and on the complicating diseases and the peculiarities of the affected organs, is far from being exhausted by the cases we discussed; but not only in the clinic, not even in a systematic text-book of internal diseases, unless it be in a special monograph, can this multiplicity be exhausted, or a complete picture of all the various forms of chronic tuberculosis of the lungs to be seen in medical practice, be presented.

I consider it, however, useful to outline before you now, after we have discussed the previous cases, a clinical picture of those varieties of the disease, which we had no opportunity to bring before you, at least the most important ones; my aim in doing this is, that the cases presented to you alongside with these forms, as well as these last side by side with the presented cases, might become more salient, and might be better and more strongly impressed on your memory, than is usually the case when pathology is studied systematically, but without the aid of a clinio. 
The last stage of chronic tuberculosis of the lungs is pulmonary phthisis, phthisis declarata. You had occasion to see a sufficient number of such patients both in our clinic and in the propædeutic clinic when you were engaged in studying the objective examination of the diseases of the organs of respiration; you are familiar with the sharp local changes, with the characteristic abundant expectoration, containing great numbers of tuberculous and other bacteria and elastic fibres of the brokendown pulmonary tissue, and also with the characteristic habitus of such patients : their extreme emaciation, their great debility (the patients, when seen, are always in the recumbent posture), the high jumps and the low falls of the temperature, the exhausting sweats. How great is the difference between these and the cases of chronic tuberculosis of the lungs in its incipient, or frequently even in its advanced development, when the patients, only slightly or not at all emaciated, with but a slight cough, without a constant or considerable fever (only a trifling rise of the temperature toward the evening without any considerable fall in the morning), - are not only not confined to bed, but are actively occupied with their affairs, and look simply like men whose health is not quite up to the ordinary, but by no means like pronouncedly sick people. I will stop here to discuss the importance of the exhaustive feverthe febris hectica - seen in phthisical patients, which is so characteristic by the high jumps and the low falls of the temperature, and also by the sweatings which accompany these last. As is well known, the expectoration of phthisical patients contains, besides the tubercle bacilli, also numbers of other micro-organisms to which is ascribed the pernicious influence on the organism of the patient, and which are considered as the cause of the last, phthisical stage of chronic pulmonary tuberculosis; but until recently the nature of the special microorganism, which is accountable for this pernicious influence, has not been thoroughly investigated. Exact investigations touching upon this subject have lately appeared from the Institute for Contagious Diseases under the supervision of Prof. Koch at Berlin. (Petrushky, Tuberculose und Septiccemie, Deutsche med. Wochenschrift, 1893, No. 14.) 
In the expectoration, which was subjected to a certain process, by which everything that was mixed with it in the cavity of the mouth was removed, there were found much smaller numbers of bacteria, than in the expectoration which was mixed with the saliva; it was found that this expectoration, free from saliva, contains principally tubercle bacilli, and of the others the most constant and the most abundant were the pyogenic organisms, namely, the streptococci. They were also found in the tissue of the lungs and other organs, and also in the blood of dead phthisical persons, so that, according to Koch, the last stage of chronic pulmonary tuberculosis presents already a complication of this last by a secondary infection of the organism through streptococci, which enter the body through the ulcerated spaces (the cavernæ) in the lungs, in a way very similar to that in which they infect the organism when entering through wounds. It thus becomes clear, why the fever in the last stage of chronic tuberculosis of the lungs has the identical features of that seen in septic wound infections. Koch calls the temperature curve in this fever, for brevity's sake, streptococcus curve (Streptococcencurve.)*

Pneumonice complicating chronic pulmonary tuberculosis. - Fibrinous (croupous) pneumonia oceurs, although very rarely, in chronic tuberculosis of the lungs and is easily diagnosticated, especially if it affects a patient whom the physician knew before: in such a case the physician will be able to detect, beside the old, well-known tuberculous focus in the apex, say, of the right lung, also an affection of the whole of the inferior lobe of the same or of the other lung, as evidenced by the dull percussion sound, by crepitation, bronchial breathing, subcrepitation; the expectoration will be tinged with blood and will contain along with the tubercle bacilli, pneumonic diplococci, while

* Not all, however, entertain a similar view on this subject: Leyden does not believe that other micro-organisms, besides the tubercle bacilli, play any part in the phthisical period; he points out the fact that in miliary tuberculosis and in tubercular caseous pneumonia the fever is very high, and still we find only tubercle bacilli, and no other micro-organisms in these cases : Fränkel states, that in many cases of tuberculous pneumothorax the pus contains only tubercle bacilli (Berlin. Flinische Wochenschrift, 1893, No. 42, pp. 1029-1030). 
the temperature will run the course so peculiar to croupous pneumonia. The disease may terminate in recovery or death, but if recovery takes place, there is usually observed an aggravation in the course of the tuberculous process.

But more frequently chronic pulmonary tuberculosis is complicated by catarrhal and caseous pneumonice, which are eminently peculiar to it. Here also the diagnosis is easier, if the patient was known before. The physician then observes simultaneously with the sudden rise and the further pneumonic course of the temperature, a rapid increase of the local changes : the wellknown sign of tuberculous affection, for instance, of the apex of the right lung, the slight dullness under the very clavicle, with prolonged expiration and in the presence of râles, is observed to be spreading to a greater distance down to the third, and then to the fourth rib, and later on further, becoming more intense all the while; the same is observed in the posterior surface of the chest; with this there are heard over the area of the dull percussion sound râles and bronchial breathing. A whole lung may thus become affected in a short time; but generally the affection spreads at a slower rate than in the case of croupous pneumonia, and, besides, usually from above downward, and not in the opposite direction, as ordinarily occurs in croupous pneumonia. Concerning the termination of the disease, there are cases in which the pneumonic fever keeps on but for a short time, the affection of the lung is not considerable and passes away with the fall of the temperature, leaving traces of the former tuberculous focus, at times slightly increased; in such cases the pneumonia is very likely of a catarrhal nature. In other cases the affection of the lungs spreads to a considerable extent and does not become limited, the high temperature of a pneumonic character, with its slight difference between morning and evening does not fall, and the patient succumbs after a few weeks (four to eight) from the commencement of the pneumonia. Autopsy shows in these cases a considerable caseous pneumonia, which terminated in the destruction of pulmonary tissue and the formation of cavities. These are the cases of what the Frenchmen call galloping pulmonary phthisis.

There was one such case that has left a particularly strong 
impression on my mind. The patient, whom I have known for several years, had small tubercular foci in both pulmonary apices, at times expectorated blood and was fererish, but had nevertheless enjoyed fair health and was very active. After one certain period of time, unfavorable to his health (after a fatigue and a cold), his temperature rose suddenly and the area of dullness under the right clavicle began to spread downward and also on the posterior surface of the right half of the chest. During six weeks, until the patient's death, the temperature vacillated constantly between $39^{\circ} \mathrm{R} .\left(102^{\circ} \mathrm{F}\right.$ ) and $39.5^{\circ} R$. $\left(103.1^{\circ} \mathrm{F}\right.$.) in the morning and $39.5^{\circ} \mathrm{R}$. (103.1 $\mathrm{F}$.) to $40^{\circ} \mathrm{R}$. $\left(104^{\circ} \mathrm{F}^{\circ}\right)$ in the evening, while the right lung was almost completely affected.

It is thus evident that the clinical pictures presenting the pneumonic complications of pulmonary tuberculosis, the ordinary course of this last and its last phthisical stage, differ one from the other, and enable one to recognize these various morbid conditions.

Acute miliury tuberculosis of the lungs may also be superadded to chronic tuberculosis (i. e. to chronic tubercular broncho-pneumonia), and its clinical picture differs also from the clinical pictures of the usual course, of the last stage and of the pneumonic complications of this last; it is rather similar in its clinical aspects to an irregular typhoid fever; but it is more opportune to discuss acute miliary tuberculosis in general and that of the lungs in particular when we chance to come across, if not a case of this comparatively rare disease, then one of typhoid fever.

The most important complications of chronic tuberculosis of the lungs by affections of other organs, ontside of those we discussed above, are pueumothorax and pyopneumothorax, tuberculosis of the larynx, and intestinal tubereulosis. Clinical pictures of these diseases enable one to fully recognize their presence.

As regards the peculiarities in the clinical picture and in the course of chronic pulmonary tuberculosis, which are determined by the peculiarities of the affected organs, I will only note the following. Persons who fall victims to chronic tuberculosis of the lungs may be subdivided into three classes: there are those 
who are by nature of a weak constitution, with a narrow and flat, under-developed chest (in proportion to their height); they frequently come from as weak and even tuberculous parents, and present but too evident a predisposition to tuberculosis of the lungs; others, on the contrary, who were formerly of a strong constitution, robust, and enjoyed the best of health, become affected by pulmonary tuberculosis after their health had been previously undermined, most frequently by syphilis, alcoholism, prolonged and severe malaria, or diabetes mellitus; quite often these persons succumb sooner than those belonging to the other categories (in those previously affected with diabetes mellitus the course of pulmonary tuberculosis is always liable to be unusually rapid, more so than in any other case); lastly, persons of the third class do not present the characteristic peculiarities of either of the above two classes.

Prognosis of chronic tuberculosis of the lungs. - I must repeat what I took occasion to say two years ago while comparing the treatment of pulmonary tuberculosis by injections of Koch's tuberculin (discussed at that time) with the treatment of this disease by the usual method, with the aid of creosote, arsenic, koumyss and cod-liver oil, in connection with the necessary symptomatic treatment, for the removal of difficult and exhausting symptoms, and with correct hygienic measures, including, of course, the climate. I said then: "What results did we obtain from this treatment? What experienced physician has not observed, even in the advanced - but certainly not in the last stage of pulmonary tuberculosis a considerable improvement for a period of years, and in the incipient period, a complete recovery of the general health ad integrum, while all the local symptoms, subjective and objective, disappear unless there remains only a slight difference between the respiratory murmur over the apex of the healthy lung and that of the affected one? What experienced physician has not noticed that such a recovery of health, such a cure, may last for years? I had occasion to keep under observation such recovered patients for periods of from five to ten years, before I lost track of them. It is true, that at times, after several years of such a favorable condition, the patient will reappear again with symptoms of pul- 
monary tuberculosis; but it can usually be proved that this fresh reappearance of the symptoms has been preceded by a weakening in the general condition of health, because of an irregular mode of living, unsatisfactory circumstances, or an attack of some painful disorder; it thus remains, in such cases, to decide whether all this was due to the lighting up of the old focus, or to the appearance of a fresh infection. Finally, such a method of treatment, whether it result in the above-outlined favorable issue or not, is neither risky nor fraught with any danger, as is so frequently the case during the treatment by injections of tuberculin."

The slighter the local, i. e. the pulmonary affection, and the better the general condition of health, and the less the number of complications, - the better is, of course, the prognosis, and contrariwise (although there are exceptions to this).

As to what concerns the influence on the prognosis of diseases developed before the occurrence of the chronic pulmonary tuberculosis, we may say that it is particularly unfavorable in cases where the pulmonary tuberculosis seizes upon an organism previously affected by diabetes mellitus, and also by syphilis. However, in the last case, in persons, of a strong constitution, we may sometimes succeed, after having influenced the syphilis effectively by the iodides or less frequently by mercury, in obtaining some good results by the usual treatment of the pulmonary tuberculosis. (Vide article on "Syphilis of the Lungs.")

\section{TREATMENT OF CHRONIC PULMONARY TUBER- CULOSIS.}

Do we possess a remedy against chronic pulmonary tuberculosis, which may be called specific? Before we present an answer to this question, we must say that the nature of a specific has not been, as yet, clearly defined. Thus, as the most typical specific remedy, we consider quinine in malaria, which last is an infectious disease; whereas modern medicaliescn ce searches for specific remedies against infectious diseases in the products of the vital activity of the micro-organisms, which 
are considered as the causative agencies of these diseases; consequently these remedies are of a nature totally different from that of quinine.*

Such being the condition of things, I can only define the nature of a specific remedy in the words I spoke while discussing the treatment of cholera: "a specific remedy is not one which exerts its effect upon one certain symptom of the disease, but one that strikes, so to say, the very centre of this last, and by acting at once on all the symptoms, breaks them up, causing a cessation of the disease, as quinine does in malaria, and sodium salycilate in acute articular rheumatism." If we are to regard a specific drug from such a point of view, then there is no doubt that we have no drug whose effect in tuberculosis could be comparable to the action of quinine in malaria; but the effect produced by creosote in tuberculosis is such that it cannot be called symptomatic only; its action is, besides, evident only in tuberculosis or in cases of predisposition to the same, and, as far as I know, in no other disease.

Let us now turn to the treatment of tuberculous patients as based upon facts.

Symptomatic treatment, by which we mean the removal of all the distressing and debilitating symptoms, plays an important part. During the discussion of the previously presented cases of chronic tuberculosis of the lungs I had occasion to speak of the treatment of all the symptoms of this nature, except hæmoptysis and fever, which I will take up presently.

During hæmoptysis the patient must preserve quietude and be silent. If there are no contra-indications, in the form of a diarrhœa or extreme debility of the patient or in the degree of the hæmoptysis, we must first of all administer a laxative (an eccoprotic, not a drastic) and then secale cornutum, an

* Let me recommend you an interesting, although somewhat verbose and lengthy (a common fault of the majority of modern writers) article of Behring's (Prof. Behring, "Die ätiologisch-therapeutische Bestrebungen der Gegenwart," Deutsche med. Wochenschrift, 1893), in which the author, being a partisan of Koch's, criticises sharply, but nevertheless judiciously, the general pathological and therapeutic positions of Virchow, which once played an important rôle, and discusses the question of the specific treatment in its past and present condition. 
infusion of $3 \mathrm{ij}$ to $\overline{\mathrm{z}} \mathrm{vj}$, a tablespoonful every hour; to quiet the cough codeine or morphine. For habitual, repeated hæmoptysis two to three leeches to the coccyx (vide article on "Blood-letting" ").

If the fever is not considerable and the rise of temperature does not exceed $38^{\circ}\left(100.4^{\circ} \mathrm{F}\right.$.), then there is usually no necessity for the employment of special measures in combatting it: the creosote treatment, by causing a general improvement, causes also a cessation of the fever. But in case the fever is more considerable, then, at the commencement of the creosote treatment, before it has as yet an opportunity to exert its effects, I prescribe for the morning, during the fall of the temperature, five to ten grains of quinine, and in the evening, during the rise of the temperature, four to eight grains of phenacetine, less frequently three to five grains of antifebrine, and still less so ten to twenty grains of sodium salycilate, and very rarely antipyrin. Most frequently we have to administer antipyretic drugs in the last stage of chronic tuberculosis of the lungs and during its pneumonic complications, to administer them of course until a marked lowering of the temperature is effected, but not until it be brought down to the normal; this last is frequently impossible, always useless, and besides risky, as it requires large doses of the mentioned antipyretics, and may thus cause a dangerous weakening of the heart.

The most important part is played by improvement in the nutrition (and this is usually accompanied by an increase of patient's strength), whether it result, in connection with the successful employment of the symptomatic treatment, from the application of hygienic measures only or from them and the additional use for this purpose of various remedial agencies, the most important of which are koumyss and creosote. It is only with the aid of improved nutrition and strength, that we obtain the good results to which we alluded in discussing the prognosis.

Koumyss. - Until the time when large doses of creosote were introduced into medical practice for the treatment of chronic pulmonary tuberculosis, koumyss was considered the best means for improving, in a very short time, the nutrition 
and strength, and also the local symptoms. But since then, koumyss has become less important. First, because creosote, although somewhat slower, gives the same results; and whereas the koumyss treatment is only possible during the three summer months (from the middle of May till the middle of August) after which it must be interrupted for nine months, during which time the obtained results are either partly or entirely lost, the treatment with creosote can be continued constantly, only with those short (about one month) interruptions, which are required by the peculiarities of the creosote treatment itself.

Secondly, the treatment with ereosote is incomparably more accessible than a trip to the koumyss sanatarium, which involves considerable expense. worry, and finally the risk of not being able to obtain good koumyss, or good board, lodging, etc.

It would, however, be erroneous to assume that koumyss has entirely lost its importance. There may occur many cases in which the koumyss treatment is both more convenient for the patient and indicated either during the interruption in the creosote treatment or when this last is not well borne or does not give the expected result, which last circumstance occurs, however, only in the minority of cases treated by creosote. Moreover, koumyss is beneficial in the treatment of other diseases, ontside of chronic pulmonary tuberculosis. It constitutes an unreplaceable medicinal agency for effecting a rapid recovery of health which has suffered from serious maladies or from a prolonged residence under unfavorable hygienic conditions. Finally, koumyss is useful not only for improving the health, but, by causing a sound and prolonged sleep, for its strong effect upon the nervous system: koumyss is an excellent remedial agent in neurasthenia. If the neurasthenic patient is not thin, the more so if he be stout, then the best thing for him is bathing in the river, and, even better still, in the sea (up to a certain age, after which baths at the temperature of $27^{\circ} R$. $\left(92.8^{\circ} \mathrm{F}\right.$ ) are more suitable; of this we shall speak in due time); but if the nutrition of the neurasthenic patient has failed considerably, then bathing may become very harmful to him, while the baths at $27^{\circ} R$. (92.8 $\mathrm{F}$.), even if harmless, will be but of little benefit 
and but for a short time, during the employment of these baths ; whereas koumyss in such cases, by improving the nutrition and by exerting a powerful influence on the nervous system, may bring about a complete recovery of health. In the same cases of neurasthenia, which are accompanied by a failure of nutrition, koumyss by its effects is superior to the best apothecary drugs used against neurasthenia, as arsenic and silver nitrate; (these last are preferred to koumyss only because they may be used at any time of the year). I will add here, that we meet very often cases of neurasthenia complicating chronic pulmonary tuberculosis.

While speaking of the koumyss treatment, I mentioned trips to the koumyss sanatoria. This must be explained. The fame of koumyss as a curative agency, a fame which is fully merited, as I can testify also by my own practical experience, has originated in the locality between the Volga and the Oural. There existed before, and there exist to an extent now, vast feeding grounds, which make it possible to breed horses in great herds; the summer there is mostly dry and warm, even hot. Only during such a summer can the patients use the considerable quantities of koumyss, so necessary for obtaining good curative results; only at such localities, from such an abundance of young healthy mares, which are not used for labor, but feed on the abundant pasture-grounds, will the patients obtain good koumyss in a sufficient quantity. The attempts to resort to koumyss treatment in the absence of such conditions, even in the cities (compare the air of the city with that of the field) or even during the winter (!) months, give, of course, miserable results, and are usually harmful (as the koumyss is not borne well and causes a diarrhœa), and has surely no claim to be regarded as koumyss treatment.*

Treatment with cod-liver oil (during the cold season of the year), with keffir, less frequently with milk and the farinaceous preparations, may serve as a good aid, in improving the nutrition, to the other treatment, but it certainly cannot be compared to the genuine koumyss treatment.

* Prof. N. F. Goluboff has written a great deal on the subject of koumyss treatment. 
The treatment with arsenic was spoken of before, and particularly its use in chlorosis and chloranæmia with a predisposition to pulmonary tuberculosis.

Creosote, guaiacol, and guaiacol carbonate.- How these remedies exert their effects - whether directly on the tuberculous bacilli (which is denied by some) or only by strengthening the organism, and thus diminishing the predisposition to the development of these last - is not known; but the fact that they are effective is beyond any doubt, and is recognized as such by a majority of most competent practitioners. My own practical experience speaks in favor of the subject in a similar manner. There are also heard dissenting opinions, which are due partly to insufficient observations, and partly to the fact that the creosote, guaiacol and its carbonate are not always of the best quality, and do not always have the same constituents. The effect itself of these remedies consists in this, that as a result of their prolonged and gradually increased use, the appetite is improved, the former slight dyspeptic symptoms disappear, the nutrition and strength improve considerably, at times strikingly so, and together with these, also the local symptoms.

The above-named remedies are used at the beginning in small and later on in gradually increasing doses, as, for instance, creosote or guaiacol at first five drops (guaiacol carbonate five grains), once a day, and later, more and more frequently, always after a meal, and, when taking frequent doses, if not after a meal, then in a half-glassful of milk; if they are taken in cachets or capsules, then one-fourth of a glass of milk is taken before the capsule and another one-fourth after it. The rapidity with which the dose is to be increased depends on how well the remedies are borne and on their effects; if, after taking them, there is felt an unpleasant heaviness in the stomach and the appetite is not improved, then the dose remains the same; but if, as usually happens, the appetite is improved, and there is no heaviness in the stomach, but on the contrary even the formerly present - before the beginning of the treatmentdyspeptic symptoms disappear, then the dose is increased up to thirty, sixty, and more drops in the twenty-four hours. At times the treatment must be interrupted, say, for two to four 
weeks after two months of treatment; we must, of course, in doing so, take into consideration the patient's individuality and the successful results of the treatment: the more considerable these last, the less necessity there is for hurrying on with the treatment. The interruptions are made with the aim in view of not injuring the stomach and the kidneys; at times, although it may be rarely, during the treatment with the above-named remedies, there is observed the appearance of albuminuria, which usually passes away after the cessation — in such cases necessary — of their empioyment.

Creosote and guaiacol do not present any notable difference in their effects : the guaiacol carbonate is at times better borne by the stomach, but it is high-priced and evidently exerts a weaker effect than creosote or guaiacol ; such is at least my impression, received from its use in several cases.

We are rarely compelled to introduce creosote or guaiacol into the system through other ways than the stomach. The slight dyspeptic symptoms, so frequently seen in patients with chronic pulmonary tuberculosis, depend usually upon the general weakening of nutrition, of the function of hæmatopoiesis and of the nervous system, and are not due to a chronic gastric cataris; a gradual and carefully conducted treatment with guaiacol or creosote not only usually ameliorates these, but with the improvement in the general condition, the symptoms pass away entirely. But if these dyspeptic symptoms be considerable, then we can usually succeed in removing them by preliminary treatment, and bring the stomach into a condition which would enable it to bear well creosote, guaiacol, or at least guaiacol carbonate, as was the case with our patient who suffered with chloranemia and incipient tuberculosis (vide 18th case). But still I had occasion to observe a case of chronic pulmonary tuberculosis complicated by a very obstinate chronic gastric catarrh of an independent origin, not due to the pulmonary trouble, in which creosote was not borne by the stomach, even after the latter had been subjected to a preliminary treatment, and the patient improved only after a prolonged introduction of great quantities of creosote - dissolved in olive oil — by subcutaneous injections, usually into the buttocks. 
The introduction of creosote through the respiratory tract is usually badly borne (it causes severe coughing), and does not produce eren a shadow of the effect which is obtained by the continuous and gradually increasing administration of the drug through the stomach. The inhalations of small quantities of creosote are sometimes well borne, but outside of a slight relief in loosening the expectoration and some improrement of this last (patients sometimes find that the expectoration. during the inhalation of creosote, is of a less disgusting taste and odor), it induces no marked change for the better in the local symptoms and in the condition of the affected portions of the lungs. I may add that the same may be said also regarding the other remedies of the inhalation therapy, as eucalyptus oil, ol. terebenth. rectific.. - which is more beneficial in bronchial catarrh complicating chronic pulmonary tuberculosis, and certainly also in a plain bronchitis, _ menthol, Perurian balsam and others.

All that was said of the use of koumyss, creosote and other remedies in chronic pulmonary tuberculosis, does not refer to the last stage of this disease : at this stage these, as well as all other, remedies are but little effective; we have as yet no reliable means for the treatment of the disease at this period. When chronic pulmonary tuberculosis is complicated by catarrhal and caseous pneumonia, then koumyss is of course of no avail; of the use of creosote in cases of these pneumonic complications I can say nothing: since I am using creosote in considerable doses, I observed such complications of chronic pulmonary tuberculosis but rarely; there are no sufficient data in the literature on the subject.

The tendency, so peculiar to modern medical bacteriology and which promises so much for the future, of searching in the pathogenic micro-organisms themselves, in the products of their life-activity, for means for immunization against the diseases produced by these micro-organisms, and also for the treatment of these diseases, has not resulted as yet, as far as tuberculosis is concerned, in anything of practical importance. I have spoken elsewhere (in an article entitled " Treatment of tuberculosis by Koch's tuberculin" appended to the Russian edition of the 
lectures) of the thoughtless, I may say precipitous treatment of tuberculosis by tuberculin - an attempt the failure of which can only be measured by the world-wide hopes its appearance has excited.

In passing from the therapy of the patients suffering with chronic pulmonary tuberculosis to their hygienic treatment, we must principally take up their climatic hygiene, which is usually, but less correctly, called climatic therapy; but the importance of this subject and the approaching close of the lecture hour compel us to postpone it till the next lecture.

\section{THE CLIMATIC THERAPY OF CHRONIC PULMONARY TUBERCULOSIS.}

\section{LECTURE OF APRIL 16, 1893.}

For a long time I have made it the business of my clinical instruction to discuss this subject not only in view of its importance and the entire ignorance on the part of the students about it, but also because it cannot be denied, that the majority of the practitioners are but slightly acquainted with the subject, and do not therefore prescribe the climatic treatment correctly. On the contrary, as we very frequently see by the physicians' advices, the climatic therapy is expected to yield what it cannot, and no advantage is taken of what it can give.

Later on I shall illustrate my assertions by sufficiently conclusive examples.

Before we speak of the climatic therapy of chronic pulmonary tuberculosis, we must say a few words regarding climate and climatic therapy in general. Climate (Gr. $\kappa \lambda i^{\prime} \mu \alpha[\tau-]$, a region, zone) denotes, properly speaking, a distance between two circles parallel to the equator. In connection with climatic therapy, climate is considered as the sum total of all the influences of the given place, which include its quantity of sunlight, its temperature, air, waters, soil and vegetation; besides these, in choosing a certain locality with a climato-therapeutic aim in view, we must also take into account the various changes in the above-named influences which take place during the various seasons of the 
year, and also the conditions of life in the given locality, the peculiar traits of its population, the residence, the diet, etc.

You thus comprehend that climate is something of a very complex and changeable nature; neither climatotherapy, nor even climatology are therefore much advanced. Thus, for instance, meteorology (the most important basis of climatology) can foretell the condition of the weather for the next twentyfour hours, but is unable to predict its condition, for the given locality, for the approaching season of the year, for instance, for the winter, which would be a matter of great importance for climatotherapy, because the climate differs not only during the various seasons of the year, but during one and the same season of the year it is liable to be variable in different years: one year, for instance, the winter is cold, the next year it is warm, or with a clear and quiet weather, or rainy, snowy, windy, etc.

The complexity and inconstancy of the climatic influences, in the undeveloped state of climatology, make it impossible to establish any satisfactory climatotherapeutic classification of the climates. Webber, the author of the article on climatotherapy in the well-known collective edition of Ziemssen's Handbuch der allgemeinen Therapie, states that he was tempted to describe the climatic sanatoria in alphabetical orcler, but the necessity of avoiding repetitions so inconvenient in a text-book prevented him from doing this. As a great many others, he divides the climatic sanatoria into those situated near the seashore (these again being subdivided into warm, cool, damp and dry ones), and those situated inland (with subdivisions into mountainous and low); but this subdivision certainly does not do away with the necessity, while selecting any climatic sanatorium, for carefully considering not only its seaside or inland position, its high or low altitude, its warmth or coolness, but also all the remaining numerous climatic and life peculiarities and the manner of their combination, by availing oneself of the aid found in literature, or better still, if possible, of the opportunity of a personal acquaintance with such places.

Such being the condition of climatology and of climatotherapy, in discussing the special climatotherapeutic question, namely, that of the climatic treatment of chronic pulmonary 
tuberculosis, I consider it best, without touching upon the unsatisfactory division and the characteristics of the climates, and giving a short review of the elements of climate and its therapeutic, or, rather, hygienic, effect in general, to give an historical exposition touching at least the last decades of the climatic treatment of chronic pulmonary tuberculosis. I am led to think that this will be the best method for explaining the nature of the climatic treatment not only of this disease, but of the whole idea in a general way, in so far as we can at least judge of this subject in the present condition of our knowledge; I think thus, because, although the question of the climatic treatment of chronic pulmonary tuberculosis is but a special one in the domain of climatotherapy, it is undoubtedly the most important one.*

\section{THE ELEMENTS OF CLIMATE.}

The temperature of the locality. - Heat weakens the nervous system (causing fatigue, disinclination to work, sleepiness), the appetite and nutrition. Cold excites the appetite keenly; it is therefore unsuitable for debilitated persons with poor digestive powers, who also evince an inclination to colds and to diseases dependant thereon. Such patients are best benefited by localities with a moderate temperature, one which is neither hot nor cold, and withal as constant as possible. undergoing but few changes. because a rapidly and pronouncedly changeable temperature tends to aggravate and to cause in such patients cold and diseases resulting therefrom. On the contrary, for neurasthenics a moderately changeable temperature is suitable, with moderate winds. as, for instance, the temperature of seaside localities; of this we shall speak when discussing balneotherapy.

* The hand- and text-books of climatotlerapy, besides the climatic therapy of chronic pulmonary tuberculosis, usually nention this therapy for the following diseases: catarrhal and "rheumatic," gout, scrofulosis, various anæmic and nervous diseases (neurasthenia, hypochondriasis, etc.); however, there are certainly but few diseases in which correct climatic conditions would not be of importance. 
2. Moderate sunlight acts beneficially on the mental condition, on the nervous system, and on nutrition. For winter, therefore, the localities where the day is mostly clear are particularly suitable; during summer, on the contrary, when there is a superabundance of sunlight, shady, woody places are preferable.

3. The air. - Moisture in the air. - Damp air is generally deleterious: damp and hot air increases the noxious effect of heat; damp and cold air has even a greater tendency to cause cold and diseases due to it, than cold air which is not damp. Very dry air, either warm or cold, irritates the mucous membranes and increases the tendency to cough and hrmoptysis. An average degree of moisture in the air is therefore a necessary attribute of a good climatic sanatorium. Considerable cloudiness of the sky and severe rains are harmful, as they increase the amount of moisture in the air and in the soil, and tend to decrease the amount of sunlight, which last is generally slight during the winter; but moderate rains are useful because they purify the atmosphere. Snow is beneficial for the same reason, and also because the covering of snow prevents the air from becoming contaminated by the various kinds of dust which rise from the ground.

The density of the air, the pressure of the air. - It is thought that the air of the seaside localities, under the greatest pressure and therefore the densest and the richest in oxygen, excites the appetite more readily than the rarefied air of mountainous localities at high altitudes; but this circumstance is usually counterbalanced by the necessity of a greater amount of physical exertion, of greater muscular labor as spent in frequent ascensions in a mountainous locality. The considerable alterations in respiration and circulation observed on ascending very high mountains or on ascending in a balloon have no significance for climatic therapy, because the climatic sanatoria are mostly located at altitudes between 500 and 1500 meters; the rarefaction of the air peculiar to such altitudes does not produce to any marked extent such influence on respiration and circulation, and on the organism in general, as is produced by the rarefied air of the high mountains. The great development of the chest, so frequently observed in inhabitants 
of the mountains, must be ascribed not to the rarefaction of the mountainous air and the increased respiratory movements consequent thereon, but rather to increased depth of the same movements as necessitated by frequent ascensions.

The motions of the air, the winds, are of enormous importance climatically : some winds carry with them warmth, moisten the dry air, and clear the atmosphere of dust; others bring with them cold or heat, superfluous moisture (dampness), or, on the contrary, dryness, dust. A good climatic sanatorium must be protected from noxious winds by an elevation of the land, or by a forest.

The clearness of the air, the freedom from all kinds of dust, inorganic, organic dead and living (as micro-organisms), is the highest, most necessary requisite of a climatic sanatorium.

The presence of ozone in the air is beneficial.

4. The soil. - The relief of the soil, in the shape of elevations or mountam ranges, which.serve as a protection against noxious winds, is directly important in determining the value of a climatic sanatorium. A damp soil predisposes to malaria, is deleterious to those suffering from catarrhal and "rheumatic" affections, as well as for those afflicted with chronic pulmonary tuberculosis, and for many other patients. A dry but bare soil, only sparsely covered with vegetation, which fills the air with dust, is particularly harmful for patients with chest troubles, for whom the best is a dry soil, but one covered with vegetation, or, during the cold season of the year, with snow.

5. Waters. - A good water for drinking purposes is certainly a necessity at a climatic sanatorium. The neighborhood of the sea, which moistens the atmosphere, purifies it by the aid of an almost constant wind, modifies the extremes of temperature, at times warms the locality, as by warm sea-currents, and presents frequently the possibility of profiting by the beneficial - as for neurasthenics - sea-bathing - - through all these influences forms a very important preference for a climatic sanatorium.

6. The vegetation, by covering the soil, prevents the formation of dust, and by absorbing the carbonic acid and yielding up the oxygen, purifies the air and assists in the formation of ozone. Forests form an excellent protection against the winds 
and, during the summer, against the excessive heat of the sun.

7. The beauty of the locality (the view on the sea, mountains, etc.) exerts a favorable psychical influence and plays by no means an unimportant part in the selection of a sanatorium.

8. The value of a trip to a sanatorium, that is, the importance of removing the patient from the habitual conditions of life (at times unfavorable, as, for instance, an unhealthy locality, excessive occupation, worries, etc.) and of a change in the mode of living, by instituting exercises in the open air, and also the importance of the conditions surrounding the life at a climatic sanatorium - partly needs no explanation, and was, to some extent, already spoken of when we discussed the internal use of mineral waters; we shall also touch upon the subject later on, as well as when we come to discuss the subject of balneotherapy.

We will now take up the climatic treatment of chronic pulmonary tuberculosis.

$\mathrm{Up}$ to within the fifties of the present century its treatment consisted chiefly in this, that the patients, during the cold season of the year, were sent away to localities with a prevailing warm climate, at first to places on the shore of the Mediterranean Sea protected by the Alps from the cold winds (the Riviera), as Cannes, Nice, Mentone, San Remo, etc., and later on to neighboring places around Cairo (Heluan), Algeria and to the Madeiras. In these localities, besides the warmth and the protection from the cold winds, there was greatly appreciated the absence of sharp variations in the temperature. Finally patients with a dry cough and with an inclination to hæmoptysis were advised to avoid localities with dry air, as, for example, the environments of Cairo, and to go to places where the air is sufficiently moist (as, for instance, Madeira), while for patients with abundant expectoration, on the contrary.

Some thirty-five years ago, the physicians of Europe turned their attention to the treatment of chronic pulmonary tuberculosis by residence in the mountains. Observations in mountainous localities induced them to do so: thus it was found that 
in Peru, where at the seaside localities the above-named disease is seen frequently, and where it rapidly brings on a fatal termination, a residence for the patients during a long period in the mountainous plains - at the altitude of 3000 meters - in the neighboring Andes has been considered since a long time as the ordinary and only successful treatment.

Meeting with scarcely any cases of chronic pulnonary tuberculosis at such high altitudes, observers came to the conclusion that the success of this treatment, consisting of a prolonged residence in plains situated at high altitudes, depends on the presence in such localities of a peculiar "immunity" as regards the above-named disease. The principal element, which was considered as exerting a favorable influence on the patients suffering from chronic pulmonary tuberculosis during their residence on high altitudes, was the rarefaction of the air, which, as it were, excited increased respiratory movements, brought about a better ventilation of the air, and tended to develop the chest, etc. But neither supposition was found to be true. It was proved that pulmonary tuberculosis is also met with in mountainous localities, although there it is of much rarer occurrence thanks to the absence of factory life and similar conditions, the population not being huddled together, as in localities with a dense population, under unfavorable conditions of life; while at the same time it was found, that even in Switzerland, in the factory districts, situated as they are on high altitudes, there is quite a number of consumptives. The importance of rarefied air in climatic mountain sanatoria has been spoken of before.

The German physician Brehmer, deeply convinced of the curative properties of the climate of mountainous localities for tuberculous patients, was the first to establish, or he thought he established, such a sanatorium; I said he thought because the place selected by him, in the vicinity of the village of Görbersdorf, in Silesia, at an altitude of hardly 550 meters, can not be called a truly mountainous locality. But even without the features of a really mountainous place, Görbersdorf is distinguished by its excellent climate during summer, which is not hot, but cool, almost without rains, and is protected from noxious winds; in connection with this the air is remarkably pure, free 
from dust and rich in ozone, thanks to the abundance of trees, especially of firs: it abounds therefore in many pleasant, shady walks; the soil is hard.

Brehmer was convinced of both the "immunity" of mountainous localities as regards tuberculosis and of the beneficial influence of the rarefied air of the mountains on tuberculous patients, although the environs of Görbersdorf present cases of consumption; and as regards the rarefaction of the air - not to repeat what was said before, that there was no foundation for ascribing to it any special benefit for the consumptives we can not speak of the presence of any considerable rarefaction of the air on such but slightly elevated locality as Görbersdorf. Besides that, Brehmer entertained a theory of his own regarding the beneficial influence of mountainous localities on tuberculous patients, namely: tuberculosis attacks persons whose lungs are large, but whose hearts are of small dimensions, and in whom therefore the lungs are not sufficiently supplied with blood and are poorly nourished, and who through such conditions become predisposed to tuberculous affections; on the mountains the pulse becomes more frequent, which means that the activity of the heart is increased, the lungs receive a greater supply of blood, they are better nourished, and therefore are better able to withstand tuberculosis and can sooner be cured of it. This theory was of course proved to be without any pathologo-anatomical foundation in fact, not to speak of the insignificant influence of such a slightly elevated locality as Görbersdorf on the increase of the frequency of the pulse.

But all this notwithstanding, Brehmer achieved a greater success in the treatment of patients afflicted with chronic pulmonary tuberculosis, than was obtained by the ordinary, at that time, treatment of such patients. This fact was recognized by competent German physicians and clinicists and has led to the establishment of sanatoria after that of Görbersdorf, with the application of Brehmer's method of treatment. This "method of treatment," to which Brehmer is so much indebted for his successes, consists properly speaking of a strict observance of hygiene. Thus, in the other climatic sanatoria patients, under the impression that the climate will do everything and that every- 
thing is possible in such a climate, neglected the necessary hygiene as much as they did in their own homes, permitted themselves to smoke, to drink too much wine, did not observe the diet regularly, spent the nights in playing cards, in visiting the theatre, etc., and therefore took no advantage of the pleasant hours during the day while the sun is up, nor of the fresh air and out-door exercises. Brehmer was remorseless in requiring of his patients the strictest observance of hygiene; he insisted upon the avoidance of pernicious habits in the mode of living, upon going to bed in due time, upon as prolonged out-door life as possible, upon exercise compatible with the patient's strength; he required a correct diet (in the form of frequent administration of nutritious food) and the use of wine ; and, besides all these, he ventured to introduce hydrotherapy into the treatment of tuberculous patients. It is true, that both the use of wine and the application of hydrotherapy were conducted without a plan and with too great impulsiveness (thus, for instance, the patients were given too strong douches) and at times they were productive of harm; but it was nevertheless shown, that wine and such moderate hydrotherapeutic procedures as sponging, when not prescribed aimlessly but by observing the individual peculiarities of every patient, are of benefit to many suffering with chronic pulmonary tubereulosis.

Of the various sanatoria, which were established in imitation of that at Görbersdorf, we must stop to consider the one best known of all, namely, Davos in Switzerland. Davos is a wide tableland situated at an altitude of 1960 meters, protected by mountains from northerly and northwesterly winds. The best season of the year is the advanced winter, when the covering of snow is constant, the air is pure when there is no wind, the sky is almost always clear and the sun sends down sufficient warmth; thanks to this, as well as to the dryness and the quietude in the air, it is so warm that the patients are able to be up and about out-of-doors in comparatively light garments. During the other seasons of the year there arrive the unpleasant local winds from the mountains and plains and then, in the autumn, when the snow begins to fall, and in the spring when it thaws, the climate is damp and inconstant; and the summer is warm, there is but lit- 
tle shade, and it is dusty. The climate of Görbersdorf is therefore better during the summer, but there the winter is worse, because during it the weather is often damp and changeable. The majority of other European health-resorts, established on the plan of that at Görbersdorf, have not the same distinctly mountainous character as Davos has, and resemble in this respect Görbersdorf.

Many physicians when comparing Davos with the warm climatic places, for instance with those on the Riviera, give the preference to Davos for the following reasons: the air at Davos. thanks to the snow-covered ground, is so pure that it stands in this respect above comparison with the air at the above-named warm places; at Davos there is no wind during the winter and the climate is therefore more constant than in the warm localities where noxious winds prevail; in these places the winter is not only warm, but even hot, whereas at Davos it is just warm enough to enable one to be up and about dressed lightly: thanks to this the appetite of the patients living at Davos is better than in those living at the above-named warmer sanatoria.

From the above you can clearly perceive what an important change took place during the last decades in the climatic treatment of chronic pulmonary tuberculosis: whereas formerly the salvation of the patients living in the middle and the northern parts of Europe and generally in countries with a similar climate, was considered to lie in a trip to the South and a prolonged residence there during the cold season of the year, - a trip which was often beset with difficulties and entailed hardships on the patients and their families, which was frequently attended with the risk of a failure, and often impossible for many, at the present time such patients are enabled to apply the climatic therapy during the winter season much nearer to their own homes.

There is also a possibility for this treatment with us, not only in our mountainous localities, but also in the plains so largely prevalent in Russia. The slight relief of the soil in the form of a forest, especially of fir-trees, serves as a sufficient protection from cold winds and exerts a modifying influence on the winter cold, if we desire to select a place which is located 
on the inclines of the soil facing the south. The cover of snow insures pure air. If during the winter, in the severe colds, the patients will be compelled to confine themselves within the house for a longer time than in Western Europe, then during the autumn and spring, when we have yet some frost, while there it is already thawing and damp weather, the condition of things will be reversed. If, finally, on account of the severity of our climate its influence during winter will be inferior to that of IV estern Europe (and this I question!), then how many times will this disadvantage be outweighed by the possibility of avoiding the above alluded to difficulties and risks, which are inseparable from far journeys and prolonged sojourn in remote countries; it will remove the necessity for expenses permitted but to a few and frequently bringing ruin to the welfare of many families, necessitating separation from the familiar surrounding circumstances and business on the one hand, and compulsory, oppressive idleness in a strange land on the other hand.

Unfortunately with us it is as yet an affair of the future. Up to now there prevail though to a slighter extent than formerly, advices for trips to "the South" and "over the border" (meaning Western Europe). Many physicians prefer the ready advice "Take a trip to Cannes, Mentone, San Remo, etc., or to Görbersdorf, Davos," to the one which requires more labor to explain as to how the patient should conduct himself in the climatic circumstances which surround him or, in case these be unsatisfactory, how to take advantage of those near by, frequently of the very near and directly neighboring localities with favorable climatic conditions. Besides, it frequently happens that the advice to go to the South or to Europe is given with such abruptness and so negligently, that the patient decides to undergo the necessary expenses for the trip, under the impression that the trip itself will bring about a cure; he perceives later on, that the trip failed to cure (it frequently does not even bring about an improvement in health), and that not only a cure but even a satisfactory improvement in health requires a long time, treatment, and a constant observance of hygiene; having given up the trips which became impossible, his means and affairs 
ruined, he ends by what the physician should have advised him to begin, namely by attempting to improve his health not through temporary, fruitless measures, as trips to the south, but by changing his mode of living in accord with the conditions of his health and by taking ably advantage of the climatic opportunities presented by the local place of residence or by the one nearest to it.

A few cases from my practice will serve as illustrations of what I said.

1. This case, which I observed some twenty-five years ago, has remained indelibly in my mind, as it produced a strong impression, and has been the means of my inaugurating the present practice in the domain of climatic therapy. The patient, forty years old, of average constitution, sober and nonsyphilitic, was a grain merchant on the Moscow baggage station of the Moscow Ryazan Railroad. He passed the whole day either in talking (usually loudly, rapidly, without rest, and frequently also shouting) out in the dusty or cold air, or in drinking tea in the tavern. When I saw the patient, he had been suffering already for about a year and a half from chronic pulmonary tuberculosis of the right lung (approximately the upper third of the superior lobe was attacked), had frequently expectorated blood, was often feverish, grew thin, but was as yet strong.

After having removed by symptomatic treatment (leeches to the coccyx, quinime, pulvis Doveri) the acute condition for which the patient consulted me, I made it clear to him that a satisfactory improvement of his health is impossible with his present mode of living, and inquired whether he would be able to take a trip to the South (it was during winter then), warning him, however, that there might arise a necessity for more than one trip. Patient replied that neither his competency nor his family relations permitted him to even attempt such a thing. Further inquiries elicited the fact, that he had in his possession, some 200 miles to the southeast of Moscow, a pleasant country place which is neither dusty nor damp, and a fair little house; some vegetation, various household buildings and a barn could form a distinct pro- 
tection from the winds; it was also found that the patient could give up his present occupation, and having settled down in his country place, would be able to find also some occupation there.

Having prescribed for the patient treatment with cod-liver oil (we did not, at those times, give creosote in large doses) and the drinking, in the morning, of Obersalzbrunnen with milk, and having instructed him as to what he was to do in case of the appearance of fever and the increase of the cough, I positively advised him to leave Moscow and to move to the country place, explaining to him the hygiene in particular: to permit a temperature in the room which should not be hot, to have a warm water-closet, to use but lukewarm and no hot drinks, to replace the greater part of tea by milk; nor must his food be hot; to take a bath, not a hot one, during the warm season of the year only, and that not frequently; during the cold season to sponge the body with a mixture of warm water and brancly; but the chief thing - not to go out-ofdoors during severe frosts, when the wind is cold and when there is no protection against its blasts, or when the air is very damp, or occasionally dusty; and on the other hand to take advantage at any time of staying out-of-doors, when the frost is not severe, say between eight or ten degrees (patient was always habituated to cold), when the weather is quiet and protection against the wind may be obtained along the various country buildings and around the garden or the grove, when the air is neither damp, nor dusty; not to talk during the cold season of the year while out-of-doors, and generally to talk only as much as necessary, not loudly, and drawing the breath carefully; finally, to devote himself to some occupation, but not to work beyond his strength, never permitting himself to become fatigued.

The patient, being an intelligent, careful man with a strong will power, followed my advice to the letter and appeared before me a year later (during May of the next year), astonishing me by such a marked improvement in his health than which I saw no greater, not only from one winter's sojourn, but from that of several winters spent by such patients in the South: 
the nutrition was completely improved, he gathered considerable strength, there was no hæmoptysis; fever and aggravation of the cough occurred but at rare intervals, incomparably less frequently than before, the cough in general and the quantity of expectoration diminished, the dullness at the area of the affected place changed but little, but the area over which râles had been heard decreased considerably. I must add that the patient did not give up his accustomed occupation, and not more than during fifty days was he compelled to confine himself within the house, partly on account of the weather, partly because of the fever and the aggravated cough. During the next seven or eight years, he showed himself to me at first every year, and then every other year, usually in May, always in the same good condition as after the first year of his country life. The further condition of the patient is not known to me; but whoever is familiar with the miserable results generally brought about by the trips to various health-resorts, trips which require constant moving around, from the home to the sanatorium and back, and which cause so much ruin to the family, to the affairs and to the well-being of the patient - whoever knows all this will certainly perceive the impression produced on my mind by the above-described results of the climatic treatment (or to be more precise, by the generally hygienic treatment) at one's own home.

Let me tell you of some eases, peculiar in another respect, observed in my recent practice.

2. In July of last year I was called to see the wife of a country merchant, thirty-one years old, who complained of cough, dyspnœa, emaciation and general weakness, and who desired me to inform her as to whether or not she should go to Yalta (in the Crimea), as advised by her physicians.

The locality where the patient resides'(in the Tula Government) is a poor one, and except winter-time it is either dusty or muddy. The residence is satisfactory, the water-closet cold. The board is good, her beverage consists of liot tea with milk, no alcoholic drinks. Patient has been married for twelve years, has nursed in all eight children, of whom five are alive. No syphilis. Notwithstanding the frequent births, the nursing of the 
children and a considerable amount of housework, the patient assists also her husband, spending daily some five hours in a cold flour-store, situated on a dusty street. Patient is very much attached to the children and the family, and is a typical specimen of a good wife and mother, and of a very painstaking housekeeper.

Until five years ago she had enjoyed good health. Five years ago during autumn patient passed through an attack of pleurisy (severe pains in the left side of the chest, cough, fever; the principal treatment consisted in the application of Spanish flies, which relieved her), after which she began to cough, at first but little, and later on more and more, and became also feverish; but of this she took no notice and continued to work as usual. Two months ago the patient began to feel very ill and took to bed: there appeared severe pains in the left side and a considerable fever, while the formerly moderate cough became aggravated and the expectoration began to show the presence of blood. In the course of three weeks the ferer decreased, but the severe cough, the abundant expectoration and the dyspnœa remained until now, and the patient continues to emaciate and weaken. Her treatment consisted in the application of flies, and during two weeks, until the middle of June, she has been taking six drops of creosote daily; later on, at the beginning of July, patient was advised to take a trip to Yalta.

Status. - Her appetite is poor ; dyspeptic phenomena absent; she is constipated (patient takes two or three times during the week, thirty or more drops of extr. fluid. cascaræ sagradæ). The urine contains a small quantity of albumen and a few hyaline cylinders. Menstruation is regular, of normal quantity and painless. The liver and the spleen are normal; so is the heart, but the pulse is frequent and weak. There is some dyspnoa, congh, expectoration of about two tablespoonfuls a day, at times with an almixture of blood; coughing causes pain in the left side of the chest. On the lower half of the left side of the chest, posteriorly and laterally, there is dullness on percussion. while auscultation elicits bronchial respiratory murmur and some râles; the apex of the left lung shows a characteristic focus with abundant and loud moist râles; objective examination 
of the right side gives normal data. The patient is somewhat feverish, her sleep is sound, and not interrupted by the cough, the head aches when the fever is increased.

The patient and her husband stated to me that the advice to take a trip to Yalta causes them a great deal of anxiety; that such a trip was for them, plain country people, a matter of unusual importance, as it might possibly result in many unpleasant accidents; that it was impossible for the husband to accompany his wife, and, which is most important of all, she would be unable to take along the children, all of whom were very young as yet; that the mother was so attached to them, and they to her, that separation would be intolerably painful and harmful for the mother's health. The husband told me that the wife having gone without the children to live in a country place, she could not stand the separation but for two days and was compelled to return, while the "children were crying all the time during her absence." Further inquiry elicited the fact that the husband possessed, some four or five miles from his constant residence, a section of forest in a pleasant, dry locality, and that he had built there a spacious and clean little house in a place completely protected from wind and dust.

My advice was as follows : I could not approve of a residence of two months' duration at Yalta; on the contrary I feared the possible heat, so harmful for the appetite, and the dust, and I advised the woman to remove together with her children to the above-named country place and to remain there until the appearance of snow, when she might return to her constant residence. I explained to the patient and to her husband that it was not only necessary for her to recover from the disease which attacked her tivo months ago, namely, inflammation of the left lung, but also to get rid of the other affection, which has been ruining her health during the last five years and which settled also in the same lung (tuberculosis of its apex); I made it clear to them that she must therefore remain at the place of her constant residence only during the winter, when thanks to the snow and frost both dust and dampness are absent, while the rest of the time she is to live in the above-named country place, where a more spacious residence is to be built at once, with 
conveniences for winter, with good stoves, and chiefly with double floors and double frames. The husband replied at once that he could do all this very readily, that by autumn the house would be ready for occupancy. To this I added, of course, that the patient must take care of herself, must refrain from fatiguing labor, must drink more milk and less hot tea, and must wear drawers (which she did not do before and which were required in view of a possible nephritis); as what concerns her outdoor life, I repeated to her my instructions as given to the case described above. I further instructed her to resort to the use of clysters in case of constipation, and to take a laxative only in case the clyster proves ineffectual; for the cough to drink Obersalzbrunnen with milk; to take guaiacol in the order explained in the treatment of chronic pulmonary tuberculosis, not exceeding the dose of twenty to twenty-five drops per day (because of the albuminuria, even though it be trifling); in case of weakness to take the stimulating drops (tra. valer. æth. and liqu. Anod. Hoffm. āà).

3. At the beginning of last autumn a land proprietress in one of the country places around Moscow requested of me advice about her daughter. The girl, twelve years old, of a weakly constitution and slightly hysterical, passed a year ago through a severe febrile affection, which left her with a cough and debilitated. The family physician found tuberculous bacilli in the sputum and advised Mentone for the winter. The first two months' residence at Mentone exerted no influence on her health, and only after she commenced taking creosote did her health begin to improve markedly. On her return to Russia in the spring the improvement continued (the treatment with creosote was discontinued by this time), and by autumn only the cough remained, without any expectoration.

Status. - The appetite is excellent, the digestion normal, all the abdominal organs, as well as the heart and the circulation, are in good condition, the nutrition, sleep and strength good. Outside of a moderate dry cough the organs of respiration present nothing abnormal : objective examination fails to elicit any dyspncea, pains or any other pathological changes. The physicians have however again advised her to go to Mentone for the winter. 
The girl's mother declared, that in view of the large family, of her husband's affairs and the serious pecuniary expenses incurred by such a trip, this last presents extremely embarrassing difficulties; that the improvement in the girl's health began not from the time they took up their residence at Mentone. but only two months later, when she began the creosote treatment; that Mentone is crowded and dusty, and that the whole family, removed forcibly from the habitual conditions and eircumstances of life (they always led a country life in a healthy locality, in an excellently arranged country residence. protected from the winds by the woods) felt as if they had been exiled. I told them that I considered the life at Mentone as unsuitable for such a family, and that in my opinion the trip to Mentone and back with all the possible accidents attached thereto, as well as the patient's attempt to disaceustom herself from a climate in which she will after all have to live, were much more risky than to remain over winter at home. in the accustomed, favorable place of residence; I therefore advised to constantly remain in the latter, keeping the same points in riew -- mutatis mutandis - as explained by me in the foregoing cases. As the girl was formerly studying hard, and had at times to pass examinations, which circumstance acted deleteriously on her nervous system. and being of a lively disposition she talked a great deal while out-of-doors, I adrised to entirely give up the examinations conducted in the manner harmful for the nerrous system, to study without unnecessary effort, to talk less, and to strictly refrain from talking when outof-doors during cold weather. As for treatment I prescribed creosote.

Thê above described eases may serve as examples of my habitual climatotherapeutic practice. The results of such practice as regards the lasting recovery of health are incomparably better, in my observation, than the results obtained by frequent trips to far-away places with a totally different climate, and by a prolonged sojoum there. I will not speak of the cares and worries that one avoids in the treatment at home. without these trips. It would howerer be narrow-mindedness to completely deny the benefit derived from trips with climatic treatment in view. 
Each given case must be individualized, as should always be done in therapy: if in the majority of cases such trips are connected with considerable inconvenience and frequently even with serious disturbances, there are on the other hand cases where all these are absent, where on the contrary it becomes beneficial for the patient, not only for climatotherapentic purposes, but also otherwise, to be removed away from various unfavorable conditions surrounding him, and from his habitual mode of living. But it may be stated in a general way, that a trip with climatic treatment in view is particnlarly indicated in those cases, in which a single trip and but a short sojourn may achieve the purpose of bringing about a recovery of health, as, for instance, in cases of debility after an attack of a severe disease. I had already once occasion to mention the indications for such a trip while analyzing our first case. (Vide the lecture of October 18, 1889, at the end.) But in cases of chronic diseases, principally in chronic pulmonary tuberculosis, in which lasting improvement in and preservation of health requires not only a prolonged, but a constant residence among favorable climatic and generally hygienic surroundings, a trip to a far-away, the more so to a foreign, land is indicated only at the beginning, when it becomes necessary to at once put the patient into favorable climatic conditions, and when such are not easily found at home; but later on it is necessary to search for such conditions in the place of residence or near by, at any rate in the native land, in one's fatherland, where the patient is after all enabled to have some occupation proportionate to his strength (patients with chronic pulmonary tuberculosis are entirely incapable of any activity only in the last. phthisical stage), which would to some extent satisfy him and give him means for a livelihood, which is very rarely possible in a foreign land.

We have discussed at sufficient length the general hygiene of patients with chronic pulmonary tuberculosis several times before, and also while discussing the therapy of this disease. 


\section{TWENTY-SECOND CASE.}

\section{LECTURE OF MARCH 3, 1893.}

Patient, a peasant, nineteen years old, was admitted to the clinic March 1st, complaining of severe dyspnœa, of pain in the left side of the chest, of headache and general weakness.

Mode of living and anammesis. - For the last five years patient has lived at Moscow. His rooms are dry and warm, but being a clerk in a green-grocer's shop, he spends his whole day in a basement floor where the store is located. Bathes himself at times during the summer, takes a hot bath once in two weeks, drinks a sufficient quantity of tea, but no brandy; does not smoke. His board is that of a workingman. Patient is single, never had any venereal diseases.

Patient comes from a healthy family; he had smallpox during childhood, but since then up to the present illness enjoyed good health. February the 27th he remained for a long time in an ice-cellar, was thoroughly chilled and in the evening felt a pain in the left side, had a severe chill and later fever which continued up to the time he entered the clinic, March 1st. On the day of his admission patient was given twelve onegrain powders of calomel, one every hour (before that he did not move his bowels for two clays). He had four abundant characteristic calomel evacuations, attended with slight pain : he was then given five drops of tra. opii and his abdomen was wrapped in some flannel; after this the intestinal pains quieted down and the loosenesss of the bowels ceased. He was given twice a day twenty-five stimulant drops (tra. valer. xether. and liqu. anod. Hofmanni $\bar{a} \bar{a}$ ) and the left side of the chest was liept warm by rubbing with warm oil, and then wrapping it with cotton-wadding and flannel.

Status. - Average constitution. He has no appetite, there 
are no dyspeptic phenomena, nor were there any previous to the disease. Had regular stools before he fell sick; had no stool during the first two days of the disease, then was given calomel, later opium. During the last twenty-four hours he had one stool, somewhat liquid but without pain. The urine passes freely, 500 c.c. during the twenty-four hours, is rich in urates, contains neither sugar, nor albumen. The abdomen, as well as the whole body, is slightly sensitive to pressure; there is a hyperasthesia cutis in comnection with a high temperature. The liver and spleen are normal. The heart, except for the frequency of the contractions (pulse 100), is normal; the pulse, which was somewhat weak on admission, is now good. There is some dyspnota, — respiration 32, on admission 36 per minute, some trifing pain in the left side of the chest, a slight cough, with some thick riscid expectoration of a rusty color. There is a dull percussion sound under the left scapula, bronchial breathing and vocal fremitus are more pronounced than under the right scapula. Objective examination in the other regions of the chest elicits nothing alonormal. Headache and dizziness on getting up. His sleep is fair. There is general debility. The course of the temperature: the morning of the day before yesterday, on admission, 40. $2^{\circ} R$. (104.4 $F$.), evening (he had before this some calomel stools), $39^{\circ} R .\left(102.2^{\circ} F\right.$.), yesterday morning, $38.7^{\circ} R$. (101.6 $F$.), in the evening, $40^{\circ}$ R. $\left(104^{\circ} \mathrm{F}\right.$.), this moming, 39. $5^{\circ}$ R. (103. $2^{\circ} \mathrm{F}$.).

Inamosis. - Here, as in the case of the pleurisy with effusion (nimeteenth case), we evidently have to do with an acute disease in the cavity of the chest which causes a febrile condition, this last appearing simnltaneously with the local symptoms, namely pains in the left side of the chest. This disease may be either pneumonia or pleurisy with effusion. If we review the comparison between the symptoms of both diseases, which we did while discussing the above mentioned case, we shall soe that the symptoms of the present case speak against pleurisy with effusion, and for pnenmonia, namely, for eroupous pneumonia (fibrinous): the pains in the side are not severe; together with a dull pereussion sound there is observed elear bronchial respiration, and not the weak respiratory mur- 
mur of indefinite quality, as in the above-named case of pleurisy with effusion; the vocal fremitus is increased (there it is weakened), the cough is not dry, as it is there, but with expectoration and characteristic at that of fibrinous pneumonia; the affection of the lower lobe of the ling is also characteristic of the same. The course of the temperature, when it became established after its fall caused by calomel passed away, also speaks for pneumonia, namely, the temperature is high, and the difference between the morning (to-day $39.5^{\circ} R$. $-103.2^{\circ} F$.) and the evening (last evening $40^{\circ} R .-10+F$.) is trifling, of one-half of a degree, whereas in the above-named case of pleurisy, at the beginning of the patient's stay in the clinic before antipyretics had been prescribed for him, the morning temperature was 38.1 $R$. $\left(100.6^{\circ} F\right.$.), that of the evening $39.6^{\circ} R$. (103.2 $2^{\circ}$.). The microscopical examination of the sputum, so easy of accomplishment in the clinic, but difficult in private practice, is unnecessary in the present case, as the diagnosis is evident beyond doubt. It is otherwise, if we have to deal with a pneumonia in the upper pulmonary lobe, and especially in a patient in whom we are led to suspect the presence of tuberculosis: then it is certainly necessary to examine the expectoration for prognostic as well as therapeutic purposes; it may contain in one case tubercular bacilli, in another pneumonic diplococei, and in a third both.

Prognosis. - The case is serious; it may terminate in recovery, but may also end in death; which of the two it is impossible to say, but we may certainly with greater probability hope for recovery, in view of the patient's youth, his fair constitution and the good condition of his cardiac activity, and also because of the fact that during the two days that the patient has been in the clinic we did not observe any further spread of the pneumonic process. In addition to this, the patient has been placed since the day before yesterday into favorable conditions by his life in the clinic, and is subjected to treatment: the calomel lowered the temperature considerably for more than twenty-four hours, and it was probably the cause why the pneumonia did not spread.

Treatment. - We possess as yet no specific remedies for pneu- 
monia, and have to rely on the symptomatic treatment. We must combat the symptoms of the disease, and prevent any of them from developing to any dangerous extent, until the disease will terminate itself. As ordinarily dangerous symptoms in croupous pneumonia may be considered a very high temperature, a very weakened cardiac activity, and dyspnoea the cough (and very rarely pain in the side) may be considered not as a directly dangerous, but as a very embarrassing symptom which exerts a harmful influence on the patient's condition.

I prescribe treatment for high temperature in croupous pneumonia only when I see that its effects are fraught with danger. As a measure of the degree of danger of these effects are considered the patient's sensations, the condition of the pulse and the respiration: the higher the fever, the worse the patient feels, and the worse are the pulse and the respiration, and on the contrary, so that with the fall in the temperature and with the termination of the crisis, the patient usually feels well, the pulse becomes good, or yields easily to the effect of cardiac stimulants, and there is no dyspnoea even by the time when the local affection presents as yet no changes and remains in the former extent.

The worse therefore the patient feels, as evidenced by extreme anxiety and a feeling of distress, the weaker the pulse and the greater the dyspnoa, the more necessary does it become to lower the temperature.

I have long ago discarded digitalis, $\uparrow$ cold baths and quinine as means for lowering the high temperature of croupous pneumonia; quinine I give only in the very last stage of the disease, when resolution is prolonged, and when the febrile condition is of a strongly remittent nature in the mornings. I also give it, as described above, in chronic pulmonary tuberculosis, thus: for the moming remission quinine, and for the evening exacerbation phenacetine or antifebrine; but to lower the

* The attempts to treat croupous pneumonia, as well as the attempt to create immunity towards it, by the products of the life activity of the micro-organisms consiclered as the causative agency of the disease, are as yet not ripe enough for practical application.

$\dagger$ See article on the treatment of croupous pneumonia by calomel. 
temperature of croupous pneumonia I resort to calomel, only in patients of a strong constitution, and only at the incipiency of the disease when the strength is in good condition; especially do I give it when there is an indication for calomel as evidenced by the condition of the gastro-intestinal canal (as constipation, meteorism, and a high position of the diaphragm, tending to increase the dyspnœa), and by that of the biliary passages. The effect of calomel in croupous pneumonia is explained in the article alluded to, and is partly also seen from the present case. To repeat calomel in croupous pneumonia is usually mnecessary in view of the short duration of the disease and the danger of weakening the patient. To further lower the temperature, to bring it down from an undesirable elevation. I usually give phenacetime or antifebrine, ordinarily in the evening when the fever is increased, and in small doses (three to eight grains of phenacetine, or three to five grains of antifebrine), which usually prove satisfactory. I avoid the use of sodium salycilate and of antipyrine in view of their weakening effect on the heart, which effect is especially dangerous in pneumonia.

To keep up the weakened cardiac activity I administer the stimulating drops mentioned here more than once, wine, coffee with cognac, whiskey, less frequently camphor (to persons not habituated to alcohol and unable to bear it).

Treatment of dyspnoen, pains and cough. - There occur infrequent cases in which the dyspnoea is at the beginning of the disease so severe that it requires local bloodletting, less frequently general; these are cases presenting a tempestuous onset of croupous pneumonia, when in conjunction with severe pains which embarrass respiration, a consiclerable portion of one lung becomes suddenly affected, and simultaneously with this, because of the impeded circulation in the affected lung and of the weakened cardiac activity (on account of the very high temperature), there takes place an cedema of the other lung which develops rapidly, and is at times accompanied by hæmoptysis. * But ordinarily, a sufficient relief of the dyspnca is obtained by lowering the high temperature. by

* Vide article on "Bloodletting." 
strengthening the weakened activity of the heart, and by re- lieving the pains and the cough. In case the dyspnoea is very severe and dangerous, as a result of an cedema of the lungs developed in acute cases at the termination of the croupous pneumonia, - in a patient, already debilitated and presenting symptoms of an approaching collapse, — then bloodletting is not to be resorted to (as recommended by many text-books), as it is then a very risky measure under such conditions, but stimulants must be administered, also senega, and large Spanish flies are applied.

For relieving the pain it is usually sufficient to keep the painful side of the chest warm (by the aid of warm oil, batting and flannel), to relieve the cough, and the coughing spells which tend to aggravate the pains. It is only on rare occasions that we have to resort for the relief of the pain to local bloodletting or to an hypodermatic injection of morphium.

As the congh aggravates the pain, and through this as well as by embarrassing respiration and circulation, it also aggravates the dyspncea, and prevents sleep, it must, therefore, be reduced to the possible minimum. As long as the expectoration is scanty, and the râles are either few or entirely absent, we should give codeine, opium, in the form of pulvis Doveri, rarely morphine. I had observed many times that the first thing a physician administers to a patient suffering with pneumonia, is infusum ipecacuanhe which is continued for several days; this is undoubtedly an error: there is at that time as yet nothing in the bronchi that can be brought away, and the ipecacuanha only tends to aggravate the cough, and if administered for a long time, it deranges the appetite and prevents its timely return. On the other hand, we must exercise extreme caution during the administration of the above-named narcotic remedies. Changes in the course of croupous pneumonia are liable to take place rapidly: codeine, opium and morphine, which relieved the dry cough, and therefore also the dyspnoea and the pain, thus benefiting the patient greatly until say the fifth or sixtl day of the disease, may become harmful on the morning of the following day, usually at the time cluring which the crisis occurs, by aggravating the dyspnoea, at a time, 
when the respiratory passages are filled with liquid contents (as evidenced by abundant subcrepitant and more prominent moist râles, whose appearance is to be carefully watched), on account of the entrance into them partly of the liquefied pneumonic infiltration (although this is usually absorbed), but principally of the adematous fluid. Now we must put aside the above-named narcotics, which we gave only yesterday, and while continuing the stimulants, we resort to expectorants, usually to senega in an infusion, $3 \mathrm{j}-3 \mathrm{ij}$ to $\overline{5} \mathrm{rj}$, and in severe cases also to large Spanish flies.

We will now turn to the treatment of our patient. From the data outlined above you will perceive that there are no indications at present for bloodletting, nor were there any before. The indication for calomel, as well as its effect in the given case, were already spoken of. What shall we give the patient to-day? We will first of all give him again five drops of the tincture of opium, as he had some loose stools, although they were painless and appeared once during the twenty-four hours. The opium will also be beneficial in quieting the cough. We will continue the stimulant drops. Their action is good: twenty-five drops without wine, twice a day, produce a fair pulse. Pain in the left side is sufficiently relieved by the part being kept warm. For the cough - tra. opii, and in case of constipation, one-fourth graim of corleine. As the temperature is quite ligh and the dyspnœa very pronounced, we will give him, in view of the probable evening rise of the temperature, three grains of phenacetine.

\section{FROM THE LECTURE OF MARCH 5, 1893.}

The day before yesterday, at seven P. x. the temperature was $39.8^{\circ} R .\left(103.6^{\circ} F\right.$.), and after he took three grains of phenacetine, it fell to $38.8^{\circ} R$. (101.8 F.) by ten o'clock in the evening; this morning it is $39.5^{\circ} R$. $\left(103.1^{\circ} F\right.$.). Yesterday morning the area of dullness seems to have spread somewhat upward, to-day it is of the same extent, as it was yesterday ; there is no further spread. Auscultation over the region of dulhess elicits bronchial breathing, no râles anywhere. The pains in the left side, 
as also the cough, are trifling and cause the patient no inconvenience; the dyspncea is moderate. The pulse is good; he gets twenty-five stimulating drops four times a day. $\mathrm{He}$ is constipated. The sleep is fair, he feels better, the hyperæsthesia cutis disappeared.

The prognosis remains favorable, because all the symptoms are slight, the strength is good, and at the present time there is no further spread of the pneumonia; this is the seventh day of the disease and we may soon expect the crisis.

Treatment: a elyster, coffee with milk (as a cardiac stimulant, at the same time counteracting the constipation), stimulants, phenacetine and codeine, as necessity requires; finally, in case adema of the lung supervenes during the crisis, senega, or even a Spanish fly.

\section{FROM THE LECTURE OF MARCH 16, 1893.}

The day when the last lecture was delivered, the seventh day of the disease, the temperature did not rise in the evening $\left(39.2^{\circ} R .-102.7^{\circ} \mathrm{F}\right.$., as against $39.5^{\circ} R .-103.1^{\circ} \mathrm{F}$. in the morning) ; on the next, eighth day, the moming temperature was $39.6^{\circ} R .\left(103.2^{\circ} \mathrm{F}\right.$.), but it rapidly fell to $38^{\circ} R .\left(100.4^{\circ} \mathrm{F}\right.$.) in the evening; on the ninth day $38.7^{\circ} R .\left(101.6^{\circ} \mathrm{F}\right.$.) in the morning, after three grains of phenacetine $38.4^{\circ} R .\left(101.1^{\circ} \mathrm{F}\right.$.) in the evening, and on the tenth day in the morning, with the appearance of considerable sweating, $36.2^{\circ} R .\left(99.01^{\circ} \mathrm{F}\right.$.), and since then there is no fever, the appetite appeared and is continuously getting stronger. There was no further spread of the pneumonic process during the seventh, eighth, and ninth day of the disease; but with the advent of the crisis the local phenomena underwent a rapid change: the dullness began to clear up, the bronchial breathing disappeared, there appeared abundant râles and the cough increased. Patient took two bottles of an infusion of senega, $3 \mathbf{j}$ to $\tilde{3} \mathrm{vj}$ a tablespoonful every hour, and by the twelfth day of the disease - March 10th - the cough disappeared, the lâles vanished and the percussion sound at the region of the former pneumonia cleared up entirely. To-day the patient, who, as you see, is completely improved, leaves the clinic. 


\title{
A FEW NOTES ON OBJECTIVE EXAMI- INATION.
}

\author{
(From Trurions Lectures.)
}

While speaking of the general examination of a patient I said: "By far not everything obtainable by examination is necessary and important for medical conclusions; we must be particularly careful not to confound diagnostic aims with those necessary to semiotics, for not everything of interest to the semiotician is necessary to the diagnostician."

As a matter of fact, every morbid phenomenon is of interest in semiotics as an independent science, as a branch of natural science, whereas for diagnostic purposes only that is of importance, which forms a symptom, a sign of disease; and the more certain the indication presented by the morbid phenomenon as regards the disease, the more reliable is this phenomenon as a symptom, the more important does it become for diagnosis. Not all the morbid phenomena possess the same value as regards diagnosis; on the contrary, the number of morbid phenomena important for diagnostic purposes is by far smaller than the number of all the morbid phenomena in general. If we were to compare the sum total of these last, the knowledge of which the student must acquire during his study of semiotics, with the limited number of those, which are made use of not only by very experienced and conscientious physicians in their practice, but even by clinical instructors, then we would find a great difference. I may also say. that not only in private practice, but even in the clinic, are the methods of examination less complicated, without losing thereby any of their correctness, than those usually recommended by text-books and by the instruction in semiotics.

This is the reason why students, who have become familiar 
with semiotics and who come to the clinic, have usually a general idea of the nature of many morbid phenomena, probably of all, but in the majority of cases they are unable to appreciate their comparative value for diagnosis, and cannot separate the most important of these from the rest; quite frequently they are able to note in the patient some trifling signs, at the same time failing to perceive valuable symptoms.

It is certainly the business of the clinical instructor to put prominently before the mind of the future physicians whatever is most important; this is comparatively but little, but ther must be taught to firmly possess this little: it is however desirable that both the text-books and the instruction in semiotics have the same ain in view. Some abbreviation of the course of instruction in semiotics, and stricter choice in the matter to be communicated to the students and in what is necessary to be acquired by them, would aid materially in reaching such an aim and would be more than timely now, when, with the rapid progress of the various medical sciences, the students are less and less able to satisfy all the requirements of a serious examination.

I will bring forward a few observations which I usually make, with the aim in view of pointing out what is most important on various occasions, in connection with the objective examination of the patient, namely. in percussion, auscultation and palpation, except auscultation in diseases of the heart, on which I touched but slightly in the preceding lectures, and which, as well as the examination of this organ, I expect yet to take up in the future. 'To make the matter clearer I shall instance such a case. I call out a student, and invite him to auscultate the patient and tell me what he hears. In reply he says that he hears rough breathing. I ask for the meaning of this term, and what condition of the respiratory passages it indicates, but fail to obtain a satisfactory answer. I then remind the students and impress it on their minds to always remember the following data concerning respiratory murmurs :

Tesiculur respiratory mumu indicates, that the area over which it is heard, contains normal pulnonary tissue, and consequently it has a diagnostically definite significance. [Each 
and every symptom, to the importance of which I call the attention of the students, is described by me, produced, if possible, by voice imitation, and is demonstrated on the patient to as great a number of students as practicable; while the resident physicians, the assistants in the clinics, as well as the privatdocenten engaged therein, instruct during the evening hours the students in the skill of determining practically on the patients those diagnostic symptoms, to which I called their attention.]

Bronchial respiratory mumur indicates that the pulmonary tissue over which it is heard contains no air (the exception refers to the bronchial respiratory murmur transmitted from the larynx and trachea, but then it is heard usually with equal distinctness over both sides of the chest). Besides the bronchial and the vesicular respiratory murmurs, which possess a characteristic distinctness in their aconstic qualities and which have a definite diagnostic significance, there are other respiratory murmurs which resemble neither the vesicular nor the bronchial murmurs, and which indicate nothing definite (they can be found both in perfectly healthy respiratory passages, as, for instance, in men when breathing quietly, and in various affections of the bronchi, lungs and pleuræ); these therefore possess no definite significance, and are for this reason called the indefinite respiratory murmurs. To this class must be referced the so-called rough breathing; and to distinguish this is for the diagnostician but an unnecessary burdening of the memory by a rhetorical, but not a diagnostic term. Once the respiratory murmur is neither vesicular nor bronchial, it has no diagnostic significance, and is therefore of an indefinite quality; whether it be rough or otherwise is of no importance. Under certain conditions, but not independently by itself, the indefinite respiratory murmur (i. e. the one resembling neither the bronchial nor the vesicular, and whether it be rough or otherwise is not important) may possess a diagnostic importance; thus, for example, if under one clavicle we hear a vesicular respiratory murmur, and under the other constantly an indefinite one, for instance, the so-called prolonged expiration, one of the varieties of the indefinite respiratory nurmur, especially in a patient, 
who excites a suspicion of tuberculosis, then we have a foundation to conclude, even before the appearance of the more positive symptoms, such as râles and alterations in the pereussion sound, that that pulmonary apex is affected, over which we hear the indefinite respiratory murmur.

In connection with the same we must also make the following observations :

About râles. - We must distinguish dry râles (usually whistling ones) and moist râles; of the latter, the very fine ones, crepitant, as typically represented in the beginning of a pneumonia, then somewhat more prominent, subcrepitant, as typically represented at the stage of resolution of a pneumonic process and at the beginning of an cedema of the lungs, and finally the medium and the coarse râles, as typically represented by a pulmonary cedema which spread upward, and by a bronchial catarh with abundant expectoration.

Then I advise to memorize well the nature of the râles which characterize the most frequent affections of the respiratory organs.

1. Abundant moist râles. at the beginning subcrepitant, later more coarse, which appear simultaneously on both sides of the chest, at first under the scapule and later spreading further and further upward (and parallel with this the dyspnea becomes aggravated), point with certainty to pulmonary cedema, which increases rapidly and requires immediate treatment. 2. Less abundant moist râles on both sides under the scapulæ. which remain constantly subcrepitant and do not spread upward, and which are usually heard on deep inspiration, ordinarily in persons with a distended abdomen and a highly elevated diaphragm, - which condition prevents a full expansion of the lower pulmonary parts, - point partly to a condition of atalectasis, partly to a slight stationary adema of these parts. 3 . Râles which are heard on either side only orer the middle portions of the lungs. and which are not perceived in the apices and the lowest portions, now more dry, now more moist, medium and large bubbling ones, point to a bronchial catarrh, either dry or moist. As a very rare exception I have seen an uncomplicated bronchial catarrh (without any affection of the pulmonary tissue) on one side. which lingered from the very 
childhood of the patient, at times quieting down, at others, during the cold season, taking on an acute form, the predisposition to which catarrhal condition was evidently determined by an insufficient expansion of the affected half of the chest during the early part of life (semiatalectasis of the lung?). On the other hand the above-mentioned râles, if considerably spread over both halves of the chest, may be heard in bronchopneumonia most frequently of tubercular origin — which attacked both lungs (the differential diagnosis between bronchial catarrh and tubercular bronchopneumonia was spoken of in connection with the twenty-first case). 4. Moist râles, observed over a limited area, in one focus or in several, point to an affection of the lung tissue, - croupous pneumonia, pneumonia complicating grippe, pertussis, etc.; such a focus exhibiting moist râles in the apex of one or the other lung or of both forms one of the most certain symptoms of tuberculous affection of the lungs.

In some cases - as in pneumothorax and in cavernæ - the respiratory murmurs and râles may possess a particular quality of resonance (simple and metallic), at times of such a pronounced and characteristic nature, as to plainly point to the above-named morbid conditions.

The percussion sound. - The principal quality of this, which must chiefly be taken into account, is its force : a clear, a dull or a flat sound. In rare cases the percussion sound over the chest, ordinarily not resonant, may possess a similar pronounced resonant quality as the phenomena observed in auscultation; it then has a similar diagnostic significance.

Determination of the dimensions of the heart, liver and spleen. The dimensions of the heart are firstly determined by palpating the region of the heart-impulse at the apex (I recommend most earnestly exercise in such examination, as it gives most important data), and secondly by percussion over the sternum : in the normal condition the lower half of the sternum gives almost as clear a percussion sound, as the upper one; while in case of an enlarged heart, and especially of its right half, the percussion sound over the lower half of the sternum and to its right is duller than over the neighboring parts - upward and to the right - of the chest wall and the upper half of the 
sternum. The area of the apex beat of the heart and the right border of the dull sound at the cardiac region, present entirely sufficient data by which we can judge of the dimensions of this organ.

The lower border of the liver and of the spleen is determined by palpation, and the upper border by percussion. While performing the last it is hardly worth while to search for the rib to which it extends. The statement of the text-books "from this rib to that" are but average deductions from many observations. Every individual case will present departures from these mean dimensions. Consequently, if such departures be small, then we cannot deduct therefrom any conclusion as regards the alteration in the size of the organ; but if they be considerable, then there is no necessity to occupy oneself with recounting the ribs: for it is plainly apparent, that the area between the superior and the inferior borders is markedly greater, or pronouncedly less extensive, than is peculiar to the organ in its normal condition. It also suffices an experienced look to judge by this area as to whether the organ is replaced or not. This is the reason why I insist, for the sake of acquiring such experience, on exercising as much as possible in defining the borders of the liver and of the spleen, and thus acquire a strong mental hold on the dimensions as well as the localization of the area contained within the abovenamed borders in the normal condition of these organs, as well as when they are enlarged, diminished and replaced.

The importance of feeling the pulse, at one time somewhat forgotten, thanks to the enthusiasm generated by the method of percussion and auscultation and also to sphygmography, but at the present time appreciated rightly, requires certainly no necessity for discussion. Of the many peculiarities of the pulse we must at all times define its three principal qualities: its frequency, rhythm and, most important of all, its strength. The strength of the pulse depends on the quantity of blood, on the force of the cardiac contractions and on the elasticity of the arteries. Feeling the pulse enables one to judge certainly only of the results of interaction of these factors. To determine the condition of these last we must consider the other data, the 
condition of nutrition and hæmatopoiesis, and the results of the examination of the heart and of the arterial system. Sphygmography and sphygmomanometry, not to speak of the great inconvenience their use occasions in private practice, cannot replace the determination of the qualities, principally of the strength, of the pulse, nor of the condition of the arteries as obtained by touch. We shall yet have occasion to speak of the pulse, as well as of auscultation of the heart, when analyzing clinically the diseases of this organ. 


\section{ELEMENTS OF BALNEOTHERAPY}

FOR THE PRACTICAL PHYSICIAN.

\section{LECTURES OF APRIL 20 AND 21, 1893.}

While giving clinical, that is, practical instruction in pathology and therapeutics, the clinician embraces the opportunity, in prescribing the treatment, to touch on all the means possessed by this last, and in doing this he is enabled to classify these means from his own special clinical, principally individualizing point of view. I therefore since long, almost from the very beginning of my clinical activity, always touched upon the subject of balneotherapy. In former times this was the more necessary, as the student at the clinic had almost no idea about this subject, because it formed no part of the curriculum of university instruction. During recent years among the various courses of our medical schools there were also established courses in balneotherapy, but I still keep to my custom of discussing the subject in my lectures; in doing this I am also urged on by my former students, who attest to the value of these lectures by their own experience. As far as the internal employment of mineral waters is concerned, I spoke of the necessity of its clinical characteristic ; but there is another reason for the utility of such a characteristic of balneotherapy, which is common to both the external and the internal employment of mineral waters.

The reason for this lies in the practice, common to all handbooks, text-books and courses of balneotherapy, of expounding together the employment of mineral waters both for external and internal uses, while discussing the subject in general, as well as in the description of special kinds of mineral waters. The subject of balneotherapy in general, that is, the science of the application of mineral waters for external and internal 
use, is very extensive, extremely complicated and varied; there is hardly a department of special pathology on which it does not touch. By the internal use of the mineral waters we aim principally to influence the mucous membranes, the blood. the nutrition and the various secretions, and through all these, the nervous system; while by their external use we act through the skin on the nervous system, and then through this last on the other parts of the organism. It is thus apparent, that the numerous and various diseases, for which the mineral waters are administered internally, differ from the great number and variety of diseases, in which they are applied externally; it is consequently but too evident how different may be the indications for the internal use of mineral waters from those for the external use of the same. To this we must subjoin the following: that the experience proving the effectiveness of the mineral waters was obtained at the places where these are located, and consequently their curative efficacy, as shown in certain diseases, was due not only to their employment, but also to the peculiar characteristics of these localities, to the climatic, dietetic and general conditions, as well as to the change in the mode of living of the patients who took up their residence there. To correctly appreciate the proper action of the mineral waters, balneotherapy must take into consideration also these last influences, climatic and otherwise, generally hygienic. All these balneotherapy must consequently touch upon. This many-sidedness of the subject, which is determined by the great variety of its contents, constitutes a great obstacle in penetrating into the true nature of balneotherapy, especially so for the beginner, who in a general way finds it difficult to differentiate the enormous mass of material offered to him for acquisition during the few years of his university (medical) course.

Therefore to better define the so important therapeutic value of the mineral waters proper, I continue to discuss this subject also at the present time during my clinical instruction; and for the sake of still greater clearness I discuss the internal use of the mineral waters separately, and the external use, or balneotherapy in the narrow acceptation of the term, also separately, after you had been shown during the course of the year the applica- 
tion of various balneotherapeutic agencies, - as the warm baths, hot air-baths, douches, cold and warm compresses, etc. ; all these were demonstrated and explained to you, as well as the indications for the use of other similar agencies, as, for instance, alkaline baths in the cases we came across (thus in the thirteenth case).

In speaking of balneotherapy in the narrow sense of the word (the external use of waters) I only aim to give its fundamental principles, the most important which shouldalways be fresh in the memory and clear to the understanding of the practical physician, and which are not always clearly defined, but rather made vague in the above-mentioned complicated and diversfied treatment of the subject by handbooks, text-books and various courses. The exposition of this most important matter is subdivided in to three parts: the action of the waters, the varieties of the same, and a short sketch of diseases in which the waters are applied externally.

The action of waters in their external application is principally determined by their temperature, and to a lesser extent by their composition and mechanical effect.

\section{The Effect of the Temperature of the Waters.}

1. On the nervous system. - Waters, the temperature of which approaches that of the surface of the body $-27^{\circ} R .\left(92.8^{\circ} \mathrm{F}\right.$.) to $28^{\circ} R$. $\left(95^{\circ} F\right.$. - are known as thermically indifferent; with a higher temperature - $29^{\circ} R$. $\left(97.2^{\circ} \mathrm{F}\right.$.) and more - as warm or hot; with a lower temperature - $26^{\circ} R .\left(90.6^{\circ} F\right.$.), and less - as cool or cold.

The thermically indifferent waters exert by themselves no effect on the nervous system; but by keeping the body in an equable temperature, they protect it from the irritation produced by the constantly changing external temperature, and thus induce a quieting effect. Cold baths exert a stimulating effect on the central nervous system, calling forth a spirited mental state and a desire for mental and physical activity, and remove contrary conditions. Warm baths act on the nervous system in a weakening manner, causing fatigue, unwillingness to work and inclination to sleep; they quiet the condition of excitement. Such an effect of the cold and of the warm baths 
is observed in the majority of cases, but there occur also numerous exceptions; in describing the external use of waters in diseases of the nervous system I shall speak of this more.

The effect of baths on the central nervous system may take place through various ways: directly in a centripetal way through the sensory nerves of the skin, through reflex action on the vasomotors of the cerebral and the cerebro-spinal vessels, and consequently through alterations in the cerebral and cerebro-spinal distribution and circulation of blood, through the vaeillations produced in this last by changes which are caused by the baths in the general circulation (as, for instance, by the influx of blood to the external parts after warm baths), later on through alterations produced by baths in the general nutrition. By influencing the nervous centres through the skin, the baths may exert an effect through these last on all the nervous functions - sensation and motion, nutrition and secretion. The mechanism of this action is not known with certainty, but the fact of its existence is beyond any doubt; thus: warm baths quiet pains and convulsions, cold baths may call out muscular contractions, as evidenced in the treatment of constipation by cold douches on the abdomen or into the rectum; later on we shall discuss the important changes which may be produced by baths of various temperatures on the temperature of the body, on the body metabolism, on the secretions, on the calibre of the vessels, distribution and movement of the blood and lymph, and the activity of the heart and the respiration; all these changes, as I said before, are reflected in their turn in the condition of the nervous system.

2. On the temperature of the body. - The thermically indifferent baths produce no marked effect on the loss and production of heat by the body. In cool and cold baths, up to a certain degree of cold and within a certain limit of time, the body is at first chilled, but later on it becomes rapidly warm and the internal bodily temperature is not lowered, but remains normal, or is even raised. Similar to the action of cold baths is that of other cooling hydrotherapeutic procedures, as douches, semibaths, sponging with a wet towel. The constancy of the internal bodily temperature in the presence of a considerable loss of 
heat in a cold bath is determined by the diminution of this loss as caused by the contraction of the peripheral vessels and by the increased production of heat in the body. But if the degree of cold and the time during which the bath is being taken exceed certain limits - which are, of course, variable in different cases - then the body, having become warmed after the first chill, is chilled anew, does not any more become warmed either in the bath or out of it. and is subjected to an abnormal state, which is made apparent by a condition of general weakness, instead of stimulation, by loss of appetite, and by a red saturated urine containing a great amount of urea and uric acid. If the use of such baths or of similar hydrotherapeutic procedures be resorted to repeatedly during a careless and negligent treatment, then there is established a genuine febrile condition, accompanied by emaciation of the body, which may lead eventually to a serious disturbance of health; any curative effect of such a treatment is certainly out of the question.

Warm and hot baths prevent the loss of heat by the body and even bring warmth to the body, and therefore the internal bodily temperature is raised while in them; thus Mosler observed that in baths of $40^{\circ} \mathrm{C} .\left(104^{\circ} \mathrm{F}\right.$.) to $44^{\circ} \mathrm{C}$. (111.2 $2^{\circ} \mathrm{F}$.) the temperature in the cavity of the mouth was raised to $38.6^{\circ} R$. $\left(118.8^{\circ} \mathrm{F}\right.$.) and Bartels noted that after a ten minutes' stay in the steam bath at the temperature of $53^{\circ} \mathrm{C}$. (about $127^{\circ} \mathrm{F}$.) the temperature in the rectum was raised from $38^{\circ} R$. to $40.4^{\circ} R$. (about $122.5^{\circ}$ F.).

3. On the tissue metabolism and an the secretions. - The thermically indifferent baths, not influencing the loss and production of heat by the body, exert no marked effect on the tissue metabolism and on the secretions.

Cold baths, and generally the loss of heat, increase the excretion and consequently the production of carbonic acid, i. e. the combustion of fats, in proportion to the degree of this loss, and consequently to the increased production of heat; the body becomes lean. If the degree of cold and the duration of the bath are kept within the above-mentioned limits, so that the body, after becoming warm after the first chill, is not chilled later on, then the excretion of urea, representing the disinte- 
gration of the albumens, is not altered by such baths; but if these limits are exceeded, that is, if the body, having become warm after the first chill, is later on chilled again and does not become warmed any more, then there develops a morbid condition which passes into fever, and with this the excretion of urea and of uric acid, representing the disintegration of the albumens, becomes increased.

Hot baths, by raising the temperature of the body, increase both the excretion of urea, which means the disintegration of the albumens, and the excretion of carbonic acid, i. e. the combustion of fats. After warm, and particularly after hot baths, the body perspires (consequently it loses heat), while the amount of urine is diminished and its concentration is increased.

4. On the circulation and respiration. - The thermically indifferent baths exert no marked effect on the calibre of the vessels, and consequently also on the distribution of blood throughout the body, on the frequency and force of the cardiac contraction (the pulse), nor on the frequency and depth of the respiratory movements.

The body becomes first pale in a cold bath, as its vessels are constricted, then the color changes to red, the vessels dilating and the circulation growing quicker, these phenomena remaining after the bath; in the unnecessarily cold and prolonged baths, the skin takes on a blue tinge, which is a sign of venous hyperæmia and of slackened circulation.

In a hot bath the skin grows red, on account of a dilatation of the vessels, which effect lasts for some time also after the bath. Very hot water, the application of which is, of course, possible only in a local way, determines a contraction of the vessels; this is the basis for certain therapeutic measures in gynæcology, as the use of hot vaginal douches for metrorrhagiæ and to assist in the absorption of exudations formed as a result of a parametritis.

In a cold bath the contractions of the heart, the pulse, become stronger and are at first more frequent (because the simultaneous contraction at the beginning of the peripheral vessels increases the blood-pressure and thus excites the activity of the left ventricle), but grow less frequent later on. 
In a hot bath the contractions of the heart, the pulse, become more frequent and weak in proportion to the rise of the internal bodily temperature.

In a cold bath the respiratory movements become deeper and more frequent in proportion to the excretion of carbonic acid. In a hot bath the respiratory movements become more frequent in proportion to the increase of the internal bodily temperature and to the increased excretion of carbonic acicl.

\section{The Effects of the Component Parts of the Waters.}

The most important component parts of the waters used externally are common salt, carbonic acid and sodium carbonate.

Experiments have shown, that a salt bath, containing three per cent of common salt, increases (as compared with a bath of water not containing any salt, at the same temperature and of the same duration) the consumption of oxygen and the excretion of carbonic acid; this effect does not take place, if the nerve-endings be paralyzed by curare. From this it becomes apparent, that the sodium chloride in the above-named concentrated solution irritates the nerve endings of the skin and in this way exerts a strong influence on the metabolism and on the secretions. Medical observations concerning the effectiveness of salt baths, as in scrofulosis, rickets, unabsorbed exudations, etc., have long ago proved the fact of such an influence. Salt baths of the above-named concentrated solution cause the superficial vessels first to contract (the skin becomes pale), and later on to strongly and continuously dilate (the skin grows red and remains so for quite a considerable time after the bath); thus the salt baths, besides influencing the circulation, and consequently the nutrition of the general integuments, may also exert an influence on the distribution of the blood throughout the body, and through this means as well as by the above-mentioned irritation of nerves of the skin, also on the nervous system.

The carbonic acill. when present in the bath in a sufficient quantity, irritates the peripheral endings of the nerves and in this way acts as a stimulant to the nervous system. 
The sodium carbonate, in the quantity found in the alkaline waters employed for bathing purposes, causes but a slight irritation of the peripheral endings of the nerves, rather contrariwise, quieting them, improving the condition of the skin by the fact that it assists in the removal of the fats (by saponifying them) as they accumulate in the mouths of the glands of the skin, and also in the removal of the epidermis.

Sulphur seems to exert some effect on the skin, because sulphur baths proved to be beneficial in certain cutaneous affections; any further effect than that the sulphur baths do not apparently exert, and they are also considered as chemically indifferent waters.

Other bodies found in mineral waters employed for batbs, do not cause any marked effects.

The substances found in the mineral muds, as the organic acids, sodium chloride, iron sulphate and other salts, exert an irritating effect on the peripheral nerve endings, as may be inferred from the curative effect of the muds, but we possess no positive knowledge concerning this subject.

Even if absorption, in the bath, of certain substances by the body does take place, it is of such trifling extent, that it possesses no practical significance.

\section{The Effect of Mechanical Influences during the External Use of Waters.}

The influences of such a nature, as in out-door bathing the current in the river and the waves in the sea, the concussions of the skin in the fan-like douches under a high pressure, the friction in the half-baths and in the wet spongings, and various other massage-like procedures, act as irritants on the skin, i. e. in the same sense as the thermic and the chemical agencies, and consequently they enhance the effect of these last. The. movements made by the patient, as in swimming and other movements in sea-bathing, friction of one's own body in the front in half-baths and wet spongings, exert an influence on the metabolism and on the secretions, on the circulation and respiration in the same way as the above-mentioned agencies. 
1. The chemically indifferent waters, usually warm (acratotherme), contain but one or less to a thousand of component parts - usually of alkaline carbonates, chlorates or sulphates namely, not more than any river water, and therefore they act only by their temperature. They are used either as thermically indifferent waters, which protect the body from irritation by the continuously changing external temperature, which means that they quiet the nervous system in various nervous disorders, especially in those in which the symptoms of hyperæsthesia prevail, principally in neurasthenia and in hysteria ; or they are employed in the form of warm and hot baths, deriving the blood towards the periphery in hyperæmia of the intermal organs, and exert a pronounced effect on metabolism and absorption in the several varieties of chronic polyarthritis, gout, syphilis, and metallic poisonings. Of the well known waters of this class I shall name as examples, Schlangenbad, * Ragatz, $\dagger$ Gastein, $\ddagger$ Teplitz,\$ Leuk, || Plombières, $₫$ Abas-Touman.*** To this class

* In the province of Hesse-Nassau, Prussia, six miles west of Wiesbaden.

$\dagger$ In the canton of St. Gall, Switzerland, situated on the Tamina in lat. $47^{\circ} \mathrm{N}$, long. $9^{\circ} 30^{\prime} \mathrm{E}$. It is noted for its hot springs, and has about fifty thousand visitors annually.

$\ddagger$ A valley in the crown land of Salzburg, Austria-Hungary, south of Salzburg. It is famous for its picturesque scenery. At Wilbad-Gastein there are hot springs.

$\$$ A town and watering place in Northern Bohemia, situated in the valley of the Biela, near the mountains, forty-six miles northwest of Prague. It is one of the most frequented places in Europe, and has been the scene of several conferences of princes. Population in 1890 (commune), 17,526.

If Leuk (loik), F. Loü̈che (lö-esh), a village in the canton of Valais, Switzerland, situated on the Rhone, fourteen miles northeast of Sion.

- In the department of Vosges, France, situated on the Augrogne, fifteen miles sonth of Eyinal. It has the most important mineral springs in the Vosges, with thermo-mineral, iron and alkaline baths. It was known to the Romans and was greatly developed by Napoleon III.

**** A watering place on the Caucasus. 
also belong the sulphur waters, as, for instance, those of Aachen * and of Pyatigorsk. $\dagger$

I must now make some general remarks concerning not only the enumerated watering places, but any others as well. It were a great, unpardonable error, in view of the fact that the above-named (and any other) watering places are classified in one category, to consider them as identical and to send patients there indiscriminately, as, for instance, to send a nervous, emaciated female patient, for whom the thermically indifferent baths are suitable, to Teplitz or Pyatigorsk, where the warm and hot springs are situated, and to send a gouty patient, with a multiple arthritis, who would be benefited by warm or even hot baths, to Schlangenbad with its thermically indifferent baths. This would be as great an error as if we were to prescribe for a patient Ems while he needs Marienbad, or on the contrary. Every watering place possesses certain peculiarities which must be taken into consideration. These peculiarities depend on numerous conditions. The difference in the temperature of the baths was spoken of before. The method of employing them is also of importance. Let us take, for the sake of comparison, Franzensbad and Saki (in Crimea, near Eupatoria). In both places mineral moor is employed, but in Franzensbad it is added to a water bath of a mostly thermically indifferent temperature, and the bath is taken within a closed space, while in Saki the patient is put directly into a mass of mud which has been prepared since morning in the open air and which was strongly heated by the rays of the sun, and he is covered up with a similar mass of mud, so that the temperature of such a bath is raised high, and the bath is a hot one. It is self-understood that Franzensbad benefits a different class of patients, than are benefited by Saki. To Franzensbad flock nervous, emaciated men and

* Aachen is the German name for Aix-la-Chapelle, in the Rhine province, Prussia, about lat. $50^{\circ} 46^{\prime} \mathrm{N}$., long. $6^{\circ} 5^{\prime} \mathrm{E}$., an important commercial and railway centre. It was founded by the Romans as a watering place.

$\dagger$ A town in the Terek Territory, Ciscaucasia, Russia, situated on an affluent of the Kuma in lat. $44^{\circ} 4^{\prime} \mathrm{X}$., long. $42^{\circ} 8^{\prime} \mathrm{E}$. It is noted as a watering place on account of its sulphur springs. Population in 1889, 13,114 . 
women (we shall have occasion later to speak of the use of moor-baths in Franzensbad for diseases of women), because many observers have proved that the thermically indifferent moor-baths exert frequently even a greater quieting effect on the nervous system, than the thermically indifferent baths of the chemically indifferent springs (acratothermæ). In Saki you will see gouty and syphilitic patients with inveterate affections of many joints, and others in whom it is expected the hot baths will exert a strong influence on the metabolism and absorption.

A particularly important part in determining the special peculiarities of the various watering places is to be attributed to their climate. To better explain its influence, let us continue the former comparison between Franzensbad and Saki. Patients with weak nerves, who usually bear heat badly, would be harmed by a sojourn during the extremely hot summer at Saki, but on the contrary they would profit by the cool summer of the Franzensbad valley; whereas for patients, who are benefited by the hot mud-baths (and these in the open air), the hot summer in Saki would be very suitable, but not the cool summer at Franzensbad, that may bring on a cold in a locality much visited and exposed to every wind.

Another example: both Gastein and Pyatigorsk possess warm springs of a similar temperature; but the patients who come to Gastein, who are frequently advanced in years, are emaciated, with failing nutrition, with weak nerves, and whose nervous condition and nutrition improve so well under the influence of the mountainous, cool climate and pure air of Gastein, would fare poorly at Pyatigorsk with its hot summer, and dusty and malodorous air; whereas for patients, who are treated by baths, mercury and iodides, and who therefore are easily predisposed to cold, the hot summer and the warm soil of Pyatigorsk are very suitable.* Another example: it is a quite different thing to order salt baths in Stara Russa (in the Novgorod

* I do not speak here of the peculiar characteristics of the water of the warm Gastein springs to conduct an electric current, as proved recently by exact physical investigations, because the nature of its therapeutic value (and this last we have no foundation to deny) is not known with certainty. 
Government) with its damp and cool summer, or in Slavyansk (in the Charkoff Govermment) with its dry and hot summer or at the Odessa estuary, where the summer heat is modified by the influence of the Black Sea.

Then for the patient who, for the sake of a trip to the watering place, leaves his habitual surroundings, his usual mode of living and his ordinary diet, the degree of comfort and the conditions of life at this place are of no small importance: one enjoys a quiet, restful locality, rich in natural beauties; the other is more benefited by a great, populous, lively centre, as the most frequented watering places become during the "season." Finally, it is of course of very great importance as to what sort of a physician will supervise and direct the treatment at the watering place.

The above is sufficient to show how necessary it is for the physician to act thoughtfully not only when he advises a trip to a watering place, which is in the majority of cases so burdensome for the patient, but also when he recommends a certain watering place. It is necessary to become thoroughly acquainted with such places, at least with the most important of them, either through the medium of literature, or, which is better, by a personal visit. As a summer trip with the purpose of resting or of travelling, the physician will find nothing more pleasant than a trip to the watering places for a personal acquaintance with them, as well as for the observation and treatment of diseases, the sufferers with which flock thither. The watering places form a sort of a clinic of such diseases.

I will turn now to the further examination of the waters.

-2. Salt waters (containing common salt). - Their action is of a double nature - thermic and chemical; the benefit derived therefrom is apparent: that the thermic agency may by itself exert a strong effect on the processes of metabolism and absorption, we must have a recourse either to hot baths $-31^{\circ} R$. $\left(101.8^{\circ} \mathrm{F}\right.$.) and higher, or to cold ones $-21^{\circ} R$. $\left(79.2^{\circ} \mathrm{F}\right.$.) and lower, for which the majority of cases will present contraindications; the addition to the thermic effect of a chemical agency - the common salt - which acts in the same manner as the thermic one, enables us to confine ourselves in such cases 
to either thermically indifferent baths, $28^{\circ} R .\left(95^{\circ} F\right.$. $)$ to $27^{\circ} R$. $\left(92.7^{\circ} F\right.$. $)$, or to warm, $29^{\circ} R$. $\left(97.2^{\circ} F\right.$. $)$ to $30^{\circ} R$. $\left(99.5^{\circ} F\right.$.), or to cool ones, $26^{\circ} R$. $\left(90.5 F\right.$.) to $22^{\circ} R$. $\left(81.5^{\circ} F\right.$.), and it certainly increases also the action of both the hot and the cold baths. The percentage of common salt in such baths is usually from two to four, seldom greater, while at the beginning, for a very sensitive skin and in the case of children, it is even smaller, beginning with one per cent. The chief indications for the use of salt baths are: scrofula, rickets, chronic exudations, chronic multiple arthritis (chronic articular "rheumatism"), less frequently diseases of the nervous system. The salt water springs are very abundant in Western Europe; the places particularly frequented are Kreutznach, IV iesbaden, $\uparrow$ Soden, $+\underset{\dagger}{ }$ Ischl, $\$$ Gmunden, |f Reichenhall, $\uparrow$ and others. Of our springs --Stara Russa, Slavyansk and the Black Sea estuary at Odessa (their action, in a general way, is similar to that of the salt springs).

3. Carbonated waters, containing a great quantity of free carbonic acid, as Schwalbach, ${ }^{*}$ or our Narzan in Kisslovodsk. These are used in the form of cool baths; in the thermically indifferent, and especially in the warm ones the carbonic acid evaporates rapidly. The effect is partly of a thermic nature, but principally, thanks to the carbonic acid, strongly stimulating. They are indicated in cases of neurasthenia and hysteria (also

* In the Rhine province, situated on the Nabe, twenty-one miles west southwest of Maintz.

$\dagger$ In Hesse-Nassau, Prussia, three miles from Rhine and six miles north by west of Maintz. It was known in Roman times; is frequented annually by about ninety thousand visitors.

$\ddagger$ In Hesse-Nassau, nine miles west northwest of Fraukfort on the Main.

$\$$ In Upper Austria, at the junction of the rivers Ischl and Framn, twenty-seven miles east by south of Salzburg. It is the favorite resort of the Austrian royal family and nobility. Population in 1891 (commune), 8,473 .

II In Upper Austria, situated on the lake of Traun, thirty-three miles southwest of Linz.

I In Upper Bavaria, situated on the Saalach, nine miles southwest of Salzburg.

*** Or Langenschwalbach in Hesse-Nassau, Prussia, eight miles northwest of Wiesbaden. Population (1890), 2,698. 
in conjunction with chlorosis and anæmia) with the character of depression, and contraindicated in these diseases if they present the character of excitement. They are also employed after treatment by warm and hot baths to remove the condition of debility and the predisposition to colds, created by these last, as for instance, the baths of Narzan after treatment at Pyatigorsk.

4. Salt waters which are at the same time carbonated, as the artesian springs of Rehme-Oeynhausen in Prussia. Their action is of a complicated nature, - thermical, chemical, and, thanks to the carbonic acid, strongly stimulating to the nervous system; their employment therefore requires particularly careful consideration: empirically they are recommended in diseases of the nervous system, especially in the different varieties of tabes dorsalis.

5. Mineral muds, added to baths of mineral waters, $i$. e. employed in the form of dissolved mud-baths, are employed at many foreign watering places; with us in the Pyatigorsk group of mineral waters the mud of the lake of Tambuktan is employed for diluted mud-baths. Franzensbad has been particularly famous for a long time for its moorbäder. To what was already said about their nse we must add their employment in diseases of women, in which they are very frequently applied. The effect of the Franzensbad moor-baths in diseases of women is usually explained and described in the following manner: The mud-bath causes uterine contractions, these being the more pronounced in proportion to the state of flabbiness and enlargement of the nterus. Thus, if the mud-baths be used in a case of insufficient involution of the uterus soon after normal delivery or after abortion, then the identical phenomena are observed as accompany in the normal course of the lying-in period the process of involution of the uterus, namely, labor-like sensations and an increased secretion resembling lochia, and containing formative elements peculiar to this last. And just as afterpains cause the narrowing and obliteration of the uterine vessels, thus weakening the nutrition of the uterine muscle and bringing about the transformation of its albuminous substances into easily absorbable fats, in the same manner the con- 
tractions of the uterus caused by the mud-baths determine the identical process in case of uterine subinvolution. If as a result of a prolonged state of subinvolution of the uterus its mucous membrane has also suffered, then a prolonged employment of mud-baths causes a return to the normal condition not only of the uterine muscle, but also of its mucous membrane. 'The absorption of exudations in the parametrium is also assisted by the use of the baths in question. The drinking of the waters of Franzensbad springs, which are slightly laxative and are beneficial in digestive disturbances to which these women are usually subjected, assists in the treatment by mud-baths.

6. The mineral muds employed divectly as such.-- Those of Saki, spoken of above, at T'chocra, near Kertch, at 'Tinack, near Astrachan. The foreign physicians, as far as we are aware, do not employ these methods of treatment.

7. Sea-bathing.-- The effective agencies in this are as follows:

The psychical effect of the sea-view, especially on those whose constant residence is far from the seashore, is usually beneficial by inducing a restful, hearty mental state, and inspiring love of life; other beneficent factors are: the salt and the low temperature of the sea-water (usually below $21^{\circ} \mathrm{R}$. $-79.2^{\circ} \mathrm{F}$.so that it acts like a cold salt bath); the continuously interchanging effects of the temperature of the water and of that of the air as the body is either wholly or partly immersed in the water: these temperatures are usually variable, the water being fresher, the air warmer, and on the contrary; besides, the air is usually in motion (the winds), and so is the water, which fact tends to even more increase the variability of the temperature; and a changeable temperature stimulates the peripheral nerves much quicker than one that is constant, whether it be low or high ; considerable mechanical influences: the wind, the waves, the motions of the bather; the humid, pure air of the seashore, which is under the greatest atmospheric pressure and consequently rich in oxygen.

It is evident from the above, that the effect of sea-bathing is very pronounced on the nervous system as well as on nutrition (as the appetite is very much increased by it, sea-bathing is con- 
traindicated for patients with poor digestive powers). Seabathing is recommended principally for cases of neurasthenia and hysteria with the character of depression, and contraindicated in the same affections with the character of excitement. For the indications and contraindications to sea-bathing in the colder climates (in the Baltic Sea, Northern Sea, and LaManche) and in the warmer (Bay of Biscay, Mediterranean, Black and Caspian Seas) compare below, in the treatment of diseases of the nervous system, the general indications and contraindications to cold and warm baths. Sea-bathing is also recommended after treatment with the warm and hot baths to remove the weakness and predisposition to cold created by them; thus after treatment with the Saki mud-baths, the patients are recommended sea-bathing, for which purpose is very suitable the seashore near Eupatoria (in the Crimea) with its warm water and warm air during the summer. Sea-bathing is also sometimes prescribed for suitable cases of scrofula in place of salt baths; and also in some certain cases of superfluous fat or general obesity.

I will say a few words on some other varieties of baths. The hot-air baths. Their strong effect was sufficiently explained in the analysis of the sixteenth case of chronic parenchymatous nephritis.

Fir-tree baths. - These are prepared by adding to baths of plain or mineral water a distillation by steam of pine and fir needles, containing ethereal oils, and a decoction of these last containing tar, turpentine and organic acids. The effect is that of a stimulant. The electric baths, together with the other methods of applying electricity, are discussed with the subject of electrotherapy.

8. Hydrotherapy. - All the hydrotherapeutic procedures are generally applied in such a manner, that the patient becomes at first chilled, and is later on warmed or, in view of the fact that the cooling of the body acts the stronger the warmer the body before the cooling began, he is at first warmed up, then chilled, and later is warmed up finally. This final warming up is known as the reaction. For the treatment to succeed, the reaction must be complete: if it be absent or incomplete, a condition similar to fever ensues, and in case the hydrotherapeutic proce- 
dures are frequently repeated without any reaction following them, a genuine febrile condition is developed which continues also after the cessation of the hydrotherapeutic treatment, and which debilitates the patient, as was explained already in connection with the effects of cold baths. I will state here that the same may occur to the patient also while bathing in the sea or anywhere else, if the bather remains in the water for a long time; therefore, having become chilled on immersing in the water and warmed up later, the bather must emerge from his bath while he is warmed, before secondary chilling takes place.

The hydrotherapeutic procedures act both thermically and mechanically; their effect is very strong, especially on the nervous system. They are principally recommended for diseases of the nervous system, particularly for neurasthenia and hysteria ; they are also recommended for the removal of the predisposition to colds, for superfluous fat and in some other cases.

The hydrotherapeutic procedures, namely, the various forms in which hydrotherapy is applied, are general and local.

Of the general forms the best is the fan-like douche under a high pressure. The construction of such a douche enables one to change the temperature of the water rapidly, almost instantly, and therefore not only can the patient be first warmed up and then cooled off, but this process can be repeated as many times as necessary. The form of the water as it strikes the body in spurts increases the stimulating effect of the douches. Finally, the water strikes the body with a force which the construction of the douche permits of varying and causing it to become, if necessary, very considerable. Consequently such a douche exerts simultaneously both a thermic and a mechanical effect, whereas in the other most frequently applied procedures, as rubbings and half-baths, a mechanical effect is brought about by the aid of massage, i. e. by the assistance of a very skillful manipulator. The douche is directed all over the body, from below upward and backward, except on the head.* The antiquated

* (Addition to the first edition.) All that was said of the fan-like douche refers to cool and cold-water douches. In some cases hot-water douches are very beneficial : local, from $35^{\circ} R$. $\left(110.8^{\circ} F\right.$.) to $40^{\circ} R .\left(122^{\circ} \mathrm{F}\right.$.), in the form of a single stream, most frequently in obstinate " rheumatic" affections (usually arthritis and neuritis, less frequently myositis of 
douches which are unfortunately not dismissed as yet by laymen, in which the principal mass of the spurting water strikes the head, are very harmful.

Of the other general procedures the most used and undoubtedly effective and beneficial ones are the rubbings and the halfbaths. At times, to be sure in but rare cases, they are borne better than the fan-like douche under high pressure, although the majority of patients express a preference for the latter.

Of the local forms of applying hydrotherapy the most frequently used ones are: cooling of the head, in hyperæmia of the brain, heat to it in anæmia of the brain, watery clysters, a warming compress, etc.

9. It is evident from the above-outlined sketch that it is not necessary for balneotherapeutic treatment always, in every case, to send the patient away to a watering place, to the seashore, or to some large city with a well constructed hydrotherapeutic institution. The most important varieties of baths, baths of variable temperatures and salt baths, at times with the addition of soda, may be prescribed and carried out with the necessary implements at the patient's residence. Sea-bathing may be replaced by bathing in the river or in a lake. Hydrotherapeutic rubbings and half-baths may be carried out everywhere by instructing a servant how to do it. For the fan-like douches there are portable apparatuses, which give a sufficiently high pressure, thus: the portable douches of Piêt and the much cheaper pumps of Allweiler, which are conveniently affixed to the douches.

I must say here a few words concerning the use of our popular bath (Russian bath). Its hygienic value, as evidenced by its purifying the skin, removing the fats and dirt which obstruct the glands of the skin, and by keeping up the normal functional activity of the skin, is very great, and especially so for the laboring class of the community; but its employment

gouty, syphilitic, catarrhal, traumatic and other origin) in persons unable to bear cold - usually in conjunction with massage (and, if necessary, with the contemporaneous treatment of the gout and the syphilis), and also in diseases of women; general, from $33^{\circ} R$. $\left(106.2^{\circ} F\right.$.) to $38^{\circ} R$. $\left(117.2^{\circ} \mathrm{F}.\right)$, in the form of a fan-like douche under high pressure, for neurasthenia and hysteria in debilitated, anæmic and chilly persons. 
during the cold season of the year, in bad weather, entails the risk of catching cold on returning from the bath-house. Without therefore dissuading healthy people habituated to it from enuploying it, and advising only to avoid hot baths and bathing during bad weather, I must say that the use of these baths must be strictly limited, or even prohibited, to persons who are weakly and predisposed to colds, the more so to ailing ones. The therapeutic effect of the bath and of the usual massage connected therewith, in cases of slight ailments due to a cold, but of a "rheumatic" and not of a catarrhal origin, is beyond doubt beneficial, but during the cold season of the year and in bad weather it is accompanied by considerable risks.

Bahneotherapeutic procedures employed in a warm room may be applied during all the seasons of the year; but the best season for such treatment is certainly the warm one, when a prolonged stay out-doors is possible without risking a cold. As regards the time of the day, all the balneotherapeutic applications must be made not on an empty stomach, but best after the morning coffee or tea, after a previous evacuation of bowels.

'The duration of the balneotherapeutic course of treatment depends certainly on the peculiarities of the given case, but in the majority of cases it does not require inore than one and a half or two months. An unnecessarily prolonged hydrotherapeutic treatment (and I have seen it prolonged not only over half a year, but even over one or two years) is much oftener harmful than beneficial, by debilitating the nutrition and the condition of the nervous system, and by inculcating in the patients habits of constant artificial stimulation by the aid of hydrotherapeutic procedures, of which, later on, they can but with ditficulty rid themselves.

A short outline of the diseases in which the waters are used externally, will give you some idea of the extent of balneotherapeutic applications and of the importance of this method of treatment.

1. Diseases of the nervous system. - In neurusthenia and hysteria with their multiform manifestations balneotherapy is considered to be the most important method of treatment, both on ac- 
count of the effectiveness of its action and the variability and extent of its application. The chief rule to be observed here is as follows: in proportion as we have a young patient with a strong constitution, in whom the condition of the internal organs need excite no apprehension, whose nutrition is good, and in whom the nervous disturbances bear the character of debility, the more suitable it is to use cool and cold water, such as contains a large proportion of carbonic acid, and the balneotherapeutic forms of application of a stimulating nature, as douches, sponging, half-baths, sea-bathing ; all these necessitating motion of the water, movements on the part of the patient and massage. On the contrary, if our patient is older, possessing a weak constitution, in whom the condition of one or another of internal organs excites grave apprehensions, while his nutrition is poor and the nervous disturbances have the character of excitability, the more suitable are thermically indifferent waters and balneotherapeutic applications of a quieting nature, such as do not necessitate any movement either on the part of the patient or of the water, namely, baths.

But this rule is only applicable to the majority of cases; in the considerable minority, in which the above-enumerated conditions do not coincide (as, for instance, when the patient is young, but weak constitutionally, or young, of a strong constitution, but emaciated, debilitated and chilly, and so on ; or the patient is not young, but of a strong constitution and nutrition, while his nervous disturbances have the character of debility etc.), after inquiring carefully of the patient as to the former effect on him of a cold and a warm bath (as bathing outside and indoors, warm baths), we must act in a manner reverse to this rule. Finally, there may occur cases which present no positive indications for either of the methods and in which a careful trial will determine the method to be resorted to.

Diseases of the brain, principally disturbances of the circulation as determined by a condition of atheromatosis of the arteries (general and those of the brain in particular) and by other causes. For these the chemically and thermically indifferent baths are suitable; at times also the warm ones (not over $29^{\circ} R$. $97.2^{\circ} \mathrm{F}$.) and the slightly alkaline, namely, those containing but 
a small quantity of sodium chloride and of soda, and also cooling of the head (in some cases warming it); cold and hot baths, as well as the applications tending to stimulation, are contraindieated.

Diseases of the spinal cord, principally tabes. For these the thermically indifferent, salt and salt-gaseous (with a moderate quantity of common salt and carbonic acid) baths are suitable, as well as slight hydrotherapy (sponging). Hot and cold baths, baths containing great quantities of salt and carbonic acid, as well as strongly stimulating forms of application, as douches and sea-bathing, are contraindicated.

2. Diseases of the digestive organs.-- For gastro-intestinal neurasthenia, which is expressed in a weakened condition of the nervo-muscular apparatus of the intestines and stomach, various douches, cool, cold, and of changeable temperature, or a warming compress on the abdomen, in connection of course with simultaneous general hydrotherapy for the general neurasthenic condition, are very beneficial. In catarrhal conditions of the stomach, intestines and the biliary passages, as well as in biliary calculi, warm baths form an excellent adjunct to the ordinary treatment of these diseases.

3. Warm baths are equally beneficial for renal gravel, for pyelitis, catarle of the ureters and the urinary bladder, while cases of acute and chronic nephritis are benefited also by hot baths.

4. For hypercmia of the spleen douches over the left hypochondrium, of the same nature as those employed in gastro-intestinal neurasthenia.

5. Different varieties of peritoned inflammation. - Some patients bear ice better, others warm applications, others again a warming compress on the abdomen.

6. Diseases of the male genital oryans, principally the various forms of impotentia virilis. In these the general balneotherapeutic treatment, directed against the general neurasthenic condition, is of more importance than the local treatment; while locally, after the general neurasthenia has been removed, careful electrotherapy is particularly efficacious.

7. Diseases of the female genital orgens. - We spoke of the peritoneal inflammations above. For painful menstruation a 
rubber bag filled with hot water and applied to the lower part of the abdomen is beneficial. For light cases of metritis, warm sitz-baths. For subinvolution of the uterus and the endometritis dependent thereon, and also for exudations in parametrium, the thermically indifferent, salty and moor baths and hot vaginal injections are useful.

8. Diseases of the respiratory organs. - The inhalation of various atomized liquids for catarrhal conditions of the larynx and trachea. Hot baths followed by sweating in bed while covered by warm blankets are at times resorted to in cases of bronchial catarrh accompanied by very abundant secretions, for the sake of alternating with other methods of treatment, if the patient became so habituated to these last that they fail to exert any marked effect on him; these baths may be employed only when the condition of the heart permits it, and generally extreme care must be exercised. In pleuritis and in pleuropneumonitis, a warming compress applied over the affected part of the chest is beneficial ; however, in the majority of cases the patients prefer better to keep this part warm by the aid of warmed oil, cotton-batting and flannel. In chronic pulmonary tuberculosis the patients, if not feverish, at times bear well the somewhat cool sponging prescribed with a view of assisting the function of the skin and simultaneously combating the neurasthenic condition.

9. Diseases of the heart. - Neuroses of the heart - palpitation, cardiac asthma, angina of nervous character (not the true angina pectoris coronaria), as well as the cardiac, angioneurotic and other neurotic conditions associated with morbus Basedowi, as well as the weakness of the cardiac muscle determined by it, as evidenced by a dilated heart and a weak pulse, -- all these present indications for the employment of balneotherapeutic treatment, the nature of which depends on the character of the general nervous condition present and of all the peculiarities of the given case (vidle above concerning balneotherapy in neurasthenia and hysteria); this treatment is indicated not only when the heart and the arteries are in a normal condition, but even when they present incipient organic alterations; extreme caution is certainly to be exercised in these cases, and the treatment is to be instituted 
only when there exists a complete compensation of the organic disturbances.

10. Diseases of nutrition and hematopoiesis. - Chlorosis and ancemia accompanied by their usual neurasthenic and hysterical symptoms, present indications, in slight cases, for the use of war'm sea or river bathing - $20^{\circ} R$. ( $77^{\circ} \mathrm{F}$.) to $18^{\circ} R .\left(72.5^{\circ}\right.$ $F$.) - and also of slightly carbonated baths ; for more pronounced cases plain or salty, thermically indifferent baths, while for strongly pronounced ones balneotherapeutic treatment is contraindicated. For general obesity cool salt baths and river and sea bathing are suitable. For diabetes mellitus, which is etiologically so closely connected with the nervous system, thermically indifferent and slightly cooling spongings are indicated in accord with the condition of this last. For gout thermically indifferent baths: of these the best are salty, alkaline ones.

11. For scrofulosis. - Thermically indifferent and cool salt baths, and sea-bathing.

12. For syphilis. - Warm baths to assist cleanliness and the functional activity of the skin (which in its turn aids in the better absorption of the mercury). and hot baths to enhance the activity of the metabolic processes, usually given simultaneously with the administration of the specific treatment; later on, to remove the debility and the predisposition to colds engendered by such treatment, strong carbonated baths (Narzan after Pyatigorsk), sea-bathing and hydrotherapy are resorted to.

13. In metallic poisoning, most frequently in mercury and lead poisoning, hot baths and later strengthening balneotherapeutic procedures are also used.

14. What concerns the application of balneotherapeutic procedures for febrile conditions and febrile affections it was spoken of and discussed by me in connection with the several analyzed cases.

15. Diseases of the skin and of the subjacent tissues. - Cold fomentations and ice in traumata, hyperæmia and inflammations. Baths with alkaline carbonates and sulphates for certain eruptions.

16. Chronic myosites, neurites (most frequently ischias) and arthrites, as multiple chronic arthritis and chronic articular 
"rheumatism." For these are suitable warm and hot, salt and alkaline baths, as well as the muds, and later, to strengthen the patient who became weak from the treatment and to remove the predisposition to cold, strongly carbonated baths, warm seabathing, as in Eupatoria after treatment by the Saki muds, and careful hydropathy. I must note the following: Strange as it may seem, I have often observed that treatment of "catarrhal" diseases, namely, of the above-named chronic affections of the organs of locomotion and also catarthal conditions of the respiratory and other passages, is often confouncled with the treatment of an existing predisposition to colds. Thus the patient tells his physician, that he has "rheumatics" and is frequently subjected to colds, although he suffers from none at the present time. The physician catches at the wor'd "rheumatics," prescribes warm or even hot baths, plain or salty ones (I had occasion to see the Saki mud adrised in view only of a predisposition to cold, without any cold or "rheumatic" affection being present at the time of the advice), which certainly only tend to increase this predisposition. This last condition must be treated not by hot baths and muds, but by removing its causative agencies, which are usually found to lie in gout, syphilis, abuse of alcoholic drinks, in a habit for abundant hot drinks and warn clothing, in neurasthenia, etc.; while for the treatment of neurasthenia and at the same time to inure the body to cold, hydrotherapy and bathing are suitable, and certainly not hot baths or muds. 


\section{CHOLERA, PARTICULARLY ITS TREATMENT.}

LECTURE OF MARCH 19, 1893.

Whether or not we shall be visited by cholera this year is uncertain. The history of the past presents no positive precedents in this regard. During the summer of 1883 Egypt was visited by a very severe epidemic of cholera, the one during which Koch succeeded in finding the cholera bacillus, while the summer of the following year in Egypt was perfectly free from it.

But it is certainly probable that we may also have an epidemic this present year. It is true that cholera, which remained over winter and came to life again in the given locality, may not possess the same virulence nor be diffused so extensively as when carried over from another locality; but still it is possible that many of you will have to face the necessity of combating this disease, and I therefore consider it my duty, the duty of a clinician, to assist you as far as it lies in my power by my advice.

Cholera, as many other diseases, is familiar to you from the systematic course of special pathology; but firstly, this course you attended a year or more ago, and science never remains in one place; it is never stationary : its level is becoming continuously higher and higher and is, besides, constantly changing, presenting not a smooth, but a constantly undulating surface: one point falls, the other rises to the surface. The year just passed, a year of a considerable cholera epidemic, was particularly instrumental in compelling us to revise our knowledge concerning cholera and especially its treatment. It is therefore imperative that you become acquainted with the results of this revision. But it chiefly devolves on the clinician, in view of a possible epidemic, 
to discuss the subject of cholera before his students for another reason. After attending on your course of special pathology, you meet later in the clinics all the forms of diseased conditions peculiar to our local and climatic conditions of life; but ordinarily you do not chance across cases of cholera. For the long period of my clinical activity, only once, in the sixties, when to my clinic was annexed a department for cholera, did I have the opportunity to discuss cholera cases before the students. But ordinarily, either there are no cases of cholera during the academic year, or, as it happened last year, during the epidemic the senior students of the last year are absent, having been sent away to combat the invasion of the disease. But the systematic course of special pathology cannot educate a physician without the aid of the clinic: the information obtained from this course, the therapy (not to speak of its other peculiarities) are of but an approximate nature, and only by individualizing this information, applying it to all the peculiarities of the given case, as taught by the clinic, can we hope for a successful treatment. As regards cholera it is the duty of the clinician to assist as much as possible in what the clinic is lacking, paying particular attention to treatment, to discuss as far as the lack of clinical individualization will permit, at least the principal forms of the disease, which may be seen in patients and which may require treatment.

The object and character of my lecture is thus determined by what I have said above. The question of the nature of cholera, and also. the picture of the disease, will be touched by me only so far as may become necessary for elucidating the prophylaxis and the therapy.

\section{The Nature of Cholera.}

Cholera belongs to the class of infectious diseases. These diseases are at the present time considered to be caused by micro-organisms, by microbes which invade the organism and produce the diseased condition therein. The microbes of all the infectious diseases have not as yet been discovered, but the very marked similarity, in their chief features, of those diseases the 
microbes of which have not been identified, with those where these have been found, compels us to class both varieties under one category.

It is not long since there prevailed a classification of infectious diseases into miasmatic, contagious, and miasatic-contagious.

By the miasmatic diseases were meant such as are not transmitted from one infected by them, to another, and the cause of which, the pathogenic microbes, enter the affected organism from the surrounding elements. The malarial diseases were considered as types of such affections.

As contagious diseases were considered such as are capable of being transmitted from one patient to another in a direct manner, without the participation of the surrounding nature. Among these were classified : syphilis. small-pox, measles, scarlatina, typhus fever, diphtheria, etc.

By miasmatic-contagious diseases were meant those, which, to become infectious, need the assistance, so to speak, of the surrounding elements and the presence therein of some special favorable conditions, without which the person affected by this disease within the given locality is incapable of causing an epidemic ; and on the other hand, the above-named diseases will not develop in the given locality, notwithstanding the presence in the surrounding elements of all the conditions favorable for their development, as long as this locality is not visited by a person who carries within his system the germs of the disease.

It was therefore assumed, that these, after leaving the patient's organism, are not yet capable of causing infection, but become so after remaining under favorable conditions for a certain time outside of the organism. To this class of diseases belong cholera. typhoid ferer, etc.

At the present time it is impossible to entirely rely upon the classification just outlined: successful experiments have been made, though it is true that they need further confirmation, of inoculation with the malarial diseases directly from the patient to a healthy individual; syphilis can be transmitted not only directly, but also through the medium of dishes, instruments, etc., consequently after it has remained for a certain 
time under the influence of the surrounding nature; the same is true also of diphtheria. It seems that at certain times, rare though such cases may be, cholera causes a direct infection. Nevertheless, the principal characteristic peculiarities of the different classes of infectious diseases, as outlined ahove, do exist and are to be kept constantly in mind: malaria is, after all, non-contagious outside of experimentation, and syphilis, measles, small-pox, scarlatina, typhus fever, diphtheria are of an extremely contagious nature ; while cholera is but slightly contagious and, in direct contradistinction to the very contagious diseases, it depends for its development entirely on the conditions present in the surrounding nature.

Outside of the "seed" of the disease and the conditions of the surroundings, an important rôle in infection, as proved by observation, is played by the individual's predisposition to cholera, or by the absence of the same.

I will take a brief survey of our knowledge concerning the three factors which play the chief rôle in the development of the cholera epidemics.

The individual predisposition to cholera has been shown by observation to be determined by digestive disturbances and by failure in nutrition, as eridenced by the fact that a population exhausted by starvation falls an easy prey to the epidemic. It is hardly probable that this fact is the only one determining the individual's susceptibility to cholera. but we know nothing' more definite about the subject.

It was Pettenkofer who called particular attention to the conditions of the surrounding elements, the local (meaning the soil and climate) and the temporary, the season of the year and the condition of the weather; but we know nothing positive regarding this matter: thus, for instance, Pettenkofer's theories concerning the influences of the vacillations of the soil-water on the development of the cholera epidemics have not been substantiated by facts. As an example of the prevalent idea as touching on the influence of the surroundings on the development of cholera epidemics, I will present before you the results of the observations and investigations made by the well-known bacteriologist, Prof. Hueppe, during the recently prevalent severe epi- 
demic at Hamburg.* I shall have yet another occasion to touch upon Prof. Hueppe's statements; and I will therefore only say, as far as they are concerned, that notwithstanding their interest, the facts presented lack verification, while the hypotheses propounded must be proved by facts.

After analyzing the local conditions, Hueppe considers as the cause of the sudden and very severe breaking out of the cholera epidemic in Hamburg not the conditions in the soil, but the condition of the Hamburg aqueduct. The water is derived from the Elbe, above the city, while the city impurities are conducted into the Elbe below the city; during the high tide of the sea, these last are carried up the river and reach the place from which the aqueduct starts, without passing through any purifying filter. According to Hueppe, the cholera was imported into Hamburg from Russia or France on sailing vessels by patients whose evacuations, without any previous disinfection, polluted the river. As a general fact, Hueppe assumes, basing this assumption on his own investigations and that of others, as Fränkel, that the cholera bacilli are rapidly destroyed in the rivers of Europe (although this is somewhat contradicted by investigations of other bacteriologists ), and that if cholera spreads along the course of rivers, it does so not by the aid of the cholera bacilli carried by the waters, but through cholera patients sailing on river vessels; but he points out that during the month of August, 1892, when the Hamburg epidemic began, there were exceptional conditions, as extreme heat, shoaliness of the river and the warm temperature of the water, which contributed to the fact that the cholera bacilli, as he supposes, were enabled to remain longer in the water, were carried into the aqueduct, in some parts of which, as the house reservoirs where the water not being in motion leaves a precipitate and has a warm temperature, they again became virulent and even began to multiply. It is hardly necessary to dilate on the fact that this supposition, however great its probability, lacks verification by facts. $\uparrow$

* Hueppe, Die Cholera-Epidemie in Hamburg, 1892. Berliner Klinische Wochenschrift, 1893, Nos. 4-7.

$\dagger$ Later addition: The spreading of cholera. principally through water, is very strongly substantiated by the last investigations of Prof. 
The seed of cholera, the cholera bacterium (the so-called " comma bacillus"), was discovered by Koch. He did not pay due attention to the conditions of the surrounding nature, whether local or temporary. He and his followers are justly reproached also for this, that the term " contagiousness " was employed by them in the sense in which the term "infectiousness" is ordinarily used, and thus cholera, in spite of other investigations (among which I may class also my own, in accord with which cholera is but slightly contagious, $i$. e. it is but very seldom transmitted directly from one person to another), was considered as a directly contagions disease ; it is attributed to them that this fact has engendered a sort of panicky feeling toward cholera. and that it assisted in the adoption of but very one-sided measures for its prevention. According to Hueppe, the observations made during the recent epidemic of cholera at Hamburg. have succeeded in completely eradicating the teaching of the contagiousness of cholera. This has also been verified by the observations of Dr. Wermel during the recent epiclemic in Nijni Novgorod (vide "Meditzina," 1892, Nos. 36 to 37 ).

According to Hueppe, the cholera bacilli are destroyed en masse in the large intestine (by the acidity of its contents, as he assumes), while those which remain and escape from the affected organism alive, do not possess any virulency, any power to infect, on account, as Hueffe thinks, of the deficiency of oxygen in the biological processes of the small intestine (a condition approaching that of anærobiosis). The cholera bacteria become again virulent and multiply outside of the affected organism under conditions of which we know nothing. The observations, made during the former, as well as during the recent epiclemic, to the effect that cholera is but slightly contagious and is but rarely transmitted directly from a sick person to a healthy one, speak certainly in favor of Hueppe's assertion, thus constituting his chief bulwark. But the case brought forward by Hueppe in proof of the slight contagiousness of the

Koch. R. Koch, Ueber die Cholera in Deutschland während des Winters 1892 bis 1893 (in the Deutsche Medic. Wochenschrift, No. 36, 1893); also R. Koch. Cholera und IV asserfiltration (in Berliner Klinische Wochenschrift, No. 27,1893$)$. 
cholera bacilli expelled from the sick organism (in this case at child devoured the excreta of his father affected by cholera, as they resembled rice soup. and remained unaffected). (an hardly prove anything, because of its singularity; it is possible that the child possessed an individual non-predisposition to cholera. a natural immunity toward it. I may as well state here that the various experiments of swallowing pure cultures of cholera bacteria, as has been done by Pettenkofer. Emmerich and some Vienna physicians. without any bad results occurring therefrom. can also hardly be considered as proving anything : not to speak of the individnal immunity (although it would be straining the point to constantly consider the same), we are hardly justified in judging by the degree of virulence of the cholera bacilli developed under artificial conditions of pure eultures, of the viruleney of the cholera bacteria which left the affected organism and underwent a development among unknown. to us. conditions of the surrounding nature.

The rare eases in which there was a direct transmission of cholera from the sick to the healthy, Hueppe explains by the fact that in the presence of a severe diarthoe the bacteria are enabled to rapidly traverse the intestines. and therefore. as he thinks, remaining but for a short time in a medium deficient in oxygen. they emerge from the affected organism with a sufticiently preserved degree of virnlence. The comparatively frequent occurrence of cholera to be seen among the washerwomen who handle linen not sufficiently disinfected. Hueppe considers as due partly to the presence in the linen of enormous numbers of bacteria of cholera. as well as of cholera toxine. and also to the fact that the choleraic bacteria. remaining in the linen mixed with the excreta. that is. living ontside of the organism. may becone anew sufficiently virulent.

'The miversally adopted theory of the contigiousness of cholera, as based on the above-disenssed facts and considerations, is as follows: the cholera bacteria enter the organism together with the food and drink. per os. multiply exceedingly in the small intestines. invading also their walls, produce a toxine, cause an extremely acute and severe affection of the stomach and intestines, as evidenced by nausea and diarrhea, and then. on ac- 
count of the great and rapil loss of the fluid portions of the organism and especially on account of the absorption of the above-named toxine, they eall out the phenomena of the algid stage of cholera, so peculiar to the disease, and of which we shall speak later.

\section{The Ford and Cougse of the Disease.}

In accord with the plan of my lecture, I shall not touch on the epidemiology of cholera. I will only say, that during the cholera epidemies there are observed, more frequently than at other times, intestinal catarrhs (diarhore), nervous disturbances of a nemrasthenic character, thanks, of comrse, to the panicky feeling towards cholera prevalent in the population, and also gastric disturbances of a dyspeptic nature, partly also of nervous origin, partly as a result of the sudden change in dietetic habits. of the monotonous food, of the constant use of boiled water, and also as a result of the employment of various " preventive" drops, as hydrochloric acid and others.

I will now take up the various choleraic affections, but I will call your attention only to their most important characteristics, the symptoms and the course, by which we must be guided in making a diagnosis and in prescribing treatment.

The incubation period, occupied by the time when the infection took place until the appearance of the disease, is short, usually between one and three days.

We distinguish three varieties of choleraic affections.

Choleraic diarhoc. - With a condition of general malaise the patient has abundant fluid stools but slightly colored with bile, usually of a painless nature, but accompanied by considerable abdominal gurgling. The disease either passes away in a few days (with or even without treatment), or passes into cholerine, or directly into cholera. The character of the suddenly appearing diarrhoea, during an epidemic of cholera, as to whether it be of a choleraic nature or not, can with certainty be decided only by a bacteriological examination of the excreta; without this last a probable diagnosis only is possible, but not a positive one. But the bacteriological examination, so impor- 
tant for defining the general character of an epirlemic affection. is not practicable for the purpose of diagnosticating cholera in private practice during an epidemic: the results of such an examination are not obtained soon enough. Jut at a comparatively late time. so that is impossible. while waiting for them. to leare the sick person without any treatment at a time when every hour is precions. and if he happens to suffer from cholerine or cholera, then every moment is invaluable. Besides, it is mmeasonable to waste time and energy on a minute bacteriological examination during a cholera epidemic. when the physician is time is 11sually wholly occupied and his energy is in a state of extreme tension. ('onsequently in cases of diarhota of which we speak now. we must be content with a probable diagnosis. As what concerns treatment in suclu cases. it will he spoken of later.

Cholerine. - Cieneral malaise, a slight felmile condition. romiting and diarlura of abmolant liquid discharges. at first colorerl. eren though slightly. by hile, later colorless; great thirst. very little mine. pulse weak, a rapid decrease in the body fat. cramps in the calres. general wealness. and reakness of the roice. In a few days the diseaste passes away. the patient is convalescent. but for a long time. for weeks together. he remains rery weak. In other less frequent cases. with a lack of proper treatment and nursing. the morlid plenomena increase and there appear sinptoms of the algirl stage of gennine cholera, during which. or during that of reaction following the algid stage. the patient sucoumbs: but with assistance at the proper time even such ases may tend to teminate farorably.

Gomime abolon develops either from the above-outlined ailments or more fireduently directly: after one or more dass of slight and painless diamhura (* the prodromal "). there suddenly takes place an etturk of afolnen: the evacuations hecome all at once frequent and abmurdant. they lose their yellow coloring and take on a resemblance to rice-sompl : to this there is soon superadded romiting. which consists in the beginning of the gastric contents. then of biliary matter. and tinally of similar. rice-sonp-like liquid masses. Eren a few hours of such a condition may be followed by the phenomena of the so-called cold 
period of cholera (stadi 1 m algidum, s. cyanoticun, s. asplyyeticums): the patient lies in a conclition of extreme weakness. speechless, at times moaning and whining with a weak roice from the pain caused by the muscular cramps. which however pass rapidly; when asked anything he replies slowly (conscions. ness weak) and in an exceedingly weat voice; the body is cold to the touch, the bodily fullness (turgor vitalis) is sharply dintinished, the cheeks fallen in, the nose sharp. the eyes deep in their sockets, the skin is cyanotic and winkled, and if caught between the fingers does not return to its previous condition; there is an insatiable thisst, no mine, pulse hardly perceptible. tones of the heart extremely weak. With the appearance of the algid stage the vomiting and diamhrea usually diminish.

The phenomena of the algid stage are undonbtedly, but only to some extent. determined by the great loss of the fluid portions of the organism, and by the results of this loss, as thickening of the blood, disturbances of the circulation. deficiency of oxygen in the blood and tissues. etc., caused by such a great transudation into the intestines: the loss of the fluid parts of the organism and the results dependent thereon are insulficient to explain the above-named phenomena. There occur cases, and very severe ones at that. which present an extreme development of the algid stage. the patients dying during it, while they have neither diarhoea nor vomiting (cholera sicca) and autopsy reveals but a trifling exudation into the intestines.

Moreover, every physician who has ever seen a goodly number of cholera patients, is well aware of the fact that very often there is to be observed a disproportion between the quantity of the liquid evacuations and the symptoms of the algid stage: the first may be very considerable, while the latter are insignificant, and contrariwise. It is thus evident that outside of the great loss of the fluid parts of the organism there must also be some other cause to account for the algid stage of the disease. As such was considered the toxine produced by the cholera bacteria. As a matter of fact both Nicati and Rietsch obtained from pure cultures of bacteria a poisonous body, the introduction of which into the systems of animals caused a fall in the temperature, paralytic phenomena and finally death. ()ut of the 
contents of the small intestines, as found on autopsies. Hueppe obtained a toxine which he considers to be a peptone produced from the albuminoid constituents of the intestinal contents under the influence of the cholera bacteria and of deficiency in oxygen. He also obtained an identical toxine from the contents of the large intestine and from the intestinal secretions of cholera patients. but in a much smaller quantity; this leads him to think that the above referred to toxine is partly absorbed by the small intestine, and is partly destroyed by the acidity of the large intestine. Introduced into the systems of animals, as guinea-pigs, rabbits and dogs, the cholera toxine produced a fall of the temperature, paralytic symptoms, and finally death; the paralytic phenomena were sometimes preceded by severe dyspnos and cyanosis: the autops of the animals so killed revealed the fact that the kidneys, liver, and the heart have undergone, according to Hueppe, changes similar to those found in the same organs of patients who died during the algid period of cholera, and on whom autopsies were performed. The absorption of the cholera toxine is certainly very probable, and may be considered as a generally accepted fact, otherwise it would be difficult to explain the so prominent and apparently toxic symptoms of the algid stage. (Hueppe assumes that he succeeded in proving the presence of this toxine in the first urine excreted during the reaction stage of cholera, which follows the anuria of the algid stage.) It is beyond doubt that it is not so much the loss of the liquid portion of the organism as the choleraic toxine, which determines the acute affection of the nervous system in general and that of the vaso-motor in particular, as observed during the algid stage, as well as the changes of the protoplasm, such as the clondiness and the coagulation-necrosis of the muscular heart fibres. of the hepatic and renal epithelia, which are all found at the autopsies of persons dying during this stage of the disease. The affection of the last (the epithelium of the kidney) causes a nephritis. the symptoms of which become prominent during the next reaction stage. on the pathological anatomy of which I may as well touch now : the vessels and the interstitial substance of the renal parenchyma are not affected. but the morbid process (coagulation-necro- 
sis) attacks the epithelium of the glomeruli and of the convoluted tubules, and it (the epithelium) becomes detached; in cases of recovery, the epithelium is rapidly regenerated and the nephritis does not pass into a chronic condition (Leyden, Berl. Klinische Wochenschrift, 1893). Without stopping to consider, in accord with the plan of my lecture. all the anatomopathological data peculiar to the algid stage of cholera and known to you from the systematic course of internal pathology, as the thickening of the blood, the drying up of the tissues and the like, I will only call your attention to the ehanges in the spleen so characteristic in cholera (Prof. stiller. in the Berlin. Klin. Wochenschrift, 189:3); this last is found in patients dying during the algid stage as climinished in size and wrinkled. a condition analogous to that of the vesisels and depending, quite probably, on identical causes. According to certain investigations, the spleen in the prodromal period of cholera is hypertrophied, just as it is in other infectious diseases. I)uring the stage of reaction the spleen again increases in size.

Patients die frequently while in the algid stage. especially during the very height of the epidemic. If they recover, then the clearly and unmistakably pronounced algid stage is never followed by an immediate recovery. lut there take place new and very variable morbid phenomena, which are collectively designated under the nitme of reaction period or under that of choleraic typhoid; although the incorrectness of this last term is admitted by everybody, still it became so popular that it is employed by all. These phenomena are of three varieties:

1. Most frequently there is an acute nephritis (as evidenced by a small quantity of urine containing much albumen, numbers of cylinders and epithelial cells), accompanied by severe uræmic symptoms.

2. Much less frequent is a febrile condition, accompanied by typhoid symptoms, without any renal affection, due evidently to the considerable alterations produced during the preceding stages in other organs, - in the intestines, liver, heart, spleen, blood, etc.

3. Various morbid couditions, their origin apparently due to other infections which found such a favolable soil in an organ- 
ism attacked by cholera: diphtheria of the intestines, of the urinary and biliary bladders, of the vagina, and also of the pharnyx (in conjunction with parotitis), of the larnyx with bronchitis, and, even if not frequently, with pneumonia and inflammation of the serous membranes, various affections of the skin (as eruptions), ulcers, abscesses, erysipelas.

All the three varieties of the morbid processes occurring during the reaction period may occur simultaneously in the most variable combinations.

A fatal issue is of frequent occurrence also during this period. Recovery after a severe pronounced attack of cholera is a slow process.

\section{Prevextefe Meastres.}

Following the plan laid ont for my lecture, I cannot stop to thoroughly discuss this most important subject, but will only make a few observations.

Quarantines, as employed heretofore, proved to be inefficient; but it does not occur to me, that the question about quarantines has been decided and that they had been recognized as inefficient or even impossible. To be sure it is difficult to picture to one's self the establishment of an efficient and rer'y strict quarantine along all the boundaries of the European governments. which are thickly populated and keep up such a lively communication among themselves: this would cause great embarrassment and could only be obtained at the cost of considerable expenditures. But the quarantine along the highways leading from the birthplace of cholera, from Asia, is a different matter. Our present Central Asiatic boundaries, through which the recent epdiemic of cholera forced its way, extend mostly through wilderness, and do not present any possible means for the establishment of a strict quarantine; but it is pointed out, that not far from these boundaries there are located mountain-chains which admit of a passage only through certain places, where it would be a very easy matter to establish the strictest quarantine which would embarrass only the insignificant caravan trains. We certainly are not to conclude from the fact that it is inpossible to establish reliable quarantines over all the Asiatic 
highways, that it is useless to do so wherever it is possible. I more direct application of the quarantine over the most important road from India, the Suez Canal, would probably hear hetter results, if not for the selfish opposition of England, which makes it impossible of realization. The brevity of the incubation period of cholera does not require any very prolonged quarantining. It is lardly probable, that cholera is transferred through goods. unless it be certain spectial ones, as old clothes and others, the admission of which is usually forbicklen by self-protecting governments. Special attention must be paid to the dirty linen of the passengers. by submitting it to strict disinfection or even destroying it by fire. It is true. that notwithstanding all the quarantine measures, if the quarantine is kept but for a limited time. which would otherwise canse extreme embarrassment in places with such an extensive travel as for instance the Suez Canal, * there may remain unolserved and may pass on persons sick with but a slight choleraic affection, choleraic diarrhea, but who may however carry with them the seed of infection and under favorable circumstances become the cause of an epidemic. Furthermore, from olservations made in Hamburg, it is made certain, that in persons who recovered from cholera, there are to be found for a long time, even after the intestinal secretions have entirely taken on their normal form, in these secretions the characteristic comma bacilli. However, the fact that the quarantine measures do not act as an absolute bar to the invasion of cholerd, need not mean that we must neglect their undoubted usefulness to diminish to a very great extent the possibility of its being transferred.

To thoroughly interrogate every single passenger, as was practised during the recent epidemic on the railroad lines, is of course a superfluous procedure; but the watching over the travelling public, at least along tracts with a considerable travel, on railroad and on river lines, the separation of the evidently

* (A later addition.) The last (1894) international sanitary conference concluded that in place of the quarantine at the snez, the sanitary inspection of the sailing vessels by physicians be enforced more strictly, if they arive from unreliable and doubtfnl ports, at the time they leave them, as well as during their jonrney. 
affected ones from the healthy, the application of disinfection and the rendering of necessary aid, are of course necessary measures.

I will not stop here to discuss the subject of disinfection, its means and ways, its application to the patient, to his linen and his residence, to the affected locality and houses, to the tramway cars, steamboats, ete., as all this is discussed for your benefit by a competent person in special lectures.

Nor will I speak here of the measures touching upon the reestablishment of the normal conditions in the affected localities; this is certainly desimable, but it must not be done in a hurry, as is usually done during the approach or even during the attack of the epidemic, but in a substantial manner, requiring a long time for its completion in accord with a matured, thought-out plan.

I camnot help stating here, that at the sight of the abovenamed hurrying measures adopted for the re-establishment of former conditions of health, the use of which measures is doubtful while their cost is great, seeing the frequently unreasonable and unnecessary expenditure of means for disinfection, which also entails considerable expenses, the thought occurs that it were better to devote the financial outlays thus expended to obtaining for the indigent population of healthy food and drink. as the benefit derived from these during a cholera epidemic is of inestimable value.

It is absolutely necessary, that a most thorough disinfection be effected around the patient, not only in cases of a clearly pronounced choleraic ailment. but in the presence of every doubtful diarrhoen; that the patient's excreta, his linen (if it be but slightly soiled, otherwise burn it) and objects surrounding him should be disinfected.

An important preventive measure is the careful supervision over persons arriving in the given locality, the isolation of those who present signs of an apparently choleraic and even of a doubtful affection, the inmediate application of thorough disinfection and also the immediate rendering of the necessary assistance. Every case to which we succed in applying thoroughly these moisures, diminishes the possibility of developing 
or of increasing the epidemic. The fortunate condition of things in Moscow during the last epidemic is undoubtedly to be ascribed in a great measure to the careful and skillful application of the enmmerated measures.

In July of last year, in view of the serious application of the above-named and other measures. and also on account of other considerations. I expressed a hope (vide the Moscow Gazette, July 16, 1892) that the further course of the epiclemic would be of moderate severity, at least in the northern and the northwestern provinces of Russia, heretofore not visited by the clisease. The course of the epidemic in Nijni Novgorod, Moscow and St. Petersburg and in other localities of the above-named provinces in Russia has completely justified my expectations: while on the southwest the epidemic was severe. I have no doubt, but that if in Baku and other places of Transcaucasia, in Astrachan. Tzarytzin. Saratov and Samara, the same measures had been adopted as were practised in Moscow. instead of the absence, not only at the beginning but also later on, of any preventive measures, and instead of the street riots which assisted so much in spreading the cholera, the course of the epidemic would have been different; it would not have developed to such a degree in the cities and would have been prevented from invading the villages and country places, where preventive as well as any curative measures are so difficult of application. while the population, which is generally poorly fed, was exhausted by a previous failure of the crops.

The individual measures of precaution can be summed up in this, that one must not in any way alter his habitual mode of living, by which we mean a mode which preserves one's health in good order: in case there be any disturbance of health one must at once look for a physician's advice, also on the return of the habitual disturbances, and especially those of the digestive organs; he must follow a certain once prearranged plan of treatment and hygiene, but under no circumstances is he to be allowed to treat himself by various " cholera" measures, drops, etc., so extensively advertised during the prevalence of an epidemic; when perfectly healthy he must avoid the use of the socalled (psuedo) preventive measures, as for instance hydro- 
chloric acid. quinine, etc. which will only tend to disturb digestion, thus creating a predisposition to choleraic affections. Pure spring water need not necessarily be boiled for drinking purposes. while water of doubtful purity. if its use cannot be a voided. must previously be allowed to settle down. be strained and boiled, and. I must say, it should he drunk only when recently boiled. while water which lad been boiled a long time previously should not be used for drinking purposes.

\section{The Treathext.}

I alluded in the beginning of the lecture to the fact, that, to replace as far as it is in my power (hecause of the absence of cholera patients) the lack of clinical individualization, I will discuss at least the most important situations in which a cholera patient may find himself. and to which treatment must be applied: I must also add that this is the more necessary, because of the rapid comse of the disease. and the lack of time at the physician's clisposal, for the number of sick is usually great; a calm and circumstantial analysis of the patient's condition is much more difficult in cholera. than in any other disease. and it is therefore necessary to have become previously acquainted with the peculiarities of treatment of the various conditions in which the patient may be found. I must however take a preliminary review of some of the general therapentic statements in connection with the treatment of cholera. I consider it as of special utility for you as beginners: a correct view and a clear intelligence constitute the most important requisites of successful activity.

We are frequently told, to be sure not by medical men, that, as the statistical data prove the mortality from cholera to be the same under all systems of treatment, which goes to show the impotence of every kind of treatment of cholera, it is useless to waste any means on it, and it were better to devote the means on hand to obtaining nourishing food to that portion of the population. which i. particularly liable to fall a prey to the disease on accomnt of insufficient nourislment. This assertion wan be refiated in a few words: only a layman, who in 
juclging of the effectiveness of treatment relies on such doubtful data as those lent by statistics. could pronounce himself against the treatment of cholera : but every physician who had the opportunity of seeing a sufficient number of cholera patients. and who perceived clearly the difference in the comse and the issue of the risease between the cases in which treatment was begun early. and those that were treated late. cannot but be fully persuaded of the effectiveness of and the crying urgency for treatment of cholera patients.

It is with a certain degree of pomposity that the assertion is made that it is not the cholera. but the cholera patient who must he treated. Yon who are well informed of the fundamental prineiple of clinical. i. e. practical activity. namely. that in all diseases. without any exceptions, we treat the patient and not the disease, will be astonished and taken aback by such an assertion. and rou will naturally ask: how otherwise? and when can we see and treat the disease. if not in the patient?

As you know. there exists no specific remerly for cholera, i. e. such a remedy as wonld not act upon some one separate symptom of this disease, but striking. so to sat the rery centre of the affection. would thus act on all the symptoms. and by destroying them. would bring about a cessation of the disease. as quinine does in malaria, sodim salycilate in acnte articular rheumatism, and to a lesser extent, as the results of the treatment are not so positive, mercury and the iodides in syphilis. As we have no such remedy, it remains for us here. ds in other diseases for which there exist no specific remedies. to pursue a symptomatic treatment, combating separately the symptoms which exhanst the patient, and subject his life to danger, and thus we enable the patient to tide orer the disease. and save his life.

Still it is strange to hear donbts expressed regarding the possibility of a specific treatment of cholera. Where can there be a basis for such doubts in view of the ahove-namerl existing specific remedies for certain diseases? And especially at the present time. when there bad been opened to therapy so many new and promising ways creating a possibility of treating the most serious diseases by the aid of the secretions and of the 
tissues of the organism itself, and also by the products of the life-activity of the pathogenic microbes, the greatest enemies of the organism. Tho is able to foretell the various means, the remedies, the combinations of various means and remedies, that the therapy of the future will make use of?"

Stranger still appears such an assertion: "For other (outside of cholera) infectious diseases, as typhoid, pneumonia, etc., we have long ago rejected specific remedies, having concluded, that symptomatic treatment only (?) will assist the organism in getting rid of the infectious agency." But this is not true; nobody ever rejected then ; let there be found to-morrow as reliable a remedy for typhoid and pnemmonia, as quinine is for malaria or salycilate of soda for acute articular rheumatism, and every physician. including the anthor of the above statement, will at once resort to it, reducing to a minimum, if not discharging altogether, the usnal symptomatic treatment; thus, for instance, it happened with the symptomatic treatment of acute articular rheumatism, which was being employed at the time before the specific effect of sodium salycilate became known. It is rather odd to hear it stated, that " the symptomatic treatment only will enable the organism to free itself from the infection," in view of the effects of the above-named specific remedies in malaria and acnte articular rheumatism, effects which excel so much by their accuracy and rapidity the symptomatic treatment of those diseases, for which we have no specific treatment.

Equally strange is the identification of the specific treatment with the routine one.

This is a confounding of ideas. Routine is not a peculiarity of any method of treatment, but surely a deplorable quality in the physician himself: hoth the specific and the symptomatic methods of treatment may be applied in a routine mamer, and again they may alio be employed in a non-routine way, so as to pay dne attention to the characteristic peculiarities of the given case, of the treated patient, which would hut mean to subject him to a systen of individualization in treatment.

I will turn now to the discussion of the treatment as applied to the vallious conditions in which the patient may be found. 
1. The diurhere. - It is self-understood. that not every cise of diarrhoea met with during a cholera epirlemic is to be treated as a choleraic diarthed. During such a time we may run across patients suffering with a chronic intestinal catarth which causes constipation intermittent with diarrhoea, or even a continuous diarrhora; if the diarrhora from which such patients sutfer, remains during the cholera epidemic in the same condition as it was before, then there is no reason for changing the usual treatment of such a diarrone, for attributing to it a certain "anticholeraic" shade. thus inordinately frightening the patient. I will add right here. that not only in such cases, but also in cases of doubtful or even undisguised choleral ailment, it is necessary to insistently keep up the patient's spirits by eneouraging him that the disease he suffers from is a "simple," and not a "genuine choleraic" affection, thus averting as much as it lies in our power the oppressive mental state. which is produced in the patients by the anxiety of falling a prey to cholera, and which tends to make their condition so much worse.

We may also see during the cholera epidemic cases of acute diarrhoea. but apparently of a non-choleraic nature, as for instance, acute catarrh of the large intestine, as evidenced by frequent inclination to stool, tenesmus, pains. and scanty evacuations with an admixture of nucus and blood.

However, such eases are rather of infrequent occurence during a cholera epirlemic, and the acute diarrhces met with at such times are usually, or in all probability. of a choleraie nature (as described above), or of such a character, as not having an apparently non-choleraic nature, and they therefore give rise to suspicion of their being of a choleraic origin (" the doubtful cases"). A positive diagnosis, as we explained above, is impossible; the bacteriological examination, which would positively diagnosticate either class of the cases. is not applicable in practice.

What then is the treatment of such eases? Having first of all informed yourself by a short inquiry of the kind and quantity of food and drink the patient had been taking recently and of the character of intestinal evacuations, and having inspected and palpated the abdomen, you should decide as to 
whether or not the intestinal canal is sufficiently emptied of its contents. If not, it must be emptied by the aid of laxatives: this precedure, so necessary in any diarrhca in case of insufficient emptying, the more so of overfilling of the intestines, is the more necessary in cholera affections, in which case the laxative may he expected to remove together with the other intestinal contents the cholera bacteria which so greatly multiply in the small intestines, and the toxine produced by them.

Which laxative is the most suitable for such cases? Castor oil or calomel with castor oil. I will take up calomel first. As a laxative calomel is particularly useful, as my observations during the former epidemies of cholera, principally of 185.3 and 1854 , testify, in patients of a robust or at least not of a weak constitution, with a large abdomen; in those who are subjected to dyspeptic gastric phenomena (in such cases the castor oil is frequently badly borne and is vomited up), to habitual constipation. jaundice, to biliary colic attended with constant tenderness on pressure over the region of the gall-bladder. I administer a four or five grain dose of calomel, repeat it in two hours, and again in two hours (if by that time there have not taken place abundant evacuations sufficient to empty the intestines); then I give a tablespoonful of castor oil. which is usually better borne after calomel. To patients who do not present the above-named peeuliarities. I only administer castor oil.

I will also touch here on calomel as a generally "' anticholeraic" remedy. It was recommended as such not only in the hegimning of the disease when the intestines are overfilled, but also for the later stages attended by a continuous, even severe diarrhoa, as well as for the algid stage of true cholera, under the assumption. that if taken internally, it will be converted into corrosive sullimate. thus exerting a fatal effect on the cholera bacteria found in the small intestines. Calomel is particularly recommended for cholera by the well-known Miinich clinician \%iemssen. But I will repeat here what I said in 1885 , concerning the nse of calomel, namely : in cholera I did not see the slightest success from the employment of calomel during the two severe epidemies at Mosenw in the years 185:? and 1854 , when, in the eapacity of a resident physician of the 
temporary cholera department in the University clinic, I had the opportunity of seeing a great many patients, and when calomel formed the ordinary treatment of the disease. Calomel was also tried during the recent epidemic and it was proved, that its use was not only of no positive, but not eren of a doubtful value. Besides, it is quite probable, that its continuous employment results in producing a harmful effect on the intestines which had already become strongly affected by the cholera, and on the kidneys, the affection of which, appearing during the period of reaction, is of such frequent occurrence in cholera, and is fraught with grave danger to an organism severely shocked by the preceding course of this disease. The above-named cases, in which. according to my observations, calomel in the beginning of the disease exerts an excellent effect as a laxative remedy, without, I may add, the least danger to the patient, are the only ones, for which I administer the drug in cholera.

I will add that for the identical purpose of directly acting on the cholera affection, by destroying in the intestinal tract the cholera bacteria and the toxine or toxines produced by them. there were recommended, besides calomel, many other drugs, notably salol. Experience proved that they were of the same value as calomel, as far as their direct anticholeraic effect is concerned, and that they also exerted a harmful effect. Hueppe, from his observations during the recent cholera epidemic, recommended specially tribromphenolbismuth; but data communicated by him, as well as the observations of other physicians, fail to show that this remedy deserves any greater credit than calomel, salol, and a host of others recommended until now.

I return now to the treatment of the diarrhcea. What is to be done for cases, in which, after an evacuation of the intestines had been effected by a laxative, the diarrhoea still continues, and also for those cases which present no necessity for the administration of a laxative, as the physician sees them at a time when the intestinal tract has already been evacuated, but meanwhile the diarrhoea continues, thus certainly debilitating the patient? In either class of cases we must first of all give opium, namely, the tincture (tinctura opii simplex s. thebaica), as the most suitable 
opium preparation, and as one, besides, which ean be divided in doses most conveniently, not requiring any weighing, like opium purum and extr. opii aquosuns. The employment of opium at the present time must certainly be explained.

During the former cholera epidemies opium may be said to have been the most generally employed remedy in doubtful as well as in undisguised cases of choleraic diseases. At the present time the matter is considered in a different light: starting with the supposition, that opium, by weakening the peristaltic efforts of the intestines, assists in the retention and multiplication (as determined by the greater amount of rest allowed to them) in them of the cholera bacteria, thus at the same time tending to increase the quantity of the produced toxine and the general intoxication of the system, many observers insist upon completely renouncing opium as a remedial agency for choleraic affections.

But my observations, as I mentioned above, were sufficient for my formulating the following: I had the opportunity during the cholera epidemics to cure by moderate doses of opium (of this later on) many cases of diarrhea, after a preliminary evacuation of the intestinal tract, or without the same when there were no indications for it. The exact nature of these diarrhoeas remained then, and remains now uncertain : it is just as impossible to assert that they had all been choleraic diarrhœas, as that they had not. In other, much less frequent, cases, notwithstanding this method of treatment, in conjunction of course with the appropriate hygienic measures, the diarrhoea did not stop and there developed cholerine or genuine cholera.

With such observations in view I consider it erroneous to reject a remedy which acts so successfully and so rapidly in the majority of cases under discussion, and I recommend, after the intestines have been evacuated, to administer at once to an adult patient fifteen or twenty drops of the tincture of opium, and later on, if necessary, in accord with the effect of the opium and the course of the disease, to give the same in five-drop doses and always in warm (boiled) water, or with the addition of strong wine or good clarified brandy. The ordinary dose is from thirty to forty or even sixty drops during twenty-four hours. 
This treatment I usually continue for one, one and a half, or the longest, for two days. And what is to be done next? In the greater majority of cases, this treatment will bring about a cessation of the diarrhoa. In other, less frequent, cases, the diarrhoea is only diminished, but does not cease altogether, and without taking on in the least the character of a choleraic affection, it is prolonged, and shows an inclination toward becoming chronic; in such cases, leaving aside opium, I resort to bismuth, especially if the large intestine become affected, or tra. coto, if the small intestine is the seat of the disease, or both together; I also administer ordinary elysters, or with bismuth, tannin and boric acid. Finally, there are still less frequent cases in which the diarrhøa, in spite of the treatment, becomes aggravated, and the affection takes on a choleraic character. Then, but not before one or two days of opium treatment, I set aside the opium, principally because in moderate doses it is ineffective, while the administration of large doses is fraught with danger: for such doses will overcome the irritant influence of the choleraic affection on the intestinal peristalsis, which may result in constipation - a condition certainly of extreme danger, and a moderate diarrhoea is by far preferable to a non-evacuated intestinal tract; but even if such be not the case, then in view of the possible approach of the algid stage, when the nervous system is most severely affected by the choleraic toxine (with the resulting thickened blood and a lack of oxygen), I consider it very dangerous to still more weaken this system by the introduction of large doses of opium.

Having then set aside opium, I administer internally bismuth subnitrate in doses sufficient to put a stop to the excessive diarrhoea which deprives the organism of so much fluid, namely, ten to fifteen grains at a dose, from four to six times a day; I recommend bismuth as a remedy reliable in non-choleraic diarrhœas, which acts at the same time as a disinfecting agency, is well borne by the intestines and does not exert any deleterious effect on the nervous system, as would be the case from large doses of opium.

But as regards the expectations reposed in bismuth as a positive anticholeraic agency, exerting an effect on the cholera 
bacteria and their toxines, which effect would be evidenced by a rapid and decided change of the choleraic affection to the better, these expectations were but little substantiated by observations, just as was the case with calomel, salol, etc. Together with the bismuth I arlvise for patients suffering from the cholera affection in the condition under discussion large (from 1000 c. c. and more). and hot ( $38^{\circ}$ to $40^{\circ} \mathrm{C}$.) watery clysters with tannin (one per cent solution), as recommended by the well-known Neapolitan clinician Cantani.

Cantani * in his treatment of cholera chiefly aims: first, to destroy the cholera bacteria and their toxines in the intestines, and secondly. to diminish the thickening of the blood and to assist in the elimination from the organism of the cholera toxine absorbed by the blood.

To effect his second purpose he recommends the introduction hypodermically of a solution of common salt (the so-called hypodermoclysis, of which we shall speak in connection with the treatment of the algid period): while for the first purpose he resorts to the above-named clysters (enteroclysmata), in the belief that they may reach beyond the ileo-coeal valve (valvula Banhini) into the small intestine and there exert an influence on the bacteria and their toxines. I personally had as yet no opportunity of treating cholera patients since the time (1884) that Cantani called attention to his method; but there are abundant proofs in the literature of the subject which speak of the merits of Cantani's treatment: hoth his own and the enthusidstic reports of many other Italian physicians fell short of the expectations excited, and the percentage of deaths has hardly changed perceptibly since the introduction of this method; nevertheless, the enteroclysmata as well as the hypodermoclysmata are recognized as useful aids in the treatment of cholera.

The principal effect of large hot enteroclysmata consists in that they communicate warmth to the body. which means that they commmicate the exeiting influence of the heat to an organism weakened by diarthos and the cholera affection in general: the diarrher and the vomiting diminish, the pulse grows stronger. the self-feeling becomes better. The entero-

* Tide his communication in Berl. Klin. Wochenschrift, No. 37, 1892. 
clysmata exert also some effect by acting on the cholera bacteria and their toxines in the large intestine, and by removing them.*

What other measures, outside of those enumerated above. must we resort to in the treatment of the affections under discussion? I refer of course only to such patients as enjoyed fair health up to the time they fell victims to cholera (we surely cannot touch here on the treatment of persons afflicted with any chronic or aoute affection previous to their being attacked with cholera, as that will lead us too far). In such patients, besides the intestinal therapy, we must also treat the cardiac and the general conditions. To combat these last symptoms, as well as those presented by the intestines, the chief measures resorted to, besides those mentioned above, consist in keeping the patient warm (as by a warm bath. by covering the patient well, and particularly by keeping the abdomen warm, and by giving warm drinks - the best is weak tea), and in the administration of alcohol in the form of wine. cognac, rum and distilled brandy. The hot baths ( $30^{\circ} R$. and higher), so useful in the algid stage of cholera, are of comrse unnecessary in the affections under consideration: these measures and the employment of enteroclysmata, repeated as necessary, will keep the patient sufficiently warm.

2. Cholerine or the period of cholera proper, precoding the alyid stage. - The same heat-generating measures and the enteroclysmata employed repeatedly. For internal use, as far as vomiting permits it, bismuth and alcoholics. There are no sure measures against vomiting: we try ice. ether, and in very rare exceptional cases, as, for instance, when the romiting takes on a dangerous character in cholera patients who are afflicted with cardiac or arterial disease, we resort to hypodermic injections of morphine (one-eighth or one-fourth grain at a time) or cocaine (Bx. cocaini muriatici gr. $x v$, aque destil. 5 ss). A whole or one-

* (A later addition.) Prof. Genersich recently recommended washing out the whole alimentary canal (so that the fluid introduced per anum is ejected by vomiting per os) in cholera and other diseases (Progrès Médical and Deutsche Medicinische Wochenschrift, 189:3). It remains for the future to determine the value and the application of this method of treatment. 
half syringe at a time), the introduction of which into the organism, with the consequent weakening effect on the nervous system, is not desirable at all in view of the possible approach of the algid stage.

But usually vomiting, and its effects on the organism, do not require the use of such strong measures ; moreover, as mentioned above, the enteroclysmata diminish the vomiting to an extent. Nor do the cramps necessitate the employment of strong measures, such as hypodermic injections of morphine. The best means for combating the cramps is to immediately put the affected part into a position opposite to that into which it was contracted; most frequently the cramps take place in the muscles of the calves extending the foot; it is sufficient to flex the foot and the cramps cease; however, as was mentioned above, the cramps tend to rapid disappearance by themselves.

3. The alyid stage of cholera proper. - The above-named measures contributing heat, including the enteroclysmata, also hot baths: in a general way the body should be kept warm by all suitable means at hand. Later on, with the first symptoms of the approaching algid stage, to counteract the thickening of the blood, the lack of oxygen and the anuria, and also to excite by warmth the heart and the nervous system, resort must be had to Cantani's large (from 300 to 1,000 c. c. at a time) hot ( $38^{\circ}$ to $40^{\circ} \mathrm{C}$.) hypodermoclysmata, containing a solution of common table-salt ( 7.5 to 1,000$)$, injected into places where the skin is easily raised in folds and the subcutaneous layer affords a convenient receptacle for the introduced fluid (except in the region of the neck, into which the introduction subcutaneously of a large quantity of fluid is known to be fraught with danger). Usually after such a hypodermoclysma has been administered, the pulse, hitherto hardly perceptible, grows stronger, and the general weakness of the patient diminishes. It is true that this improvement does not last long, and on the hypodermoclysma being repeated, it - the improvement - is not as considerable as it was the first time; nevertheless, the value of the hypodermoclysina is beyond doubt, and its employment is unconditionally indicated. The injection into the veins of a salt solution does not offer any advantages over the hypoder- 
moclysmata; it is moreover applied with greater difficulty, unless it be in the hospital, and besides is not devoid of danger. Finally, during the algid stage, it is of the greatest importance to introduce stimulants both internally, such as hot tea, the various alcoholics, ether, camphor, and hypodermically; for this last we use oleum camphoratum fortius $(1: 5)$, one syringeful at a time, and caffeinum natrobenzoicum, from one-third to twothirds of a syringeful (it is best used thus: R. natri benzoici, caffeini āâ 3 ij, aquæ destil. $\bar{z}$ ss.; such a considerable concentration will assist in keeping the solution in better condition, nor will it become turbid). It needs no explanation that both the hypodermoclysmata, as well as the hypodermic injections of the various stimulants, should be made under the strictest antiseptic precautions.*

4. The period of reaction (typhoid cholera). - The treatment of the nephritis caused by cholera consists in warm baths and abundant drinks.

The treatment of the typhoid and uræmic symptoms: signs of congestion (as a flushed face) require the application of cold to the head or even the application of leeches to the root of the nose, if the patient is not very weak; for symptoms indicating excitability of the nervous system (as delirium, etc.) sodium bromide; and for symptoms of nervous oppression, moschus and washing of the head with fresh water.

If the body temperature be very high (about $40^{\circ} \mathrm{C} ., 104^{\circ} \mathrm{F}$.) small doses (three to five grains) of phenacetine or antifebrine will reduce it to an extent.

* (A later note.) Prot. Shkliarefisky ( “On the Treatment of Cholera luring the Algid Stage," a reprint from Nos. 16 and 17 of the SonthRussian Medical Gazette, 1893) calls attention to the long forgotten, but according to the observations of many physicians, very effective treatment of the algid period of cholera by the method of Dr. Chapman. "The gist of this method consists in this, that with the aid of cold or heat an effect is produced on the patient's spinal column, while the other parts of the body are subjected to heat in the first case, and to cold in the second by compresses. Thus a rubber bag filled with ice is placed along the spinal column of the patient during the algid stage, and during the typhoid period a similar bag is filled with water at the temperature of $38^{\circ} R$." Considering the algid period of cholera as an "infectious neurosis," Prof. Shkliareffsky explains from this point of view the effect of Chapman's method of treatment. Prof. Shkliareffsky's article is full of interesting points. 
The treatment of the various complications - as erysipelas, ulcers, abscesses and diphtheritic affections (as dysentery, etc.) - is to be conducted according to the indications of these complications.

Having spoken thus far of the genuine Asiatic or India cholera. I will now say a few words on cholera nostras.

The attacks of this disease are similar by their nature and course to those of the genuine cholera, and may also occur with a similar intensity, but are not as extensive as these last: cholera nostras occurs only as an endemic, never as an epidemic disease. The microbe which produces it is as yet unknown: they failed to find the comma bacilli of the genuine cholera in the evacuation of patients suffering from ou (or European) cholera. During the prevalence of this last Finkler and Pryor found a bacterium similar to, but not identical with. the comma bacillus of genuine cholera; but in the following epidemics, the Finkler-Pryor bacilli have not been found. Last spring, there occurred in the vicinity of Paris (at the beginning only there, and at no other place in France) severe cholera-like attacks, but withont any notable tendency to further spreading. Considering them of the nature of cholera nostras and noting that they occurred in the localities which had formerly suffered severely from the true Asiatic cholera, some physicians assumed that cholera nostras was but a degenerate descendant of the Asiatic cholera. The treatment of the attack of cholera nostras does not in the least differ from that of the genuine Asiatic cholera. 
SUPPLEMENTS. 


\section{ON BLOODLETTING.}

\section{AN ADDRESS DELIVERED AT THE ANNUAL MEETING OF THE MOSCOW PHYSIO-MEDICAL SOCIETY, JANUARY, 1889.}

Gentlemen:- I propose to speak of bloodletting - a remedial measure the value and importance of which an experience extending over many years taught me to appreciate. I shall discuss bloodletting only as applied in the domain of internal medicine, without touching on its use in surgery, gynæcology, pediatrics, etc.

This is not the place to dilate on the history of bloodletting, which begins, probably, with the history of medicine itself. I shall only touch on that period in its history, of which I have been an eye-witness myself; I shall do that, that I may show how my own experience was formed concerning the eniployment of this measure. During my student years and my three years' clinical assistantship, bloodletting, general as well as local, was resorted to frequently and unsparingly: the influence of Broussais was as yet prevalent, although not strongly, and I must add, to a constantly diminishing extent. I visited later, in 1856, Westem Europe, and found there a complete reaction against venesection, both in literature and in practice: during my three years' sojourn there, I did not see, in any of the clinics in Germany, Austria and France, the employment of bloodletting, and only very rarely indeed did I witness the use of cupping-glasses and leeches. On my return to Moscow, I also found here the beginning of a similar reactionary movement, which resulted in this, that eight or ten years later a similar negative stand was taken against the employment of bloodletting in practice ( $\mathrm{I}$ do not speak of my own), as obtained at that period in Western Europe, as well as in England.

Let us consider the position occupied by this subject at the present time. In Western Europe we hear complaints of the unjust condemnation and neglect with which the subject of bloodletting is being treated; and from such men as, for instance, in England, Fenwick, Broadbent and Oliver: in France. 
Hayem; in Germany, the well-known clinical teachers and authors of special pathology and therapeutics, Liebermeister, Strümpell, and Eichhorst. As all these complain of neglect, this seems to be continuing. Liebermeister, Strümpell and Eichhorst recommend bloodletting, but they do not give any sufficiently clear indications for its employment, thus showing but a poor personal experience. Regarding these same recommendations it is interesting to note the opinion of another clinical teacher and author of a text-book of special pathology and therapeutics, Jürgensen, who confesses openly to being sceptical towards bloodletting, and who expresses himself as follows: "Personally I am, perhaps, too much afraid of blood, and I will therefore rather not enter my opinion." * He further makes a statement, which is evidently intended for Liebermeister, Striimpell and Eichhorst, authors of the most widely read text-books of special pathology and therapeutics, to this effect: "We observe a tendency in our text-books of to-day not to wholly neglect the use of bloodletting. even if the author in his practice does not employ it at all, or very rarely." $\dagger$

As regards medical practice with us, with the exception of Moscow and the physicians who are the followers of Moscow methods in medicine, everywhere else, in the principal medical centres, the university cities, as well as in the regions embraced by their influence, bloodletting, and abstraction of blood in general, is looked upon, as far as I know, with disfavor. I am unable to bring before you any literary material on the subject, as I am not aware of the existence of any such; but as far as is known from practice, the stand taken against bloodletting is of a pronouncedly negative character, at least up till now, in the above-named localities. Thus, for instance, a patient from a provincial city who applied a few leeches by the advice of his physician, a graduate of the Moscow University, and who was considerably relieved by them, later on, while consulting a University professor, not in Moscow, heard him ask, after inquiring about his disease and the treatment, "Whoever uses leeches now?" Or another example: a

* Ziemssen, Handbuch d. allgemeinen Therapie. I. B., 2 u. 3 Th., Antiphlogistische Heilmethoden v. Jïrgensen, S. 159: "Ich fïr meine Person bin vielleicht iibertrieben blutscheu und möchte daher mit meinem Urtheil zuriickhalten.

$\dagger$ Tbidem, S. 219: "Man erkennt in unseren heutigen Lehrbiuchern das Bestreben die Blutentziehung nicht ganz fallen zu lassem, auch dann, wemn der Verfasser in praxi seltenen oder gar keinen Gebrauch davon macht." 
patient, who was suffering with pain in the stomach and with nausea due to extreme hyperæmia of the liver caused partly by weak cardiac activity, partly by abuse of wine, and who has been taking various drugs without any relief, called in the aid of a Moscow physician. This last ordered leeches to the coccygeal region: on the very next day the above-named suffering's disappeared, without in the least weakening the patient. One month later the patient returns to his constant place of residence, and tells his former physician, a clinical professor, of the improvement in his health, whereat this last remarks: "But this improvement is only temporary." Patient however replies : - Whether temporary or not. I cannot say. but I appreciate it dearly, whereas the former medicinal means have not done for me even that much." Or here is another: a patient, who at the advice of his physician. a Moscow man, applied leeches and was relieved by them, was asked by another doctor, not from Moscow, as to the age of his physician. When told that he the physician — was thirty years of age. he observed : I thought he was seventy years old, as he employs such ancient remedies." I had occasion to hear a great many similarly expressed opinions; but I have not heard such as would favol the employment of bloodletting. It is also curious to note, that the unfavorable opinions come from persons, who have never employed bloodletting, and who therefore had no personal experience with this method of treatment.

Turning now to Moscow and the region influenced by its teachings. I will first of all touch upon my personal relation to the subject nnder discussion. As I said before. during my student years and my clinical service, bloodletting was practised frequently and unsparingly, so that I had many an opportunity to witness its undoubted ralue, when it could not be replaced in many cases, while in others I also saw great harm caused by it. Of course, only later on, as ny practical experience began to mature, did I learn to appreciate with greater exactness its beneficial as well as its harmful effects. The errors of the medical practice of those times consisted in this, that it was taught that bloodletting would cure diseases - - the inflammations" - considering as an inflammation pneumonia. acute articular rhemmatism. etc.; consequently, without taking into account the condition of the patient's strength, they resorted not only to one, but to repeated bloodlettings (the socalled "coup sur coup" of Bouillot), not curing the disease, but debilitating the patient, thus increasing to a fatal extent the 
conditions favoring a bad telmination of the disease. Another cause of the unfortunate employment of bloodletting was to be found in the imperfectness of diagnostic skill at those times: thus, for instance, in headache, in which out of ten cases we will probably in one resort to bloodletting in the coccygeal region, we then did it if not in all the ten, surely in nine, and we did not put five to eight leeches as is done now, but from ten to twenty and more. As one of the harmful influences of those times must be considered the universal custom of the laity to resort to local, and at times even to general bloodletting. without the physician's advice, a custom which fortunately does not exist at the present time, thanks, we must openly confess, to the negative stand taken by the medical profession toward the subject of bloodletting.

But I must say again, that at the same time, at the beginning of my medical career, I had occasion to observe the undisputed benefit from bloodletting and even the impossibility of replacing it, and I therefore never left off this method of treatment; but in accord with my experience extending over many years, with the continually developing medical maturity and the rapid progress in the domain of diagnosis, general pathology and therapeutics, I worked out a different practice of bloodletting. As regards the practice in Moscow, I can remember the timethe acme of the period of opposition to bloodletting - when I met ahmost no colleagues who resorted to bloodletting as a derivative measure, and I had to defend my position when advising its application in consultations. At the present time the matter stands otherwise: my own practice and that of a number of the attending physicians at my clinic, as well as personal assistants who became later on independent practitioners and partly also clinical instructors, have brought it about that the employment of bloodletting as a derivative measure in the way I am applying it, has become firmly established in Moscow and the circle of its medical influence.

I will explain now the method used in my present practice of bloodletting. I shall be concise, as I intend to speak only of what is proved by facts to be certain, without touching upon theories which explain and prove the benefit derived from bloodletting. In the present condition of physiology and pathology such theories are as yet impossible: there exists no theory that could not be contradicted; and in doing this, there is always a danger, - to be sure, only when the subject is but superficially diseussed, - while disproving the 
theory on which the facts are based, to consider also these last as refuted. My conviction regarding the benefit of bloodletting as based upon facts has been reached by the same method of analyzing and criticising, as my personal conviction of the benefit derived from any other indisputably efficient remedial agencies of our therapy.

With every bloodletting there takes place a more or less considerable depletion of the circulatory system: this effect of bloodletting surely no one will deny. The so-called derivative effect of bloodletting is not recognized by all: I am personally convinced of this last effect, and I will explain later on what I mean by it and will show why I believe in it.

\section{Bloodletting, where the CHIEF EFFECT Is DEPLETION, although} at the same time there may be also derivation.

Venesection is resorted to by me, when in disturbances of the brain or chest circulation there occurs a vital indication, i. e. an indication to save the life from threatening death; this occurs in the following most important cases.

Disturbances of the cerebral circulation.--1. In the presence of evident symptoms of a threatening or an inevitable cerebral apoplexy in patients with athermatous arteriss, usually caused by rupture of the cerebral arteries: in embolism and thrombosis the indications for bloodletting are much less frequent. I must say here a few words concerning the condition of the pulse. Bloodletting in such cases is to be done not only when the pulse is full and strong, but also if it be weak, as long as we have to do with a patient who is not anæmic. Let me say, that the majority of patients present the following appearance; the pulse is weak, but the patient's nutrition is in a good condition, he does not look anæmic, lather plethoric, and the inquiry from among the surrounding friends elicits the fact, that the patient enjoyed a good appetite and digestion, has never suffered from any loss of blood, has usually complained of feeling warm and not chilly, etc. In such cases the small pulse points not to anæmia, but to a weak condition of the cardiac muscle, namely of the left ventricle, or to a disturbance of the innervation of the beart, which in its turn may be due to the disturbance of the cerebral circulation, which occasioned the apoplectic condition. Here we must employ bloodletting and administer at the same time stimulants per os or hypodermically: frequently at once after or even during the bloodletting, the pulse becomes fuller and stronger. Bloodletting in these as well as 
in the cases which will be spoken of later, should always be performed in the presence of a physician. As regards the amount of blood to be abstracted. in this as in other cases, I abstract from adults twelve omnees. but frequently, guided by the condition of the constitution, nutrition and hematopoiesis of the patient and other peculiarities of the given case, eight or six ounces.

2. In the presence of evident symptoms of threatening or already orcuring cerelnal apoplexy in patients with a chronic nephitis and hypertrophy of the left ventricle. The text-books often recommend bloodletting in the treatment of uremia, but they fail to give any definite indieations, the absence of which among the recommended therapentical measures forms, however. the usual weak point of many a text-book. In a given case it may lead the young physician into committing a fatal error. namely: if with the urremic symptoms there are also present signs of threatening cerebral apoplexy, and with these there is a full and strong pulse because of a hypertrophy of the left ventricle, a flushed face, etc., then we must of course employ bloodletting; lout if the symptoms of threatening apoplexy are absent, if the face is pale, the pulse weak, and if there is considerable cedema, then bloodletting may harm the patient greatly, if it does not directly kill him by hastening the development of cerebral redema. In such a case we should not resort to bloodletting, but we should administer stimulants, and principally calomel. as a laxative and diuretie remedy whose effect diminishes directly the uremic poisoning of the blood.

Disturbances of the dirnlation within the chest.

1. There oceur cases. though rarely, of diseases of the heurt. most frequently with stemosis of the left venous opening, when the patient, as yet not anemic and generally not exhausted, presents a condition of extreme disturbance of the circulation. principally of the pulmonary, as evidenced by severe dyspnoa and by hiemoptysis, and of the portal, as seen by the enlargement and sensitiveness of the liver: here we liave no time to wait for the effects of remedies which regulate the cardiac activity and the circulation ; besides, the best of them, namely digitalis, is at times badly borne in such cases and acts slowly: it is slowly absorbed, as you can easily comprehend, on account of the overfilling by blood of the liver, and. consequently, also of the stomach. Here we must employ hloodletting, administer stimulants (as the pulse is usually weak) — at times calomel, if there be an indication for it, - and later on digitalis, 
which by this time will usually be well borne and will exert its favorable effects.

2. There are cases, though not frequent ones. presenting a tumultuous onset of croupous pneumonia, when there becomes affected at once a considerable portion of one lung, and simultaneously with this, in view of the embarrassed circulation in the affected lung, there appears an cedema of the other healthy lung, which spreads rapidly, and is accompanied at times by bæmoptysis. If the patient is not old, and is possessed of a sound constitution and has enjoyed good health before he was attacked by the pneumonia, then we must do bloodletting; if the pulse, as happens rather unfrequently. be weak. then we must certainly administer simultaneously stimulants. The textbooks usually recommend, after explaining that the cedema of the lungs depends on the weakened cardiac activity, for treatment stimulants. and for the relief of the right ventricle bloodletting from the overfilled venous half of the circulation.

Again I must point to such a want of precision in the inclications, in recommending in such a general way bloodletting for pulmonary redema. If we deal with a case as described above. then we must of course alsstract some blood. But if we have a case of pulmonary cedema which appeared at the termination of an attack of croupous pneumonia. after the seventh or eighth day and later. in a patient already debilitated and presenting symptoms of collapse, then we should not resort to bloodletting, a rather risky procedure in such a case, but we must administer stimulants. resort to large blister's and to the use of senega. However. as regards the recommendation by the text-books of bloodletting for julmonary cedema we cannot help recalling to our mind again the observation of Jirgensen, that the authors of the text-looks recommend bloodletting, but never employ it themselves.

Local Bloodletting, cupping-glasses with scarification. and leeches, are employed by me with the amm in riew of deplotiny the bloorl-vessels. in the following. most frequent, cases.

1. In acute pleuropnemonia and acute pleurisy. - In pure pericarditis uncomplicated by a plenriss of any consiclerable extent, the resort to bloodletting is rather rare: the therapeutic indication can usually be fulfilled by a large blister. 2. In acute nephritis. 3 . In acnte inflammation of the great nervous trunks, principally of the sciatic nerves -as in ischias ex neuritide acuta. For all these enumerated cases, I employ cupping-glasses with scarification, not leeches. I prefer them 
to leeches in all eases in which the local conditions permit their employment - for the following reasons: their application, as well as their effect, is more rapid; bleeding stops at once after the cups are taken off, and there is no consequent bleeding, so that we are enabled to extract the desired amount of blood with greater exactness without weakening the patient, as happens frequently with leeches after which it is difficult to stop the bleeding, and there may occur more bleeding later on ; the depleting effect is also accompanied by derivation, which is greater than in the ease of leeches, and which is also beneficial in the above-enumerated eases ; finally eupping-glasses with scarifications are cheaper than leeches and require less care on the part of the patient after they are applied.

The number of cupping-glasses for the above-considered cases is usually six. seldom four, at times eight; this last number may be employed. for instance. in pleuropneumonia. when to the pleuritic phenomena, such as severe pains in the side embarrassing breathing. and therefore increasing the dyspncea. which is itself caused by the pnemmonia, there are superadded symptoms of a tempestuous attack of pnemmonia with a simultaneous redema of the other healthy lung, - symptoms, which were spoken of above. in tonching on bloodletting: in such cases. which are not clearly defined. cupping may replace bloodletting.

Local abstraction of blood, namely. as I said before, wet cups, are resorted to by me in the above-mentioned cases in the presence of the following conditions: when the patient has not as yet become weakened, therefore usually only at the beginning of the disease, during the first two or three days; in ischias ex neuritide also later : if the local symptoms. especially the pains, are severe and far advanced. is regards pleuritis I must say that with it also bloodletting is indicated prineipally loy the acuteness of the attack when the patientis condition is as yet not weakened. and not by the causation of the pleurisy: to be sure. we are much more frequently compelled to do bloodletting for pleurisy cansed by a cold, or for pleurisy of a pneumonic nature, than for purulent or tuberculous pleurisy ; but these two forms of pleurisy do not by any means present an absolute contraindication to local bloodletting. The effect of bloodletting is usually manifested by the diminution of the excruciating pains and the dyspncea in cases of ehest troubles, by the improvement in the condition of the urine, in acute nephritis, by the decrease of the febrile condition, by the more 
quiet sleep, and --as I can attest from my own observations - by a more favorable consequent course of the disease.

Where are the cups to be applied? In the above-enumerated diseases of the chest they are usually applied to the posterior and lateral surfaces of the thorax; in nephritis to the back, in the region of the kidney; in ischias ex nemritide, on the buttocks, some four or five cupping-glasses of medium size around the incisura ischiadica, leaving the central part for the Spanish fly, which must usually be applied soon after, a few hours after the bloodletting. I do not think that this last procedure is a trifling affair: in ischias ex neuritide a large fly exerts its best effect when applied to the sides of the incisura ischiadica and to its circumference, and especially if it be applied soon, in a few hours after the bloodletting. But if there be scattered over the part several incisions over the above-named region. as a result of the inaccurate directions of the physician, and to apply a fly directly on these would be extremely painful and would tend to cantharis poisoning, then we must either wait or apply the fly to another place; in either of which cases the patient gains.

In connection with this I cannot help observing, that in a general way, due attention is often not paid to the intelligent treatment of the surface of the body at the affected place. and it - the surface - - is exploited l"dther thoughtlessly, forgetting' that it is on this surface and with its assistance. that we are sometimes compeller to withstand the last positive struggle against the disease.

Thus, for instance, in the rarious chest affections dry cups are applied over the chest to effect a relief from dyspnoda relief usually of but a slight nature and short duration - and these are at times applied in such numbers and with such immoderate zeal, that at the decided lour, when the consulting physician is usually called in. _ in case for instance, an redema of the lungs is developed, and the patient can be saved only by the aid of stimulants. by senega, and principally, by large flies applied to the chest (if applied to any other portion of the body. we cannot obtain even a shadow of that effect), -- the chest is seen to be covered by so many severe ecchymoses resulting from dry cupping, that there is no place over it even for a small fly. whereas, as we said, we must apply a large one, and usually more than one. The same may be said of painting the skin with the iodine tincture, which is just as little useful in the above-named cases and which, later on, serves as an olstacle to the application of Spanish flies. 
t. For local blood-extraction with depletion in view in diseases of the abdomen, usually in eircumseribed peritonitis (most frequently for perityphlitis, at times for perieystitis of the gallbladder). I resort to leeches (as cupping is inconvenient over the abdomen), from six to ten in number.

(of loeal clepleting bloodletting - the application of leeches -- in the region of the head, I shall speak later. for convenience of exposition, in connection with derivative bloodletting.

II. BLoondet'Tisa, where, although there is also depletion, THE PLINCIPAL EFFECT IS THAT OF A IOERIVATIVE.

To elearly elucidate what 1 mean by derivative bloodletting. I must first say a few words on the subject of hemorrhoids.

What are hemorrhoids? Aml does there exist in reality a morbid condition which must be distinctively named "hæmorrhoids"? The ancient medical works deroted much space to the subject of hemorrhoids. Later the eriticism of the views of the ancient writers resulted in a complete rejection of the same, so that for some time hemorrhoids were not treated of by any textbooks on pathology. At the present time they again begin at least to speak of the symptoms peculiar to this condition, even if they do not consider it as an independent morbid state: thus the text-book of Eichhorst. the only one of all the modern German text-books of pathology. in describing the diseases of the rectum and amus. describes haemorrhoidal molmmina preceding hremorrhages from the back passage. and consequently hæmorrhoidal phenomena are alluded to in this connection as a peeuliarity of the above-named local affections. I held similar views at the beginning of $m y$ medical practice. assuming, namely, that there exist no hiemorrhoids as a morbid condition of an independent nature. and withal of a general character, but that all so-called hremorrhoidal symptoms are but determined by certain diseases of the rectum and anus in the presence of an impeded cinculation in the portal system. as caused by constipation, intestinal meteorism. abdominal obesity. hepatic diseases, ete. But experience taught me otherwise : it proved that haemorrhoids constituted a morlid condition by itself, totally independent of diseases of the rectum, anus or the abdomen in general; that it may be seen in comnection with these, as well as with diseases of any other parts of the organism, but it may also occur without these, in their complete absence; in fact, it is more frequently absent. when these affections are present. This morbid condition determines the periodical appearance of morbid attacks in the head, spine. chest 
and abdomen, which take on a more or less rapid or slow development. and which disappear suddenly with the appearance of haemorrhage. at times from the nose (more frequently in childhood and boyhood). less frequently from the chest. i. e. haemoptysis (more often in youth), but most frequently of a "* haemorrhoidal " character, from the back passage. The nature of the appearance and of the disappearance of the attacks is distinctly angioneurotic. peculiar to other neuroses of the eirculatory system. such as migraine. urticaria and other's. and I therefore consider haemorrhoids as an anginueurosis.

Here is the first case. which. together with similar ones later on. led me to formulate my idea about the nature of hæmorrhoids. The patient tells me that when a boy he suffered with nose-bleed which was precerled by heaclaches and a flushed face: with the bleeding the headlache would disappear. When a youth he had slight hrmoptysis. lut unaccompanied with any constant cough or generally with any constant attacks in the chest.

Patient is now forty years old : he is of a robust constitution. with an entirely healthy chest. He complains of periodic malaise: there appear heaviness in the head. pain in the neck. disturbed sleep, gloomy and irritible mental state. and indisposition to work; he experiences pains along the spinal column, especially in the lumbar region, a feeling of oppression in the chest and palpitation. intestinal pains, and the stool is somewhat difficult; then there appears hæmorrhage from the back passage and all the symptoms vanish. Outside of these attacks. which occur three or four times during the course of the year, and last one or two weeks, and which hinder him in his oceupation, the patient is entirely healthy and does not present absolutely any morbid symptoms either in the rectum and anus or in the abdominal organs in general. or in any other part of the organism.

Such cases of pure. uncomplicated hemorrhoids are certainly. very rare: during the thirty-nine years of my practice. I have seen only about twenty. I must however state here, that if the symptoms of such uncomplicated hæmorrhoids be less pronounced, they are usually borne without difficulty, not forming a sufficiently strong reason for calling in the assistance of the doctor. While the cases of hrmorrhoids to be seen together with other morbid states of the abdominal as well as of any other organs, are far from being rare: in these mutually complicating each other morbid conditions, it is at times possible to 
distinguish which part of the attack is to be referred to the haemorrhoids, and which is to be ascribed to other causes; but at times they are so intermingled that it is impossible to effect this distinction.

The canse of haemorrhoids is to be looked for in the inborn peculiarity of the organism. This is indicated by the ordinarily observable hereditary predisposition to haemorrhoids, as well as by the absence of other causative agencies. In the abovenamed cases of pure haemorrhoids I failed to find such agencies either in the condition of the organism itself or outside of it: nor did these cases present the effect of causes which increase the inborn predisposition to haemorrhoids, causes which I shall touch upon later on. As regards the condition of the haematopoietic faculties in persons presenting symptoms of pure haemorrhoids. some could be considered as plethoric, others. again. as anaemic. while the majority presented all the signs of normal haematopoiesis. I may add that some of the abovenamed cases of uncomplicated haemorrhoids presented also other phenomena of angionemrotic nature (such as migraine and urticaria), and quite frequently the coincident occurrence of the haemorrhoidal bleeding with the menstrual flow - an angioneurotic process : in cases of pure, as well as of complicated haemorrhoids in women, the haemorrhoidal bleeding takes place most frequently during or immediately after the menstruation, and also immediately before it. Of the influences which increase the predisposition to haemorrhoids, the use, or even more, the abuse, of alcoholic drinks is evidently one, and quite likely a sedentary life and immoderate horseback riding, especially galloping and trotting on a wild horse. In the abovenamed cases of pure haemorrhoids, the bleeding, while it determined the disappearance of the morbid symptoms, did not in the very least weaken the patient, neither did it cause any anaemia, or any generally bad consequences; whereas haemorrhoidal bleeding aggravated by the abuse of alcohol or by diseases of the rectum or anus (as, for instance, by rupture of the dilated veins), may cause dangerous anaemia and debility of the organism.

I will turn now to derivative blood-letting. It stands to reason beyond doubt, that the observation of cases in which the morbid symptoms in the head, chest, and other organs disappeared consequent on haemorrhoidal bleeding gave rise to the idea of bloodletting at the circumference of the back passage for affections of the head, chest etc., the aim in view being not de- 
pletion alone; to effect depletion ouly. it would be much more natural, in case. say. of affections of the head, to choose as a place for hlood-extraction one much less distant from the head. As a matter of fact. bloodletting from the circumference of the back passage has since long been considered as of a derivative nature. It is certain that at the beginning there occurred frequent mistakes. when haemorrhoidlal bloodletting was recommended for such affections of the liear, chest, and other organs. in which not only haemorrhoidal. but any other bloodletting was contraindicated, and it was only in the course of time that experience showed where the former was suitable. To explain the nature of observations on which I base my personal opinion as to a special. not only a depletive. effect of blood-extraction from the circumference of the back passage, I will cite a case in my practice which I had under my obserration twenty-five years ago, and about which I delivered an address before this society in October. 1864. Althongh I have had many occasions before as well as since. to see cases proving without doubt a special influence and value of haemorthoidal blood-extraction. and pointing to indications for it. still this observation is particularly strong in its conclusiveness.

I was called to attend a woman suffering from severe nosebleed: she lost a full deep-bottomed plate of blood. and the bleeding still continued in spite of all the adopted measures. Patient was a woman of about forty years of age, of very robust constitution. living in favorable conditions of life; she had until recently enjoyed good health. and at the time presented nothing abnormal, outside of the bleeding and some trifling general weakness and that of the pulse; everything was in good order; there was only a feeling of heaviness in the head and an oppressive mental state. Inquiry elicited the fact that some time ago, while driving in a sled. the patient's feet were severely chilled, but on her return home they became warm again and she continued to feel well; however, the menstrual flow which followed soon after this occurence and which was formerly regular and abundant, was at that time very scanty and terminated much sooner than at the usual period. By the end of the menstruation patient began to feel heaviness in the head, and in a few days there appeared the nose-bleed, the feeling of heaviness not abating. I ordered ice on the head, and for intermal administration infusum secalis cornuti ex $3 \mathrm{ij}$ ad $\bar{j} \mathrm{vj}$; in the course of the day and night the bleeding ceased somewhat, but it again increased in the morning and she lost another plateful of blood. 
I then put aside the ice and the ergot and ordered four leeches to the circumference of the back passage. By the time the leeches had fallen off the bleeding ceased, and did not return again. On the moming of the next day the heaviness in the head disappenred, the patient felt as usual hale and strong; she did not even complain of weakness: the following menstrual periods were, as formerly, regular and abundant.

It is apparent that in this case the action of the four leeches applied to the circumference of the back passage consisted in producing a depletion of the circulatory system : the abundant depletion through the nose - the blood filling a deep-bottomed plate - did not remove the sensation of heaviness, nor did it avert the severe haemorrhage on the next day; whereas but an insignificant haemorrhoidal blood-extraction (as the four leeches, together with the bleeding following their bites, could at a maximum abstract but eight ounces of blood) stopped the haemorrhage, relieved the liead symptoms, and all this so rapidly and so clecidedly, that to ascribe this fact to accident would be equivalent to closing one's eyes to an obvious fact, and to manifesting one's incapacity for observation.

If the effect of the haemorrhoidal bloodletting in the described case was not of a depletive nature, what was it then? I call this effect derivative, so that it may not be confounded with depletion, and may be named by a special term; I employ for this purpose the term derivative, as one long in'use, but I do not connect with this name, for the sake of explaining the nature of the alluded effect, any theory, which is an impossible thing in the present condition of our science.

I will note here another thing. In the above described case I did not resort to tamponade of the nasal cavity, and the termination of the case has completely justified my mode of action. I will also say, that there occur cases of nose-bleed, in which a tamponade of the nasal cavity may become directly dangerous: thus I had occasion to see patients over sixty or seventy years of age who since childhood had a predisposition to bleeding; this last is usually preceded by and at times it relieves cerebral congestion. There occur cases in which such haemorrhages become excessive, without however affording any relief to the symptoms in the head: in such I consider tamponade as an extremely risky procedure, for it may tend to cerebral extravasation of blood, and I resort to slight haemorrhoidal blood-letting, - usually with the same success as in the abovedescribed case. 


\section{The Indications for Derivative Bloodletting.}

1. In the region of the head. - Cerebral congestion, without a simultaneous affection of the circulatory system, and also in comnection with diseases of the heart and blood-ressels, especially in those cases where along with the constantly present phenomena of cerebral congestion (as disturbed sleep, gloomy and irritable mental state. heaviness in the head, pain in the neck and at times in the temples, reeling during walking, dizziness on stooping down. etc.) there oceur at times also sudden fluxes of blood to the head (the face flushes rapidly and deeply), reminding one of haemorrhoidal molumina, and threatening with an approaching apoplectic attack. If the phenomena of cerebral congestion are of moderate severity and not of an urgent character, I at first regulate the mode of living, prescribe less of mental work. more of exereise, walking, and massage of the feet which are usually cold and chilly in such patients. I regulate the diet, remove various morbid conditions which tend to keep up the cerebral congestion, such as constipation, cough (usually due to a catarrhal coudition of the larynx or the bronchi), and only then do I resort to the derivative bloodletting. when the above-named measures fail to produce the desired effect. But if the phenomena of cerebral congestion be strongly developed and the patient be of robust constitution with good nutrition and good haematopoiesis, and particularly if the abovementioned influxes oceur, then I at once apply a few leeches (in very threatening cases, as I explained above. I do bloodletting), and together with this I resort to the above-enumerated measures. I will state here that an ice-bag applied in such cases to the head exerts but a trifling effect which passes as soon as the bag is taken off. Of the stability and duration of the effects of the derivative bloodletting in the above-named affections of the head, as well as in diseases of other organs. I shall speak later on, when expounding the method of derivative bloodletting in connection with the question of repeating the same.

For the above-described cases of cerebral congestion I do not resort to the application of leeches behind the ears and to the nasal septum, i. e. with a direct depletive purpose. We can apply but a few leeches to the nasal septum (two to four). so that we are unable to get any sufficient effect: and according to some observations we are led to think, that such an insufficient depletion from the given place may even tend to increase the phenomena of cerebral congestion. The application of leeches behind the ears may certainly relieve the attacks of cerebral congestion; 
but first, we shall be compelled, to effect this, to apply at least twice as may leeches as we would do for the derivative (haemorrhoidal) bloodletting, i. e. we shall find it necessary to cause twice as great a loss of blood, and secondly, even after such a loss of blood we fail to obtain as complete, stable and lasting an effect. as we do with the derivative bloodletting.

There is only one class of cases for which I continue to apply, as I consider it an undoubtedly correct and raluable practice, my former method of bloodletting in the region of the head with a direct depletive aim in view. I refer to the application of two to four leeches to the nasal septum in cases of cerebral hyperaemia in infectious diseases, most frequently. of course, in typhus and in typhoid ferer. There occur cases of these diseases, in which during several dars there continue symptoms of cerebral congestion ver distressing to the patient. namely: serere headache, insomnia, at times riolent delirium, all these accompanying a distinctly flushed face; then there occurs nasal bleeding and the symptoms are considerably relieved (there is usually with this also a considerable fall in the temperature. which continues for some time), without however causing any marked weakness in the patient. If in such cases the bleeding from the nose does not occur for a long time. while the further developinent of the symptoms and their deleterious effect on the condition of the patient continue, yielding but slightly to ice applications to the head, then if the patient be at all strong, it is necessary to apply two, rarely three or four leeches to the nasal septum : bloodletting. even if it be inconsiderable (and this is, of course. desirable. in such cases). is sufficiently effective in removing the abore-mentioned symptoms. The application of leeches to the circumference of the back passage in such patients would be extremely inconvenient: and besides the effect of the derivative bleeding in these cases is uncertain.

2. In the region of the spinal colnemn. - Hypercemia of the spinal cord and of its membrones. as evidenced by pain along the tract of the spinal columm and in the region of both sciatic nerves (the trunks of the last presenting no tenderness on pressure. thus showing the absence of symptoms of neuritis), occurring in the majority of cases simultaneously with symptoms of cerebral congestion. rarely by itself or to a more predominating extent. It is well understood. that before ordering the application of leeches we inust by a careful diagnosis determine exactly the nature of the affection, so as not to confound with it other diseases 
of the spinal cord and its membranes, as well as spinal synovites neurites and myosites, and also various functional nervous disturbances, such as neurasthenia and hysteria, and abdominal diseases, as hepatic and renal colics, constipation, etc. With a correct diagnosis on hand, the effect of the haemorrhoidal bloodletting on the symptoms of hyperaemia of the spine and its members is just as stable and complete, as it is in cerebral congestion. It is of course difficult to determine as to how much of this effect is, in these cases, of a derivative character, and how much of a depletive one; be as it may, it is incomparably more efficacious than bloodletting, even if more abundant, but produced anywhere higher up along the tract of the spinal cord.

3. In the region of the chest. - (a) In haemoptysis not due to any organic causes, in the presence of healthy lungs and heart. Such haemoptysis, as is well known, usually occurs in young persons predisposed to pulmonary tuberculosis, and also in such young persons, who are not predisposed to this disease, but who have a weakened nervous system, especially as a result of sexual irregularities, and who are at the same time inclined to pure haemorrhoids, i. e. haemorrhoids under which, as I explained above, we understand an angioneurotic condition. If a haemoptysis of this nature does not rapidly yield to other measures, then the most reliable means is slight haemorrhoidal bloodletting. This will of course bring about only a cessation of the haemoptysis for the time being; later on the repetition of haemoptysis must be met with by radical measures, which consist in strengthening the organism, in removing the neurasthenia and the sexual irregularities, etc.

(b) Haemoptysis in pulmonary tuberculosis, excepting those cases in which it is caused by destruction of large blood-vessels in cavernous regions. If the haemoptysis be at all considerable, without yielding quickly to other measures (among other things, in the presence at the same time of constipation with a distended abdomen, which is not relieved by a laxative), and in case the patient does not present any signs of great weakness, then I order two, seldom three leeches to the coccyx, and I must here testify to the successful effect of such bloodletting; the haemoptysis ceases without causing any particularly noticeable weakness of the patient.

(c.) Haemoptysis in diseases of the heart, principally in affection of the left venous opening. - In these cases, especially if at the same time the liver, as a result of blood-stasis, be enlarged and sensitive, and after the other indications, such as the relief of 
constipation, the regulation of cardiac activity, etc., have been fulfilled without bringing about the desired results, then we must resort to bloodletting. I will recall to your mind what I said before in connection with bloodletting, that in such cases of a pronouneed distmbance of compensation we must employ bloodletting. even before fulfilling the other indieations ; in case disturbances of compensation be absent. and the moderate haemoptyses recur frequently, withont yielding to other treatment, then we must apply a few leeches to the coccyx.

t. In the abdominal region. - For blood-stasis in the liver and consequently in cases of impeded cireulation in the portal system, as eaused by diseases of the heart, by immoderate eating and by the use of alcoholic drinks, etc. If in such cases of enlarged and sensitive liver there predominate biliary disturbances pointing to an insufficient secretion of bile (as a bitter taste, at times vomiting of bile, an insufficient coloring of the excreta : symptoms of biliary pigment in the mine), in connection with a particular tenderness in the region of the gall-bladder and with a simultaneous constipation, then it is advisable to give calomel. But if the enumerated biliary symptoms are absent, then there is an indieation for the application of leeches to the coceyx. It is hard to determine as to how much of the effect of the leeches in these cases is of a derivative nature and how much of a depletive one; but the principal thing is that it is effective, incomparably more so, with a smaller number of leeches, than the effect produced by applying the same along the right hypochondrium, which procedure wonld be an umpardonable mistake in such cases, just as it would be erroneous in pericystitis of the gall-bladder to apply leeches to the coccyx, instead of along the right hypochondrium.

5. In inflammution of larye hamorrhoidal nodes, leeches when applied to the coccyx, acting at one and the same time both as a depletive and as a derivative measure, serve in very pronounced caser as the most effective means.

In conclusion let we sia here a few words regarding the method of employing derivative bloodletting.

As any other blood-extraction, it must he performed in the presence of the physician, and espeeially so in the above-named cerehral and chest cases. In other cases, in patients who are unt weak, we may rely on a male or female assistant-surgeon (Feldscher) if they are well known to the physician, reliable, familiar with the alteration of the pulse, and are able, when 
necessary, to administer stimulants, as valeriana or Hoffman's drops, which may at times become necessary for patients with a weakened nervous system, or in those who have become frightened even before the leeches are applied. I will state here that a weak pulse does not always constitute a contraindication for derivative bloodletting, as was partly also explained in connection with the subject of the more considerable bloodletting - venesection. Bloodletting should be performed in as spacious and airy a room as possible.

The time of the day. - The best is during the evening, before going to bed, so that after the application of the leeches the patient nay remain in bed until morning: however, in urgent cases it may be done at any time.

No leeches are to be applied immediately after taking food, but some time later in accord with the quantity of the same. The intestinal tract should be evacuated as a preliminary measure: if this be sufficiently effected by an injection, then it should be made directly before the leeches are applied; but if there is a necessity for the employment of a laxative, then, haring administered this last, we must of course wait for its effects and then wait for a few hours before we apply the leeches, and before doing this re administer some stimulant so as to aroid any weakening on the part of the patient.

The position of the patient must be lying, or at least reclining. (in dyspncea) on the side. The practice formerly in rogue of performing haemorrhoidal bloodletting with the patient in a sitting posture may tend to call out dangerous fainting.

The site of the derivative bloodletting is, as explained above. pointed out by nature itself, namely, the circumference of the back passage; but I order leeches to the coccyx, and not all over the cireumference of the anus: for this last is much more painful and serves later as an obstacle to defecation; it is moreover more difficult to stop the bleeding in this locality and to preserve the necessary cleanliness.

The day following the bloodletting the patient should remain at home, so that the wounds caused by the leeches may heal the sooner, and in case he feels any weakness - which, if the indication be correctly understood and the number of leeches carefully considered, will occur but very rarely, and even then during the first day only - then he should take two or three doses of the above-named stimulant drops.

The number of leeches. - During my early practice I had frequent occasions to see usually ten leeches applied, seldom less. 
more frequently a greater number, as twelve to fifteen, at times twenty and even more. I was then convinced of the undoubted harm of such considerable extraction of blood, and I began to apply five leeches, thinking that in case of necessity I can repeat the bloodletting. Experience has taught me that there are but very rare cases where we have to repeat, i. e. to apply twice five leeches, in the course of a few days: in the majority of cases five, in exceptional cases six or seven leeches. produce a sufficient effect. The number of five (or six to seven) refers to all the above-enumerated indications, with the exception of the chest indication - the haemoptyses : for these I never use more than five, more often four or three, and in pulmonary tuberculosis, as alluded to above, even two.

To keep up the bleeding after the leeches fall off. -- Experience has proved that derivative bloodletting is attended with the best effects when, after the leeches fall off, the bleeding is kept up by washing the wounds with warm water until the blood, at the beginning usually very dark, becomes bright red in color.

The repetition of derivative bloodletting. - Among the objections against this last, — raised, however, usually by persons who do not themselves resort to it, and thus lack the necessary experience, - we hear this one, that derivative bloodletting once produced determines by this fact alone the necessity for its being repeated. From long experience I will state the following: if, for instance, cerebral congestion (and this may be said of the other indications for derivative bleeding) was relieved at the given time by the application of leeches to the coccyx, if with this the causes producing the cerebral congestion were removed, and the patient follows strictly the physician's advice which will result is setting aside these causes, then there is usually no necessity for a repetition of the derivative bleeding. But if the patient neglects the physician's instructions, and consequently thus permits the causes of the congestion to further exert their deleterious mfluence, or if these causes be not removable, then certainly, to avoid the worst consequences, we are compelled to repeat the application of the leeches once or twice during the year, - rarely, in very exceptional cases, more frequently, usually in the course of time, in a smaller number than at the beginning; but if the number of leeches is ordered with care, in accord with the patient's idiosyncrasies, his constitution, haematopoietic ability, and so on, then we never observe any andemia, nor any weakening of the patient resulting from derivative bloodletting. 


\section{CALOMEL.}

\section{IN HYPERTROPHIC CIRRHOSIS OF THE LIVER AND IN GENERAL THERAPY.*}

The principal mercurial compound used in the treatment of internal diseases, ontside of syphilis, is certainly calomel. As is well known, there exist various views regarding its effectiveness. In England calomel is highly valued and is one of the ordinary, commonly used drugs, although less so than it was at the beginning of the present century, when it became known. In France it is not so much valued and its employment is limited; but in a general way there is a considerable similitude in its use with the English practice. But it is quite different in Germany. According to Nothnagel and Rossbach there is to be noted a progressive scepticism in the direction of internal mercurial treatment (except in syphilis), so that at the present time there is hardly one positive indication for the use of the drug. The above-named authors consider calomel as a convenient laxative at times. As far as I am acquainted, from literature and personal experience, with the modern practice of the German physicians and their attitude toward calomel, I can but substantiate the correctness of the authors' statement. It however occurs to me that calomel is not fully appreciated by the modern German practitioners in its application to the treatment of diseases of the liver, namely, of the biliary tract. Thus, even the work of Binz ( "Arzneimittellehre"), the tendency of which can hardly be called sceptical, contains no such indication for the employment of this drug. The tendency of the Russian practice is similar to what we find among the Germans, namely, the same progressive scepticism that began in the fifties; the condition at present, as far as I am aware, is the same as in Germany. I do not speak of myself and those of my former students who had the opportunity to see me use calomel in the clinic.

*Address delivered before the Moscow Physio-Medical Society, January, 1884 . 
During the fifties, when I was a student, and later on as an assistant in the clinic. I had frequent opportunities to observe the use of calomel. I failed to see any successful results, but saw a great deal of harm done. The causes of this were to be found in the poor diagnostication of those times. in the insufficient exactness of the indications for the use of the remedy. namely, in what diseases it should be used, when to begin and when to stop its administration (failure to properly indicate all this led to munecessary diarrhoas and exhaustion of the patient): then in the inability to prevent the occurrence of stomatitis, as the use of potassium chlorate as a gargle was unknown then. But at the same time I happened to observe once a case outside of the clinic. where an old physician, without making a diagnosis, but simply on account of the presence of the "status biliosus," administered calomel in a small. slightly laxative. dose: the immediate marvellous improvement in the patient's condition has impressed itself upon my memory. since 1860 I conducted an independent clinical and private practice, at the beginning of which, under. the influence of the general " progressive scepticism " toward calomel. I almost never" used it; but some four year's later I had to resort to it. and since that time I bave learned to appreciate the value of this remedy. In the following lines I intend to bring forward proofs of my conviction, that calomel in diseases of the biliary passages is a valuable drug which, in the present condition of therapy, cannot be replaced. In connection with this I will also tonch. in a few lines, on the use of calomel in erysipelas, typhoid fever, pneumonia, and acute Bright's.

I must touch, in a few words, on what pharmacology calls the physiological action of the drug, namely, the influence of calomel on the liver, on the secretion and excretion of bile. The development of scepticism toward calomel, outside of the above-named clinical causes, was undoubtedly aided by the fact, that whereas the physicians have derived the greatest good from the use of calomel in diseases of the liver, and in view of the well-known dark-greenish evacuations following its use lave put it down as a cholagogue, pharmacological investigations, by the aid of artificial biliary concretions, have led to the conclusion that not only is the quantity of secreted bile not increased by calomel, but on the contrary, after a continuous use of the same, it - the secretion - tends to diminish. But the doubtful conclusions of these pharmacological data as regards the calomel therapy in diseases of the liver are, to say the 
least, premature. It was firstly proved that the dark-greenish color of the usually so abundant calomel evacuations is due to a rapid descent of bile from the superior portion of the intestine, this descent being caused by the employment of calomel (but of no other laxative). Further, let us take the following from the above-mentioned work of Binz: "If, in accord with the investigations of Shiff, we consider that the liver not only prepares. but also excretes the bile already prepared and again absorbed by the intestines, then the diminution of the latter after a calomel diarrhoea becomes clear"; and again: "In this manner calomel frees the juices of a possible superfluity of any component parts of the bile, and can be called a cholagogue from a point of view totally different from the former." We will add that Shiff's theory, on which the physiologists, thanks to the contradictory results of Sokoloff"s work, looked with distrust, has received lately considerable support, if not complete confirmation. Weiss has repeated Sokoloff's experiments in the laboratory of Prof. Bonlyginsky, and after three years of labor has arriced at conclusions contradicting those of sokoloff, but sulsstantiating shiff's theory.* And if this theory be correct, then we may assume that calomel, by causing a considerable excretion of bile and thus diminishing its absorption and its return to the liver, cannot but influence also the formation of bile in the liver, as according to Shiff's theory the quantity of bile secreted by the liver is made up of that which was reabsorber and of that which was newly formed. However I do not at all intend to enter upon useless hypotheses. I only desired to show that the present condition of the pharmacological data cin in no manner serve as a basis, as they sometimes did and do now, for the sceptical view as to the effectiveness of calomel therapy in hepatic diseases. I again repeat. I do not intend to elucidate the pharmacology of calomel. but to bring forward clinical testimony as to the effectiveness of this remedy, - rather to determine the diseases, in which its use is successful, and to clearly point out the indication for it in the sphere of such diseases.

The English practice, to which calomel owes its reputation, has among other things given a very indefinite indication for the use of calomel, namely, torpor of the liver, an indication, by the way, that remains even up to the present time almost the most frequent one. The following combination of symptoms is embraced under this designation: constipation or a general

* Vide The Physiology of Bile. Moscow, 1883. 
irregularity of the intestinal functions, insufficient coloring of the excreta, a too great derelopment of the intestinal gases, a pale, sad face, low spirits, etc. Such a picture of disease may undoubtedly be seen in liseases of the liver, in which neither calomel nor anything else. in the present state of therapy, will be of any avail. as for instance in cancer, in multilocular echinococcus. in simple (Laennec, non hypertrophic) cirrhosis of the liver. Again this picture may also be observed in other diseases of the liver. as in hyperaemic conditions of that organ, in which calomel may be effective. but in which other drugs will do the same and better. and must be for certain reasons preferred. as. for instance, mineral waters containing Glauber's salt (Marienbad). My investigations lead me to think that there are only two diseases of the liver, or properly speaking, of the biliary passages, in which calomel is not only efficient, but will do what no other remedy, in the present condition of therapy, will, be it a laxative or otherwise.

These diseases are: severe. especially febrile ases of colic from biliary calculi (colical hepatica), and hypertrophic cirrhosis of the liver.

Severe, especially febrile cases of colic from biliary calculi. - During the first four years of my practice, as I said before. I never used calomel. but some twenty years ago I took it up. Here is the first case which induced me to employ it. I will relate it hriefly. as I have harl since many more typical cases and had the opportmnity of better observation (I will communicate one of them later in extenso).

Patient was about fifty years old. of good constitution. lived in favorable cireumstances and led quite a regular mode of living; but he was at times careless with his food and also with the use of alcoholic drinks. ('hest and nervous system normal. Lrine reddish in color (no albumen). 'There were some slight gouty phenomena in the small joints. He was troubled with dyspepsia (there was no evillence of any gastric catarh ). had a tendency to constipation. slight attacks of liver colic. and moderate tenderness on pressure in the region of the gall-bladder. Fever absent: condition of strength good. I regulated his mole of living. especially the diet. and prescribed Vichy (C'élestins) in the molning on an empty stomach, three doses a $\bar{z}$ va at the temperature of freshly drawn milk. and at times, in case of an insufticient enptying of the intestines, the third dose of Vichy was replaced by a similar dose of bitter Friedrichshall water, also warmed somewhat. There 
followed rapid improvement during the first two weeks: ap. petite, sleep and mental condition became better, the dyspeptic phenomena and the pains in the region of the liver disappeared; less tendency to constipation.

Then, after this, a considerable error in diet, and a cold at the same time; the patient called on me in five days, complaining that everything became worse, although he continued the former treatment. To be sure, the appetite was gone; the pain in the region of the liver grew much sharper and was aggravated after drinking the mineral waters, especially the bitter ones; there appeared slight icteric symptoms and obstinate constipation. I stopped the mineral water and administered castor oil; he romited up the first teaspoonful, but had a stool after the second; but his condition was not ameliorated. There appeared febrile condition with an evening exacerbation. I gave him three three-grain doses of quinine in the course of the day. He grew worse by the next day: pains and icteric symptoms aggravated, temperature higher, the patient is weaker and more irritable. I then administered twelve powders of calomel grain $\mathrm{j}$ ( + five grains of sugar) every liour, and at the same time a gargle of potassium chlorate; verily, the effect was magical : the patient had abundant, characteristic calomel evacuations, both the fever and the pains disappeared on the next day, the sleep and the mental condition bectme normal, the appetite reappeared. Two days later, the patient returned to his former treatment with the mineral water, and, punished by experience, kept a strict diet, and soon improved completely.

I will now communicate to you a very typical case, the last of a similar class of cases seen by me, and one of those that I had a good opportunity to observe: the patient was in my clinic last summer and had more than once served as a subject of my clinical lectures before students and physicians.

Mr. N., a member of the circuit court, forty-two years of age, entered the clinic January 2.5, 1884, complaining of severe pains in the region of the stomach, under the right hypochondrium and all over the abdomen, of jaundice, feverishness, and extreme debility.

Mode of life and anamnesis. - Patient is married, has no syphilis, almost never used any alcoholic drinks formerly, while during the last year and a half drank none at all. The principal irregularities in his mode of living were as follows: insufficient sleep (some six hours in the twenty-four), a great amount of mental labor (fourteen hours a day), little exercise 
out of doors, summer in the city: smoked about fifty cigarettes daily; had irregular meals - but once a day - and was careless in selecting his food. The last year and a half the patient was compelled by poor health to work less (three hours a day), gave up smoking, and is very careful with his diet; drinks tea and coffee moderately, without cream or milk, and with but a small quantity of sugar (formerly he used about three-fourths of a pound of sugar in twenty-four hours). Ten years ago there appeared the first symptoms of neurasthenia: low spirits, quick fatigue from mental and physical labor, constipation, although the gastric digestion was satisfactory. The patient took silver nitrate, somewhat improved for a time, but continued to work harl, slept and rested little. and the neurasthenic symptoms became more and more aggravated each year. The constipation particularly increased the last four years and the patient resorted to clysters and at times to castor oil. During June, 1882, he had the first attack of liver colic; in October, the second. The patient drank Vichy and Ems for a week and a half each. In November another attack of colic with nallsea; again Vichy and Ems for three weeks. In April, 188:, another attack of colic, and Vichy and Ems for three weeks, and from the latter half of .July until the end of August (for about ten weeks) Karlsbad. three glasses per day. September 30th and October 1st, while riding for a long time in a jolting carriage, he experienced a severe attack of colic, for the first time accompanied by jaundice and ferer (chills and later heat). since then and up to the time he entered the clinic his condition was as follows: every lay, some two hours after dinner, he experiences a painful sensation in the region of the stomach and in the right bypochondrium. The pain is usually of moderate severity and lasts for about one hour; but once during the week it is quite severe, lasting for several hours, and is accompanied by an increase in the jaundice; and once in about every two weeks (of late more frequently) there appear attacks of severe colic lasting three to four days, with pronounced jaundice and fever $\left(40^{\circ}\right)$, which begins with a severe chill and lasts during the three or four days. The treatment for this time was as follows: he drank continuonsly first Vichy, later Ems in small doses ( $\overline{3} \mathrm{v}$ three times a day), and used laxatives constantly: either an additional teaspoon of Karlsbad salt to the mineral water, or a teaspoonful of powdered rhularb in a glass of water, and at times castor oil. During the attacks of colic accompanied by fever ho was given quinine until he experienced slight buzzing in the 
ears, but this failed to produce any effect on the fever. The result of the treatment unsatisfactory: the attacks of colic and fever continued to increase and became more frequent: there appeared intestinal pains, which became frequent and more severe on the continuous use of the mineral waters; the patient became very emaciated, debilitated, lost his sleep and was in bad spirits.

Status January 24, 1884.-- Patient of medium constitution, and, as mentioned above, emaciated and weak. There is a moderate jaundice of the skin and of the sclerotics. He lias almost no appetite, some thirst. Has belching after dinner, and at first heaviness in the stomach, later pains. as described in the anamnesis; with this there is nausea, and at times vomiting. after which the pains grow less severe for some time. Abdomen somewhat distended; moderate pressure elicits pain in the stomach, and much more of it in the region of the gall-bladder; liver and spleen not enlarged. Notwithstanding the constant use of laxatives, a stool takes place only after a clyster of warm water (and even then not always satisfactory) every other day; only castor oil can replace the clyster. The evacuations are paler than normal, pale yellow. Besides the attacks of liver colic, i. e. of pains which appear two hours after a meal, in the stomach and under the right hypochondrium, accompanied at times by nausea, the patient is suffering from other pains, evidently of intestinal origin, in the umbilical region and along the course of the large intestine, accompanied by gurgling and relieved after an emission of gases or after a clyster. The urine, 500 c. c. in twenty-four hours, is red, contains biliary pigment, but neither albumen, nor sugar. Chest healthy. Pulse 80-90, very weak. Poor sleep, on account of abdominal pains. Mental work causes headache. Lately fatigue brings on dizziness, during which the patient grows pale. Oppressed low spirits. Patient was admitted on the third day of his usually severe attack of colic with fever. Temperature in the evening $38.6^{\circ} R .\left(101.5^{\circ} \mathrm{F}\right.$.) ; in the morning of the next day $38^{\circ} R$. $\left(100.4^{\circ} \mathrm{F}\right.$.), evening $38.8^{\circ} R$. (101.8 $\mathrm{F}$.). He is lodged in a dry, warm room, and ordered at first to be confined there, not to sit for a long time, to lie down at once on feeling fatigued, not to tire himself with reading, and to keep the abdomen warm (Provence oil and a double layer of flannel). Diet: weak, warm tea, without milk or sugar; between two or three tablespoonfuls of port wine per day; chicken soup, chicken meat and some white bread; he is to eat several times 
per day, but never to satiety. When feeling weak and dizzy, he is given twenty-five drops of Trae. Ammon. Valer. and Liq. Anodyni Hoffmanni āā, twice or three times a day. If necessary a clyster of warm water is administered.

. Jomary $2 v^{2}+h$. - In the beginning calomel (one grain + five grains of sugar) every hour. and after the fourth close every two hours; from this day on constantly gargling his mouth with potassium chlorate ( $3 \mathrm{ij}$ to $\Xi \mathrm{vj}$ ). After the seventh grain, the patient had a thorough clearing of the bowels with characteristic calomel evacuations. without intestinal pains; the calomel was stopped at once.

Jamury 99th. - Morning temperature $37^{\circ} R .\left(98.6^{\circ} \mathrm{F}\right.$.), evening $37.2^{\circ}$ R. $\left(99^{\circ} \mathrm{F}.\right)$ Patient is much better: he has some appetite, almost no colic, intestinal pains less severe and less frequent; amount of urine 1000 c. c.. color paler: sleeps well, feels generally better and stronger.

Fehruary 1st. - Condition same. but colic somewhat more severe; stool only after a clyster: had five grains of calomel in the manner as above; two hours after the last dose, the interne, fearing to weaken the patient more with a superfluous dose of calomel, ordered a spoonful of castor oil. Then followed evacuations as formerly and also a similar improvement.

February 9th and 10th. - Patient has no ferer. feels stronger and heartier, eats better; but the colic has been worse these days ; eight grains of calomel in the former manner.

February 11th. - No stool. Temperature 39.2 R. (102.5० F.). Had a spoonful of castor oil (this was the last close: he was given no more of it). I good calomel evacuation.

February 12th. - Morning temperature $36.8^{\circ} R$. (98. $2^{\circ}$ F.). evening $37.2^{\circ} R .\left(99^{\circ} F.\right):$ urine 1500 c. c. of almost normal color; colic again insignificant. improvement in everything else. Thus up to February 17 th.

Febrary $1 \%$ th. - An evident error in diet; ate too much. Colic much stronger.

Felmary 18th. - A severe chill. Temperature in the norning $40.1^{\circ} R .\left(104.1^{\circ} F\right.$. $)$. Was given calomel, one grain every hour. He had a stool after the fourth dose. and did not get any more. Evening temperature $37.6^{\circ} R$. $\left(99.6^{\circ} \mathrm{F}\right.$.).

Fehwary 19th. - Morning temperature $37^{\circ} R$. (98.6 F.). evening $39.2^{\circ} R$. $\left(102.5^{\circ} F\right.$.). No further laxation. Was given six grains of calomel. as before.

Felruary 20th. - A good calomel stool. Morning temperature $36.90^{\circ}$ R. $\left(98.2^{\circ} \mathrm{F}.\right)$, evening $37.1^{\circ} R .\left(98.8^{\circ} F.\right)$ No colic. 
From this day on the patient's condition continued improving: the fever disappeared, the patient ate more and digested well (no heaviness and belching); the clyster acted better. and at times the bowels moved withont any clyster; the intestinal pains appeared at rarer intervals, and were much less severe than formerly; the color of the urine became normal and the general jaundice began to disappear; he began to sleep better, has grown stouter and stronger. The attacks of liver colic appeared now and then. but each time they grew weaker and weaker. At such times he was always given calomel: February 29th. Narch 3d. 9th. 15th. eight grains on each day, and the last time, March 24th, four grains. with good effects, without ever causing any laxative action.

Since March $2 \pm$ th he had no attacks of liver colic. the sensitiveness in the region of the stomach and gall-bladder disappeared even before, and a little later the intestinal pains also ceased. The patient began the use of condurango since April 1st, $\bar{z}$ ss. in $\xi \mathrm{vj}$. two tablespoonfuls per day. He left our clinic April 21st entirely improved: the appetite and digestion became normal, he had stools without the aid of clysters, and all the abdominal pains disappeared: he has grown somewhat stouter, quite strong and hearty. Since the latter half of February he had warm baths twice a week: at first at $30^{\circ} R$. as he was quite chilly _ six baths : and before leaving, when with the improvement in sleep, he became less chilly, three baths at $28^{\circ}, 27.5^{\circ}$ and $27^{\circ} R$. The baths at $30^{\circ} R$. quieted the patient, while cool ones strengthened him.

Twenty years passed in the interval between these two cases the first and the last. During this period I had the opportmity of observing many such patients. and of employing calomel. With all their various peculiarities these cases all present. in their principal traits. the same characters as the two related above. My practice is as follows: the simplest cases of liver colic accompanied by some dyspeptic phenomena require only a strict diet and a generally regulated mode of living; but such cases are certainly rare. For those that we ordinarily run $\mathrm{ac}_{\mathrm{r}}$ oss, with more or less severe attacks of liver colic. gastric ca tarrh and jaundice. I prescribe a strict diet, a regular mode of living and Ems, or Vichy, or Yessentucki. or Karlsbad (less frequently Marienbad). in accord with the peculiarities of the case. Calomel is less frequently administered in fresh. recent cases; much oftener for patients who had suffered for a long time from liver colic and deranged digestion, who have more 
than once gotten well, who had been subjected to recurrent attacks of the disease, have grown weak and thin and are not benefited any more by the use of mineral waters. As the principal indication for the use of calomel I consider not so much the severity of the individual attacks, as the presence of the constant pain in the region of the gall-bladder, generally in the liver, and the fever: calomel actministered in such cases has always produced results as splendid as it did in the abovementioned cases. In a small minority of cases (less than in one out of every twenty), without iundoubted symptoms of pericystitis, I ordered leeches - from six to ten alongside the right hypochondrium.

For the sake of precision I must tell you also of a case which was not benefited by calomel ; however, this case, which etiologically belongs perhaps to the class of cases under consideration, is certainly one of a different nature. The patient, a female of sixty, strongly built, had since her fortieth year suffered from liver colic, had frequently used the mineral waters, but did not complain of any colic lately. Without anything to account for them, there appeared febrile attacks of a pyaemic nature, against which enormous doses of quinine proved ineffectual (the salycilates were as yet not in use at those times). As no cause for these attacks could be determined, we, guided by the anamnestic data, concluded them to be probably due to abscesses of the liver, as caused by biliary calculi, although there was neither. jaundice nor pains in the region of the liver, neither spontaneous nor on pressure (the liver was felt to be diminished in size). The appetite becane somewhat livelier and the next attack of fever came later; however the attacks continued as before without cessation up to the time she died. The case was one in private practice, and no autopsy was permitted.

I usually prescribe one grain of calomel every hour, and after the sixth dose (either before or after it, in accord with the peculiarities of the case) every two hours, and after the first good calomel evacuation I stop the drug.

Ordinarily I give no more than twelve grains in succession, and in case the patient has no loose stools I give a tablespoonful of castor oil two hours after the last dose; but this is rarely necessary. I have observed that patients in whom castor oil causes nausea, bear it better after calomel. Diarrhœa after calomel is of rather rare occurrence and is easily checked by a lew drops of simple tincture of opium. Larger doses of calomel and more frequently administered I found unsuitable: they 
reaken the patient who usually suffers already with poor health. and cause obstinate diambea. With the very first dose of calomel that I prescribe, I advise gargling with a solution of potassium chlorate ( $3 \mathrm{ij}$ to $\overline{\mathrm{v} j}$ ), and continue this last for a few days after the use of calomel has been stopped. I never observed any trouble in the gums while taking such precautions.

We hear at times that calomel acts only as a laxative. Neither the pharmacological nor the clinical data present a basis for such a view. But even if calomel acts only as a laxative, are then all the laxatives alike? Ignorant indeed would be the practitioner who would consider all the laxatives alike, without differentiating the cases where each of them may be employed.

Facts have made it sufficiently clear to me. that whether as a laxative or otherwise. calomel will in certain cases do what, in the present condition of therapeutics. no other laxative, nor generally any other drug, can do. Many a time have I seen cases which, just as the second related case, were not benefited by the various laxatives, including castor oil, when the condition of the patient remained the same, or became frequently worse, while calomel turned the scales.

As far as the particular comparison between calomel and castor oil is concerned. I will say this much: In the above-described diseases of the biliary passages. castor oil can hardly at all be compared to calomel: it only causes a loose stool, without producing the characteristic, in the case of calomel. dark-greenish, abundant bile-colored eracuations ; nor does it produce that effect on the morbid phenomena in the domain of biliary passages. which is so pronounced and so constant after the use of calomel. Besides, it is badly borne in such cases. ofttimes indncing romiting and aggravating the disease. In erysipelas of the face, in typhoid fever, croupons pneumonia and acute Brights. there is some similarity in the nature of the effect, but an enormous difference in the degree of this last. And to administer - with the purpose of obtaining an effect similar to that obtained from calomel — doses of castor oil, larger than those used for ordinary laxative effects. would be an unfortunate procedure, as even the ordinary doses are not bome as well as those of calomel. while larger ones would affect injuriously the stomach and the intestines. which eircumstance would result not in improving. but in aggravating the condition of the patient.

Hypertrophic cirrhosis of the liver. - I observed only one 
case, to which I shall refer here. November 8,1883 , I was called for the first time to see N. N. The patient, twenty-three years old, had long since ceased to obey his elders, was extremely irritable, formerly neglected his health entirely, and during his illness lost his faith in medicine, did not follow his physician's instruetions, and is in addition to this very weak, so that it is almost impossible to obtain from him any information as to his past. All the data, which we succeeded in obtaining from him and from his relatives after he improved, may be summed up as follows. When fifteen years of age, patient had an attack of diphtheria, after which there remained some hoarseness (paresis of the left vocal cord). During several summers he suffered from intermittent fever, one or two attacks, which yielded quickly to quinine. Patient took to drinking during the last four years. and exclusively wine at that. Madeira (never drank any brandy). in great quantities, from three to five bottles per day, so that he was tipsy almost every day. The last year and a half he became very imitable and lost his appetite. In the summer of 1883, he began to have daily vomiting in the moming, after the first glasses of Madeira. During the month of September patient spent much of his time, late in the evenings, in excursions, garden-parties and during bad weather was subjected to attacks of cold. By the middle of September he became feverish, lost his appetite completely, and the whites of his eyes became yellow. A physician was called in September 2.5th, and detected an enormons enlargenent of the liver. At the advice of another physician he gave up chinking wine, and from October 11th drank natural Karlsbad water, one and a half glasses per day, somewhat warmed. During the time that he was treated with the Karlsbad water, patient had every day ten to fifteen loose stools, not more than a teaspoonful at a time (hardly one half a glassful during the twenty-four hours), of a painful character: since then the abdomen became constantly sensitive, especially so in the left lower half. The jaundice became aggravated during this period, the abdomen increased in size (the physician diagnosed abdominal dropsy), and there appeared redema of the legs. The sleep became very poor. October 22d and $2: 3$ d the patient took infusion of digitalis (from ten grains), but without any result. October 28 th, he began to take adonis vernalis, but did not even take the whole of the first bottle. since then and until November 8 l, he took pills made of extr. rhei compos. jj, extr. hyosciami gr. ij (after he stopped taking the liarlsbad water he became constipated), which produced loose 
stools, similar to those caused by the Karlsbad water: some ten to fifteen stools per day, not more than one teaspoonful at a time. From November 1st to $10 \mathrm{th}$, patient took potassium bromide (ten to fifteen grains at bed-time) for his poor sleep, without any success, and during both days of November ith and 6th, he took fifteen grains of potassium iodide.

Status November $8 t h, 1883$. - Patient is genelally surrounded by excellent conditions of life ; but the room which he preferably occupies at the present time, is situated in a corner, and has many windows, while near by the patient's very bedstead is a door. through which there blows a strong draught (alongside of this is a cold watercloset). This notwithstanding, patient stubbornly refuses to change his room ; besides, he is very weak. Only two weeks later, when he improved somewhat and grew more obedient. we succeeded in removing him into a large, spacious, entirely satisfactory room, and this removal had induced a farorable change in his condition. Ontside of four glasses of milk a day, patient reither eats, nor drinks anything: drinks neither wine nor tea, does not smoke (formerly he drank tea and smoked).

Patient is of a weak constitution : he is tall, and has a narrow chest. Is extremely weak and emaciated; the abdomen is enormously enlarged. the legs are cedematous almost up to the knees. The whites of the eyes are moderately yellow; the skin' is yellowish at some places. light-olive colored in others. At the present time (August. 188t), when the patient, as we shall see, improved greatly, and the evacuations for the last six months became normally colored, the color of the skin is light olive. Patient asserts that his skin was thus colored for a very long time. long before the appearance of the evident jaundice in September, 188.3. The tongue is normal. There are no gastric disturbances, such as belching, pyrosis, nausea, vomiting. sensation of heaviness and pain in the region of the stomach. either spontaneous or induced by pressure. As mentioned already, he has some ten to fifteen stools daily. a teaspoonful at a time : the evacuations are loose, whitish, slightly but still surely colored by bile. and contain some mucus. There are undoubted symptoms of accumulated fluid in the abdominal cavity. The circumference of this last at the level of the umbilicus is 95 c. m., the distance between the xyphoid cartilage and the symphysis pubis is $42 \mathrm{c} . \mathrm{m}$. But the abdominal walls are flabby, not distended, and extremely thin (on account of the extreme general emaciation). so that examination of the abdomen is eas- 
ily made. The subcutaneous abdominal veins are not distended. There is constant pain in the left lower half of the abdomen, which is increased on pressure and at times after evacuations. The amount of urine is 350 to 400 c. c. a day : it is very concentrated. gives the reaction of the biliary pigments and contains some albumen; at times there are found in the urinary sediment uric acid cylinders. The spleen is slightly enlarged, not sensitive. The liver is enormous: everywhere equable, preserving its normal shape, and reaching beyond the border of the ribs and down to the umbilicus. The surface of the liver is perfectly smooth, not presenting the least protuberance; the anterior border is sharp. The liver feels very hard and is entirely insensitive to pressure; there never were nor are there now any spontaneous pains in the region of this organ. I must repeat again that the extreme thinning out (with the general emaciation) and flabbiness of the abdominal walls enabled us to palpate the liver with an ease, that is only rarely possible. Percussion of the superior border of the liver shows it to be somewhat higher than normally. The respiratory organs are normal; the condition of the larynx was alluded to above. The heart-beat, perceptible in the mammillary line, is diffuse, but weak; there is a dull sound in the region of the heart in the normalline; the tones are clear. The pulse is 110 to 120 , extremely weak; the arteries are, however, felt without difficulty, they are somewhat tortuous and apparently slightly thickened. The patient is feverish: the evening temperature is between $37.8^{\circ} R$. $\left(100.4^{\circ} F\right.$.) and $38.5^{\circ}$ R. (101.3० F.), the morning the same or more frequently lower. The skin is dry and covered here and there by extensive ecchymotic spots, which reappear easily after a slight pressure. His sleep is restless and lasts only from three to four hours out of the twenty-four. The head does not ache and is generally free.

The final diagnostic conclusions are reached by me during the exposition of the course of the disease and of the effects of the treatment, for in this way, as will be seen later, we obtain valuable data for the diagnosis. Let me only mention here my first suppositions. I certainly could not stop on simple hyperaemia of the liver, whether active or passive, as such an assumption could not adequately explain the presence of jaundice, the impaction of bile, the enomous enlargement of the liver and the absence of tenderness in the same. ('ancer of the liver was excluded on account of the absence of pains on pressure, and by the results of palpation. Closure of the ductus choledochus was 
excluded by me in view of the absence of attacks of hepatic colic in the past and the absence of pain and sensitiveness on pressure at the present time, but chiefly on account of the fact, that notwithstanding the enormously enlarged liver, the evacuations were not entirely colorless, and contained some biliary pigment, while the icterus of the face, of the general integuments and of the urine was very moderate, being far less considerable than when seen in cases of closure of the principal biliary duct.

Outside of the hypertrophic cirrhosis one would most certainly suspect either multilocular echinococcus or syphilis. Against echinococcus there was the shape of the liver. while syphilis was excluded by the fact that neither the patient nor his parents ever had this disease. The presence of alodominal dropsy I had to refer to peritonitis, which was caused by a cold caught in the patient's rooms; the following facts lead to such reasoning: the abdominal pains appeared about the midclle of October; for a whole month previous to this the patient was confined in his room. lying most of the time in bed clad only in his undergarments, either covered with a quilt, or entirely uncovered when feverish ; while near by the berlstead there was the door, either half or entirely open, through which there entered a strong draught from the watercloset alongsicle of it. The pains appeared at the time when the patient began having, thanks to the Karlskad, ten to fifteen loose stools per day; at first they occurred only with each inclination to stool, but later on they became constant. As will be seen further on, from the diary of the disease, the matter stood thus :

When the danger from further colds ceased, when the abdomen was constantly kept warm, and when, after the use of calomel, the diarrhoa disappeared and consequently at once with it there also passed away the irritation of the peritoneum by the intestinal movements, then the abdominal pains began to diminish, so that even in two weeks from the time I began my observation there was a noticeable diminution of the abdomen contemporaneous with the disappearance of the pains. At that time it was as yet impossible to diagnosticate a diminution in the volume of the liver even to the slightest extent: the beginning of this process of diminution was only observed two and one half weeks later. Finally, notwithstanding the considerable abdominal dropsy, the patient did not present the least sign of any distension of the veins on the abdominal surface, although in view of his extreme emaciation this condition could surely be 
very easily perceived; this condition of the veins, as you know, is observed in cases of abdominal dropsy caused by hepatic disease, on account of impeded circulation in the portal system.

Thus I put it down as a hypertrophic (biliary) cirrhosis of the liver, catarrh of the large intestine, a peritonitis of moderate severity, particularly in the vicinity of the sigmoid flexure, and also a possible cirrhosis of the kidneys, this last in view of the former abuse of alcohol, the cardiac and arterial phenomena, and the presence of albumen and cylinders in the urine. The considexable development of the cedema of the legs and partly the abdominal dropsy I ascribed to the failure of the cardiac activity induced by the sudden cessation of wine drinking, by the fever and emaciation; the same may be said partly in regard to the poor sleep. Having made the patient as comfortable as it was possible in his own room, and later, at the first opportunity, having removed him into another more satisfactory one, I advised to keep the abdomen warm by the use of Provence oil and a double abdominal flannel binder, and for nourishment and drink to continue the warm milk (later on, when the appetite improved, we added roasted woodeock, without the skin, and some white bread), and to support the cardiac activity and the nervous functions in general, he was given his habitual nervines: a glass of sutticiently strong tea during the day, some six to eight tablespoonfuls of Madeira wine, and three to four times a day twenty-five drops of the mixture of the tinctura valer. aethereae and liquoris anodyni Hoffmanni āă. Guided by my experience in the use of calomel for hepatic colic cansed by biliary calculi, I decided to try this remedy in the present case. I also thought of applying Spanish flies to the lower part of the left abdomen, but the considerable ecchymoses just in this region prevented me from doing this: while lying on the left side patient pressed his hand on this region and the ecchymotic spots presented true reprints of the palm and of the fingers.

Nov.9. - Gargling of the mouth with potas. chlorate: this the patient contimued throughout the whole period of treatment with the calomel, up to the end of June, 1884. Six powders of calomel, containing one grain each: the first three every hour; the others every two hours. The sixth was followed by abundant and characteristic calomel evacuations of a dark-green color, and painless. 'This time, as well as at other times later' on, the patient had one or at most two loose stools from calomel, but never more, always more or less abundant and painless. 
Nov. 10. - No stool: the former frequent and useless attempts at defecation ceased from this time on. The abdominal pain subsided somewhat. both the pulse and the sleep are markedly better : everything else as before.

Nov. 11. - Three one-grain powders of calomel, one every hour. A good calomel evacuation.

Tov. 19. - The same treatment. the same result.

Nov. 1.3. - Two powders - the same result.

Nov. 1\%, 1\%. 16. and 1\%. - He was not given any calomel during these dars. No spontaneous stool; a clyster caused a slightly formed evacuation, not of the same dark-greenish color as after calomel. but colored more markedly than it was before, though still less than normal. The appetite began to reappear. the abdominal pains diminished. the sleep is better. the pulse fuller and stronger, although still 120 per minute. Everything else as before.

Nov. 19. 20, 22. and 2.5.- Three grains erery day in the former order. The usual effects.

Tus. 2.;. - The patient is evidently improved: the appetite is livelier, the evacuations are more markedly colored (but stool takes place unly after a clyster of warm water which is given every other day). the jaundice is less pronounced. the abdominal pains begin to disappear, the abdomen not so large $(30$ and 40 (.m.), the sleep and pulse better.

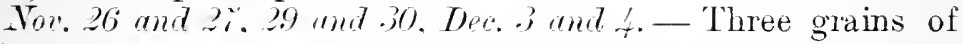
calomel as before. The usual calomel stool, less abundant than formerly. The improvement continues little by little.

Dee. $i$ and s.-_Four grains of calomel daily : no stool.

Dec. 9. - Thre doses of calomel. two grains every two hours. Four hours after the last dose a tablespoonful of castor oil. Abundant calomel eracuations.

Dec. 11. 1. and 1.).- Appetite markedly better. Stool either spontaneous, or by clyster, fairly well colored, but by far not normally. The jaundice of the eres and skin is disappearing; but the olive color has remained until now. No abdominal pain. But here I will note a most important fact: from this time on the amount of urine has suddenly increased, up to 900 or 1.000 c.c. in the twenty-four hours. and it became limpid and free from any biliary pigment; still there is some albumen as yet; the febrile condition diminished. The fever continued for another six weeks up to January $22 \mathrm{~d}$; but instead of the former evening temperature. $37.8^{\circ} \mathrm{R} .\left(100.4^{\circ} \mathrm{F}\right.$.) to $38.5^{\circ} \mathrm{R}$. $\left(101.3^{\circ} F\right.$.), and at times the same in the morning, the even- 
ing temperature was only $37.6^{\circ} R .\left(99.6^{\circ} F\right.$.) to $37.8^{\circ} R$.

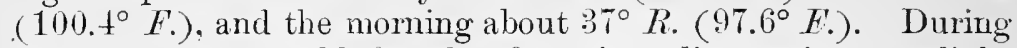
these days we could for the first time diagnosticate a slight diminution in the size of the liver. Sleep and pulse (110) still better. No fresh ecchymotic spots appeared again. As regards treatment we must add that during the past month the patient was given four times quinine, twice in five-grain doses on the days when he did not get any calomel. The quinine was well borne by the stomach. but failed to exert any influence on the fever.

Dee. 95. - The dimensions of the abdomen are is and 83 c.m. The cedema of the legs is rapidly diminishing. Stool again only by the aid of clysters. the evacuations less in number and their color paler.

Dee.26 and $2 \%, 29,30$ and 31 . - Three one-grain doses of calomel, one every hour. A moderate calomel stool.

Jan. 3, 1854. - Three one-grain doses of calomel, one every hour. No stool.

Jan. 4.-Three doses of calomel, two grains every two hours, later a teaspoonful of castor oil. Abundant calomel evacuation.

From January 5th up to 22d, equable improvement continues. On that day the symptoms of accumulation of fluid in the abdomen, the redema of the legs, as well as the fever, have all entirely disappeared. The liver continues decreasing in size. During this time the patient was given two soap-baths so as to clean his skin; this weakened him. During the last days the stool is again worse: it is necessary to resort to a clyster, the evacuation is insufficient and its coloring is again lighter.

Jan. 2.3. - Four doses of calomel, one grain every hour. No effect.

Jan. 2.4. - Three doses of calomel. two grains every two hours. Abundant calomel evacuations. From that time until the present (August, 188t) the patient's condition continued to improve. Little by little the appetite grew better than it ever was during the last three years. The liver contimued to shrink in size. although slowly so. The albumen in the urine amounts only to a slight reaction, but it did not lisappear entirely. The sleep became entirely normal, while the mental state, as compared with what it was before. is marvellously good. Patient began to take on flesh and gather strength little by little. The evacuations, after the abundant calomel stool, were for a few days spontaneous, in sufficient 
quantity and fairly well colored; but later on they grew less abundant and became less colored, till finally a resort to clyster was had again. We then administered calomel again - from now on always in three two-grain doses, every three hours without any castor oil - and obtained the usual good results. Thus calomel was given February 6th, 16th and 26th, March 6th and 30th, April 14th, May 1st and 16th, and the last time June 20th. Between March 6th and 30th, when calomel was not administered, he was given for about two weeks $\bar{j} 1 \mathrm{~V}$ of Ems-Kesselbrunnen, three times in the morning. slightly warmed, without the least effect on the patient's condition. At the same time, after the Ems, in view of the fact that notwithstanding the absence of fever the pulse was still frequent (100), we tried digitalis ( $2+$ grains in an infusion during three days), but this also failed to cxert any effect. The pulse grew less and less frequent only contemporaneously with the improvement in health, but even now it is about 90. Finally for the sake of completeness me must add that. thanks to the prolonged recumbent posture, usually on the back, with legs flexed at the knees, the patient developed contractures and paresis of certain muscles of both legs and feet. Gradual exercises by walking, massage and the induced current remored. though slowly, these conditions. and with this the muscles have markedly increased in size. Since March the induced current was applied to the abdomen so as to act on the intestines and liver. This has apparently also exerted some influence on the constipation; whether or not the liver was influenced, it is difficult to say, as the liver had diminished in size even before. and since this time it has diminished but little (we also continued the use of calomel).

At the present time (the end of August, 188t) the patient's condition is as follows: while living during the summer in the country he walks a great deal. For more than two months he had taken no calomel, nor any other treatment. The appetite is good, the digestion is perfectly regular (he resorts to clysters but very seldom), his sleep is excellent, the mental condition good, the patient has grown stout and strong. The urine still contains some albumen, but in a very trifling amount; the arteries and the heart are in the former condition, the pulse is still about 90 . The liver is much smaller in size than it was before, but still certainly larger than the normal liver; it reaches two fingers' wilth beyond the border of the ribs and is firmer than normally. The light olive-like color of the skin has not changed. 
I will return now to the diagnosis, the interest in which in the present case is quite evident. The markedly favorable course of the disease compels us to entirely exclude multiple eohinococcus. It is otherwise with syphilis : did we not treat a syphilitic liver with a mercurial preparation, calomel? This supposition cannot be entertained for the following reasons: neither the patient's father (now dead, but whom I knew well) nor his mother had syphilis. The patient himself, who had now acquired boundless implicit confidence in the physician, and who became very docile, declares that he was generally averse to sexual intercourse, had never suffered from anything, not even gonorrhcea, that he never had any falling out of the hair (this is now remarkably thick). nor any eruptions or uleers, nor any nocturnal or other pains. The results obtained by palpating the liver. namely: the perfect smoothness of its surface. the regularity of its shape and its sharp, free border, also speak against syphilis; the slight coloring of the evacuations and the jaundice are also unlikely to be seen in syphilis of the liver.

Finally, the treatment itself: calomel was always administered so as to cause loose stools. Which it did: and it was given at intervals which became more and more prolonged. Every experienced physician is well aware of the fact. that success in treating syphilis of the liver. or generally any severe case of syphilitic affection, cannot be attained by such intensive mercurial treatment applied in such a manner, and that it can hardly be expected without a simultaneous use of large doses of potassium iodide. Therefore, even with greater probability than at the beginning of my observation. can I view this case as one of hypertrophic cirrhosis of the liver. It is worth our while to note the following : the etiology of hypertrophic or biliary cirrhosis of the liver made clear the fact. that contrary to the simple Laennec cirrhosis, it is seen in those who abuse wine, and not whiskey. The anamnesis of the case described by me substantiates this fact: our patient always drank Madeira, never any whiskey.

A few words concerning the prognosis. Is the improvement in the condition of the liver of a lasting nature? If the patient will lead as regular a mode of living as he does now, then the recurrence of the icterous symptoms seems to me but slightly probable, and even if it will occur, then in the presence of the effective influence thereon of calomel, it will present no particular danger. It is otherwise if we were to ask, whether or not 
the other variety of hepatic cirrhosis - the Laennec cirrhosis - could develop; this however is a question we are unable to answer at the present time. The albuninuria and the arterial and cardiac phenomena have certainly a very serious significance as regards the future state of the patient. Be as it may, the improvement in the patient's condition under the given circumstances is marvellous: the patient's relatives, who last autunn lost all hopes for his recovery, inquire of the physician about the advisability of having the patient married.

Facial Erysipelas. - For young persons with a good heart and regular digestion, I give quinine until a slight noise in the ears is produced, and if the conditions permit I order general baths of $24^{\circ}$ to $20^{\circ} R$., and apply cold compresses to the parts affected by erysipelas. Quinine exerts a favorable effect in lowering the temperature; the same may be said of the cold compresses, which, together with the quinine, may even directly interrupt the process, when the fever and the further spread of the erysipelas cease. But there occur cases of facial erysipelas in persons of advanced age whose cardiac activity and digestive powers are below normal, who suffer from dyspepsia or gastric catarth with a tendency to constipation, with attacks of hepatic colic, either in the past or at the present time, and in whom the evacuations are not normally colored. In such cases quinine is badly borne by the stomach, and so are the cold baths, in view of the abdominal distension cansed by meteorism and the weak cardiac activity. Neither of the above drugs gives as good results in such cases, as in those of the preceding class. I replace them by calomel, in one-grain doses every hour (the last doses every two hours), till looseness of the bowels is established; if this does not take place after twelve grains, I administer castor oil. I never omit in these eases the potassium chlorate gargling. A good loose stool is followed by improvement in the meteorism, the respiration and the condition of the head are made easier, the temperature is lowered for one or two days, the patient is enabled to sleep quietly one or two nights. At times the temperature will fall to the normal without ever rising again; then the further spread of the erysipelas ceases. But if the temperature rises again, if the erysipelas spreads further and the condition of the patient's strength is satisfactory, then I administer calomel for the second time in the former fashion. We never have to administer the calomel more than twice, either because the erysipelas ceases, or, in case it does continue, because the fever is slight, and the patient's strength is affected by the 
continuance of the disease. I then prescribe moderate doses of quinine, which is better borne then, after an improvement in the condition of the digestive organs has taken place, thanks to the use of calomel.

Typhoid Fever. - Like many others, I also witnessed the value of calomel in this disease. In accord with others, I administer it during the first week and usually not later than on the eighth or ninth day of the disease. I give it when there is no diarrhœa, when the strength of the patient permits it, and I must say again I particularly insist on giving it to patients with a condition of the digestive organs as described above in touching on the second class of erysipelatous patients. The loose stool after calomel diminishes the meteorism, relieves the breathing and the condition of the head, causes a fall of temperature for one or two days, and enables the patient to spend one or two quiet nights (he sleeps better and is less delirious). The favorable effect of two good days at this period on the further course of the disease is not to be underestimated. I give the calomel here in the same manner as I administer it for erysipelas, but I do not repeat it, out of apprehension of weakening the patient; this apprehension is of even greater importance in typhoid fever than in erysipelas. I cannot help observing here that the assumed specific effect of calomel on the intestinal microbes of the typhoid fever does not seem to me particularly probable. We fail to observe a sudden interruption of the fever by calomel, as we do in the case of quinine in malaria; we only witness here a favorable effect on the further course of the disease, just as we did in erysipelas, and, as we shall see presently, in pneumonia, and in acute Bright's, — in processes which have nothing to do with intestinal microbes. On the contrary in cholera, in which we would most certainly expect an effect from the calomel. I failed to see the very least effect from it during the two severe epidemics in Moscow, of 1853 and 1854 , when in my capacity as a resident physician in the temporary choler"d ward of the university clinic, I had charge of a great many patients, and calomel constituted the ordinary treatment of cholera.

Croupous Pneumonia. - During the first two years of my medical practice I userl digitalis, but it did not prove efficacious, at times it was even harmful, and since then I never use it in pneumonia. In several cases I tried cold baths and concluded that, although the patient may bear them, they hardly profit by them, if we except a trifling fall of the temperature of but short 
duration. My acquaintance with cairine did not tempt me into trying this drug. From quinine I only obtained a fall of temperature of short duration, and this only when administered in enormous doses which are but with difficulty borne by the stomach and the nervous system; I therefore discarded this drug, except in some protracted cases, when the fever is of a strongly remittent type in the morning, and moderate doses of quinine become effective. Even less suitable for pneumonia do I consider sodium salycilate, especially in view of its action on the heart. My principal remedy in the treatment of pneumonia, outside of the usual symptomatic drugs (as Dover's powders for the congh in the beginning when there is but little or no expectoration, wine for the heart, etc.). has been calomel until now, and is even at the present time when antipyrin and antifebrin are introduced into practice. I administer it in the same manner as for erysipelas, namely in sthenic cases of pneumonia, to patients of strong constitution, when the condition of their strength is good, mostly during the first days of the disease, and particularly when the condition of the digestive organs resembles that described above in connection with erysipelatous patients of the second category. The effect is the same as in facial erysipelas and typhoid fever: the looseness of the bowels is followed by a decrease in the size of the abdomen, by relief of the respiration and of the condition of the head. by a fall of the temperature and by at least one quiet night. Such a relief at this stage of a clisease of the limited duration of croupous pneumonia, has, according to my observations, the same favorable influence on the further course of the affection, as it has in typhoid fever, if not a better one: at times the fall of temperature caused by the calomel passes directly into the crisis. We usually do not have to repeat the calomel in view of the rapid course of the disease and the danger of weakening the patient.

Acute Nephritis. - In those cases which developed independently of any preceding infectious disease, and also in those seen during the puerperal period, but not connected with puerperal septicaemia, I administer calomel for the first days of the disease, in the same manner as described in connection with erysipelas, with the identical good result, as shown by the considerable fall of the temperature and the diminution of the local phenomena: the pains in the region of the kidneys grow weaker, the urine increases in amount and becomes limpid. If the temperature after this rises again, and the condition of the strength is fair, then I sometimes resort to a repeated use of calomel, just as I do in erysipelas. 
As contraindications to the use of calomel, outside of the weakness of the patient (as evidenced principally by the pulse), are considered severe diarrhceas with abundant exhausting evacuations, but not such as were described when we discussed the case of the patient suffering from hypertrophic cirrhosis of the liver: such a diarrhoa is on the contrary an indication for the use of calomel.

Ax Admitional Note Appended to the Edition of 1895.

I will tonch now on the indications for the use of calomel in diseases of the biliary passages.

In my formes communication I mentioned two affections of the biliary passages in which calomel should be used, namely, acute, particularly febrile, cases of hepatic colic, and hypertrophic cirrhosis of the liver. Further observations compel me to widen the range of these indications: calomel should be used, for hypertrophic cirrhosis of the liver, in all (not only acute) cases of biliary calculi and in eatarrhal jaundice.

The indication for the use of calomel in biliary calculi and in catarrhal jaundice I must formulate thus: if the ordinary measures employed in the treatment of the disease, such as a regulated regimen, diet, at times warm baths, and chiefly the use of mineral waters, either alkaline or earthy (Contrexéville), improve the condition slowly, if we observe constant tenderness in the region of the gall-bladder (in biliary calculi) or over the whole liver (as in catarrhal jaundice), the more so if it be attended with a febrile condition (as well as without it), and if the above alluded to contraindications to the use of calomel are absent, then we interrupt the use of the mineral waters and administer calomel in the manner explained in the first communication; later on we return again to the mineral waters and, if necessary, repeat the calomel; every time we do that we must of course put aside the mineral waters.

To illustrate these propositions I shall speak here briefly of the following cases.

1. Biliary Calculi. - This patient was first treated for an exacerbated chronic catarrh of the large intestine.

The patient, a woman sixty-seven years old, passed through an attack of dysentery four years ago. She usually complains only of slight dyspeptic symptoms (heaviness and belching after meals), of pain in the region of the gall-bladder, and of irregular stools since the attack of dysentery : either a constipation or a diarrhoea for two or three days. The present condition: an 
accidental cold was followed by loss of appetite, by the appearance of nausea, of intestinal pains, and a feverish condition. On the next day vomiting and diarrhœea accompanied by severe intestinal pains : some insufficient liquid stools mixed with mucus, but without any blood, three to four per hour; the abdomen is distended and sensitive, particularly in the region of the colon. Evening temperature $39.2^{\circ} R .\left(102.5^{\circ} \mathrm{F}\right.$.); the urine shows the presence of albumen. In the evening we began to give her one-grain doses of calomel every hour. After the third powder the vomiting ceased; the ninth dose was followed by abundant painless dark-green evacuations; the calomel was stopped. Patient began to feel better at once. In the course of three days the temperature fell to the normal; with this there disappeared also the albumen. The first three days after the calomel she had two to four stools a day; later, one. The darkgreen color of the evacuations continued decreasing during a. whole week. Tannin clysters which were used on the fourth day after the calomel brought to a speedy termination what there was left of the intestinal catarrh.

In a year and a half the patient presented herself to me in the following condition : the nervous system, the chest (outside of a slight atheromatosis of the aorta) and the kidneys normal ; she complains of a constant bitter taste, frequent belching of bile, a feeling of fullness in the stomach, moderate pain in the region of the gall-bladder, aggravated on pressure, and of constipation with intermittent diarrhœa. Warmed Ems and Vichy in slight quantities proved of no avail; while the bitters (nux vomica and condurango) brought but slight relief. I then prescribed one-grain doses of calomel every hour; after the third powder there appeared abundant dark-greenish stools (painless), three times the first day, twice the next (she was only given three powders of calomel); the above-enumerated symptoms have entirely disappeared both from the stomach and the biliary passages for a long time. Only in the course of another year did the patient present herself again with the same symptoms, but in a much lighter degree; the same plan of treatment, three one-grain doses of calomel, and the same success.

In connection with the above-described case I consider it necessary to touch upon the diagnosis of biliary calculi. Some consider as a reliable symptom only undoubted attacks of biliary colic, without paying due attention to the presence of pain in the region of the gall-bladder, of which the patients 
complain, or which is detected by pressure. My observations are of the following nature: in all the cases of biliary calculi which are attended by frequent or infrequent attacks of biliary colic, there is observed a constant, $i$. e. occurring in the intervals between the attacks, pain, as above-described, in the region of the gall-bladder; exceptions to this are very rare indeed. Further, there occur cases of biliary calculi where the above-mentioned pain is seen without any attacks of liver colic. We also meet various gradations between these two classes of cases: thus it happens that for a long time there is observed only a constant pain in the region of the gall-bladder, and later there may also appear attacks of biliary colic; these last may be of variable severity, from the lightest to the very severe ones: then the attacks of biliary colic may cease, thanks to treatment or to a regulated mode of living, and there remains only the constant pain in the gall-bladder, which in its turn may also disappear. In view of all this I consider the above-named constant pain in the region of the gall-bladder as a certain symptom of the presence of biliary calculi therein.

This pain depends very likely on a chronic inflammation of the walls of the gall-bladder caused by the presence of the calculi: while the attacks of biliary colic are determined by spasms of the nervo-muscular apparatus of the bladder and by the accompanying neuralgia, and is called forth by such influences as traumata (a ride over a jolting pavement, inconvenient clothing), mental anguish. dietetic errors, and cold, as when sitting with right side exposed to the window or the street side of the house during the cold season of the year, or while staying out-of-doors with the right side of the body against the cold wind.

2. Catarrhal Jaundice. - I will describe here one of the cases observed by me. November 12, 1890, there entered our clinic a peasant, thirty years of age, complaining of pain in the region of the stomach, fetid belching, nausea, at times vomiting, and of jaundice and general debility.

Conditions and mode of living. - The locality is healthy. the residence is good, the working-room is very hot (patient is stoker by occupation). and this in connection with a cold watercloset, and the necessity of going out of the room often, gives rise to frequent colds. During the summer the patient bathes in the river; during the remaining seasons of the year, he takes a weekly bath; he is subject to sweating. Smokes a little; is a moderate tea-drinker, does not abuse brandy. Is 
married; is not affected with syphilis. The meals are those of a workingman, consisting of dinner and supper. Works some twelve hours a day; until the appearance of the present disease the work never tired him.

Anamnesis. - Patient comes from a healthy family, and until the last four years enjoyed good health. Then he suffered from masked malaria appearing in the form of attacks of excruciating pain in the right half of the head, which at times terminated in a severe nose-bleed from the right nostril, causing him to faint. These pains and nose-bleed continued for two years and almost disappeared when the patient moved to another locality. He then improved, although he did not enjoy his former health, and was free from any suffering for about one year. But for the last year he has been suffering from dyspeptic symptoms pointing to a gastric catarrh (pain in the stomach after a meal, belching and pyrosis). One week ago, as a result evidently of a cold, the patient began to have a febrile attack, pain in the larynx, hoarseness, the habitual slight cough became aggravated and at the same time there appeared vomiting and diarrhoea, and soon after that also jaundice.

Status November 1.3, 1890. - Patient is of average constitution, poor nutrition. with jaundice of the skin and of the eyes. The appetite is very poor, the meals are followed by pain in the stomach, fetid belching, pyrosis and nausea; while in the clinic he had no vomiting. The region of the stomach and the liver, i. e. the subgastric and the right hypochondriac, are sensitive to pressure. The liver is somewhat enlarged. The spleen is normal. Had no stool for the last two days (formerly he had diarrhoea). To-day, a clyster brought away a few hard scybala, almost without any biliary pigment, of the color of clay. The urine is of the color of beer, gives a decided reaction of biliary pigment; contains neither albumen nor sugar. The pulse is not frequent, but somewhat weak. The organs of circulation and respiration are normal. The pain in the throat, the hoarseness and the severe cough disappeared and there remained only the habitual slight cough due to chronic laryngeal catarrh. Patient has no fever. The sleep is usually good, but last night he could not sleep on account of the itching of the skin. The right half of the head is somewhat painful even now (the supra- and infraorbital nerves of the right side are sensitive to pressure). Fatigue is at times accompanied by dizziness, which points to the fact that the former 
abundant losses of blood left the patient in a somewhat anaemic condition. Further than the general debility, there is nothing abnormal in the nervous system and the organs of locomotion.

The course of the disease. - November 13th, five one-grain powders of calomel every hour. and then five again every two hours. Potas. chlor. for gargling the mouth, four tablespoonfuls of wine, diet, warmth (flannel) to the abdomen, and rest. Loose, soft, dark-greenish stools, after which the urine became limpid. Since November 15th he was given Ems-Kesselbrunnen, half a glassful three times a day, one hour before a meal, warmed to the temperature of $28^{\circ} R$., and when necessary in case of constipation, clysters, and to allay the itching, to improve the sleep, and also to act on the stomach and the biliary passages, warm baths. By November 21st the dyspeptic phenomena diminished, the sleep became better, and the patient felt somewhat stronger; but the icterous phenomena which yielded to calomel reappeared again in their former condition since the 15th: the evacuations became colorless, and the urine dark, just as they were before the calomel treatment. November 21 st we repeated calomel, of course setting aside during the 21st and 22d the Ems water, five one-grain powders every hour, and then two more every two hours. The effect was temporary, although there followed a more prolonged, relief of the icterous symptoms and of the pains in the region of the liver, similar to what took place after the first doses of calomel. Then until December 1st, notwithstanding the use of the Ems water. the extr. fl. condurango (twenty drops twice a day, after a meal), the warm baths and a regulated diet, neither the icterous symptoms nor the pain in the liver diminished to any marked extent. I will say that after the second dose of calomel the patient had a toothache, especially on the right side, probably on account of carelessness in gargling his mouth; but there were no symptoms of mercurial stomatitis. December 1st calomel was administered for the third time; five one-grain powders every hour, then three every two hours, and in two hours after the last powder a tablespoonful of castor oil. the mouth being all this time carefully rinsed with the potassium chlorate solution. Patient had abundant, loose, soft, dark-greenish evacuations, the last of which began to be gradually colored by biliary pigment, at first somewhat weaker than normally, and later perfectly normal; with this the amount of biliary pigmentation in the urine began to diminish rapidly, and the general integuments began to pale. The pain in the liver also disappeared 
rapidly after the last abundant calomel evacuation. The patient returned again to the treatment with Ems water, condurango and sometimes baths, which was interrupted the last time by the calomel treatment, and December Stl left the clinic entirely recovered.

For the sake of precision I must add that one week before the patient left our clinic an accidental cold (from a draught through the window) caused the appearance of a severe attack of his habitual pain in the right half of the head in connection with a slight febrile condition $\left(37.8^{\circ} R\right.$.). A elyster containing ten grains of quinine was administered (in view of the previous gastric catarrh), after which, accompanied by severe noises in the ears, the fever and the headache disappeared rapidly, the patient felt bimself much stronger. and the improvement continued its ordinary course. 


\section{SYPHILIS OF THE LUNGS.}

For the last two years we have had no opportunity of obserring any cases of pulmonary syphilis. Meanwhile at the present time, in connection with the discussion in our lectures of chronic pulmonary tuberculosis, to which syphilis predisposes so readily and together with which it is at times seen, it is but proper to say a few words regarding the syphilitic affection of the lungs (pneumonia syphilitica, lues pulmonum). I shall make use, for this purpose of my former communications and of the cases observed by me after these had been made.

Iy communications were made at the meetings of the Moscow Physio-Medical Society, April 11, 1877, and January 16,1878 (they were afterwards published), consequently several years before the discovery by Koch of the tubercle bacillus - a discovery which determined the specificity of tuberculosis, which up to that time, under the influence of Virchow's theories concerning the subject, was generally considered as an inflammatory process and was usually known in the clinics under the name of chronic pnemmonia. The diagnosis of pulmonary diseases was not possessed at that time of such an important means as the detection of tuberculous bacilli in the sputum. This notwithstanding, the abovementioned communications preserved, as you shall see from the extracts, their clinical, i. e. medico-practical importance.

\section{From the First Commexication.}

Not every physician has had the opportunity to observe an uncomplicated, positively certain syphilitic affection of the lungs; but who has not heard, or does not hear at times of certain persons affected with some chest disease, who were for a long tine supposed to have suffered from an ordinary pulmonary phthisis and were treated as phthisical persons. but who were cured finally by potassium iodide and mercury"? Excited curiosity will naturally search for the determining differences these cases present as compared with ordinary 
pulmonary tuberculosis, but it fails to obtain any definite indications. Such indications are absent not only in the literature of the past, but even in that of very recent date.

Ordinary pulmonary tuberculosis, as is well known, is often seen in syphilitic patients. Those persons who had been infected by syphilis and were subjected to the necessary treatment therefor, under unfavorable circumstances usually suffer from a radical impairment of their health, which frequently terminates in pulmonary tuberculosis. I had frequent occasions to observe, that not only persons who have passed through syphilis, but even those who presented its early or late manifestations, were sufferers from pulmonary tuberculosis in its ordinary form, presenting febrile symptoms, haemoptysis, cough, expectoration, changes in the percussion sound, in the respiratory murmur, and certainly more or less abundant constantly present râles. In such cases the attempt to resort to iodides or to mercury (if the syphilitic manifestations at the time present required it) not only produced an improvement in the chest trouble, but usually tended to its rapid and very pronounced aggravation, which compelled one to give up the specific treatment.

I chanced to observe two cases of chest troubles in syphilitic persons, which are decidedly different from those I have just outlined. I saw the first case fourteen years ago. Patient, thirty years old, of a good constitution. with a well-developed chest, coming from a family of healthy, sturdily built people, with no case of tuberculosis in the family, and living among favorable conditions of life, had been suffering during the last six or seven years with attacks of undoubted syphilitic infection, manifested for the last three years by deep serpiginous ulcers of the skin and periostitis of the tibiae and ulnae, which yielded to the treatment by potassium iodide, and reappeared again after the cessation of the treatment. Several weeks before I saw the patient he was complaining of pain and a feeling of oppression in the chest, slight dyspncea and general weakness; one week before, thanks to an accidental cold, he began to cough slightly and became somewhat feverish. (The patient became during the last years very susceptible to colds, and his attacks of colds usually take the form of a febrile bronchitis of short (luration and slight intensity). During the last days patient was confined to his room, the cough quieted down, and the fever disappeared entirely.

Status. - Patient is costive, his abdomen is somewhat dis- 
tended by intestinal gases, the urine contains neither sugar nor albumen; there is a slight cough, some expectoration (once or twice in the morning; patient always fails to preserve it for physician's examination), dyspncea, pain and a feeling of oppression in the chest; both clavicles are very prominent, the sub- and supraclavicular fossae are very marked, and the sound is duller in these places (especially on the right side) than in the area below; the rocal fremitus is weakened, while auscultation gives an indefinite respiratory murmur, approaching bronchial respiration. Everywhere are heard non-abundant râles, characteristic of bronchial catarrh. Patient lost flesh. His sleep is poor. He has slight nocturnal pains in the tibial and ulnar bones.

During the course of one week patient was confined to his room, took no treatment, and observed the diet prescribed for him in view of the meteorism and his tendency to constipation. From this time the cough and expectoration ceased and the râles disappeared. After weighing all the data as found in the case, I concluded that the pulmonary trouble was of a syphilitic nature, and required the use of mercury, because during recent years the patient had been constantly treated by potassium iodide and had observed that it failed to exert the same decided action it had done formerly: moreover, he had not been treated by mercury for a long time, and generally had but little mercurial treatment. Circumstances compelled me to administer the mercurial treatment in a form different from what I usually do. During the first week while he was under my observation. before I commenced the mercurial treatment, patient began to suffer from severe, intolerable itching which became aggravated at night and prevented his sleep. Scratching caused an eruption in the form of very minute red papules, and aggravated the itch; the spots that the patient could not reach by his hands, and which he therefore did not scratch, were not covered by the eruption. Baths and various external applications were of no avail; only applications of a corrosive sublimate solution brought considerable relief. In view of this the general mercurial treatment was ordered as follows: externally applications of a solution of bichloride for the itching and the eruption. and also certainly for its internal effect; as the eruption was widely disseminated, the applications of the bichloride solution had to be done over an extensive surface; internally strong Zittmann's decoction (Decoctum Sarsaparillae Compositum Fortius P. G.), as a slight laxative, and at the 
same time as a mercurial preparation. The itching and the eruption disappeared rapidly; this was followed by a return of the sleep, and after four weeks of such treatment. the dyspnœa, pain and the sensation of oppression in the chest passed away, the sub-and supraclavicular fossae became less marked, for, thanks to the improved appetite and digestion. the patient grew a little stouter, while the above-described symptoms which had been elicited by palpation, percussion and auscultation, could not be observed again : the chest in these regions, as everywhere else, presented nothing abnormal. I had several occusions to run across the patient during the next eight year's: he complained somewhat of gastric and nervous disturbances, but his chest, outside of slight, rapidly passing bronchitic troubles, was healthy.

The second case, which I had nnder observation six years ago (during the winter of 1870-71), is a complete duplicate of the foregoing one. Patient was also thirty years old, of a very robust constitution, living under favorable conditions; he had been suffering from syphilis for nine years. the last five years principally from various periostites and from ozcena. Was once treated with mercury at the beginning of the disease. but since this took only potassium iodide, which has ceased recently to exert its formerly good effect. For the last few years patient began also to suffer from slight disturbances of digestion and of the nervous system, and also from bronchitis of a febrile type of a slight nature and rapidly yielding to rest at home and the use of quinine. For some time before I saw the patient first, he was taken sick with his usual febrile bronchitis, but this time it was accompanied by pain in the chest, and by dyspncea; these were not present formerly. Patient confined himself within. the house, and when I was called to see him for the first time he had no fever, and the cough was just passing away.

Status. - Patient has a slight cough without expectoration : dyspncea, a feeling of oppression and pain in the chest; the clavicles are very prominent, and the sub and supraclavicular fossae are sharply marked, especially on the right side; over these places the sound is duller and the vocal fremitus weaker than in the area below. especially on the right side. while the respiratory murmur is of an indefinite quality, approaching bronchial respiration; over the whole chest there are heard râles here and there, peculiar to bronchial catarrh; there is no fever. patient became somewhat emaciated. As in the first case. patient was given no treatment during the first week: for the 
same reasons as guided me in the first case, I wanted to examine the disease more thoroughly, before prescribing specific treatment in the presence of such chest symptoms; during this time the cough disappeared entirely, as well as the râles, while the other symptoms remained unchanged. Guided by the same reasons as in the first case, mercurial treatment was ordered, in the form of inmetions with mercurial ointment. Altogether twenty-five inunctions were made: five of ten grains each, five of fifteen grains, and fifteen of twenty grains each. During the course of the treatment the morbid symptoms began to disappear, and at the termination of the same there was neither dyspnoea nor any feeling of oppression or pains in the chest; the abovedescribed symptoms, which were observed on the objective examination of the chest have also disappeared, the sub- and supraclavicular fossa became much less prominent, partly, of course, on account of the fact that, thanks to the corrected diet, the appetite and the digestion were in good order during the treatment, and the patient grew somewhat stout.

The first case impressed me, not because the chest symptoms which could excite a suspicion of pulmonary tuberculosis, yielded so completely to the effects of the mereurial treatment, but because the whole group of these symptoms presented such a sharp distinction from the collection of symptoms of ordinary pulmonary phthisis. and the patient himself resembled a consumptive so little; it was only the second observation, in which all the characteristics of the first case were repeated with photographic accuracy, which led me to the conclusion that I had to deal not with a group of coincidently connected symptoms, but with a permanent form of syphilitic pneumonia, in its genuine, uncomplicated form; a diseased condition, which semiotically and diagnostically differs greatly from the picture of ordinary pulmonary tuberculosis in non-syphilitics, as well as in syphilitics. The principal criterion by which we could judge that in both cases the affection was undoubtedly of a syphilitic nature, the criterion ex juvantibus was but too apparent: under the influence of the mercurial treatment the morbid phenomena disappeared. My conviction was even more strengthened by all we know concerning the anatomical alterations in the pulmonary tissue when it becomes affected by syphilis, the interstitial and gummatous character of these alterations which result in the thickening of the pulmonary tissue, and in the expulsion of air therefrom - all of which coincided so fully with the nature of the symptoms observed in the above-described cases on their objective examination. 
Putting now together the peculiarities of these cases which enable us to recognize syphilitic pneumonia in its pure, uncomplicated form, as distinguished from chronic pulmonary tuberculosis seen in non-syphilitic patients, as well as in syphilitics, we get the following:

1. Anamnestic and other data, pointing to an affection of the patient's organism by syphilis.

2. The robust constitution of the patient. It is very likely that persons with weak constitutions and with a predisposition to ordinary - tuberculous - consumption, are rather sooner affected by the disease, their lives not being prolonged until the appearance of late syphilitic manifestations, to which belongs syphilitic pneumonia.

3. The objective symptoms of thickening of the lungs are as follows : dull percussion sound, weakened vocal fremitus, alteration in the normal respiratory murmur. A sharp marking out of the sub- and supra-clavicular fossae, due very likely to an adhesive pleurisy, which accompanies syphilitic pneumonia as frequently as adhesive perihepatitis accompanies syphilitic hepatitis. Dyspncea. a feeling of oppression and pain in the chest.

4. Absence of haemoptysis, cough, expectoration and râles.

5. Absence of febrile condition.

6. The decided effect of the specific treatment.

That such cases of syphilitic pneumonia as described above may in their further course, if not recognized and treated at the beginning, or if not presenting any possibility for treatment under given conditions, become complicated by other affections of the respiratory organs, such as a severe bronchitis, a pleurisy with effusion, an acute and chronic pneumonia of non-syphilitic nature, and then appear in another form, is both possible and probable; but such complicated cases do not permit a deduction to be drawn therefrom in regard to the diagnostic, prognostic, and therapeutic conclusions about syphilis of the lungs.

\section{Fron the Second Connunication.}

Patient, thirty-two years old, entered the faculty clinic October 21, 1877, complaining of dyspncea, cough, loss of voice, swelling of the legs, and enlargement of the abdomen.

Mode of living and anamnesis. - Patient lives in a damp locality. Residence and board good. He was addicted to much smoking, and to immoderate whiskey drinking (neither smoked 
nor drank lately), and being a superintendent of an estate, be talked much and loudly, and fatigued himself.

Patient comes from a healthy family. In February, 1870 (when twenty-five years old), he had post-coitum ulcera penis. which passed away in the course of two months and a half with the aid of local treatment solely. In November of the same year there appeared a papular eruption, and later on ulcers on the skin. With the aid of thirty inunctions with mercurial ointment, and eight bottles of potassium iodide, everything disappeared by February, 1871.

During March, 1872 , there appeared nocturnal pains and slight swellings of the clavicles and of the tibiae; these lasted for eight months and disappeared without treatment. During the next four years our patient enjoyed good health. In March, 1877, there appeared a cough with dyspnœa and expectoration; during June various cedemata and general weakness; in July, hoarseness of the voice and pain on deglutition. Later on, by the time he entered the clinic, all the enumerated symptoms increased rapidly, with the exception of the cough which diminished during summer and remained so (since autumn the patient was contined within doors); there were but trifling febrile symptoms, if we are to judge from the inquiry.

Status. - The appetite is poor, there are slight dyspeptic phenomena; diarrhoea; ascites. The liver and spleen do not admit of palpation. The urine is 600 c.c. in quantity during twenty-four hours; it is reddish, turbid, and contains much albumen and many cylinders.

There is pain in the throat on swallowing and coughing; complete aphonia; laryngoscopic examination shows a condition of thickening and softening of the mucous membrane of the true and false vocal cords and of the epiglottis, and later on also the presence of ulcers in these parts. There is severe dyspncea; the cough is but slight, expectoration of a mucopurulent character, with an admixture of blood, the quantity of the expectoration at the beginning being about one-fourth of a glassful for twenty-four hours ; later on the quantity of expectoration and the cough became very insignificant. On the right side the sound is dull over the clavicle and from there down to the third rib; thence downward it grows clearer: in the subscapular fossa and also between the scapula and the vertebral columm upward, the sound is also dull. (On the left sicle, immediately above and below the clavicle, the sound is somewhat dull, but excepting these regions it is everywhere clear. All over the 
lungs there are heard whistling râles; besides these, in the area of the dull sound on the right side, there is heard a respiratory murmur approaching bronchial respiration, and very few loud moist râles; on the right side prolonged expiration. - The impulse of the heart is not displaced, but it is weak; its tones are clear.

The pulse is 96 , somewhat weak. The evening temperature reaches $38^{\circ} R$. (100. $4^{\circ} \mathrm{F}$.). There is everywhere a slight anasarca, which is considerable on the foot and the leg. The skin presents traces of serpiginous ulcers. The sleep is poor. There is general debility.

The course of the disense. - During the first days the patient was given Dover's powder and some quinine. The cough rapidly diminished after this, and so did the quantity of expectoration and the number of râles; but the diarrhcea increased somewhat, and quinine was therefore put aside, and tra. opii simplex was given. A week after the patient's admission to the clinic the fever began to increase somewhat. the pulse grew weak, notwithstanding the use of stimulants (as wine and tra. valer. aether.), the dropsical phenomena became more marked, and there appeared uraemic symptoms: heariness and pain in the head, and nausea. Norember 8th there appeared erysipelas accompanied by considerable fever $\left(39.7^{\circ} R .-103.6^{\circ} F\right.$.), and November 12 th patient died. During all the time that the patient remained in the clinic, the area of the dull sound over the surface of the chest increased gradually from above downward ; during the last days it was impossible to examine the chest posteriorly on account of the patient's extreme debility.

The patient was under the observation of our clinical assistant, Dr. V. G. Nesteroff. The first communication made by me in regard to syphilitic pneumonia led him to think of the possible presence of such in the present case. in view of the fact that the symptoms of thickening of the lungs, and especially of the right one. were so considerable, while the ferer was slight and moist râles were observed only over a very limited area (under the right clavicle). while the whistling ones disappeared during the first week of the patient's sojourn in the clinic.

While analyzing the case before my students, I arrived at the following diagnostic conclusions: 1. Chronic inflammation of the kidneys (large kidney, i. e. chronic parenchymatous nephritis). 2. The ascites and the diarrhoea may possibly have their special causation in an alcoholic or syphilitic cirrhosis of the liver. 3. A simple or degenerative atrophy of the heart. 4 . As 
regards the pulmonary affection, I agreed with Dr. Nesteroff that the peculiarities of the case, as observed by him, together with the anammestic data, and the nature of the laryngeal changes, lead us to recognize in the present case a syphilitic pneumonia; but as fever, haemoptysis, cough, expectoration, and moist râles, although observed in a small degree, are still not entirely absent, as was the case with the patients who suffered from a pure syphilitic affection of the lungs, and who were spoken of in my first communication, we may then assume that the present case presents neither a pure syphilitic pneumonia, nor a pure ordinary ehronic one (the usual pulmonary consumption), but very possibly a contemporaneous affection of the lungs by both processes. As regards treatment, the specific antisyphilitic - treatment was contraindicated by the multitude of painful affeetions, and by the extreme failure of nutrition and strength, as well as by the fact that the syphilitic affection of the lungs was not pure, but complicated by phenomena of ordinary consumption.

The autopsy was performed by the then docent of pathological anatomy (now professor of general pathology), A. B. Vogt. The results of an extensive report and of the microscopical examination are as follows: syphilitic disease of the larynx (ulcers, scars, diffuse papillary growths), agglutinated pleura, syphilitic (interstitial and gummatous) affection of the upper lobes of both lungs (especially of the right one), a chronic catarrhal pneumonia also of the right lung (of its upper and middle lobes), an acute, developed during the last days of patient's life, catarrhal inflammation of the lower portions of both lungs, "brown" atrophy (atrophia fusca) of the cardiac muscle (the cardiac valves and orifices are not altered); a syphilitic interstitial hepatitis, amyloid degeneration of the spleen: the increased size of the kidneys presents sharply defined signs of Bright's disease.

While communicating this case at the annual (.Jamuary, 1878) meeting of the Physio-Medical Society, I arrived at the following conclusions: 1. What I alluded to in my first communication of April, 187T, as possille and probable, became a real fact in this case: namely, the syphilitic pnemmonia was proved to have become complicated by bronchitis, adhesive pleurisy and by chronic catarhal pneumonia, ${ }^{*}$ as well as by acnte. 2. This case proves that it is possible to diagnose not only a pure syphilitic pneumonia, but a complicated one as well.

* Such was the name at that time, uncler the influence of Virchow's teaching, of chronic pulmonary tuberculosis. 
Observations made by me in the domain of diseases of the respiratory organs, and especially of syphilis and pulmonary tuberculosis, during the last fifteen years - since my second communication on syphilis pulmonum - strengthen my assertions which were put forward by me in the above communications, touching upon the semiotics and the diagnosis of simple and complicated syphilitic pneumonia. As what concerns the treatment of this last I must make a correction. In the above communications I spoke against specific treatment in syphilitic pneumonia complicated by tuberculosis, in view of the fact, that during such a treatment this last is liable to rapid advances. For the immense majority of such cases this position holds good even now. But with the aid of modern diagnosis (as the presence of tuberculous bacilli in the sputum enables one at times to recognize early even very slightly diffused affections of the lungs by tuberculosis. and consequently early measures of treatment can be adopted in time), and modern treatment, such as by creosote in large doses, there occur, although rarely, exceptions, as a type of which may serve this case observed by me.

The patient, who became infected with syphilis about ten years ago and who presented at the time seen undoubted manifestations of syphilis of the nerrous system, begins to cough more frequently than formerly (he usually coughs because of a laryngeal catarrh brought on by smoking), and complains of pain in the side of the chest; at the same time he grows somewhat thin and weak, but has no febrile symptoms. Objective investigation shows considerable solidification in the upper lobe of the right lung, and with this there is heard over the solidified area a respiratory murmur of a character approaching bronchial respiration, and at times infrequent, nonabundant râles; the expectoration, which is very scanty, shows the presence of tuberculous bacilli. Thanks to a sufficiently thorough iodide treatment the symptoms of solidification and pain in the right side disappeared, while the patient himself grew stronger; with the aid of creosote following this treatment, the cough diminished to the degree usual with the patient, the râles, as well as the bacilli in the expectoration, disappeared, while the patient himself became so stout and strong, that his health could be called florid. Later on the patient underwent also calomel and iodide treatment, which was ordered because of the other above-mentioned manifestations of syphilis, without any marked impairment of his health and without the slightest symptoms of a recurrence of the pulmonary tuberculosis. 


\title{
SYPHILIS OF THE HEART.
}

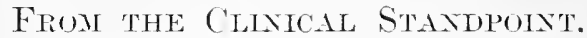

The short report of my address* which was publisher in the Contributions to the Physio-Medical Society (No. 1, January. 1887 ) contains only my conclusions; the remaining part of the report is not written by me and it, as well as the references which appeared in many other editions, present certain inaccuracies due to somewhat hurried observations.

Having some time at my disposal during the summer of 1889, I decided to prepare for publication also my own communication on syphilis of the heart, as I can see from medical literature that the subject is just as interesting now as it was two and a half year's ago: but this communication appears at the present time in a somewhat changed form: first, because the number of cases observed by me has increased, and one of these, which had been under my care in the clinic during the last spring, will be discussed by me later on, as it presents some peculiar characteristics: and secondly, because I will only touch slightly on the general therapy of the diseases of the heart (to this I had to resort in complicated cases of cardiac syphilis; I therefore spoke of it in particular during my first communication), as I intend to take up the subject and discuss it more fully in the edition of my lectures when treating of the subject of diseases of the heart in general.

Turning to the literature on the pathology of the subject. I must say that with the exception of some additional matter of a casuistic nature, the subject under discussion did not advance during these two years and a half.

As regards the pathological anatomy, we have long been informed of the existence of gummatous myocarditis, and we know now of syphilitic affections of both the endocardium and pericardium; but we possess no definite information re-

* Delivered at the annual meeting of the Moscow Physio-Medical Society, January, 1887. 
garding syphilitic affections of the cardiac vessels and nerres. and meanwhile clinical observations, as we shall see later, lead us to the conclusions, that in some cases the syphilitic process attacks the cardiac nerves even to a preëminent degree as compared with the other tissues of the organ. As far as the clinical data are concerned, the modern text-books of special pathology and therapeutics do not pay due attention to syphilis of the heart. To be sure, among the causative agencies of cardiac diseases syphilis is mentioned as one; gummatous myocarditis is treated of by pathological anatomy; but there is no mention made of cardiac syphilis in either symptomatology or diagnosis ; while in discussing the treatment some allude to the possibility of a specific treatment in cardiac diseases, while others do not mention even this. Thus the study of the modern text-books will rather tend to cultivate a neglect toward the subject of cardiac syphilis, failing to bestow on it the attention it merits. An impression is abroad that syphilis is but an accidental discovery during autopsies, and that it is rather a subject for pathological anatomy than for the clinic. Be as it may, the study of these text-books fails to convey the idea of the consiclerable practical importance of this affection. In vain will the student search in these text-books for answers to the questions: What is the clinical aspect of syphilis of the heart? In what forms does it present itself? Has this cardiac affection any practical significance so that it can be recognized in time and subjected to successful treatment? How effective may such treatment prove?

Up to the present time I have had occasion to observe already more than ten cases. I will first describe those which present a smaller variety of attacks and are less complicated by other diseases.

The First Case. - Patient is about forty years old, was infected with syphilis, and does not abuse alcoholic drinks. For the last few years he has been suffering with dyspnoea, palpitation, and at times with symptoms of distmbed circulation (as evinced by wedema of the legs and lungs and by enlargement of the liver), which is but slightly and for a short time relieved by digitalis. The left ventricle is enlarged, but the heart sounds are clear and there are no symptoms of atheromatosis of the arteries: i. e. not only is there no systolic murmur at the sternum, but the arteries are not stiff, and there are no symptoms of a disturbed cerebal circulation. The kidneys are healthy. Treatment by sodium iodide, commencing with ten and increasing up to 100 
grains per day during the period of six weeks, brought about a complete improvement in the patient's condition : all the morbid symptoms clisappeared, and in the three or four years during which the patient ras constantly under my observation, he only had at times attacks of dyspnuea and palpitation of much less frequent oceurrence and of slighter intensity than formerly, which yielded rapidly to digitalis: but he never again had any redema. The left rentricle diminished in size (the apex beat of the heart is on the left mammillary line, whereas formerly it reached beyond it) and the pulse became less frequent, stronger and more regular.

Nutrition and strength improved considerably. Patient repeated twice the treatment with sodium iodide.

The second case is in all respects similar to the foregoing, except that the patient suffered. not from a disturbance of the general circulation, but from frequent attacks of cardiac asthma which culminated in cedema of the lungs and did not yield to either digitalis or to any other remedies. After a course of energetic treatment with sodium iodide, the attacks disappeared entirely for a long time. and even when they reappeared later on they were much less frequent and of slighter intensity, and yielded rapidly to digitalis.

Third Case. - Patient is fifty-five years old; is infected with syphilis: suffered before with acute articular rheumatism, and abused to some extent alcoholic drinks. Has been suffering for more than a year with torturing attacks of angina pectoris, for which he took a great variety of palliative remedies, lately always nitro-glycerine, several times a day, with but a trifling, rapidly passing, relief. Patient has stiff arteries and presents evident symptoms of insufficiency of the aortic valves (sharp diastolic murmur at the sternum, a jerking pulse, enlargement of the left rentricle); but outside of the attacks of angina pectoris he presents nothing morbid: neither dyspnœe, nor palpitation, nor any odena, - all the functions are normal. The sodium iodide treatment relieved the patient considerably and later on inunctions of mercurial ointment brought about a complete cessation of the attacks of angina pectoris; the objective signs of insufficiency of the aortic valves and of general chronic arteritis remained unchanged.

The fourth cuse is similar to the third: there were also attacks of angina pectoris, which yielded to specific treatment.

The above-described cases of syphilis of the heart must be considered, from their aspects, as exceptions, because the other 
cases, i. e. the consiclerable majority of such, present usually another aspect, differing by a great variety of attacks, and by being usually considerably complicated, namely: by alcoholism or gout, by chronic arteritis. gastro-intestinal disturbances, and also by simultaneons syphilitic affections of other organs, most frequently of the nervous system and of the liver. The cardiac symptoms in such cases, as I said above. take on a great variety of forms and are usually all easily discoverable: such as a constant dyspnœea with palpitation and a sensation of oppression in the region of the heart, attacks of cardiac asthma, as well as of angina pectoris, considerable cedema and accumulations in the cavities as a result of disturbed circulation. The heart is ustally enlarged, especially its left ventricle, and the pulse is weak, frequent and arythmical; a systolic murmur is frequently heard at the sternum. Examination of both the present and the past conditions of the patient either elicits syphilitic infection directly, or makes it very probable.

The general character of all these cases is such that the most careful and conscientious non-specific treatment fails to bring about any marked improvement. while a specific treatment consisting of the employment of sodium iodide and of mercurial inunctions results in a lasting improvement: the morbid phenomena disappear. there remain only the objective signs of some enlargement of the heart, and at times a weak systolic murmur over the sternum.

As a type of the above alluded to majority of cases of cardiac syphilis as seen by me. I will now describe more fully the one spoken of before, which was under my observation in the clinic cluring the last spring, and served as a subject for my clinical lectures during the beginning, the middle and the end of the patient's sojourn in the clinic.

Patient, thirty-four years old, entered the clinic March $T$, 1889 , in a very aggravated condition, complaining of extreme weakness, dyspncea. and swelling of the whole body, and especially of the legs.

Mode of life and ancmnesis. - Patient has no constant place of residence, but lives in turn in three different cities, several months in each. His rooms, in a hotel, are not always satisfactory; the watercloset is always cold. Patient drinks some four glasses of tea per day; aroids drinking coffee. as it gives rise to palpitation; has one meal at day, always containing some meat; smokes much, and is addicted to alcoholic drinks : not less than seven wine-glasses of brandy, and seven bottles of beer 
per day; and three times a month even more than this. With the exception of the last month, such drinking has continued for ten years. but during the last month he entirely discontinued the use of alcoholic drinks; his occupation is not of a fatiguing nature, outside of considerable travel - in winter on sleds, in summer mostly on the steamer.

The patient is tall and broadly built; was even when young inclined to corpulence and was subject to attacks of slight dyspncea, but since he began to abuse alcoholic drinks both the corpulence and dyspnoea increased: barring this, the patient does not recollect having liad any diseases until two years ago. It that time he became infected with syphilis: there appeared an ulcer on the penis, exuptions, and falling out of the liair. Patient took pills of mercury during three months, and after a two months' interval. for another month and a half; as a result all the above-named symptoms of syphilis disappeared, but the patient began to feel weak and fatigued by his work, which he formerly bore well. Some eight months ago, after an ordinary day's work, he had at night an attack of angina pectoris without any ascribable cause: there was pain along the sternum and in the region of the lieart transmitted to the back. left shoulder, and the left arm down to the elbow ; the pain was not severe, but lasted for a whole night and passed away in the morning without any treatment. Since then there occurred nocturnal pains in the legs, in the region of the tibial bones. Patient again took pills of mercury during one month, and the pains in the legs disappeared.

The second attack of angina pectoris similar to the first one took place on the night of February 1, 1889; it was not severe, but also continued during the whole night, for about eight hours. Patient at once left off drinking brandy and beer, after which he rapidly lost his appetite and grew thin and weak. Three weeks ago the dyspnoed began to increase. and there appeared cedema of the legs. Patient took no treatment. and the described attacks, having reached the above-mentioned severe state, compelled him to enter our clinic.

Status Murch" " 1889. - Appetite not as good as it was before; eating is accompanied by pyrosis, eructations, nausea, and occasional vomiting. Since the patient gave up drinking beer and brandy he suffers from constipation; has every other day some hard evacuations. Until that time he used to have three to four soft, gruel-like, painless stools. The urine is secreted freely, to the amount of 1,000 c.c. in twenty-four hours, of a red 
color, containing neither albumen nor sugar. The liver is enlarged and painful. The spleen presents nothing abnormal. There are symptoms of fluid accumulations in the abdominal cavity, and, as mentioned above, considerable cedema of the arms, legs, and of the entire subcutaneous tissue. Patient complains of palpitation. On account of the present corpulence, of the cedlema of the subcutaneous tissue, and of the weak cardiac activity, the results obtained by the objective examination of this last are not pronounced: there is a weak cardiac impulse felt at the left mammillary line, but percussion elicits a dull sound over the left half of the sternum, and auscultation a weak systolic nurmur. The pulse is 120 , very weak and arythmical. The dyspncea is very pronounced. 46 to 52 per minute: patient is unable to lie down. There is no cough. The condition of the lungs. as shown by percussion. is normal (only the diaphragm is somewhat raised). and auscultation shows abundant moist râles under both scapulæ. The temperature is $38^{\circ} R$. $\left(98.4^{\circ} F\right.$.). He hardly sleeps at all on account of the dyspncea. His mental state is extremely depressed, and at times very irritable. There is considerable weakening of the memory. No dizziness, headache, nor any pains, except some in the region of the calves when they are compressed. Extreme weakness of all the morements, and especially of those of the lower extremities.

Having put the patient in a condition of rest, having limited the quantity of drink to what was absolutely necessary. and after regulating the diet (at the beginning all his food and drink consisted of a glass of tea without sugar in the morning, a half-glass of bouillon and a small cutlet for dimner, and six glasses of milk during the whole day; later on, with the improvement of the patient. the diet was accordingly changed), we prescribed eight tablespoonfuls of strong Crimea wine per day, the mixture of the aether-valerianate and Hoffman's drops $\bar{a} \bar{a}$ at the beginning up to 200 drops per day, digitalis in an infusion, altogether twenty-four grains cluring the first two days. a large Spanish fly over the region of the heart, and a clyster in case of necessity. By the third day there occurred some slight improvement: the pulse was 98, the dyspnoa somewhat less severe and the amount of urine was 2500 c.c. ; but on the next day the dyspncea became again aggravated, and the urine fell to only 1500 c.c. As the clyster evacuated the bowels insufticiently and the abdomen was very much distended, we gave him calomel, one grain every hour: after the fifth dose the 
patient had abundant loose dark-green evacuations, the respiration became easier. but the amount of urine did not increase. We then resorted again to the infusion of digitalis. twenty-four grains during two days; the stimulants. namely the wine and the above-named drops. were given to the patient during all the time that he remained in the clinic, - later on in smaller doses in accord with the improvement of the pulse: only during the last days before patient left the clinie did we leare off these drops.

The second dose of digitalis given together with the stimulants in the first large dose, failed to markedly improve either the pulse or the respiration, or to increase the amount of urine over 2000, and did not produce any marked decrease of the signs of cedema. Putting then aside the digitalis and continuing the stimulants, sodium iodide ( $\mathrm{jij}$ to $\overline{\mathrm{vj}}$ ) was ordered in Ems water (in view of the continuing dyspeptie symptoms). a tablespoonful in a quarter of a glass of the water (altogether between one and one and a half glasses of Ems per day). Mareh 15th, twenty grains of sodium iodide; 16th. thirty grains ; 17th and 18th, forty grains each: the urine rose to 3000 c. c. in quantity, the signs of cedema luegan to diminish, the pulse became less frequent ( $78-72)$. breathing grew easier, sleep better, the temperature fell to the nomal. From March 19 th to 23 d he had fifty grains of sodium iodide per day. from the $23 \mathrm{~d}$ to the 28th sixty grains; with the former quantity of drink the urine amounted to $\$ 000$ c.c.; the redema passed away, the breathing became free (20-18). the sleep quiet; the liver has diminished considerably in size and is not sensitive, the pulse beeame regular and strong, but is still frequent (96$100)$. He was again given infusion of digitalis (twenty-four grains of digitalis) during two days, but without any appreciable result. We then again resorted to sodium iodide.

We must now say a few words concerning the condition of the patient's nervous system. Notwithstanding the considerable decrease in the extent of the cedema and the increase of the patient's strength, the weakness in the movements of the legs, which was ascribed to general debility and to the great weight of the legs on aecount of the redema, remained in the former condition: the patient was unable to either walk, or to even stand erect: we eould not bring out any tendon reflexes in the legs (the sensibility was preserved). Massage failed to improve the condition of the lower extremities. Such a paretic state of the legs and a considerable weakening of 
the memory, in the presence of an undoubted syphilitic infection of the organism, have naturally led us to suspect a syphilitic infection also of the nervous system; we therefore continued the specific treatment the more persistently. constantly increasing the dosage of the sodium iodide, from 60 grains per day to 90,100 and 120. By April 19th the patient's condition, outside of that of the legs, was entirely satisfactory: the appetite was good, the digestion regular, the amount of urine was always 1800 c. c. in twenty-four hours. the dimensions of the liver almost normal (the signs of ascites. as mentioned above, disappeared even before). breathing free. no palpitation. the pulse is to 80, regular and strong. the dimensions of the heart decreased (the percussion sounds on the inferior part of the chest are clear): there remained the weak'systolic murnur at the sternum; the sleep is good. the mental state hearty, the memory better.nutrition and strength are normal; but the weakness of the lower extremities has not passed away. although it has diminished somewhat. April 19th. he was given eighty grains of sodium iodide, and since the onth. sixty grains a day and at the same time inunctions with merculial ointment. $5 \mathrm{j}$ per day. in conjunction of course with a mouth wash of a potassium chlorate solution. With the air of inunctions the condition of the lower extremities began to improve rapidly. May the first, the patient was compelled by his official duties to leave the clinic; by this time he was able to walk almost as well as any healthy man, and the tendon reflexes retumed; but he was advised to continue the specific treatment for another three weeks so as to obtain a lasting benefit from this treatment which he bore so well.

In this case, ontside of syphilis of the heart and of the nervous system, there was probably also a syphilitic affection of the liver. It is true, that enlargement as well as tenderness of this last might also have been due to blood stagnation, in the presence of a general disturbance of the circulation. and also to alcoholism; but the considerable ascites indicates not only a condition of stagnation. but a more serious affection of the liver, and that this last was of syphilitic. rather than of an alcoholic nature. is seen from the rapid improvement in the condition of the liver under the influence of the iodicle treatment.

As far as the diagnostication of the cardiac condition in the above-described cases is concerned, we are compelled to infer in the first case, if not a pure, then a preëminent affection of the cardiac muscle, namely syphilitic myocarditis; in the second, third. 
fourth, a preëminent syphilitic affection of the cardiac nerves: and in the last as well as in those of which it serves as a type. namely, in the majority of the cases of cardiac syphilis, as observed by me, a simultaneous syphilitic affection of both the muscle and the nerves of this organ. I did not come across a case of cardiac syphilis, which would present evident objective symptoms of an affection of the valves and of the cardiac orifices and in which, with the improvement of the heart activity and circulation. these symptoms would disappear.

To judge from the foregoing, the chief basis for the diagnosis of cardiae syphilis, as well as of syphilis of the other internal viscera, is found in the anamnestic data and in the simultaneously occurring symptoms of syphilis in other parts of the organism. Is regards the anamnesis, we are far from relying upon what the patient asserts or denies concerning syphilitic infection. Both the assertion and the denial are to be considered critically ; while denying, the patient may hide the truth purposely, or he may do so bona fide, withont any suspicion on his part of ever having been infected by syphilis : on the other hand the patient may mistake a syphilitic infection, althongh this happens but seldom. To verify these assertions and to obtain reliable data for the diagnosis we must resort to a thorough investigation of the past history, of the phenomena which the patient considered as syphilitic, of whether there was any falling out of hair of the head, and especially of the eyelashes and beard, of characteristic eruptions, nocturnal pains, etc., and also as to whether the patient subjected himself to iodide and mercury treatment and as to the influence of such treatment on his condition. As indications for the diagnosis may also serve the absence of other causes of the cardiac affection (as in our first case), and the slight effectiveness of non-specific treatment.

The specific treatment instituted for the sake of verifying the diagnosis of cardiac syphilis, is the same as applied in other cases of late syphilitic affections; if the patient has formerly subjected himself to a sufficient mercurial treatment, then the iodide preparations usually exert a favorable effect. and the iodide treatment alone may be sufficient to improve the patient's condition; otherwise, it becomes necessary to resort to mercury, alone or in combination with the iodide preparations, which last are in such cases administered in smaller doses than when the iodides alone are used. As what concerns the non-specific treatment of the cardiac therapy in general, I intend to return to it at some other time. However the last fully described case 
of cardiac syphilis gives a sulficiently clear idea as to what remedies of general cardiac therapy are to be employed in syphilitic affection of the heart. The same case makes it clear, that if the indications of the presence of syphilis be sufficient, then there is no need to hesitate with the ordering of a specific treatment.

I will take the opportunity to touch now on two remedial agencies in the domain of cardiac therapy, the so-called milk treatment of diseases of the heart. and the application of Spanish flies to the region of the heart.

As far as the first is concerned, its name contains an inaccuracy which may be the cause of mistaken application of the milk treatment for such cases of cardiac disease. in which it is both unnecessary and unsuitalle. The milk treatment exerts its effect not on the diseases of the heart (except in one case, of which presently). but on their complications, such as ascites, abdominal disorders (dyspepsia. constipation, enlarged abdomen due to gastro-intestinal meteorism. and abdominal obesity) and. less frequently, general obesity. It is true that in cases of ascites caused by cardiac disease, milk serves as the best diuretic, when it becomes necessary to act through the kidneys (and when we desire to act through the heart. we resort to digitalis and to similar drugs): it is also true that the complications of cardiac diseases in the form of abdominal disturbances are very frequent and serve as the chief cause for the presence of obstinate dyspnoa, cardiac asthma, angina pectoris, and the circulatory disturbances, and that the removal of these complications by the aid of a strict milk treatment (at the beginning milk only, and later on milk with meat, fish, and eggs, but no carbohydrates) is attended with splendid results; however, the milk treatment acts solely on the above-named complications, and not on the heart itself. and to prescribe this treatment for cases of cardiac diseases unattended with such complications - which is often done, thanks to the erroneous idea conveyed by the name (" of the diseases of the heart" ) - is a procedure devoid of any purpose and tending to weaken the patient. There is only one case in which the milk treatment may exert a direct action on the heart, namely in the condition of fatty heart resulting from general obesity: it is admissible to assume that with the general emaciation which usually follows the milk treatment, there also occurs a diminution in the fat of the heart. If the syphilitic affection of the heart becomes complicated by ascites. abdominal disturbances and obesity, then in prescribing the 
treatment we should take into aecount such a condition of affairs, and so we did in the last of the described cases of cardiac syphilis.

I will now take up the use of Spanish flies. Some seven years ago (February, 188.2) I called the attention in a communication before the Physio-Medical Society to the value of Spanish flies in peripheral neurites. Since that time I had frequent occasions to observe a considerable and lasting improvement from the application of flies to the region of the heart, to either the right or the left side of it, as well as above or below the left nipple (in women, to the left mammary gland) in angina pectoris, usually in cases of such a nature : in patients no longer young, mostly gouty, sometimes with symptoms of chronic arteritis. The attacks of angina pectoris were usually caused by colds, appearing when the chest was subjected to a cold blast, or after the inhalation of cold air.

The examination of the chest in the region of the heart in such cases usually elicits, skin hyperaesthesia being absent, pain on pressing the ribs (periostites of the ribs) and the intercostal spaces (intercostal neurites). Quite frequently the other parts of the chest, especially its left side, present the same phenomena; but the pain is usually most marked on pressing those intercostal spaces which correspond to the locality of the heart, which circumstance leads us to assume the existence also of a neuritis of the cardiac plexus in such cases. The attacks themselves of angina pectoris are quite characteristic, the pain being transmitted to the left arm down to the very fingers. Outside of Spanish flies and other necessary treatment, I usually advise such patients to wear a woolen or silk chest protector so as to avoid any possibility of catching cold. But in cases of attacks of angina pectoris in syphilitic cardiac affection, the application of Spanish flies to the affected spot produces but an insignificant and rapidly passing relief, and should not therefore be recommended.

The observations brought forth in this communication give one an idea of the clinical picture of cardiac syphilis, and stand witness to the fact that this last may yet be recognized early enough to be treated successfully and lastingly to no lesser degree than is the case with syphilis of other internal organs. 


\section{Additional Notes.}

First. As is well known, the iodides are recommended and used also in simple, non-syphilitic sclerosis and atheromatosis of the arteries and the myocarditis following thereon. I have seen a great many patients suffering with the above-named diseases, who had been taking for a long time the iodides of potassium and sodium; but never, not in one single instance, even when large doses, as thirty to forty grains per day, of these remedies were used, have I observed such a positive, incontrovertible success, as I saw in the treatment by the iodides and by mercury of the same affections when of syphilitic origin. I do not consider it improbable, that in the cases in which certain observers noted such a success, syphilis was an element in the development of these affections.

Second. During the year I had occasion to observe a few more cases of cardiac syphilis; but as they present nothing different from the above conclusions, I do not consider them here. I think however, that the following case of aneurism of the aorta (and probably of the innominate artery) as resulting from syphilitic aortitis, is of sufficient interest to be brought forward.

February 6, 1890, a patient, thirty-seven years old, entered our clinic, complaining of pains in the right half of the head and in the right shoulder, and also of palpitation and dyspnœa.

He lives in a good locality and in a fairly good house. $\mathrm{He}$ does not bathe in the river; takes a hot bath weekly and perspires freely. Does not smoke, drinks always brandy and sometimes to excess, also a great deal of hot and strong tea; has good board. Patient has a great many occupations of a troublesome nature; has to talk loudly, to travel in jolting carriages and sleighs over poor roads, and conducts a considerable correspondence. He was infected with syphilis, later got married; out of six children three were still-born, the fourth died in early infancy, the fifth is sickly, the sixth is as yet well.

Anamnesis. - When twenty-four years old, patient was infected with syphilis, was treated for two months by mercury, and to some extent by the iodides, and since then, considering himself recovered, he resorted to no antisyphilitic treatment. During the last four years patient had at times pains in the legs day, and night: while one year ago there appeared pains in the right half of the head and in the right shoulder, which 
gradually increased. Lately these pains have prevented good sleep and patient grew emaciated. Five months ago there appeared palpitation of the heart and a swelling on the lower portion of the right side of the neck, which kept continually increasing in size, so that lately, on account of the swelling, he has been unable to button his shirt-collar.

Status Feb. 6, 1890. - Patient is of strong constitution and good nutrition. The appetite is fair, digestion regular, the liver, spleen, kidneys, and the urinary bladder are in a normal condition. There is palpitation of the heart, some dyspnøea, and a slight dry cough. Objective examination of the chest shows nothing abnormal in the respiratory passages, while the organs of circulation present the following: a strong, visible impulse of the cardiac apex two fingers' width outside the left mammillary line, between the sixth and seventh ribs; a pulsating tumor in the region of the right sterno-clavicular articulation; on percussion a dull sound over the tumor and over the upper third of the sternum; percussion over the other two-thirds of the same elicits normal clearness; on auscultation, a systolic murmur along the whole line from the apex of the heart up to the above-mentioned tumor, especially so over the sternum and to its right; there is also heard over the sternum a slight diastolic murmur pointing to an affection also of the aortic valves, namely, insufficiency; over the carotid arteries a systolic murmur, but no heart tone is heard. The pulse is between 80 and 90, of regular rhythm, somewhat weak, weaker on the left than on the right hand. No fever. The abovedescribed pains on the right side of the head and right shoulder prevent sleeping; he is unable to rest on the right side; the right arm is so weak that patient is mable to write. These pains and weakness depend evidently on pressure of the tumor on the right brachial plexus.

Outside of regulating the regimen and diet, he was given the following : some wine, tra. valer. aether. and liqu. anodyn. Hoffm. $\bar{a} \bar{a}$, when the prlse is weak, and constantly increasing doses of sodium iodide, beginning with ten grains a day up to eighty (during the last week before he left the clinic). As we intended to administer large doses of sodium iodide, and as this iodide, just as that of potassium, is better borne by the stomach and intestines in alkaline solutions, we gave it to him dissolved in Vichy; but as this caused some looseness of the bowels, we replaced after two days the Vichy by Ems water. and with this the iodide was very well borne. Both the Vichy (Célestins) and the Ems (Kessel- 
brunnen) were used as natural waters, at the temperature of warm milk, to the quantity of half a glassful during the day. Simultaneously with the administration of the sodium iodide there were made twelve mercurial inunctions, at first in onehalf drachm doses, and later in drachm doses. In the intervals between the inunctions the patient was sponged with a mixture of water and brandy at the temperature of warm milk and somewhat cooler, which diminished the sweating and strengthened him.

March 12, 1890, patient left the clinic in the following condition: all the pains disappeared, he can lie on the right side; the sleep is good; the right arm is completely serviceable, patient can write freely; the tumor in the region of the right sterno-clavicular articulation has diminished so much that he can freely button his shirt; the pulsation of the swelling is so insignificant that it can be detected only by touch, but not by the eye, as was the case formerly; the pulse of the right arm has become fuller; the symptoms obtained by percussion and auscultation have not changed; the palpitation does not trouble the patient any more, and he declares that in a general way he feels well and cannot complain of anything.

The patient was advised, outside of preserving a regular mode of living and caution required by the condition of his chest, to continue the sodium iodide for a few more days (up to six weeks), and to repeat the treatment in summer. Up to recently (November, 1890), as we have heard, the condition of the patient continues as satisfactory as when he left the clinic.

My last observations (1891) on cardiac syphilis, in the clinic as well as in private practice, completely substantiate all the above assumptions. 


\section{TREATMENT OF FEVER.}

\section{(A Therapeutic Note.)}

Shall we treat fever, and if yes, how?

At the present time there are those who assert that the fever accompanying the acute infectious diseases must not be treated. because it forms, as it were, a protecting agency to the organism against the pathogenic factor. Without denying not only the possibility, but even the probability of such a process of selfprotection, we nevertheless are unable to draw conclnsions therefrom to the effect that the fever must be left untreated. Is not, for instance, the cough in certain cases (as in bronchial catarrh with abundant secretion, in œelema of the lung's and so on) a self-protecting agency to the organism, withont which the patient would suffocate, - a beneficial symptom which in such cases must not be weakened. but on the contrary strengthened, and are we not compelled in other caser, as even in this same bronchial catarrh. to relieve the too severe cough, if it tends to impair the sleep, and to act injurionsly or even dangerously on the cerebral circulation, on the cardiac activity, on the conclition of the abdominal organs, etc.? The same may be said of diarrhea, and of other symptoms which are beneficial to a certain degree, and become injurious as soon as this degree is orerstepped; the same applies to fever in connection with infectious diseases, and consequently the physician must consider it in the same light in which he considers the other symptoms of a similar nature, namely, not to treat it, if he has reason enough to consider its presence beneficial and as long as it does not overstep certain bounds. and to treat it in the contrary case.

How is fever to be treated? In cases where the radical treatment. not only of the febrile symptoms, but of the disease itself (as, for instance, in malaria. in acute articular rheumatism) is possible, the modus operandi for the physician needs no explanation.

In the acute infections diseases for which we possess no rad460 
ical treatment. as, for instance, in typhus, typhoid and recurrent fevers, influenza, croupous pneumonia, erysipelas, etc., I act as follows: If the fever is moderate, and if by analyzing the patient's condition I am unable to detect any undoubted deleterious influence on the patient therefrom, then I wait; otherwise I resort to treatment. What is the degree of temperature that constitutes a moderate fever? IVe must, of course, first of all individualize our case: aged people are less able to bear a temperature of $39^{\circ} R$. (102. $2^{\circ} F$.) than children will bear one of

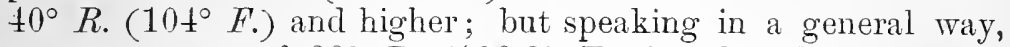
a temperaturc of $39^{\circ} R$. $\left(102.2^{\circ} K\right.$.) in adult, but not aged persons, need but seldom trouble the physician, whereas one of $40^{\circ} R$. $\left(104^{\circ} \mathrm{F}\right.$.) and above excites apprehensions. The greatest care and most thorough inquiry about the condition of the patient is required in case of a temperature ranging between

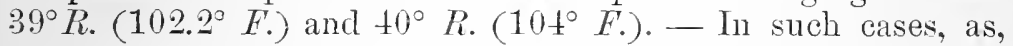
for instance, in typhoid fever, if there be present no other troublesome symptoms, I do not treat the fever. If with such a temperature there be also present some dangerous symptom, then, before taking up the treatment of the fever, I seek for other possible causes of this symptom outside of the high temperature: if I observe. for instance, depression of the cerebral activity, and simultaneously with this symptoms indicating an influx of blood to the brain (as a flushed face), as frequently occurs in typhoid fever (and often passes away with a spontaneous nose-bleed), then I order not antipyretics, but two leeches to the nasal septum. and an ice-bag on the head, which is often sufficient to bring relief; if I note mental depression with a pale face, then I wash the head in cold water, administer musk, and sometimes - usually during the further course of the disease, when there appear's a threatening nedema of the brain, - I apply a Spanish fly to the nape of the neck; for a weak pulse, especially in persons habituated to the use of alcoholic drinks, I give wine, cognac and stimulints; in other cases, to remove the troublesome symptoms, we are compelled to administer laxatives (in cases of constipation and intestinal meteorism), and so on. But if, after analyzing the patient's condition, I find no reason to expect any benefit from the use of the above-enumerated and similar means, or, on applying the same, I do not obtain any sufficient relief and am compelled to refer the dangerous symptoms to the rise in temperature, then I resort to antipyretic treatment - calomel, cool bathing, antifebrine (less frequently antipyrine), phenacetine, sodium salycilate, and quinine. 
The indications for the use of calomel in certain febrile affections, as typhoid fever, croupous pneumonia. erysipelas, and acute nephritis have been spoken of before (vide p. +27 ).

Cool baths - temperature $24^{\circ}$ to $22^{\circ} R$., lowering it while the bath is being given to $20^{\circ}-18^{\circ}$. The duration of the bath must be determined by the peculiarities of the given case; the first bath is to be taken in the presence of a physician, the following ones under the supervision of a skilled assistant. The patient is lowered into the bath and raised therefrom on a sheet. The chief contraindications for this procedure are: diarrhoa, intestinal hæmorrhages, poor cardiac activity, embarrassed respiration, as from a severe bronchitis with hypostasis or from a superadded pneumonia, and great excitement of the nervous system. The chief indication for its use, besides the lowering of the temperature, is a depressed state of the nervous system. Cool spongings of the whole body with vinegar and water are necessary for cleanliness' sake. and are usually pleasant to the patient, for they cause a slightly stimulating effect on the nervous system, but their influence on lowering the temperature is certainly very insignificant.

Let me add that at times, most frequently in typhoid fever, we must resort to another hydrotherapentic measure, namely, in the following cases, usually during the further course of the typhoid, when the patient has become considerably exhausted: burning heat in the trunk, cold extremities, a very weak pulse, great mental excitement, as evidenced by insomnia and constant delirium. In such cases I administer bromides and place the body, as it were, in a warm compress: several flannel comforters are spread over the bed, on top of which is put a sheet soaked in water cooler than lukewarm; the patient, completely naked, is placed on the sheet, which is wrapped around him, and over this the comforters. Such treatment is in the majority of cases successful: the patient is quieted down, the delirium ceases and he falls asleep. the burning heat in the back and the cold in the extremities pass away, and the pulse improves.

Antipyrine, antifebrine and phenacetine I use ordinarily in the following manner: I give a five-grain dose (less frequently three grains); in the majority of cases I obtain a lowering of the temperature down to $39^{\circ} R$. and lower, and as long as this lasts I do not administer any more. As soon as the temperature has risen again to over $39^{\circ}$, I repeat the same dose, and so on; consequently I do not in the least attempt to bring the temperature down to its normal condition, but am content to 
lower it sufficiently to exclude any possible danger (the same is to be said of the use of cool baths).*

I use most frequently phenacetine or antifebrine, and if they lose their effect or the patient becomes habituated to them, then I resort to antipyrine or sodium salycilate (in ten-grain doses).

Outside of malaria, I give quinine in those cases in which the febrile condition is of a strongly exhausting or directly intermittent nature (in the febrile condition of the continual fever, quinine acts only when given in very large doses, which are borne rather difficultly, and even then it acts slightly and for a short time), most frequently in febrile catarrhs of the respiratory passages, in prolonged pneumonia, in influenza and typhoid fever, if the condition of the intestines permits it, when from being constant the fever becomes a severely exhausting or an intermittent one; for constant fever I give antifebrine, phenacetine and antipyrine. I administer it usually during the period of exhaustion or cluring the intermittence, and therefore in the majority of cases in the morning, not on an empty stomach, but after the tea or coffee, and not in the evening, as is usually recommended. When given during the above-mentioned periods the quinine acts better, probably because it is more readily absorbed. I do not increase the dose of quinine until the appearance of cinchonism, as we do in malaria, but I give usually fivegrain doses once or twice, rarely three times a day.

For pulmonary tuberculosis the chief antipyretic and, generally speaking, the chief remedial agent is certainly creosote, guaiacol, or guaiacol carbonate; but before we are able to obtain a good effect from them, we are at times compelled to give quinine in the exhausting and intermittent fever, and for the constant fever, antifebrine, antipyrine or phenacetine, while sodium salicylate is but little effective in small doses, and is liable to im-

* The lowering of the temperature down to its normal condition during the whole comrse of typhoirl fever by the aid of cold baths and the above-named antipyreties was attempted, but did not become a generally adopted practical procedure. and is recommender but by very few, because, first, it does not benefit the patient, as the duration of the disease is not shortened; and secondly, it may become injurious, for continuous cold baths, by determining an increased production of heat, increase the exhaustion of the organism; while the use of the above-named antipyretics, which, to keep the temperature constantly down to its normal eondition, must be given in large rloses, may tend to affect the lieart injuriously or even to a dangerous extent: not to speak of the possible harm from the cessation of the fever, which may act as a self-protecting agency to the organism. Thus some observers assert that in eases of typhoid fever, which were persistently treated by the application of cold water, recurrences were more frequent. 
pair digestion in large ones, and I therefore prefer the above antipyretics.

In septicremia and pyæmia, not having any better agents, I give creosote, or guaiacol and guaiacol-carbonate, as, for instance, in the treatment of empyema (outside of operative interference). 


\section{N D E X.}

AAchex, a German watering-place . . . . . . . 341

Abas-Touman, a Caucasian watering-place . . . . . 340

Abdomen, inquiry about, in examining a patient. . . . 13

" method of palpating . . . . . . . 4t5

Acid, boric, in choleraic diarrhœa . . . . . . . . . . 379

" earbolic, in chronic articular affections . . . . . . 222

“. carbonic, action of, in the stomach . . . . . 90

..

“ hydrochloric, employment of, in cliseases of the stomach . 83, 84

" . " "pseudo" preventive of cholera . . 371, 372

" .. $\quad$ secretion of, in ehronic gastric catarrh . . . 5?

" $\quad$. $\quad$. $\quad$ in gastric ulcer . . . . . 125

Acids, organic, effects of, in hydrotherapy . . . . . . . $\quad 339$

Accumulations in the peritoneal cavity, treatment or . . . . 199

Agencies, curative, in mineral waters . . . . . . . . 90

Air, compressed, in pleurisy . . . . . . . . 272

Air-baths in parenchymatous nephritis . . . . . $\quad 210,245,246$

" effects of, vide Climatotherapy.

Albuminates, peptonized, in gastric diseases . . . . . . 8.5

Albuminuria in the chronic nephrites . . . . . . 242,243

Alcohol a predisposing factor in hæmorrhoids . . . . . . . 398

" in the algid stage of cholera . . . . . . . . 383

. in debility . . . . . . . . . . . 43

" in the treatment of choleraic diarrhœa . . . . . . 381

Alcoholic drinks, results of abrupt abstinence from . . . . 203

Algeria as a health resort . . . . . . . . . . 303

Algid stage of cholera, causation of . . . . . . . . . 365, 366

" . 4 . . . . . . . . . 382, 383

Aloe as a laxative . . . . . . . . . . . . . . . . . . . . . . . .

Anæmia, mineral waters in. . . . . . . . . . . . . . . 116

Anamnesis . . . . . . . . . . . . . . 8

" definition of . . . . . . . . . . . . . . . 17

.. health of parents and relatives . . . . . 17

" as regards syphilitic infection. . . . . . . 454

Andes, the, treatment of tuberculosis in . . . . . . . . 304

Aneurism of the aorta, case of . . . . . . . . . . 457

Angina pectoris, Spanish flies in . . . . . . . . . . $\quad . \quad 456$

". . with cardiac syphilis . . . . . . 448,449 
Angioneurotic nature of hæmorrhoids

Antifebrine in hectic fever .

" in reaction period of cholera

. $\quad$ in treatment of fever

462

212

292

in hectic fever.

268

، in pleurisy

462

in treatment of fever

398,399

Anus, bloodletting from circumference of, reasons for

inquiry about, in examination of patient
lorta, abdominal, sensitiveness of, on palpating the abdomen

" aneurism of, case of

Apoplexy, venesection in

391,392

Arsenic in chlorosis

$$
\text { " " " } \text { " contraindications to use of }
$$

" " neurasthenia

261,262

Arthritis urica, vide Gout.

Articular affections, chronic, local treatment of

Atelectasis, pulmonary, râles in .

Atrophic gastric catarrh (with cancer of the duodenum), 4th case .

BACTERIUM of cholera

Balneotherapy, elements of

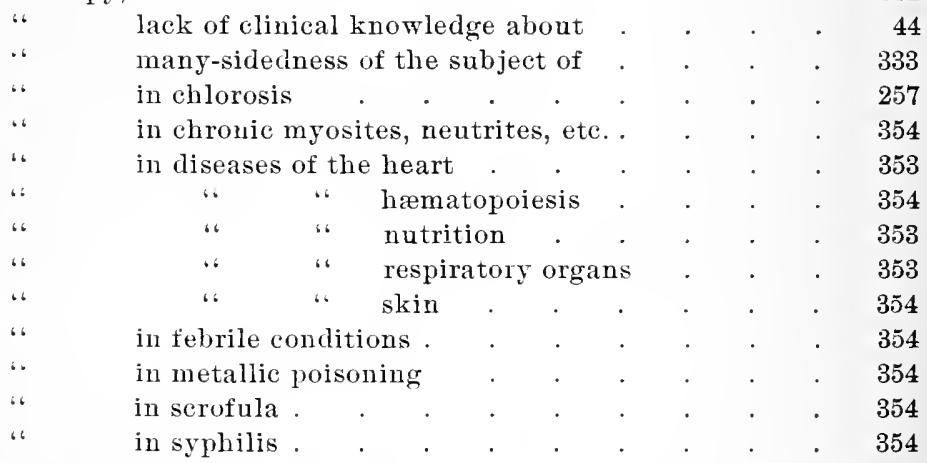

( ride also Hyclrotherapy, Mineral waters, Watering-places, etc.)

Baltic Sea, a trip to, in neurasthenia. . . . . . . . 101

Bathing. river, in neurasthenia . . . . . . . . 102

" sea, diseases in which it is indicated. . . . . 346,347

" " $"$ effect of mechanical influences in . . . . 339

" " . effective agencies in . . . . . . . 346

Baths, action of, on the central nervous system . . . . . 335

" " . . temperature of the body . . . 335,336 
Baths, cool, in the treatment of fever

"، determination of necessary tem

mperature of

" effects of cold and warm .

334,335

cold and hot, influence of, on circulation, etc.

336,337

" uselessness of, in pneumonia . . . . . . 320

hot, in the treatment of choleraic diarrhœa . . . . . 381

"air.... 347

347

Russian . . . . . . . . . . 349,350

warm, drawbacks in use of . . . . . . . 52

" in biliary calculi. . . . . . . . 177

"6 for promoting sleep

51,52

228 for pains

Biarritz, a trip to, in neurasthenia . . . . . . . . 101

Binz on calomel

407,409

Biliary calculi, vide Calculi.

Bilirubin, formation of (foot-note)

Bismuth in choleraic diarrhœa.

" in ulcer of the stomach (foot-note) . . . . . . 131

" phenolate, vide Tribromphenolbismuth.

Bitters, use of, in chronic catarrh of the stomach . . . . . $\quad 78$

Black Sea, a trip to, in neurasthenia. . . . . . 101

Bladder, urinary, inquiry about, in examination of patient . $\quad 12$

Blisters, use of, in pleurisy.

" " in pericarditis . . . . . . . . 390

Blood, quantity of, extracted in derivative bloodletting . . . $\quad 392$

Bloodletting, former practice of . $\quad . \quad$. $\quad . \quad$. $\quad . \quad 387,389,390$

Moscow practice of . . . . . . . . 390

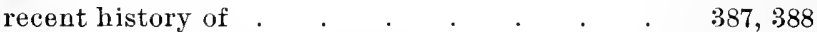

views of different authorities on . . . . . . 388

depletive (vide also Venesection) . . . . . 391

derivative . . . . . . . . . . 396

" case where employed . . . . 399,400

" indications for: in large hæmorrhoidal nodes, 404

" " $"$ in region of abdomen . 404

"6 " " " 4 chest . . 403

" " " " 4 head . 401, 402

" " " " spine . 402, 403

" methods of use : care in performing . $\quad 404,405$

" " $\quad$ number of leeches . . 405

" " $"$ position of patient . . 405

. . . $\quad$. . 405

". $\quad$ where applied . . . 405

. $\quad$ reasons for its employment . . $\quad 398,399$

". $\quad$ repetition of $. \quad . \quad . \quad . \quad . \quad . \quad 406$

“ to the nasal septum . . . . . . . 402

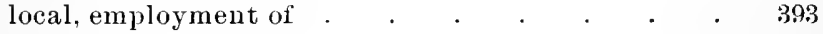


Bloodletting, local, with depletion in view

Brain, diseases of, balneotherapy in .

Breathing, rough, as a symptom

Brehmer's experiments with elimatotherapy

Bright's disease, vide Nephritis.

Bromicle of sodium in reaction stage of cholera . . . . . 383

Bromides in nemasthenia . . . . . . . . . . . . 148

Bronchopneumonia, chronic tuberculous . . . . . . . $\quad$. 277,282

Broussai's influence on the practice of bloodletting. . . . . 387

Brown-Sequard's theories on glandular secretion . . . . 252

CAFFerne as a diuretic . . . . . . . . . . . . 246

.. natrobenzoicum in the algid stage of eholera. . . $\quad 383$

Cairo as a health resort . . . . . . . . 303

ralenli, biliary, Ems water in . . . . . . . . . . 79, 166, 430

. $\quad$. formation of . . . . . . . . . . $169,170,176$

.. $\quad . \quad$ peeuliar symptom of . . . . . . . 170,171

.. $\quad . \quad$ relation to neuralgia of the hepatic plexus . $171,173,174$

.. . diagnosis of . . . . . . . . 431, 432

. $\quad . \quad$ treatment of, when discontinued . . . . 180

" " and elolangitis catarrhalis . . . . . 194

Calomel, action of, physiological . . . . . . . . . . 4 408, 409

" as a diuretic. . . . . . . . . . . . . 423

. " laxative. . . . . . . . . . 153

.. compared with castor oil . . . . . . . . . 417

.. Nothnagel (and Rossbach) on the use of, in Germany . 407

. in biliary calenli. . . . . . . . . 430

. in catarrhal jaundice. . . . . . . . . 430

" in acute nephritis . . . . . . . . 439

" in eirrhosis of the liver . . . . . . . . . . . . . . . 407, 417-427

" in cholera . . . . . . . . . . . . . . 376,377

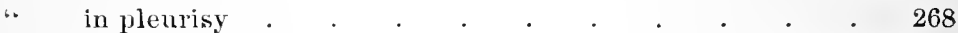

.. in pneumonia . . . . . . . . . . $317,320,321,428,429$

. in tacial erysipelas . . . . . . . . . . . 427, 428

" in typhoid fever. . . . . . . . . . . 4 428

. use of, in Germany, France and England . . . . . 407

. mode of employing . . . . . . . $415,416,417$

. indication for use of . . . . . . . . . 416

" contraindication to use of . . . . . . . . . . 430

Camphor in the algid stage of cholera . . . . . . . . . 383

Cannes, a French health resort . . . . . . . . 303

Cancer, gastric, differential diagnosis of . . . . . . . . . . . . . . . . .

" duodenal (4th case) . . . . . . . . . 160

. of paucreas (5th case) . . . . . . . . . . . 162

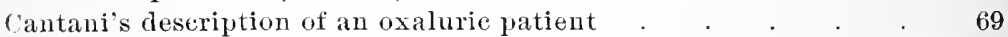

.. treatment of choleraic diarrhca . . . . . $\quad 380,382$ 
Catarrh, gastric, atrophic

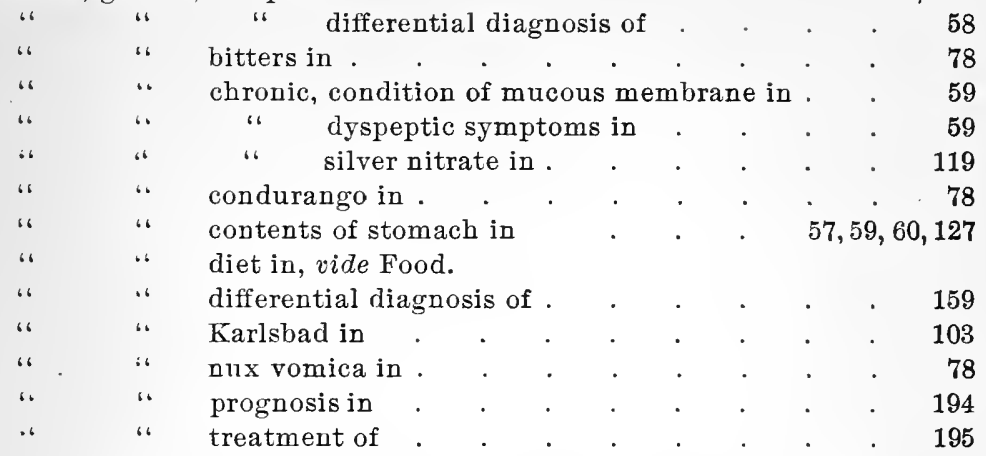

simple, of the large intestine, differentiated from tuberculous ulcerations

Catarrhal conditions, contrasted with predisposition to same, treatment of

Célestins, vide Vichy.

355

Cerebral congestion, derivative bloodletting in . . . . 401

Cliapman's treatment of cholera (foot-note) . . . . . 383

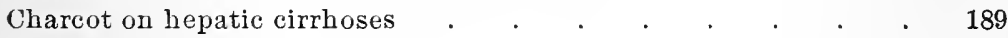

Chest, inquiry about, in examining a patient . . . . . . . 13, 14

Chloranæmia, treatment of . . . . . . . . . 261

Chlorosis (17th case). . . . . . . . . . 248

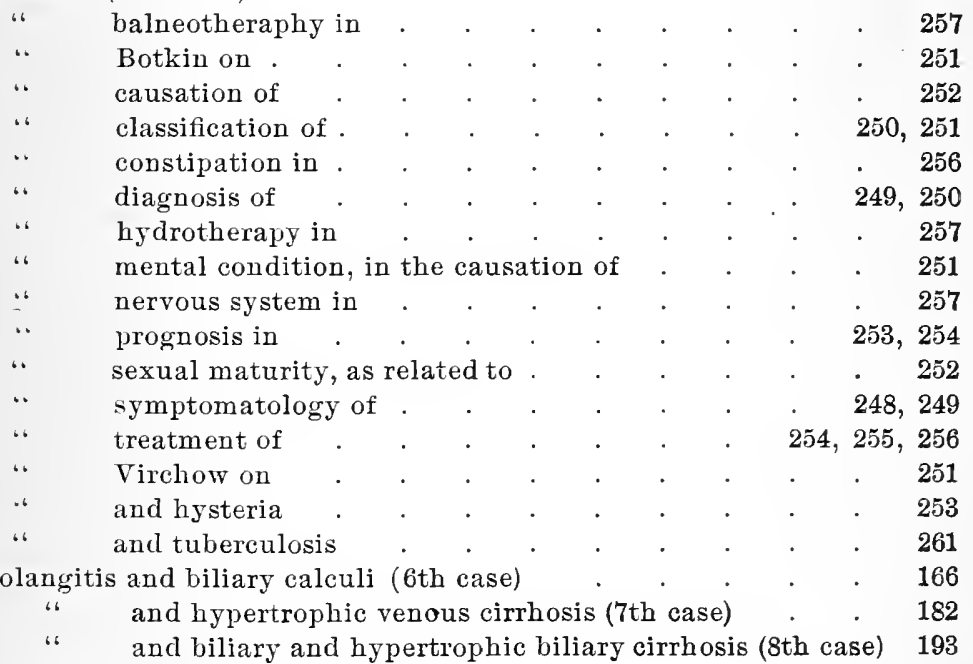

Cholelithiasis, vide Calculi, biliary. 
Cholera .

algid stage of .

arrivals, supervision of, in epidemics

attack, symptoms of an .

304,365

causation of, according to Pettenkofer

359

contagiousness of

$35 \% 9,361,362,363$

complications of

384

Hueppe's investigations about . . . . . 303, 301, 302

infectiousness.

359

immunity toward, Pettenkofer's experiments concerning .

362

" " unreliability of experimentation.

362

individual measures of precaution against .

371,372

nature of

357,358

nephritis of the algid stage .

366. 367

Nicati (and Rietsch) toxine from cholera bacteria

366

predisposition to

359

preventive measures against

371,372

reaction stage of

367,368

spleen, condition of, in .

367

treatment of (vide also special headings) .

". effectiveness of

372,373

necessity for systematic.

diarrhœa.

375-379

nostras

Circulation, as influenced by baths

Cirrhosis of the liver, hypertrophic, biliary

$193,419,420$

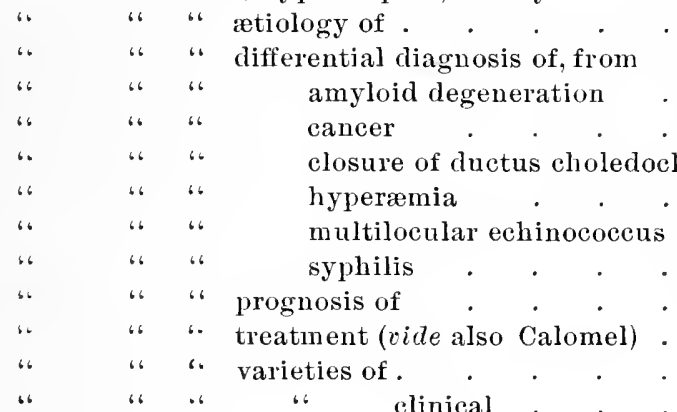

Climate, definition of

elinical

$$
\text { " . " . " density of . . . . . } 301
$$


Climatotherapy, elements of : air, moisture in

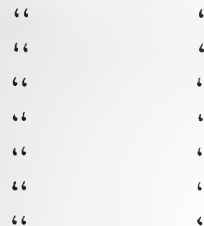

" " " beauty of locality

" of internal diseases, vide General clinic.

" and medical education

Condition of patient, interrogation about $\quad . \quad$. $\quad . \quad$. $\quad$. 10

Condurango in diarrhca . . . . . . . . . . . . . 78

Constant electric current in atonic gout . . . . . . . . 222

Constipation, cause of, in gastric catarrh . . . . . . . . $\quad 67$

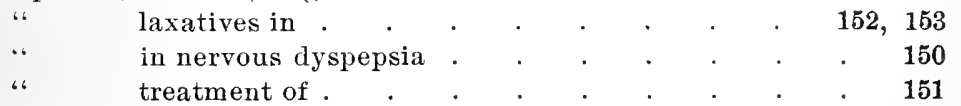

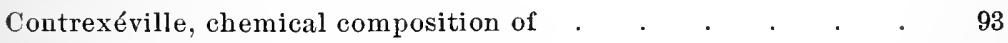

" contraindication for use of . . . . . . . . 80 
Contrexéville in atonic gout

222,228

" in oxaluria and renal colic

Coto, tincture of, in diarrhœa

Cough in pneunronia, treatment of

321,322

Cramps in cholerine, treatment of

Creosote compared with koumyss in pulmonary tuberculosis

" introduction of, through respiratory tract . . . $\quad 297$

" in tuberculosis . . . . . . . . . . $\quad 295,296,315$

“ in septicæma and pyæmia . . . . . . . . . 464

" subcutaneous injections of . . . . . . . . . 296

Cupping-glasses, care in application of, for apoplexy $\quad$. $\quad$. $\quad$. 395

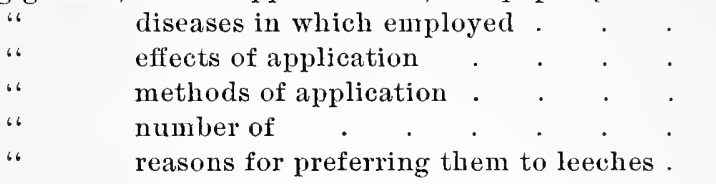

393

394,395

395

394

394

DAvos compared with other sanatoria

306,307

Debout d'Estrées on the forner use of mineral waters . . . 108

Deficiencies in students' knowledge, making up of . . . . . 26

Diabetes mellitus, relation of, to oxaluria . . . . . . 54

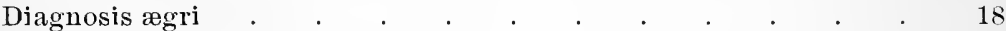

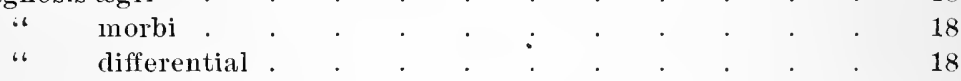

" how arrived at . . . . . . . . . . . . . $15,18,42$

Diaphoretics in pleurisy $\quad . \quad$. $\quad . \quad$. $\quad . \quad$. . . . $\quad$. 267,269

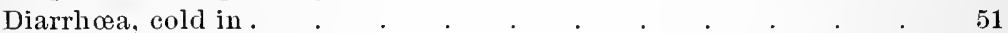

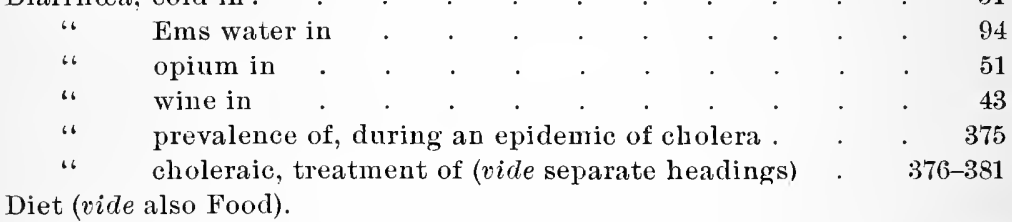

" during the administration of arsenic in anæmia

" in gastric catarih . . . . . . . . . . . . . 150

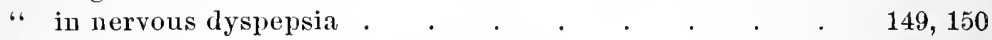

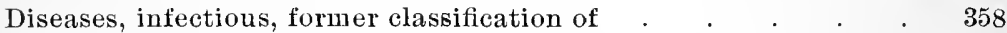

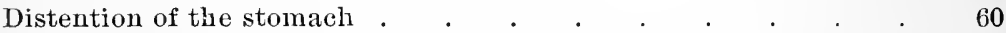

Disinfection in the prevention of cholera outbreaks . . . $\quad 370$

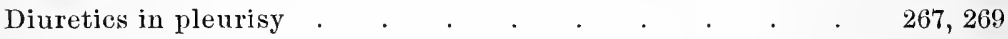

Douches in nervous dyspepsia . . . . . . . . . . 151

Dover's powder in pleurisy . $\quad . \quad$. $\quad . \quad$. $\quad . \quad . \quad . \quad . \quad 208$

" " $\quad$ in pneumonia . . . . . . . . . . . 322

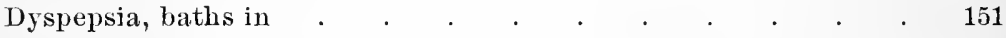

". constipation ill . . . . . . . . . . . . 150

" diet in . . . . . . . . . . . . . 144,150

" Ems water in . . . . . . . . . . . . . 151 
Dyspepsia, erroneous employment of lavage in

" mineral waters in . . .

" nervous, gastric symptoms of .

$57,127,158,159$

$$
\therefore 6
$$

diagnosis, differential

Dyspeptic symptoms, preliminary treatment of, in tuberculosis

Ebstein on gout .

Echinococcus cyst of the liver (9th case)

Effusion in pleurisy, absorption of

269

"6

“

character of

265,266

\section{" operative removal of .}

269

Eichhorst, treatment of pleurisy

" on hæmorrhoids .

396

Electricity in neurasthenia .

Emmerich's experiments on cholera.

362

Ems, chemical composition of

compared with Marienbad .

" Obersalzbrunnen

96,97

dosage of

111,112

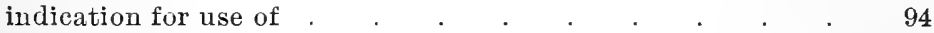

in biliary calculi .

79

in catarrhal conditions of the respiratory tract

96,97

in gout (atonic)

222

in oxalic acid gravel.

79

in pulmonary tubereulosis

washing of the stomach with

124

with sodium iodide

208,456

$$
\text { ، salycilate }
$$

Kesselbrunnen

$$
\text { compared to Kränchen }
$$

130,131

\section{" $\quad$ in nervous dyspepsia}

in hypertrophic biliary cirrhosis

“ Kränchen

Enteroclysmata in choleraic diarrhœa

Ether in the algid stage of cholera 
FEBRILe condition, inquiry about, in examining a patient

Fever, hectic, in pulmonary phthisis

" " "

$\begin{array}{lll}. . & . & \text { ". } \\ . & \text {.. } & \end{array}$

cause of

285

286

treatment of . . . . 292

undesirability of bringing it down to the normal

292

“ a protecting agency to the organism . . . . . 460

" treatment of . . . . . . . . . 460

Finkler-Pryor, bacillus of cholera uostras $\quad . \quad$. . . . . $\quad . \quad 384$

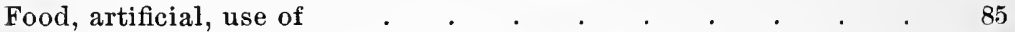

" in chronic gastric catarrh . . . . . . . . . 75

".

"6 6 "6 " 6 " 6 quantity and apportionment of . 75

. $\quad$ " $\quad$ " $6 \quad$ " 6 instructions to patients about . 76

. 6 " $4 \quad$.

Fragaria vesca (strawberry) in atonic gout . . . . . $\quad . \quad 236$

Fränkel on phthisical stage of tuberculosis (foot-note) . . . . 286

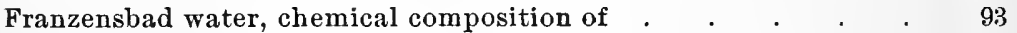

" ". diseases in which it is used . . . . 104

" " " (Salzquelle) Moorbäder . . . . . 143,345

" $\quad$ " temperature of . . . . . . . . . . . 455

" " " $\quad$ " . . . . . . 115

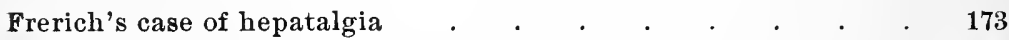

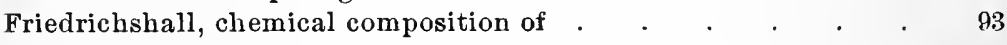

Gasteis, an Austrian watering-place . . . . . . 340

Gastric splash, unreliability of, as a symptom . . . . . . $\quad 60$

" lavage, abuse of . . . . . . . . $\quad$. $\quad 61,62,63,132,137$

" sound in differential diagnosis of diseases of the stomach . 63,64

Genital organs, male, inquiry about, in examination of patient $\quad 32$

Gmunden, an Austrian watering-place . . . . . . . 344

Görbersdorf compared to Davos . . . . . . . . 306, 307

" as a climatotherapeutic sanatorium . . . . . . 305

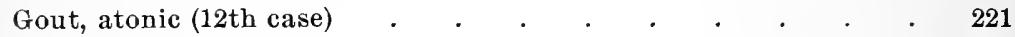

" causation of . . . . . . . . . . . . . . . 213

.. differentiation from nocturnal syphilitic pains . . . 227

" milk treatment in . . . . . . . . . . 236

“ predisposition to . . . . . . . . . . . . 221

" relation of, to acute articular rheumatism . . . 213, 214

" 6 to articular diseases . . . . . . . . . . 221

" strawberry tea in . . . . . . . . . . . . . 236

" symptoms of . . . . . . . . . . . . . 214,226

" treatment of $. \quad . \quad . \quad . \quad . \quad . \quad . \quad . \quad .222,228,229$

“ Vichy water in . . . . . . . . . . . . 104

Grande-Grille, vide Vichy.

Gravel, oxalic acid, Erus in . $\quad . \quad$. $\quad . \quad$. . . . $\quad$. 79

" " . . . . . . . 80

“ phosphatic, Contrexéville in . . . . . . . . 106 
Guaiacol and its carbonate in pyæmia and septicæmia

" " "

295, 296,314

carbonate in incipient tuberculosis

Gumma, syphilitic, of the liver, differential diagnosis of

HeMATIN in ulcer of the stomach

Hæmatopoiesis, balneotherapy in diseases of

Hæmoptysis in tuberculosis, bloodletting in

$$
6
$$

$$
\text { .. } \quad \text { leeches in . }
$$

Hæmorrhoids, case of, substantiating author's views on

".
..
..
.
causation of . former views on nature of (an angioneurosis) pure, uncomplicated. views concerning .

Hamburg, epidemic of cholera in, origin of Hahnemann in the history of therapeutics Hauôt on hepatic cirrhoses .

Headache, inquiry about, in examining a patient Heart, determination of dimensions of

“ diseases of, balneotherapy in.

.. " milk treatment of

. syphilis of the, angina pectoris in .

. " $"$ " diagnostic data of .

.. $\quad$.. $\quad$.. lack of knowledge on

. $\quad$. $\quad$. $\quad$ treatment of

. $\quad$.. $\quad$. $\quad$ type of the majority of cases

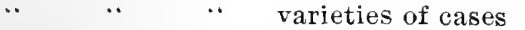

Helouan, a health resort near Cairo, Egypt

Hepatalgia, relation of, to biliary calculi .

\section{Frerich's case of}

292

$291,292,403$

397

398

396

397

397

397

360

23

189

15,16

329,330

353

455

448,449

454

447

454

449

449

303

$171,173,174$ 173

Horseback riding as a predisposing factor in hæmorrhoids 398

Hospital therapy, characteristics of

Hueppe's investigations on cholera

$.359,360,361,362$

toxine of cholera bacteria.

366

use of bismuth phenolate in cholera

Hydrobilirubin, formation of (foot-note)

Hydrotherapy, effects of

\section{general}

methods of application

Hygiene in the treatment of neurasthenia 
PAGE

Hyperæmia of the spinal cord and its membranes, bloodletting in, 402, 403 Hypodermoclysis in choleraic diarrhœa . . . . . . . . $\quad 380$ " in the algid stage of cholera proper . . . . 382

Hysteria, balneotherapy in .

" carbonated waters $\mathrm{i}$

344,345

" sea-bathing in

347

and chlorosis

253

ICE in the vomiting of cholerine . . . . . . . . 381

Impotence, male, hydrotherapy in . . . . . . . . . $\quad . \quad 352$

Individualization in clinical work $\quad . \quad$. $\quad . \quad$. . . . . 3

Infectious diseases, former classification of . . . . . . . 358

" " $\quad$ " treatment of fever in . . . . . . 460,461

Influences, effect of mechanical, in hydrotherapy . . . . . . $\quad 339$

Interrogation of patient, special points about . . . . . $\quad 8,9,10$

Intestines, particular inquiry about the condition of $\quad . \quad$. 10, 11

Investigation, objective $\quad . \quad$. . . . . . . . . 17

Iodides in syphilitic affections of the heart . . . . . . 448, 457

" " " " . . . . . . . 206

Iodine in the treatment of pleurisy . $\quad . \quad$. $\quad . \quad$. $\quad$. $\quad 267,269$

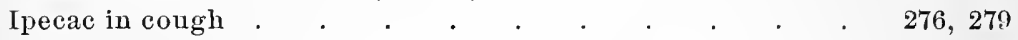

" " $\quad$ of pneumonia, erroneous administration of . . 322

Iron in chlorosis.

$254,255,256$

" in neurasthenia . . . . . . . . . . 147

" mineral waters in chlorosis . . . . . . 256

" sulphate, effect of, in hydrotherapy . . . . . . . 339

Ischias ex neuritide (sciatica), bloodletting in . . . . 393, 395

Ischl, an Austrian watering-place . . . . . . . . . 344

JUERGENSEN on bloodletting . . . . . . . . . 388

Jaundice, catarrhal . . . . . . . . . . . . . $\quad . \quad 432,433$

" ، calomel in . . . . . . . . . . 430

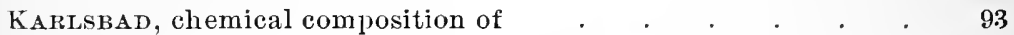

" compared with Ems . . . . . . 9 95, 103, 177

" (Mihlbrunnen) compared with Marienbad . 102, 103, 187

" in cholelithiasis . . . . . . $\quad$. $167,176,177,179$

" in gastric catarrh . . . . . . . . . 103

" in renal gravel . . . . . . . . . 105

" with "Karlsbad" salt . . . . . . . 104

" mode of administration . . . . . . . 177

Keffir in tuberculosis . . . . . . . . . . 294

Kesselbrunnen, vide Ems.

Kidney, movable (for diseases of, vide Nephritis) . 69,70

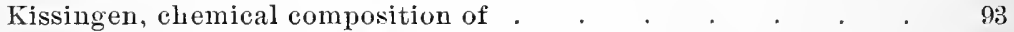

" compared with Marienbad . . . . . . . 103

" contraindications to use of . . . . . . . 95 
Kissingen, dosage of

dosage of . . . . . 113

Koch on "contagiousness" of cholera

Koumyss in neurasthenia

Kränchen, vide Ems.

Kreutznach, a German watering-place

LENNEC, atrophic cirrhosis of the liver

Lavage, gastric, employment of

Laxatives in constipation .

$$
\text { " in pleurisy . }
$$

152,153

267,269

Leeches in pulmonary hæmoptysis

. $\quad$ in reaction stage of cholera . . . . . . . . . 383

“ for depletive bloodletting . . . . . . . 393

Leucocytes in the gastric contents . . . . . . . . 127

Leuk, a watering-place in Switzerland . . . . . . 340

Leyden on the phthisical period of tuberculosis (foot-note) . . $\quad 286$

Liquor ammoniæ anisatus . . . . . . . . . . 279

Eithium in gouty pains . . . . . . . . . . 229

Liver, blood-stasis of, bloodletting in . . . . . . . 404

" cirrhosis of, ride Cirrhosis.

" determination of dimensions of . . . . . . . 330

“ $\quad$ echinococcus of $(9$ th case). . . . . . . . 197

" syphilitic affection of . . . . . . . . 204

" " " " . . . . . . 205

" " " $"$ "

" torpor of, calomel in. . . . . . . . . . . . . 409,410

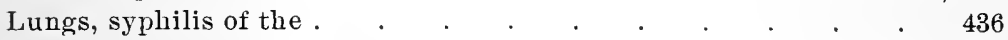

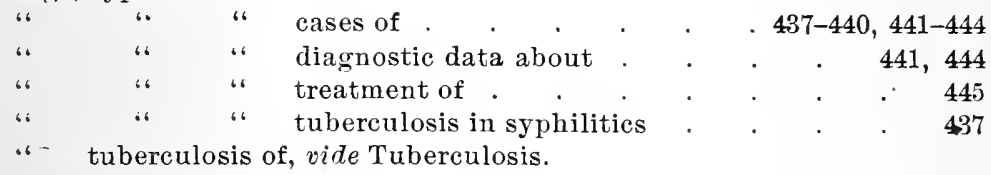

MADEIRA wine, in the etiology of the case of biliary cirrhosis . $\quad 426$

Madeiras, the, as health resorts . . . . . . . . 304

.Magnesia usta for pyrosis . . . . . . . . . . . . . . 124

" " a a laxative . . . . . . . . 152

Marienbad water, chemical composition of . . . . . . . 93

\begin{tabular}{|c|c|c|c|c|c|c|c|c|c|}
\hline 86 & 6 & compared & with & Ems . & . & & & $\therefore$ & . \\
\hline “، & “ & ، & " & Karlsbad & . & • & • & . & 102 , \\
\hline 6 & “. & 6 & "، & Kissingen & . & . & & . & \\
\hline & . & .6 & .. & Vichy. & . & $\cdot$ & . & 102 & 104 \\
\hline & .. & ، & ‘ & Tessentucki & & & & . & 105 \\
\hline
\end{tabular}


Marienbad water, contraindications for use of .

. “ in Dr. S. W. Mitchell's treatment of neurasthenia, 53, 54

$$
\begin{array}{lll}
\cdot & \\
\cdots & \cdots
\end{array}
$$
in atonic gout

222

$$
\text { * }
$$
in muscular rheumatism

Meat-powder, use of, in gastric disorders .

146,147

Menstruation, relation of, to hæmorrhoidal bleeding

Mercurial inunctions in syphilis of the liver

$303,314,315$

Metallic poisoning, hydrotherapy in .

263,268

Method requisite in clinical work

Miasmatic diseases, nature of

" contagious diseases

358

358

Milk treatment in atonic gout

236

455

" as a diuretic

in diseases of the heart

246,455

Mitchell's, Dr. S. W., treatment of neurasthenia

Morphine in pleurisy

in the vomiting of cholerine

. 53,54

268

381

383

Moschus in the reaction period of cholera

388,389

Moscow practice of bloodletting .

Muds, mineral, use of .

Miihlbrunnen, vide Karlsbad.

Murmur, bronchial respiratory, as a symptom .

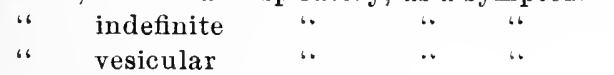

Iyocarditis, syphilitic, case of .

NARcotics, care in using, in pneumonia . 
Nephritis, interstitial, senile

" parenchymatous

" " "

course of

244

diagnosis of

245

Nephrolithiasis, warm baths in (vide also Biliary calculi)

Nerves, cardiac, syphilitic affection of, cases

Nervous system, condition of, in chlorosis

Neurasthenia, arsenic in

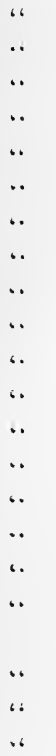
balneotherapy in .

Mitchell's treatment of . . . . . . $\quad$. 53

prognosis of . . . . . . . . . . 145

sea-bathing in . . . . . . . . . 347

sexual intercourse, immoderate, as a causative agen-

cy, treatment of . . . . . . . . 145,146 silver nitrate in . . . . . . . . . . . 147

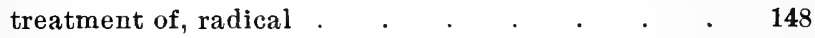

trips to various seashores for . . . . . . 101

Neuritis, treatment of, in articular rheumatism . . . . . 223

Nicati (and Rietsch), cultures of choleraic toxine . . . . . 365

Nice, a health resort in Southern France . . . . . . 305

Noorden, v., on the formation of hydrobilirubin (foot-note) . . $\quad 253$

Nothnagel (and Rossbach) on the use of calomel in Germany . $\quad 407$

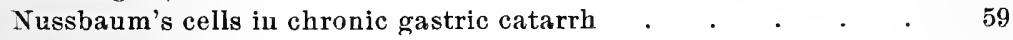

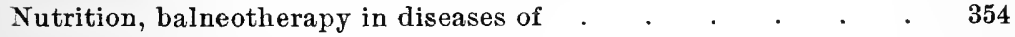

" importance of, in tuberculosis . . . . . . 292

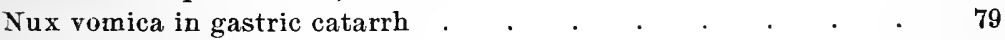

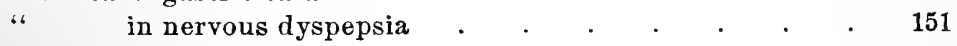

OBEsrTy, general, milk treatment in . . . . . . . . 455

Obersalzbrunnen, chemical composition of $\quad . \quad$. $\quad . \quad . \quad . \quad . \quad 93$

" compared with Ems . . . . . . 96

" dosage of . . . . . . . . . . 111

" use of . . . . . . . . . . . . . . 96,97

. $\quad$ in pulmonary tuberculosis . . . . . . 96, 97

.. with milk (for cough) . . . . . 275, 279, 310,314 
Observation of the course of the disease

Edema of the lungs, bloodletting in .

Odessa estuary, a health resort .

Oleum terebenthinæ rectif. in tuberculosis

Opium in choleraic diarrhœa

377,378

" in intestinal pains.

. 50,51

" reasons for employing

378,379

Oxalic acid gravel, vide Gravel.

" " $\quad$ sediment in relation to uric acid during treatment $\quad . \quad 69$

Oxaluria, Contrexéville in .

" relation to diabetes mellitus . . . . . . . . 54

"6 as a symptom . . . . . . . . . . . . 70

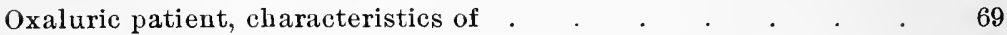

PAINs in pneumonia, treatment of . . . . . . . . 321,322

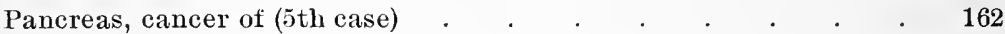

" " a absence of symptoms of . . . . 159,160, 164

" " d diagnosis, differential . . . . . 164

Pepsin, condition of, in chronic gastric catarrh $\quad . \quad . \quad . \quad . \quad 5 \quad 59$

" use of, in diseases of the stomach . . . . . . . . . 84

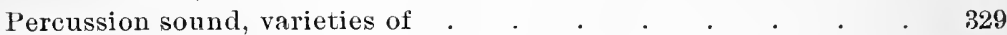

Pericarditis, blisters in . . . . . . . . . . . . . . 393

Pericystitis, leeches iu. . . . . . . . . . . . . . 396

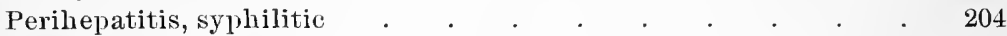

Peritoneal inflammation, hydrotherapy in . . . . . . $\quad 352$

Peritonitis, leeches in . . . . . . . . . . . . . . 396

Peruvian balsam in tuberculosis . . . . . . . . 297

Phenacetine in hectic fever . . . . . . . . . . 268

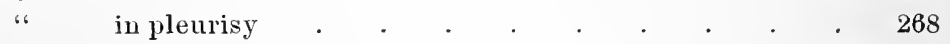

" in preumonia . . . . . . . . . . . . 320,321

" in reaction stage of cholera . . . . . . . . 383

Phthisis declarata (pulmonary phthisis), appearance of patients with, 285

$\therefore$ care of . . . . . . . . . . . . . 288

" galloping . . . . . . . . . . . . . . . 289

". hectic fever in . . . . . . . . . . . . . 285

.. mixed infection in . . . . . . . . 286

Pilocarpine hydrochlorate in pleurisy $\quad . \quad$. . . . . . . $\quad$. 267

Piperazine in gouty deposits . . . . . . . . . . 229, 230

Pityriasis versicolor in emaciated persons _ . . . . . . . . . 45,46

Pleurisy, acute (19th case, with tuberculosis) . . . . . . 263

" bloodletting in . . . . . . . . . . . $\quad 268,393,394$

. breaking up of the acute stage of . . . . . . 267

" causation of . . . . . . . . . . . 265

" " cold as a factor in the . . . . . 265, 266

. compressed air in. $\quad . \quad$. $\quad . \quad . \quad . \quad . \quad . \quad . \quad .272$

، differentiated from pneumonia . . . . . $\quad 265,318,319$ 
Pleurisy, hygiene in

prognosis of

.. symptoms and physical signs of . . . . 264, 265, 267

.. treatment of (vide drugs under special headings), 267, 268, 269, 270

.. " "ambulance". . . . . . 270

.. “

.. 6 6 fever . . . . . . . . . . . 268

.. $\quad$ " $\quad$ in syphilitic patients . . . . . 267

.. $"$ in tuberculous patients . . . . . 267

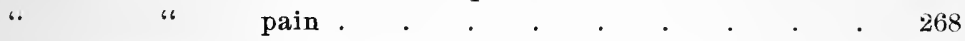

" with effusion, determination of character of . . $\quad 265,266$

" " . . . . . . 269, 270

Pleuropneumonia, acute, bloodletting in . . . . . . . $\quad 393,394$

Plombières, a French health resort . $\quad . \quad$. $\quad . \quad$. $\quad . \quad 340$

Pneumonia, catarrhal (caseous), complicating chrouic pulmonary tuberculosis . . . . . . . . 287

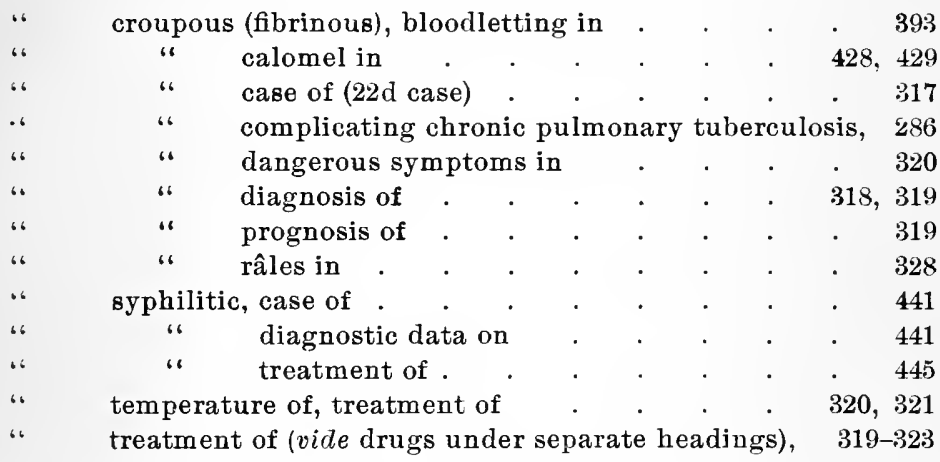

Podophyllin as a laxative . . . . . . . . . . . . . $\quad$. 153

Polyarthritis chronica (chronic articular rheumatism) . . . 221

Popoff, Dr. P. M., on gastric catarrh · · . 57, 59, 84

Potassium chlorate, gargling with a solution of, when using calomel, 417

" tartrate as a laxative . . . . . . . . . 152

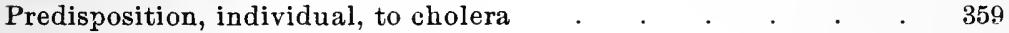

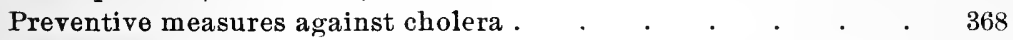

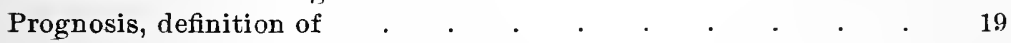

physician's duty concerning . . . . . . . . $\quad$. 21

precision in wording the . . . . . . . . . 22

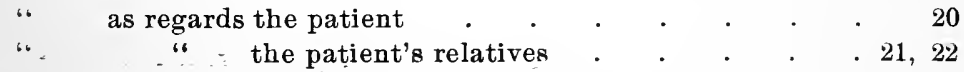

$\because$ in extreme cases . . . . . . . . . 21

Püllna spring (Bitterwasser), chemical composition of . . . $\quad 93$

Pulse, condition of, when employing venesection for apoplexy $\quad$. $\quad 391$

" importance of, in objective examinations . . . . . $\quad 330$

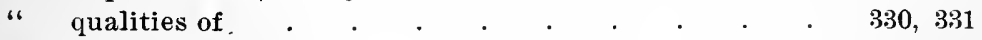

Pyatigorsk springs, chemical composition of . $\quad . \quad$. $\quad . \quad$. 93 
QUARAntining along the Asiatic borders during cholera epidemics, $\quad 369$ " impossibility of a thorough application of . . 368

Quinine, a "pseudo" preventive measure in cholera . . . . 372

" compared with sodium salicylate . . . . . 268,269

" in erysipelas . . . . . . . . . . . . . 427

" in feverish conditions . . . . . . . . . . . 463

". in hectic fever . . . . . . . . . . . . 292

" in pleurisy . . . . . . . . . . . . . . 268

" in pnenmonia . . . . . . . . . . . . 320

RAGATZ, a health resort in Switzerland . . . . . . . $\quad$. 340

Rakoczy, vide Kissingen.

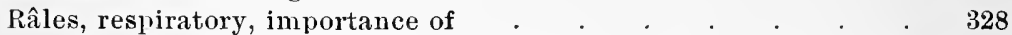

" " $\quad$ " . . . . . . . . . 328,329

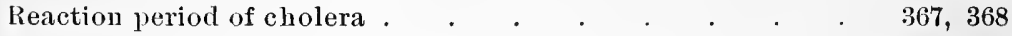

" in hydrotherapy, significance of $\quad . \quad$. $\quad . \quad . \quad 347,348$

Rehme-Oeynhausen, an artesian spring in Prussia . . . . 345

Reichenhall, a German watering-place . . . . . . . . 344

Remedies, simultaneous employment of . . . . . . . . $\quad$. 24

Respiration as influenced by baths . . . . . . . . . $\quad . \quad 337$

Respiratory organs, balneotherapy in diseases of . . . . . $\quad 353$

Rhamnus frangula (black dogwood) as a laxative . . . . . 153

Rheumatism, acute articular (11th case) . . . . . . . 209

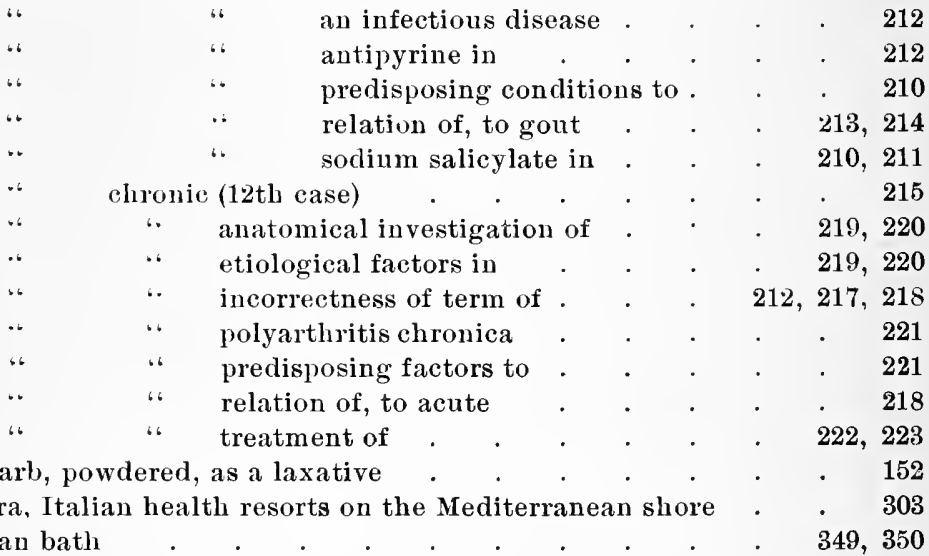

Rhnbarb, powdered, as a laxative $\quad . \quad$. 152

Riviera, Italian health resorts on the Mediterranean shore . $\quad 303$

Russian bath . . . . . . . . . . . 349,350

SAKI, a health resort in Southern Russia, compared with Franzens-

bad

341,342

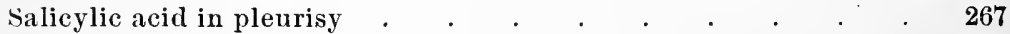

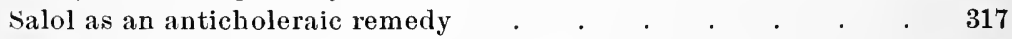

Salt, common, percentage of, in salt baths . . . . . . . $\quad$. 344

Salts, laxative, contained in the mineral waters, effects of . . $\quad 91$

Sanatoria, trips to, with climatotherapentic aims in view . . 310

San Remo, an Italian health resort . . . . . . . . . 303 
Schlangenbad, a Prussian watering-place .

Schwalbach, a Prussian watering-place

Scrofula, hydrotherapy in .

" sea-bathing in . . . . . . . 354

Semiatalectesis of the lungs, physical symptoms of $\quad . \quad$. $\quad 328,329$

Senna as a laxative

Sévigné, Mme., treated at Vichy . . . . . . . . 108

Sexual intercourse, immoderate, as a causative factor in neurasthenia, 145

" maturity in relation to chlorosis . . . . . . 252

Shiff's theory regarding the effects of calomel . . . . . 409

Shkliareffsky's treatment of the algid stage of cholera (foot-note) . 383

Silver nitrate in gastro-intestinal catarrh . . . . . . $\quad$. $118,119,120$

" " in nervous dyspepsia . . . . . . . . . 151

" " in neurastlienia . . . . . . . . . . 147

Skin, diseases of, hydrotherapy in . . . . . . . . . 354

Slaryansk, a Russian watering-place . . . . . . . . . 344

Soden, a German watering-place . . . . . . . . 344

Sodium bicarbonate, action of in the body $\quad . \quad$. $\quad . \quad$. $\quad .90,91$

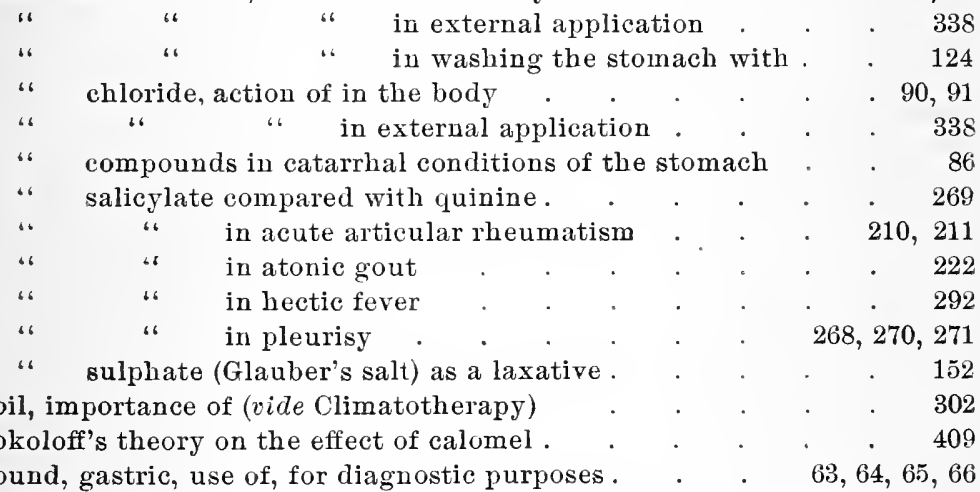

Sound, gastric, use of, for diagnostic purposes . $\quad$. $\quad$. $\quad 63,64,65,66$

" " " " objections to, for purposes of verification . $\quad 77$

Spanish flies in dyspnoa of pneumonia . . . . . . . 322, 323

" " in angina pectoris . . . . . . . . . 456

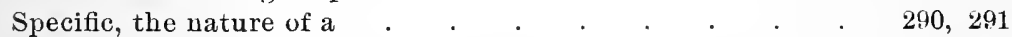

Spinal cord, diseases of, balneotherapy in $\quad . \quad 3 . \quad . \quad 3 \quad . \quad 352$

" " " . . . . . 4loodletting in . 402, 403

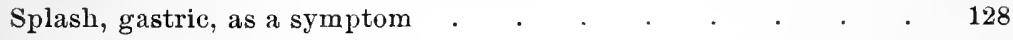

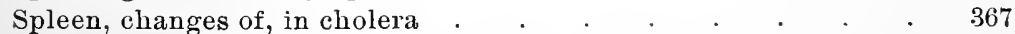

" determination of dimensions of . . . . . . 330

. hyperæmia of, hydrotherapy in . . . . . . . 352

Sputum, examination of, in pneumouia . . . . . . . . . $\quad . \quad 319$

Stara-Russa, a Russian resort . . . . . . . . . . . 344

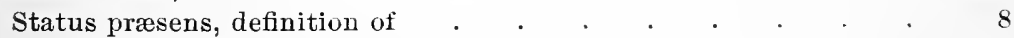

Stimulants, habitual, result of enforced abstineuce from . . $\quad 74$

" " $"$. . . . . . . . . . . . 74

" in pleurisy $\quad . \quad . \quad . \quad . \quad . \quad . \quad . \quad . \quad . \quad 268,270$ 
Stomach, atrophic catarrh of, vide Catarrh, atrophic.

" dilatation of causation . . . .

128,129

" lavage in

133

" symptoms of .

127,128

" treatment of .

131

distention of, distinguished from dilatation

. differential diagnosis of

58,129

127

" symptoms of .

131

$\begin{array}{lllllllllll}\text { Strawberry tea (fragaria vesca) in atonic gout } & . & . & . & & & & & & & 236 \\ \text { "Streptococcencurve" in phthisis } & . & . & . & . & . & . & . & 286\end{array}$

Sunlight, effect of, vide Climatotherapy.

Sulphur, action of, in hydrotherapy .

339

Symptoms, importance of, in semioties and diagnosis

325,326

Syphilitic pains differentiated from gouty

226,227

56,267

354

" hydrotherapy in.

12

". inquiry about, when examining a patient.

446

" of the heart (vide also Heart)

436

TABES DoRsALIS (locomotor ataxia), carbonated waters in

345

Tamponade of the nasal cavity for nosebleed, care in employing

Tannin in diarrhœa

400

"6

"

of cholera.

51

374

Tea as a stimulant drink in diarrhœa

74,75

Temperature, influence of, vide Climatotherapy.

" of the body as influenced by baths

335,336

6 of croupous pneumonia, treatment of

320,321

(Vide also Fever.)

Teplitz, a health resort in Bohemia

Terpine hydrate for cough .

279,283

Therapy, hospital, character of . . . . . . . . . . 25

" special . . . . . . . . . . . . . . 25,29

Tinnitus aurium while employing sodium salicylate . . . 211

Tissue metabolism as influenced by baths . . . . . 336, 337

Toxine of choleraic bacteria (Nicati and Rietsch, Hueppe) . . 365

" " " its effect on the system . . 366, 367

Treatment, employment of many remedies in . . . . . . . $\quad 24$

" individualization and method requisite in . . . 22

" hospital methods of . . . . . . . . . 25

" hygienic, neglect of . . . . . . . . . . 23

" necessity for conducting before the students . . 42

" symptomatic, abuse of . . . . . . . 148,149

"Trial" breakfast . . . . . . . . . . 61

“ dinner . . . . . . . . . . . . . . . . 61

'ribromphenolbismuth (bismuth phenolate) as an anticholeraic . 377 
Trips to health resorts with climatotherapeutic aims in view .

Trypsin in diseases of the stomach . . . . . . . . . . . 84

Tscherbakoff's table of mineral waters . . . . . . . 92,83

Tuberculosis, acute miliary, complicating chronjc . . . . 288

" chronic pulmonary . . . . . 274-278, 309-316

". complications of . . . . . . . . . 288

“ $\quad$ creosote in . . . . . . . . $271,272,295,296,297$

.. diagnosis of chronic pulmonary . . . . 283, 284

“ guaiacol and guaiacol carbonate in . . 295, 296, 297

". hæmoptysis in the incipient stage of . . . . 260

“ how the lungs become affected by . . . . 277

" Obersalzbrunnen in . . . . . . . . . . 96,97

“ pneumoniæ complicating chronic pulmonary, 286, 287, 288

. prognosis of chronic pulmonary . . . . 289,290

.. treatment of . . . . . . . . . . 290,316

". " " climatic (vide Climatotherapy) . 303-316

" " symptomatic (vide Ergot, Keffir, Koumyss, Creosote, Guaiacol, etc.) . $\quad$. 291

“. types of persons affected by . . . . . . 288, 289

. varieties of chronic pulmonary . . . . . . 283

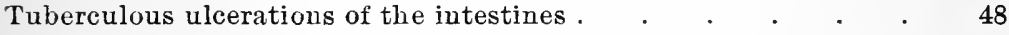

Typhoid cholera, vide Reaction period of cholera.

". fever, body compress in . . . . . . . . 462

" $"$ calomel in . . . . . . . . . 420

ULCER, gastric, vide Stomach, ulcer of.

Urethritis a causative agency in neurasthenia . . . . . 144

Uric acid diathesis, vide Gout.

Urobilin (foot-note)

253

Vegetation, importance of, vide Climatotherapy.

Venesection in disturbances of cerebral circulation . . . $\quad 391,392$

" " circulation within the chest . 392,393

(Vide also Bloodletting.)

Ventricle, left, condition of, in interstitial nephritis . . . . 242

" " " in parenchymatous nephritis . . 242

Vertigo, inquiry about, in examining a patient . . . . . 16

Vichy, chemical composition of . . . . . . . . . . . . $\quad .93$

“ as compared with Marienbad . . . . . . . . 102

“ Célestins . . . . . . . . . . . . . . . . . $\quad .95,102$

" " with sodium salicylate . . . . . . 210

" dosage of . . . . . . . . . . . . . 111

" Grande Grille . . . . . . . . . . . .95, 102

" indications for . . . . . . . . . . . 104

" in gout . . . . . . . . . . . . 228

Virchow's influence on therapeutics . . . . . . . . 213

" views on chlorosis . . . . . . . . . 251

Volga and Oural, koumyss sanatoria on . . . . . . . . 294 
WATER, action of

"drinking, at a sanatorium

" mineral, temperature of

Watering-places, trips to, as a remedial agency _ . . . . 110

Waters, mineral, aims in employment of .

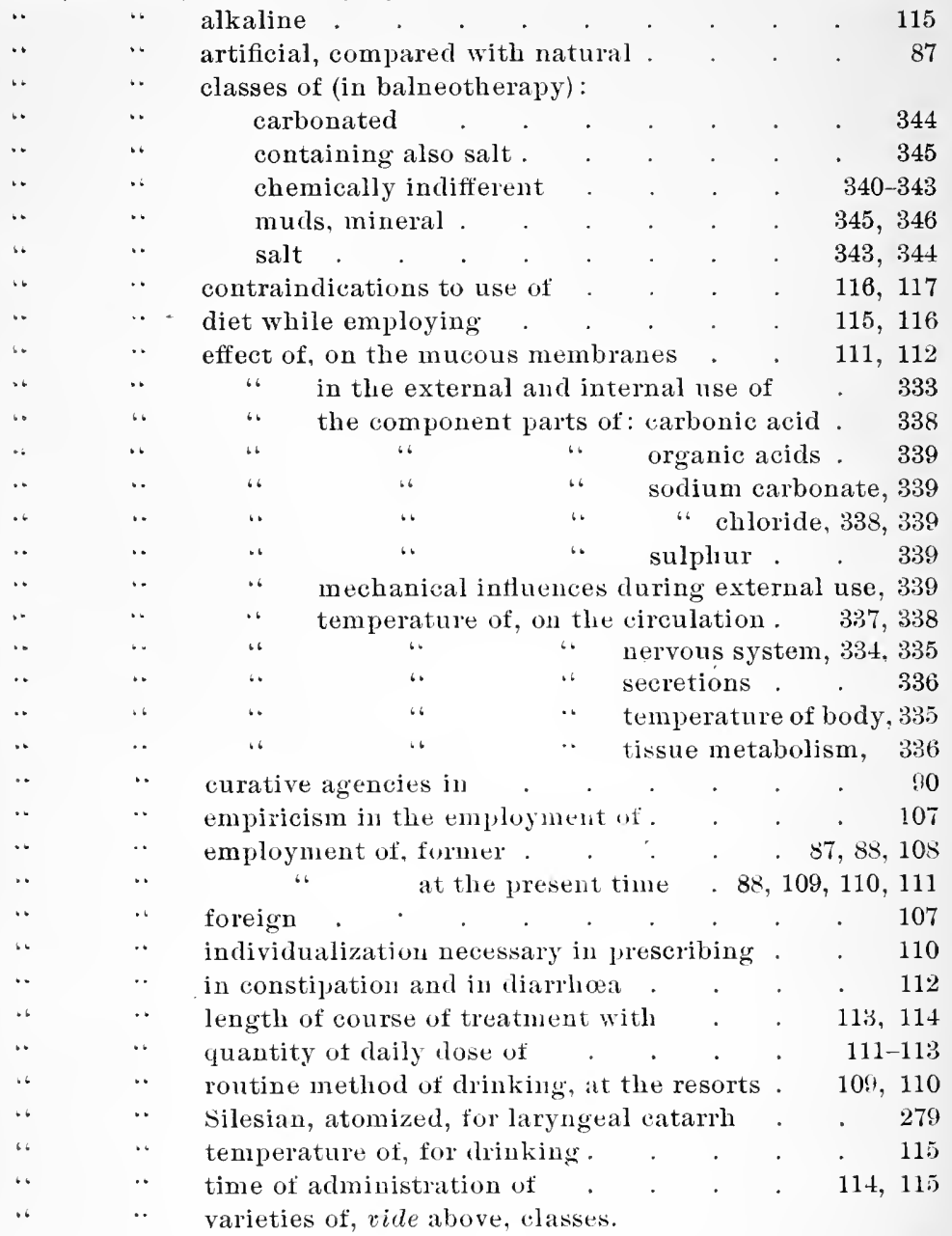

Webber's classification of climates 
Wiesbaden, a German watering-place

Winds, importance of, vide Climatotherapy.

Wine in cardiac debility

.. in diarrhœa

- as a causative agency in hypertrophic biliary cirrhosis

Women, diseases of, Franzensbad-Moorbäder in

YALTA, a health resort in the Crimea

Yessentucki, chemical composition of compared with Ems

$$
\text { " " Vichy }
$$
contraindication to use of

.. dosage of

$\therefore$ indications for use of . 111,112

.. in atonic gout

105,108

.. $\quad$ temperature of

222,228

ZIEUSSEN on calomel in cholera 





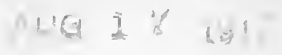

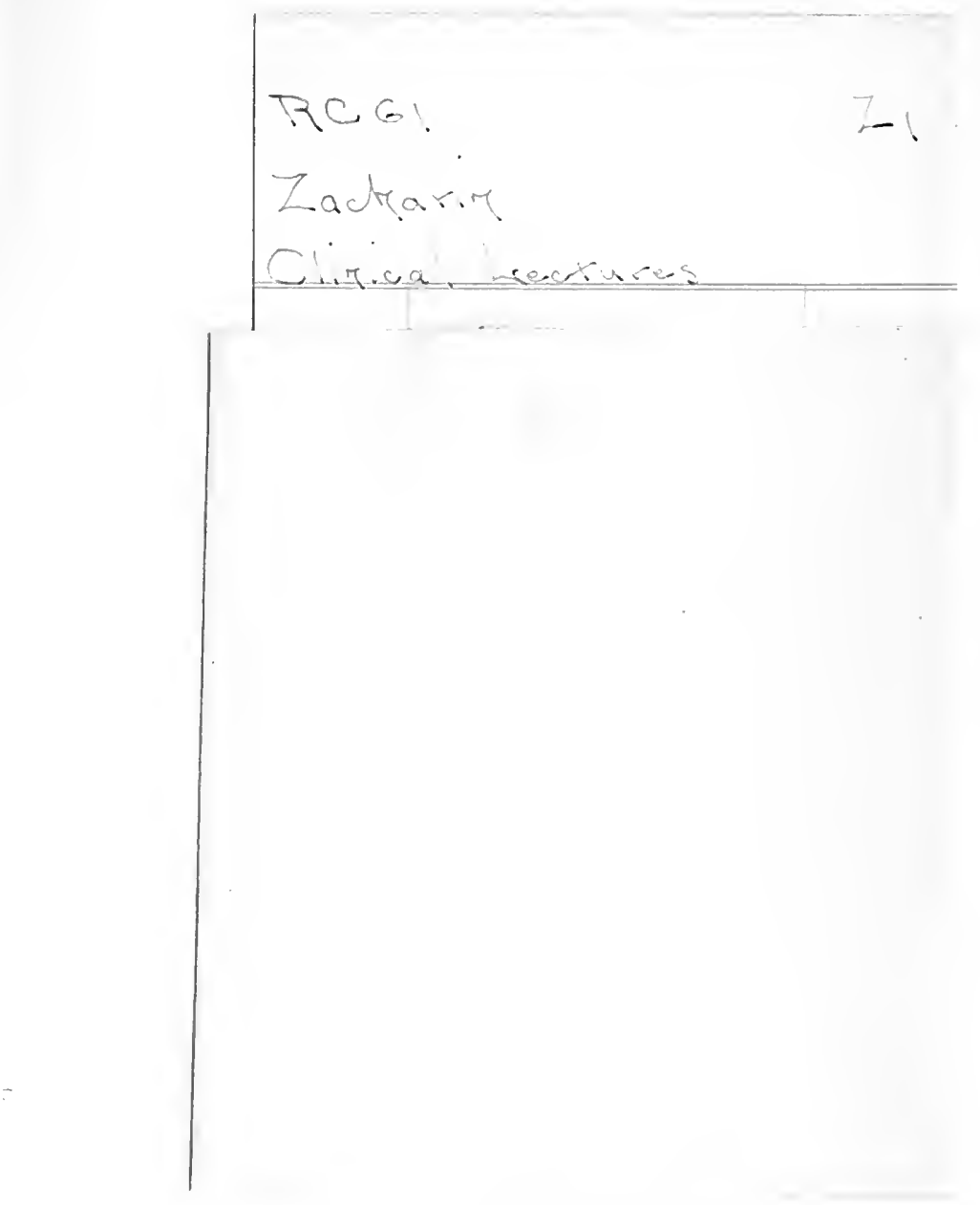


now

Wo

so

ow

\%

$85 \%$

$3 \%$

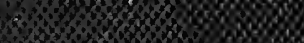

$3 \% 0 \%$

36

no

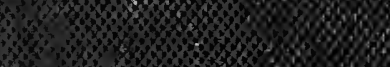

s.6.6\%

,

-

H.

$x^{2}+2$

3rok

3.

S.

8

s.t. 